FERNANDO SILVA DE MOURA

\title{
ESTIMAÇÃO NÃO LINEAR DE ESTADO ATRAVÉS DO UNSCENTED KALMAN FILTER NA TOMOGRAFIA POR IMPEDÂNCIA ELÉTRICA
}

São Paulo 

FERNANDO SILVA DE MOURA

\section{ESTIMAÇÃO NÃO LINEAR DE ESTADO ATRAVÉS DO UNSCENTED KALMAN FILTER NA TOMOGRAFIA POR IMPEDÂNCIA ELÉTRICA}

Tese apresentada à Escola Politécnica da Universidade de São Paulo para obtenção do título de doutor em engenharia

São Paulo 



\title{
ESTIMAÇÃO NÃO LINEAR DE ESTADO ATRAVÉS DO UNSCENTED KALMAN FILTER NA TOMOGRAFIA POR IMPEDÂNCIA ELÉTRICA
}

\author{
Tese apresentada à Escola Politécnica da \\ Universidade de São Paulo para obtenção \\ do título de doutor em engenharia \\ Área de Concentração: Engenharia \\ Mecânica \\ Orientador: Dr. Raul Gonzalez Lima \\ Co-Orientador: Dr. Jari Kaipio
}

São Paulo 
Este exemplar foi revisado e alterado em relação à versão original, sob responsabilidade única do autor e com a anuência de seu orientador.

São Paulo, de de

Assinatura do autor

Assinatura do orientador

FICHA CATALOGRÁFICA

Moura, Fernando Silva de

Estimação não linear de estado através do unscented

Kalman filter na tomografia por impedância elétrica / F.S.

de Moura. - versão corr. - São Paulo, 2013.

$293 \mathrm{p}$.

Tese (Doutorado) - Escola Politécnica da Universidade de São Paulo. Departamento de Engenharia Mecânica.

1. Tomografia 2. Filtros de Kalman 3. Estimação não linear I. Universidade de São Paulo. Escola Politécnica. Departamento de Engenharia Mecânica II. t. 


\section{Agradecimentos}

Inicialmente agradeço aos meus orientadores Raul Gonzalez Lima e Jari Kaipio pelo auxílio ao longo do caminho sinuoso que é necessário percorrer para finalizar uma tese. Agradeço à fundação de amparo à pesquisa do estado de São Paulo, FAPESP, pelo apoio financeiro durante a realização deste trabalho.

Agradeço aos amigos da bat caverna que estiveram sempre por perto e que foram peças fundamentais para o desenvolvimento desta tese. Meus agradecimentos aos amigos e amigas Júlio Aya, Erick Camargo, Olavo Luppi, Michelle Mellenthin, Miguel Montoya, Natalia Herrera, Paulo Sabia, Thais Samed e Talles Rattis por todas as conversas que tivemos relacionadas, ou não, à pesquisa.

Gostaria de agradecer o apoio que tive durante a minha estadia na cidade de Kuopio, Finlândia, durante os seis meses de permanência neste país conhecido pelo intenso inverno, porém aquecido de boas almas. Meus agradecimentos aos amigos Aku Seppänen, Antti Lipponen, Antti Nissinen, Janne Huttunen, Kimmo Karhunen, Simo-Pekka Simonaho, Ville Kolehmainen e Ville Rimpiläinen. Kiitos kaikesta kaverit, toivottavasti tapaamme uudestaan tulevaisuudessa! Meu especial agradecimento ao amigo Arto Voutilainen por todo o apoio logístico e de infraestrutura que obtive mesmo antes de pousar naquele país. Gostaria de agradecer ao departamento de física aplicada da University of Eastern Finland - Kuopio por aceitar me receber em suas instalações para realizar parte do meu doutorado.

Agradecimentos não podem faltar a Marcelo Amato e todo o grupo de pesquisa do Laboratório de Investigação Médica em Pneumologia Experimental (LIM-09) da Faculdade de Medicina da Universidade de São Paulo pelo apoio incondicional à minha pesquisa.

Por último, quero agradecer à minha família. Deixo apenas uma citação de George Santayana como explanação "The family is one of nature's masterpieces". 



\section{Dedicatória}

Para Laura e Álvaro. 



\section{Epígrafe}

"The scientist has a lot of experience with ignorance and doubt and uncertainty, and this experience is of very great importance, I think. When a scientist doesn't know the answer to a problem, he is ignorant. When he has a hunch as to what the result is, he is uncertain. And when he is pretty damn sure of what the result is going to be, he is still in some doubt. We have found it of paramount importance that in order to progress, we must recognize our ignorance and leave room for doubt. Scientific knowledge is a body of statements of varying degrees of certainty - some most unsure, some nearly sure, but none absolutely certain. Now, we scientists are used to this, and we take it for granted that it is perfectly consistent to be unsure, that it is possible to live and not know. But I don't know whether everyone realizes this is true. Our freedom to doubt was born out of a struggle against authority in the early days of science. It was a very deep and strong struggle: permit us to question - to doubt - to not be sure. I think that it is important that we do not forget this struggle and thus perhaps lose what we have gained."

Richard P. Feynman (1918 - 1988)

"As scientists all we can report is the likelihood. Everyone should put their own prior in and then produce their own posterior. " 



\section{Resumo}

A Tomografia por Impedância Elétrica tem como objetivo estimar a distribuição de impedância elétrica dentro de uma região a partir de medidas de potencial elétrico coletadas apenas em seu contorno externo quando corrente elétrica é imposta neste mesmo contorno. Uma das aplicações para esta tecnologia é o monitoramento das condições pulmonares de pacientes em Unidades de Tratamento Intensivo. Dentre vários algoritmos, destacam-se os filtros de Kalman que abordam o problema de estimação sob o ponto de vista probabilístico, procurando encontrar a distribuição de probabilidade do estado condicionada à realização das medidas. Para que estes filtros possam ser utilizados, um modelo de evolução temporal do sistema sendo observado deve ser adotado. Esta tese propõe o uso de um modelo de evolução para a variação de volume de ar nos pulmões durante a respiração de um paciente sob ventilação artificial. Este modelo é utilizado no unscented Kalman filter, uma extensão não linear do filtro de Kalman. Tal modelo é ajustado em paralelo à estimação do estado, utilizando um esquema dual de estimação. Um algoritmo de segmentação de imagem é proposto para identificar as regiões pulmonares nas imagens estimadas e assim utilizar o modelo de evolução. Com o intuito de melhorar as estimativas, o método do erro de aproximação é utilizado no modelo de observação para mitigar os erros de modelagem e informação $a$ priorié adicionada na solução do problema inverso mal-posto. O método é avaliado através de simulações numéricas e ensaio experimental coletado em um voluntário. Os resultados mostram que o método proposto melhora as estimativas feitas pelo filtro de Kalman, propiciando a visualização de imagens absolutas, dinâmicas e com bom nível de contraste entre os tecidos e órgãos internos.

Palavras-chave: Tomografia por impedância elétrica, unscented Kalman filter, estimação não linear de estado, modelo de evolução, informação a priori 



\begin{abstract}
Electrical impedance tomography estimates the electrical impedance distribution within a region given a set of electrical potential measurements acquired along its boundary at the same time that electrical currents are imposed on the same boundary. One of the applications of this technology is lung monitoring of patients in Intensive Care Units. One class of algorithms employed for the estimation are the Kalman filters which deal with the estimation problem in a probabilistic framework, looking for the probability density function of the state conditioned to the acquired measurements. In order to use such filters, an evolution models of the system must be employed. This thesis proposes an evolution model of the variation of air in the lungs of patients under artificial ventilation. This model is used on the Unscented Kalman Filter, a nonlinear extension of the Kalman filter. This model is adjusted in parallel to the state estimation, in a dual estimation scheme. An image segmentation algorithm is proposed for identifying the lungs in the images. In order to improve the estimate, the approximation error method is employed for mitigating the observation model errors and prior information is added for the solution of the ill-posed inverse problem. The method is evaluated with numerical simulations and with experimental data of a volunteer. The results show that the proposed method increases the quality of the estimates, allowing the visualization of absolute and dynamic images, with good level of contrast between the tissues and internal organs.
\end{abstract}

keywords: Electrical impedance tomography, unscented Kalman filter, nonlinear state estimation, evolution model, prior information 



\section{Sumário}

Lista símbolos e siglas . . . . . . . . . . . . . . . . . . . . . . . . 19

I Introdução

1 Introducão

1.1 Objetivos . . . . . . . . . . . . . . . . . 35

2 Formulacão do Problema 37

2.1 Modelo do domínio . . . . . . . . . . . . . . . . . . . . . 37

2.2 Condicão de contorno e modelo do eletrodo . . . . . . . . . . . . 41

2.3 Estratégia de excitacão e de medição . . . . . . . . . . . . . 45

2.4 Discretizacão do domínio . . . . . . . . . . . . . . . . . . . . 53

2.5 Determinacão dos potenciais elétricos nos nós virtuais dos eletrodos . . 53

2.6 Modelo linearizado de observação . . . . . . . . . . . . . . . 55

2.7 Fantoma numérico . . . . . . . . . . . . . . . . . . 57

3 Problema mal-posto 59

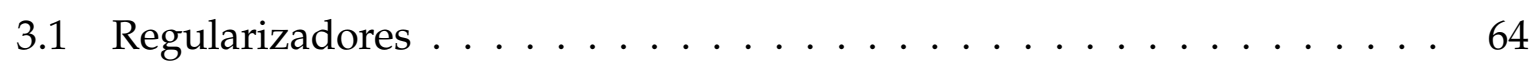

3.2 Análise da solução regularizada . . . . . . . . . . . . . . . . 66

4 Inversão Bavesiana e Filtros de Kalman 69

4.1 Inversão Bavesiana $\ldots \ldots \ldots \ldots \ldots \ldots$. . . . . . . . . . . 70

4.2 Filtragem Bavesiana $\ldots \ldots \ldots \ldots \ldots \ldots \ldots \ldots$

4.3 Filtro de Kalman . . . . . . . . . . . . . . . . . . . . 78

4.4 Informacão a priori nos métodos Bayesianos . . . . . . . . . . . . . 80

4.5 As variantes do filtro de Kalman e seu uso na TIE . . . . . . . . . . . 85

4.6 Método do erro de aproximação . . . . . . . . . . . . . . 93 
4.6.1 Cálculo das estatísticas do método do erro de aproximação . . . . 95

\section{Método}

5 Método Proposto

5.1 Implementacão do UKF na TIE para monitoramento de pacientes . . . 100

5.2 Estimacão dual com filtro de Kalman . . . . . . . . . . . . . . . . . . 103

5.3 Variante Raiz Quadrada do UKF . . . . . . . . . . . . . . . 105

6 Restringindo o espaco solução 111

6.1 Vínculo de intervalo . . . . . . . . . . . . . . . . . . . . . . 111

6.2 Densidade de probabilidade a priori . . . . . . . . . . . . 115

7 Método do erro de aproximacão para o modelo de observação 119

7.1 Efeitos não modelados no modelo fidedigno . . . . . . . . . . . . 120

8 Ensaios numéricos para avaliacão do método do erro de aproximação $\quad 125$

8.1 Fantoma numérico para os ensaios . . . . . . . . . . . . . . . . . . 125

8.2 Amostragem do ruído do método do erro de aproximação . . . . . . . 127

8.3 Descricão dos ensaios . . . . . . . . . . . . . . . . . . . . . . . . 131

8.4 Critérios de convergêncial . . . . . . . . . . . . . . . . . . . . 135

8.5 Resultados dos ensaios . . . . . . . . . . . . . . . . . . . . . 138

9 Modelo dinâmico $\quad 145$

9.1 Modelo dinâmico dos pulmões . . . . . . . . . . . . . . . . . . . . 145

9.2 Número de graus de liberdade no modelo dinâmico . . . . . . . . . . . 153

10 Segmentação das imagens 155

11 Ensaios numéricos para avaliacão do método com modelo de evolução 163

11.1 Fantoma numérico de um tórax humano . . . . . . . . . . . . . . . 163

11.2 Descricão dos ensaios . . . . . . . . . . . . . . . . . . . . . . . 167

11.3 Resultados dos ensaios . . . . . . . . . . . . . . . . . . . . . 173

12 Avaliacão do método com dados experimentais 185

12.1 Protocolo experimental, coleta e filtragem dos dados . . . . . . . . . . 185 
12.2 Método do erro de aproximacão ． . . . . . . . . . . . . . . . . . 188

12.3 Parâmetros utilizados na solução do problema inverso . . . . . . . . . . 189

12.4 Resultados do experimento . . . . . . . . . . . . . . . . . . . . 193

III Discussões e conclusões 197

13 Discussões e conclusões 199

IV Apêndice 205

A Formulacão da matriz local dos elementos finitos 207

A.1 Elementos que compõem o interior do domínio . . . . . . . . . . . . . . 207

A.1.1 Domínio 2D - Elementos Triangulares . . . . . . . . . . . . 207

A.1.2 Domínio 3D - Elementos Tetraédricos . . . . . . . . . . . . . 211

A.1.3 Domínio 3D - Elementos prismáticos de base triangular. . . . . . 213

A.1.4 Matriz Global . . . . . . . . . . . . . . . . . . . . . . . . . 215

A.2 Elementos que compõem a interface dos eletrodos . . . . . . . . . . . 215

A.2.1 Modelo da interface dos eletrodos 2D . . . . . . . . . . . 216

A.2.2 Modelo da interface dos eletrodos 3D . . . . . . . . . . . . . . 219

A.2.3 Matriz Global . . . . . . . . . . . . . . . . . . . . . . . . 222

B Autoadjuntividade do operador $A_{A}$

C Detalhes de implementacão do SR-UKF 227

C.1 Equacões do cálculo da matriz de covariância do algoritmo SR-UKP. . . 227

C.2 Equacões do SR-UKF para estimacão dos parâmetros do modelo de evolução 229

C.3 Modelo linearizado de observacão utilizando a mudança de variável] . . 232

C.3.1 Regra da cadeia para derivativos matriciais . . . . . . . . . . 232

C.3.2 Modelo linearizado de observação . . . . . . . . . . . . . . . . 233

D Atualização e desatualização de Cholesky] 235

D.1 Rotacão de Givens . . . . . . . . . . . . . . . . . . . . . . . 236

D.2 Reflexão de Householder . . . . . . . . . . . . . . . . . . . . . . . . 240

D.3 Atualização de Cholesky . . . . . . . . . . . . . . . . . . . . . . . 243 
D.4 Desatualização de Cholesky . . . . . . . . . . . . . . . . . . . 244

E Método Level Set para segmentacão de imagens 247

E.1 Funcões explícitas e implícitas . . . . . . . . . . . . . . . . . . . . 247

E.1.1 Propriedades . . . . . . . . . . . . . . . . . . . . 249

E.1.2 Ferramentas . . . . . . . . . . . . . . . . 250

E.1.3 Funcão distância com sinal . . . . . . . . . . . . . . . . . . 252

E.2 Método level set e segmentação de imagem . . . . . . . . . . . . . . . . 254

E.2.1 Reinicializacão . . . . . . . . . . . . . . . . . . . 257

E.2.2 Formulacão sem reinicialização . . . . . . . . . . . . . . . . . . 258

E.2.3 Critério de parada . . . . . . . . . . . . . . . . . 259

E.2.4 Detalhes da implementação . . . . . . . . . . . . . . . . . . . 261

F Resultados dos ensaios $\quad 265$

F.1 Ensaios do método do erro de aproximação . . . . . . . . . . . . . . . . 265

F.1.1 Sem o uso de densidade a priori . . . . . . . . . . . . . . . 265

F.1.2 Com o uso de densidade a priori . . . . . . . . . . . . . . 274

$\begin{array}{ll}\text { Referências Bibliográficas } & 283\end{array}$ 


\section{Lista símbolos e siglas}

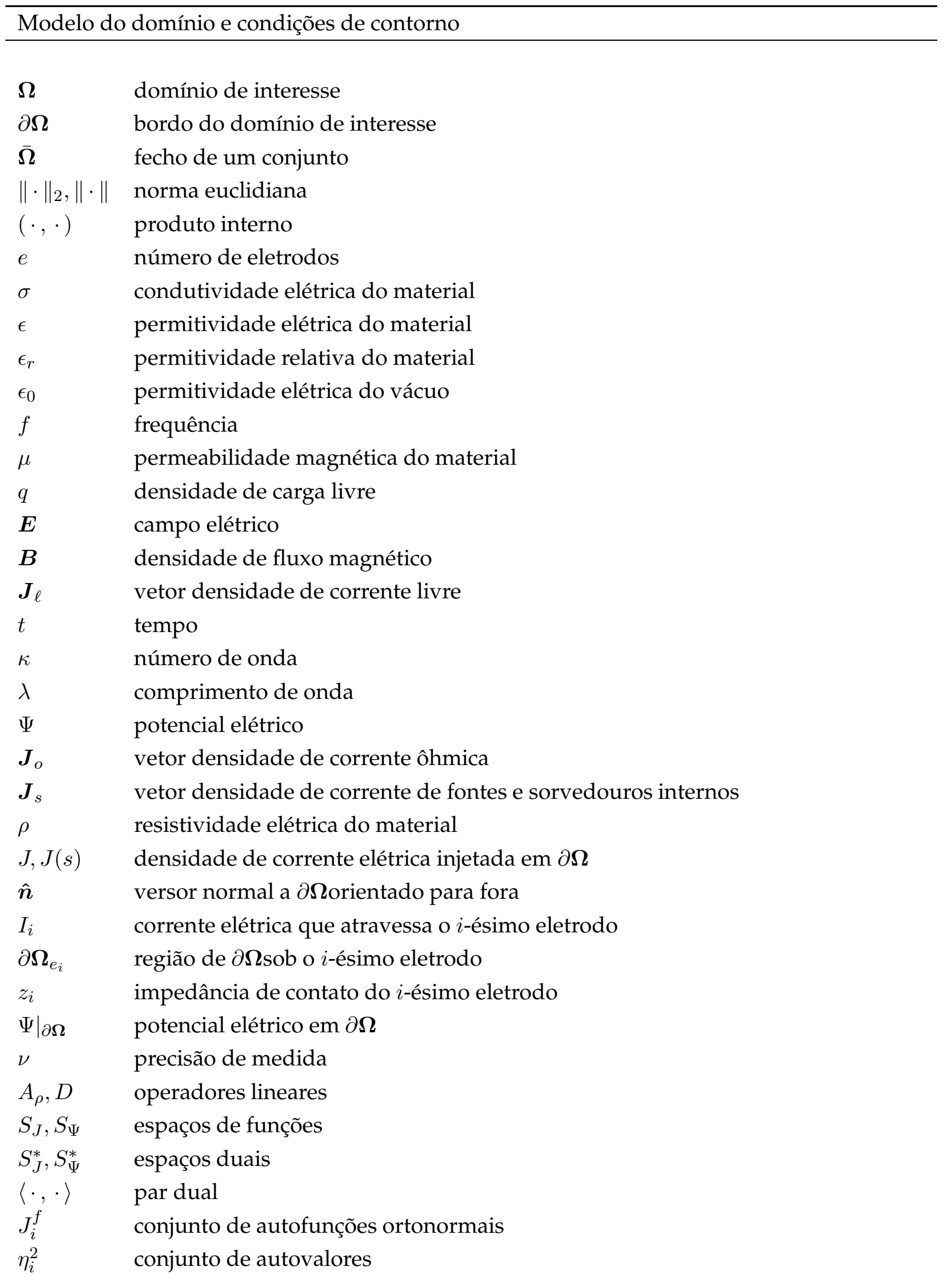




\begin{tabular}{ll}
\hline Estratégia de excitação e de medição \\
\hline $\boldsymbol{v}_{j}^{e}, \boldsymbol{v}^{e}$ & vetor de potenciais elétricos medidos nos eletrodos \\
$\boldsymbol{i}_{j}^{e}, \boldsymbol{i}^{e}$ & vetor de corrente elétrica imposto nos eletrodos \\
$\boldsymbol{Y}^{e}$ & matriz de admitância mútua \\
$S$ & subespaço vetorial \\
$\boldsymbol{V}^{e}, \boldsymbol{D}^{e}$ & decomposição em autovalores de $\boldsymbol{Y}^{e}$ \\
$d_{i}$ & i-ésimo autovalor de $\boldsymbol{Y}^{e}$ \\
$\boldsymbol{y}_{i}$ & i-ésimo autovetor de $\boldsymbol{Y}^{e}$ \\
$\boldsymbol{A}^{\dagger}$ & pseudo inversa da matriz $\boldsymbol{A}$ \\
$\boldsymbol{A}_{a n t i-s i m .}$ & componente antissimétrica da matriz $\boldsymbol{A}$ \\
$p$ & número de padrões de corrente \\
$\boldsymbol{v}^{e, d i f}$ & vetor de potenciais diferenciais entre eletrodos \\
$\boldsymbol{C}_{d}$ & matriz de conversão de potenciais single-ended para diferenciais
\end{tabular}

Discretização do domínio

$n \quad$ número de elementos da malha de elementos finitos

$m \quad$ número de nós da malha de elementos finitos

$\rho_{i} \quad$ resistividade elétrica do $i$-ésimo elemento da malha de elementos finitos

$v_{i}, v_{i}(x, y, z) \quad$ potencial elétrico no $i$-ésimo elemento da malha

$x_{k}, y_{k}, z_{k} \quad$ coordenadas nodais do $i$-ésimo elemento da malha

$v_{i k} \quad$ potencial elétrico no $k$-ésimo nó do $i$-ésimo elemento da malha

$a_{i}, b_{i}, c_{i}, d_{i} \quad$ coeficientes polinomiais interpoladores

$\alpha_{k}, \beta_{k}, \gamma_{k}, \delta_{k} \quad$ coeficientes geométricos

$\mathbb{A}_{i} \quad$ área do $i$-ésimo elemento $2 \mathrm{D}$ da malha

$f_{k} \quad$ função de forma do $i$-ésimo elemento da malha

$f_{i} \quad$ vetor de funções de forma do $i$-ésimo elemento da malha

$\tilde{\boldsymbol{v}}_{i} \quad$ vetor de potenciais elétricos nodais do $i$-ésimo elemento da malha

$\pi_{i} \quad$ energia do $i$-ésimo elemento da malha

$\mathbb{V}_{i} \quad$ volume do $i$-ésimo elemento da malha

$\hat{\boldsymbol{n}}_{i} \quad$ versor normal às arestas do $i$-ésimo elemento da malha orientado para fora

$\boldsymbol{J}_{i} \quad$ vetor densidade de corrente imposta no bordo do $i$-ésimo elemento

$\boldsymbol{F}_{i} \quad$ matriz gradiente das funções de forma do $i$-ésimo elemento da malha

$\tilde{\boldsymbol{Y}}_{i} \quad$ matriz de condutividade local do $i$-ésimo elemento da malha

$\tilde{\boldsymbol{I}}_{i} \quad$ vetor de correntes externas local do $i$-ésimo elemento da malha

$h \quad$ espessura dos elementos finitos triangulares

$\boldsymbol{Y} \quad$ matriz de condutividade global

$\rho \quad$ vetor de resistividades

$\boldsymbol{I}_{,} \boldsymbol{I}_{j} \quad$ vetor de correntes externas impostas, na numeração global

$\boldsymbol{v}, \boldsymbol{v}_{j} \quad$ vetor de potenciais elétricos, na numeração global 
$a, b, t \quad$ dimensões lineares do eletrodo

$\boldsymbol{f}_{r}, \boldsymbol{f}_{h}$

vetor função de forma do elemento retangular e hexaédrico respectivamente

$\boldsymbol{F}_{r}, \boldsymbol{F}_{h}$

matriz gradiente das funções de forma do elemento retangular e hexaédrico

$J$ densidade de corrente elétrica injetada em $\partial \boldsymbol{\Omega}$

$\tilde{\boldsymbol{Y}}_{r}, \tilde{\boldsymbol{Y}}_{h}$ matriz de condutividade local de um elemento retangular e hexaédrico

$\tilde{\boldsymbol{I}}_{r}, \tilde{\boldsymbol{I}}_{h}$ vetor de correntes externas de um elemento retangular e hexaédrico

$\tilde{\boldsymbol{v}}_{r}, \tilde{\boldsymbol{v}}_{h}$

$\tilde{\boldsymbol{Y}}_{i}^{\text {elet }}$ vetor de potenciais elétricos nodais do elemento retangular e hexaédrico

$\tilde{\boldsymbol{I}}_{i}^{\text {elet }}$ matriz de condutividade local do $i$-ésimo eletrodo vetor de correntes externas local do $i$-ésimo eletrodo

$\rho_{i}^{\prime}$ parâmetro de eletrodo do $i$-ésimo eletrodo

\begin{tabular}{|c|c|}
\hline \multicolumn{2}{|c|}{ Modelo linear e não linear de observação } \\
\hline $\mathbb{R}^{m} /\{\mathbf{1}\}$ & espaço quociente $\mathbb{R}^{m}$ pelo espaço gerado por $\mathbf{1}$ \\
\hline $\boldsymbol{h}_{j}$ & equação não linear de observação do $j$-ésimo padrão de corrente \\
\hline$\ell$ & número total de medidas, considerando todas as injeções de corrente \\
\hline$h$ & equação não linear de observação considerando todas as injeções de corrente \\
\hline$y$ & vetor de medidas \\
\hline$\rho_{0}$ & resistividade de referência na linearização \\
\hline$\left.\tilde{\boldsymbol{H}}_{j}\right|_{\rho_{0}}$ & $\begin{array}{l}\text { matriz jacobiana do modelo de observação no } j \text {-ésimo vetor de corrente } \\
\text { em torno de } \rho_{0} \text { para potenciais single-ended }\end{array}$ \\
\hline$\left.\tilde{\boldsymbol{H}}_{j}^{e}\right|_{\boldsymbol{\rho}_{0}}$ & $\begin{array}{l}\text { matriz jacobiana do modelo de observação no } j \text {-ésimo vetor de corrente } \\
\text { em torno de } \rho_{0} \text { para potenciais single-ended nos nós virtuais dos eletrodos }\end{array}$ \\
\hline $\boldsymbol{H},\left.\tilde{\boldsymbol{H}}^{e, d i f}\right|_{\boldsymbol{\rho}_{0}}$ & $\begin{array}{l}\text { matriz jacobiana do modelo de observação no } j \text {-ésimo vetor de corrente } \\
\text { em torno de } \rho_{0} \text { para potenciais diferenciais entre eletrodos }\end{array}$ \\
\hline $\boldsymbol{y}^{\prime}$ & vetor de medidas diferenciais nominais \\
\hline$\rho^{s i m}$ & vetor de resistividade utilizado no fantoma numérico \\
\hline \multicolumn{2}{|c|}{ Problema mal-posto e regularização } \\
\hline$\left(X, d_{x}\right),\left(Y, d_{y}\right)$ & espaços métricos e suas normas \\
\hline$x, y$ & vetores nos espaços $\mathrm{X}$ e $\mathrm{Y}$ \\
\hline$A(x)$ & operador \\
\hline$A$ & operador linear de dimensão finita \\
\hline $\boldsymbol{x}, \boldsymbol{y}$ & vetores de dimensão finita \\
\hline$r$ & posto de $\boldsymbol{A}$ \\
\hline $\boldsymbol{u}_{i}, \boldsymbol{v}_{i}$ & vetores singulares esquerdo e direito respectivamente \\
\hline$\sigma_{i}$ & valor singular \\
\hline$\tilde{A}$ & aproximação da matriz original $\boldsymbol{A}$ \\
\hline $\boldsymbol{y}_{0}$ & componente ortogonal ao espaço gerado por $\{\boldsymbol{u}\}_{i}^{r}$ \\
\hline
\end{tabular}


$\delta A$

$\delta x, \delta y$

$\boldsymbol{x}^{\delta}, \boldsymbol{y}^{\delta}$

$\kappa(\boldsymbol{A})$

$J(\boldsymbol{x})$

$\boldsymbol{L}_{r}$

$\boldsymbol{x}^{*}$

$\alpha$

$\boldsymbol{x}_{\alpha}$

$\nu$

$\mathbb{I}$

$\gamma_{i}$

matriz de perturbação

vetores de perturbação

vetores perturbados

número de condição da matriz $\boldsymbol{A}$

função custo

operador de regularização

vetor de referência

parâmetro de regularização

solução regularizada

precisão das medidas

matriz identidade

valor singular generalizado

Inversão Bayesiana e filtros de Kalman

$\begin{array}{ll}\mathbf{x}, \mathbf{y}, \mathbf{p}, \mathbf{e} & \text { variáveis aleatórias } \\ \boldsymbol{x}, \boldsymbol{p}, \boldsymbol{e} & \text { realização das variáveis aleatórias } \\ \boldsymbol{y}, \boldsymbol{y}_{\text {medido }} & \text { medida realizada } \\ f_{\mathbf{x}, \mathbf{y}}(\boldsymbol{x}, \boldsymbol{y}), f_{\mathbf{x}, \mathbf{y}} & \text { função densidade de probabilidade conjunta de } \mathbf{x} \text { e } \mathbf{y} \\ f_{\mathbf{x} \mid \mathbf{y}}(\boldsymbol{x} \mid \boldsymbol{y}), f_{\mathbf{x} \mid \mathbf{y}} & \begin{array}{l}\text { função densidade de probabilidade condicional de } \mathbf{x} \text {, condicionado à } \\ \text { realização } \boldsymbol{y} \text { da variável aleatória } \mathbf{y}\end{array} \\ E\{\mathbf{x}\} & \text { operador esperança de uma variável aleatória } \mathbf{x} \\ E\{\mathbf{x} \mid \mathbf{y}=\boldsymbol{y}\} & \text { operador esperança de uma variável aleatória } \mathbf{x} \text {, condicionado à } \\ & \text { realização } \boldsymbol{y} \text { da variável aleatória } \mathbf{y} \\ \hat{\boldsymbol{x}} & \text { estimativa pontual da variável aleatória } \mathbf{x} \\ t_{k} & \text { instante de tempo } \\ k & \text { índice de tempo discreto } \\ \mathbf{x}_{a}^{b}, \mathbf{y}_{a}^{b} & \text { processos estocásticos entre os instantes } t_{a} \text { e } t_{b} \\ \boldsymbol{x}_{a}^{b}, \boldsymbol{y}_{a}^{b} & \text { realização do processo estocástico entre os instantes } t_{a} \text { e } t_{b} \\ T_{s} & \text { período de amostragem do tempo discreto } \\ \boldsymbol{\Phi}_{k} & \text { modelo de evolução do processo Markoviano no instante } t_{k} \\ \mathbf{x}_{k}, \mathbf{y}_{k}, \mathbf{v}_{k}, \mathbf{e}_{k} & \text { processo estocástico no instante } t_{k} \\ \boldsymbol{x}_{k}, \boldsymbol{y}_{k}, \boldsymbol{v}_{k}, \boldsymbol{e}_{k} & \text { realização do processo estocástico no instante } t_{k} \\ \overline{\boldsymbol{x}}_{k} & \text { valor esperado de } \mathbf{x} \text { em } t_{k}, \text { condicionado às medidas realizadas } \boldsymbol{y}_{1}^{k} \\ \overline{\boldsymbol{x}}_{k+1 \mid k} & \text { valor esperado de } \mathbf{x} \text { em } t_{k+1}, \text { condicionado às medidas realizadas } \boldsymbol{y}_{1}^{k} \\ \boldsymbol{\Gamma}_{k}^{x x} & \text { matriz de covariância de } \mathbf{x} \text { em } t_{k}, \text { condicionada às medidas realizadas } \boldsymbol{y}_{1}^{k} \\ \boldsymbol{\Gamma}_{k x+1 \mid k}^{x x} & \text { matriz de covariância de } \mathbf{x} \text { em } t_{k+1} \text {, condicionada às medidas realizadas } \boldsymbol{y}_{1}^{k} \\ \boldsymbol{\Gamma}_{k+1 \mid k}^{x y} & \text { matriz de covariância cruzada entre } \mathbf{x} \text { e } \mathbf{y} \text { em } t_{k+1} \text {, condicionada } \\ \mathbf{x} \sim \mathcal{N}\left(\overline{\boldsymbol{x}}, \boldsymbol{\Gamma}^{x}\right) & \text { às medidas realizadas } \boldsymbol{y}_{1}^{k} \text {. } \\ J_{k} & \text { fistribuição Gaussiana } \\ \boldsymbol{S}_{k}^{x}, \boldsymbol{S}_{k}^{e} & \end{array}$




$\begin{array}{ll}f_{\mathbf{x}_{k}}^{\text {priori }} & \text { densidade de probabilidade a priorida variável aleatória } \mathbf{X} \text { no instante } t_{k} \\ \boldsymbol{x}^{*} & \text { estado de referência conhecido } \\ \boldsymbol{z}_{k} & \text { vetor de medidas de não conformidade no instante } t_{k} \\ \boldsymbol{y}_{k}^{\alpha} & \text { vetor de medidas aumentado } \\ \boldsymbol{h}^{\alpha} & \text { modelo de observação aumentado no instante } t_{k} \\ \boldsymbol{e}_{k}^{\alpha} & \text { ruído de observação aumentado no instante } t_{k} \\ \overline{\boldsymbol{e}}_{k}^{\alpha} & \text { valor esperado do ruído de observação aumentado no instante } t_{k} \\ \boldsymbol{\Gamma}_{k}^{e^{\alpha}} & \text { matriz de covariância do ruído de observação aumentado no instante } t_{k} \\ \boldsymbol{u} & \text { vetor de entradas conhecidas } \\ \boldsymbol{h}^{a} & \text { aproximação da função } \boldsymbol{h} \\ \boldsymbol{x}^{a}, \boldsymbol{p}^{a}, \boldsymbol{e}^{a} & \text { aproximação dos vetores } \boldsymbol{x}, \boldsymbol{p}, \boldsymbol{e} \\ \boldsymbol{T} & \text { Operador de projeção } \\ \boldsymbol{\varepsilon} & \text { ruído do método do erro de aproximação } \\ \overline{\boldsymbol{\varepsilon}}, \boldsymbol{\Gamma}^{\varepsilon} & \text { média e covariância do ruído do método do erro de aproximação }\end{array}$

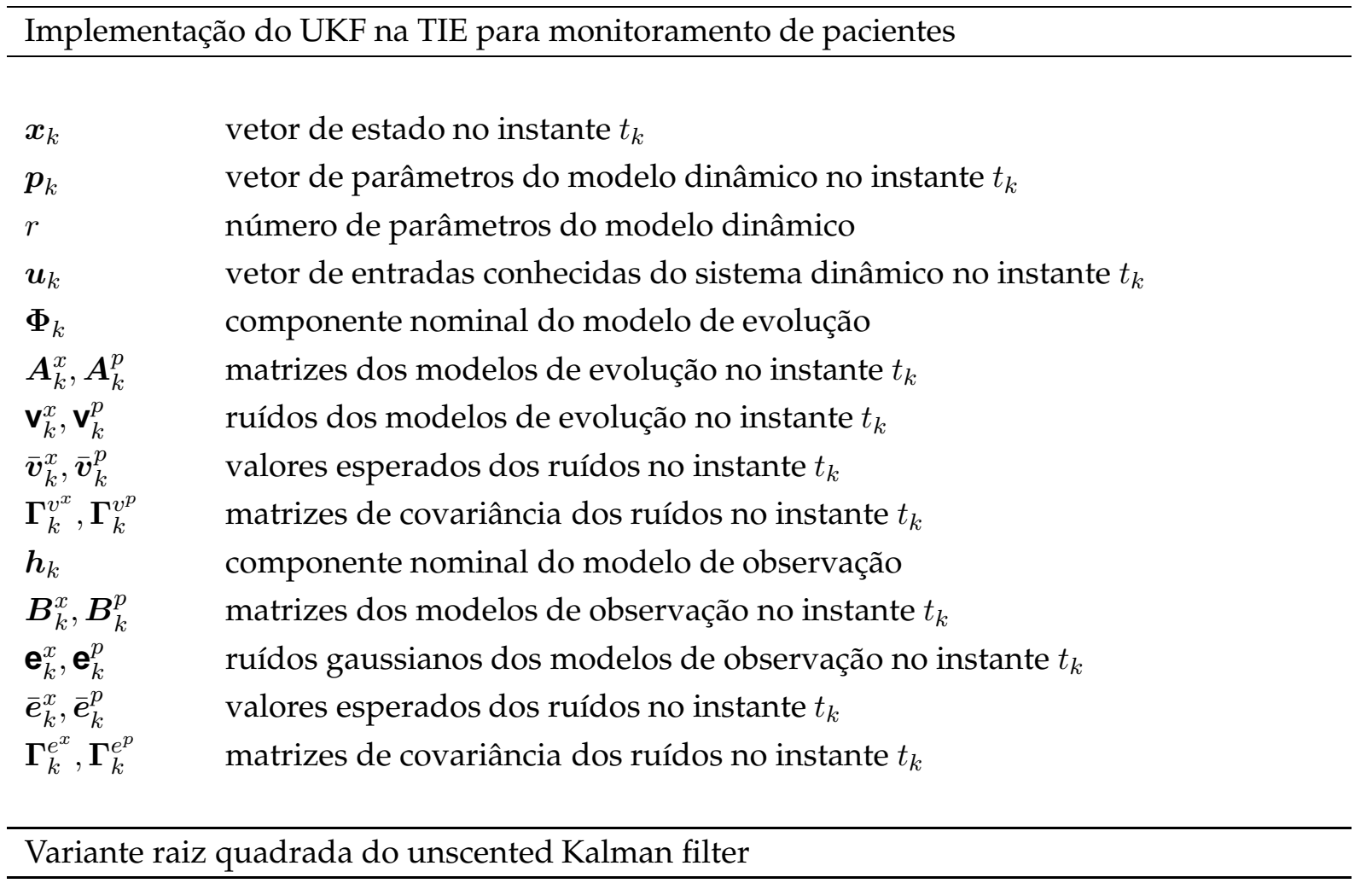

$L \quad$ dimensão do estado $x$

$\boldsymbol{S}_{k}^{x} \quad$ fator de Cholesky da matriz $\boldsymbol{\Gamma}_{k}^{x x}$

$\left\{\mathcal{X}_{k}^{i}\right\}_{i=0}^{2 L} \quad$ conjunto de pontos sigma no instante $t_{k}$

$W_{i}^{m} \quad$ peso associado ao $i$-ésimo ponto sigma para o cálculo do valor esperado

$W_{i}^{c} \quad$ peso associado ao $i$-ésimo ponto sigma para o cálculo da covariância

$\alpha, \beta, \gamma, \lambda \quad$ parâmetros de dispersão do filtro

$\left\{\mathcal{X}_{k+1 \mid k}^{i}\right\}_{i=0}^{2 L}$ conjunto de pontos sigma propagados no tempo

$\left\{\mathcal{Y}_{k+1 \mid k}^{i}\right\}_{i=0}^{4 L} \quad$ conjunto de pontos sigma referentes à observação em $t_{k+1}$

$\boldsymbol{K}_{k} \quad$ ganho de Kalman no instante $t_{k}$ 


\begin{tabular}{|c|c|}
\hline \multicolumn{2}{|c|}{ Restringindo o espaço solução } \\
\hline$\varphi_{i}$ & função sigmóide do $i$-ésimo elemento \\
\hline$\rho_{i}^{\min }, \rho_{i}^{\max }, s_{i}$ & parâmetros da sigmóide do $i$-ésimo elemento \\
\hline$\zeta_{i}$ & metaestado referente ao $i$-ésimo elemento \\
\hline$\zeta$ & vetor de metaestado referente ao estado $\rho$ \\
\hline$\varphi$ & transformação vetorial do metaestado \\
\hline$d(i, j)$ & distância euclidiana entre os centróides do $i$-ésimo e $j$-ésimo elementos \\
\hline \multicolumn{2}{|c|}{ Método do erro de aproximação } \\
\hline$h$ & modelo fidedigno de observação do sistema \\
\hline $\boldsymbol{h}^{a}$ & modelo aproximado de observação do sistema \\
\hline $\boldsymbol{d}^{a}$ & parâmetros aproximados do modelo de observação \\
\hline$e^{a}$ & ruído aproximado do modelo de observação \\
\hline $\boldsymbol{h}^{v}$ & equação verdadeira de observação do sistema \\
\hline$r$ & vetor de parâmetros ocultos do sistema \\
\hline $\boldsymbol{r}^{a}$ & vetor aproximado de parâmetros ocultos do sistema \\
\hline$\eta$ & vetor de ruído residual do modelo \\
\hline$\sigma_{\eta}$ & desvio padrão do ruído residual \\
\hline \multicolumn{2}{|c|}{ Ensaio numérico do método do erro de aproximação } \\
\hline$\varepsilon_{i}$ & realização do ruído do modelo do erro de aproximação \\
\hline $\boldsymbol{d}_{i}$ & realização do vetor de parâmetros do modelo de observação \\
\hline $\boldsymbol{\vartheta}_{i}$ & realização do vetor de parametrização de $\partial \boldsymbol{\Omega}$ \\
\hline$\vartheta^{a}$ & aproximação da parametrização de $\partial \boldsymbol{\Omega}$ \\
\hline $\boldsymbol{\vartheta}_{c}$ & vetor de parametrização de um domínio circular \\
\hline $\boldsymbol{\vartheta}_{\delta}$ & perturbação em porcentagem do vetor de parametrização de $\partial \boldsymbol{\Omega}$ \\
\hline$\sigma_{\vartheta}$ & desvio padrão da perturbação $\vartheta_{\delta}$ \\
\hline $\mathbf{x} \sim \mathcal{N}^{3 \sigma}\left(\overline{\boldsymbol{x}}, \boldsymbol{\Gamma}^{x}\right)$ & distribuição Gaussiana truncada em três desvios padrão \\
\hline $\boldsymbol{p}_{e, i}$ & realização do vetor de posição dos eletrodos \\
\hline$p^{a}$ & aproximação da posição dos eletrodos \\
\hline$p_{0}$ & vetor de posição dos eletrodos equidistantes \\
\hline $\boldsymbol{p}_{\delta}$ & vetor de perturbação de posição dos eletrodos \\
\hline$p^{-}, p^{+}$ & limites de perturbação da posição de cada eletrodo \\
\hline$\delta_{i}$ & realização do refinamento da malha \\
\hline$\delta^{a}$ & aproximação do refinamento da malha \\
\hline$\delta_{0}$ & refinamento de referência \\
\hline$\delta_{\delta}$ & variação do refinamento da malha \\
\hline$\delta^{-}, \delta^{+}$ & limites de perturbação do refinamento \\
\hline
\end{tabular}




$$
\begin{array}{ll}
M_{i} & \text { realização de uma malha } \\
M^{a} & \text { malha aproximada } \\
\boldsymbol{T}_{i}, \boldsymbol{G} & \text { interpolador de resistividades gaussiano } \\
\mathbf{x}_{u} & \text { resistividade média } \\
\mathbf{x}_{\delta} & \text { variação de resistividade } \\
\boldsymbol{e}^{a} & \text { aproximação do ruído de medida } \\
\boldsymbol{\rho}^{\prime a} & \text { aproximação dos parâmetros dos eletrodos } \\
\mathbf{r}_{k} & \text { inovação do filtro de Kalman } \\
\overline{\boldsymbol{r}}_{k}, \boldsymbol{\Gamma}_{k}^{r} & \text { estatísticas da inovação do filtro de Kalman } \\
J_{m}, J_{d}, J_{r} & \text { parcelas do custo logarítmico do filtro de Kalman } \\
C_{v}, C_{m} & \text { critérios de convergência do filtro, baseados na inovação do filtro }
\end{array}
$$

\begin{tabular}{ll}
\hline \multicolumn{2}{l}{ Modelo dinâmico dos pulmões } \\
\hline$N_{c}$ & numero de compartimentos do modelo dos pulmões \\
$V_{i}$ & volume \\
$\dot{V}_{i}$ & vazão \\
$P_{i}$ & pressão \\
$K_{i}$ & rigidez do modelo dinâmico \\
$c_{i}$ & constante de proporcionalidade da força viscosa \\
$a_{i}, b_{i}, d_{i}, e_{i}$ & coeficientes da curva Volume-Pressão \\
$L$ & função lagrangiana \\
$R$ & função de dissipação de Rayleigh \\
$Q$ & força generalizada \\
$C$ & complacência do pulmão \\
$A_{i}$ & área de cada elemento da malha \\
$\tilde{\rho}_{k, i}$ & resistividade média da região pulmonar
\end{tabular}

Ensaios Numéricos de um tórax humano

$\begin{array}{ll}P_{0}, P_{r p} & \text { pressões do ventilador mecânico } \\ T_{f} & \text { duração de tempo em que a pressão no ventilador é } P_{r p} \\ T & \text { período do ciclo ventilatório } \\ \tau_{1}, \tau_{2} & \text { constantes de tempo }\end{array}$

\begin{tabular}{ll}
\hline Atualização e Desatualização de Cholesky \\
\hline $\boldsymbol{G}$ & matriz de rotação de Givens em $\mathbb{R}^{2}$ \\
$\theta$ & ângulo de rotação \\
$c, s$ & cosseno e seno do ângulo de rotação \\
$\boldsymbol{G}_{i, j}$ & matriz de rotação de Givens no plano $(i, j)$ \\
$\boldsymbol{T}$ & matriz triangular superior \\
$\boldsymbol{P}$ & matriz de projeção ortogonal resultante
\end{tabular}


$\boldsymbol{v} \quad$ vetor de Householder

$\boldsymbol{H}_{i} \quad$ matriz de reflexão de Householder

$\hat{e}_{1} \quad$ primeiro versor da base canônica

$\boldsymbol{Q}, \boldsymbol{R}$ matrizes da fatoração QR de $\boldsymbol{A}$

$\boldsymbol{u}$ vetor de modificação

$\boldsymbol{A}^{\prime} \quad$ matriz modificada

$\boldsymbol{Q}^{\prime}, \boldsymbol{R}^{\prime}$ matrizes da fatoração QR de $\boldsymbol{A}^{\prime}$

Método Level Set

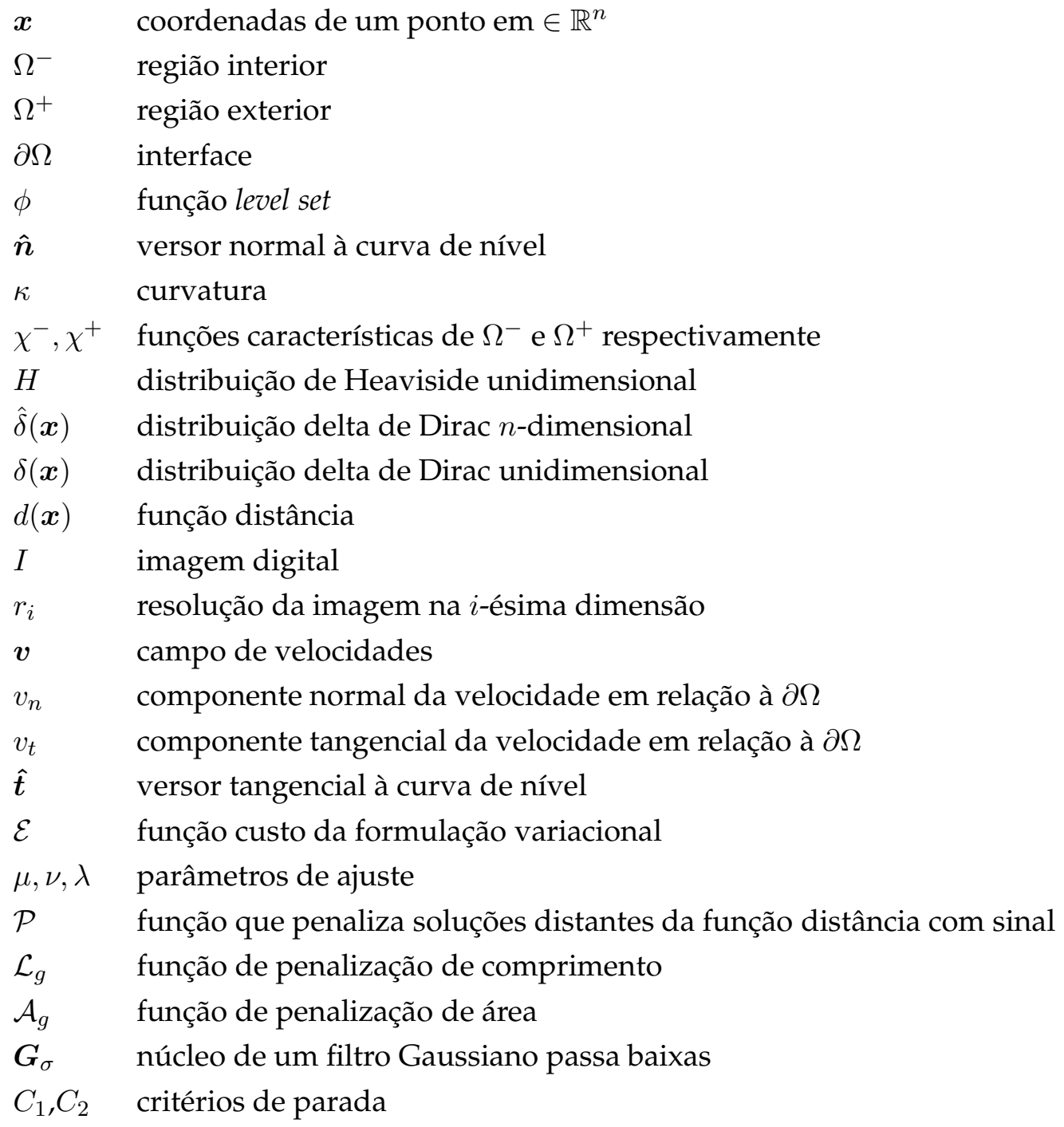


Lista de Siglas

CPT capacidade pulmonar total

CRF capacidade residual funcional

CV capacidade vital

FK filtro linear de Kalman

FEK filtro estendido de Kalman

GSVD decomposição generalizada em valores singulares

IUKF improved unscented Kalman filter

MAP máximo a posteriori

SI Sistema Internacional de Unidades

SR-UKF square root unscented Kalman filter

SVD decomposição em valores singulares

TIE tomografia por impedância elétrica

UKF unscented Kalman filter

UT unscented transform

UTI unidades de tratamento intensivo

VC volume corrente

VR volume residual 



\section{Parte I}

\section{Introdução}





\section{Capítulo 1}

\section{Introdução}

A Tomografia por Impedância Elétrica (TIE) é um método não invasivo de obtenção de imagens da distribuição de impeditividade elétrical no interior de uma região de interesse, doravante chamada de domínio, utilizando a distribuição do potencial elétrico medido na interface deste domínio com o meio exterior quando uma distribuição de corrente elétrica de baixa potência é imposta nesta mesma interface, ou vice-versa. Esta tecnologia pode ser utilizada para visualizar a distribuição interna, espacial e temporal, de alguma propriedade física, contanto que exista uma relação entre a distribuição de impedância elétrica e a propriedade de interesse. Com a distribuição espacial e temporal de impedância obtida pela TIE é possível montar uma sequência de imagens para o usuário que podem ser relacionadas com o fenômeno de interesse.

Por tratar-se de um método de observação não invasivo, a TIE vem sendo estudada para aplicações em diversas áreas. Uma pequena lista de aplicações da TIE é encontrada em Cheney et al. [1]. Entre as aplicações não clínicas, os autores citam o monitoramento de escoamento multifásico, localização de depósitos de minerais no solo, monitoramento da proliferação de contaminantes (radioativos ou não) no solo ou água, ensaios não destrutivos para a deteç̧ão de falhas em componentes mecânicos, para o acompanhamento do processo de cura de adesivos e controle de processos químicos. Entre as aplicações clínicas, cita-se a possibilidade de utilizar a TIE em monitoramento não invasivo das funções cardíacas e de fluxo sanguíneo, detecção de hemorragias internas, exame de câncer de mama, estudo do esvaziamento

\footnotetext{
${ }^{1}$ Em diversas aplicações, apenas a componente real, a resistividade elétrica, é necessária.
} 
do estômago, detectar acúmulo de fluído pélvico e para determinar o contorno que separa tecidos vivos e mortos.

Pacientes com danos severos nos pulmões estão sujeitos à ocorrência de mudanças significativas no processo respiratório como a atelectasia2, pneumotórax 3 ou derrame pleural, que podem causar inflamações do parênquima. Nestas circunstâncias, segundo Amato et al. [2], a detecção precoce a fim de evitar o colabamento e o sobre distendimento dos alvéolos é fundamental para uma estratégia eficaz de proteção dos pulmões, resultando em um aumento das funções pulmonares e aumento do índice de sobrevivência destes pacientes. Esta estratégia é fundamental em Unidades de Tratamento Intensivo (UTI), onde os médicos precisam ajustar manualmente os parâmetros dos equipamentos de ventilação mecânica (vazão de ar e pressão). Segundo Trigo et al. [3], as condições do paciente podem mudar drasticamente durante o dia e até mesmo após um intervalo de tempo de poucos ciclos respiratórios. Nestes casos um ajuste inapropriado da pressão imposta pelo equipamento é suficiente para causar inflamação severa ou mesmo ruptura dos tecidos pulmonares. Infelizmente, métodos convencionais de exposição com raios-X e métodos clínicos corriqueiros em leitos de UTI falham em conseguir detectar tais ocorrências anormais nos pulmões em tempo hábil.

Atualmente, a tomografia computadorizada por raios- $X$ continua sendo o melhor método para detectar tais anormalidades, apesar dos problemas associados a ele: o transporte do paciente em seu leito juntamente com os equipamentos de suporte à vida é muito arriscado e a natureza estática das imagens da tomografia por raios- $X$ não traz a informação dinâmica sem que haja uma manipulação sincronizada entre o equipamento de ventilação e o equipamento de raios-X4. Algumas vezes, quando o médico decide que tal procedimento é necessário, já é tarde demais para reverter os danos nos pulmões. Considerando isto, a TIE pode ser uma ferramenta atrativa pois é de fácil utilização em leitos de UTI, evitando os problemas de transporte e o uso de substâncias potencialmente nocivas ao organismo ou radiação.

\footnotetext{
${ }^{2}$ Atelectasia é o estado clínico em que há um colabamento dos alvéolos de parte ou de todo o pulmão. Colabamento é o fechamento dos alvéolos em virtude da baixa pressão do ar em seu interior.

${ }^{3}$ Pneumotórax é o estado clínico caracterizado pela presença de ar no espaço pleural, região existente entre a cavidade torácica e os pulmões.

${ }^{4} \mathrm{O}$ procedimento nestes casos consiste em regular a frequência de obtenção das imagens de raios- $X$ aproximadamente igual à frequência do ventilador de modo a causar um aliasing entre os dois e, assim, obter imagens aproximadas de um ciclo ventilatório.
} 
Tabela 1.1: Condutividade e permitividade elétrica relativa de diversos tecidos biológicos in vitro a $125 \mathrm{kHz}$. Extraído de Gabriel et al. [6].

\begin{tabular}{lll|lll}
\hline Tecido & $\sigma$ & $\epsilon_{r}$ & Tecido & $\begin{array}{l}\sigma \\
(\mathrm{S} / \mathrm{m})\end{array}$ & $\begin{array}{l}\epsilon_{r} \\
\text { (adim. })\end{array}$ \\
\hline Aorta & $(\mathrm{S} / \mathrm{m})$ & (adim.) & & $8.335 \mathrm{e}-2$ & $1.793 \mathrm{e}+3$ \\
Bexiga & $3.193 \mathrm{e}-1$ & $7.846 \mathrm{e}+2$ & Massa branca cerebral & & \\
Cartilagem & $2.197 \mathrm{e}-1$ & $1.085 \mathrm{e}+3$ & Massa cinzenta cerebral & $1.358 \mathrm{e}-1$ & $2.750 \mathrm{e}+3$ \\
Cerebelo & $1.794 \mathrm{e}-1$ & $2.515 \mathrm{e}+3$ & Medula óssea & $3.382 \mathrm{e}-3$ & $9.608 \mathrm{e}+1$ \\
Coluna vertebral & $1.558 \mathrm{e}-1$ & $3.043 \mathrm{e}+3$ & Músculo esquelético & $3.671 \mathrm{e}-1$ & $7.550 \mathrm{e}+3$ \\
Coração & $8.446 \mathrm{e}-2$ & $4.238 \mathrm{e}+3$ & Osso cortical & $2.086 \mathrm{e}-2$ & $2.191 \mathrm{e}+2$ \\
Dente & $2.220 \mathrm{e}-1$ & $8.351 \mathrm{e}+3$ & Osso trabecular & $8.409 \mathrm{e}-2$ & $4.404 \mathrm{e}+2$ \\
Estômago & $2.086 \mathrm{e}-2$ & $2.191 \mathrm{e}+2$ & Pulmão desinflado & $2.751 \mathrm{e}-1$ & $4.426 \mathrm{e}+3$ \\
Fígado & $5.371 \mathrm{e}-1$ & $2.710 \mathrm{e}+3$ & Pulmão inflado & $1.090 \mathrm{e}-1$ & $2.225 \mathrm{e}+3$ \\
Fluido cerebrospinal & $2.000 \mathrm{e}+0$ & $1.090 \mathrm{e}+2$ & Sangue & $1.761 \mathrm{e}-1$ & $6.821 \mathrm{e}+3$ \\
Gordura & $9.011 \mathrm{e}-2$ & $6.702 \mathrm{e}+3$ & Rim & $7.044 \mathrm{e}-1$ & $5.076 \mathrm{e}+3$ \\
Gordura do seio & $2.447 \mathrm{e}-2$ & $7.792 \mathrm{e}+1$ & Tendão & $3.888 \mathrm{e}-1$ & $4.066 \mathrm{e}+2$ \\
Linfa & $2.509 \mathrm{e}-2$ & $6.109 \mathrm{e}+1$ & Traqueia & $3.406 \mathrm{e}-1$ & $3.097 \mathrm{e}+3$ \\
\hline Ar/Vácuo & $5.384 \mathrm{e}-1$ & $3.132 \mathrm{e}+3$ & Vaso sanguíneo & $3.193 \mathrm{e}-1$ & $7.846 \mathrm{e}+2$ \\
\hline
\end{tabular}

Sob o ponto de vista clínico, a TIE é eficaz pois a dinâmica dos fluidos no interior do corpo humano afeta a distribuição de impeditividade elétrica. A Tabela 1.1 mostra valores de condutividade $\sigma$ e permitividade relativa $\epsilon_{r}$ dos principais tecidos, obtidos de amostras in vitro. Uma tabela de valores in vivo é apresentada em Sousa et al. [4]. Segundo Nopp et al. [5], o pulmão aerado apresenta uma grande diferença nas propriedades elétricas comparado com o pulmão colabado graças à presença de ar, que possui alta resistividade, contrastando com os tecidos do corpo humano. Além disto, o trabalho mostra que muitas patologias pulmonares também alteram estes valores, como os diversos tipos de pneumonia e enfisemas, e conclui que seria possível utilizar as propriedades elétricas dos pulmões como meio de auxiliar o diagnóstico do paciente.

\footnotetext{
${ }^{5}$ A permitividade relativa de um material $\epsilon_{r}$ é definida por $\epsilon_{r}=\frac{\epsilon}{\epsilon_{0}}$, onde $\epsilon$ é a permitividade elétrica do material, $\epsilon_{0}=\frac{1}{4 \pi \cdot 10^{-7} c_{0}} \mathrm{~F} / \mathrm{m}$ é a permitividade elétrica do vácuo e $c_{0}$ é a velocidade da luz no vácuo.
} 
Para que a TIE possa auxiliar o médico no diagnóstico e no ajuste dos equipamentos, esta deve ser capaz de fornecer imagens em taxa suficiente para retratar a fisiologia do paciente. Deve ainda ser capaz de detectar a tempo a ocorrência das anormalidades a que os pulmões estão sujeitos, como colabamento e pneumotórax. Atualmente a resolução espacial das imagens absolutas 6 da TIE são inferiores quando comparadas às imagens geradas por outros métodos, tais como a ressonância magnética e a tomografia computadorizada por raios-X. Apesar disto, a TIE apresenta algumas vantagens, a saber:

1. Não é invasiva e não utiliza nenhum tipo de radiação, possuindo então grande potencial em diagnósticos médicos pois é inofensiva ao paciente contanto que a corrente injetada obedeça aos limites de segurança definidos;

2. Baixo custo, em razão de um hardware relativamente simples;

3. Portabilidade devida às pequenas dimensões do equipamento;

4. Apresenta elevada resolução temporal.

O trabalho desenvolvido no doutorado decorre do Projeto Temático FAPESP 01/05303-4, que estudou a aplicação da TIE para o monitoramento das condições pulmonares. Os trabalhos publicados pelo grupo de pesquisa varrem diversos aspectos da TIE. Dentre estes trabalhos, pode-se citar uma lista parcial: [2, 7, 8, 9] mostrando os benefícios da TIE no monitoramento de pacientes em leito de UTI; trabalhos sobre modelagem numérica e métodos numéricos [10, 11]; trabalhos sobre métodos de estimação de imagens, como o Newton-Raphson [12, 13, 14, 15, 16], otimização topológica [17, 18, 19, 20, 21, 22, 23, 24, 25, 26], back-projection [27], Simulated Annealing [28, 29], matriz de sensibilidade [30, 31], filtros de Kalman $[32,33,3,34,35,36,37,38]$, método D-bar [39, 40] e desenvolvimento de atlas anatômico [4, 41].

${ }^{6} \mathrm{Em}$ TIE, imagens absolutas são aquelas cuja intensidades dos pixels indicam o valor da propriedade física. Imagens de diferenças ou relativas são imagens cuja intensidades dos pixels indicam as variações da propriedade física em relação a um valor de referência, diferente de zero. Alguns autores chamam-nas imagens estática e dinâmica, mas esta nomenclatura entra em conflito quando o domínio em observação apresenta uma dinâmica temporal. 


\subsection{Objetivos}

A TIE é um método de obtenção de imagens tomográficas de uso em muitas áreas, nesta pesquisa é dada atenção às aplicações médicas da TIE, mais especificamente no monitoramento das condições pulmonares.

Apesar das vantagens teóricas do Unscented Kalman Filter (UKF) sobre as outras variantes dos filtros de Kalman, ainda não existe uma investigação sobre a utilização do UKF na TIE para monitorar condições pulmonares. O objetivo principal desta pesquisa é investigar o desempenho do UKF na TIE para estimação não linear do estado do sistema variante no tempo.

Para a implementação do UKF (mais especificamente sua variante raiz quadrada), um modelo de evolução do sistema deve ser adotado. É sabido que os diferentes órgãos e tecidos internos do tórax humano possuem comportamentos temporais diferentes ao longo do ciclo respiratório. Neste trabalho um modelo de evolução do estado é proposto. Este modelo contém parâmetros que são ajustados conforme as condições clínicas do paciente ao mesmo tempo que o estado do sistema é estimado.

Um algoritmo de segmentação de imagem é desenvolvido com o objetivo de identificar o contorno dos principais órgãos internos para assim selecionar o modelo de evolução correto e o método do erro de aproximação é utilizado para reduzir os erros do modelo de observação utilizado no UKF.

O UKF é avaliado usando dados simulados de um fantoma numérico e usando também dados experimentais, coletados previamente no departamento de pneumologia da Faculdade de Medicina da Universidade de São Paulo, dentro do Projeto Temático FAPESP 01/05304-4. 


\section{Capítulo 2}

\section{Formulação do Problema}

\subsection{Modelo do domínio}

Sejam o domínio $\Omega$ um conjunto aberto e limitado em $\mathbb{R}^{3}$ e o bordo $\partial \Omega$ o conjunto formado pela intersecção do fecho $\bar{\Omega}$ e o fecho do conjunto complementar $\overline{\left(\mathbb{R}^{3} \backslash \Omega\right.}$. Assume-se que $\Omega$ possui uma distribuição estacionária de carga, permitividade $\epsilon(f)$ e condutividade $\sigma(f)$. Por simplificação da notação, a dependência destas grandezas com relação à frequência $f$ (em hertz) de oscilação dos campos elétricos e magnéticos dentro do domínio será omitida. Admite-se também que as propriedades elétricas sejam isotrópicas, lineares e não dispersivas.

As equações de Maxwell do eletromagnetismo nas formas diferenciais e integrais, considerando as hipóteses estabelecidas acima são:

$$
\begin{array}{ll}
\nabla \cdot \epsilon \boldsymbol{E}=q & \oint_{\partial \boldsymbol{\Omega}} \epsilon \boldsymbol{E} \cdot d \boldsymbol{A}=\int_{\boldsymbol{\Omega}} q d V \\
\nabla \cdot \boldsymbol{B}=0 & \oint_{\partial \boldsymbol{\Omega}} \boldsymbol{B} \cdot d \boldsymbol{A}=0 \\
\nabla \times \boldsymbol{E}=-\frac{\partial \boldsymbol{B}}{\partial t} & \oint_{C} \boldsymbol{E} \cdot d \boldsymbol{l}=-\frac{\partial}{\partial t}\left(\int_{S} \boldsymbol{B} \cdot d \boldsymbol{A}\right) \\
\nabla \times \mu^{-1} \boldsymbol{B}=\boldsymbol{J}_{\ell}+\epsilon \frac{\partial \boldsymbol{E}}{\partial t} & \oint_{C} \mu^{-1} \boldsymbol{B} \cdot d \boldsymbol{l}=\int_{S} \boldsymbol{J}_{\ell} \cdot d \boldsymbol{A}+\frac{\partial}{\partial t}\left(\int_{S} \epsilon \boldsymbol{E} \cdot d \boldsymbol{A}\right),
\end{array}
$$

onde (2.1) é a lei de Gauss, (2.2) é a lei de Gauss para o magnetismo, (2.3) é a lei de Faraday e (2.4) é a lei de Ampère. Nestas equações, $\boldsymbol{E}$ é o campo elétrico, $q$ é a densidade de carga livre, $\boldsymbol{B}$ é a densidade de fluxo magnético, $\boldsymbol{J}_{\ell}$ é a densidade de corrente livre, $\mu$ é a permeabilidade magnética do material, $\epsilon$ é a permitividade elétrica 
do material, $C$ é uma curva fechada simples qualquer dentro do domínio e $S$ é uma superfície qualquer dentro do domínio, limitada por $C,(\nabla \times)$ é o operador rotacional e $(\nabla \cdot)$ é o operador divergência.

Em TIE é usual considerar um modelo isotrópico para o interior do domínio. Tal simplificação é questionável em algumas aplicações, como imagem de tecidos musculares, ósseos e cerebrais pois sabe-se que estes tecidos são altamente anisotrópicos. Tecidos musculares esqueléticos por exemplo, apresentam razão entre condutividade longitudinal e transversal (em relação às fibras musculares) entre $2<\frac{\sigma_{L}}{\sigma_{T}}<15$, conforme estudo publicado em Gielen et al. [42]. Nestes casos, onde a anisotropia é um fator importante, esta deve ser incluída, o que aumenta consideravelmente a complexidade do problema. Alguns resultados considerando a anisotropia em EIT podem ser vistos em Abascal et al. [43, 44], Glidewell e Kwong [45]. Neste trabalho, a anisotropia dos tecidos presentes na caixa torácica não são considerados.

Pode-se avaliar também a influência da variação do campo magnético sobre o campo elétrico (indução magnética), aplicando o operador rotacional em (2.4) na forma diferencial e calculando a norma dos vetores resultantes [46, p. 539]. Feito isto, chega-se à seguinte condição para que os efeitos de indução magnética no campo elétrico possam ser desconsiderados

$$
\frac{2 \pi f \mu(f) \sigma}{\kappa^{2}} \sqrt{1+\left(\frac{2 \pi f \epsilon}{\sigma}\right)^{2}} \ll 1
$$

onde $\kappa=\frac{2 \pi}{\lambda}$, é o menor número de onda característico no domínio. Valores compatíveis para a aplicação biomédica são: $\mu \approx \mu_{0}=1,25 \cdot 10^{-6} \mathrm{Vs} / \mathrm{Am}, \lambda=1,0 \mathrm{~m}$ (comprimento de onda característico dentro do corpo humano). O maior valor desta razão, usando os valores da Tabela 1.1é o fluido cerebrospinal com razão 0.049, que significa que o efeito de indução é, no máximo, 5\% do efeito do campo elétrico nesta frequência. Neste trabalho os efeitos de indução magnética serão desprezados.

Parte-se da terceira equação de Maxwell (2.3) na forma diferencial

$$
\nabla \times \boldsymbol{E}=-\frac{\partial \boldsymbol{B}}{\partial t}
$$


Desprezando os efeitos de indução magnéticas, o termo da direita é eliminado, restando então

$$
\nabla \times \boldsymbol{E}=0
$$

e na sua forma integral

$$
\oint_{C} \boldsymbol{E} \cdot d \boldsymbol{l}=0
$$

Esta integral é igual a zero para qualquer curva fechada $C, \log$ $E$ é um campo conservativo e, por consequência, existe uma função escalar potencial (elétrico) $\Psi$ que se relaciona com $\boldsymbol{E}$ da seguinte forma

$$
-\nabla \Psi=\boldsymbol{E}
$$

Aplicando o operador divergência $(\nabla \cdot)$ em ambos os lados da quarta equação de Maxwell (2.4) na forma diferencial, obtém-se

$$
\nabla \cdot\left(\nabla \times \mu^{-1} \boldsymbol{B}\right)=\nabla \cdot \boldsymbol{J}_{\ell}+\frac{\partial(\nabla \cdot \epsilon \boldsymbol{E})}{\partial t}
$$

Sabe-se que $\nabla \cdot\left(\nabla \times \mu^{-1} \boldsymbol{B}\right)=0$ pois o divergente do rotacional de um campo vetorial em $\mathbb{R}^{3}$ é zero. Então, usando (2.1) na sua forma diferencial, chega-se a

$$
\nabla \cdot \boldsymbol{J}_{\ell}=-\frac{\partial q}{\partial t}
$$

Assumindo que não haja acúmulo de carga dentro de $\Omega$, o termo da direita é nulo, então

$$
\nabla \cdot \boldsymbol{J}_{\ell}=0
$$

A densidade de corrente livre $\boldsymbol{J}_{\ell}$ é a soma da corrente ohmica $\boldsymbol{J}_{o}$ com as fontes de corrente internas $\boldsymbol{J}_{s}$.

$$
\boldsymbol{J}_{\ell}=\boldsymbol{J}_{o}+\boldsymbol{J}_{s}
$$


No caso da TIE, não há fontes ou sorvedores de corrente elétrica no volume, portanto $\boldsymbol{J}_{s}=\mathbf{0}$. A densidade de corrente livre resultante, com propriedades resistivas e capacitivas, no caso em que o campo elétrico oscila com frequência fixa é dada por

$$
\boldsymbol{J}_{\ell}=[\sigma+i 2 \pi f \epsilon] \boldsymbol{E},
$$

onde $i$ é a unidade imaginária. Uma simplificação extra nas equações é desconsiderar o efeito capacitivo do meio. Esta simplificação é questionável, já que em muitos tecidos a componente capacitiva não é desprezível. Um extenso estudo a respeito de valores de condutividade e permitividade pode ser encontrado em Gabriel et al. [47, 48, 6, 49]. O efeito capacitivo do material pode ser desprezado se a razão entre corrente capacitiva e corrente resistiva for pequena [46, p. 158]

$$
\frac{\text { corrente capacitiva }}{\text { corrente resistiva }}=\frac{2 \pi f \epsilon}{\sigma} \ll 1 \text {. }
$$

O maior valor desta razão, usando os valores da Tabela 1.1 é o fígado com razão 0.52 , que significa que a corrente capacitiva é aproximadamente $50 \%$ da corrente resistiva! 1 . Na caixa torácica, o tecido com maior razão é o músculo cardíaco, que apresenta razão 0.26 . Neste trabalho a componente capacitiva será desprezada por simplificação, logo

$$
\boldsymbol{J}_{\ell}=\sigma \boldsymbol{E} .
$$

Substituindo (2.16) e (2.9) em (2.12), obtém-se a equação generalizada de Laplace

$$
\nabla \cdot(\sigma \nabla \Psi)=0
$$

que descreve a distribuição de potencial elétrico dentro de $\Omega$. Neste trabalho, optou-se por utilizar a resistividade $\rho$, que é relacionada com a condutividade por $\rho=\sigma^{-1}$ quando a componente complexa é desprezada.

\footnotetext{
${ }^{1}$ Neste caso, duas são as possibilidades: reduzir a frequência de excitação do sistema a procura de um nível aceitável de erro ou trabalhar com as equações sem esta simplificação.
} 


\subsection{Condição de contorno e modelo do eletrodo}

A condição de contorno é encontrada integrando (2.12) em todo o domínio

$$
\int_{\Omega} \nabla \cdot J_{\ell} d V=\int_{\Omega} 0 d V=0
$$

e usando o teorema da divergência

$$
\oint_{\partial \boldsymbol{\Omega}} \boldsymbol{J}_{\ell} \cdot \hat{\boldsymbol{n}} d A=0
$$

onde $\hat{\boldsymbol{n}}$ é o versor normal à $\partial \boldsymbol{\Omega}$ orientado para fora. Esta equação é comumente chamada de modelo contínuo de eletrodo. Esta expressão pode ser vista como a equação de conservação de carga livre dentro de $\Omega$. O termo $\boldsymbol{J}_{\ell} \cdot \hat{\boldsymbol{n}}$ é á componente normal da densidade de corrente elétrica que atravessa $\partial \Omega$. Esta corrente deve ser, pelo princípio da conservação de carga, a mesma que é imposta na superfície de $\Omega$. Define-se a densidade de corrente elétrica injetada $J$ como o negativo desta componente normal2

$$
J=-\boldsymbol{J}_{\ell} \cdot \hat{\boldsymbol{n}}=-\left.\left(-\frac{1}{\rho} \nabla \Psi\right)\right|_{\partial \boldsymbol{\Omega}} \cdot \hat{\boldsymbol{n}}=\frac{1}{\rho} \frac{\partial \Psi}{\partial \hat{\boldsymbol{n}}}
$$

A maioria dos equipamentos de TIE impõe as condições de contorno por um conjunto de eletrodos afixados ao longo de $\partial \Omega$. Com a colocação dos eletrodos, o uso do modelo contínuo de eletrodos não é aceitável já que as lacunas entre os eletrodos não impõem corrente elétrica e na região das extremidades dos eletrodos, a densidade de corrente injetada varia abruptamente para $J=0$ (região da lacuna). Esta singularidade tem comportamento $O\left(r^{-1 / 2}\right)$, onde $r$ é a distância até a borda do eletrodo [50, p. 9]. Outros dois efeitos não modelados por este modelo são (i) a superfície de contato do eletrodo é uma isopotencial e (ii) existe uma impedância de contato na interface entre o eletrodo e $\partial \boldsymbol{\Omega}$. Tal impedância elimina a singularidade, porém a densidade de corrente elétrica nas extremidades dos eletrodos ainda é muito elevada.

Neste trabalho, o modelo de eletrodo segue o modelo apresentado em Cheng et al. [51]. Este modelo, chamado de modelo completo de eletrodos, supõe um eletrodo com área de contato finita, cobrindo uma área apreciável do domínio. Segundo Hua et al.

\footnotetext{
${ }^{2}$ Esta definição é feita para acomodar a convenção de que correntes que entram em $\Omega$ são positivas.
} 
[52], eletrodos com área de contato grande permitem uma distribuição de corrente elétrica mais uniforme em $\Omega$ e possuem menor impedância de contato pois esta última é inversamente proporcional à área de contato. Isto aumenta a sensibilidade das medidas em relação às variações de resistividade internas.

Entretanto, é importante salientar que, na prática, eletrodos com grande área de contato causam problemas na imagem estimada se as distâncias entre os eletrodos não estiverem corretamente representadas no modelo matemático ou se estas distâncias não forem corrigidas de alguma forma. Nestes casos, usar eletrodos menores reduz o problema.

Este modelo supõe também uma impedância de contato $z_{i}$ entre a superfície metálica dos eletrodos e a superfície de $\partial \Omega$. Em geral esta impedância varia ao longo da superfície sob os eletrodos, mas este efeito não é modelado. Além disto, será considerado que a interface apresenta apenas efeitos resistivos. Em aplicações médicas, esta resistência é em geral elevada e variável ao longo das horas pois mudanças fisiológicas ocorrem na epiderme do paciente. Por último, este modelo admite que a superfície metálica do eletrodo seja isopotencial. As equações finais do modelo completo são

$$
\begin{aligned}
\int_{\partial \boldsymbol{\Omega}_{e_{i}}} \frac{1}{\rho} \frac{\partial \Psi}{\partial \hat{\boldsymbol{n}}} d A & =I_{i}, \quad \text { no } i \text {-ésimo eletrodo } \\
\left.\Psi\right|_{\partial \boldsymbol{\Omega}_{e_{i}}}+z_{i} \frac{1}{\rho} \frac{\partial \Psi}{\partial \hat{\boldsymbol{n}}} & =v_{i}, \quad \text { no } i \text {-ésimo eletrodo } \\
\frac{1}{\rho} \frac{\partial \Psi}{\partial \hat{\boldsymbol{n}}} & =0, \quad \partial \boldsymbol{\Omega} \backslash \bigcup_{i} \partial \boldsymbol{\Omega}_{e_{i}},
\end{aligned}
$$

onde $\partial \boldsymbol{\Omega}_{e_{i}}$ é a parte do bordo do domínio sob o $i$-ésimo eletrodo, $i=1, \cdots, e$, e é o número de eletrodos e $I_{i}$ é a corrente total injetada no $i$-ésimo eletrodo. Neste modelo, (2.21) representa a corrente injetada em cada eletrodo, (2.22) representa o potencial elétrico efetivamente medido nos eletrodos $v_{i}$ como sendo a soma do potencial $\Psi$ em $\partial \boldsymbol{\Omega}_{e_{i}}$ mais a queda de potencial elétrico em resultado da resistência de contato da interface $z_{i}$ e (2.23) representa a ausência de corrente elétrica atravessando o resto do bordo do domínio.

Em TIE, utiliza-se o termo padrão de corrente para exprimir o modo como as correntes elétricas são impostas ou medidas e o termo padrão de medição para exprimir o modo 
como os potenciais elétricos são impostos ou medidos. Neste trabalho, e na maioria dos equipamentos de impedância, considera-se que o domínio seja excitado por correntes elétricas e que sejam feitas medidas de potencial elétrico ao longo de $\partial \Omega$.

A forma como a corrente elétrica é imposta ao longo de $\partial \boldsymbol{\Omega}$ influencia na sua sensibilidade para determinar a distribuição de resistividade em $\Omega$. Como regra geral, para maximizar a sensibilidade das medidas em relação à resistividade em uma sub-região, deve haver suficiente densidade de corrente elétrica nesta região. A dificuldade surge do fato de que a distribuição de densidade de corrente é função da forma de $\Omega$, tamanho, posição e natureza da região onde a perturbação 3 está localizada. Estas informações são geralmente desconhecidas, ou apenas aproximadamente conhecidas. Para tratar do problema de sensibilidade das medidas, convém apresentar o conceito de distinguibilidade entre duas distribuições de resistividades, que foi introduzido inicialmente em Isaacson [53] como critério de avaliação dos padrões de corrente e medição de potenciais elétricos.

Seja $\left.\Psi\right|_{\partial \boldsymbol{\Omega}}(\rho, J(s))$ a função potencial elétrico em $\partial \boldsymbol{\Omega}$ quando há um padrão de corrente $J(s)$, com $\|J(s)\|_{2}=1$ e $s \in \partial \boldsymbol{\Omega}$ sendo imposto em um domínio com distribuição de resistividade $\rho$.

Diz-se que $\rho_{1}$ e $\rho_{2}$ são duas distribuições de resistividade distinguíveis por medidas de precisão $\nu>0$ se e somente se existir um padrão de corrente $J(s)$ tal que

$$
\left\|\left.\Psi\right|_{\partial \boldsymbol{\Omega}}\left(\rho_{1}, J(s)\right)-\left.\Psi\right|_{\partial \boldsymbol{\Omega}}\left(\rho_{2}, J(s)\right)\right\|_{2}>\nu
$$

Estas distribuições são indistinguíveis, no mesmo sentido, se para todo $J(s)$, com $\|J(s)\|_{2}=1$, a expressão acima for falsa. É possível determinar a forma do padrão de corrente ótimo ao maximizar esta função.

Seja o operador linear autoadjuntd $4 A_{\rho}: S_{J} \rightarrow S_{\Psi}$

$$
\left.A_{\rho} J \triangleq \Psi\right|_{\partial \boldsymbol{\Omega}}(\rho, J(s))
$$

onde $S_{J}$ e $S_{\Psi}$ são os espaços de funções da corrente $J(s)$ e potencial elétrico $\left.\Psi\right|_{\partial \boldsymbol{\Omega}}$.

\footnotetext{
${ }^{3}$ Perturbação refere-se a uma diferença de resistividade entre o valor real de resistividade e o valor aproximado assumido inicialmente nos algoritmos de TIE, localizada em alguma sub-região de $\Omega$.

${ }^{4} \mathrm{~A}$ demonstração da autoadjuntividade do operador $A_{\rho}$ encontra-se no Apêndice B
} 
Com esta definição, a distribuição de corrente ótima é aquela que também maximiza o funcional

$$
\left\|A_{\rho_{1}} J-A_{\rho_{2}} J\right\|_{2}^{2}=\left(J, D^{2} J\right)
$$

onde $D \triangleq A_{\rho_{1}}-A_{\rho_{2}}$ e $\|J\|_{2}=1$. Nota-se que esta função é maximizada quando $J(s)$ for a autofunção de maior autovalor associado pois neste caso

$$
\max _{\|J\|_{2}=1}\left(J, D^{2} J\right)=\left\|D^{2}\right\|_{2}=\eta_{1}^{2}
$$

onde $\eta_{1}^{2} \geq \eta_{2}^{2} \geq \ldots \geq 0$ são os autovalores de $D^{2}$. Voltando agora à maximização da distinguibilidade (2.24),

$$
\max _{\|J(s)\|_{2}=1}\left\|A_{\rho_{1}} J-A_{\rho_{2}} J\right\|_{2}=\left\|D J_{1}^{f}\right\|_{2}=\sqrt{\eta_{1}^{2}}>\nu
$$

onde $J_{i}^{f}$, com $i=1,2, \ldots$ é o conjunto completo de autofunções ortonormais referentes aos autovalores. Este resultado mostra que as distribuições de resitividades $\rho_{1}$ e $\rho_{2}$ são distinguíveis se $\sqrt{\eta_{1}^{2}}>\nu \mathrm{e}$, neste caso, $J(s)=J_{1}^{f}$. O próximo melhor padrão de corrente seria $J(s)=J_{2}^{f}$ se $\sqrt{\eta_{2}^{2}}>\nu$, e assim por diante, até que $\sqrt{\eta_{j}^{2}} \leq \nu$, para algum $j$.

Ainda em Isaacson [53], os autores determinam, como exemplo, uma expressão para o limite de distinguibilidade para uma anormalidade concêntrica a um domínio circular e também o melhor padrão de corrente para este caso, chamado pelos autores de padrão trigonométrico e que será apresentado mais a frente no texto. O maior desafio neste tipo de padrão de injeção é a necessidade de um equipamento complexo, com múltiplas fontes de corrente. Neste tipo de equipamento, o problema de capacitância parasita entre todos os canais deve ser considerado devido à proximidade dos cabos. Um equipamento com injeção de corrente bipolar, isto é, onde apenas dois eletrodos estão injetando corrente elétrica é mais simples de ser construído.

Em Kyriacou et al. [54] foi estudado o melhor distanciamento angular entre os eletrodos de injeção para um equipamento de injeção bipolar, quando não se conhece a posição da perturbação dentro de um domínio circular. Neste trabalho, os autores determinam o melhor distanciamento considerando que (i) todo o domínio apresenta 
a mesma probabilidade de conter uma anormalidade e (ii) o valor médio da melhor distância angular entre os eletrodos para todos os pontos do domínio resultará em uma melhor sensibilidade geral. Como resultado, os autores chegam no valor de aproximadamente $48^{\circ}$ em um sistema com 16, 32 ou 64 eletrodos.

Para mais informações a respeito de distinguibilidade, ver Seagar et al. [55], Cheney e Isaacson [56], Eyüboğlu e Pilkington [57], Eyüboğlu et al. [58].

\subsection{Estratégia de excitação e de medição}

Sejam $e$ eletrodos posicionados em $\partial \boldsymbol{\Omega}$. Seja também $\boldsymbol{v}^{e} \in \mathbb{R}^{e}$ o vetor contendo os potenciais elétricos medidos nos eletrodos, e $\boldsymbol{i}^{e} \in \mathbb{R}^{e}$ o vetor contendo as correntes elétricas impostas nos eletrodos. Estes dois vetores estão relacionados com o equivalente discreto do mapa Dirichlet-Neumann

$$
\boldsymbol{Y}^{e} \boldsymbol{v}^{e}=i^{e}
$$

onde $\boldsymbol{Y}^{e} \in \mathbb{R}^{e x e}$ é chamada matriz de condutância mútua. Existe então um número máximo de $e^{2}$ parâmetros, ou graus de liberdade, que podem ser determinados pelas medidas elétricas, que irão compor a distribuição final de resistividade.

Nota-se entretanto que o vetor $\mathbf{1} \triangleq\left[\begin{array}{llll}1 & 1 & \ldots & 1\end{array}\right]^{\mathrm{T}} \in \mathbb{R}^{e}$ está no núcleo do operador $\boldsymbol{Y}^{e}$ pois potenciais elétricos idênticos em todos os eletrodos não permitem corrente elétrica atravessando $\partial \boldsymbol{\Omega}$. Este é o único vetor com esta propriedade além do vetor nulo. Qualquer outro vetor $\boldsymbol{v}^{e}$ linearmente independente de 1 impõe alguma diferença de potencial entre eletrodos, resultando em corrente atravessando o bordo. A existência de um núcleo não trivial causa uma redução dos graus de liberdade de $\boldsymbol{Y}^{e}$.

Seja então $S$ o subespaço perpendicular ao vetor 1 . Mostra-se que $\boldsymbol{Y}^{e}$ é invertível de $S$ em $S$ e, portanto, apresenta $(e-1)^{2}$ parâmetros que podem ser determinados a partir de pares $\left(\boldsymbol{v}^{e}, \boldsymbol{i}^{e}\right)$. Este número é facilmente obtido ao computar a decomposição 
em autovalores 5 de $\boldsymbol{Y}^{e}$

$$
\boldsymbol{Y}^{e}=\boldsymbol{V}^{e} \boldsymbol{D}^{e}\left[\boldsymbol{V}^{e}\right]^{\mathrm{T}}
$$

onde $\boldsymbol{V}^{e} \in \mathbb{R}^{e \times e}$ é a matriz contendo os autovetores em suas colunas e $\boldsymbol{D}^{e} \in \mathbb{R}^{e \times e}$ é a matriz diagonal contendo os autovalores associados. Sabendo que o vetor 1 está no núcleo de $\boldsymbol{Y}^{e}$, pode-se reescrever a decomposição como

$$
\boldsymbol{Y}^{e}=\left[\begin{array}{llll}
\boldsymbol{y}_{1} & \ldots & \boldsymbol{y}_{e-1} & \frac{1}{\|\mathbf{1}\|_{2}}
\end{array}\right] \operatorname{diag}\left(\left[\begin{array}{llll}
d_{1} & \ldots & d_{e-1} & 0
\end{array}\right]\right)\left[\begin{array}{llll}
\boldsymbol{y}_{1} & \ldots & \boldsymbol{y}_{e-1} & \frac{1}{\|\mathbf{1}\|_{2}}
\end{array}\right]^{\mathrm{T}}
$$

onde cada autovetor $\boldsymbol{y}_{i} \in \mathbb{R}^{e}$ deve ser determinado, resultando em $e(e-1)$ graus de liberdade 6 . Entretanto, sabendo que os autovetores são ortogonais, obtém-se $e-1$ vínculos do tipo

$$
\boldsymbol{y}_{i} \cdot \frac{\mathbf{1}}{\|\mathbf{1}\|_{2}}=0 \Rightarrow \sum_{j=1}^{e} y_{i, j}=0 \quad i=1,2, \ldots,(e-1)
$$

onde $y_{i, j}$ é a $j$-ésima componente do autovetor $\boldsymbol{y}_{i}$. $\mathrm{O}$ número total de graus de liberdade cai então para $e(e-1)-(e-1)=(e-1)^{2}$.

O número de graus de liberdade é reduzido ainda mais pelo princípio da reciprocidade, que atesta que ao inverter os eletrodos de medida com os eletrodos de injeção obtém-se os mesmos valores de condutância mútua. A reciprocidade impõe uma estrutura simétrica para $\boldsymbol{Y}^{e}$, o que reduz o número de graus de liberdade para $e(e-1) / 2$. Este é, portanto, o número máximo de medidas independentes que podem ser realizadas para formar uma base para o espaço $S$. Este número é também o número máximo de graus de liberdade da solução que é possível encontrar com $e$ eletrodos. Qualquer tentativa de aumentar o número de incógnitas da solução necessitará de informação a priori adicional.

Um sistema de TIE deve obter pares $\left(\boldsymbol{v}^{e}, \boldsymbol{i}^{e}\right)$ que serão usados para determinar uma base de $S$. Com esta base, a matriz $\boldsymbol{Y}^{e}$ pode ser determinada ou estimada.

\footnotetext{
${ }^{5}$ Considerando $\boldsymbol{Y}^{e}$ real, a decomposição em autovalores desta matriz resulta em matrizes reais pois, pelo princípio da reciprocidade, $\boldsymbol{Y}^{e}$ deve ser simétrica. Isto será explorado mais a frente.

${ }^{6}$ Os autovalores não entram no número de graus de liberdade pois uma vez determinados os autovetores, os autovalores associados são calculados a partir de sua definição $\boldsymbol{Y}^{e} \boldsymbol{y}_{i}=d_{i} \boldsymbol{y}_{i}$.
} 
Uma vez coletados $p$ pares de medidas $\left(\boldsymbol{v}^{e}, \boldsymbol{i}^{e}\right)$, uma estimativa para $\boldsymbol{Y}^{e}$ pode ser obtida por regressão múltipla7

$$
\boldsymbol{Y}^{e}=\boldsymbol{I}_{p} \boldsymbol{V}_{p}^{\dagger}
$$

onde $\boldsymbol{V}_{p}, \boldsymbol{I}_{p} \in \mathbb{R}^{e \times p}$ são matrizes cujas colunas são compostas pelos pares $\left(\boldsymbol{v}^{e}, \boldsymbol{i}^{e}\right) \mathrm{e}$ $\boldsymbol{V}_{p}^{\dagger}$ é a pseudoinversa de $\boldsymbol{V}_{p}$.

A solução deste sistema não garante que $\boldsymbol{Y}^{e}$ seja simétrica em decorrência dos erros numéricos e de instrumentação. Para contornar isto, pode se usar a média sobre o erro de reciprocidade, que corrige a solução de (2.33) pela remoção de sua componente antissimétrica 8

$$
\boldsymbol{Y}_{\text {corrigida }}^{e}=\boldsymbol{Y}^{e}-\boldsymbol{Y}_{\text {anti-sim. }}^{e}
$$

onde $\boldsymbol{Y}_{\text {anti-sim. }}^{e}=\frac{1}{2}\left(\boldsymbol{Y}^{e}-\left(\boldsymbol{Y}^{e}\right)^{\mathrm{T}}\right)$ pode ser usada para avaliar os erros na instrumentação do sistema de coleta de dados.

Muitas vezes o número de medidas independentes é menor que $e(e-1) / 2$ por conta da dificuldade de medição dos potenciais elétricos nos eletrodos onde a corrente elétrica está sendo imposta, quando algum canal de medida está com problema e precisa ser desligado ou no caso em que as medidas não formem um conjunto linearmente independente. No caso de um conjunto de pares $\left(\boldsymbol{v}^{e}, \boldsymbol{i}^{e}\right)$ que somente forma uma base para um subespaço de $S$, a solução de (2.33) será a projeção de $\boldsymbol{Y}^{e}$ neste subespaço. Caso haja mais medidas do que o necessário para completar a base de $S$, a solução será a que minimiza o erro quadrático de todas as medidas, o que reduz a covariância de $\boldsymbol{Y}^{e}$. Geralmente $p=e$, resultando em $\boldsymbol{V}_{p}$ quadrada porém de posto incompleto.

Em Malmivuo e Plonsey [59, cap. 26] algumas formas de padrões de injeção e medição são apresentadas. Duas formas bastante frequentes de impor a corrente elétrica são o padrão de corrente trigonométrico e o pula-m, onde $m \geq 0$ é escolhido. Estes padrões podem ser vistos na Figura 2.1. No padrão de corrente trigonométrico,

\footnotetext{
${ }^{7}$ Regressão múltipla pode ser aplicada caso $\boldsymbol{v}^{e}$ ou $\boldsymbol{i}^{e}$ for conhecido com precisão suficiente. Caso contrário é necessária uma análise de correlação múltipla. Neste trabalho assume-se que as correntes elétricas são conhecidas com suficiente precisão e acurácia.

${ }^{8}$ Toda matriz quadrada pode ser decomposta em uma componente simétrica e uma componente antissimétrica. $\boldsymbol{A}=\boldsymbol{A}_{\text {sim. }}+\boldsymbol{A}_{\text {anti-sim. }}=\frac{1}{2}\left(\boldsymbol{A}+\boldsymbol{A}^{\mathrm{T}}\right)+\frac{1}{2}\left(\boldsymbol{A}-\boldsymbol{A}^{\mathrm{T}}\right)$.
} 


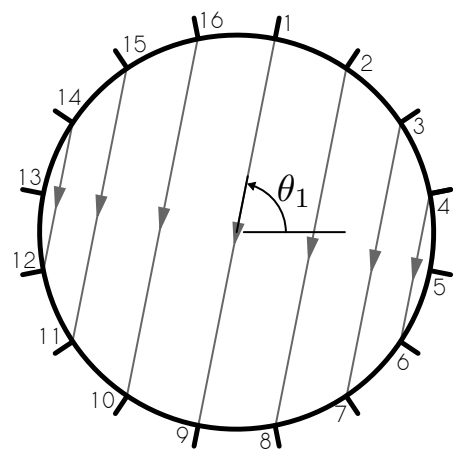

padrão trigonométrico

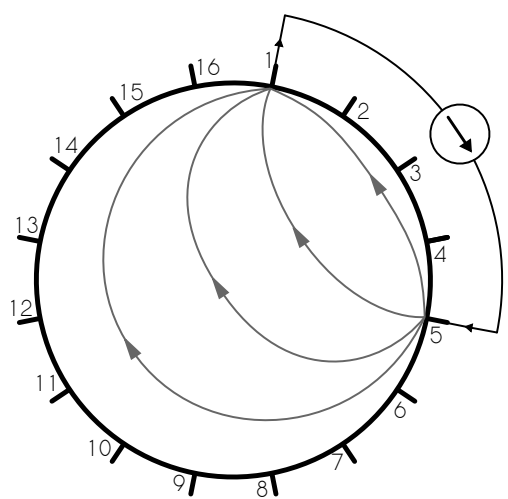

padrão pula- $m$, para $m=3$

Figura 2.1: Exemplos de padrões de corrente. Adaptado de Malmivuo e Plonsey [59].

a corrente elétrica é imposta através de todos os eletrodos. O intuito deste padrão é tentar produzir uma densidade de corrente uniforme 9 em um domínio circular. Neste padrão, a corrente imposta nos eletrodos é função da posição angular dos eletrodos $I_{i, j}=I_{\max } \cos \left(\theta_{i}-\theta_{j}^{\max }\right)$, onde $\theta_{i}$ é a posição angular do $i$-ésimo eletrodo e $\theta_{j}^{\max }$ é a posição angular onde ocorre o pico de corrente $I_{\max }$ no $j$-ésimo padrão.

Na Figura 2.1, pode-se ver o primeiro vetor $\boldsymbol{i}_{1}^{e}\left(\theta_{1}^{\max }=\theta_{1}\right)$. No segundo vetor, $\boldsymbol{i}_{2}^{e}$, o ângulo de referência passa a ser a posição angular do eletrodo 2 e assim por diante até o eletrodo $e / 2$, pois além deste valor todas os padrões serão linearmente dependentes dos anteriores.

O outro tipo de padrão de corrente, pula-m, utiliza apenas dois eletrodos para injeção de corrente. Neste padrão, o par de eletrodos ativos é separado por $m$ eletrodos, como pode ser visto na Figura 2.1 para $m=3$. O $j$-ésimo vetor $\boldsymbol{i}_{j}^{e}$ utiliza como pares ativos os eletrodos $j$ e $\bmod (m+j, e)+1$.

O caso em que $m=0$ é também chamado padrão de eletrodos vizinhos e o caso em que $m=e / 2-1$ é também chamado de padrão diametral. O padrão pula-0 tem a desvantagem de concentrar a maior parte da corrente elétrica na vizinhança do par de injeção, tornando as medidas de potencial menos sensíveis à variações de resistividade em seu interior. Este efeito é reduzido conforme $m$ aumenta, porém a razão sinal/ruído torna-se mais desfavorável.

A medição dos potenciais pode ser realizada de duas formas: (i) medidas single-ended, ou seja, medidas de potencial elétrico referentes a um referencial fixo, usualmente o terra, ou (ii) medidas diferenciais entre eletrodos. No caso de medidas

\footnotetext{
${ }^{9} \mathrm{~A}$ uniformidade de corrente só ocorre em um domínio cilíndrico e com resistividade uniforme.
} 
diferenciais, é usual utilizar o padrão de medição pula-m. A Figura 2.2 apresenta os dois casos.

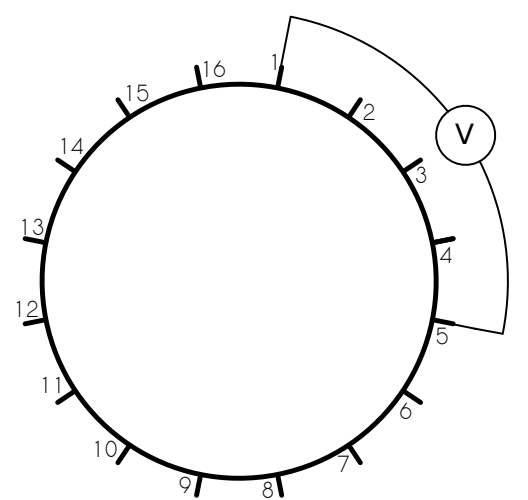

padrão pula- $m$, para $m=3$

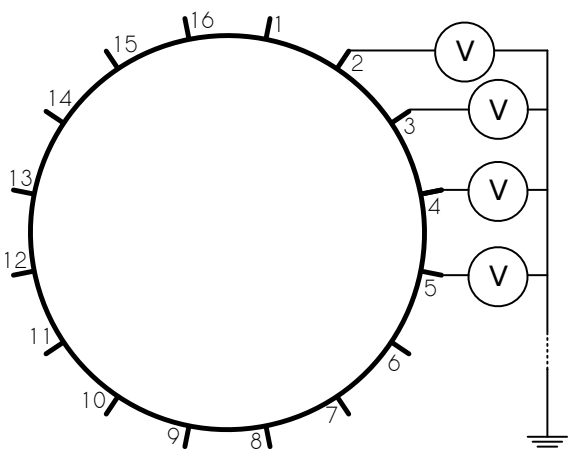

single-ended

Figura 2.2: Exemplos de padrões de medição. Adaptado de Malmivuo e Plonsey [59].

O equipamento utilizado nos ensaios experimentais desta tese possui $e=32$ eletrodos, impõe a corrente elétrica através do padrão pula-3 e os potenciais elétricos medidos pelo equipamento são single-ended. O equipamento é capaz de fazer medidas de potencial elétrico também nos eletrodos de injeção, contanto que as medidas não saturem o conversor analógico $\rightarrow$ digital. Por conta desta possível saturação, pode ser necessário reduzir o valor da corrente injetada no tórax do paciente, resultando em uma razão sinal/ruído mais desfavorável em todas as medidas. Assim, dependendo do caso, torna-se mais vantajoso desprezar estas medidas em favor de manter uma boa razão sinal/ruído nas medidas dos demais eletrodos.

Caso o interesse seja estimar os parâmetros de eletrodos, explicado na Seção 2.4, é necessário utilizar os potenciais elétricos que envolvem o par de injeção pois as medidas nos eletrodos passivos são pouco sensíveis a variações na impedância de contato. Nos desenvolvimentos das próximas seções, serão consideradas e medidas por par de injeção. As alterações das equações são triviais nos casos em que o número de medidas utilizadas for menor do que $e$.

Cabe uma observação importante a respeito do número de pares $\left(\boldsymbol{v}^{e}, \boldsymbol{i}^{e}\right)$ efetivamente informativos para compor o espaço $S$ usando um padrão pula- $m$, tanto para a injeção quanto para a medida dos potenciais elétricos. 
Iniciando pela corrente, em padrões pula- $m$, com $m \geq 0$, a matriz de correntes $\boldsymbol{I}_{p} \in \mathbb{R}^{e \times e}$ para $p=e$ é dada por

$$
\left[\boldsymbol{I}_{p}\right]_{(i, k)}=\left\{\begin{aligned}
I, & i=k \\
-I, & i=\bmod (k+m, e)+1 \\
0, & \text { demais }
\end{aligned}\right.
$$

onde $I$ é o valor da corrente injetada nos eletrodos. O posto desta matriz, variando-se o valor de $m$ é apresentado na Figura 2.3 para $e=32$.

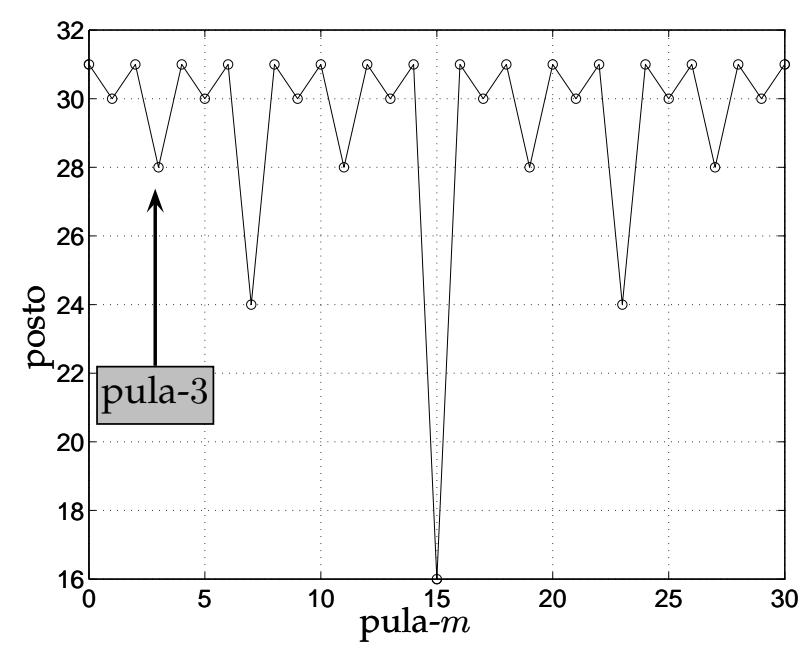

Figura 2.3: Posto da matriz de correntes $\boldsymbol{I}_{p}$ em função de $m$ para 32 eletrodos.

$\mathrm{O}$ posto desta matriz indica o número de vetores de corrente $\boldsymbol{i}^{e}$ linearmente independentes do conjunto de $p=32$ vetores. Pode-se observar então que no caso do pula-3, o número de vetores independentes é 28 , ao passo que se fosse escolhido pula-4, o número subiria para 31.

Analisando agora os potenciais elétricos diferenciais, pode-se adaptar os resultados da análise feita no início da Seção 2.3, que considera apenas medições single-ended, para medidas diferenciais definindo uma matriz $\boldsymbol{C}_{d} \in \mathbb{R}^{e \times e}$ de conversão single-ended para pula-m. Esta matriz é dada por

$$
\left[\boldsymbol{C}_{d}\right]_{(i, k)}=\left\{\begin{aligned}
-1, & i=k \\
1, & i=\bmod (k+m, e)+1 \\
0, & \text { demais }
\end{aligned}\right.
$$


Esta matriz apresenta a mesma estrutura da matriz $\boldsymbol{I}_{p}$, portanto o posto de $\boldsymbol{C}_{d}$ em função do valor $m$ é o mesmo e pode ser visto na Figura 2.3. É fácil notar que em todos os casos diferenciais, o vetor 1 está no núcleo de $\boldsymbol{C}_{d}$.

Montando agora o mapa Dirichlet-Neumann para medidas diferenciais nos moldes de (2.29)

$$
\boldsymbol{Y}^{e} \boldsymbol{v}^{e, d i f}=\boldsymbol{i}^{e} \Leftrightarrow \boldsymbol{Y}^{e}\left[\boldsymbol{C}_{d} \boldsymbol{v}^{e, s e}\right]=\boldsymbol{i}^{e}
$$

nota-se que o uso de potenciais diferenciais $\boldsymbol{v}^{e, d i f}$ é equivalente a usar potenciais single-ended, adicionando a matriz $\boldsymbol{C}_{d}$, que possui posto incompleto. O resultado disto é que alguma informação pode ser perdida ao utilizar medidas diferenciais. No caso de medidas pula-3, três outros vetores estarão no núcleo de $\boldsymbol{C}_{d}$ além do vetor $\mathbf{1}$ ao passo que no pula-4 apenas o vetor 1 estará presente.

O número de pares $\left(\boldsymbol{v}^{e}, \boldsymbol{i}^{e}\right)$ efetivamente úteis para compor o espaço $S$ depende do valor $m$ escolhido. Por esta análise, equipamentos que utilizam padrões pula-3 poderiam ser modificados para pula-4 de modo a aumentar o número de medidas por vetor de corrente e aumentar também o número de vetores de corrente linearmente independentes, sem grandes mudanças no nível de sensibilidade das medidas à variações de resistividade.

A informação perdida das medidas ao utilizar o pula- $m$ está relacionada ao número de circuitos fechados de medidas. A Figura 2.4 mostra as medidas dos 32 eletrodos no padrão pula-3 organizadas em quatro circuitos fechados, cada um indicado em uma cor.
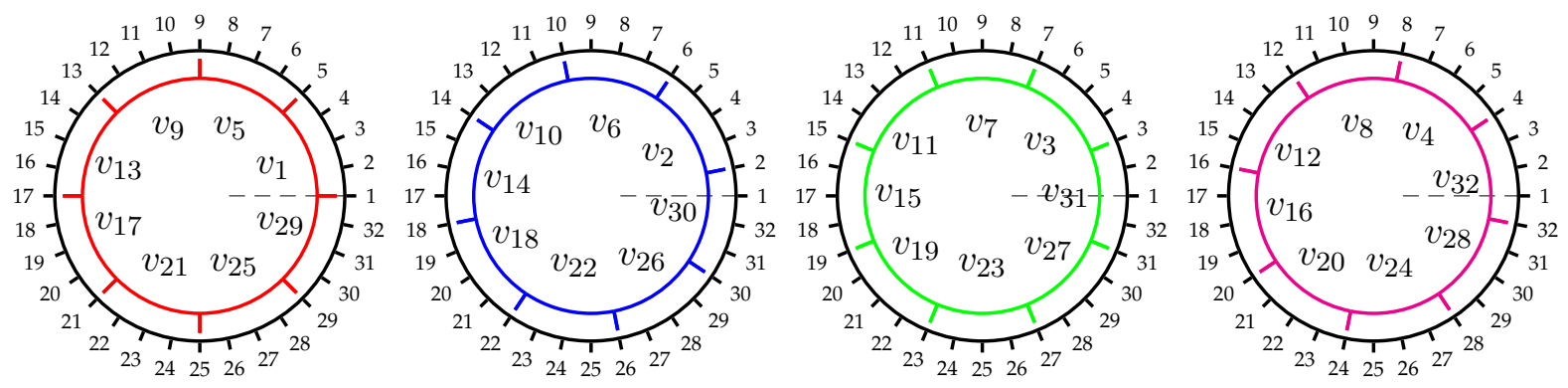

Figura 2.4: Medidas realizadas no padrão pula-3.

A Figura 2.5 mostra as medidas dos 32 eletrodos no padrão pula-4 e destaca que há apenas 1 circuito de medidas, ou seja, iniciando em um eletrodo qualquer e avançando 
de cinco em cinco eletrodos, o eletrodo inicial só é novamente alcançado após todas as 32 medidas.
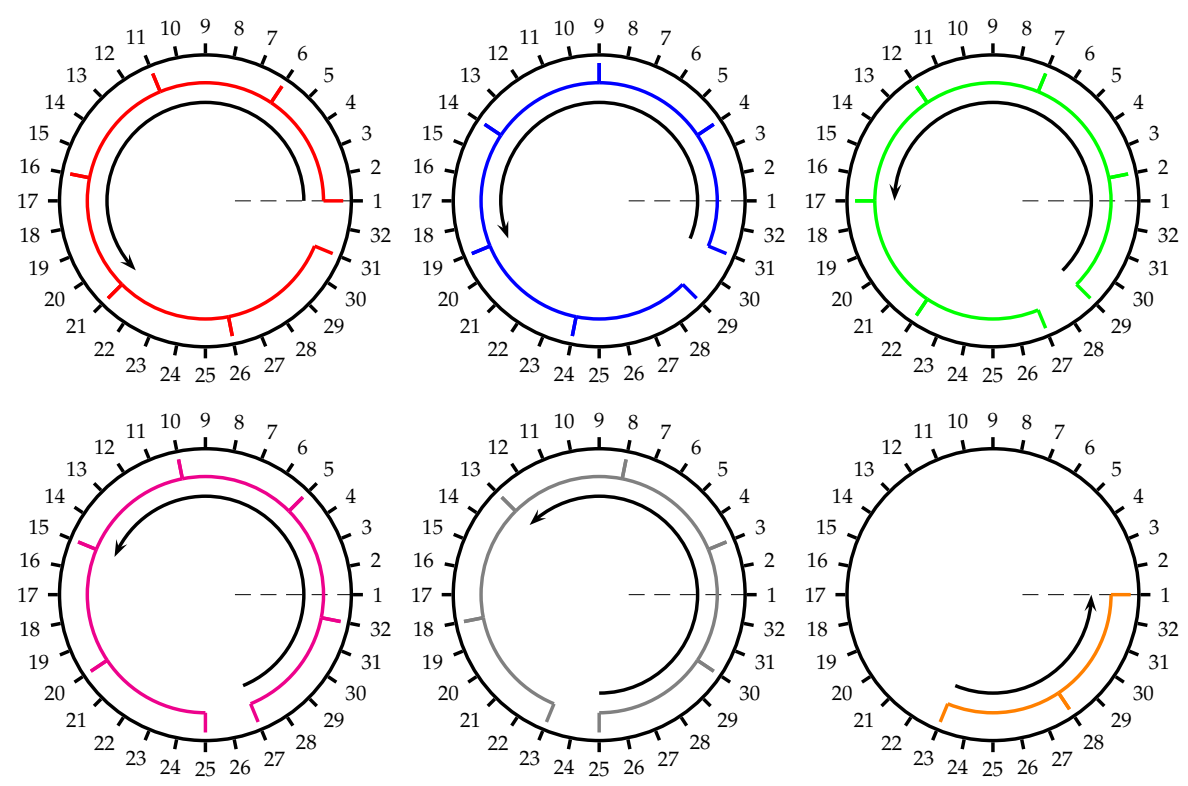

Figura 2.5: Medidas realizadas no padrão pula-4.

A Figura 2.6 apresenta os quatro vetores pertencentes ao núcleo de $\boldsymbol{C}_{d}$ no padrão pula-3. Estes vetores são aqueles que estabelecem a diferença de potencial entre os circuitos. Ou seja, ao realizar medidas pula-3, a referência entre cada um dos circuitos fechados é perdida. No caso de medidas pula-4, o único vetor não nulo no núcleo é o vetor 1 que representa a referência do potencial elétrico em relação a um terra comum.
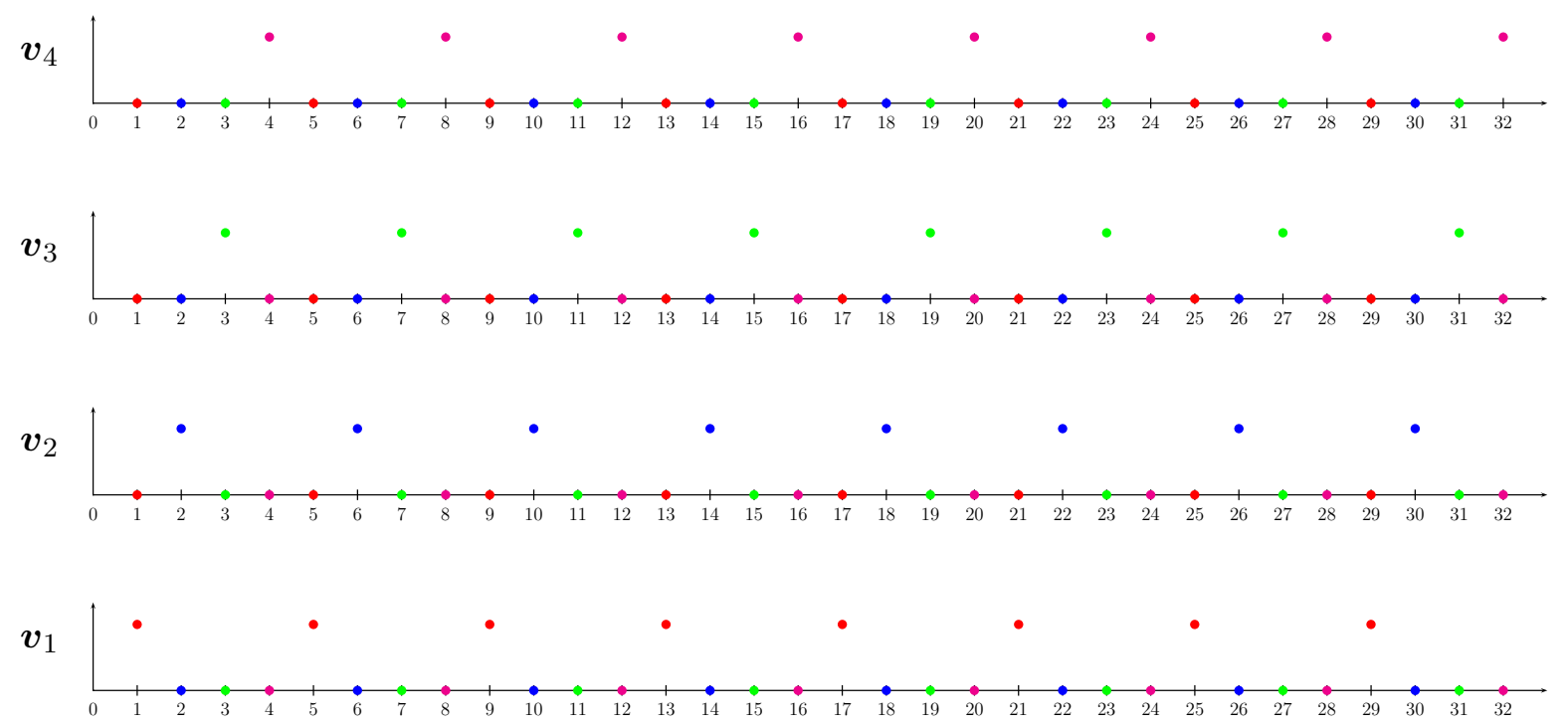

Figura 2.6: Vetores do núcleo de $\boldsymbol{C}_{d}$ no padrão pula-3. 


\subsection{Discretização do domínio}

O domínio $\Omega$ é discretizado em elementos finitos para a solução numérica das equações (2.17), (2.21)-(2.23). O domínio é dividido em uma malha com $m$ nós e $n$ elementos finitos de resistividade $\rho_{i}, i=1,2, \ldots, n$, constante por elemento. As interfaces dos $e$ eletrodos também são discretizadas utilizando elementos finitos. As equações dos elementos finitos são desenvolvidas no apêndice A.1 A.2, para o caso 2D e 3D.

Após a discretização, o sistema de equações pode ser reescrito na forma matricial

$$
\boldsymbol{Y}(\boldsymbol{\rho}) \boldsymbol{v}=\boldsymbol{I}
$$

onde $\boldsymbol{Y} \in \mathbb{R}^{m \times m}$ é a matriz de condutividade global, $\boldsymbol{v} \in \mathbb{R}^{m}$ é o vetor de potenciais elétricos nodais, $\boldsymbol{I} \in \mathbb{R}^{m}$ é o vetor de corrente nodal imposta em $\partial \boldsymbol{\Omega}$ e $\boldsymbol{\rho} \in \mathbb{R}^{n+e}$ é o vetor composto pelas resistividades dos elementos e pelos parâmetros dos eletrodos $\rho^{\prime}$ das interfaces, conforme apresentado no apêndice A.2.

\subsection{Determinação dos potenciais elétricos nos nós virtuais dos eletrodos}

A determinação dos potenciais elétricos nodais via método dos elementos finitos (MEF) é feita resolvendo o sistema (2.38) para um determinado vetor de corrente $j=1,2, \ldots, p$

$$
\boldsymbol{Y}(\boldsymbol{\rho}) \boldsymbol{v}_{j}=\boldsymbol{I}_{j}
$$

onde $\boldsymbol{v}_{j} \in \mathbb{R}^{m}$ é o vetor de potenciais elétricos nodais referentes ao $j$-ésimo vetor de corrente $\boldsymbol{I}_{j}$. Esta equação é resolvida conhecendo-se a resistividade elétrica dos $n$ elementos da malha dos $e$ parâmetros dos eletrodos e também do vetor de corrente usado $\boldsymbol{I}_{j}$.

A matriz $\boldsymbol{Y}$ também apresenta núcleo não trivial. Este núcleo tem dimensão um,

contendo os vetores nulo e $\mathbf{1}=\left[\begin{array}{llll}1 & 1 & \ldots & 1\end{array}\right] \in \mathbb{R}^{m}$. Por conta deste núcleo, se $\boldsymbol{v}^{*}$ é solução de (2.39), $\boldsymbol{v}^{*}+c \mathbf{1}$ também é solução, onde $c$ é uma constante qualquer. Para contornar este problema, é necessário trabalhar no espaço quociente $\mathbb{R}^{m} /\{\mathbf{1}\}$, onde a 
solução é uma classe de equivalência $\left[\boldsymbol{v}^{*}\right]=\boldsymbol{v}^{*}+c \mathbf{1}, \forall c$. Na prática procura-se, como solução de (2.39), um representante desta classe de equivalência que contenha uma das componentes com valor fixado. Usualmente escolhe-se o valor zero para alguma componente, o que fisicamente representa aterrar um dos nós da malha de elementos finitos. Salienta-se que na solução de (2.39) obtém-se potenciais single-ended.

Após definir o nó com potencial elétrico de referência, pode-se reescrever (2.39) como uma equação não linear de observação $\boldsymbol{h}_{j}: \boldsymbol{\rho} \mapsto \boldsymbol{v}_{j}$

$$
\boldsymbol{v}_{j}(\boldsymbol{\rho})=\boldsymbol{h}_{j}(\boldsymbol{\rho})=[\boldsymbol{Y}(\boldsymbol{\rho})]^{-1} \boldsymbol{I}_{j} \quad, \quad j=1,2, \ldots, p
$$

Como somente é possível fazer a medida de potencial elétrico nos eletrodos, apenas os elementos de $\boldsymbol{v}_{j}$ referentes aos nós virtuais 10 dos eletrodos são considerados medidas. Desta forma, pode-se montar um vetor $\boldsymbol{v}_{j}^{e} \in \mathbb{R}^{e}$ contendo apenas estes potenciais. Caso seja necessário converter os potenciais single-ended para potenciais diferenciais, uma matriz de conversão $C_{d}$ é utilizada.

Por uma questão de uniformidade de notação, os potenciais elétricos dos nós virtuais dos eletrodos calculados com (2.39), para $j=1,2, \ldots, p$, e convertidos para potenciais diferenciais são agrupados em um vetor $\boldsymbol{y} \in \mathbb{R}^{\ell}$, onde $\ell=p$.e é o número total de medidas realizadas, considerando todos os vetores de corrente

$$
\boldsymbol{y}(\boldsymbol{\rho})=\boldsymbol{h}(\boldsymbol{\rho}) \triangleq\left[\begin{array}{c}
\boldsymbol{C}_{d} \boldsymbol{v}_{1}^{e}(\boldsymbol{\rho}) \\
\boldsymbol{C}_{d} \boldsymbol{v}_{2}^{e}(\boldsymbol{\rho}) \\
\vdots \\
\boldsymbol{C}_{d} \boldsymbol{v}_{p}^{e}(\boldsymbol{\rho})
\end{array}\right]
$$

Em alguns casos é conveniente linearizar (2.39) em torno de uma resistividade qualquer. Isto é explicado a seguir.

\footnotetext{
${ }^{10}$ Os nós virtuais dos eletrodos são apresentados no Apêndice A.2.1e Apêndice A.2.2
} 


\subsection{Modelo linearizado de observação}

Expandindo (2.40) em série de Taylor em torno de uma resistividade $\rho_{0}$ qualquer 11 , tem-se

$$
\boldsymbol{v}_{j}(\boldsymbol{\rho})=\boldsymbol{v}_{j}\left(\boldsymbol{\rho}_{0}\right)+\left.\frac{\partial \boldsymbol{h}_{j}(\boldsymbol{\rho})}{\partial \boldsymbol{\rho}}\right|_{\boldsymbol{\rho}_{0}}\left(\boldsymbol{\rho}-\boldsymbol{\rho}_{0}\right)+\mathcal{O}\left[\left(\boldsymbol{\rho}-\boldsymbol{\rho}_{0}\right)^{2}\right]
$$

Desprezando os termos de ordem superior e substituindo (2.40), esta expansão fica

$$
\boldsymbol{v}_{j}(\boldsymbol{\rho})=\boldsymbol{v}_{j}\left(\boldsymbol{\rho}_{0}\right)+\left.\frac{\partial\left([\boldsymbol{Y}]^{-1} \boldsymbol{I}_{j}\right)}{\partial \boldsymbol{\rho}}\right|_{\boldsymbol{\rho}_{0}}\left(\boldsymbol{\rho}-\boldsymbol{\rho}_{0}\right)
$$

Usando o resultado da álgebra matricial para a derivada da inversa de uma matriz com respeito a um vetor [60, p. 8], tem-se

$$
\boldsymbol{v}_{j}(\boldsymbol{\rho})=\boldsymbol{v}_{j}\left(\boldsymbol{\rho}_{0}\right)+\left.\left(-\boldsymbol{Y}^{-1} \frac{\partial \boldsymbol{Y}}{\partial \boldsymbol{\rho}} \boldsymbol{Y}^{-1} \boldsymbol{I}_{j}\right)\right|_{\boldsymbol{\rho}_{0}}\left(\boldsymbol{\rho}-\boldsymbol{\rho}_{0}\right)
$$

O termo avaliado em $\boldsymbol{\rho}_{0}$ é a matriz jacobiana referente ao vetor de corrente $\boldsymbol{I}_{j}$ :

$$
\left.\tilde{\boldsymbol{H}}_{j}\right|_{\boldsymbol{\rho}_{0}}=\left.\left(-\boldsymbol{Y}^{-1} \frac{\partial \boldsymbol{Y}}{\partial \boldsymbol{\rho}} \boldsymbol{Y}^{-1} \boldsymbol{I}_{j}\right)\right|_{\boldsymbol{\rho}_{0}}=-\left.\left[\begin{array}{lll}
{\left[\boldsymbol{Y}^{-1} \frac{\partial \boldsymbol{Y}}{\partial \rho_{1}} \boldsymbol{Y}^{-1} \boldsymbol{I}_{j}\right]} & \ldots & {\left[\boldsymbol{Y}^{-1} \frac{\partial \boldsymbol{Y}}{\partial \rho_{n}} \boldsymbol{Y}^{-1} \boldsymbol{I}_{j}\right]}
\end{array}\right]\right|_{\boldsymbol{\rho}_{0}}
$$

Somente as linhas de $\left.\tilde{\boldsymbol{H}}_{j}\right|_{\rho_{0}}$ relacionadas com os nós virtuais da interface dos eletrodos precisam ser calculados, logo pode-se excluir de $\left.\tilde{\boldsymbol{H}}_{j}\right|_{\rho_{0}}$ as demais linhas. Após a remoção, a matriz Jacobiana torna-se, $\left.\tilde{\boldsymbol{H}}_{j}^{e}\right|_{\rho_{0}} \in \mathbb{R}^{e \times n}$ e o vetor de medidas $\boldsymbol{v}_{j}^{e}(\boldsymbol{\rho}) \in \mathbb{R}^{e}$.

A conversão de single-ended para diferencial é feita pré-multiplicando os potenciais por $\boldsymbol{C}_{d}$

$$
\boldsymbol{v}_{j}^{e, d i f}(\boldsymbol{\rho})=\boldsymbol{C}_{d} \boldsymbol{v}_{j}^{e}(\boldsymbol{\rho})=\boldsymbol{C}_{d} \boldsymbol{v}_{j}^{e}\left(\boldsymbol{\rho}_{0}\right)+\left.\boldsymbol{C}_{d} \tilde{\boldsymbol{H}}_{j}^{e}\right|_{\boldsymbol{\rho}_{0}}\left(\boldsymbol{\rho}-\boldsymbol{\rho}_{0}\right)
$$

Considerando todos os $p$ vetores de corrente utilizados, pode-se agrupar todas as matrizes jacobianas $\left.\boldsymbol{C}_{d} \tilde{\boldsymbol{H}}_{j}^{e}\right|_{\rho_{0}}$ e todos os vetores de potenciais elétricos $\boldsymbol{C}_{d} \boldsymbol{v}_{j}^{e}\left(\boldsymbol{\rho}_{0}\right)$ e

\footnotetext{
${ }^{11}$ Aqui considera-se que $\rho \in \mathbb{R}^{n}$, ou seja, os parâmetros dos eletrodos não são incógnitas. Caso sejam, a dedução é idêntica, apenas mudando-se as dimensões das matrizes.
} 
escrever (2.44) da seguinte maneira:

$$
\boldsymbol{v}^{e, d i f}(\boldsymbol{\rho})=\boldsymbol{v}^{e, d i f}\left(\boldsymbol{\rho}_{0}\right)+\left.\boldsymbol{H}^{e, d i f}\right|_{\boldsymbol{\rho}_{0}}\left(\boldsymbol{\rho}-\boldsymbol{\rho}_{0}\right),
$$

onde

$$
\boldsymbol{v}^{e, d i f}\left(\boldsymbol{\rho}_{0}\right)=\left[\begin{array}{c}
\boldsymbol{C}_{d} \boldsymbol{v}_{1}^{e}\left(\boldsymbol{\rho}_{0}\right) \\
\boldsymbol{C}_{d} \boldsymbol{v}_{2}^{e}\left(\boldsymbol{\rho}_{0}\right) \\
\vdots \\
\boldsymbol{C}_{d} \boldsymbol{v}_{p}^{e}\left(\boldsymbol{\rho}_{0}\right)
\end{array}\right],\left.\quad \boldsymbol{H}^{e, d i f}\right|_{\boldsymbol{\rho}_{0}}=\left[\begin{array}{c}
\left.\boldsymbol{C}_{d} \tilde{\boldsymbol{H}}_{1}^{e}\right|_{\rho_{0}} \\
\left.\boldsymbol{C}_{d} \tilde{\boldsymbol{H}}_{2}^{e}\right|_{\rho_{0}} \\
\vdots \\
\left.\boldsymbol{C}_{d} \tilde{\boldsymbol{H}}_{p}^{e}\right|_{\rho_{0}}
\end{array}\right]
$$

$\left.\operatorname{com} \boldsymbol{H}^{e, d i f}\right|_{\boldsymbol{\rho}_{0}} \in \mathbb{R}^{\ell \times n}$ e $\boldsymbol{v}^{e, d i f}(\boldsymbol{\rho}) \in \mathbb{R}^{\ell}$. Isolando o termo $\left.\boldsymbol{H}^{e, d i f}\right|_{\boldsymbol{\rho}_{0}} \boldsymbol{\rho}$ na direita

$$
\boldsymbol{v}^{e, d i f}(\boldsymbol{\rho})-\boldsymbol{v}^{e, d i f}\left(\boldsymbol{\rho}_{0}\right)+\left.\boldsymbol{H}^{e, d i f}\right|_{\boldsymbol{\rho}_{0}} \boldsymbol{\rho}_{0}=\left.\boldsymbol{H}^{e, d i f}\right|_{\boldsymbol{\rho}_{0}} \boldsymbol{\rho}
$$

e definindo uma medida nominal $\boldsymbol{y}^{\prime}$

$$
\boldsymbol{y}^{\prime}(\boldsymbol{\rho})=\boldsymbol{v}^{e, d i f}(\boldsymbol{\rho})-\boldsymbol{v}^{e, d i f}\left(\boldsymbol{\rho}_{0}\right)+\left.\boldsymbol{H}^{e, d i f}\right|_{\boldsymbol{\rho}_{0}} \boldsymbol{\rho}_{0}
$$

obtém-se o modelo linearizado de observação

$$
\boldsymbol{y}^{\prime}(\boldsymbol{\rho})=\left.\boldsymbol{H}^{e, d i f}\right|_{\boldsymbol{\rho}_{0}} \boldsymbol{\rho}
$$

Para facilitar a notação, daqui em diante $\left.\boldsymbol{H}^{e, d i f}\right|_{\boldsymbol{\rho}_{0}}$ será denotado por $\boldsymbol{H}$ ou por $\boldsymbol{H}\left(\boldsymbol{\rho}_{0}\right)$ quando for necessário explicitar o ponto de linearização 12

\footnotetext{
${ }^{12}$ Por simplificação de notação, $\boldsymbol{y}^{\prime}$ será também denotada por $\boldsymbol{y}$, porém nestes casos o leitor será informado.
} 


\subsection{Fantoma numérico}

Uma parte dos ensaios utiliza dados gerados numericamente para simular a aquisição dos dados elétricos em um indivíduo.

A simulação da aquisição consiste em gerar uma malha de elementos finitos, escolher uma distribuição de resistividade no domínio e parâmetros de eletrodos $\rho^{\text {sim. }}$, resolver o problema direto para todos os vetores de injeção de corrente e armazenar apenas os valores dos potenciais elétricos dos nós que representam a parte metálica dos eletrodos. Em outras palavras, resolve-se (2.41). Por último, adiciona-se um ruído gaussiano não correlacionado no tempo nos potenciais elétricos obtidos para simular os erros de medição. Nesta tese o ruído tem média zero e desvio padrão de $1 \mathrm{mV}$.

O vetor resultante $\boldsymbol{y}\left(\boldsymbol{\rho}^{\text {sim. }}\right)$ contém as medidas simuladas. As medidas são então utilizadas nos filtros de Kalman e os resultados podem ser então comparados com $\boldsymbol{\rho}^{\text {sim. }}$. Por conta da natureza Gaussiana da densidade de probabilidade condicionada às medidas proveniente do filtro de Kalman, utiliza-se a média desta distribuição como estimativa pontual para comparações.

A fim de minimizar o efeito da discretização do domínio, a malha utilizada na solução do problema direto deve ser substancialmente mais refinada do que a malha utilizada na solução do problema inverso. O uso de duas malhas também reduz o efeito do crime de inversãd 13 .

A corrente injetada e os potenciais medidos seguem o padrão pula-3, como descrito na Seção 2.3 em todos os ensaios numéricos. Doravante, o processo descrito será denominado fantoma numérico.

\footnotetext{
${ }^{13}$ Crimes de inversão consistem em tornar o problema inverso menos mal-posto adicionando informação artificial a este. Eles ocorrem quando o modelo adotado e a realidade coincidem [61, p. 5]. Na prática ele ocorre principalmente em duas situações: (1) Os dados gerados através da solução do problema direto são produzidos com o mesmo modelo utilizado para a solução do problema inverso sendo que este modelo difere da realidade e (2) quando a discretização na simulação numérica é a mesma usada na solução do problema inverso.
} 


\section{Capítulo 3}

\section{Problema mal-posto}

Sob o ponto de vista matemático, a TIE procura resolver um problema inverso, mal-posto e não linear. Em apenas poucas situações são conhecidas fórmulas fechadas para a solução das equações envolvidas no problema, para as demais são necessários métodos numéricos para sua solução. Na TIE, a principal fonte de dificuldades é a natureza mal-posta [62] do problema inverso:

Considere os espaços métricos $\left(X, d_{x}\right)$ e $\left(Y, d_{y}\right)$ e o operador $A: X \mapsto Y$. Então o problema de encontrar $x \in X$, dado $y \in Y$, tal que

$$
A(x)=y
$$

é dito bem posto se $A$ tem uma inversa contínua $A^{-1}: Y \mapsto X$. Em outras palavras, um problema é bem posto se e somente se cumpre as três condições de Hadamard:

1. A é sobrejetor: para $y \in Y$, existe $x \in X$ tal que $A(x)=y$;

2. $A$ é injetor: para $y \in Y$ e $A\left(x_{1}\right)=A\left(x_{2}\right)=y$, então $x_{1}=x_{2}$;

3. A solução tem uma dependência contínua (suave) com os dados de entrada: para todo $\epsilon>0$, existe $\delta(\epsilon, y)>0$ tal que $d_{y}\left(y, y_{1}\right)<\delta$ implica que $d_{x}\left(x, x_{1}\right)<\epsilon$.

Note que não é suficiente a existência da inversa de $A$ para que o problema seja bem posto. No caso da TIE, a injetividade de $A$ foi demonstrada em algumas situações. Astala e Päivärinta [63] mostraram a injetividade de $A \operatorname{com} \sigma \in L^{\infty}(\boldsymbol{\Omega})$ sobre os reais e $\Omega \in \mathbb{R}^{2}$. Para o caso complexo, Francini [64] mostra a injetividade se $\Omega \in \mathbb{R}^{2}$ for Lipschitz-contínua e $\sigma, \epsilon \in W^{2, \infty}$. Ainda neste trabalho, a autora faz uma ressalva 
dizendo que a parte complexa precisa ser pequena mas não fica claro o motivo de tal afirmação. Uma hipótese para tal afirmação é o uso de uma linearização da matriz de potencial em torno de $\epsilon=0$. Para $\Omega \in \mathbb{R}^{n}, n \geq 3$, o resultado mais abrangente é de Brown e Torres [65] para valores reais, onde $\sigma \in W^{3 / 2, p}(\Omega), p>2 n$.

Estes resultados foram obtidos para problemas isotrópicos. No caso de materiais anisotrópicos, foi demonstrado que não existe injetividade, ou seja, duas distribuições de condutividade (real ou complexa) diferentes, podem resultar em um mesmo conjunto de medidas em $\partial \boldsymbol{\Omega}$ [66]. Para mais detalhes sobre estes e outros casos, entre eles a possibilidade de "invisibilidade", ver o artigo de revisão Uhlmann [67].

Em princípio, o problema da injetividade pode ser resolvido ao definir o espaço quociente $\tilde{X}=X / \operatorname{ker}(A)$ e definir um operador $\tilde{A}: \tilde{X} \rightarrow Y$ como $\tilde{A}[x]=A x$ pois todos os elementos do núcleo de $A$ são "unidos" no espaço quociente. A sobrejeção poderia ser resolvida ao substituir $Y$ pela imagem de $\tilde{A}$, apesar de que este espaço não é completo e uma medida realizada $y_{\text {med }} \in L^{2}$, que é um espaço completo, pode estar fora da imagem $\tilde{A}[x]$. Neste caso, $\tilde{A}[x] \neq y_{\text {med }}$ para todo $[x] \in \tilde{X}$. Das três condições de Hadamard, a mais difícil de ser tratada é a terceira, que versa sobre a continuidade da inversa de $A$. É possível mostrar que $A^{-1}$ não é contínua no problema não linear da TIE através de uma generalização de um teorema para operadores lineares que atesta que se $X$ e $Y$ são espaços normados e $X$ tem dimensão infinita, então o operador bijetor compacto $A$ não apresenta inversa contínua. Para detalhes sobre o teorema para problemas não lineares, ver Engl et al. [68].

Diz-se resolver o problema direto quando se deseja encontrar $y$, conhecendo-se $A$ e $x$. Já o problema inverso é resolvido quando se conhece $y$ e a função $A$ e procura-se encontrar $x$. Sob o ponto de vista da teoria de controle, pode-se ver (3.1) como uma equação de observação de estado, onde $x$ é o vetor de estado e $y$ é o vetor de medidas dos sensores e $A$ é o modelo de observação. Neste caso, o problema direto consiste em determinar as medidas dos sensores a partir de um estado conhecido, ou seja, determinar as consequências (medidas) a partir das causas (estado e modelo de observação). O problema inverso é resolvido quando se conhece o vetor de medidas e determina-se o estado que geram as medidas, ou seja, a partir das consequências (medidas e modelo de observação), determinar parte das causas (o estado). $\mathrm{O}$ problema inverso costuma ser mal-posto. 
Sob um ponto de vista puramente matemático, problemas mal-postos em espaços de dimensão infinita, quando discretizados e dispostos em forma matricial $\boldsymbol{A x}=$ $\boldsymbol{y}$, apresentam solução de mínima norma estável e, portanto, são bem-postos (regularização por discretização). Entretanto, ao se resolver numericamente tais problemas, os erros de arredondamento causam resultados desastrosos devido ao mau condicionamento da matriz $\boldsymbol{A}$. Tais problemas, comumente chamados problemas mal-postos discretos, apresentam características similares aos dos problemas mal-postos em dimensão infinita, como apresentado ao longo de Kaipio e Somersalo [61], Hansen [69, 70].

Um problema discreto é dito mal-posto se

1. os valores singulares da matriz $\boldsymbol{A}$ decaírem para zero, ou quase zero, de forma gradual;

2. o número de condição da matriz $\boldsymbol{A}$ for grande.

Estes comportamentos podem ser facilmente visualizados utilizando a decomposição em valores singulares (SVD, do inglês singular value decomposition) do sistema $\boldsymbol{A} \boldsymbol{x}=\boldsymbol{y}, \boldsymbol{A} \in \mathbb{R}^{m \times n}, \boldsymbol{x} \in \mathbb{R}^{n}$ e $\boldsymbol{y} \in \mathbb{R}^{m}$.

A matriz $\boldsymbol{A}$ com posto $r \leq \min (m, n)$ pode ser decomposta em um sistema de valores singulares

$$
\boldsymbol{A}=\sum_{i=1}^{r} \boldsymbol{u}_{i} \sigma_{i} \boldsymbol{v}_{i}^{\mathrm{T}}
$$

onde $\boldsymbol{u}_{i} \in \mathbb{R}^{m}$ e $\boldsymbol{v}_{i} \in \mathbb{R}^{n}$ são vetores singulares de $\boldsymbol{A}$, ortonormais em seus espaços e $\sigma_{1} \geq \sigma_{2} \geq \ldots \sigma_{r}>0$ são os valores singulares associados. Os vetores singulares apresentam mais oscilações em sua forma com o aumento do índice $i$ [69, p. 8].

Com simples substituição, nota-se que

$$
\boldsymbol{A} \boldsymbol{v}_{j}=\sum_{i=1}^{r} \boldsymbol{u}_{i} \sigma_{i} \boldsymbol{v}_{i}^{\mathrm{T}} \boldsymbol{v}_{j}=\sigma_{j} \boldsymbol{u}_{j} \quad \Rightarrow \quad\left\|\boldsymbol{A} \boldsymbol{v}_{j}\right\|_{2}=\sigma_{j}
$$

o que mostra que se um determinado valor singular $\sigma_{j}$ se aproxima de zero, $\boldsymbol{v}_{j}$ se aproxima do núcleo de $\boldsymbol{A}$. Esta matriz apresenta, portanto, posto numericamente incompleto se $\sigma_{j}$ for suficientemente próximo de zero. Para tentar contornar este 
problema, pode-se truncar a SVD em um índice $s<r$ tal que $\sigma_{s+1}<\epsilon$, para algum $\epsilon>0$ com o intuito de encontrar uma aproximação $\tilde{A}$ da matriz original $\boldsymbol{A}$ com posto menor, porém bem condicionado

$$
\tilde{\boldsymbol{A}}=\sum_{i=1}^{s} \boldsymbol{u}_{i} \sigma_{i} \boldsymbol{v}_{i}^{\mathrm{T}}
$$

Porém, se os valores singulares decaírem para zero de forma suave, tal truncamento não produzirá um operador $\tilde{\boldsymbol{A}}$ em espaço de dimensão menor que seja similar a $\boldsymbol{A}$ original pois a soma dos termos associados aos índices $s+1, \ldots, r$ não considerados em $\tilde{A}$ não será desprezível. Esta é uma das maiores críticas dos métodos de solução de problemas mal-postos utilizando a decomposição truncada em valores singulares.

Continuando com a SVD, pode-se escrever o vetor $\boldsymbol{y}$ em função dos vetores da base ortogonal $\left\{\boldsymbol{u}_{i}\right\}_{1}^{r}$ da decomposição

$$
\boldsymbol{y}=\sum_{i=1}^{r}\left(\boldsymbol{u}_{i}^{\mathrm{T}} \boldsymbol{y}\right) \boldsymbol{u}_{i}+\boldsymbol{y}_{0}
$$

onde $\boldsymbol{y}_{0}$ é uma possível componente ortogonal ao espaço gerado por $\left\{\boldsymbol{u}_{i}\right\}_{1}^{r}$.

Pode-se também calcular a pseudoinversa de $\boldsymbol{A}$ usando a decomposição

$$
\boldsymbol{A}^{\dagger}=\sum_{i=1}^{r} \boldsymbol{v}_{i} \sigma_{i}^{-1} \boldsymbol{u}_{i}^{\mathrm{T}}
$$

A solução de $\boldsymbol{A} \boldsymbol{x}=\boldsymbol{y}$ pode ser então escrita

$$
\begin{aligned}
\boldsymbol{x}=\boldsymbol{A}^{\dagger} \boldsymbol{y} & =\left(\sum_{i=1}^{r} \boldsymbol{v}_{i} \sigma_{i}^{-1} \boldsymbol{u}_{i}^{\mathrm{T}}\right)\left(\sum_{j=1}^{r}\left(\boldsymbol{u}_{j}^{\mathrm{T}} \boldsymbol{y}\right) \boldsymbol{u}_{j}+\boldsymbol{y}_{0}\right) \\
& =\left(\sum_{i=1}^{r} \boldsymbol{v}_{i} \sigma_{i}^{-1} \boldsymbol{u}_{i}^{\mathrm{T}}\right)\left(\sum_{j=1}^{r} \boldsymbol{u}_{j}\left(\boldsymbol{u}_{j}^{\mathrm{T}} \boldsymbol{y}\right)\right)+\left(\sum_{i=1}^{r} \boldsymbol{v}_{i} \sigma_{i}^{-1} \boldsymbol{u}_{i}^{\mathrm{T}}\right) \boldsymbol{y}_{0} .
\end{aligned}
$$

Usando agora a informação de que $\boldsymbol{y}_{0} \perp \boldsymbol{u}_{i}$ e a ortonormalidade da base $\left\{\boldsymbol{u}_{i}\right\}_{1}^{r}$

$$
\boldsymbol{x}=\sum_{i=1}^{r} \boldsymbol{v}_{i} \sigma_{i}^{-1}\left(\boldsymbol{u}_{i}^{\mathrm{T}} \boldsymbol{y}\right)
$$


Para entender o problema de sensibilidade das medidas, considere agora um vetor de medidas contendo erro $\boldsymbol{y}^{\delta}=\boldsymbol{y}+\boldsymbol{\delta} \boldsymbol{y}$. Substituindo na solução do problema tem-se

$$
\begin{aligned}
\boldsymbol{x}^{\delta}=\sum_{i=1}^{r} \boldsymbol{v}_{i} \sigma_{i}^{-1}\left(\boldsymbol{u}_{i}^{\mathrm{T}} \boldsymbol{y}^{\delta}\right) & =\sum_{i=1}^{r} \boldsymbol{v}_{i} \sigma_{i}^{-1}\left(\boldsymbol{u}_{i}^{\mathrm{T}} \boldsymbol{y}\right)+\sum_{i=1}^{r} \boldsymbol{v}_{i} \sigma_{i}^{-1}\left(\boldsymbol{u}_{i}^{\mathrm{T}} \boldsymbol{\delta} \boldsymbol{y}\right) \\
& =\boldsymbol{x}+\sum_{i=1}^{r} \boldsymbol{v}_{i} \sigma_{i}^{-1}\left(\boldsymbol{u}_{i}^{\mathrm{T}} \boldsymbol{\delta} \boldsymbol{y}\right)=\boldsymbol{x}+\boldsymbol{\delta} \boldsymbol{x}
\end{aligned}
$$

onde $\boldsymbol{x}^{\delta}=\boldsymbol{x}+\boldsymbol{\delta} \boldsymbol{x}$ é a solução utilizando o vetor de medidas $\boldsymbol{y}^{\delta}$ e $\boldsymbol{x}$ é a solução no caso onde não há erros de medida.

Se o erro $\boldsymbol{\delta} \boldsymbol{y}$ não for ortogonal a $\left\{\boldsymbol{u}_{i}\right\}_{1}^{r}$, o que é o caso geral, as suas projeções $\left(\boldsymbol{u}_{i}^{\mathrm{T}} \boldsymbol{\delta} \boldsymbol{y}\right)$ serão multiplicadas por $\sigma_{i}^{-1}$. Se o valor absoluto destas projeções convergirem para zero mais rapidamente do que $\sigma_{i}$, o ruído não acarretará muitos problemas, mas se $\sigma_{i}$ convergir mais rapidamente para zero, estas projeções serão amplificadas e a solução será fortemente influenciada pelo ruído. Este critério de análise da sensibilidade é chamado Critério de Picard.

Lembrando que $\boldsymbol{v}_{i}$ apresenta forma mais oscilatória conforme $\sigma_{i}$ tende a zero, a solução $\boldsymbol{x}^{\delta}$ apresentará ruído de alta frequência dominando a solução. A relação com o número de condição na norma euclidiana, $\kappa(\boldsymbol{A})=\|\boldsymbol{A}\|_{2} \cdot\left\|\boldsymbol{A}^{\dagger}\right\|_{2}=\frac{\sigma_{1}}{\sigma_{r}} \geq 1$ pode ser vista quando se calcula a norma do erro relativo da solução [71, p. 60], considerando perturbações em $\boldsymbol{y}$ e em $\boldsymbol{A}$

$$
\frac{\|\boldsymbol{\delta} \boldsymbol{x}\|_{2}}{\|\boldsymbol{x}\|_{2}} \leq \frac{\kappa(\boldsymbol{A})}{1-\kappa(\boldsymbol{A}) \frac{\|\boldsymbol{\delta} \boldsymbol{A}\|_{2}}{\|\boldsymbol{A}\|_{2}}}\left(\frac{\|\boldsymbol{\delta} \boldsymbol{y}\|_{2}}{\|\boldsymbol{y}\|_{2}}+\frac{\|\boldsymbol{\delta} \boldsymbol{A}\|_{2}}{\|\boldsymbol{A}\|_{2}}\right)
$$

onde $\boldsymbol{\delta} \boldsymbol{A}$ é uma perturbação na matriz $\boldsymbol{A}$. Para $\boldsymbol{\delta} \boldsymbol{A}=\mathbf{0}$

$$
\frac{\|\boldsymbol{\delta} \boldsymbol{x}\|_{2}}{\|\boldsymbol{x}\|_{2}} \leq \kappa(\boldsymbol{A}) \frac{\|\boldsymbol{\delta} \boldsymbol{y}\|_{2}}{\|\boldsymbol{y}\|_{2}}
$$

Este resultado mostra que erros em $\boldsymbol{y}$ são amplificados por $\kappa(\boldsymbol{A})$ na solução $\boldsymbol{x}^{\delta}$. Em sistemas em que $\sigma_{r}$ tende a zero, o número de condição $\kappa(\boldsymbol{A})$ tende a infinito, que é o caso de sistemas mal-postos discretos.

Uma vasta literatura a respeito de problemas inversos mal-postos pode ser encontrada, como Ramm [72], Aster et al. [73]. A forma mais utilizada para resolver o problema mal-posto é pelo uso de regularizadores. Isto é apresentado a seguir 
para o caso de problemas lineares mal-postos. Para a análise de regularizadores em problemas não lineares, ver Kaltenbacher et al. [74], Engl et al. [75].

\subsection{Regularizadores}

Em função do conjunto de valores singulares de $\boldsymbol{A}$ próximos de zero, a solução do sistema $\boldsymbol{A x}=\boldsymbol{y}$ é numericamente indeterminada. É necessário então adicionar outras informações a respeito da solução desejada de forma a estabilizar o problema e extrair uma solução útil.

A forma mais usual de adicionar informação e regularizar o problema é requerer que a solução apresente uma norma, ou seminorma, pequena. Desta forma, procura-se uma solução do sistema que minimize também

$$
J(\boldsymbol{x})=\left\|\boldsymbol{L}_{r}\left(\boldsymbol{x}-\boldsymbol{x}^{*}\right)\right\|_{2}^{2},
$$

onde $\boldsymbol{L}_{r} \in \mathbb{R}^{q \times n}, q \leq n$ possui posto $q$ e $\boldsymbol{x}^{*}$ é um vetor opcional, contendo uma estimativa inicial da solução do problema. Tal norma deve ser convenientemente escolhida e depende do tipo de problema que se quer resolver.

Duas regularizações bastante comuns são

1. $\boldsymbol{L}_{r}$ é a matriz identidade $\mathbb{I}_{n}$. Neste caso, o vínculo torna-se $\left\|\boldsymbol{x}-\boldsymbol{x}^{*}\right\|_{2}^{2}$, que penaliza soluções $\boldsymbol{x}$ distantes de $\boldsymbol{x}^{*}$;

2. $\boldsymbol{L}_{r}$ é um filtro passa altas espacial e $\boldsymbol{x}^{*}=\mathbf{0}$. Neste caso, o vínculo torna-se $\left\|\boldsymbol{L}_{r} \boldsymbol{x}\right\|_{2}^{2}$, que penaliza soluções não suaves.

Ao adicionar o vínculo $J(\boldsymbol{x})$ no problema, a sua solução não será mais uma que garante a igualdade $\boldsymbol{A x}=\boldsymbol{y}$, mas uma que balanceie o resultado entre uma solução que minimize o resíduo $\|\boldsymbol{A} \boldsymbol{x}-\boldsymbol{y}\|_{2}$ e uma que minimize $J(\boldsymbol{x})$. Dentro desta classe de regularizadores, a mais utilizada é a regularização generalizada de Tikhonov. A idéia é aceitar como solução aproximada do problema original o vetor $\boldsymbol{x}_{\alpha}$ dado por

$$
\boldsymbol{x}_{\alpha}=\arg \min _{\boldsymbol{x}}\left\{\|\boldsymbol{A} \boldsymbol{x}-\boldsymbol{y}\|_{2}^{2}+\alpha^{2}\left\|\boldsymbol{L}_{r}\left(\boldsymbol{x}-\boldsymbol{x}^{*}\right)\right\|_{2}^{2}\right\}
$$

onde $\alpha>0$ é chamado parâmetro de regularização e tem o papel de balancear o peso 
de cada termo na solução final. Grandes valores de $\alpha$ favorecem soluções em que minimizar $J(\boldsymbol{x})$ é mais importante do que minimizar o resíduo $\|\boldsymbol{A} \boldsymbol{x}-\boldsymbol{y}\|_{2}$ e vice-versa. O parâmetro de regularização $\alpha$ e $\boldsymbol{L}_{r}$ ainda controlam a sensibilidade da solução regularizada $\boldsymbol{x}_{\alpha}$ em relação a perturbações em $\boldsymbol{A}$ e $\boldsymbol{y}$. Em Hansent1 e Hansen 2 apud Hansen [70] mostra-se que o limite superior do erro relativo da solução regularizada perturbada é proporcional à $1 / \alpha^{2}$ e ao inverso do menor valor singular de $\boldsymbol{L}_{r}$.

Um ponto importante deve ser observado. A solução $\boldsymbol{x}_{\alpha}$ de (3.13) só será única, ou estável, se $\operatorname{ker}(\boldsymbol{A}) \cap \operatorname{ker}\left(\boldsymbol{L}_{r}\right)=\{\mathbf{0}\}$. Isto pode ser facilmente observado na equação anterior: Seja $\boldsymbol{x}_{\alpha}$ a solução de (3.13) e $\boldsymbol{v} \neq \mathbf{0}$ um vetor nos núcleos 3 de $\boldsymbol{A}$ e $\boldsymbol{L}_{r}$ simultaneamente, então qualquer combinação linear $\boldsymbol{x}_{\alpha}+c \boldsymbol{v}, \forall c \in \mathbb{R}$ também será solução do problema e, portanto, o problema continua mal-posto.

Garantidas as condições apresentadas, a solução de (3.13) é

$$
\boldsymbol{x}_{\alpha}=\left(\boldsymbol{A}^{\mathrm{T}} \boldsymbol{A}+\alpha^{2} \boldsymbol{L}_{r}^{\mathrm{T}} \boldsymbol{L}_{r}\right)^{-1}\left(\boldsymbol{A}^{\mathrm{T}} \boldsymbol{y}+\alpha^{2} \boldsymbol{L}_{r}^{\mathrm{T}} \boldsymbol{L}_{r} \boldsymbol{x}^{*}\right)
$$

e sua análise é feita com a decomposição generalizada em valores singulares (GSVD, do inglês generalized singular value decomposition) do par $\left(\boldsymbol{A}, \boldsymbol{L}_{r}\right)$ a ser apresentada a seguir.

A escolha do parâmetro de regularização é crítica para a solução do problema. Uma vez que esta escolha é realizada antes da solução do problema, esta deve ser feita utilizado métodos que utilizem informação a priori a respeito do problema.

Dentre os métodos para a seleção, cita-se o princípio da discrepância de Morozov, que atesta que não é razoável esperar uma norma do resíduo $\left\|\boldsymbol{A} \boldsymbol{x}_{\alpha}-\boldsymbol{y}\right\|_{2}$ menor que a norma esperada do erro de medida $\nu$. Desta forma, soluções aceitáveis são aquela em que

$$
\left\|\boldsymbol{A} \boldsymbol{x}_{\alpha}-\boldsymbol{y}\right\|_{2} \leq \nu
$$

ou seja, a solução não deve tentar ajustar as medidas mais precisamente do que o nível de ruído. O parâmetro de regularização é calculado ao resolver (3.15) no caso

\footnotetext{
${ }^{1}$ P. C. Hansen. Regularization, gsvd and truncated gsvd. BIT, 29(3):491-504, august 1989. ISSN 0006-3835. doi: http:/ /dx.doi.org/10.1007/BF02219234.

${ }^{2}$ P. C. Hansen. Perturbation bounds for discrete tikhonov regularisation. Inverse Problems, 5(4):L41-L44, august 1998. doi: 10.1088/0266-5611/5/4/002.

${ }^{3} \mathrm{Ou}$ no $\varepsilon$-núcleo numérico, apresentado em Hansen [69, p. 45].
} 
de igualdade com $\nu$ com o uso de (3.14).

A escolha do nível de ruído $\nu$ pode ser feita considerando as estatísticas esperadas do ruído. Em princípio existem diversos possíveis candidatos [61, p. 20], como $\nu=E\left\{\|\boldsymbol{\delta} \boldsymbol{y}\|_{2}\right\}$ ou $\nu^{2}=E\left\{\|\boldsymbol{\delta} \boldsymbol{y}\|_{2}^{2}\right\}$, que avaliados para um erro aleatório do tipo Gaussiano $\delta \boldsymbol{y} \sim \mathcal{N}\left(\mathbf{0}, \sigma^{2} \mathbb{I}\right)$ resultam em valores proporcionais a $\sigma$. Para mais critérios para a escolha do parâmetro de regularização, ver Hansen [69, cap. 7].

\subsection{Análise da solução regularizada}

Sejam $\boldsymbol{A} \in \mathbb{R}^{m \times n}$ e $\boldsymbol{L}_{r} \in \mathbb{R}^{q \times n}$, satisfazendo $m \geq n \geq q$, $\operatorname{ker}(\boldsymbol{A}) \cap \operatorname{ker}\left(\boldsymbol{L}_{r}\right)=\{\mathbf{0}\}$ e que o posto de $\boldsymbol{L}_{r}$ é $q$. A GSVD do par $\left(\boldsymbol{A}, \boldsymbol{L}_{r}\right)$ é dada por

$$
\begin{aligned}
& \boldsymbol{A}=\boldsymbol{U}\left(\begin{array}{cc}
\boldsymbol{\Sigma} & 0 \\
\mathbf{0} & \mathbb{I}_{n-q}
\end{array}\right) \boldsymbol{X}^{-1} \\
& \boldsymbol{L}_{r}=\boldsymbol{V}\left(\begin{array}{ll}
\boldsymbol{M} & \mathbf{0}
\end{array}\right) \boldsymbol{X}^{-1}
\end{aligned}
$$

onde as colunas de $\boldsymbol{U} \in \mathbb{R}^{m \times n}$ e $\boldsymbol{V} \in \mathbb{R}^{q \times q}$ são ortonormais, $\boldsymbol{X} \in \mathbb{R}^{n \times n}$ não é singular e possui colunas ortogonais em relação à $\boldsymbol{A}^{\mathrm{T}} \boldsymbol{A}$ e as matrizes $\boldsymbol{\Sigma}=\operatorname{diag}\left(\sigma_{1}, \sigma_{2}, \ldots, \sigma_{q}\right) \in \mathbb{R}^{q \times q}$ e $\boldsymbol{M}=\operatorname{diag}\left(\mu_{1}, \mu_{2}, \ldots, \mu_{q}\right) \in \mathbb{R}^{q \times q}$ são tais que

$$
\begin{aligned}
& 0 \leq \sigma_{1} \leq \ldots \leq \sigma_{q} \leq 1, \quad 1 \geq \mu_{1} \geq \ldots \geq \mu_{q}>0 \\
& \sigma_{i}^{2}+\mu_{i}^{2}=1
\end{aligned}
$$

Os valores singulares generalizados do $\operatorname{par}\left(\boldsymbol{A}, \boldsymbol{L}_{r}\right)$ são definidos por

$$
\gamma_{i}=\frac{\sigma_{i}}{\mu_{i}}
$$

aparecendo em ordem não decrescente. Mostra-se que $\left(\boldsymbol{A}, \boldsymbol{L}_{r}\right)$ são diagonalizados se escritos na base $\boldsymbol{U}, \boldsymbol{V} e \boldsymbol{X}$.

Observando (3.17), nota-se que as últimas $n-q$ colunas de $\boldsymbol{X}$ formam uma base para o núcleo de $\boldsymbol{L}_{r}$. Ainda, se $L_{r}=\mathbb{I}$, a GSVD do par é idêntica à SVD de $\boldsymbol{A}$. 
Em Hansent apud Hansen [70] mostra-se que em geral a matriz $\boldsymbol{X}$ é aproximadamente tão bem condicionada quanto $\boldsymbol{L}_{r}$ e que os elementos de $\boldsymbol{\Sigma}$ mostram o mau condicionamento de $\boldsymbol{A}$.

Substituindo (3.16) e (3.17) em (3.14) e assumindo $\boldsymbol{x}^{*}=\mathbf{0}$ por simplicidade

$$
\begin{aligned}
& \boldsymbol{x}_{\alpha}=\left(\boldsymbol{X}\left(\begin{array}{cc}
\Sigma^{2} & \mathbf{0} \\
\mathbf{0} & \mathbb{I}_{n-p}
\end{array}\right) \boldsymbol{X}^{-1}+\boldsymbol{X}\left(\begin{array}{cc}
\boldsymbol{\alpha}^{2} \boldsymbol{M}^{2} & \mathbf{0} \\
\mathbf{0} & \mathbf{0}
\end{array}\right) \boldsymbol{X}^{-1}\right)^{-1} \boldsymbol{X}\left(\begin{array}{cc}
\boldsymbol{\Sigma} & \mathbf{0} \\
\mathbf{0} & \mathbb{I}_{n-p}
\end{array}\right) \boldsymbol{U}^{\mathrm{T}} \boldsymbol{y} \\
& =\left(\boldsymbol{X}\left(\begin{array}{cc}
\boldsymbol{\Sigma}^{2}+\alpha^{2} \boldsymbol{M}^{2} & \mathbf{0} \\
\mathbf{0} & \mathbb{I}_{n-p}
\end{array}\right) \boldsymbol{X}^{-1}\right)^{-1} \boldsymbol{X}\left(\begin{array}{cc}
\boldsymbol{\Sigma} & \mathbf{0} \\
\mathbf{0} & \mathbb{I}_{n-p}
\end{array}\right) \boldsymbol{U}^{\mathrm{T}} \boldsymbol{y} .
\end{aligned}
$$

Usando agora a propriedade matricial $(\boldsymbol{A B})^{-1}=\boldsymbol{B}^{-1} \boldsymbol{A}^{-1}$ duas vezes e simplificando, chega-se a

$$
\boldsymbol{x}_{\alpha}=\boldsymbol{X}\left(\begin{array}{cc}
\frac{\boldsymbol{\Sigma}}{\boldsymbol{\Sigma}^{2}+\alpha^{2} \boldsymbol{M}^{2}} & \mathbf{0} \\
\mathbf{0} & \mathbb{I}_{n-q}
\end{array}\right) \boldsymbol{U}^{\mathrm{T}} \boldsymbol{y}=\sum_{i=1}^{q} \frac{\sigma_{i}^{2}}{\sigma_{i}^{2}+\alpha^{2} \mu_{i}^{2}} \frac{\left(\boldsymbol{u}_{i}^{\mathrm{T}} \boldsymbol{y}\right)}{\sigma_{i}} \boldsymbol{x}_{i}+\sum_{i=q+1}^{n}\left(\boldsymbol{u}_{i}^{\mathrm{T}} \boldsymbol{y}\right) \boldsymbol{x}_{i}
$$

onde a segunda somatória contém a parcela da solução não regularizada, pois está no núcleo do operador $\boldsymbol{L}_{r}$. Os componentes da parcela regularizada são agora multiplicadas por um termo $\frac{\sigma_{i}^{2}}{\sigma_{i}^{2}+\alpha^{2} \mu_{i}^{2}}=\frac{\gamma_{i}^{2}}{\gamma_{i}^{2}+\alpha^{2}}$ que converge para zero suavemente, conforme a assíntota $\gamma_{i}^{2} / \alpha^{2}$, filtrando a solução.

${ }^{4}$ P. C. Hansen. Regularization, gsvd and truncated gsvd. BIT, 29(3):491-504, august 1989. ISSN 0006-3835. doi: http:/ /dx.doi.org/10.1007/BF02219234. 


\section{Capítulo 4}

\section{Inversão Bayesiana e Filtros de Kalman}

A TIE depende de potenciais elétricos e correntes, medidos nos eletrodos posicionados ao longo do bordo do domínio para determinar uma estimativa da distribuição de resitividade elétrica em seu interior. Para tal, a solução do problema direto associado se faz necessária, geralmente utilizando-se uma malha de elementos finitos como descrito na Seção 2.4.

Para a construção da malha é necessário o conhecimento da posição dos eletrodos e suas dimensões, geometria e tamanho do tórax e nível de discretização suficiente para obter uma boa aproximação. Esta é a primeira fonte de incertezas em uma aplicação real, como na monitoração da ventilação pulmonar. A geometria do tórax varia de paciente para paciente e, em um mesmo paciente, a geometria muda ao longo do ciclo respiratório e também ao longo do tempo em decorrência da perda/ganho de massa corpórea no leito de UTI. Além disto, a posição dos eletrodos não é conhecida perfeitamente e estes podem mover-se ao longo do tempo. Já a coleta de dados está sujeita a interferências externas devidas aos demais equipamentos conectados ao paciente, fugas de corrente entre os equipamentos, erros de leitura e variações de impedância de contato entre o eletrodo e a pele do paciente, decorrentes do descolamento destes ou mudanças devido à sudorese ou (des)hidratação.

Pode-se argumentar que algumas destas fontes de incerteza poderiam a princípio ser monitoradas, como a forma e tamanho do tórax e posição relativa entre os eletrodos, porém ainda assim algum nível de incerteza permanece. Outras fontes de incerteza são de difícil monitoramento, por exemplo a interferência eletromagnética causada por outros equipamentos e efeitos térmicos no equipamento de medição. 
A teoria dos métodos de inversão Bayesiana lida com problemas cujo nível de incerteza nos modelos está presente e não pode ser desconsiderado. O objetivo da inversão Bayesiana é extrair informação a respeito das incógnitas e determinar o seu nível de incerteza baseado em toda informação disponível do processo de medição e modelos disponíveis sobre as incógnitas antes da realização das medidas.

Métodos de regularização clássicos, como Tikhonov, produzem uma estimativa única com o uso de informação adicional na forma do operador $\boldsymbol{L}_{r}$ visando tornar o problema bem posto. Ao contrário do método de Tikhonov, a inversão Bayesiana não produz uma única estimativa, mas procura remover a natureza mal posta do problema redefinindo o problema mal-posto original em um espaço mais amplo de distribuição de probabilidade, onde o problema original torna-se bem posto. Além disto, nos permite ser explícitos a respeito de toda informação a priori que é geralmente oculta em outros tipos de regularização.

\subsection{Inversão Bayesiana}

Em inversão Bayesiana, os parâmetros são vistos como variáveis aleatórias. Considerando o problema $\boldsymbol{A x}=\boldsymbol{y}$ anteriormente apresentado, $\boldsymbol{x}$ e $\boldsymbol{y}$ são vistos como realizações das variáveis aleatórias $\mathbf{x}$ e $\mathbf{y}$. Como todo sistema de medição, existem incertezas nas medidas $\boldsymbol{y}$. Esta incerteza pode ser modelado como uma terceira variável aleatória e, cuja realização é $\boldsymbol{e}$. Pode ainda ser o caso em que $\boldsymbol{A}$ depende de uma série de parâmetros que podem também não ser completamente conhecidos. Estes parâmetros podem ser agrupados em um vetor $\boldsymbol{p}$ cuja variável aleatória associada é p. Considera-se aqui que todas as variáveis são contínuas e portanto apresentam função densidade de probabilidade.

Estas quatro variáveis aleatórias combinam-se em uma equação de observação da forma

$$
\mathbf{y}=\boldsymbol{h}(\mathbf{x}, \mathbf{p}, \mathbf{e}),
$$

que relaciona $\mathbf{y}$ com todas as demais variáveis. Rotineiramente, nomeia-se $\mathbf{y}$ como medidas ou observação, $\mathbf{x}$ como incógnita ou estado, $\mathbf{p}$ como parâmetros e e como ruído. 
O interesse em inversão Bayesiana é determinar a densidade de probabilidade a posteriori de $\mathbf{x}$, dada a realização $\mathbf{y}=\boldsymbol{y}_{\text {medido }}$ (medida realizada), denotada por $f_{\mathbf{x} \mid \mathbf{y}}\left(\boldsymbol{x} \mid \boldsymbol{y}_{\text {medido }}\right)$. Isto é obtido através a fórmula de Bayes

$$
f_{\mathbf{x} \mid \mathbf{y}}\left(\boldsymbol{x} \mid \boldsymbol{y}_{\text {medido }}\right)=\frac{f_{\mathbf{x}, \mathbf{y}}\left(\boldsymbol{x}, \boldsymbol{y}_{\text {medido }}\right)}{f_{\mathbf{y}}\left(\boldsymbol{y}_{\text {medido }}\right)}=\frac{f_{\mathbf{x}}(\boldsymbol{x}) \cdot f_{\mathbf{y} \mid \mathbf{x}}\left(\boldsymbol{y}_{\text {medido }} \mid \boldsymbol{x}\right)}{f_{\mathbf{y}}\left(\boldsymbol{y}_{\text {medido }}\right)}
$$

se $f_{\mathbf{y}}\left(\boldsymbol{y}_{\text {medido }}\right) \neq 0$. Nesta equação, $f_{\mathbf{x}}(\boldsymbol{x})$ é chamada densidade de probabilidade a priori de $\mathbf{x}$. Ela contém toda a informação conhecida a respeito de $\boldsymbol{x}$ antes da realização das medidas. O termo $f_{\mathbf{y} \mid \mathbf{x}}\left(\boldsymbol{y}_{\text {medido }} \mid \boldsymbol{x}\right)$ é a função de verossimilhança e expressa a probabilidade de realização de uma medida, dado um estado conhecido $\boldsymbol{x}$. O denominador é visto como um fator de escala e possui pouca importância para o problema contanto que $f_{\mathbf{y}}\left(\boldsymbol{y}_{\text {medido }}\right) \neq 0$. Daqui em diante denota-se $\boldsymbol{y}_{\text {medido }}$ por $\boldsymbol{y}$ para simplificação da notação.

Seja a função densidade de probabilidade conjunta de todas as variáveis aleatórias apresentadas $f_{\mathbf{y}, \mathbf{x}, \mathbf{p}, \mathbf{e}}(\boldsymbol{y}, \boldsymbol{x}, \boldsymbol{p}, \boldsymbol{e})$. Pode-se expandi-la de duas formas

$$
f_{\mathbf{y}, \mathbf{x}, \mathbf{p}, \mathbf{e}}(\boldsymbol{y}, \boldsymbol{x}, \boldsymbol{p}, \boldsymbol{e})=f_{\mathbf{y}, \mathbf{p}, \mathbf{e} \mid \mathbf{x}}(\boldsymbol{y}, \boldsymbol{p}, \boldsymbol{e} \mid \boldsymbol{x}) \cdot f_{\mathbf{x}}(\boldsymbol{x})
$$

$\mathrm{ou}$

$$
\begin{aligned}
f_{\mathbf{y}, \mathbf{x}, \mathbf{p}, \mathbf{e}}(\boldsymbol{y}, \boldsymbol{x}, \boldsymbol{p}, \boldsymbol{e}) & =f_{\mathbf{y} \mid \mathbf{x}, \mathbf{p}, \mathbf{e}}(\boldsymbol{y} \mid \boldsymbol{x}, \boldsymbol{p}, \boldsymbol{e}) \cdot f_{\mathbf{x}, \mathbf{p}, \mathbf{e}}(\boldsymbol{x}, \boldsymbol{p}, \boldsymbol{e}) \\
& =f_{\mathbf{y} \mid \mathbf{x}, \mathbf{p}, \mathbf{e}}(\boldsymbol{y} \mid \boldsymbol{x}, \boldsymbol{p}, \boldsymbol{e}) \cdot f_{\mathbf{p}, \mathbf{e} \mid \mathbf{x}}(\boldsymbol{p}, \boldsymbol{e} \mid \boldsymbol{x}) f_{\mathbf{x}}(\boldsymbol{x})
\end{aligned}
$$

Identifica-se então que

$$
f_{\mathbf{y}, \mathbf{p}, \mathbf{e} \mid \mathbf{x}}(\boldsymbol{y}, \boldsymbol{p}, \boldsymbol{e} \mid \boldsymbol{x})=f_{\mathbf{y} \mid \mathbf{x}, \mathbf{p}, \mathbf{e}}(\boldsymbol{y} \mid \boldsymbol{x}, \boldsymbol{p}, \boldsymbol{e}) . f_{\mathbf{p}, \mathbf{e} \mid \mathbf{x}}(\boldsymbol{p}, \boldsymbol{e} \mid \boldsymbol{x})
$$

e pode-se calcular agora a verosimilhança que é a densidade de probabilidade marginal

$$
f_{\mathbf{y} \mid \mathbf{x}}(\boldsymbol{y} \mid \boldsymbol{x})=\iint f_{\mathbf{y}, \mathbf{p}, \mathbf{e} \mid \mathbf{x}}(\boldsymbol{y}, \boldsymbol{p}, \boldsymbol{e} \mid \boldsymbol{x}) d \boldsymbol{e} d \boldsymbol{p}=\iint f_{\mathbf{y} \mid \mathbf{x}, \mathbf{p}, \mathbf{e}}(\boldsymbol{y} \mid \boldsymbol{x}, \boldsymbol{p}, \boldsymbol{e}) . f_{\mathbf{p}, \mathbf{e} \mid \mathbf{x}}(\boldsymbol{p}, \boldsymbol{e} \mid \boldsymbol{x}) d \boldsymbol{e} d \boldsymbol{p}
$$


Observa-se que ao fixar $\boldsymbol{x}$, $\boldsymbol{e}$ e $\boldsymbol{p}$, o vetor de medidas $\boldsymbol{y}$ fica completamente especificado com o modelo (4.1) e a primeira função na integral torna-se

$$
f_{\mathbf{y} \mid \mathbf{x}, \mathbf{p}, \mathbf{e}}(\boldsymbol{y} \mid \boldsymbol{x}, \boldsymbol{p}, \boldsymbol{e})=\delta(\boldsymbol{y}-\boldsymbol{h}(\boldsymbol{x}, \boldsymbol{p}, \boldsymbol{e}))
$$

onde $\delta(\cdot)$ é a distribuição delta de Dirac.

Caso e possa ser modelado como ruído aditivo e independente de $\mathbf{p}$ e $\mathbf{x}$, o modelo estatístico (4.1) é simplificado para $\mathbf{y}=\boldsymbol{h}(\mathbf{x}, \mathbf{p})+\mathbf{e}$ e

$$
f_{\mathbf{y} \mid \mathbf{x}, \mathbf{p}, \mathbf{e}}(\boldsymbol{y} \mid \boldsymbol{x}, \boldsymbol{p}, \boldsymbol{e})=\delta(\boldsymbol{y}-\boldsymbol{h}(\boldsymbol{x}, \boldsymbol{p})-\boldsymbol{e})
$$

de forma que a integral em $\boldsymbol{e}$ pode ser feita

$$
f_{\mathbf{y} \mid \mathbf{x}}(\boldsymbol{y} \mid \boldsymbol{x})=\int f_{\mathbf{p}, \mathbf{e} \mid \mathbf{x}}(\boldsymbol{p}, \boldsymbol{e}=\boldsymbol{y}-\boldsymbol{h}(\boldsymbol{x}, \boldsymbol{p}) \mid \boldsymbol{x}) d \boldsymbol{p}
$$

Se $\mathbf{p}$ e e forem independentes,

$$
f_{\mathbf{y} \mid \mathbf{x}}(\boldsymbol{y} \mid \boldsymbol{x})=\int f_{\mathbf{p} \mid \mathbf{x}}(\boldsymbol{p} \mid \boldsymbol{x}) \cdot f_{\mathbf{e} \mid \mathbf{x}}(\boldsymbol{y}-\boldsymbol{h}(\boldsymbol{x}, \boldsymbol{p}) \mid \boldsymbol{x}) d \boldsymbol{p}
$$

Em geral $\mathbf{p}$ não pode ser modelado como processo aditivo e a integral em $\boldsymbol{p}$ deve ser calculada numericamente. Após este processo, (4.2) pode ser usada para determinar a densidade a posteriori.

Quando a dimensão do espaço x é elevada, torna-se difícil a visualização da densidade de probabilidade a posteriori como solução do problema. Nestes casos é conveniente utilizar estimativas pontuais $\hat{\boldsymbol{x}}$ ao invés da estimativa de intervalo. Uma estimativa de intervalo é útil para responder questões do tipo Em qual intervalo estão os valores da solução, com 90\% de confiabilidade, dada a informação a priori e as medidas? Uma estimativa pontual é útil para responder questões do tipo Qual é a solução mais provável, dada a informação a priori e as medidas?

Observa-se aqui que métodos determinísticos apresentam soluções pontuais, sem referência a nenhuma base estatística e sem evidenciar quais hipóteses estão sendo assumidas. Apresentam, portanto, apenas parte da informação disponível. Os métodos Bayesianos podem ser utilizados para analisar métodos determinísticos, evidenciando quais hipóteses estatísticas estão por detrás destes métodos. Para mais 
informações, ver Kaipio e Somersalo [61, cap. 3]. Outro ponto importante é o caso em que a densidade de probabilidade é multimodal. Neste caso estimadores pontuais podem ser insatisfatórios.

Uma forma usual de extrair uma estimativa pontual a partir da densidade de probabilidade a posteriori é o critério Máximo a Posteriori (MAP)

$$
\hat{\boldsymbol{x}}_{M A P}=\arg \max _{\boldsymbol{x}} f_{\mathbf{x} \mid \mathbf{y}}(\boldsymbol{x} \mid \boldsymbol{y}),
$$

se tal máximo existir e for único. Outro critério é a esperança condicional do estado $\mathbf{x}$, condicionada à medida realizada $\boldsymbol{y}$ da variável aleatória $\mathbf{y}$

$$
\hat{\boldsymbol{x}}_{E C}=E\{\mathbf{x} \mid \mathbf{y}=\boldsymbol{y}\}=\int \boldsymbol{x} f_{\mathbf{x} \mid \mathbf{y}}(\boldsymbol{x} \mid \boldsymbol{y}) d \boldsymbol{x}
$$

se tal integral convergir. Este critério tem a vantagem de não depender tanto da suavidade de $f_{\mathbf{x} \mid \mathbf{y}}$ por conta da operação de integração. A desvantagem é que se a dimensão de $\boldsymbol{x}$ for elevada e não houver um meio de evitar a integração numérica, haverá um grande custo computacional. Um último exemplo de estimador pontual é a máxima verossimilhança

$$
\hat{\boldsymbol{x}}_{M V}=\arg \max _{\boldsymbol{x}} f_{\mathbf{y} \mid \mathbf{x}}(\boldsymbol{y} \mid \boldsymbol{x})
$$

Este estimador maximiza a probabilidade de ocorrência da medida $\boldsymbol{y}$ realizada. Este estimador é inadequado em alguns casos, como na TIE, que frequentemente tem mais incógnitas do que equações, gerando múltiplos máximos locais. Além disto, não permite a inclusão de informação a priori do espaço de soluções do problema inverso.

Quando o sistema sob observação apresenta características dinâmicas, é conveniente adicionar tal informação no problema de estimação na forma de um modelo de evolução. Para isto é introduzida a filtragem Bayesianal.

\footnotetext{
${ }^{1}$ Por motivo de simplificação de notação, daqui em diante a função densidade de probabilidade de uma variável aleatória $f_{\mathbf{x}}(\boldsymbol{x})$ será denotado na forma simplificada $f_{\mathbf{x}}$, sem expressar o valor da realização nos casos onde o valor da realização for evidente. Caso contrário, a notação completa será utilizada.
} 


\subsection{Filtragem Bayesiana}

Seja agora um sistema em que o estado varia no tempo $\boldsymbol{x}(t)$. O problema agora é estimar o estado ao longo do tempo $\boldsymbol{x}_{0}^{K}=\left\{\boldsymbol{x}_{0}, \boldsymbol{x}_{1}, \ldots, \boldsymbol{x}_{K}\right\}$, utilizando o conjunto de medidas $\boldsymbol{y}_{1}^{K}=\left\{\boldsymbol{y}_{1}, \boldsymbol{y}_{2}, \ldots, \boldsymbol{y}_{K}\right\}$ coletadas a intervalos de tempo $T_{s}$ (assumido constante por simplificação), $t_{k}=k T_{s}, k=1,2, \ldots$, a partir de uma estimativa inicial $\boldsymbol{x}_{0}$. Tais problemas são geralmente referenciados como problemas inversos não estacionários e os métodos Bayesianos para sua solução são chamados de métodos de filtragem Bayesianas.

Em tais sistemas, um modelo de evolução é adotado, geralmente no tempo discreto

$$
\boldsymbol{x}_{k+1}=\boldsymbol{\Phi}_{k}\left(\boldsymbol{x}_{k}, \boldsymbol{v}_{k}\right)
$$

onde $\boldsymbol{v}_{k}$ é um vetor de parâmetros ou erros do modelo.

Sob o ponto de vista Bayesiano, considera-se que $\mathbf{x}_{0}^{K}$ e $\mathbf{y}_{1}^{K}$ são processos estocásticos 2 , mais especificamente processos de Markov com as seguintes propriedades

$$
\begin{aligned}
f_{\mathbf{x}_{k+1} \mid \mathbf{x}_{0}^{k}}\left(\boldsymbol{x}_{k+1} \mid \boldsymbol{x}_{0}^{k}\right) & =f_{\mathbf{x}_{k+1} \mid \mathbf{x}_{k}}\left(\boldsymbol{x}_{k+1} \mid \boldsymbol{x}_{k}\right) \\
f_{\mathbf{y}_{k} \mid \mathbf{x}_{0}^{k}}\left(\boldsymbol{x}_{k+1} \mid \boldsymbol{x}_{0}^{k}\right) & =f_{\mathbf{y}_{k} \mid \mathbf{x}_{k}}\left(\boldsymbol{y}_{k} \mid \boldsymbol{x}_{k}\right) \\
f_{\mathbf{x}_{k+1} \mid \mathbf{x}_{k}, \mathbf{y}_{1}^{k}}\left(\boldsymbol{x}_{k+1} \mid \boldsymbol{x}_{k}, \boldsymbol{y}_{1}^{k}\right) & =f_{\mathbf{x}_{k+1} \mid \mathbf{x}_{k}}\left(\boldsymbol{x}_{k+1} \mid \boldsymbol{x}_{k}\right) .
\end{aligned}
$$

O índice de $\mathbf{x}$ inicia em zero e de $\mathbf{y}$ inicia em um pois considera-se que no instante $t_{0}$, $\mathbf{x}_{0}$ possui a densidade $a$ priori do problema $f_{\mathbf{x}_{0}}$ e, por definição, não contém informação das medidas.

Usando o modelo de evolução (4.15), monta-se o seguinte modelo de Markov para o problema

$$
\mathbf{x}_{k+1}=\Phi_{k}\left(\mathbf{x}_{k}, \mathbf{v}_{k}\right)
$$

onde $\mathbf{v}_{k}$ é o ruído de processo.

\footnotetext{
${ }^{2} \mathrm{~A}$ notação $\mathbf{x}_{a}^{b}$ representa o processo estocástico, do instante $t_{a}=a T_{s}$ até o instante $t_{b}=b T_{s}$ e a notação $\boldsymbol{x}_{a}^{b}$ representa a realização do processo estocástico, do instante $t_{a}=a T_{s}$ até o instante $t_{b}=b T_{s}$. A notação $\mathbf{x}_{a}$ representa o processo estocástico no instante $t_{a}=a T_{s}$ somente e a notação $\boldsymbol{x}_{a}$ representa a realização do processo estocástico no instante $t_{a}=a T_{s}$ somente.
} 
As equações (4.1) e (4.19) em conjunto com $f_{\mathbf{x}_{0}}\left(\boldsymbol{x}_{0}\right)$ especificam o problema para a filtragem Bayesiana. Assume-se que $\mathbf{v}_{k}$ e $\mathbf{e}_{k}$ sejam processos brancos, independentes entre si e em relação a $\mathbf{x}_{0}$.

Em métodos de filtragem Bayesiana, pode-se listar alguns tipos de problemas de interesse:

1. Determinar $f_{\mathbf{x}_{k+p} \mid \mathbf{y}_{1}^{k}}, p>0$ é chamado problema de predição;

2. Determinar $f_{\mathbf{x}_{k} \mid \mathbf{y}_{1}^{k}}$ é chamado problema de filtragem;

3. Determinar $f_{\mathbf{x}_{k-p} \mid \mathbf{y}_{1}^{k}}, p<k$ é chamado problema de suavização com atraso $p$;

4. Determinar $f_{\mathbf{x}_{k+1} \mid \mathbf{y}_{1}^{K}}, 0 \leq k \leq K$ e com todo $\boldsymbol{y}_{1}^{K}$ disponível, é chamado problema de suavização.

O primeiro diz respeito ao problema de predizer o estado em um momento no futuro (não necessariamente apenas em $t_{k+1}$ ) baseado em informações do sistema até $o$ instante atual $t_{k}$. O segundo é de interesse quando se quer monitorar em tempo real um sistema, como por exemplo problemas de controle onde o sistema de controle deve agir baseado no estado atual da planta. O terceiro problema é de interesse quando um atraso é permitido para a estimação do estado. O quarto é o problema em que todas as medidas são coletadas antes da estimação, ou seja, é a estimação realizada após a finalização do processo. A vantagem do terceiro e quarto problemas é que eles permitem obter estimativas com erros menores do que os demais casos em tempo real já que utilizam medidas passadas e futuras a um instante $t_{k}$.

A solução do problema de filtragem Bayesiana pode ser dividida em 2 fases. A fase de propagação é responsável por avançar a distribuição de probabilidade ao longo do tempo, entre $t_{k}$ até o instante que estará disponível uma nova medida dos sensores $t_{k+1}$. A fase de atualização é responsável pela incorporação de uma nova medida na estimativa do estado $\boldsymbol{x}_{k+1}$.

A linha do tempo da Figura 4.1 mostra as duas fases entre o instante $t_{0}$ e $t_{1}$ e também entre o instante $t_{k}$ e $t_{k+1}$. Partindo de um instante $t_{k}$, após a incorporação da medida $\boldsymbol{y}_{k}$, tem-se $f_{\mathbf{x}_{k} \mid \mathbf{y}_{1}^{k}}$. A fase de propagação determina a densidade de probabilidade do processo de Markov $f_{\mathbf{x}_{k+1} \mid \mathbf{y}_{1}^{k}}$, usando o modelo de evolução (4.19). Neste instante, um novo vetor de medidas está disponível $\boldsymbol{y}_{k+1}$ e a fase de atualização pode ser 
efetuada, usando o modelo de observação (4.1). Esta fase determina a densidade de probabilidade $f_{\mathbf{x}_{k+1} \mid \mathbf{y}_{1}^{k+1}}$, incorporando a informação contida no novo vetor de medidas. Estas fases são alternadas indefinidamente, enquanto novas medidas estiverem sendo geradas 3

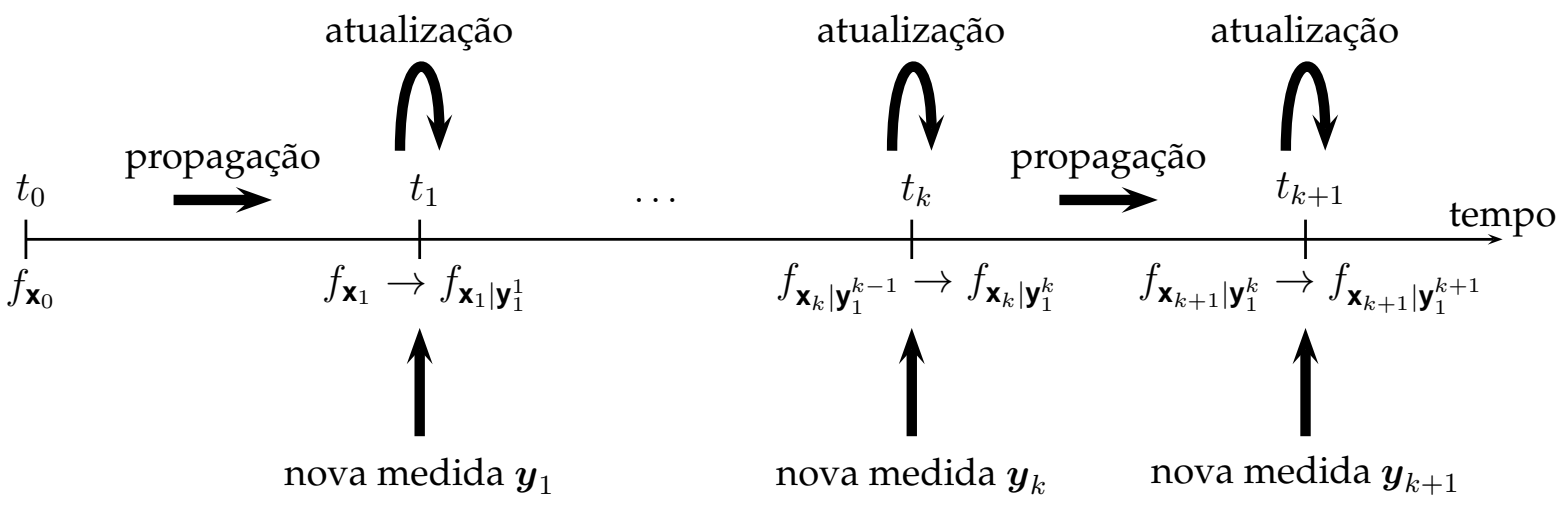

Figura 4.1: Linha do tempo entre instantes sucessivos no tempo discreto.

A fase de propagação é determinada a partir da densidade de probabilidade conjunta

$$
f_{\mathbf{x}_{k+1}, \mathbf{x}_{k}, \mathbf{y}_{1}^{k}}=f_{\mathbf{x}_{k+1} \mid \mathbf{x}_{k}, \mathbf{y}_{1}^{k}} \cdot f_{\mathbf{x}_{k}, \mathbf{y}_{1}^{k}}=f_{\mathbf{x}_{k+1} \mid \mathbf{x}_{k}, \mathbf{y}_{1}^{k}} \cdot f_{\mathbf{x}_{k} \mid \mathbf{y}_{1}^{k}} \cdot f_{\mathbf{y}_{1}^{k}},
$$

calculando a densidade marginal em ambos os lados

$$
\begin{aligned}
\int f_{\mathbf{x}_{k+1}, \mathbf{x}_{k}, \mathbf{y}_{1}^{k}} d \boldsymbol{x}_{k} & =f_{\mathbf{y}_{1}^{k}} \int f_{\mathbf{x}_{k+1} \mid \mathbf{x}_{k}, \mathbf{y}_{1}^{k}} \cdot f_{\mathbf{x}_{k} \mid \mathbf{y}_{1}^{k}} d \boldsymbol{x}_{k} \\
f_{\mathbf{x}_{k+1}, \mathbf{y}_{1}^{k}} & =f_{\mathbf{y}_{1}^{k}} \int f_{\mathbf{x}_{k+1} \mid \mathbf{x}_{k}, \mathbf{y}_{1}^{k}} \cdot f_{\mathbf{x}_{k} \mid \mathbf{y}_{1}^{k}} d \boldsymbol{x}_{k} \\
f_{\mathbf{x}_{k+1} \mid \mathbf{y}_{1}^{k}} & =\int f_{\mathbf{x}_{k+1} \mid \mathbf{x}_{k}, \mathbf{y}_{1}^{k}} \cdot f_{\mathbf{x}_{k} \mid \mathbf{y}_{1}^{k}} d \boldsymbol{x}_{k} .
\end{aligned}
$$

Por fim, usando a propriedade (4.18)

$$
f_{\mathbf{x}_{k+1} \mid \mathbf{y}_{1}^{k}}=\int f_{\mathbf{x}_{k+1} \mid \mathbf{x}_{k}} \cdot f_{\mathbf{x}_{k} \mid \mathbf{y}_{1}^{k}} d \boldsymbol{x}_{k}
$$

Na fase de atualização, a informação da medida $\boldsymbol{y}_{k+1}$ é incorporada para calcular a densidade a posteriori $f_{\mathbf{x}_{k+1} \mid \mathbf{y}_{1}^{k+1}}$. Por consequência, a fórmula de Bayes (4.2), apresentada na seção anterior, pode ser usada diretamente, apenas substituindo-se a

\footnotetext{
${ }^{3}$ Por motivos de recursividade dos índices do algoritmo, considera-se que no instante inicial
} $t_{0}: f_{\mathbf{x}_{0} \mid \mathbf{y}_{1}^{0}}=f_{\mathbf{x}_{0}}$ e em $t_{1}: f_{\mathbf{x}_{1} \mid \mathbf{y}_{1}^{0}}=f_{\mathbf{x}_{1}}$. 
densidade a priori $f_{\mathbf{x}}$ por $f_{\mathbf{x}_{k+1} \mid \mathbf{y}_{1}^{k}}$

$$
f_{\mathbf{x}_{k+1} \mid \mathbf{y}_{1}^{k+1}}=\frac{f_{\mathbf{y}_{k+1} \mid \mathbf{x}_{k+1}} \cdot f_{\mathbf{x}_{k+1} \mid \mathbf{y}_{1}^{k}}}{f_{\mathbf{y}_{k+1} \mid \mathbf{y}_{1}^{k}}}
$$

Neste trabalho, a seguinte notação será utilizada:

- $\boldsymbol{x} \in \mathbb{R}^{L}$ : estado a ser estimado, onde $L$ é a dimensão do vetor;

- $\overline{\boldsymbol{x}}_{k}=E\left\{\mathbf{x}_{k} \mid \mathbf{y}_{1}^{k}=\boldsymbol{y}_{1}^{k}\right\}$ : média de $\mathbf{x}$ em $t_{k}$, condicionada às medidas realizadas $\boldsymbol{y}_{1}^{k}$;

- $\overline{\boldsymbol{x}}_{k+1 \mid k}=E\left\{\mathbf{x}_{k+1} \mid \mathbf{y}_{1}^{k}=\boldsymbol{y}_{1}^{k}\right\}$ : média de $\mathbf{x}$ em $t_{k+1}$, condicionada às medidas realizadas $\boldsymbol{y}_{1}^{k}$;

- $\Gamma_{k}^{x x}=E\left\{\left(\mathbf{x}_{k}-\overline{\boldsymbol{x}}_{k}\right)\left(\mathbf{x}_{k}-\overline{\boldsymbol{x}}_{k}\right)^{\mathrm{T}} \mid \mathbf{y}_{1}^{k}=\boldsymbol{y}_{1}^{k}\right\}$ : matriz de covariância de $\mathbf{x}$ em $t_{k}$, condicionada às medidas realizadas $\boldsymbol{y}_{1}^{k}$;

- $\boldsymbol{\Gamma}_{k+1 \mid k}^{x x}=E\left\{\left(\mathbf{x}_{k+1}-\overline{\boldsymbol{x}}_{k+1 \mid k}\right)\left(\mathbf{x}_{k+1}-\overline{\boldsymbol{x}}_{k+1 \mid k}\right)^{\mathrm{T}} \mid \mathbf{y}_{1}^{k}=\boldsymbol{y}_{1}^{k}\right\}$ : matriz de covariância de $\mathbf{x}$ em $t_{k+1}$, condicionada às medidas realizadas $\boldsymbol{y}_{1}^{k}$;

- $\boldsymbol{\Gamma}_{k+1 \mid k}^{x y}=E\left\{\left(\mathbf{x}_{k+1}-\overline{\boldsymbol{x}}_{k+1 \mid k}\right)\left(\mathbf{y}_{k+1}-\overline{\boldsymbol{y}}_{k+1 \mid k}\right)^{\mathrm{T}} \mid \mathbf{y}_{1}^{k}=\boldsymbol{y}_{1}^{k}\right\}$ : matriz de covariância cruzada entre $\mathbf{x}$ e $\mathbf{y}$ em $t_{k+1}$, condicionada às medidas realizadas $\boldsymbol{y}_{1}^{k}$.

Por questão de recursividade de notação, assume-se que em $k=0$

- $\boldsymbol{y}_{1}^{0}$ é o conjunto vazio de medidas, ou seja, nenhuma medida realizada;

- $\overline{\boldsymbol{x}}_{0}=E\left\{\mathbf{x}_{0} \mid \mathbf{y}_{1}^{0}=\boldsymbol{y}_{1}^{0}\right\}=E\left\{\mathbf{x}_{0}\right\}$;

- $\overline{\boldsymbol{x}}_{1 \mid 0}=E\left\{\mathbf{x}_{1} \mid \mathbf{y}_{1}^{0}=\boldsymbol{y}_{1}^{0}\right\}=E\left\{\mathbf{x}_{1}\right\}=\overline{\boldsymbol{x}}_{1}$ : média de $\mathbf{x}$ em $t_{1}$, condicionada ao conjunto vazio de medidas realizadas $\boldsymbol{y}_{1}^{0}$, ou seja, é a média não condicional em $t_{1} ;$

- $\Gamma_{1 \mid 0}^{x x}=\Gamma_{1}^{x x}$ : matriz de covariância de $\mathbf{x}$ em $t_{1}$, condicionada ao conjunto vazio de medidas realizadas $\boldsymbol{y}_{1}^{0}$, ou seja, é a covariância não condicional em $t_{1}$. 


\subsection{Filtro de Kalman}

As equações (4.24) e (4.25) resolvem o problema de filtragem Bayesiana a partir da a distribuição $f_{\mathbf{x}_{0}}$ e dos modelos de observação (4.1) e evolução (4.19), entretanto apresentam algumas integrais que na maioria dos casos não apresentam solução analítica, o que implica na utilização de integrações numéricas.

Caso algumas hipóteses adicionais possam ser utilizadas, estas integrais podem ser calculadas analiticamente e a solução pode ser reduzida a um problema de fatoração matricial e operações algébricas.

Na formulação original do filtro (linear) de Kalman [76, 77], os ruídos $\mathbf{e}_{k}$ e $\mathbf{v}_{k}$ são gaussianos, não correlacionados no tempo e independentes mutualmente e de $\mathbf{x}_{0}$, que por sua vez também é gaussiano. Além disto, os ruídos em (4.1) e (4.19) são aditivos, os modelos são lineares em relação ao estado $\mathbf{x}_{k}$ e os parâmetros destes modelos são todos conhecidos, isto é, não existe p:

$$
\begin{aligned}
\mathbf{y}_{k} & =\boldsymbol{H}_{k} \mathbf{x}_{k}+\mathbf{e}_{k} \\
\mathbf{x}_{k+1} & =\boldsymbol{\Phi}_{k} \mathbf{x}_{k}+\mathbf{v}_{k} \\
\mathbf{x}_{0} & \sim \mathcal{N}\left(\overline{\boldsymbol{x}}_{0}, \boldsymbol{\Gamma}_{0}^{x x}\right) \\
\mathbf{e}_{k} & \sim \mathcal{N}\left(\overline{\boldsymbol{e}}_{k}, \boldsymbol{\Gamma}_{k}^{e e}\right) \\
\mathbf{v}_{k} & \sim \mathcal{N}\left(\overline{\boldsymbol{v}}_{k}, \boldsymbol{\Gamma}_{k}^{v v}\right),
\end{aligned}
$$

onde $\mathbf{x} \sim \mathcal{N}\left(\overline{\boldsymbol{x}}, \Gamma^{x x}\right)$ indica distribuição Gaussiana, com média $\overline{\boldsymbol{x}}$ e covariância $\Gamma^{x x}$. É importante salientar que o ruído $\mathbf{v}_{k}$ altera o valor de $\mathbf{x}_{k+1}$ sem passar por uma matriz de transição, ou seja, apesar do índice $k$, o ruído $v_{k}$ ocorre em $k+1$. Este fato é importante no desenvolvimento das equações para adição da informação a priori a ser descrito adiante.

Nestas circunstâncias, pode-se calcular analiticamente (4.24) e (4.25), chegando-se em um algoritmo iterativo de estimação dos filtros de Kalman (FK). Vasta literatura está disponível a respeito dos filtros de Kalman. Para a dedução e informações adicionais a respeito dos filtros de Kalman, ver Maybeck [78, 79, 80], Kaipio e Somersalo [61], Jazwinski [81]. 
Sob estas hipóteses, pode-se mostrar que $f_{\mathbf{x}_{k+1} \mid \mathbf{y}_{1}^{k}}$ e $f_{\mathbf{x}_{k+1} \mid \mathbf{y}_{1}^{k+1}}$ são gaussianas, portanto (4.24) e (4.25) podem ser agrupadas [61, p. 123]

$$
f_{\mathbf{x}_{k+1} \mid \mathbf{y}_{1}^{k+1}}=\frac{f_{\mathbf{y}_{k+1} \mid \mathbf{x}_{k+1}} \cdot f_{\mathbf{x}_{k+1} \mid \mathbf{y}_{1}^{k}}}{f_{\mathbf{y}_{k+1} \mid \mathbf{y}_{1}^{k}}} \propto f_{\mathbf{y}_{k+1} \mid \mathbf{x}_{k+1}} f_{\mathbf{x}_{k+1} \mid \mathbf{y}_{1}^{k}}
$$

ou

$$
\begin{aligned}
f_{\mathbf{x}_{k+1} \mid \mathbf{y}_{1}^{k+1}} \propto & \exp \left(-\frac{1}{2}\left(\boldsymbol{y}_{k+1}-\boldsymbol{H}_{k+1} \mathbf{x}_{k+1}-\overline{\boldsymbol{e}}_{k}\right)^{\mathrm{T}}\left(\boldsymbol{\Gamma}_{k+1}^{e e}\right)^{-1}\left(\boldsymbol{y}_{k+1}-\boldsymbol{H}_{k+1} \mathbf{x}_{k+1}-\overline{\boldsymbol{e}}_{k}\right)\right. \\
- & \left.\frac{1}{2}\left(\mathbf{x}_{k+1}-\boldsymbol{\Phi}_{k} \overline{\boldsymbol{x}}_{k}-\overline{\boldsymbol{v}}_{k}\right)^{\mathrm{T}}\left(\boldsymbol{\Gamma}_{k+1 \mid k}^{x x}\right)^{-1}\left(\mathbf{x}_{k+1}-\boldsymbol{\Phi}_{k} \overline{\boldsymbol{x}}_{k}-\overline{\boldsymbol{v}}_{k}\right)\right)
\end{aligned}
$$

onde $\Gamma_{k+1 \mid k}^{x x}=\Phi_{k} \Gamma_{k}^{x x} \Phi_{k}^{\mathrm{T}}+\boldsymbol{\Gamma}_{k+1}^{v v}$. Finalmente, com alguma álgebra matricial, chega-se ao conhecido conjunto de equações dos filtros de Kalman para sistemas lineares.

Calculando o logaritmo neperiano de ambos os lados, trocando o sinal e eliminando a constante multiplicativa, pode-se definir uma função custo $J_{k}\left(\mathbf{x}_{k}\right)$ que os filtro de Kalman minimizam implicitamente na solução do problema inverso

$$
\begin{aligned}
J_{k+1}\left(\mathbf{x}_{k+1}\right)= & \left(\boldsymbol{y}_{k+1}-\boldsymbol{H}_{k+1} \mathbf{x}_{k+1}-\overline{\boldsymbol{e}}_{k}\right)^{\mathrm{T}}\left(\boldsymbol{\Gamma}_{k+1}^{e e}\right)^{-1}\left(\boldsymbol{y}_{k+1}-\boldsymbol{H}_{k+1} \mathbf{x}_{k+1}-\overline{\boldsymbol{e}}_{k}\right) \\
& +\left(\mathbf{x}_{k+1}-\boldsymbol{\Phi}_{k} \overline{\boldsymbol{x}}_{k}-\overline{\boldsymbol{v}}_{k}\right)^{\mathrm{T}}\left(\boldsymbol{\Gamma}_{k+1 \mid k}^{x x}\right)^{-1}\left(\mathbf{x}_{k+1}-\boldsymbol{\Phi}_{k} \overline{\boldsymbol{x}}_{k}-\overline{\boldsymbol{v}}_{k}\right) .
\end{aligned}
$$

Sejam os fatores de Cholesky das matrizes de covariância $\boldsymbol{S}_{k+1}^{e}\left(\boldsymbol{S}_{k+1}^{e}\right)^{\mathrm{T}}=\boldsymbol{\Gamma}_{k+1}^{e e} \mathrm{e}$ $\boldsymbol{S}_{k+1 \mid k}^{x}\left(\boldsymbol{S}_{k+1 \mid k}^{x}\right)^{\mathrm{T}}=\boldsymbol{\Gamma}_{k+1 \mid k}^{x x}$. Pode-se reescrever este custo na forma padrão

$$
J_{k+1}\left(\mathbf{x}_{k+1}\right)=\left\|\left(\boldsymbol{S}_{k+1}^{e}\right)^{-1}\left(\boldsymbol{y}_{k+1}-\boldsymbol{H}_{k+1} \mathbf{x}_{k+1}-\overline{\boldsymbol{e}}_{k}\right)\right\|_{2}^{2}+\left\|\left(\boldsymbol{S}_{k+1}^{x}\right)^{-1}\left(\mathbf{x}_{k+1}-\boldsymbol{\Phi}_{k} \overline{\boldsymbol{x}}_{k}-\overline{\boldsymbol{v}}_{k}\right)\right\|_{2}^{2}
$$

Pode-se ver esta equação como uma função a ser minimizada para determinar a estimativa pontual $\hat{\boldsymbol{x}}_{k+1}$. Por tratar-se de uma distribuição normal, as estimativas por média condicional, MAP e máxima verossimilhança coincidem

$$
\hat{\boldsymbol{x}}_{k+1}=\arg \min _{\mathbf{x}_{k+1}} J_{k+1}\left(\mathbf{x}_{k+1}\right) \text {. }
$$

Nota-se aqui a similaridade entre a solução do filtro de Kalman e a solução regularizada de Tikhonov, dada por (3.13). Salienta-se contudo que na solução por Tikhonov, análise estatística não é possível de ser feita pois as hipóteses ficam ocultas 
no operador e no parâmetro de regularização, enquanto que sob ponto de vista Bayesiano todas as hipóteses são explicitamente modeladas e utilizadas.

Pode-se então associar o modelo de evolução nos filtros de Kalman a uma regularização temporal, sob o ponto de vista clássico de regularização. De fato, comparando a fase de observação (4.25) com a fórmula de Bayes (4.2), nota-se que a densidade a priori em relação às medidas é a densidade predita pela fase de evolução (4.24), ou seja, o modelo de evolução entre $t_{k}$ e $t_{k+1}$ pode ser visto como informação $a$ priori em relação à observação em $t_{k+1}$. A questão que aparece então é como adicionar outras informações no problema sob o ponto de vista Bayesiano. Isto é apresentado a seguir para informação que possa ser concebida como Gaussiana. Para os demais casos, ver, por exemplo, Kaipio e Somersalo [61, seç. 3.3].

Outra razão para a inclusão de informação extra é que quando a dimensão do estado é elevada em comparação à dimensão das medidas realizadas, a solução dos filtros de Kalman pode sofrer problemas similares aos métodos determinísticos pela natureza mal posta do problema. Diversos trabalhos mostram o problema de estabilidade da solução do filtro de Kalman com estado de dimensão elevada, como em Kaipio et al. [82], Kim et al. [83, 84]. Nestes casos é aconselhável adicionar mais informação a priori ao problema, sob o mesmo ponto de vista probabilístico.

\subsection{Informação a priori nos métodos Bayesianos}

A informação a priori nos métodos Bayesianos exprime na forma de uma função densidade de probabilidade a idéia de que para uma solução plausível $x_{a}$ e uma solução não plausível $x_{b}$, as suas probabilidades obedecem $f\left(\mathbf{x}=x_{a}\right)>f\left(\mathbf{x}=x_{b}\right)$.

Distribuições Gaussianas geram uma vasta classe de informações a priori devido a sua fácil formulação, tratamento analítico e versatilidade de uso. Além disto, amparado pelo teorema do limite central [85, p. 214], distribuições Gaussianas são boas aproximações quando vários processos aleatórios mutuamente independentes se somam.

Uma vez que minimizar um funcional $J(\boldsymbol{x})$ é o mesmo que maximizar $J^{\prime}(\boldsymbol{x})=\exp \left(-\frac{1}{2} J(\boldsymbol{x})\right)$, a função a ser minimizada relativa à solução regularizada (3.13) 
pode ser reescrita como

$$
J^{\prime}(\boldsymbol{x})=\exp \left(-\frac{1}{2}(\boldsymbol{A} \boldsymbol{x}-\boldsymbol{y})^{\mathrm{T}}(\boldsymbol{A} \boldsymbol{x}-\boldsymbol{y})-\frac{\alpha^{2}}{2}\left(\boldsymbol{x}-\boldsymbol{x}^{*}\right)^{\mathrm{T}} \boldsymbol{L}_{r}^{\mathrm{T}} \boldsymbol{L}_{r}\left(\boldsymbol{x}-\boldsymbol{x}^{*}\right)\right)
$$

Identificando as componentes desta equação com as componentes de (4.32), nota-se que o termo de regularização apresenta relação direta com a distribuição a priori $f_{\mathbf{x}_{k+1} \mid \mathbf{y}_{1}^{k}}$. Em outras palavras, a regularização pode ser interpretada estatisticamente como uma densidade gaussiana

$$
\begin{aligned}
f_{\mathbf{x}}^{\text {priori }} & \propto \exp \left(-\frac{\alpha^{2}}{2}\left\|\boldsymbol{L}_{r}\left(\mathbf{x}-\boldsymbol{x}^{*}\right)\right\|_{2}^{2}\right)=\mathcal{N}\left(\boldsymbol{x}^{*}, \boldsymbol{\Gamma}^{x x}\right) \\
\boldsymbol{\Gamma}^{x x} & =\frac{1}{\alpha^{2}}\left(\boldsymbol{L}_{r}^{\mathrm{T}} \boldsymbol{L}_{r}\right)^{-1} .
\end{aligned}
$$

O problema desta abordagem, como mostrado em Kaipio e Somersalo [61, p. 80], é que $\boldsymbol{L}_{r}$ pode não ter posto completo e, portanto, a matriz de covariância $\Gamma^{x x}$ não estaria definida.

Ignorando, sem perda de generalidade, o termo $\boldsymbol{x}^{*}$, é possível mostrar entretanto que é possível fazer a seguinte decomposição utilizando a pseudoinversa $\boldsymbol{L}_{r}^{\dagger}$

$$
\begin{gathered}
\boldsymbol{\Gamma}^{x x}=\frac{1}{\alpha^{2}} \boldsymbol{L}_{r}^{\dagger}\left(\boldsymbol{L}_{r}^{\dagger}\right)^{\mathrm{T}}+a^{2} \boldsymbol{Q} \boldsymbol{Q}^{\mathrm{T}} \\
\left(\boldsymbol{\Gamma}^{x x}\right)^{-1}=\alpha^{2} \boldsymbol{L}_{r}^{\mathrm{T}} \boldsymbol{L}_{r}+\frac{1}{a^{2}} \boldsymbol{Q} \boldsymbol{Q}^{\mathrm{T}},
\end{gathered}
$$

onde $\boldsymbol{L}_{r}$ tem posto incompleto $q$, a matriz $\boldsymbol{Q} \in \mathbb{R}^{n \times n-q}$ é composta pelos $n-q$ vetores do núcleo de $\boldsymbol{L}_{r}$ e $a>0$ é uma constante arbitrária.

Para valores grandes de $a$, a densidade (4.37) se aproxima da desejada. Porém, na direção do núcleo de $\boldsymbol{L}_{r}$, a covariância apresenta grandes variâncias e a densidade não traz informação alguma. Neste caso é dito que a densidade de probabilidade é imprópria.

Assim, como visto anteriormente que se $\operatorname{ker}(\boldsymbol{A}) \cap \operatorname{ker}\left(\boldsymbol{L}_{r}\right) \neq\{\mathbf{0}\}$ a regularização clássica não tornava o problema bem posto, no caso dos métodos Bayesianos também não melhora a condição do problema. De fato, caso esta intersecção não seja a trivial, a densidade a posteriori não define uma densidade de probabilidade e o problema permanece indeterminado. 
Todavia, existe uma forma de lidar com casos impróprios. O método consiste em fixar alguns componentes deste vetor de modo que os restantes tornem a distribuição própria. Após isto, a densidade a priori torna-se uma distribuição condicionada à realização dos componentes fixados:

Seja a divisão do vetor $\boldsymbol{x}$ em dois grupos: um com os elementos que serão fixados $\mathbf{x}^{f}$ e outro com os demais elementos $\mathbf{x}^{\prime}$. Sejam eles reorganizados em $\boldsymbol{x}$ de tal sorte que este seja particionado em dois blocos $\boldsymbol{x}=\left[\begin{array}{ll}\left(\mathbf{x}^{f}\right)^{\mathrm{T}} & \left(\mathbf{x}^{\prime}\right)^{\mathrm{T}}\end{array}\right]^{\mathrm{T}}$.

Sua matriz de covariância torna-se

$$
\left(\boldsymbol{\Gamma}^{x x}\right)^{-1}=\alpha^{2}\left[\begin{array}{ll}
\boldsymbol{K}_{11} & \boldsymbol{K}_{12} \\
\boldsymbol{K}_{21} & \boldsymbol{K}_{22}
\end{array}\right]
$$

e a densidade de probabilidade de $\mathbf{x}^{\prime}$ condicionada a uma realização de $\mathbf{x}^{f}$ é

$$
\begin{aligned}
f_{\mathbf{x}^{\prime} \mid \mathbf{x}^{f}}=\frac{f_{\mathbf{x}^{\prime}, \mathbf{x}^{f}}}{f_{\mathbf{x}^{f}}} \propto f_{\mathbf{x}^{\prime}, \mathbf{x}^{f}} & =\exp \left(-\frac{\alpha^{2}}{2}\left(\boldsymbol{x}^{f \mathrm{~T}} \boldsymbol{K}_{11} \boldsymbol{x}^{f}+\boldsymbol{x}^{\prime \mathrm{T}} \boldsymbol{K}_{21} \boldsymbol{x}^{f}+\boldsymbol{x}^{f \mathrm{~T}} \boldsymbol{K}_{12} \boldsymbol{x}^{\prime}+\boldsymbol{x}^{\prime \mathrm{T}} \boldsymbol{K}_{22} \boldsymbol{x}^{\prime}\right)\right) \\
& =\exp \left(-\frac{\alpha^{2}}{2}\left(\boldsymbol{x}^{\prime}+\boldsymbol{K}_{22}^{-1} \boldsymbol{K}_{21} \boldsymbol{x}^{f}\right)^{\mathrm{T}} \boldsymbol{K}_{22}\left(\boldsymbol{x}^{\prime}+\boldsymbol{K}_{22}^{-1} \boldsymbol{K}_{21} \boldsymbol{x}^{f}\right)\right)
\end{aligned}
$$

onde $\boldsymbol{K}_{22}$ deve ser invertível. Isto é garantido através da escolha criteriosa de um número suficiente de elementos que compõem $\boldsymbol{x}^{f}$, com a distribuição de probabilidade própria $\mathbf{x}^{f} \sim \mathcal{N}\left(\overline{\boldsymbol{x}}^{f}, \boldsymbol{\Gamma}^{x^{f}}\right)$ desenvolvida a parte.

Finalmente, a densidade a priori é encontrada através da relação

$$
\begin{array}{r}
f_{\mathbf{x}}^{\text {priori }}=f_{\mathbf{x}^{\prime}, \mathbf{x}^{f}}=f_{\mathbf{x}^{\prime} \mid \mathbf{x}^{f}} \cdot f_{\mathbf{x}^{f}} \propto \exp \left(-\frac{\alpha^{2}}{2}\left(\boldsymbol{x}^{\prime}+\boldsymbol{K}_{22}^{-1} \boldsymbol{K}_{21} \boldsymbol{x}^{f}\right)^{\mathrm{T}} \boldsymbol{K}_{22}\left(\boldsymbol{x}^{\prime}+\boldsymbol{K}_{22}^{-1} \boldsymbol{K}_{21} \boldsymbol{x}^{f}\right)\right. \\
\left.-\frac{1}{2}\left(\boldsymbol{x}^{f}-\overline{\boldsymbol{x}}^{f}\right)^{\mathrm{T}} \boldsymbol{K}_{11}\left(\boldsymbol{x}^{f}-\overline{\boldsymbol{x}}^{f}\right)\right),
\end{array}
$$

onde é possível identificar prontamente que $\boldsymbol{K}_{11}=\left(\boldsymbol{\Gamma}^{x^{f}}\right)^{-1}$. Mostra-se finalmente que a densidade a priori de $\mathbf{x}$ é

$$
\begin{gathered}
\mathbf{x}^{\text {priori }} \sim \mathcal{N}\left(\overline{\boldsymbol{x}}, \boldsymbol{\Gamma}^{x}\right) \\
\overline{\boldsymbol{x}}=\left[\begin{array}{c}
\overline{\boldsymbol{x}}^{f} \\
\boldsymbol{K}_{22}^{-1} \boldsymbol{K}_{21} \overline{\boldsymbol{x}}^{f}
\end{array}\right], \quad \boldsymbol{\Gamma}^{x x}=\left[\begin{array}{cc}
\left(\boldsymbol{\Gamma}^{x^{f}}\right)^{-1}+\alpha^{2} \boldsymbol{K}_{12} \boldsymbol{K}_{22}^{-1} \boldsymbol{K}_{21} & \alpha^{2} \boldsymbol{K}_{21} \\
\alpha^{2} \boldsymbol{K}_{12} & \alpha^{2} \boldsymbol{K}_{22}
\end{array}\right]^{-1} .
\end{gathered}
$$


Nos casos em que se sabe que a informação $a$ priori se mantém ao longo do tempo, como no caso de informação espacial, é necessário modificar a etapa de evolução (4.24) do filtro para inserir $f_{\mathbf{x}_{k+1}}^{\text {priori }}$ na etapa de propagação. Para tal, faz-se uso da regra de Bayes

$$
f_{\mathbf{x}_{k+1} \mid \mathbf{x}_{k}}=\frac{f_{\mathbf{x}_{k+1}}^{\text {priori }} \cdot f_{\mathbf{x}_{k} \mid \mathbf{x}_{k+1}}}{f_{\mathbf{x}_{k}}}
$$

onde $f_{\mathbf{x}_{k} \mid \mathbf{x}_{k+1}}$ é a denominada densidade de probabilidade do modelo reverso no tempo. $\mathrm{O}$ denominador é dado pela integração do modelo reverso no tempo

$$
f_{\mathbf{x}_{k}}=\int f_{\mathbf{x}_{k+1}^{\text {priori }}} \cdot f_{\mathbf{x}_{k} \mid \mathbf{x}_{k+1}} d \boldsymbol{x}_{k+1}
$$

Substituindo (4.45) em (4.24) resulta na nova equação de propagação

$$
f_{\mathbf{x}_{k+1} \mid \mathbf{y}_{1}^{k}}=\int \frac{f_{\mathbf{x}_{k+1}}^{\text {priori }} f_{\mathbf{x}_{k} \mid \mathbf{x}_{k+1}}}{f_{\mathbf{x}_{k}}} \cdot f_{\mathbf{x}_{k} \mid \mathbf{y}_{1}^{k}} d \boldsymbol{x}_{k}=f_{\mathbf{x}_{k+1}^{\text {priori }}} \int \frac{f_{\mathbf{x}_{k} \mid \mathbf{x}_{k+1}}}{f_{\mathbf{x}_{k}}} \cdot f_{\mathbf{x}_{k} \mid \mathbf{y}_{1}^{k}} d \boldsymbol{x}_{k}
$$

Para modelos de observação do tipo

$$
\begin{array}{r}
\mathbf{y}_{k}=\boldsymbol{h}\left(\mathbf{x}_{k}\right)+\boldsymbol{B} \mathbf{e}_{k} \\
\mathbf{e}_{k} \sim \mathcal{N}\left(\overline{\boldsymbol{e}}_{k}, \boldsymbol{\Gamma}_{k}^{e e}\right),
\end{array}
$$

onde $\boldsymbol{B}$ é uma matriz conhecida, a atualização modificada é equivalente a adicionar medidas fictícias a respeito da não conformidade com a informação a priori desejada e impor que esta seja a menor possível [61, p. 137].

Definindo um vetor de medida de não conformidade

$$
\boldsymbol{z}_{k+1} \triangleq \alpha \boldsymbol{L}_{r}\left(\boldsymbol{x}_{k+1}-\boldsymbol{x}_{k+1}^{*}\right)=\alpha \boldsymbol{L}_{r} \boldsymbol{x}_{k+1}-\alpha \boldsymbol{L}_{r} \boldsymbol{x}_{k+1}^{*},
$$

pode-se reescrever a densidade de probabilidade a priori (4.37) como

$$
\begin{aligned}
f_{\mathbf{x}_{k+1}}^{\text {priori }} & \propto \exp \left(-\frac{\alpha^{2}}{2}\left\|\boldsymbol{L}_{r}\left(\mathbf{x}_{k+1}-\boldsymbol{x}_{k+1}^{*}\right)\right\|_{2}^{2}\right) \\
& \propto \exp \left(-\frac{1}{2}\left\|\alpha \boldsymbol{L}_{r} \mathbf{x}_{k+1}-\alpha \boldsymbol{L}_{r} \boldsymbol{x}_{k+1}^{*}\right\|_{2}^{2}\right) \sim \mathcal{N}\left(\alpha \boldsymbol{L}_{r} \boldsymbol{x}_{k+1}^{*}, \mathbb{I}\right)
\end{aligned}
$$


ou ainda

$$
f_{\mathbf{x}_{k+1}}^{\text {priori }} \propto \exp \left(-\frac{1}{2}\left\|\mathbf{z}_{k+1}\right\|_{2}^{2}\right) \sim \mathcal{N}(\mathbf{0}, \mathbb{I})
$$

Define-se ainda o modelo de observação de não conformidade

$$
\begin{aligned}
& \mathbf{z}_{k+1}=\alpha \boldsymbol{L}_{r} \mathbf{x}_{k+1}-\alpha \boldsymbol{L}_{r} \boldsymbol{x}_{k+1}^{*}+\mathbf{e}_{k+1}^{z} \\
& \mathbf{e}_{k+1}^{z} \sim \mathcal{N}(\mathbf{0}, \mathbb{I})
\end{aligned}
$$

ou

$$
\begin{aligned}
\mathbf{z}_{k+1} & =\alpha \boldsymbol{L}_{r} \mathbf{x}_{k+1}+\mathbf{e}_{k+1}^{z^{\prime}} \\
\mathbf{e}_{k+1}^{z^{\prime}} & \sim \mathcal{N}\left(-\alpha \boldsymbol{L}_{r} \boldsymbol{x}_{k+1}^{*}, \mathbb{I}\right) .
\end{aligned}
$$

e aumenta-se o modelo de observação (4.48) para incluir as novas medidas $\mathbf{Z}$

$$
\mathbf{y}_{k+1}^{\alpha} \triangleq\left[\begin{array}{c}
\mathbf{y}_{k+1} \\
\mathbf{z}_{k+1}
\end{array}\right]=\left[\begin{array}{c}
\boldsymbol{h}\left(\mathbf{x}_{k+1}\right) \\
\alpha \boldsymbol{L}_{r} \boldsymbol{x}_{k+1}
\end{array}\right]+\left[\begin{array}{c}
\boldsymbol{B} \mathbf{e}_{k+1} \\
\mathbf{e}_{k+1}^{z^{\prime}}
\end{array}\right]=\boldsymbol{h}^{\alpha}\left(\mathbf{x}_{k+1}\right)+\mathbf{e}_{k+1}^{\alpha},
$$

onde $\mathbf{e}_{k+1}^{\alpha}$ é ruído gaussiano, com estatísticas

$$
\overline{\boldsymbol{e}}_{k+1}^{\alpha}=\left[\begin{array}{c}
\boldsymbol{B} \overline{\boldsymbol{e}}_{k+1} \\
-\alpha \boldsymbol{L}_{r} \boldsymbol{x}_{k+1}^{*}
\end{array}\right], \quad \boldsymbol{\Gamma}_{k+1}^{e^{\alpha}}=\left[\begin{array}{cc}
\boldsymbol{B} \boldsymbol{\Gamma}_{k+1}^{e e} \boldsymbol{B}^{\mathrm{T}} & \mathbf{0} \\
\mathbf{0} & \mathbb{I}
\end{array}\right] .
$$

Ao adicionar a informação a priori, é de se esperar que o vetor de medida de não conformidade seja $\boldsymbol{z}_{k+1}=\mathbf{0}$. Por este motivo, impõe-se que $\boldsymbol{z}_{k+1}^{\text {medido }}=\mathbf{0}$

$$
\boldsymbol{y}_{k+1}^{\alpha}=\left[\begin{array}{l}
\boldsymbol{y}_{k+1}^{\text {medido }} \\
\boldsymbol{z}_{k+1}^{\text {medido }}
\end{array}\right]=\left[\begin{array}{c}
\boldsymbol{y}_{k+1}^{\text {medido }} \\
\mathbf{0}
\end{array}\right]
$$




\subsection{As variantes do filtro de Kalman e seu uso na TIE}

O filtro de Kalman é essencialmente um filtro Bayesiano (condicionado às medidas) que é ótimo no sentido de minimizar o traço da matriz de covariância do erro de estimação condicionado às medidas. É um algoritmo conveniente para implementação em computadores pois é recursivo, ou seja, o processamento é realizado conforme novos dados a respeito do sistema são obtidos, sem a necessidade de armazenar e utilizar todo o histórico de medidas explicitamente a cada nova estimação. Se os ruídos associados às medidas e ao modelo forem gaussianos, o FK exibe propriedades de otimalidade quadráticas para sistemas lineares.

O primeiro artigo publicado que resolve o problema da TIE usando o Filtro de Kalman para observar variações temporais de resistividade é de Vauhkonen et al. [86]. O problema de estimação é tratado como um problema de estimação de estado. Os autores utilizam o filtro (linearizado) de Kalman e argumentam que abordagens clássicas (determinísticas) não levam em conta a dependência estatística entre imagens em instantes diferentes, ou seja, não são levados em conta os estados anteriores para a nova estimação. Como modelo de evolução, usaram o passeio aleatório, juntamente com a linearização da equação de observação, daí o nome filtro linearizado de Kalman.

A acuidade das estimativas do Filtro linearizado de Kalman pode ser prejudicada caso a escolha do estado de referência para a linearização, usualmente $E\left\{\mathbf{x}_{0}\right\}$ seja muito diferente do estado real e a não linearidade das equações forem pronunciadas. Para tentar contornar este problema, em Kim et al. [83] foi utilizado pela primeira vez o filtro estendido de Kalman (FEK) na TIE, que faz a linearização sempre em torno da última estimativa, $\hat{\boldsymbol{x}}_{k}=E\left\{\mathbf{x}_{k} \mid \mathbf{y}_{1}^{k}=\boldsymbol{y}_{1}^{k}\right\}$ (para linearizar o modelo de evolução) ou $\hat{\boldsymbol{x}}_{k+1 \mid k}=E\left\{\mathbf{x}_{k+1} \mid \mathbf{y}_{1}^{k}=\boldsymbol{y}_{1}^{k}\right\}$ (para linearizar o modelo de observação). O preço que se paga por isto é um maior tempo computacional devido ao cálculo da matriz jacobiana da equação não linear de observação a cada iteração. Ainda no mesmo trabalho, os autores mostram a superioridade do FEK sobre o FK principalmente na definição das imagens, porém ainda assim foi necessário utilizar regularização generalizada de Tikhonov na forma de um filtro espacial para restringir a solução. Os autores utilizaram informação de suavidade mas não especificam o tipo de regularização utilizada. 
Em Kim et al. [84] estudou-se o caso em que a resistividade de uma certa sub-região interna é conhecida, por exemplo a região da coluna vertebral e em Kolehmainen et al. [87] é estudado o caso da determinação do contorno de uma região que varia ao longo do tempo. Informação a priori na forma de distribuições gaussianas foi utilizada em Kim et al. [83, 84], Kaipio et al. [82], Kolehmainen et al. [87].

Trigo et al. [3], Trigo [34], utilizando o FEK, apresentam métodos para estimar a distribuição de resistividade em $\Omega$ e também o valor da resistência de contato de cada um dos eletrodos, método denominado duas fases. O método consiste em estimar alternadamente a resistência de contato e a resistividade de $\Omega$.

Em Moura et al. [38] um método para identificar a matriz de transição é usada no FEK. A matriz de transição é calculada usando o Método de Ibrahim no domínio do tempo [88, p. 186], [89]. A determinação da matriz de transição foi feita resolvendo-se um sistema linear mal condicionado, formado por imagens estimadas por um algoritmo Caixa-preta [30] e utilizando o método dos mínimos quadrados para estimar uma matriz de transição. Para diminuir o efeito do mau condicionamento, um método de regularização foi empregado. Além disto, um modelo de ruído foi proposto para reduzir o efeito de discretização do domínio. O uso de uma matriz de transição no FEK acelerou o processo de convergência em comparação com o passeio aleatório. Os resultados mostraram que o FEK estimou o estado com convergência estatística conforme os critérios usados em Trigo [34, p. 60].

Para que o FEK seja utilizado, uma linearização do sistema deve ser realizada a cada iteração e os efeitos de segunda ordem são desprezadost 4 . De fato, o FEK é considerado sub-ótimo para sistemas não lineares e apresenta problemas de divergência. Em 1996, Julier e Uhlmann [90] apresenta uma nova abordagem para o filtro de Kalman chamada pelos autores de Unscented Kalman Filter (UKF), que tem como vantagem tratar o problema de estimação sem a necessidade de linearizar o modelo do sistema. Com este método, os autores mostram que ao transformar uma variável aleatória através de qualquer função não linear analítica 5 , as estimativas da média e covariância apresentam erros de terceira ordem no máximo para qualquer variável aleatória e erros de quarta ordem para variáveis aleatórias simetricamente distribuídas. No FEK,

\footnotetext{
${ }^{4}$ Existem implementações de segunda ordem para o FEK, mas a carga computacional necessária é muito alta. A solução usual para estes casos é calcular a hessiana do modelo apenas em algumas poucas iterações e fixá-la, assumindo que esta não deve variar muito.

${ }^{5}$ Em Julier e Uhlmann [90] mostra-se bons resultados também para funções descontínuas.
} 
os erros são de segunda e quarta ordem respectivamente (para variáveis aleatórias gaussianas).

A idéia básica do UKF vem do filtro de partículas [61, p. 129], porém amostrando de forma determinística um número pequeno de realizações das variáveis aleatórias, chamadas de Pontos Sigma. Após a amostragem, os pontos sigma são transformados através da equação de evolução não linear e/ou a equação de observação não linear. Esta transformação é nomeada pelos autores Unscented Transform (UT).

Sejam as variáveis aleatórias $\mathbf{x} \in \mathbb{R}^{n}$ e $\mathbf{y} \in \mathbb{R}^{m}$ relacionadas através de uma função $\mathbf{y}=\boldsymbol{h}(\mathbf{x})$. Assumindo conhecidos o valor esperado $\overline{\boldsymbol{x}}$ e a matriz de covariância $\Gamma^{x x}$ da variável aleatória $\mathbf{x}$, deseja-se conhecer $\overline{\boldsymbol{y}}$ e $\Gamma^{y y}$ da variável aleatória $\mathbf{y}$ e também a matriz de covariância cruzada $\Gamma^{x y}=E\left\{(\mathbf{x}-\overline{\boldsymbol{x}})(\mathbf{y}-\overline{\boldsymbol{y}})^{\mathrm{T}}\right\}$.

O cálculo da UT é dada pelos seguintes passos:

1. Um conjunto de pontos sigma $\left\{\mathcal{X}^{i}\right\}_{0}^{2 n}$ e seus pesos $W_{i}$ são determinados

$$
\begin{aligned}
\mathcal{X}^{0} & =\overline{\boldsymbol{x}} \\
\mathcal{X}^{i} & =\overline{\boldsymbol{x}}+\gamma\left[\sqrt{\boldsymbol{\Gamma}^{x x}}\right]_{(:, i)}, \quad i=1, \ldots, n \\
\mathcal{X}^{i+n} & =\overline{\boldsymbol{x}}-\gamma\left[\sqrt{\boldsymbol{\Gamma}^{x x}}\right]_{(:, i)}, \quad i=1, \ldots, n \\
W_{0} & =\frac{\kappa}{(n+\kappa)} \\
W_{i} & =\frac{1}{2(n+\kappa)}, \quad i=1, \ldots, 2 n,
\end{aligned}
$$

onde $\gamma=\sqrt{n+\kappa}$ e a notação $\left[\sqrt{\Gamma^{x x}}\right]_{(:, i)}$ representa a $i$-ésima coluna de uma matriz raiz quadrada (usualmente o fator de Cholesky triangular inferior) da covariância do estado. O escalar $\kappa>-n$ é um parâmetro de dispersão dos pontos sigma. Para funções escalares, $\kappa=2$ resulta em erros de sexta ordem. Para funções em $\mathbb{R}^{n}, \kappa=3-n$ resulta em estimativas que minimizam o erro quadrático até a quarta ordem [90].

2. O conjunto de pontos sigma é transformado utilizando a função $\mathbf{y}=\boldsymbol{h}(\mathbf{x})$.

$$
\mathcal{Y}^{i}=\boldsymbol{h}\left(\mathcal{X}^{i}\right), \quad i=0, \ldots, 2 n
$$


3. A média e as covariâncias são então calculadas com os conjuntos $\left\{\mathcal{X}^{i}\right\}_{0}^{2 n}$ e $\left\{\mathcal{Y}^{i}\right\}_{0}^{2 n}$

$$
\begin{aligned}
\overline{\boldsymbol{y}} & =\sum_{i=0}^{2 n} W_{i} \mathcal{Y}^{i} \\
\Gamma^{y y} & =\sum_{i=0}^{2 n} W_{i}\left(\mathcal{Y}^{i}-\overline{\boldsymbol{y}}\right)\left(\mathcal{Y}^{i}-\overline{\boldsymbol{y}}\right)^{\mathrm{T}} \\
\Gamma^{x y} & =\sum_{i=0}^{2 n} W_{i}\left(\mathcal{X}^{i}-\overline{\boldsymbol{x}}\right)\left(\mathcal{Y}^{i}-\overline{\boldsymbol{y}}\right)^{\mathrm{T}} .
\end{aligned}
$$

Para entender esta transformação, considere um caso unidimensional em que $y=x^{2}$ e $x \sim \mathcal{N}(0,1)$. Os valores da média de variância de $y$ podem ser calculadas analiticamente para comparação

$$
\begin{aligned}
\bar{y} & =E\{y\}=\int_{-\infty}^{\infty} x^{2} f_{x} d x=1 \\
\sigma_{y}^{2} & =E\left\{(y-\bar{y})^{2}\right\}=\int_{-\infty}^{\infty}\left(x^{2}-1\right)^{2} f_{x} d x=2 .
\end{aligned}
$$

Agora, utilizando a UT para este mesmo problema tem-se

1. Pontos sigma $\left\{\mathcal{X}^{i}\right\}_{0}^{2}$ e seus pesos

$$
\mathcal{X}^{0}=0, \quad \mathcal{X}^{1,2}= \pm \gamma \sigma_{x}, \quad W_{0}=\frac{\kappa}{(1+\kappa)}, \quad W_{1,2}=\frac{1}{2(1+\kappa)}
$$

2. Pontos sigma $\left\{\mathcal{Y}^{i}\right\}_{0}^{2}$ calculados através da função não linear $y=x^{2}$

$$
\mathcal{Y}^{0}=0, \quad \mathcal{Y}^{1,2}=\gamma^{2} \sigma_{x}^{2}
$$

3. Finalmente, as estatísticas de $y$ podem ser estimadas, lembrando que $\kappa=2$ para funções escalares

$$
\begin{aligned}
\bar{y} & =\sum_{i=0}^{2} W_{i} \mathcal{Y}^{i}=1 \\
\sigma_{y}^{2} & =\sum_{i=0}^{2} W_{i}\left(\mathcal{Y}^{i}-\bar{y}\right)\left(\mathcal{Y}^{i}-\bar{y}\right)^{\mathrm{T}}=2 .
\end{aligned}
$$

Estes resultados coincidem com os resultados analíticos. 
Já no caso das estimativas através da linearização da função, que seria utilizado no FEK, tem-se

$$
\begin{aligned}
y & \left.\approx y\right|_{\bar{x}}+\left.\frac{d y}{d x}\right|_{\bar{x}}(x-\bar{x})=\bar{x}^{2}+2 \bar{x}(x-\bar{x}) \\
\bar{y} & =E\{y\} \approx \bar{x}^{2}+2 \bar{x}(E\{x\}-\bar{x})=\bar{x}^{2}=0 \\
\sigma_{y}^{2} & =E\left\{(y-\bar{y})^{2}\right\} \approx E\left\{\left(y-\bar{x}^{2}\right)^{2}\right\}=(2 \bar{x})^{2} \sigma_{x}^{2}=0 .
\end{aligned}
$$

Em um caso multivariável, os pontos sigma estarão distribuídos ao redor do hiper-elipsóide relacionado à forma quadrática da matriz de covariância $\Gamma^{x x}$. O fator $\gamma$ regula o tamanho desta hiper-elipsóide e controla então a dispersão dos pontos sigma. Para relações $\mathbf{y}=\boldsymbol{h}(\mathbf{x})$ altamente não lineares, como por exemplo em funções trigonométricas ou exponenciais, deve ser usado um fator pequeno para que apenas os efeitos locais sejam levados em conta.

Seja agora a estimação da média e covariância de uma variável aleatória $\mathbf{z} \in \mathbb{R}^{2}$ definida por

$$
\begin{aligned}
& \mathbf{z}=\left[\begin{array}{ll}
v_{1} \cdot v_{2} & v_{1}^{2}
\end{array}\right]^{\mathrm{T}} \\
& \mathbf{v} \sim \mathcal{N}\left(\overline{\boldsymbol{v}}, \boldsymbol{\Gamma}^{v}\right) \\
& \overline{\boldsymbol{v}}=\left[\begin{array}{ll}
1 & 2
\end{array}\right]^{\mathrm{T}}, \quad \boldsymbol{\Gamma}^{v}=\left[\begin{array}{ll}
1 & 1 \\
1 & 4
\end{array}\right] .
\end{aligned}
$$

Neste exemplo numérico inicialmente foram realizadas dez mil amostragens de $\mathbf{v}$ para uma primeira estimação da média e covariância de z. Uma fração destas amostras pode ser vista na Figura 4.2(a) como pontos de cor cinza. Nesta mesma figura, a elipse traçada é o lugar geométrico dos pontos cuja distancia de Mahalanobis $6 d_{M}(x)$ é unitária. A distância $d_{M}(x)=1$ pode ser compreendida simplificadamente como " $a$ generalização em $\mathbb{R}^{2}$ do conjunto de pontos distantes um desvio padrão da média". Ainda nesta figura, os círculos em cor verde são os pontos sigma $\left\{\mathcal{V}^{i}\right\}_{0}^{5}$ selecionados para a UT. Estes pontos estão distribuídos ao longo da elipse de Mahalanobis, porém afastados desta pelo fator $\gamma$, que neste exemplo vale $\sqrt{3}$. As posições dos pontos sigma dependem da escolha da matriz raiz quadrada e, portanto, não necessariamente localizam-se sobre

\footnotetext{
${ }^{6}$ A distância de Mahalanobis é definida por $d_{M}(x)=\left[(\mathbf{x}-\overline{\boldsymbol{x}})^{\mathrm{T}} \boldsymbol{\Gamma}(\mathbf{x}-\overline{\boldsymbol{x}})\right]^{1 / 2}$. Esta medida generaliza o conceito de distância à média em espaços de dimensão maior que um.
} 
os eixos da elipse. Neste exemplo foi escolhida a fatoração de Cholesky como raiz quadrada de $\Gamma^{v}$.

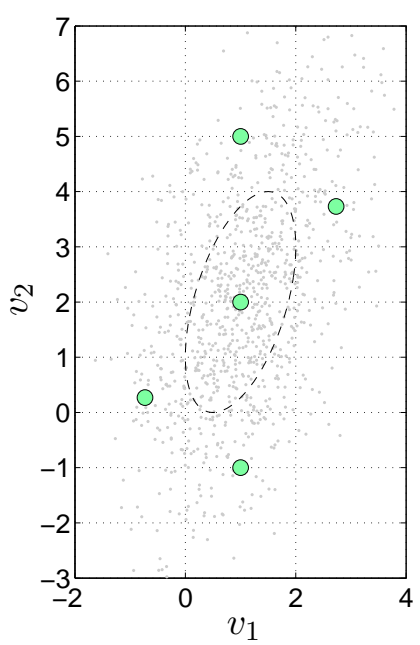

(a) Amostras de v.

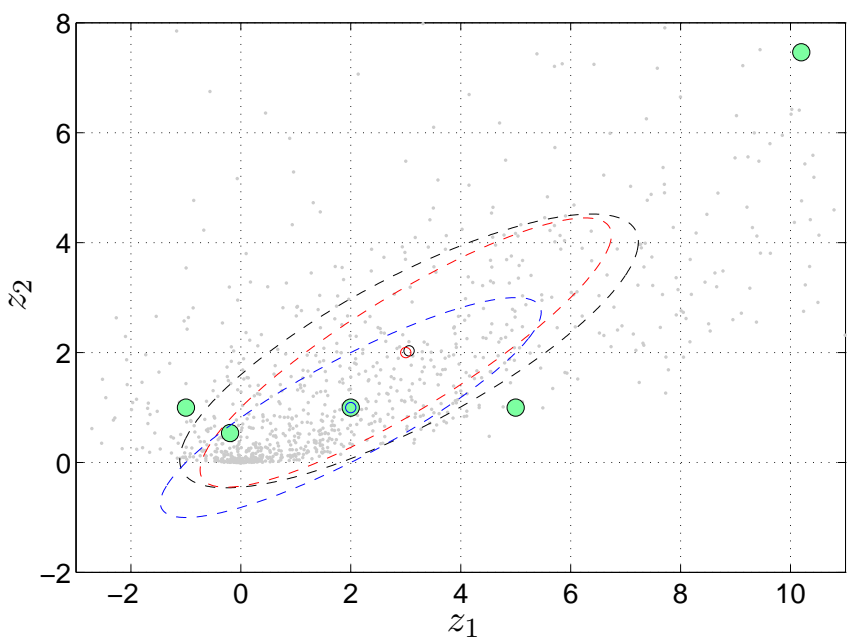

(b) Amostras de $\mathbf{z}$

Figura 4.2: Exemplo em $\mathbb{R}^{2}$. Os pontos cinza são amostras das variáveis aleatórias. Os círculos verdes são os pontos sigma e as elipses são os lugares geométricos onde $d_{M}(x)=1$ para a estimação com amostragem (preto), com o uso da UT (vermelho) e linearização (azul).

A Figura 4.2(b) apresenta a variável z. Os pontos cinzas são os valores obtidos ao transformar cada uma das amostras de $\mathbf{v}$ através da função não linear (4.77). Os círculos em verde são os pontos sigma $\left\{\mathcal{Z}^{i}\right\}_{0}^{5}$ transformados através da UT. Nesta mesma figura, a elipse de Mahalanobis preta está associada à matriz de covariância obtida através das amostras, a elipse vermelha está associada à covariância estimada com a UT e a azul à covariância estimada com a linearização de (4.77). É possível ver que a estimativa obtida pela da UT está mais próxima da estimativa utilizando o processo de amostragem.

Os valores numéricos das médias e covariâncias para este exemplo são apresentados na Tabela 4.1. Por esta tabela nota-se que os valores das médias e covariâncias entre UT e amostragem são bastante próximos. Já a linearização subestima tanto a média quanto a covariância.

Uma outra vantagem do UKF em relação ao FEK está na avaliação da função não linear $\boldsymbol{h}$ diretamente com os estados amostrados $\mathcal{X}^{i}$, não necessitando determinar as matrizes jacobianas utilizadas no FEK. Portanto possíveis efeitos de descontinuidade 
Tabela 4.1: Comparação dos resultados entre Amostragem, UT e linearização.

\begin{tabular}{lcc}
\hline método & média $\overline{\boldsymbol{z}}$ & covariância $\Gamma^{z}$ \\
\hline amostragem & {$\left[\begin{array}{l}2.98 \\
1.98\end{array}\right]$} & {$\left[\begin{array}{cc}17.29 & 8.03 \\
8.03 & 5.91\end{array}\right]$} \\
UT & {$\left[\begin{array}{l}3.00 \\
2.00\end{array}\right]$} & {$\left[\begin{array}{cc}14.00 & 8.00 \\
8.00 & 6.00\end{array}\right]$} \\
linearização & {$\left[\begin{array}{l}2.00 \\
1.00\end{array}\right]$} & {$\left[\begin{array}{cc}12.00 & 6.00 \\
6.00 & 4.00\end{array}\right]$} \\
\hline
\end{tabular}

podem ser levados em conta diretamente, sem necessidade de implementações computacionais complexas.

Entretanto o UKF também pode sofrer problemas de divergência. Os trabalhos Perea et al. [91], Perea [92] apresentam o problema de divergência no UKF para o caso de não linearidade nas observações. A divergência é causada pela rápida contração da matriz de covariância do estado estimado em comparação com a distância entre o estado verdadeiro e a média estimada, e também pelo possível aumento do seu número de condição. Com esta rápida contração e aumento do número de condição, o estado verdadeiro passa a localizar-se fora do intervalo de confiança da estimativa após algumas iterações. Com o intuito de sanar o problema de divergência, os autores propõem o método modificado UKFz com a substituição do cálculo de $\overline{\boldsymbol{y}}$ da UT pela aproximação $\overline{\boldsymbol{y}} \approx \boldsymbol{h}(\overline{\boldsymbol{x}})$. Esta modificação impõe uma inovação com maior magnitude e covariância na equação de atualização do filtro. Em Perea e Elosegui [93] é proposta uma nova modificação do UKFz, desta vez apenas modificando a equação de atualização do estado visando retardar a contração da matriz de covariância. Este método é chamado IUKF, do inglês Improved UKF. A modificação consiste em manter o cálculo de $\overline{\boldsymbol{y}}$ conforme a UT e apenas substituir o cálculo da inovação $\left(\boldsymbol{y}_{\text {medido }}-\overline{\boldsymbol{y}}\right)$ por $\left(\boldsymbol{y}_{\text {medido }}-\boldsymbol{h}(\overline{\boldsymbol{x}})\right)$. Com esta modificação, $\boldsymbol{\Gamma}^{y y}$ permanece inalterada, não reduzindo a taxa de convergência do algoritmo.

Outro problema do UKF é analisado em Leven e Lanterman [94]. Os autores mostram um exemplo de equação não linear em que os pontos sigma $\mathcal{Y}^{i}$ estão degenerados em um subespaço de dimensão menor que o espaço $\boldsymbol{y}$ (ou estão quase 
degenerando em um subespaço de dimensão menor). No trabalho é apresentado um exemplo no qual os pontos sigma $\mathcal{Y}^{i}$ transformados com $\boldsymbol{h}$ dispõem-se todos sobre um plano cruzando o espaço $\mathbb{R}^{3}$. Nesta situação, ao calcular $\Gamma^{y y}$, obtém-se uma matriz singular (ou com número de condição muito elevado), o que torna o cálculo da matriz de ganho do filtro de Kalman propenso a erros numéricos já que necessita da inversa de $\Gamma^{y y}$. Em estatística, este problema é chamado problema de multicolinearidade.

A multicolinearidade refere-se à situação em que duas ou mais componentes de um vetor aleatório $\mathbf{y} \in \mathbb{R}^{m}$ são correlacionadas, ou seja, existe um conjunto $\left\{\lambda_{i}\right\}_{1}^{m}$, não todos nulos simultaneamente, tal que

$$
\sum_{i=1}^{m} \lambda_{i} y_{i}=0 .
$$

No trabalho em questão, os autores propõem uma mudança de variáveis, ou seja, uma mudança da função $\boldsymbol{h}$ para contornar o problema. Após a modificação no exemplo tridimensional, os pontos ainda estão dispostos sobre uma superfície, mas esta não é mais plana no sentido euclidiano e o cálculo de $\Gamma^{y y}$ não resulta mais em uma matriz singular.

Em Julier [95, 96] é proposto o algoritmo UKF escalado, que remove o problema de obter uma matriz $\Gamma^{y y}$ nos casos em que $\kappa<0$. Existem variações do UKF nas quais um número menor de pontos sigma é escolhido. O UKF simplex de assimetria mínima é proposto em Julier e Uhlmann [97], que necessita apenas $n+1$ pontos sigma para a UT, e em Julier [98] é proposto o UKF simplex esférico, que é mais estável numericamente do que o UKF simplex de assimetria mínima.

Uma outra forma de reduzir o número de pontos sigma é proposto em Padilla e Rowley [99]. Os autores definem os pontos sigma após o truncamento da decomposição em autovalores da matriz de covariância $\Gamma^{x x}$. No trabalho, o truncamento é realizado de forma a captar 99\% da variância total das componentes do vetor aleatório $\mathbf{x}$.

Outras variantes do UKF podem ser encontradas, como o UKF iterado [100] ou para aplicações em que há algum tipo de vínculo de intervalo nas variáveis de estado [101]. Em Haykin et al. [102, p. 273] e Wan e Merwe [103] é introduzida a variante raiz quadrada do UKF (SR-UKF), que apresenta melhor estabilidade numérica pois garante 
que as matrizes de covariâncias sejam positivas definidas. Os autores incorporam ainda nesta implementação a formulação do UKF escalado proposta em Julier [96]. Esta variante é utilizada neste trabalho.

\subsection{Método do erro de aproximação}

Considere inicialmente um sistema cujo modeld]

$$
\boldsymbol{y}=\boldsymbol{h}(\boldsymbol{x}, \boldsymbol{p}, \boldsymbol{u}, \boldsymbol{e})
$$

descreve com precisão e acurácia suficiente os fenômenos que ocorrem nele. Nesta equação, $\boldsymbol{x}$ é o vetor de estado, $\boldsymbol{p}$ é um vetor que contém os parâmetros deste modelo, $\boldsymbol{u}$ é um vetor de entradas conhecidas e $\boldsymbol{e}$ é o vetor de perturbações.

$\mathrm{O}$ vetor $\boldsymbol{p}$ contém propriedades físicas deste sistema e normalmente é medido previamente com algum aparato experimental. No entanto, muitas vezes estes parâmetros não podem ser medidos, ou não podem ser medidos com precisão, ou ainda estes mudam de forma imprevisível como o passar do tempo. Em tais casos só é possível encontrar um vetor de parâmetros aproximados para o modelo em questão, denotado por $\boldsymbol{p}^{a}$.

Outra fonte de problemas é a necessidade de simplificações do modelo. Muitas vezes (4.81) não pode ser usado diretamente nos filtros devido ao alto custo computacional, que necessitaria de mais tempo para os cálculos do que a aplicação permite. Em tais casos, uma função simplificada $\boldsymbol{h}^{a}$ deve ser usada, geralmente um linearização ou discretização de $\boldsymbol{h}$. Nestes casos, um estado aproximado $\boldsymbol{x}^{a}$ é empregado e está relacionado $\operatorname{com} \boldsymbol{x}$ em (4.81) através de uma projeção em um subespaço por $\boldsymbol{x}^{a}=\boldsymbol{T} \boldsymbol{x}$, onde $\boldsymbol{T}$ é um operador de projeção e a mudança de $\boldsymbol{h}$ para $\boldsymbol{h}^{a}$ pode ser concebida através da adição de novos parâmetros em $\boldsymbol{p}^{a}$.

Além disso, perturbações $e$ podem agir no sistema. Por sua natureza desconhecida/imprevisível, o melhor que se pode fazer é usar algum valor aproximado $e^{a}$ para descrever a perturbação, normalmente o seu valor médio quando suas estatísticas são conhecidas.

\footnotetext{
${ }^{7}$ Salienta-se aqui que os filtros de Kalman utilizam dois modelos do sistema, um para a observação e outro para a evolução temporal. Ambos os modelos podem ser escritos no formato da equação (4.81) e podem usufruir deste método.
} 
Em geral, mais de um destes fatores ocorrem em um sistema. Seja a função aproximada $\boldsymbol{h}^{a}$ escrita em forma genérica através de

$$
\boldsymbol{y}=\boldsymbol{h}^{a}\left(\boldsymbol{x}^{a}, \boldsymbol{p}^{a}, \boldsymbol{u}, \boldsymbol{e}^{a}\right)
$$

Se esses fatores aproximados puderem ser concebidos como realizações de variáveis aleatórias $\mathbf{x}, \mathbf{p}$ e $\mathbf{e}$, o método do erro de aproximação, primeiramente proposto em [61, p. 181] pode ser empregado. Este método foi utilizado em outros problemas inversos, como em Huttunen [104], Lipponen et al. [105], Lehikoinen et al. [106].

O método do erro de aproximação lida com a questão Como levar em conta os erros inerentes da utilização do modelo aproximado $\boldsymbol{h}^{a}$ com o conjunto de parâmetros $\boldsymbol{p}^{a} \mathrm{em}$ substituição ao modelo fidedigno $\boldsymbol{h}$ cujos parâmetros $\boldsymbol{p}$ não são conhecidos? O método do erro de aproximação lida com esta questão computando as estatísticas do erro total causado por estas aproximações, dadas as estatísticas de cada elemento aproximado.

Iniciando com (4.81), pode-se reescrevê-la como

$$
\begin{aligned}
\boldsymbol{y} & =\boldsymbol{h}(\boldsymbol{x}, \boldsymbol{p}, \boldsymbol{u}, \boldsymbol{e})+\boldsymbol{h}^{a}\left(\boldsymbol{x}^{a}, \boldsymbol{p}^{a}, \boldsymbol{u}, \boldsymbol{e}^{a}\right)-\boldsymbol{h}^{a}\left(\boldsymbol{x}^{a}, \boldsymbol{p}^{a}, \boldsymbol{u}, \boldsymbol{e}^{a}\right) \\
& =\boldsymbol{h}^{a}\left(\boldsymbol{x}^{a}, \boldsymbol{p}^{a}, \boldsymbol{u}, \boldsymbol{e}^{a}\right)+\left[\boldsymbol{h}(\boldsymbol{x}, \boldsymbol{p}, \boldsymbol{u}, \boldsymbol{e})-\boldsymbol{h}^{a}\left(\boldsymbol{x}^{a}, \boldsymbol{p}^{a}, \boldsymbol{u}, \boldsymbol{e}^{a}\right)\right] \\
& =\boldsymbol{h}^{a}\left(\boldsymbol{x}^{a}, \boldsymbol{p}^{a}, \boldsymbol{u}, \boldsymbol{e}^{a}\right)+\boldsymbol{\varepsilon},
\end{aligned}
$$

onde $\varepsilon$ é a discrepância do resultado $y$ por adotar o modelo aproximado (4.82) em substituição ao modelo exato (4.81)

$$
\varepsilon=\boldsymbol{h}(\boldsymbol{x}, \boldsymbol{p}, \boldsymbol{u}, \boldsymbol{e})-\boldsymbol{h}^{a}\left(\boldsymbol{x}^{a}, \boldsymbol{p}^{a}, \boldsymbol{u}, \boldsymbol{e}^{a}\right) .
$$

Considerando que esta discrepância seja modelada como uma variável aleatória, as estatísticas de $\boldsymbol{y}$ em (4.83) são as mesmas 8 que em (4.81) se as estatísticas de $\varepsilon$ forem conhecidas. Neste sentido estatístico, os modelos (4.83) e (4.81) são equivalentes. Este modelo é muitas vezes chamado de modelo do erro de aproximação ou, se a dependência de $\varepsilon$ com relação a $\boldsymbol{x}$ puder ser ignorada, modelo de ruído melhorado (do inglês enhanced error model) [61, p. 182].

\footnotetext{
${ }^{8}$ A equivalência das estatísticas só ocorre se as estatísticas de $\varepsilon$ forem determinada com exatidão. Na maioria dos casos apenas estimativas destas estatísticas são determinadas.
} 
Somente o modelo (4.83) é utilizado iterativamente nos filtro de Kalman, porém as estatísticas do modelo fidedigno estarão inclusas na forma da variável aleatória $\varepsilon$. $\mathrm{O}$ modelo completo (4.81) é utilizado apenas para o cálculo das estatísticas de $\varepsilon$, que é realizado antes da coleta de qualquer medida, sendo independente dos resultados da estimação do sistema. As estatísticas de $\varepsilon$ dependem exclusivamente das escolhas $\boldsymbol{p}^{a}$, $\boldsymbol{e}^{a}$ e $\boldsymbol{x}^{a}$ e das estatísticas de $\mathbf{x}, \mathbf{p}$ e $\mathbf{e}$.

\subsubsection{Cálculo das estatísticas do método do erro de aproximação}

Em geral não é tarefa trivial encontrar as estatísticas de $\varepsilon$ a partir de (4.84) analiticamente, mas métodos numéricos podem ser usados para estimar suas estatísticas. É bom ressaltar novamente que todos os cálculos podem ser realizados antes de que qualquer medição seja feita. Desta forma, um algoritmo offline pode ser implementado e as estatísticas armazenadas em um computador para uso posterior, desde que os mesmos parâmetros aproximados sejam utilizados e as estatísticas de $\mathbf{x}$, p e e utilizadas no cálculo offline permaneçam válidas.

Considere que $\boldsymbol{u}$ seja conhecido e $\boldsymbol{p}^{a}$ e $\boldsymbol{e}^{a}$ sejam escolhidos. Seja também o nível de discretização do estado fixado e a relação $\boldsymbol{x}^{a}=\boldsymbol{T} \boldsymbol{x}$ determinada. Seja ainda a densidade de probabilidade conjunta $f_{\mathbf{x}, \mathbf{p}, \mathbf{e}}$, conhecida e não necessariamente com base em pressupostos gaussianos?. O método básico para aproximação das estatísticas do erro de aproximação é:

1. Amostrar um número suficientemente grande de realizações $\boldsymbol{x}_{i}, \boldsymbol{e}_{i}$ e $\boldsymbol{p}_{i}$ seguindo suas densidades de probabilidade. Calcular também a projeção $\boldsymbol{x}_{i}^{a}=\boldsymbol{T} \boldsymbol{x}_{i}$ caso haja discretização do estado envolvida na simplificação do modelo;

2. Para cada conjunto $\left(\boldsymbol{x}_{i}, \boldsymbol{e}_{i}, \boldsymbol{p}_{i}\right)$ (e também $\boldsymbol{x}_{i}^{a}$ caso haja discretização), utilizar (4.84) para calcular realizações de $\varepsilon_{i}$, utilizando também os valores fixados para $\boldsymbol{p}^{a}$ e $\boldsymbol{e}^{a}$ e o valor conhecido $\boldsymbol{u}$;

3. Com todas as realizações de $\varepsilon_{i}$, calcular aproximações das suas estatísticas.

Geralmente, supõe-se que $\varepsilon$ seja Gaussiana e, por este motivo, as sua estatísticas são completamente descritas por sua média $\bar{\varepsilon}$ e covariância $\Gamma^{\varepsilon}$. Geralmente utiliza-se os estimadores não viesados usuais para esta estimação.

\footnotetext{
${ }^{9}$ Geralmente é assumida a independência entre $\mathbf{p}, \mathbf{e}$ e $\mathbf{x}$, mas isto não é estritamente necessário.
} 
Uma desvantagem deste método simplificado é que esta amostragem é realizada antes da estimação e não considera que a densidade de probabilidade a priori $f_{\mathbf{x}, \mathbf{p}, \mathbf{e}}$ pode ser corrigida ou ajustada melhor conforme a estimação está em andamento. Para incluir esta informação extra, o cálculo das estatísticas de $\varepsilon$ deve ser corrigido conforme novas estimativas são obtidas. Para incluir tal mudança, Lehikoinen et al. [106] apresenta um método baseado nos métodos de amostragem por importância que leva em conta tal mudança e atualiza as estatísticas. Nesta tese o método mais simplificado é utilizado.

Como última observação, se o vetor $\boldsymbol{u}$ contiver entradas de controle, este método pode ser ainda utilizado. Considerando, por exemplo, um controle linear quadrático, pode-se considerar a determinação do controle $\boldsymbol{u}$ como uma função do estado atual $\boldsymbol{x}$ e de alguns parâmetros de controle, como estado de referência e matrizes da função custo, agrupados em um vetor $r$. Pode-se então considerar o cálculo do controle através da solução da equação de Riccati como uma função do tipo

$$
\boldsymbol{u}_{k}=g\left(\boldsymbol{x}_{k}, \boldsymbol{r}\right)
$$

A única alteração necessária no algoritmo descrito acima para a estimativa das estatísticas de $\varepsilon$ é calcular $\boldsymbol{u}_{i} \operatorname{com}(4.85)$ utilizando $\boldsymbol{x}_{i}^{a}$ no primeiro passo. 


\title{
Parte II
}

\author{
Método
}





\section{Capítulo 5}

\section{Método Proposto}

A seguir mostra-se um fluxograma do método proposto neste trabalho. Neste diagrama, o SR-UKF estima o estado do sistema, composto pela distribuição de resistividade elétrica dentro de $\Omega$, em paralelo com a estimação dos parâmetros dos eletrodos e estimação dos parâmetros do modelo de evolução.

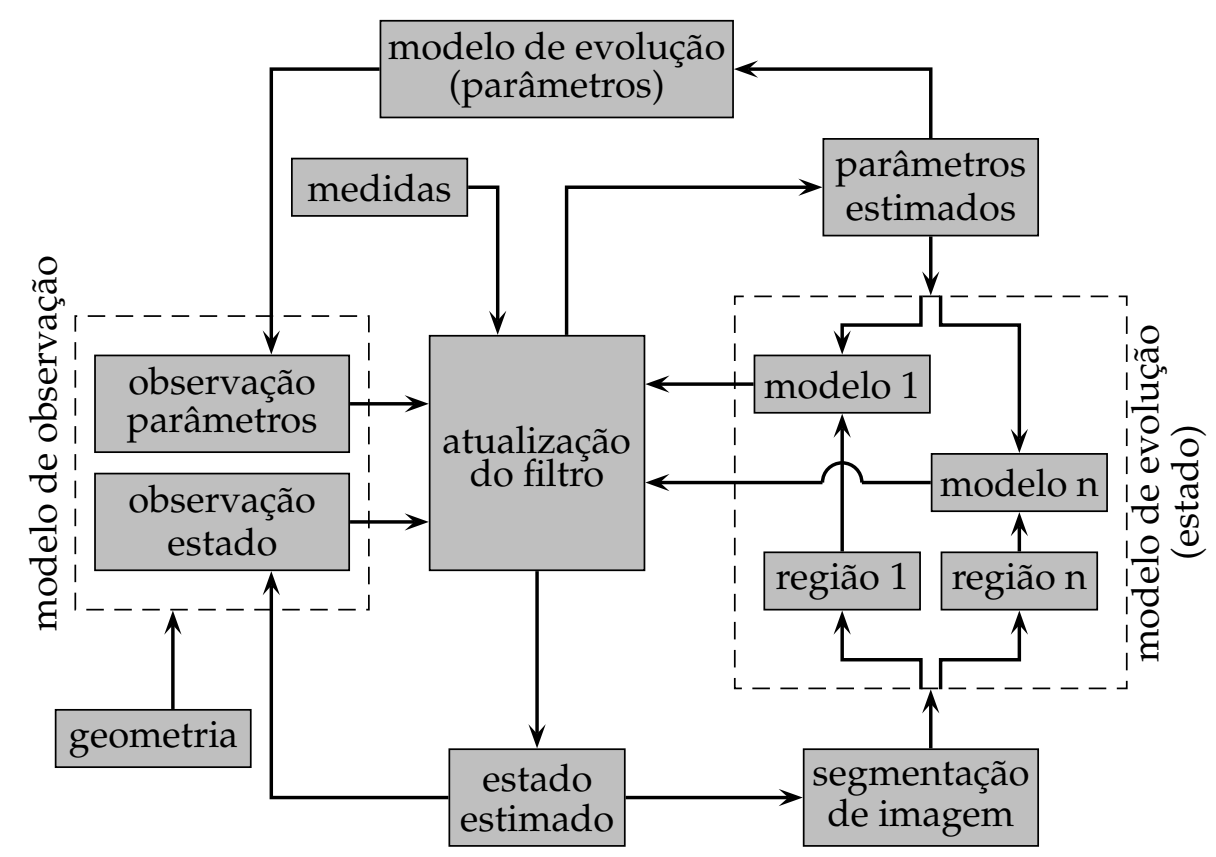

Figura 5.1: Diagrama de funcionamento do algoritmo.

A estimativa dos parâmetros do modelo de evolução ajusta o modelo adotado sobre a dinâmica do paciente sob monitoração. Desta forma, espera-se que o modelo seja mais fidedigno e erros de modelagem sejam minimizados. Uma etapa de segmentação 
de imagem identifica as principais estruturas internas e assim seleciona o modelo de evolução apropriado para cada segmento identificado.

A segmentação de imagem é feita sobre os mapas, discretizados nos elementos da malha de elementos finitos, da distribuição de resistividade em $\Omega$. O método de segmentação adotado é o level set, que é apresentado no Capítulo 10 .

\subsection{Implementação do UKF na TIE para monitoramento de pacientes}

O sistema dinâmico que o UKF deve estimar no monitoramento das condições pulmonares é não linear pois, além do modelo não linear de observação, inclui o comportamento dinâmico e não linear dos diversos órgãos e tecidos localizados na caixa torácica, como os pulmões, o coração, o tecido muscular, além dos tecidos sem variações expressivas para esta aplicação, como o tecido ósseo e o tecido adiposo. Em adição, o comportamento dos tecidos pode variar consideravelmente ao longo do tempo em virtude das mudanças das condições fisiológicas do paciente.

Sob o ponto de vista Bayesiano, considera-se que o sistema é o conjunto de tecidos no interior do tórax do paciente e o estado $\boldsymbol{x}_{k}=\boldsymbol{\rho}_{k} \in \mathbb{R}^{n+e}$ é a distribuição de resistividade elétrica dentro de $\Omega$, mais os parâmetros dos eletrodos, discretizado em uma malha de elementos finitos como descrito no Apêndice A.1 e Apêndice A.2. Considera-se que o estado em um determinado instante é uma realização do vetor aleatório $\mathbf{x}_{k}$. O modelo de evolução do estado possui um conjunto de $r$ parâmetros a serem ajustados, agrupados em um vetor $\boldsymbol{p}_{k} \in \mathbb{R}^{r}$, que por sua vez também é considerado uma realização do vetor aleatório $\mathbf{p}_{k}$.

O modelo de evolução do estado contém um ruído aditivo e pode ser escrito no tempo discreto $t_{k}=k T_{s}$, para $k=0,1 \ldots$ como

$$
\mathbf{x}_{k+1}=\boldsymbol{\Phi}_{k}\left(\mathbf{x}_{k}, \mathbf{p}_{k}, \boldsymbol{u}_{k}\right)+\boldsymbol{A}_{k}^{x} \mathbf{v}_{k}^{x}
$$

onde $\Phi_{k}$ é a componente nominal do modelo de evolução composto pelos modelos dos diversos órgãos a ser apresentado no Capítulo $9 \mathbf{p}_{k}$ é o vetor aleatório composto pelos $r$ parâmetros do modelo de evolução, $\mathbf{v}_{k}^{x}$ é um ruído não correlacionado no tempo e, 
por hipótese, Gaussiano que excita o sistema e $\boldsymbol{A}_{k}^{x}$ é uma matriz conhecida. O vetor $\boldsymbol{u}_{k} \in \mathbb{R}^{s}$ é composto pelas possíveis $s$ entradas conhecidas do sistema e, portanto, não é uma variável aleatória.

Considera-se aqui também a estimação dos parâmetros do modelo de evolução. Para esta estimação, modelos de evolução e observação devem ser propostos. O modelo de evolução dos parâmetros adotado é o chamado passeio aleatório e pode ser escrito como

$$
\mathbf{p}_{k+1}=\mathbf{p}_{k}+\boldsymbol{A}_{k}^{p} \mathbf{v}_{k}^{p}
$$

onde $\mathbf{V}_{k}^{p}$ é um ruído gaussiano não correlacionado no tempo e $\boldsymbol{A}_{k}^{p}$ é uma matriz conhecida. Neste modelo não é esperada uma variação determinística dos valores de $\mathbf{p}$, apenas variações aleatórias devido ao processo gaussiano $\mathbf{v}_{k}^{p}$.

Assume-se que $\mathbf{V}_{k}^{x}$ e $\mathbf{V}_{k}^{p}$ sejam independentes entre si e em relação a $\mathbf{x}_{k}$ e $\mathbf{p}_{k}$ e tenham estatísticas

$$
\begin{aligned}
\mathbf{v}_{k}^{x} & \sim \mathcal{N}\left(\overline{\boldsymbol{v}}_{k}^{x}, \boldsymbol{\Gamma}_{k}^{v^{x}}\right) \\
\mathbf{v}_{k}^{p} & \sim \mathcal{N}\left(\overline{\boldsymbol{v}}_{k}^{p}, \Gamma_{k}^{v^{p}}\right) .
\end{aligned}
$$

Neste trabalho, $\boldsymbol{A}_{k}^{p}$ é assumida a matriz identidade e o ruído $\mathbf{V}_{k}^{p}$ apresenta a mesma dimensão do vetor de parâmetros $\boldsymbol{p}$. A matriz $\boldsymbol{A}_{k}^{x}$ também assume a forma da matriz identidade neste trabalho, porém no caso de monitoramento em leitos de UTI, esta poderia assumir a forma de um filtro passa-baixas espacial 1 . O ruído $\mathbf{v}_{k}^{x}$, apresenta a mesma dimensão do vetor de estado $\boldsymbol{x}_{k}$.

Em adição às variáveis aleatórias apresentadas, o vetor de medidas dos potenciais elétricos $\boldsymbol{y}_{k} \in \mathbb{R}^{\ell}$ é uma realização do vetor aleatório $\mathbf{y}_{k}$ e relaciona-se com o estado através do modelo de observação. O modelo de observação proposto para estimação do estado $\boldsymbol{x}_{k}$ é

$$
\mathbf{y}_{k+1}=\boldsymbol{h}_{k+1}\left(\mathbf{x}_{k+1}\right)+\boldsymbol{B}_{k+1}^{x} \mathbf{e}_{k+1}^{x},
$$

\footnotetext{
${ }^{1}$ Esta escolha para a matriz $\boldsymbol{A}_{k}^{x}$ reflete o fato de que a evolução temporal do estado apresenta certa correlação espacial no sentido de que é de se esperar que dois pontos próximos tenham variações similares de resistividade ao longo do tempo, com exceção nas interfaces entre as diversas regiões segmentadas dentro do domínio, onde o modelo de evolução regional difere.
} 
onde o vetor aleatório $\mathbf{e}_{k+1}^{x}$ é ruído gaussiano, não correlacionado no tempo e assumido independente de $\mathbf{v}_{k}^{x}$ e $\mathbf{v}_{k}^{p}$ e $\boldsymbol{B}_{k+1}^{x}$ é uma matriz conhecida. Assume-se que $\mathbf{e}_{k+1}^{x}$ tenha estatísticas

$$
\mathbf{e}_{k}^{x} \sim \mathcal{N}\left(\overline{\boldsymbol{e}}_{k+1}^{x}, \boldsymbol{\Gamma}_{k+1}^{e^{x}}\right)
$$

A cada iteração do UKF, a equação de observação (2.40) deve ser resolvida para cada ponto sigma, que requer grande carga computacional. Para acelerar as iterações do UKF em casos onde o tempo computacional é fator crítico, o modelo linearizado de observação (2.51) pode ser utilizado, linearizado em torno de

$$
\hat{\boldsymbol{x}}_{k+1 \mid k}=E\left\{\mathbf{x}_{k+1} \mid \mathbf{y}_{1}^{k}=\boldsymbol{y}_{1}^{k}\right\}
$$

Já os parâmetros do modelo de evolução não podem ser observados diretamente, sendo necessário observá-los indiretamente com as medidas elétricas. A componente determinística da equação de observação dos parâmetros dos modelos é dada pela composição das funções nominais $\boldsymbol{h}_{k+1} \circ \boldsymbol{\Phi}_{k}$, ou seja

$$
\mathbf{y}_{k+1}=\boldsymbol{h}_{k+1}\left(\boldsymbol{\Phi}_{k}\left(\mathbf{x}_{k}, \mathbf{p}_{k+1}, \boldsymbol{u}_{k}\right)\right)+\boldsymbol{B}_{k+1}^{p} \mathbf{e}_{k+1}^{p},
$$

onde o vetor aleatório $\mathbf{e}_{k+1}^{p}$ é ruído gaussiano e $\boldsymbol{B}_{k+1}^{p}$ é uma matriz conhecida. Assume-se que $\mathbf{e}_{k+1}^{p}$ tenha média $\overline{\boldsymbol{e}}_{k+1}^{p}$ e matriz de covariância $\boldsymbol{\Gamma}_{k+1}^{e^{p}}$. A função $\boldsymbol{h}_{k+1}$ também é dada por (2.41) ou por (2.51).

A composição de funções nessa equação representa a evolução temporal de uma realização de $\boldsymbol{x}_{k}$ do instante $t_{k}$ até o instante atual $t_{k+1}$, utilizando a componente determinística do modelo (5.1), para uma realização de $\mathbf{p}_{k+1}$. Após a evolução temporal, a amostra do estado propagado $\boldsymbol{x}_{k+1}$ é observada com o modelo de observação (5.5). Salienta-se que $\mathbf{e}_{k}^{x}$ e $\mathbf{e}_{k}^{p}$ não são necessariamente iguais uma vez que há uma composição de incertezas na função $\boldsymbol{h} \circ \boldsymbol{\Phi}$.

Neste trabalho, $\boldsymbol{B}_{k}^{x}$ e $\boldsymbol{B}_{k}^{p}$ são assumidas matrizes identidade e os ruídos $\mathbf{e}_{k}^{x}$ e $\mathbf{e}_{k}^{p}$ apresentam as mesmas dimensões do vetor de medidas $\boldsymbol{y}_{k}$. 


\subsection{Estimação dual com filtro de Kalman}

A princípio, a estimação do estado $\boldsymbol{x}_{k}$ e dos parâmetros $\boldsymbol{p}_{k}$ deve ser feita de forma conjunta, ou seja, deve-se criar um estado aumentado composto pelos dois vetores e compor novos modelos de evolução e observação com os modelos originais e então determinar $f_{\mathbf{x}_{k}, \mathbf{p}_{k} \mid \mathbf{y}_{k}}$. Este método é chamado estimação conjunta e surgiu inicialmente para sistemas bilineares, com o uso do FEK em Kopp e R. J. Orford2 e Cox 3 apud Haykin et al. [102, p. 125] e posteriormente analisado em Ljungt e Niedzwiecki e Cisowski[ apud Haykin et al. [102, p.125]. Porém paga-se um preço considerável pela carga computacional necessária, o que pode ser proibitivo em problemas de predição e filtragem em tempo real.

Uma forma de contornar este problema é utilizar um método alternativo, proposto inicialmente em Nelson e Stear [107] para um sistema bilinear. O método, chamado estimação dual, consiste em usar dois filtros de Kalman em paralelo, um para estimar $\boldsymbol{x}_{k}$ e um para estimar $\boldsymbol{p}_{k}$. Nesta abordagem, a estimativa pontual mais atual de um filtro é utilizada no outro filtro como um parâmetro fixo para a estimação. Desta forma determina-se $f_{\mathbf{x}_{k+1} \mid \mathbf{p}_{k}, \mathbf{y}_{1}^{k+1}}$ e $f_{\mathbf{p}_{k+1} \mid \mathbf{x}_{k}, \mathbf{y}_{1}^{k+1}}$. Este método desconsidera possíveis correlações entre estes vetores, sendo portanto uma aproximação. O método foi aplicado para problemas não lineares em Wan e Nelson [108].

O desempenho da estimação dual ou conjunta varia conforme o problema. Como exemplo, os resultados em Hegyi et al. [109] apresentam um melhor desempenho da estimação conjunta com o FEK, já em Haykin et al. [102, p. 249] e Wan et al. [110], os resultados usando a estimação dual são melhores, tanto com FEK quanto com UKF.

A solução do problema de estimação dual pode ser deduzida da solução de Máximo A Posteriori (MAP) [102, p. 135].

\footnotetext{
${ }^{2}$ R. E. Kopp e R. J. Orford. Linear regression applied to system identification for adaptive control systems. AIAA Journal, 1(10):2300-2306, october 1963.

${ }^{3} \mathrm{H}$. Cox. On the estimation of state variables and parameters for noisy dynamic systems. IEEE Transactions on Automatic Control, 9(1):5-12, 1964.

${ }^{4}$ L. Ljung. Asymptotic behavior of the extended kalman Filter as a parameter estimator for linear systems. IEEE Transactions on Automatic Control, 24(1):36-50, january 1974.

${ }^{5} \mathrm{M}$. Niedzwiecki e K. Cisowski. Adaptive scheme for elimination of broadband noise and impulsive disturbances from ar and arma signals. IEEE Transactions on Signal Processing, 44(3):528-537, march 1996.
} 
Sejam os processos estocásticos $\mathbf{x}_{0}^{k}, \mathbf{y}_{1}^{k}$ e $\mathbf{p}_{0}^{k}$, então

$$
\left(\hat{\boldsymbol{x}}_{0}^{k}, \hat{\boldsymbol{p}}_{0}^{k}\right)=\arg \max _{\boldsymbol{x}_{0}^{k}, \boldsymbol{p}_{0}^{k}} f_{\mathbf{x}_{0}^{k}, \mathbf{p}_{0}^{k} \mid \mathbf{y}_{1}^{k}}=\arg \max _{\boldsymbol{x}_{0}^{k}, \boldsymbol{p}_{0}^{k}} f_{\mathbf{x}_{0}^{k} \mathbf{p}_{0}^{k}, \mathbf{y}_{1}^{k}} \cdot f_{\mathbf{p}_{0}^{k} \mid \mathbf{y}_{1}^{k}}
$$

A estimativa $\hat{\boldsymbol{x}}_{0}^{k}$ é obtida pela maximização do primeiro fator e $\hat{\boldsymbol{p}}_{0}^{k}$ é obtida pela maximização do segundo fator. Como o estado $\mathbf{x}$ só aparece no primeiro fator desta fatoração, a maximização deste termo para determinar $\hat{\boldsymbol{x}}_{0}^{k}$ resulta na mesma solução da maximização da distribuição conjunta, assumindo que o parâmetro $\boldsymbol{p}$ ótimo foi encontrado. Porém, a maximização do segundo termo em relação a $\mathbf{p}_{0}^{k}$ não resulta necessariamente na mesma solução da distribuição conjunta em resultado do fato de que $\mathbf{p}$ aparece em ambos os termos.

A maximização em separado das densidades determina uma solução que maximiza, ao menos localmente, a densidade conjunta $f_{\mathbf{x}_{0}^{k}, \mathbf{p}_{0}^{k} \mid \mathbf{y}_{1}^{k}}$. Por este motivo a inicialização das variáveis para a estimação é importante neste método.

As densidades de probabilidade condicionadas às medidas que devem ser determinadas para o estado e parâmetros são

$$
\begin{aligned}
f_{\mathbf{x}_{0}^{k+1}} \mid \mathbf{p}_{0}^{k}, \mathbf{y}_{1}^{k+1} & =f_{\mathbf{x}_{0}^{k+1} \mid \mathbf{p}_{k}, \mathbf{y}_{1}^{k+1}}\left(\boldsymbol{x}_{0}^{k+1} \mid \hat{\boldsymbol{p}}_{k}, \boldsymbol{y}_{1}^{k+1}\right) \\
f_{\mathbf{p}_{0}^{k+1} \mid \mathbf{x}_{0}^{k}, \mathbf{y}_{1}^{k+1}} & =f_{\mathbf{p}_{0}^{k+1} \mid \mathbf{y}_{1}^{k+1}, \mathbf{x}_{k}}\left(\boldsymbol{p}_{0}^{k+1} \mid \hat{\boldsymbol{x}}_{k}, \boldsymbol{y}_{1}^{k+1}\right),
\end{aligned}
$$

cujas igualdades em ambas vêm do fato de que os processos são Markovianos e, portanto, não dependem de estados anteriores a $t_{k}$. A inclusão da realização $\hat{\boldsymbol{x}}_{k}$ na segunda expressão deve ser interpretada como um parâmetro conhecido da função e não como uma outra variável aleatória, já que em (5.9) foi mostrado que esta densidade de probabilidade não é condicionada à uma realização de $\mathbf{x}$.

Assumindo hipóteses de Gaussianiedade, como descrito anteriormente, o filtro de Kalman pode ser usado. A estimação dual é composta por dois filtros estimando, em paralelo, o estado e os parâmetros dos modelo de evolução, daí o nome filtro de Kalman dual. Em cada um dos filtros, as estimativas pontuais da iteração anterior do outro filtro são utilizadas. O diagrama da Figura 5.2 mostra a implementação dos dois filtros em paralelo (desconsiderando a entrada conhecida $\boldsymbol{u}_{k}$ por brevidade). 


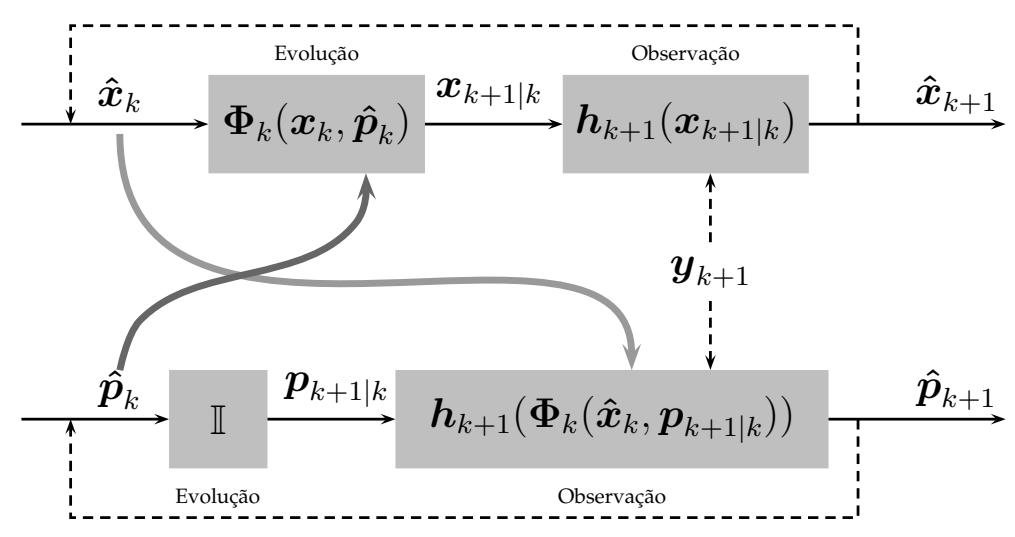

Figura 5.2: Diagrama de implementação em paralelo dos filtros.

Com um argumento análogo, pode-se resolver o problema de estimação das resistividades no domínio em separado da estimação dos parâmetros dos eletrodos. Neste caso, o algoritmo contém três filtros em paralelo estimando alternadamente o estado, os parâmetros dos eletrodos e os parâmetros do modelo. Esta abordagem de estimação foi primeiramente estudada em Trigo [34, cap. 7] e é utilizada também neste trabalho. Os modelos de observação e evolução no filtro para estimação dos eletrodos são análogos a (5.5) e (5.1), exceto que o modelo de evolução é o passeio aleatório.

\subsection{Variante Raiz Quadrada do UKF}

O UKF necessita do cálculo de uma matriz raiz quadrada de $\Gamma_{k}^{x x}$ a cada iteração. A variante raiz quadrada do UKF (SR-UKF) trabalha diretamente com esta matriz raiz quadrada $\boldsymbol{S}^{x}$ sem montar $\boldsymbol{\Gamma}_{k}^{x x}$. A carga computacional não é reduzida nesta variante para estimação de estado, mas há uma melhora numérica da solução como a garantia de positividade da matriz de covariância. Já para estimação de parâmetros, esta variante apresenta vantagem também no número de operações necessárias [103].

Esta variante utiliza a decomposição QR, (des)atualização de Cholesky e solução eficiente de sistemas lineares triangulares para calcular eficientemente as novas estimativas. 
O algoritmo, para ruídos aditivos, é apresentado a seguir 6 .

\section{Inicialização}

Seja o estado $\boldsymbol{x}_{k}=\boldsymbol{\rho}_{k} \in \mathbb{R}^{L}$, onde $L=n \mathrm{e}$

$$
\begin{aligned}
& \overline{\boldsymbol{x}}_{0}=E\left\{\mathbf{x}_{0}\right\} \\
& \boldsymbol{S}_{0}^{x}=\operatorname{chol}\left(\boldsymbol{\Gamma}_{0}^{x x}\right),
\end{aligned}
$$

onde $\operatorname{chol}(\boldsymbol{M})$ representa uma função que retorna o fator (triangular inferior) de Cholesky de uma matriz $M$.

Após a inicialização, para $k=0,1, \ldots$ as fases de propagação, predição das medidas e atualização do filtro são repetidas sucessivamente:

\section{Propagação do filtro}

1-) $\mathrm{O}$ conjunto de $2 L+1$ pontos sigma $\left\{\mathcal{X}_{k}^{i}\right\}_{i=0}^{2 L}$ e seus pesos $W_{i}$ são amostrados da seguinte forma

$$
\begin{aligned}
\mathcal{X}_{k}^{0} & =\overline{\boldsymbol{x}}_{k} \\
\mathcal{X}_{k}^{i} & =\overline{\boldsymbol{x}}_{k}+\gamma\left[\boldsymbol{S}_{k}^{x}\right]_{(:, i)}, \quad i=1, \ldots, L \\
\mathcal{X}_{k}^{i+L} & =\overline{\boldsymbol{x}}_{k}-\gamma\left[\boldsymbol{S}_{k}^{x}\right]_{(:, i)}, \quad i=1, \ldots, L \\
W_{0}^{m} & =\frac{\lambda}{(L+\lambda)} \\
W_{0}^{c} & =\frac{\lambda}{(L+\lambda)}+1-\alpha^{2}+\beta \\
W_{i}^{m} & =W_{i}^{c}=\frac{1}{2(L+\lambda)}, \quad i=1, \ldots, 2 L
\end{aligned}
$$

onde $\gamma=\sqrt{L+\lambda}$ e $\lambda=\alpha^{2}(L+\kappa)-L$. O parâmetrd $\alpha$ regula a dispersão dos pontos sigma ao redor da média, $\kappa$ é um segundo parâmetro de dispersão, usualmente $\kappa=$ $3-L$ ou $\kappa=0$ e $\beta$ é usado para adicionar informação sobre momentos de ordem superior da variável aleatória. Para distribuições gaussianas, $\beta=2$. A notação $\left[\boldsymbol{S}_{k}^{x}\right]_{(:, i)}$ representa a $i$-ésima coluna de $\boldsymbol{S}_{k}^{x}$.

\footnotetext{
${ }^{6}$ As equações do SR-UKF para estimação dos parâmetros são apresentadas no Apêndice C.2

${ }^{7}$ Salienta-se aqui que a forma como foram definidos os parâmetros $\alpha, \beta$ e $\kappa$ resulta na formulação escalada do UKF, que apresenta melhores propriedades numéricas, como discutido em Julier [96].
} 
2-) Os pontos sigma são propagados no tempo com o modelo de evolução (5.1)

$$
\mathcal{X}_{k+1 \mid k}^{i}=\boldsymbol{\Phi}_{k}\left(\mathcal{X}_{k}^{i}, \hat{\boldsymbol{p}}_{k}, \boldsymbol{u}_{k}\right)+\boldsymbol{A}_{k}^{x} \overline{\boldsymbol{v}}_{k}^{x}, \quad i=0, \ldots, 2 L
$$

onde $\hat{\boldsymbol{p}}_{k}$ é a ultima estimativa obtida com o filtro em paralelo para estimação dos parâmetros do modelo de evolução.

3-) As estatísticas de $\mathbf{x}_{k+1 \mid k}$ são calculadas com

$$
\begin{aligned}
& \overline{\boldsymbol{x}}_{k+1 \mid k}=\sum_{i=0}^{2 L} W_{i}^{m} \mathcal{X}_{k+1 \mid k}^{i} \\
& \boldsymbol{S}_{k+1 \mid k}^{x x}=\operatorname{qr}\left(\left[\sqrt{W_{i}^{c}}\left(\mathcal{X}_{k+1 \mid k}^{1: 2 L}-\overline{\boldsymbol{x}}_{k+1 \mid k}\right) \quad \boldsymbol{A}_{k}^{x} \sqrt{\boldsymbol{\Gamma}_{k}^{v^{x}}}\right]\right) \\
& \boldsymbol{S}_{k+1 \mid k}^{x x}=\text { cholupdate }\left(\boldsymbol{S}_{k+1 \mid k}^{x x}, \mathcal{X}_{k+1 \mid k}^{0}-\overline{\boldsymbol{x}}_{k+1 \mid k}, W_{0}^{c}\right) \text {, }
\end{aligned}
$$

onde $\operatorname{qr}(\boldsymbol{M})$ representa uma função que retorna apenas o transposto da parte triangular superior da matriz $\boldsymbol{R}$ da fatoração $\mathrm{QR}$ de uma matriz $\boldsymbol{M}$, cholupdate $(\boldsymbol{M}, \boldsymbol{y}, a)$ é uma função que realiza a (des)atualização de Cholesky 8 de $\boldsymbol{M} \boldsymbol{M}^{\mathrm{T}} \pm \sqrt{a} \boldsymbol{y} \boldsymbol{y}^{\mathrm{T}}$. Se $\boldsymbol{y}$ for uma matriz, então a função executa uma (des)atualização para cada coluna de $\boldsymbol{y}$. O termo $\sqrt{\boldsymbol{\Gamma}_{k}^{v^{x}}}$ é uma matriz raiz quadrada da matriz de covariância do ruído do processo $\mathbf{v}_{k}^{x}$. As duas equações para o cálculo de $\boldsymbol{S}_{k+1 \mid k}^{x x}$ são analisadas em mais detalhes no Apêndice C.1.

\section{Predição das medidas}

1-) O conjunto de pontos sigma é aumentado para contabilizar o ruído aditivo. Para $i=0, \ldots, 4 L$ os pontos sigma e seus pesos são dados por

$$
\begin{aligned}
\mathcal{X}_{k+1 \mid k}^{i} & =\mathcal{X}_{k+1 \mid k}^{i}, \quad i=0, \ldots, 2 L \\
\mathcal{X}_{k+1 \mid k}^{i+2 L} & =\mathcal{X}_{k+1 \mid k}^{0}+\gamma\left[\boldsymbol{A}_{k+1}^{x} \sqrt{\Gamma_{k}^{v^{x}}}\right]_{(:, i)}, \quad i=1, \ldots, L \\
\mathcal{X}_{k+1 \mid k}^{i+3 L} & =\mathcal{X}_{k+1 \mid k}^{0}-\gamma\left[\boldsymbol{A}_{k+1}^{x} \sqrt{\Gamma_{k}^{v^{x}}}\right]_{(:, i)}, \quad i=1, \ldots, L
\end{aligned}
$$

\footnotetext{
${ }^{8}$ A rotina para cálculo da (des)atualização de Cholesky é apresentada no Apêndice D
} 
108

$$
\begin{aligned}
W_{0}^{m} & =\frac{\lambda}{(2 L+\lambda)} \\
W_{0}^{c} & =\frac{\lambda}{(2 L+\lambda)}+1-\alpha^{2}+\beta \\
W_{i}^{m} & =W_{i}^{c}=\frac{1}{2(2 L+\lambda)}, \quad i=1, \ldots, 4 L
\end{aligned}
$$

onde $\gamma=\sqrt{2 L+\lambda}$ e $\lambda=\alpha^{2}(2 L+\kappa)-2 L$.

2-) O conjunto de pontos sigma é observado pelo modelo de observação (5.5)

$$
\mathcal{Y}_{k+1 \mid k}^{i}=\boldsymbol{h}_{k+1}\left(\mathcal{X}_{k+1 \mid k}^{i}\right)+\boldsymbol{B}_{k+1}^{x} \overline{\boldsymbol{e}}_{k+1}^{x}, \quad i=0, \ldots, 4 L .
$$

3-) As estatísticas de $\mathbf{y}_{k+1 \mid k}$ são calculadas com

$$
\begin{aligned}
& \overline{\boldsymbol{y}}_{k+1 \mid k}=\sum_{i=0}^{4 L} W_{i}^{m} \mathcal{Y}_{k+1 \mid k}^{i} \\
& \boldsymbol{S}_{k+1 \mid k}^{y}=\operatorname{qr}\left(\left[\begin{array}{cc}
\sqrt{W_{i}^{c}}\left(\mathcal{Y}_{k+1 \mid k}^{1: 4 L}-\overline{\boldsymbol{y}}_{k+1 \mid k}\right) & \boldsymbol{B}_{k+1}^{x} \sqrt{\boldsymbol{\Gamma}_{k+1}^{e^{x}}}
\end{array}\right]\right) \\
& \boldsymbol{S}_{k+1 \mid k}^{y}=\text { cholupdate }\left(\boldsymbol{S}_{k+1 \mid k}^{y}, \mathcal{Y}_{k+1 \mid k}^{0}-\overline{\boldsymbol{y}}_{k+1 \mid k}, W_{0}^{c}\right) \text {, }
\end{aligned}
$$

onde $\sqrt{\Gamma_{k+1}^{e^{x}}}$ é uma matriz raiz quadrada da matriz de covariância do ruído de observação $\mathbf{e}_{k+1}^{x}$.

\section{Atualização do filtro}

1-) A covariância cruzada entre $\mathbf{x}_{k+1 \mid k}$ e $\mathbf{y}_{k+1 \mid k}$ é determinada por

$$
\boldsymbol{\Gamma}_{k+1 \mid k}^{x y}=\sum_{i=0}^{4 L} W_{i}^{c}\left(\mathcal{X}_{k+1 \mid k}^{i}-\overline{\boldsymbol{x}}_{k+1 \mid k}\right)\left(\mathcal{Y}_{k+1 \mid k}^{i}-\overline{\boldsymbol{y}}_{k+1 \mid k}\right)^{\mathrm{T}} .
$$

2-) As atualizações de $\overline{\boldsymbol{x}}$ e $\boldsymbol{S}^{x}$ são calculadas com

$$
\begin{aligned}
\boldsymbol{U} & =\boldsymbol{\Gamma}_{k+1 \mid k}^{x y} /\left(\boldsymbol{S}_{k+1 \mid k}^{y}\right)^{\mathrm{T}} \\
\boldsymbol{K}_{k+1} & =\boldsymbol{U} / \boldsymbol{S}_{k+1 \mid k}^{y} \\
\overline{\boldsymbol{x}}_{k+1} & =\overline{\boldsymbol{x}}_{k+1 \mid k}+\boldsymbol{K}_{k+1}\left(\boldsymbol{y}_{k+1}^{\text {medido }}-\overline{\boldsymbol{y}}_{k+1 \mid k}\right) \\
\boldsymbol{S}_{k+1}^{x} & =\text { cholupdate }\left\{\boldsymbol{S}_{k+1 \mid k}^{x}, \boldsymbol{U},-1\right\},
\end{aligned}
$$

onde $\boldsymbol{B} / \boldsymbol{A}$ representa a solução eficiente do sistema linear triangular $\boldsymbol{X} \boldsymbol{A}=\boldsymbol{B}$. 
Em Perea e Elosegui [93], é proposta uma modificação para (5.37). A modificação consiste em substituir o o uso das medidas preditas $\overline{\boldsymbol{y}}_{k+1 \mid k}$ pela aproximação

$$
\overline{\boldsymbol{y}}_{k+1 \mid k} \approx \boldsymbol{h}_{k+1}\left(\overline{\boldsymbol{x}}_{k+1 \mid k}\right)+\boldsymbol{B}_{k+1}^{x} \overline{\boldsymbol{e}}_{k+1}^{x},
$$

visando reduzir o problema de divergência que pode ocorrer no UKF. Esta alteração é nomeada pelos autores de IUKF, do inglês improved UKF. Esta modificação é utilizada neste trabalho. 


\section{Capítulo 6}

\section{Restringindo o espaço solução}

Duas formas de restrição do espaço solução foram implementadas. A primeira forma impõe uma restrição no intervalo de valores possíveis para cada componente do vetor de estado na forma de uma mudança de variáveis. A segunda impõe informação $a$ priori como descrito na Seção 4.4 .

\subsection{Vínculo de intervalo}

Caso haja informação a priori da faixa de valores de resistividade dentro de $\Omega$, por exemplo proveniente de um atlas anatômico, pode-se adaptar o algoritmo para que o filtro não procure soluções fora deste intervalo. Isto é especialmente importante em problemas com múltiplos mínimos locais, em que as atualizações do estado nas primeiras iterações do filtro ao redor de um atrator podem acarretar na mudança do estado para próximo de algum outro ponto atrator. Outro caso de importância são aqueles com limitações físicas ou matemáticas, como é o caso da TIE em que os valores assumidos pelas componentes do vetor do estado devem ser estritamente positivos.

O método proposto neste trabalho considera uma mudança de variável para o estadd 1 , de forma que o filtro de Kalman trabalhe em $\mathbb{R}$, enquanto que os valores de interesse se mantenham dentro do intervalo imposto. Outros métodos para incluir vínculos de intervalo no SR-UKF são analisados em Teixeira et al. [101].

\footnotetext{
${ }^{1}$ Este método será apresentado para a distribuição de resistividade dentro do domínio, mas o método pode ser utilizado também no filtro para estimar os parâmetros dos eletrodos ou parâmetros do modelo de evolução.
} 
Seja $\rho_{i}$ a resistividade do $i$-ésimo elemento da malha sob a condição $\left.\rho_{i} \in\right] \rho_{i}^{\min }, \rho_{i}^{\max }[$. Propõe-se uma mudança de variável do tipo $\rho_{i}=\varphi\left(\zeta_{i}\right)$, tal que $\left.\varphi: \mathbb{R} \rightarrow\right] \rho_{i}^{\min }, \rho_{i}^{\max }[\mathrm{e}$ que exista inversa $\left.\varphi^{-1}:\right] \rho_{i}^{\min }, \rho_{i}^{\max }[\rightarrow \mathbb{R}$. Como propriedade adicional, procura-se uma função suficientemente suave para evitar aumentar a não linearidade do problema.

Neste trabalho, utiliza-se uma sigmóide exponencial do tipo

$$
\varphi_{i}\left(\zeta_{i}\right)=\rho_{i}^{\min }+\left(\rho_{i}^{\max }-\rho_{i}^{\min }\right)\left[1+\exp \left(-\frac{\zeta_{i}}{s_{i}}\right)\right]^{-1}, \quad i=1, \ldots, n
$$

onde $\rho_{i}^{\min }$ e $\rho_{i}^{\max }$ são as assíntotas da curva e $s_{i}$ regula a derivada de $\varphi_{i}$, portanto a suavidade da curva na transição entre $\rho_{i}^{\min }$ e $\rho_{i}^{\max }$.

$$
\left.\frac{\partial \varphi}{\partial \zeta_{i}}\right|_{\zeta_{i}=0}=\frac{\rho_{i}^{\max }-\rho_{i}^{\min }}{4 s_{i}}
$$

O gráfico desta função pode ser visto na Figura 6.1

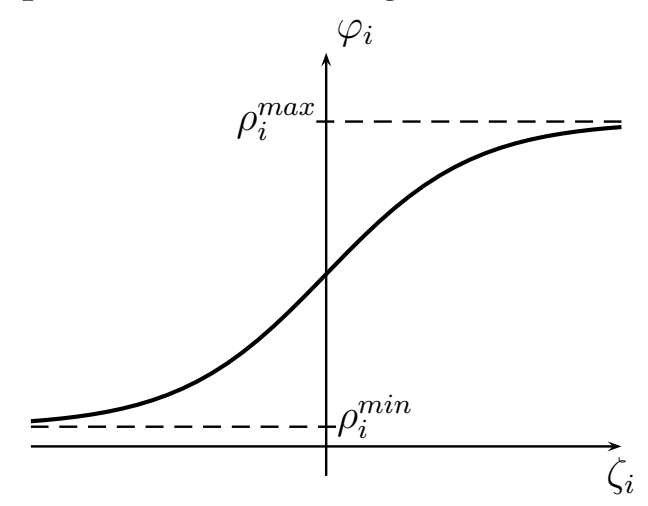

Figura 6.1: Gráfico da função sigmóide exponencial.

Sua inversa também é necessária e é dada por

$$
\left.\varphi_{i}^{-1}\left(\rho_{i}\right)=-s_{i} \ln \left(\frac{\rho_{i}^{\max }-\rho_{i}^{\min }}{\rho_{i}-\rho_{i}^{\min }}-1\right), \quad \rho_{i} \in\right] \rho_{i}^{\min }, \rho_{i}^{\max }[
$$

Neste trabalho, os limites de resistividade são adotados por região, isto é, elementos pertencentes a um mesmo tecidos utilizam um mesmo conjunto de parâmetros, compatíveis com valores clínicos obtidos de Gabriel et al. [47, 48, 6], Sousa et al. [4], Camargo et al. [41]. 
Por fim, define-se o metaestado $\zeta \in \mathbb{R}^{n}$, contendo $\zeta_{i}$ de todos os elementos e as funções vetoriais

$$
\begin{aligned}
& \rho=\varphi(\zeta) \\
& \zeta=\varphi^{-1}(\rho)
\end{aligned}
$$

que operam elemento a elemento nos vetores em questãd2.

O metaestado $\zeta$ pode ser considerado o novo estado do sistema para a estimação. Este novo estado pode assumir qualquer valor em $\mathbb{R}^{n}$, contudo $\rho=\varphi(\zeta)$ permanecerá dentro do intervalo $] \rho_{i}^{\min }, \rho_{i}^{\max }$ [ de cada elemento ou região.

As equações dos filtros de Kalman permanecem inalteradas, porém redefinindo o estado $\boldsymbol{x}_{k}=\boldsymbol{\zeta}_{k}$ e redefinindo os modelos de evolução (5.1) e observações 3 (5.5) e (5.8)

$$
\begin{aligned}
& \mathbf{x}_{k+1}=\boldsymbol{\varphi}^{-1}\left(\boldsymbol{\Phi}_{k}\left(\boldsymbol{\varphi}\left(\mathbf{x}_{k}\right), \mathbf{p}_{k}, \boldsymbol{u}_{k}\right)\right)+\boldsymbol{A}_{k}^{x} \mathbf{v}_{k}^{x}, \\
& \mathbf{y}_{k+1}=\boldsymbol{h}_{k+1}\left(\boldsymbol{\varphi}\left(\mathbf{x}_{k+1}\right)\right)+\boldsymbol{B}_{k+1}^{x} \mathbf{e}_{k+1}^{x} \\
& \mathbf{y}_{k+1}=\boldsymbol{h}_{k+1}\left(\boldsymbol{\Phi}_{k}\left(\boldsymbol{\varphi}\left(\mathbf{x}_{k}\right), \mathbf{p}_{k+1}, \boldsymbol{u}_{k}\right)\right)+\boldsymbol{B}_{k+1}^{p} \mathbf{e}_{k+1}^{p}
\end{aligned}
$$

Quanto à conversão de uma variável aleatória $\rho$, descrita por sua média $\bar{\rho}$ e covariância $\Gamma^{\rho \rho}$ para o metaestado, utiliza-se

$$
\bar{\zeta}=\varphi^{-1}(\bar{\rho})
$$

e para a matriz de covariância a aproximação

$$
\begin{gathered}
\boldsymbol{\rho}^{-}=\overline{\boldsymbol{\rho}}-\boldsymbol{\sigma}^{\rho}, \quad \boldsymbol{\zeta}^{-}=\boldsymbol{\varphi}^{-1}\left(\boldsymbol{\rho}^{-}\right) \\
\boldsymbol{\rho}^{+}=\overline{\boldsymbol{\rho}}+\boldsymbol{\sigma}^{\rho}, \quad \boldsymbol{\zeta}^{+}=\boldsymbol{\varphi}^{-1}\left(\boldsymbol{\rho}^{+}\right) \\
\boldsymbol{\sigma}^{\zeta}=\frac{\boldsymbol{\zeta}^{+}-\boldsymbol{\zeta}^{-}}{2} \\
{\left[\boldsymbol{\Gamma}^{\zeta \zeta}\right]_{(i, j)} \approx \frac{\sigma_{i}^{\zeta} \sigma_{j}^{\zeta}}{\sigma_{i}^{\rho} \sigma_{j}^{\rho}}\left[\boldsymbol{\Gamma}^{\rho \rho}\right]_{(i, j)},}
\end{gathered}
$$

onde $\sigma^{\rho}$ é um vetor com os desvios padrão das componentes de $\rho, \sigma^{\zeta}$ é um vetor

\footnotetext{
${ }^{2} \mathrm{~A}$ dependência de $\varphi$ e $\varphi^{-1}$ em relação à trinca de parâmetros $\left(\rho_{i}^{\min }, \rho_{i}^{\max }, s_{i}\right)$ foi suprimida para a simplificação da notação.

${ }^{3}$ No Apêndice C.3 mostra-se como modificar o modelo de observação linearizado (2.51) para incluir a transformação $\rho=\varphi(\zeta)$.
} 
com os desvios padrão (aproximados) do estado transformado. Estas transformações podem ser vistas na Figura 6.2 para um elemento.

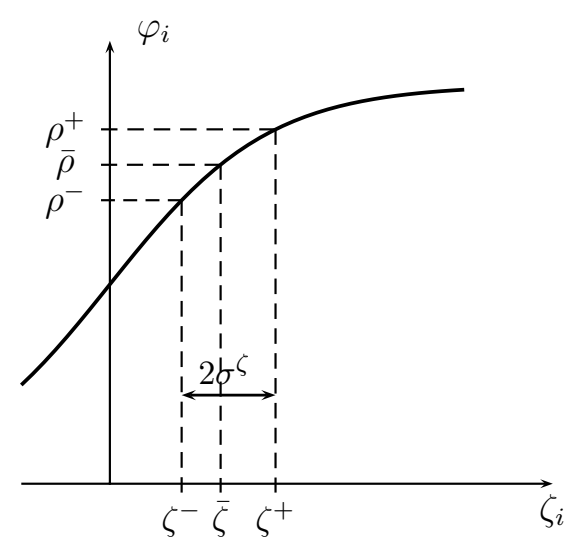

Figura 6.2: Aproximações das transformações das estatísticas.

Definindo um operador responsável pelas transformações descritas de uma variável aleatória $\rho$ como

$$
\left[\overline{\boldsymbol{\zeta}}, \boldsymbol{\Gamma}^{\zeta \zeta}\right]=\operatorname{Rho} 2 \operatorname{Meta}\left(\overline{\boldsymbol{\rho}}, \boldsymbol{\Gamma}^{\rho \rho}\right),
$$

as estatísticas iniciais do estado são transformadas por

$$
\left[\overline{\boldsymbol{x}}_{0}, \boldsymbol{\Gamma}_{0}^{x x}\right]_{\zeta}=\operatorname{Rho} 2 \operatorname{Meta}\left(\overline{\boldsymbol{x}}_{0}, \boldsymbol{\Gamma}_{0}^{x x}\right) .
$$

O cálculo das estatísticas do ruído de processo $\mathbf{V}_{k}^{x}$ segue a mesma fórmula, mas $\overline{\boldsymbol{\rho}}_{i}$ utilizado é o valor médio entre $a$ e $b$. Define-se também o operador inverso

$$
\begin{gathered}
{\left[\overline{\boldsymbol{\rho}}, \boldsymbol{\Gamma}^{\rho \rho}\right]=\operatorname{Meta2} \operatorname{Rho}\left(\overline{\boldsymbol{\zeta}}, \boldsymbol{\Gamma}^{\zeta \zeta}\right)} \\
\overline{\boldsymbol{\rho}}=\boldsymbol{\varphi}(\overline{\boldsymbol{\zeta}}) \\
\boldsymbol{\zeta}^{-}=\overline{\boldsymbol{\zeta}}-\boldsymbol{\sigma}^{\zeta}, \quad \boldsymbol{\rho}^{-}=\boldsymbol{\varphi}\left(\boldsymbol{\zeta}^{-}\right) \\
\boldsymbol{\zeta}^{+}=\overline{\boldsymbol{\zeta}}+\boldsymbol{\sigma}^{\zeta}, \quad \boldsymbol{\rho}^{+}=\boldsymbol{\varphi}\left(\boldsymbol{\zeta}^{+}\right) \\
\boldsymbol{\sigma}^{\rho}=\frac{\boldsymbol{\rho}^{+}-\boldsymbol{\rho}^{-}}{2} \\
{\left[\boldsymbol{\Gamma}^{\rho \rho}\right]_{(i, j)} \approx \frac{\sigma_{i}^{\rho} \sigma_{j}^{\rho}}{\sigma_{i}^{\zeta} \sigma_{j}^{\zeta}}\left[\boldsymbol{\Gamma}^{\zeta \zeta}\right]_{(i, j)} .}
\end{gathered}
$$




\subsection{Densidade de probabilidade a priori}

A segunda maneira utilizada neste trabalho para introduzir informação a priori no problema de estimação é através da densidade de probabilidade a priori, descrita na Seção 4.4 .

Neste trabalho considera-se que a estimativa da distribuição de resitividade $\hat{\boldsymbol{\rho}}_{k+1}$ deve ser suave, ou seja, não são esperadas mudanças abruptas de valores de resistividades entre elementos vizinhos. Esta hipótese é questionável nas regiões de interface do tecido ósseo com os demais tecidos ao seu redor e na interface de um possível pneumotórax. Todavia, a informação de suavidade é utilizada pela sua formulação linear e possibilidade de tratamento analítico. Outra possibilidade seria utilizar a regularização por variação total, que permite que variações de resistividade abruptas ocorram, porém esta regularização resulta em um operador não linear que, portanto, não pode ser escrito na forma (4.37).

Para a estimação da resitividade dentro do domínio, a informação a priori é composta por uma matriz $\boldsymbol{L}_{r} \in \mathbb{R}^{n \times n}$ que aproxima o filtro passa-altas gaussiano, cujos elementos são dados por

$$
\begin{aligned}
{[\boldsymbol{G}]_{(i, j)} } & = \begin{cases}\frac{1}{2 \pi \sigma} \exp \left(-\frac{d(i, j)^{2}}{2 \sigma^{2}}\right), & \text { se } i \text { e } j \text { pertencem à mesma região } \\
0, & \text { caso contrário }\end{cases} \\
{\left[\boldsymbol{L}_{r}\right]_{(i, j)} } & =\mathbb{I}-\frac{\boldsymbol{G}}{S} \\
S & =\sum_{i, j}[\boldsymbol{G}]_{(i, j)},
\end{aligned}
$$

onde $d(i, j)$ é a distância euclidiana entre os centróides do $i$-ésimo e $j$-ésimo elementos. O parâmetro $\sigma$ é o desvio padrão do filtro que ajusta o seu nível de suavidade. Definindo $\boldsymbol{G}$ desta maneira, leva-se em conta que a distribuição de resistividade não deve ser suave na interface entre as regiões dos pulmões e dos demais tecidos.

Já a escolha do vetor de estado de referência $\boldsymbol{x}^{*}$ depende das condições do experimento e será apresentada na descrição de cada ensaio numérico e experimental desta tese. 
Adianta-se aqui que no ensaio experimental, a montagem da matriz $\boldsymbol{L}_{r}$ levando em consideração a não suavidade de resistividade na interface entre as regiões pulmonar e demais tecidos resultou em imagens insatisfatórias. Por isto, uma matriz $G$ que não distingue interfaces foi utilizada no lugar de (6.21) neste ensaio

$$
[\boldsymbol{G}]_{(i, j)}=\frac{1}{2 \pi \sigma} \exp \left(-\frac{d(i, j)^{2}}{2 \sigma^{2}}\right) \quad \forall i, j
$$

A razão para a degradação das imagens é discutida no final da tese, no Capítulo 13 .

Para a estimação dos parâmetros dos eletrodos e parâmetros do modelo de evolução, $\boldsymbol{x}^{*}$ é um vetor uniforme com o valor médio esperado e $\boldsymbol{L}_{r}=\mathbb{I}$.

Caso o vínculo de intervalo seja também utilizado, duas possibilidades para a densidade a priori podem ser empregadas:

$$
\begin{array}{r}
f_{\mathbf{x}_{k+1}}^{\text {priori }} \propto \exp \left(-\frac{\alpha^{2}}{2}\left\|\boldsymbol{L}_{r}\left(\boldsymbol{\varphi}\left(\boldsymbol{\zeta}_{k+1}\right)-\boldsymbol{x}_{k+1}^{*}\right)\right\|_{2}^{2}\right) \\
f_{\mathbf{x}_{k+1}}^{\text {priori }} \propto \exp \left(-\frac{\alpha^{2}}{2}\left\|\boldsymbol{L}_{r}\left(\boldsymbol{\zeta}_{k+1}-\boldsymbol{\varphi}^{-1}\left(\boldsymbol{x}_{k+1}^{*}\right)\right)\right\|_{2}^{2}\right) .
\end{array}
$$

No primeiro caso, $\boldsymbol{L}_{r}$ atua no espaço de $\boldsymbol{\rho}_{k+1}$ e no segundo $\boldsymbol{L}_{r}$ atua no espaço de $\boldsymbol{\zeta}_{k+1}$. Neste trabalho o segundo método é utilizado por três motivos. Primeiro pois esta forma mantém a linearidade do operador, reduzindo o custo computacional. Segundo pois controla mais a estimativa $\hat{\boldsymbol{\zeta}}_{k+1}$ quando a conversão para $\boldsymbol{\rho}$ estiver próxima das assíntotas: Nas regiões onde $\boldsymbol{\rho}_{k+1}$ está próximo destes limites, perturbações nos componentes do estado $\boldsymbol{\zeta}_{k+1}$ não causam grandes perturbações de $\boldsymbol{\rho}_{k+1}$ e, por consequência, pouca perturbação é transmitida para as medidas preditas $\overline{\boldsymbol{y}}_{k+1 \mid k}$. No primeiro caso, mesmo com a suavidade de $\rho_{k+1}=\varphi\left(\zeta_{k+1}\right)$, grandes oscilações podem ocorrer no estado $\boldsymbol{\zeta}_{k+1}$ quando $\boldsymbol{\rho}_{k+1}$ está próximo das assíntotas, dificultando a convergência do algoritmo.

O terceiro motivo é melhor compreendido utilizando o exemplo de informação de suavidade. Uma estimativa suave $\zeta_{k+1}$ não acarreta imediatamente em uma solução suave em $\boldsymbol{\rho}_{k+1}$ caso a trinca de parâmetros $\left(\rho_{i}^{\min }, \rho_{i}^{\max }, s_{i}\right)$ das regiões ou elementos sejam diferentes. 
Um exemplo numérico clarifica o terceiro argumento. Seja um caso unidimensional, com 9 elementos alinhados, cujas trincas de parâmetros de $\varphi_{i}$ são

$$
\begin{aligned}
& \left(\rho_{i}^{\min }, \rho_{i}^{\max }, s_{i}\right)=(0,1,1), \quad i=1,2,3 \\
& \left(\rho_{i}^{\min }, \rho_{i}^{\max }, s_{i}\right)=(2,3,1), \quad i=4,5,6 \\
& \left(\rho_{i}^{\min }, \rho_{i}^{\max }, s_{i}\right)=(1,2,1), \quad i=7,8,9 .
\end{aligned}
$$

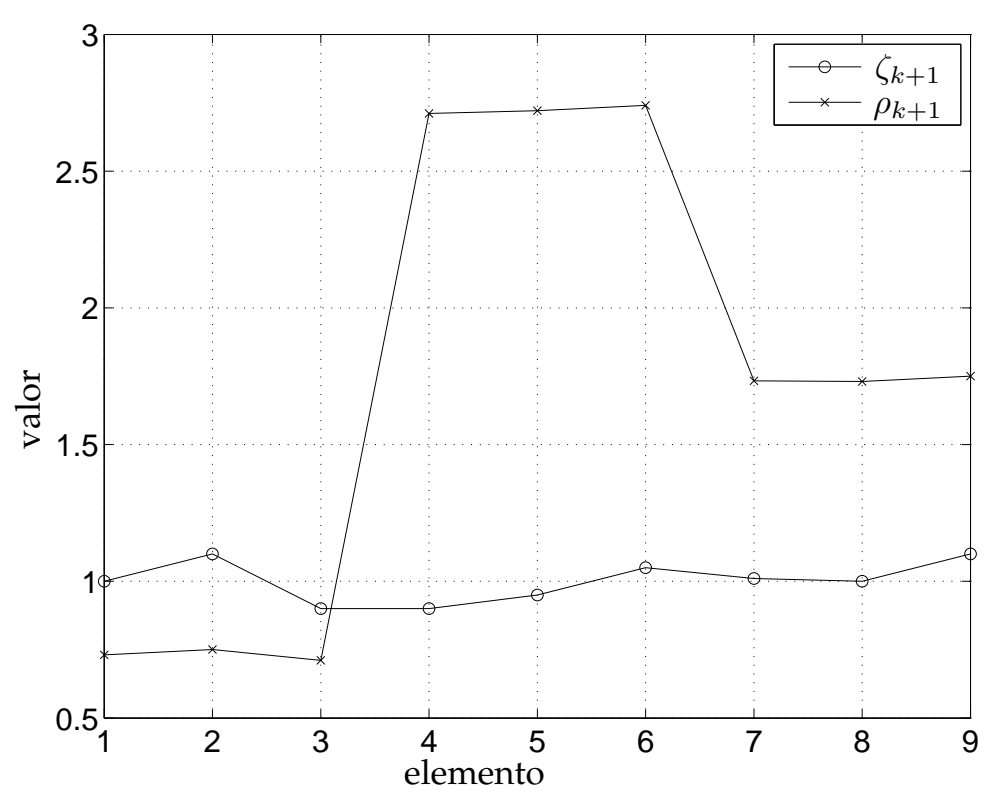

Figura 6.3: Gráfico da solução.

Seja uma solução suave $\hat{\boldsymbol{\zeta}}_{k+1}=\left[\begin{array}{lllllllll}1.0 & 1.1 & 0.9 & 0.9 & 0.95 & 1.05 & 1.01 & 1.0 & 1.1\end{array}\right]^{\mathrm{T}}$. A Figura 6.3 mostra o gráfico da estimativa $\hat{\zeta}_{k+1}$ e sua transformada $\hat{\rho}_{k+1}=\varphi\left(\hat{\boldsymbol{\zeta}}_{k+1}\right)$. Nota-se que apesar da suavidade de $\hat{\boldsymbol{\zeta}}_{k+1}$, as diferenças entre as regiões mantém-se destacadas em $\hat{\boldsymbol{\rho}}_{k+1}$.

Por fim, salienta-se que apesar do aumento da dimensão do vetor de medidas devido à adição das medidas de não conformidade (4.58), fato que a princípio aumentaria a carga computacional, muitas das etapas do algoritmo do SR-UKF podem ser simplificadas observando que a medida de não conformidade $\boldsymbol{z}_{k}$ é uma função linear do estado como apresentado em (4.54). 


\section{Capítulo 7}

\section{Método do erro de aproximação para o modelo de observação}

Utiliza-se neste trabalho o método do erro de aproximação para o modelo de observação, como em Nissinen et al. [111, 112, 113], Nissinen [114]. Este modelo é utilizado tanto para o modelo não linear (2.40), quando para o linearizado (2.51).

Sejam os modelos de observação (5.5) fidedigno e aproximado representados respectivamente por $\boldsymbol{h}$ e $\boldsymbol{h}^{a}$. Estas duas versões do modelo de observação são apresentadas novamente para facilitar a explanação. 1 .

$$
\begin{aligned}
& \mathbf{y}=\boldsymbol{h}(\mathbf{x})+\boldsymbol{B}^{x} \mathbf{e}^{x} \\
& \mathbf{y}=\boldsymbol{h}^{a}\left(\mathbf{x}^{a}\right)+\boldsymbol{B}^{x} \mathbf{e}^{x} .
\end{aligned}
$$

As funções $\boldsymbol{h}$ e $\boldsymbol{h}^{a}$ são ainda funções de alguns parâmetros, como a informação a respeito do formato de $\partial \boldsymbol{\Omega}$, posicionamento dos eletrodos e nível de discretização da malha de elementos finitos. Outras informações ainda poderiam ser: característica térmica dos componentes do circuito de injeção de corrente e medição dos potenciais elétricos, capacitância dos cabos de medidas, interferência eletromagnética no recinto onde as medições estão sendo realizadas, variações do formato do domínio ao longo do ciclo respiratório, entre outros. Seja $\boldsymbol{d}$ o vetor que contém tais informações.

\footnotetext{
${ }^{1} \mathrm{O}$ subscrito referente ao tempo discreto $t_{k}$ foi omitido para facilitar a notação.
} 
Reescrevendo ambos os modelos, incluindo os vetores de parâmetros, chega-se a

$$
\begin{aligned}
& \mathbf{y}=\boldsymbol{h}(\mathbf{x}, \mathbf{d})+\boldsymbol{B}^{x} \mathbf{e}^{x} \\
& \mathbf{y}=\boldsymbol{h}^{a}\left(\mathbf{x}^{a}, \boldsymbol{d}^{a}\right)+\boldsymbol{B}^{x} \boldsymbol{e}^{x a},
\end{aligned}
$$

onde $\boldsymbol{d}^{a}$ e $\boldsymbol{e}^{x a}=\overline{\boldsymbol{e}}^{x}$ são os vetores aproximados adotados não sendo, portanto, variáveis aleatórias.

Substituindo em (4.83), o modelo do erro de aproximação fica

$$
\begin{aligned}
& \mathbf{y}=\boldsymbol{h}^{a}\left(\mathbf{x}^{a}, \boldsymbol{d}^{a}\right)+\boldsymbol{B}^{x} \boldsymbol{e}^{x a}+\boldsymbol{\varepsilon} \\
& \boldsymbol{\varepsilon}=\boldsymbol{h}(\mathbf{x}, \mathbf{d})+\boldsymbol{B}^{x} \mathbf{e}^{x}-\left[\boldsymbol{h}^{a}\left(\mathbf{x}^{a}, \boldsymbol{d}^{a}\right)+\boldsymbol{B}^{x} \boldsymbol{e}^{x a}\right] .
\end{aligned}
$$

\subsection{Efeitos não modelados no modelo fidedigno}

Uma questão importante que se levanta no uso do método do erro de aproximação é o que acontece quando o modelo fidedigno ainda está aquém do ideal. Isto ocorre no caso da TIE, por exemplo, nos modelos de eletrodos: O modelo de eletrodos atualmente utilizado e discretizado em elementos finitos aproxima os fenômenos que ocorrem na interface entre pele e metal do eletrodo por uma região condutora, de resitividade constante. Sabe-se entretanto que a condução de cargas elétricas no corpo humano ocorre por transporte de íons e no metal a condução ocorre através de elétrons da camada de valência. A camada da interface é responsável pela mudança da forma como as cargas são transportadas. Esta mudança é uma das fontes da impedância de contato. A impedância de contato é, portanto, uma propriedade do par de materiais e de sua geometria local. Neste sentido, modelar a interface através de um contínuo é apenas uma aproximação. Outras fontes de incertezas que aparecem no caso da TIE podem ser citadas, como o comportamento térmico dos componentes do circuito de aquisição e interferência eletromagnética.

Devido a este fato, uma modificação no método do erro de aproximação é proposta neste trabalho. 
Seja a equação

$$
\boldsymbol{y}=\boldsymbol{h}^{v}(\boldsymbol{x}, \boldsymbol{p}, \boldsymbol{u}, \boldsymbol{e}, \boldsymbol{r})
$$

aquela que descreve com perfeição o fenômeno real. Esta função é, por hipótese, não conhecida. Nesta equação, considera-se que os vetores $\boldsymbol{x}, \boldsymbol{p}, \boldsymbol{u}$ e $\boldsymbol{e}$ sejam os mesmos que aparecem em (4.81), ou seja, esta parte do fenômeno está apropriadamente modelada pelo modelo chamado até então de modelo "fidedigno".

Entretanto existe um vetor a mais na equação verdadeira. O vetor $\boldsymbol{r}$ é um vetor de parâmetros adicional, não modelado em (4.81) ou (4.82). Este vetor de parâmetros representa a parte do fenômeno que é desconhecida ou não modelada e também é, por consequência, desconhecida. Denomina-se esta parte do fenômeno de fenômeno oculto. Salienta-se que o fenômeno oculto pode ou não ser desconhecido. Em alguns casos este fenômeno pode ser conhecido mas faltam ferramentas teóricas para compreendê-lo e, portanto, acaba sendo deixado de lado no modelo "fidedigno" 2 . Em alguns casos, o fenômeno oculto é conhecido, mas é ignorado propositalmente pois sabe-se que o erro cometido é pequeno.

Considerando este vetor de parâmetros extra, chamado doravante de parâmetro oculto, pode-se pensar no modelo "fidedigno" como

$$
\boldsymbol{y}=\boldsymbol{h}\left(\boldsymbol{x}, \boldsymbol{p}, \boldsymbol{u}, \boldsymbol{e}, \boldsymbol{r}^{a}\right)
$$

onde $\boldsymbol{r}^{a}$ é o vetor oculto, escolhido implicitamente ao não considerar o fenômeno associado. Pode-se considerar que a escolha implícita seria algo como $\boldsymbol{r}^{a}=\mathbf{0}$, o que indicaria que o fenômeno oculto foi ignorado.

Da mesma forma que $\boldsymbol{r}^{a}=\mathbf{0}$ foi implicitamente escolhido para (4.81) , este também é usado no modelo aproximado (4.82).

$$
\boldsymbol{y}=\boldsymbol{h}^{a}\left(\boldsymbol{x}^{a}, \boldsymbol{p}^{a}, \boldsymbol{u}, \boldsymbol{e}^{a}, \boldsymbol{r}^{a}\right)
$$

\footnotetext{
${ }^{2}$ Neste sentido, o modelo fidedigno deveria ser chamado apenas de melhor modelo disponível, porém esta renomeação não será feita neste trabalho.
} 
Computando agora o método do erro de aproximação, chega-se a

$$
\begin{aligned}
& \boldsymbol{y}=\boldsymbol{h}^{a}\left(\boldsymbol{x}^{a}, \boldsymbol{p}^{a}, \boldsymbol{u}, \boldsymbol{e}^{a}, \boldsymbol{r}^{a}\right)+\varepsilon, \\
& \boldsymbol{\varepsilon}=\boldsymbol{h}\left(\mathbf{x}, \mathbf{p}, \boldsymbol{u}, \mathbf{e}, \boldsymbol{r}^{a}\right)-\boldsymbol{h}^{a}\left(\boldsymbol{x}^{a}, \boldsymbol{p}^{a}, \boldsymbol{u}, \boldsymbol{e}^{a}, \boldsymbol{r}^{a}\right),
\end{aligned}
$$

e o processo de amostragem pode ser feito para a estimação das estatísticas de $\varepsilon$, como descrito anteriormente na Seção 4.6.1.

Nota-se entretanto que não serão realizadas amostragens de $\boldsymbol{r}$ pelo fato do vetor de parâmetros ocultos ser desconhecido. Desta forma, as estatísticas do fenômeno oculto não serão incluídas nas estatísticas do ruído do método do erro de aproximação.

Considerando a falta de modelagem para $r$, é necessário substituir (7.10) por um equivalente onde um ruído extra seria responsável pela diferença entre o modelo aproximado e o processo real, que não é completamente conhecido. Uma primeira forma de se fazer isto é propor uma modificação em (7.10) do tipo

$$
\boldsymbol{y}=\boldsymbol{h}^{a}\left(\boldsymbol{x}^{a}, \boldsymbol{p}^{a}, \boldsymbol{u}, \boldsymbol{e}^{a}, \boldsymbol{r}^{a}\right)+\boldsymbol{\varepsilon}+\boldsymbol{\eta},
$$

onde $\boldsymbol{\eta}$ é o ruído residual que balancearia a falta de modelagem do fenômeno oculto. As estatísticas de $\boldsymbol{\eta}$ precisam ser estimadas. Pode-se se assumir inicialmente que $\varepsilon$ e $\boldsymbol{\eta}$ sejam independentes.

Um questionamento válido na discussão do método do erro de aproximação no caso da ocorrência de um fenômeno oculto reside no fato de que o modelo (7.12) continua com um ruído artificial adicional $\eta$, relativo a um processo desconhecido e cujas estatísticas são, portanto, nebulosas e de difícil determinação. Ao arbitrar um valor para as estatísticas de $\boldsymbol{\eta}$, comumente na forma $\boldsymbol{\eta} \sim \mathcal{N}\left(\mathbf{0}, \sigma_{\eta} \mathbb{I}\right)$, para $\sigma_{\eta}$ suficientemente grande e ajustado empiricamente, pode-se questionar qual a vantagem do método já que continua-se com um parâmetro para ser ajustado.

$\mathrm{O}$ argumento é válido, entretanto é importante salientar que ao separar $\varepsilon$ e $\eta$, identifica-se parte das incertezas do modelo. Desta forma, parte do ruído apresenta estatísticas conhecidas (ou estimadas) e a intensidade do ruído residual é menor. 
Sob este paradigma, pode-se conceber o método do erro de aproximação como um método de identificação do ruído. Tal método tem como objetivo minimizar a intensidade do ruído $\boldsymbol{\eta}$ ao identificar componentes que podem ser passadas para $\varepsilon$, cujas estatísticas podem ser estimadas.

Por argumentos similares, é possível conceber que um modelo aproximado $\boldsymbol{h}^{a}$ também pode apresentar parâmetros ocultos em relação ao fenômeno real. Um exemplo é o caso em que a modelagem de um fenômeno sofre com condicionamento numérico, como nos casos de modelagem do modelo dinâmico de sistemas nas quais o modelo aproximado é composto por equações diferenciais rígidas (como em sistemas dinâmicos caóticos). Neste caso, mesmo que o sistema real se comporte razoavelmente bem ao longo do tempo, a rigidez das equações do seu modelo causa problemas de predição de estado. Neste exemplo seria também necessário aumentar a variância do ruído de processo para conter o comportamento arisco do modelo de predição e confiar mais nas medidas realizadas para a determinação do ganho do filtro.

Neste trabalho, o modelo (7.12) é utilizado. Mais detalhes são fornecidos no Capítulo 8 . 


\section{Capítulo 8}

\section{Ensaios numéricos para avaliação do método do erro de aproximação}

Este capítulo apresenta a avaliação do método do erro de aproximação utilizado no modelo de observação do SR-UKF através de ensaios numéricos.

Os ensaios avaliam a capacidade do método do erro de aproximação em lidar com erros nos valores dos parâmetros dos eletrodos, posicionamento dos eletrodos, formato do domínio e erros de discretização através de fantomas numéricos.

Toda a programação utilizada neste trabalho foi feita em linguagem C. O código foi compilado utilizando o compilador Gnu C Compiler 4.4 .5 (http://gcc.gnu.org/) e todos os ensaios e verificações foram realizados em Linux, distribuição Kubuntu 10.10 e superior (http://www.kubuntu.org/) e Fedora Core 11 e superior (http://fedoraproject.org/). As malhas de elementos finitos foram geradas com o programa gmsh (http://geuz.org/gmsh/).

\subsection{Fantoma numérico para os ensaios}

Este fantoma consiste em simular a aquisição das medidas em um domínio cilíndrico de altura infinita, com um objeto mais resistivo em seu interior. O objeto escolhido é um outro cilindro de altura infinita.

Com o objetivo de testar o método do erro de aproximação em diversas situações, o fantoma numérico é resolvido conforme apresentado na Seção 2.7, utilizando malhas com posição de eletrodos, formato do domínio e parâmetros dos eletrodos diferentes 
da malha utilizada no filtro de Kalman para a estimação.

A Figura 8.1 mostra as malhas utilizadas no fantoma numérico. As malhas A a D são utilizadas no fantoma para gerar os dados e a malha $E$, que é apresentada na Figura 8.2. é utilizada em todos os casos para a solução do problema inverso. Pode-se ver nas primeiras malhas o posicionamento do objeto. As malhas A, B e E são circulares, já as malhas C e D são ovaladas.

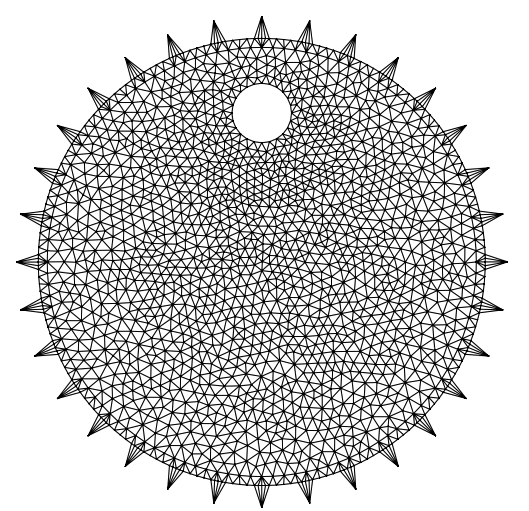

(a) Malha A: 3342 elementos, circular, eletrodos equidistantes.

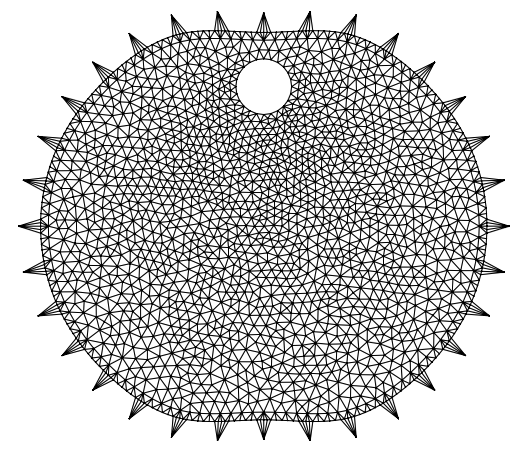

(c) Malha C: 3500 elementos, não circular, eletrodos equidistantes.

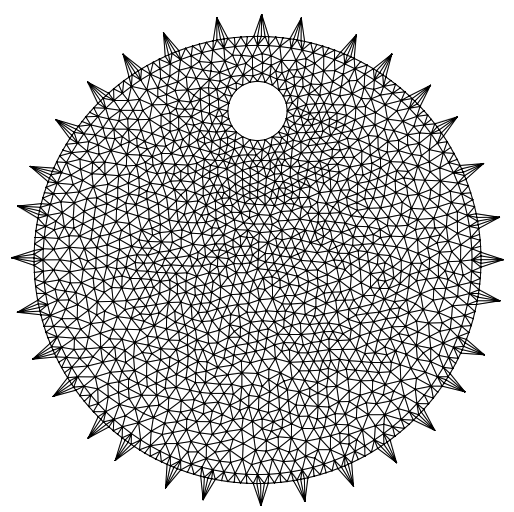

(b) Malha B: 3345 elementos, circular, eletrodos não equidistantes.

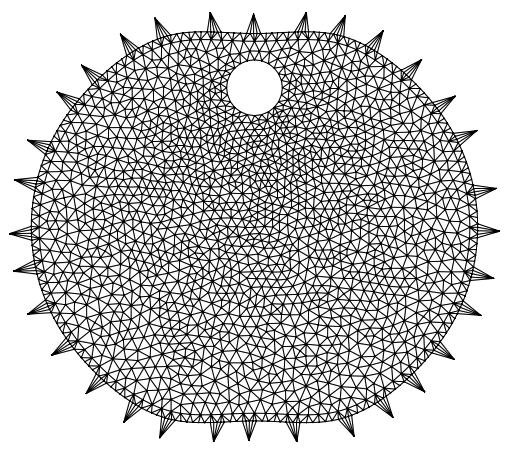

(d) Malha D: 3508 elementos, não circular, eletrodos não equidistantes.

Figura 8.1: Malhas utilizadas no fantoma numérico. Os elementos que preenchem os objetos foram ocultos para destacar o seu formato e posicionamento.

Todas as malhas possuem 32 eletrodos com quatro elementos por eletrodo, equidistantes ao longo do perímetro nas malhas A, C e E. Nas malhas B e D as posições dos eletrodos foram perturbadas em relação ao equispaçamento por deslocamentos aleatórios, seguindo uma distribuição uniforme no intervalo $\pm 5 \mathrm{~mm}$. Todas as malhas têm diâmetro de $30 \mathrm{~cm}$ (diâmetro médio, no caso das malhas $\mathrm{C}$ e D), os centros dos objetos estão posicionados a $10 \mathrm{~cm}$ do centro e possuem $4 \mathrm{~cm}$ de diâmetro. Os 
elementos que preenchem os objetos foram ocultos nas figuras para destacar o seu posicionamento na malha.

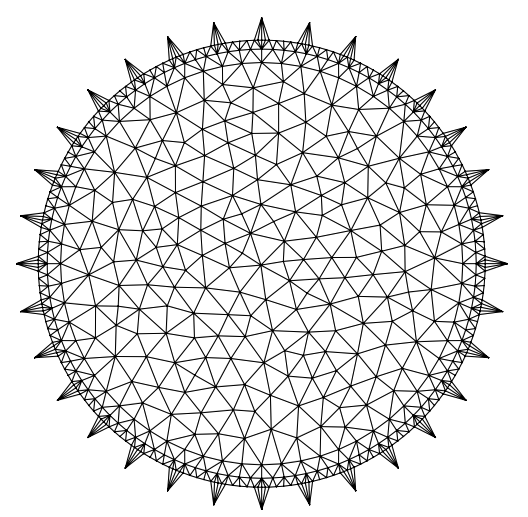

Figura 8.2: Malha E: 978 elementos, circular, eletrodos equidistantes. Utilizada no problema inverso.

A resistividade escolhida para o meio ao redor dos objetos é de $4.0 \Omega \mathrm{m}$ e os objetos possuem resistividade de $40.0 \Omega \mathrm{m}$. A amplitude 1 da corrente injetada é de $4 \mathrm{~mA}$ zero-pico. Os valores dos parâmetros dos eletrodos das malhas A a D são escolhidos aleatoriamente seguindo uma distribuição uniforme no intervalo entre 0.01 e $0.03 \Omega \mathrm{m}^{2}$.

\subsection{Amostragem do ruído do método do erro de aproximação}

Antes de prosseguir com a descrição de cada ensaio, faz-se necessário definir uma amostragem do ruído $\varepsilon_{i}(7.6)$, repetido a seguir por facilidade

$$
\boldsymbol{\varepsilon}_{i}=\boldsymbol{h}\left(\boldsymbol{x}_{i}, \boldsymbol{d}_{i}\right)+\boldsymbol{B}^{x} \boldsymbol{e}_{i}^{x}-\left[\boldsymbol{h}^{a}\left(\boldsymbol{x}^{a}, \boldsymbol{d}^{a}\right)+\boldsymbol{B}^{x} \boldsymbol{e}^{a}\right]=\boldsymbol{h}\left(\boldsymbol{x}_{i}, \boldsymbol{d}_{i}\right)-\boldsymbol{h}^{a}\left(\boldsymbol{x}^{a}, \boldsymbol{d}^{a}\right)+\boldsymbol{B}^{x}\left[\boldsymbol{e}_{i}^{x}-\boldsymbol{e}^{a}\right] .
$$

Nesta amostra, ambas $\boldsymbol{h}$ e $\boldsymbol{h}^{a}$ são soluções do problema direto (2.41) utilizando duas malhas diferentes. Estas últimas são determinadas a partir de alguns parâmetros que definem o formato do domínio, posição dos eletrodos e nível de refinamento.

\footnotetext{
${ }^{1}$ As intensidades da corrente imposta e potenciais medidos podem ser dadas em amplitude zero-pico, pico-pico ou valor RMS. O importante é que tanto a intensidade da corrente quanto dos potenciais elétricos utilizados estejam na mesma métrica.
} 
Para parametrizar $\partial \boldsymbol{\Omega}$, seleciona-se um certo número de pontos que devem pertencer a esta curva e interpola-se por estes pontos uma spline cúbica para definir o bordo. Estes pontos são chamados de nós da spline. Escrevendo-se as coordenadas destes nós em coordenadas polares e fixando-se a componente angular, pode-se variar o formato de $\partial \Omega$ ao modificar suas componentes radiais.

Sejam o vetor $\boldsymbol{\vartheta}$ composto pelos valores das componentes radiais destes pontos, $\boldsymbol{p}_{e}$ um vetor que contém a posição de cada um dos eletrodos ao longo de $\partial \Omega$ e $\delta$ um tamanho característico dos elementos da malha. A malha está definida através da escolha destes três parâmetros2 2 . Desta forma, $\boldsymbol{d}_{i}=\left[\begin{array}{lll}\boldsymbol{\vartheta}_{i} & \boldsymbol{p}_{e, i} & \boldsymbol{\delta}_{i}\end{array}\right]$ e $\boldsymbol{d}^{a}=\left[\begin{array}{lll}\boldsymbol{\vartheta}^{a} & \boldsymbol{p}_{e}^{a} & \boldsymbol{\delta}^{a}\end{array}\right]$ definem duas malhas: $M_{i}$ e $M^{a}$. Pode-se dizer que $\boldsymbol{d}_{i}$ é uma variável aleatória e muda a cada realização, já $\boldsymbol{d}^{a}$ é, por hipótese, conhecida e fixa, resultando na malha E apresentada anteriormente.

Nos ensaios, o vetor $\boldsymbol{\vartheta}_{i}$ é uma realização do seguinte vetor aleatório

$$
\begin{aligned}
& \boldsymbol{\vartheta}=\boldsymbol{\vartheta}_{c} \cdot \frac{\boldsymbol{\vartheta}_{\delta}}{100} \quad(\mathrm{~m}) \\
& \boldsymbol{\vartheta}_{\delta} \sim \mathcal{N}^{3 \sigma}\left(\mathbf{1 0 0}, \sigma_{\vartheta}^{2} \mathbb{I}\right), \quad \text { (adim.) }
\end{aligned}
$$

onde $\vartheta_{c}$ é um vetor constante e conhecido com as distância radias dos nós que descrevem um círculo, $\boldsymbol{\vartheta}_{\delta}$ são porcentagens que alteram as componentes radiais dos nós, $\mathcal{N}^{3 \sigma}\left(\overline{\boldsymbol{x}}, \boldsymbol{\Gamma}^{x}\right)$ é uma distribuição Gaussiana com média $\overline{\boldsymbol{x}}$, (co)variância $\boldsymbol{\Gamma}^{x}$ truncada em mais ou menos três vezes o desvio padrão e $\sigma_{\vartheta}$ é o desvio padrão da distribuição. Nesta equação, a multiplicação deve ser entendida como uma operação elemento a elemento.

Definindo a variável aleatória desta forma, pode-se criar malhas cujos bordos são perturbações do formato circular variando-se $\sigma_{\vartheta}$. Este valor é apresentado em cada ensaio.

O vetor $\boldsymbol{p}_{e, i}$ é uma realização do vetor aleatório

$$
\begin{aligned}
& \boldsymbol{p}_{e}=\boldsymbol{p}_{0}+\boldsymbol{p}_{\delta} \quad(\mathrm{m}) \\
& \boldsymbol{p}_{\delta} \sim \mathcal{U}\left(p^{-}, p^{+}\right), \quad(\mathrm{m})
\end{aligned}
$$

\footnotetext{
${ }^{2}$ A definição matemática de um tamanho característico de elemento não garante que a malha esteja completamente definida no sentido de ser única, porém no programa utilizado para geração de malhas nesta tese, Gmsh, a definição de $\delta$ é suficiente para determinar univocamente a malha.
} 
onde $\boldsymbol{p}_{0}$ é um vetor constante e conhecido com as distâncias dos eletrodos equidistantes, $\boldsymbol{p}_{\delta}$ são deslocamentos destas posições e $\mathcal{U}\left(p^{-}, p^{+}\right)$é uma distribuição uniforme entre $p^{-}$e $p^{+}$. Definindo-se a posição dos eletrodos desta forma, pode-se variar a disposição dos eletrodos. Os valores de $p^{-}$e $p^{+}$são apresentados em cada ensaio e nos ensaios em que $p^{-}=p^{+}=0.0$, deve-se entender como $\boldsymbol{p}_{e}=\boldsymbol{p}_{0}$.

O refinamento da malha $\delta_{i}$ é uma realização da variável aleatória

$$
\begin{aligned}
\delta & =\delta_{0} . \delta_{\delta} \quad \text { (adim.) } \\
\delta_{\delta} & \sim \mathcal{U}\left(\delta^{-}, \delta^{+}\right), \quad \text { (adim.) }
\end{aligned}
$$

onde $\delta_{0}$ é o refinamento de referência utilizado nos fantomas e $\delta_{\delta}$ é uma porcentagem de perturbação. Em todos os ensaios, $\delta^{-}=0.9$ e $\delta^{+}=1.1$.

Definidas as malhas, está definido o operador $\boldsymbol{T}_{i}$ que interpola a distribuição de resistividade entre a realização da malha e a malha aproximada

$$
\boldsymbol{x}^{a}=\boldsymbol{T}_{i} \boldsymbol{x}_{i}
$$

Neste trabalho, o operador $\boldsymbol{T}_{i}$ é dado por um filtro passa-baixas Gaussiano, cujos elementos são dados por

$$
\left[\boldsymbol{T}_{i}\right]_{(j, k)}=\frac{\frac{1}{2 \pi \sigma} \exp \left(-\frac{d(j, k)^{2}}{2 \sigma^{2}}\right)}{\sum_{j, k} \frac{1}{2 \pi \sigma} \exp \left(-\frac{d(j, k) 2}{2 \sigma^{2}}\right)}
$$

onde $d(j, k)$ é a distância euclidiana entre o centróide do $j$-ésimo elemento da malha $M^{a}$ e $k$-ésimo elemento da malha $M_{i}$ e $\sigma$ é o desvio padrão do filtro. Nestes ensaios, $\sigma=0.01 \mathrm{~m}$.

A distribuição de resistividade $\boldsymbol{x}_{i}$ é uma amostra do seguinte vetor aleatório

$$
\begin{aligned}
\mathbf{x} & =\boldsymbol{G} \mathbf{x}_{\delta}, \quad(\Omega \mathrm{m}) \\
\mathbf{x}_{\delta} & \sim \mathcal{N}^{L^{-}}\left(\mathbf{x}_{u} \mathbf{1}, 3.0^{2} \mathbb{I}, \mathbf{0}\right) \quad(\Omega \mathrm{m}) \\
\mathbf{x}_{u} & \sim \mathcal{U}(3.0,5.0), \quad(\Omega \mathrm{m})
\end{aligned}
$$


onde $G$ é um filtro passa-baixas Gaussiand 3 , 1 é um vetor cujas componentes são todas unitárias e $\mathcal{N}^{L^{-}}\left(\overline{\boldsymbol{x}}, \Gamma^{x}, L^{-}\right)$é uma distribuição Gaussiana com média $\overline{\boldsymbol{x}}$, (co)variância $\Gamma^{x}$ e com truncamento inferior em $L^{-}$. Esta distribuição pode ser vista na Figura 8.3 para um caso unidimensional.

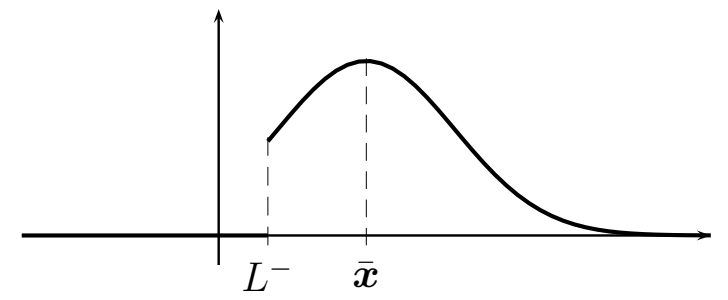

Figura 8.3: Distribuição Gaussiana com truncamento inferior.

Com esta definição de $\mathbf{x}$, pode-se obter distribuições suaves de resistividade e com valores médios equiprováveis no intervalo 3.0 a $5.0 \Omega \mathrm{m}$. Nota-se ainda que com esta definição, a ocorrência da distribuição verdadeira, isto é, aquela com um objeto circular é bastante improvável. Sorteios desta distribuição são realizados em todos os ensaios.

Os parâmetros dos eletrodos $\boldsymbol{\rho}_{i}^{\prime}$ não são considerados variáveis de estado, mas sim parâmetros do modelo de observação nestes ensaios. Desta forma, devem ser incluídos nas amostras nos ensaios em que estes erros estão presentes. Este vetor é uma realização do vetor aleatório

$$
\rho^{\prime} \sim \mathcal{U}(0.01,0.03) \quad\left(\Omega \mathrm{m}^{2}\right)
$$

O ruído $e_{i}^{x}$ neste ensaio é uma realização do vetor aleatório

$$
\mathbf{e}^{x} \sim \mathcal{N}\left(\mathbf{0},\left(1.0 \cdot 10^{-3}\right)^{2} \mathbb{I}\right)
$$

em todos os ensaios. O ruído $e^{a}$ é assumido constante e igual a média de $\mathbf{e}^{x}$, ou seja, zero em todos os ensaios.

Ao final do processo de geração das malhas e definição das propriedades elétricas, os fantomas são resolvidos e $\varepsilon_{i}$ pode ser calculado. Após a amostragem do ruído, as

\footnotetext{
${ }^{3}$ Este filtro é calculado nos mesmos molde de $\boldsymbol{T}_{i}$, como apresentado em (8.9), porém a distância $d(j, k)$ diz respeito a elementos da mesma malha.
} 
suas estatísticas podem ser estimadas com

$$
\begin{aligned}
\bar{\varepsilon} & =\frac{1}{N} \sum_{i=1}^{N} \varepsilon_{i} \\
\Gamma^{\varepsilon} & =\frac{1}{N-1} \sum_{i=1}^{N}\left(\varepsilon_{i}-\bar{\varepsilon}\right)^{\mathrm{T}}\left(\varepsilon_{i}-\bar{\varepsilon}\right) .
\end{aligned}
$$

Nos ensaios são realizadas $N=5000$ amostras de (8.1).

Uma vez que a natureza do ruído $\mathbf{e}^{x}$ é aditiva e é assumida independência estatística em relação aos demais parâmetros, pode-se gerar as amostras $\varepsilon_{i}$ sem considerar o ruído $\mathbf{e}^{x}$ e no final da estimativa utilizar o resultado

$$
\boldsymbol{\Gamma}^{\varepsilon}=\boldsymbol{\Gamma}_{0}^{\varepsilon}+\boldsymbol{B}^{x} \boldsymbol{\Gamma}^{e^{x}}\left(\boldsymbol{B}^{x}\right)^{\mathrm{T}}
$$

onde $\Gamma_{0}^{\varepsilon}$ é a matriz de covariância calculada com amostragem desconsiderando os ruídos $\mathbf{e}^{x}$ e $\boldsymbol{e}^{a}$. Esta forma é útil quando as estatísticas do ruído de medição variam de experimento para experimento, sendo possível atualizar as estatísticas do método do erro de aproximação sem a necessidade de realizar o processo de amostragem novamente.

A seguir são descritos os ensaios numéricos utilizando o fantoma do domínio circular.

\subsection{Descrição dos ensaios}

No total, oito ensaios são realizados. Cada um consiste na estimativa do estado, composto apenas pela distribuição de resistividade dentro do domínio $\rho$, utilizando dados provenientes de fantomas cujas malhas de elementos finitos e/ou parâmetros dos eletrodos diferem dos utilizados no SR-UKF. Duas estimativas são realizadas em cada ensaio, uma sem o uso do método do erro de aproximação e outra com o uso do método do erro de aproximação.

Salienta-se que o SR-UKF utilizado nestes ensaios usa o passeio aleatório como modelo de evolução do estado. Além disto, não são realizadas segmentações na imagem e apenas informação a priori de vínculo de intervalo é adicionado. 
A Tabela 8.1 mostra os erros de modelagem considerados em cada ensaio realizado. Em todos os casos, a malha utilizada no SR-UKF é a malha E, apresentada na Figura 8.2 . Além dos erros constados na tabela, todos os ensaios contém também o erro de modelagem proveniente do diferente nível de refinamento das malhas.

Tabela 8.1: Ensaios realizados para avaliar o método do erro de aproximação.

\begin{tabular}{lllllllll}
\hline Ensaio & I & II & III & IV & V & VI & VII & VIII \\
\hline malha utilizada no fantoma numérico & A & A & B & C & B & C & D & D \\
erro no formato do domínio & - & - & - & $\checkmark$ & - & $\checkmark$ & $\checkmark$ & $\checkmark$ \\
erro na posição dos eletrodos & - & - & $\checkmark$ & - & $\checkmark$ & - & $\checkmark$ & $\checkmark$ \\
erro no parâmetro dos eletrodos & - & $\checkmark$ & - & - & $\checkmark$ & $\checkmark$ & - & $\checkmark$ \\
\hline
\end{tabular}

Ensaio I: Este ensaio avalia a presença de erros devido à diferença de nível de refinamento entre as malhas. Por este motivo, o fantoma numérico utiliza a malha A. Os valores dos parâmetros dos eletrodos utilizados no SR-UKF são os mesmos utilizados no fantoma. Como o formato do domínio e posições dos eletrodos não apresentam erros neste ensaio, utiliza-se $\sigma_{\vartheta}=0, p^{-}=0 \mathrm{~mm}$ e $p^{+}=0 \mathrm{~mm}$ no processo de amostragem de $\varepsilon$.

Ensaio II: Este ensaio avalia a presença de erros devido à diferença de nível de refinamento e parâmetro dos eletrodos. Utiliza-se novamente os potenciais elétricos obtidos com o fantoma com a malha A. O valor dos parâmetros dos eletrodos utilizados no SR-UKF é $\boldsymbol{\rho}^{\prime a}=0.02 \Omega \mathrm{m}^{2}$ em todos os eletrodos. Como o formato do domínio e posições dos eletrodos não apresentam erros neste ensaio, utiliza-se $\sigma_{\vartheta}=0$, $p^{-}=0 \mathrm{~mm}$ e $p^{+}=0 \mathrm{~mm}$ no processo de amostragem de $\varepsilon$. O sorteio dos parâmetros dos eletrodos utiliza (8.13).

Ensaio III: Este ensaio avalia a presença de erros devido à diferença de nível de refinamento e posição dos eletrodos. Utiliza-se a malha B no fantoma. Os valores dos parâmetros dos eletrodos utilizados no SR-UKF são os mesmos utilizados no fantoma. Utiliza-se $\sigma_{\vartheta}=0, p^{-}=-5 \mathrm{~mm}$ e $p^{+}=5 \mathrm{~mm}$ no processo de amostragem de $\varepsilon$. 
Ensaio IV: Este ensaio avalia a presença de erros devido à diferença de nível de refinamento e formato de $\partial \boldsymbol{\Omega}$. Utiliza-se a malha $\mathrm{C}$ no fantoma. Os valores dos parâmetros dos eletrodos utilizados no SR-UKF são os mesmos utilizados no fantoma. Utiliza-se $\sigma_{\vartheta}=5 \%, p^{-}=0 \mathrm{~mm}$ e $p^{+}=0 \mathrm{~mm}$ no processo de amostragem de $\varepsilon$.

Ensaio V: Este ensaio avalia a presença de erros devido à diferença de nível de refinamento, parâmetro e posição dos eletrodos. Utiliza-se a malha B no fantoma. O valor dos parâmetros dos eletrodos utilizados no SR-UKF é $\rho^{\prime a}=0.02 \Omega \mathrm{m}^{2}$ em todos os eletrodos. Utiliza-se $\sigma_{\vartheta}=0, p^{-}=-5 \mathrm{~mm}$ e $p^{+}=5 \mathrm{~mm}$ no processo de amostragem de $\varepsilon$. O sorteio dos parâmetros dos eletrodos utiliza (8.13).

Ensaio VI: Este ensaio avalia a presença de erros devido à diferença de nível de refinamento, parâmetro dos eletrodos e formato de $\partial \boldsymbol{\Omega}$. Utiliza-se a malha $\mathrm{C}$ no fantoma. O valor dos parâmetros dos eletrodos utilizados no SR-UKF é $\boldsymbol{\rho}^{\prime a}=0.02 \Omega \mathrm{m}^{2}$ em todos os eletrodos. Utiliza-se $\sigma_{\vartheta}=5 \%, p^{-}=0 \mathrm{~mm}$ e $p^{+}=0 \mathrm{~mm}$ no processo de amostragem de $\varepsilon$. O sorteio dos parâmetros dos eletrodos utiliza (8.13).

Ensaio VII: Este ensaio avalia a presença de erros devido à diferença de nível de refinamento, posição dos eletrodos e formato de $\partial \boldsymbol{\Omega}$. Utiliza-se a malha $\mathrm{D}$ no fantoma. Os valores dos parâmetros dos eletrodos utilizados no SR-UKF são os mesmos utilizados no fantoma. Utiliza-se $\sigma_{\vartheta}=5 \%, p^{-}=-5 \mathrm{~mm}$ e $p^{+}=5 \mathrm{~mm}$ no processo de amostragem de $\varepsilon$.

Ensaio VIII: Este ensaio avalia a presença de erros devido à diferença de nível de refinamento, posição e parâmetro dos eletrodos e formato de $\partial \boldsymbol{\Omega}$. Utiliza-se a malha D no fantoma. O valor dos parâmetros dos eletrodos utilizados no SR-UKF é $\boldsymbol{\rho}^{\prime a}=0.02 \Omega \mathrm{m}^{2}$ em todos os eletrodos. Utiliza-se $\sigma_{\vartheta}=5 \%, p^{-}=-5 \mathrm{~mm}$ e $p^{+}=5 \mathrm{~mm}$ no processo de amostragem de $\varepsilon$. O sorteio dos parâmetros dos eletrodos utiliza (8.13).

A Tabela 8.2 mostra as informações apresentadas de forma comparativa. Nesta tabela, $\boldsymbol{\rho}_{f}^{\prime}$ indica vetor de parâmetros dos eletrodos iguais ao fantoma. Em todos os oito ensaios, os parâmetros utilizados no SR-UKF são apresentados na Tabela 8.3 . 
Tabela 8.2: Parâmetros usados no processo de amostragem do ruído $\varepsilon$.

\begin{tabular}{|c|c|c|c|c|c|c|c|c|}
\hline Ensaio & $\mathrm{I}$ & II & III & IV & $\mathrm{V}$ & VI & VII & VIII \\
\hline refinamento & \multirow{2}{*}{\multicolumn{8}{|c|}{$\begin{array}{c}\delta^{-}=0.9, \quad \delta^{+}=1.1 \\
\sim \mathcal{N}\left(\mathbf{0},\left(1.0 \cdot 10^{-3}\right)^{2} \mathbb{I}\right)\end{array}$}} \\
\hline ruído $\mathbf{e}^{x}$ & & & & & & & & \\
\hline malha utilizada no fantoma numérico & A & A & $\mathrm{B}$ & $\mathrm{C}$ & B & $\mathrm{C}$ & $\mathrm{D}$ & $\mathrm{D}$ \\
\hline$\sigma_{\vartheta}(\%)$ & - & - & - & 5 & - & 5 & 5 & 5 \\
\hline$p^{-}, p^{+}(\mathrm{mm})$ & - & - & \pm 5 & - & \pm 5 & - & \pm 5 & \pm 5 \\
\hline $\boldsymbol{\rho}^{\prime a}\left(\Omega \mathrm{m}^{2}\right)$ & $\rho_{f}^{\prime}$ & 0.02 & $\rho_{f}^{\prime}$ & $\rho_{f}^{\prime}$ & 0.02 & 0.02 & $\rho_{f}^{\prime}$ & 0.02 \\
\hline resistividade $(\Omega \mathrm{m})$ & \multicolumn{8}{|c|}{$\boldsymbol{\rho} \sim \mathcal{N}^{L^{-}}\left(\mathbf{x}_{u} \mathbf{1}, 3.0^{2} \mathbb{I}, \mathbf{0}\right)$} \\
\hline
\end{tabular}

Tabela 8.3: Parâmetros usados no SR-UKF em todos os ensaios.

\begin{tabular}{|c|c|c|}
\hline Ensaio & I & $\begin{array}{lllllll}\text { II } & \text { III } & \text { IV } & \text { V } & \text { VI } & \text { VII } & \text { VIII }\end{array}$ \\
\hline ruído residual $\boldsymbol{\eta}$ (sem erro de apr.) ${ }^{a}$ & 0.0 & $\sim \mathcal{N}\left(0,1.0 \cdot 10^{-3} \mathbb{I}\right)$ \\
\hline ruído residual $\boldsymbol{\eta}$ (com erro de apr.) ${ }^{b}$ & & 0.0 \\
\hline parâmetros de dispersão (eq. (5.18) ) & & $\alpha=1.0, \beta=2.0, \kappa=0.0$ \\
\hline 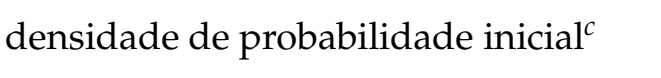 & & $\overline{\boldsymbol{x}}_{0}=4.0 \cdot \mathbf{1}, \boldsymbol{\Gamma}_{0}^{x x}=1.0 \mathbb{I}$ \\
\hline modelo de evolução & & passeio aleatório \\
\hline ruído de processo $^{c}$ & & $\overline{\boldsymbol{v}}_{k}^{x}=\mathbf{0}, \boldsymbol{\Gamma}_{k}^{v^{x}}=1.0 \cdot 10^{-6} \mathbb{I}$ \\
\hline modelo de observação & & modelo linearizado \\
\hline ruído de observação & & $\overline{\boldsymbol{e}}_{k}^{x}=\mathbf{0}, \boldsymbol{\Gamma}_{k}^{e^{x}}=\left(1.0 \cdot 10^{-3}\right)^{2} \mathbb{I}$ \\
\hline IUKF (eq. (5.39) ) & & $\operatorname{sim}$ \\
\hline densidade de prob. a priori (eq. (6.26) & & não \\
\hline vínculo de intervalo (eq. (6.4)) & & $\rho^{\min }=0.0, \rho^{\max }=50.0, s=0.1$ \\
\hline segmentação de imagem & & não \\
\hline número de medidas utilizadas & & $\ell=1024$ \\
\hline
\end{tabular}

\footnotetext{
${ }^{a}$ Ruído adicionado ao ruído de medição $\mathbf{e}^{x}$ para estabilizar o filtro nas estimações sem o método do erro de aproximação.

${ }^{b}$ Ruído residual (7.12) adicionado ao ruído $\varepsilon$ para estabilizar o filtro nas estimações com o método do erro de aproximação.

${ }^{c}$ As estatísticas foram convertidas para o metaestado com 6.14
} 


\subsection{Critérios de convergência}

Os filtros de Kalman são estimadores que maximizam a densidade de probabilidade a posteriori do estado, condicionado às realizações das medidas. Como apresentado na Seção 4.3, é possível também interpretar a média da distribuição obtida como a solução que minimiza a função custo (4.34).

Considerando inicialmente a estimação da resistividade no domínio, a inclusão de informação a priori (6.26), dos modelos de evolução e observação (5.1) e (5.5) e do método do erro de aproximação (7.12) no índice (4.34) resulta em

$$
\begin{aligned}
J_{k+1}\left(\mathbf{x}_{k+1}\right)= & \left\|\left(\boldsymbol{S}_{k+1 \mid k}^{y}\right)^{-1}\left(\boldsymbol{y}_{k+1}^{\text {medido }}-\boldsymbol{h}^{a}\left(\mathbf{x}_{k+1}, \boldsymbol{d}^{a}, \boldsymbol{e}^{x a}, \boldsymbol{r}^{a}\right)-\overline{\boldsymbol{\varepsilon}}-\overline{\boldsymbol{\eta}}\right)\right\|_{2}^{2} \\
& +\left\|\left(\boldsymbol{S}_{k+1 \mid k}^{x}\right)^{-1}\left(\mathbf{x}_{k+1}-\boldsymbol{\Phi}_{k}\left(\mathbf{x}_{k}, \mathbf{p}_{k}, \boldsymbol{u}_{k}\right)-\boldsymbol{A}_{k}^{x} \overline{\boldsymbol{v}}_{k}\right)\right\|_{2}^{2} \\
& +\alpha^{2}\left\|\boldsymbol{L}_{r}\left(\boldsymbol{\varphi}^{-1}\left(\mathbf{x}_{k+1}\right)-\boldsymbol{\varphi}^{-1}\left(\boldsymbol{x}_{k+1}^{*}\right)\right)\right\|_{2}^{2},
\end{aligned}
$$

ou, separando as três parcelas

$$
\begin{aligned}
J_{k+1}\left(\mathbf{x}_{k+1}\right) & =J_{m}+J_{d}+J_{p} \\
J_{m} & =\left\|\left(\boldsymbol{S}_{k+1 \mid k}^{y}\right)^{-1}\left(\boldsymbol{y}_{k+1}^{\text {medido }}-\boldsymbol{h}^{a}\left(\mathbf{x}_{k+1}, \boldsymbol{d}^{a}, \boldsymbol{e}^{x a}, \boldsymbol{r}^{a}\right)-\overline{\boldsymbol{\varepsilon}}-\overline{\boldsymbol{\eta}}\right)\right\|_{2}^{2} \\
J_{d} & =\left\|\left(\boldsymbol{S}_{k+1 \mid k}^{x}\right)^{-1}\left(\mathbf{x}_{k+1}-\boldsymbol{\Phi}_{k}\left(\mathbf{x}_{k}, \mathbf{p}_{k}, \boldsymbol{u}_{k}\right)-\boldsymbol{A}_{k}^{x} \overline{\boldsymbol{v}}_{k}\right)\right\|_{2}^{2} \\
J_{p} & =\alpha^{2}\left\|\boldsymbol{L}_{r}\left(\boldsymbol{\varphi}^{-1}\left(\mathbf{x}_{k+1}\right)-\boldsymbol{\varphi}^{-1}\left(\boldsymbol{x}_{k+1}^{*}\right)\right)\right\|_{2}^{2} .
\end{aligned}
$$

Analogamente, para os parâmetros dos eletrodos, tem-se

$$
\begin{aligned}
J_{k+1}\left(\mathbf{x}_{k+1}\right) & =J_{m}+J_{d}+J_{p} \\
J_{m} & =\left\|\left(\boldsymbol{S}_{k+1 \mid k}^{y}\right)^{-1}\left(\boldsymbol{y}_{k+1}^{\text {medido }}-\boldsymbol{h}^{a}\left(\mathbf{x}_{k+1}, \boldsymbol{d}^{a}, \boldsymbol{e}^{x a}, \boldsymbol{r}^{a}\right)-\overline{\boldsymbol{\varepsilon}}-\overline{\boldsymbol{\eta}}\right)\right\|_{2}^{2} \\
J_{d} & =\left\|\left(\boldsymbol{S}_{k+1 \mid k}^{x}\right)^{-1}\left(\mathbf{x}_{k+1}-\mathbf{x}_{k}-\boldsymbol{A}_{k}^{x} \overline{\boldsymbol{v}}_{k}\right)\right\|_{2}^{2} \\
J_{p} & =\alpha^{2}\left\|\boldsymbol{L}_{r}\left(\boldsymbol{\varphi}^{-1}\left(\mathbf{x}_{k+1}\right)-\boldsymbol{\varphi}^{-1}\left(\boldsymbol{x}_{k+1}^{*}\right)\right)\right\|_{2}^{2},
\end{aligned}
$$


e para os parâmetros do modelo de evolução

$$
\begin{aligned}
J_{k+1}\left(\mathbf{p}_{k+1}\right) & =J_{m}+J_{d}+J_{p} \\
J_{m} & =\left\|\left(\boldsymbol{S}_{k+1 \mid k}^{y}\right)^{-1}\left(\boldsymbol{y}_{k+1}^{\text {medido }}-\boldsymbol{h}^{a}\left(\boldsymbol{x}_{k+1}, \mathbf{p}_{k+1}, \boldsymbol{d}^{a}, \boldsymbol{e}^{p a}, \boldsymbol{r}^{a}\right)-\overline{\boldsymbol{\varepsilon}}-\overline{\boldsymbol{\eta}}\right)\right\|_{2}^{2} \\
J_{d} & =\left\|\left(\boldsymbol{S}_{k+1 \mid k}^{p}\right)^{-1}\left(\mathbf{p}_{k+1}-\mathbf{p}_{k}-\boldsymbol{A}_{k}^{p} \overline{\boldsymbol{v}}_{p}\right)\right\|_{2}^{2} \\
J_{p} & =\alpha^{2}\left\|\boldsymbol{L}_{r}\left(\boldsymbol{\varphi}^{-1}\left(\mathbf{x}_{k+1}\right)-\boldsymbol{\varphi}^{-1}\left(\boldsymbol{x}_{k+1}^{*}\right)\right)\right\|_{2}^{2},
\end{aligned}
$$

onde o modelo de observação é dado pela equação (5.8). Estas três parcelas são avaliadas para analisar a convergência do algoritmo. Conforme o ensaio, a parcela $J_{p}$ pode estar ausente.

Por outro lado, mostra-se também que a inovação $\mathbf{r}_{k} \in \mathbb{R}^{\ell}$ dos filtros de Kalman, definida como

$$
\mathbf{r}_{k+1}=\left(\boldsymbol{y}_{k+1}^{\text {medido }}-\overline{\boldsymbol{y}}_{k+1 \mid k}\right)
$$

e que representa a diferença entre as medidas realizadas e a predição do modelo de observação considerando o estado $\mathbf{x}_{k+1 \mid k}$, é um ruído Gaussiano branco com estatísticas

$$
\begin{aligned}
\overline{\boldsymbol{r}}_{k+1} & =\mathbf{0} \\
\boldsymbol{\Gamma}_{k+1}^{r} & =\boldsymbol{\Gamma}_{k+1}^{y y},
\end{aligned}
$$

como pode ser visto em Maybeck [78, p. 228].

Considerando a presença do ruído do erro de aproximação na forma de (7.12), as estatísticas da inovação passam a ser

$$
\begin{aligned}
\overline{\boldsymbol{r}}_{k+1} & =-\overline{\boldsymbol{\varepsilon}}-\overline{\boldsymbol{\eta}} \\
\boldsymbol{\Gamma}_{k+1}^{r} & =\boldsymbol{\Gamma}_{k+1}^{y y} .
\end{aligned}
$$

O segundo índice a ser observado para constatar convergência estatística dos filtros consiste em comparar as estatísticas das realizações $\boldsymbol{r}_{k}$ com as estatísticas esperadas. 
Para tal, seja a sequência $\left\{\boldsymbol{r}_{k}\right\}_{k=0}^{K}$, armazenada entre $t_{0}$ e o instante atual $t_{K}$ :

1. Calcula-se os estimadores não viesados para a média e covariância, dentro de uma janela móvel de tamanho $N$

$$
\begin{aligned}
\overline{\boldsymbol{r}}_{k}^{N} & =\frac{1}{N} \sum_{i=0}^{N-1} \boldsymbol{r}_{k-i} \\
\boldsymbol{\Gamma}_{k}^{r r, N} & =\frac{1}{N-1} \sum_{i=0}^{N-1}\left(\boldsymbol{r}_{k-i}-\overline{\boldsymbol{r}}_{k}^{N}\right)^{\mathrm{T}}\left(\boldsymbol{r}_{k-i}-\overline{\boldsymbol{r}}_{k}^{N}\right) .
\end{aligned}
$$

2. Verifica-se as inequações

$$
\begin{aligned}
C_{m} & =\max \left(\frac{\left|\overline{\boldsymbol{r}}_{k}^{N}-\overline{\boldsymbol{r}}_{k}\right|}{\sqrt{\sigma_{\eta}^{2}+\sigma_{\varepsilon}^{2}}}\right)=\max \left(\frac{\left|\overline{\boldsymbol{r}}_{k}^{N}+\overline{\boldsymbol{\varepsilon}}+\overline{\boldsymbol{\eta}}\right|}{\sqrt{\sigma_{\eta}^{2}+\sigma_{\varepsilon}^{2}}}\right) \leq 3 \\
C_{v} & =\max \left(\frac{\boldsymbol{\sigma}_{r}}{\boldsymbol{\sigma}_{y}}\right) \leq 3 \\
\boldsymbol{\sigma}_{y} & =\operatorname{diag}\left(\boldsymbol{\Gamma}_{k}^{y y}\right) \\
\boldsymbol{\sigma}_{r} & =\operatorname{diag}\left(\boldsymbol{\Gamma}_{k}^{r r, N}\right)
\end{aligned}
$$

onde as comparações e operações devem ser realizadas elemento a elemento dos vetores. Na primeira inequação, $\sigma_{\eta}$ é o desvio padrão do do ruído residual em (7.12) e $\sigma_{\varepsilon}$ é o desvio padrão (médio) do ruído $\varepsilon$. Nesta mesma equação, o denominador $\sqrt{\sigma_{\eta}^{2}+\sigma_{\varepsilon}^{2}}$ representa o desvio padrão de um ruído composto pela soma dos ruídos independentes $\eta$ e $\varepsilon$. Na segunda inequação, $\boldsymbol{\sigma}_{y}$ e $\boldsymbol{\sigma}_{r}$ são vetores contendo as diagonais das matrizes de covariância.

3. Considera-se a solução aceitável estatisticamente se ambas as inequações forem verdadeiras 4 .

A primeira inequação baseia-se no princípio da discrepância de Morozov [61, p. 18], atestando que não é esperado que a diferença entre a medição realizada e os valores preditos seja menor que a precisão das leituras. A segunda inequação compara as variâncias amostradas e esperadas

Um último comentário importante a respeito da convergência dos filtros de Kalman se faz necessário. A convergência entendida nos filtros de Kalman diz respeito à

\footnotetext{
${ }^{4}$ Salienta-se que também seria necessário avaliar se a matriz $\Gamma_{k}^{r r, N}$ é de fato não correlacionada no tempo, mas este teste não é realizado neste trabalho.
} 
redução do traço da matriz de covariância do erro de estimação, que nos casos lineares é $\Gamma_{k}^{x x}$. Este critério não garante que a estimativa pontual $\overline{\boldsymbol{x}}_{k}$ tenha um viés pequeno. Em outras palavras, o filtro de Kalman pode convergir, neste sentido, para uma solução que não retrata a solução desejada. Alguns autores consideram que tal viés também deva ser classificada como divergência.

Por razão da possível presença deste viés, analisar apenas as estatísticas apresentadas acima não garantem que a solução seja de utilidade. O contrário também é verdadeiro: mesmo sem atingir a convergência estatística, a estimativa obtida pode ser informativa. Neste trabalho, a convergência é analisada com os critérios descritos e comentários a respeito da imagem final são feitos.

\subsection{Resultados dos ensaios}

A Figura 8.4 mostra dez realizações de $\partial \Omega$ para o cálculo das estatísticas do ruído do método do erro de aproximação no ensaio VII. Nesta figura, o contorno da malha utilizada no SR-UKF é mostrado com linha tracejada azul e o contorno da malha utilizada no fantoma é mostrado com linha tracejada vermelha.

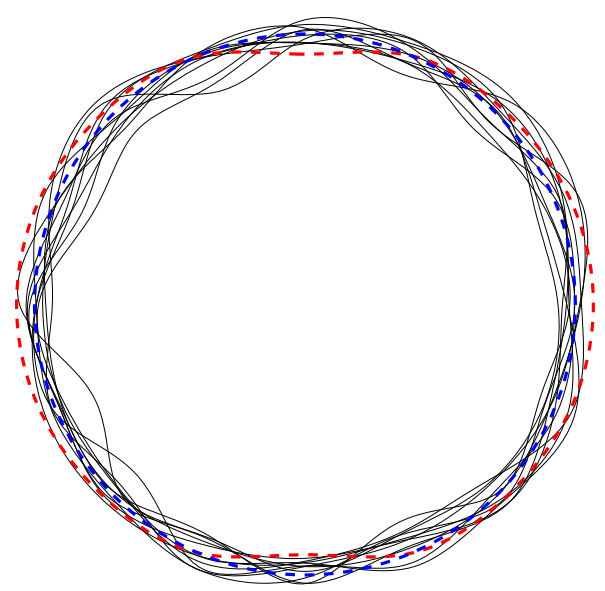

Figura 8.4: Dez realizações do contorno no Ensaio VII. A linha tracejada azul representa o contorno da malha E. A linha tracejada vermelha representa o contorno da malha utilizada no fantoma numérico.

Por esta figura é possível observar que o bordo da malha utilizada no fantoma é estatisticamente plausível, considerando as estatísticas adotadas para a composição do vetor $\vartheta_{i}$. 
A Figura 8.5 mostra quatro realizações da distribuição de resistividade dentro do domínio no ensaio IV. A primeira linha apresenta realizações de resistividade na malha $M_{i}$ e a segunda linha apresenta a projeção na malha $M^{a}$.
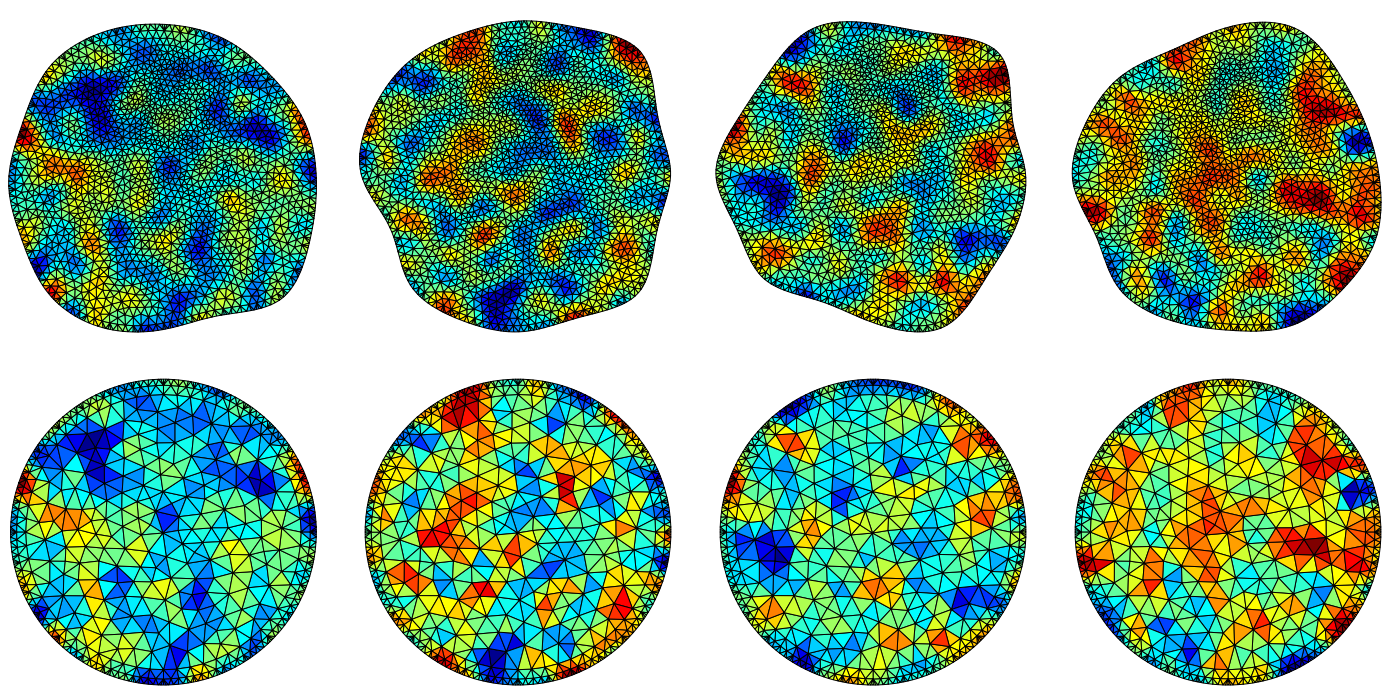

Figura 8.5: Realizações da distribuição de resistividade no Ensaio IV. Primeira linha: malha $M_{i}$, Segunda linha: malha $M^{a}$. Valores em $\Omega \mathrm{m}$.

Esta figura mostra que a amostragem do vetor de resistividades através de (8.10) consiste de uma distribuição de resistividade suave ao longo de $\Omega$ e sua projeção (8.8) mantém as características espaciais.

As figuras 8.6 e 8.7 mostram os resultados das estimações utilizando o SR-UKF no ensaio para avaliação do método do erro de aproximação. Em cada uma das figuras, a primeira linha mostra a estimação do SR-UKF sem o uso do método do erro de aproximação e a segunda linha mostra a estimação utilizando o método. Todas as imagens mostradas são as soluções da estimação na ducentésima iteração do filtro. Em cada uma das imagens, o círculo mostra a posição e tamanho correto do objeto. 


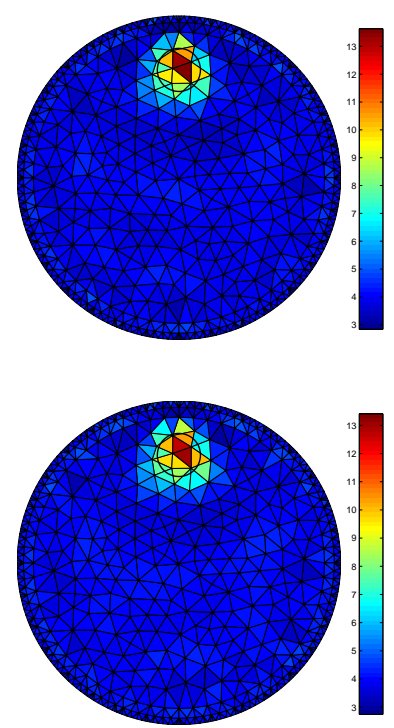

Ensaio I
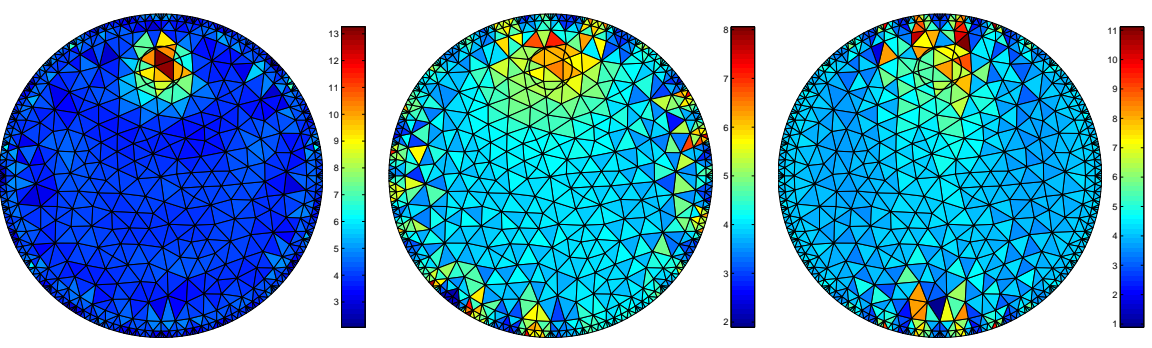

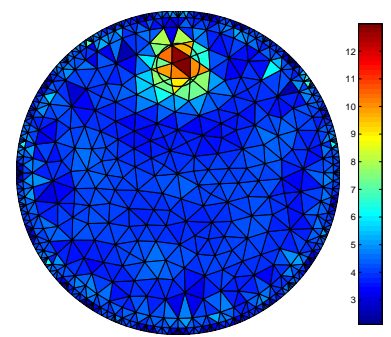

Ensaio II

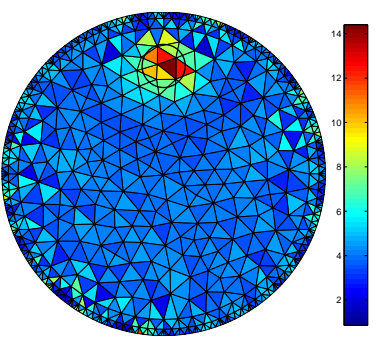

Ensaio III

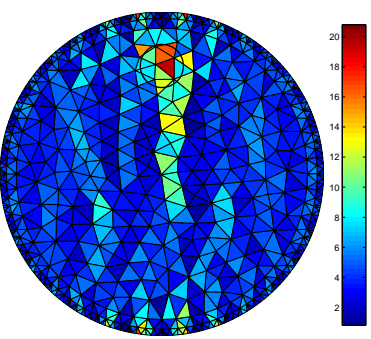

Ensaio IV

Figura 8.6: Resultados dos Ensaios I a IV. Primeira linha: estimação sem utilizar o método do erro de aproximação. Segunda linha: estimação utilizando o método do erro de aproximação. Valores em $\Omega \mathrm{m}$.

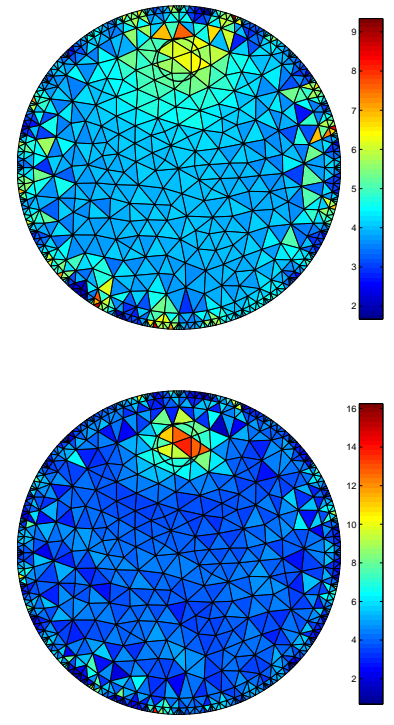

Ensaio V
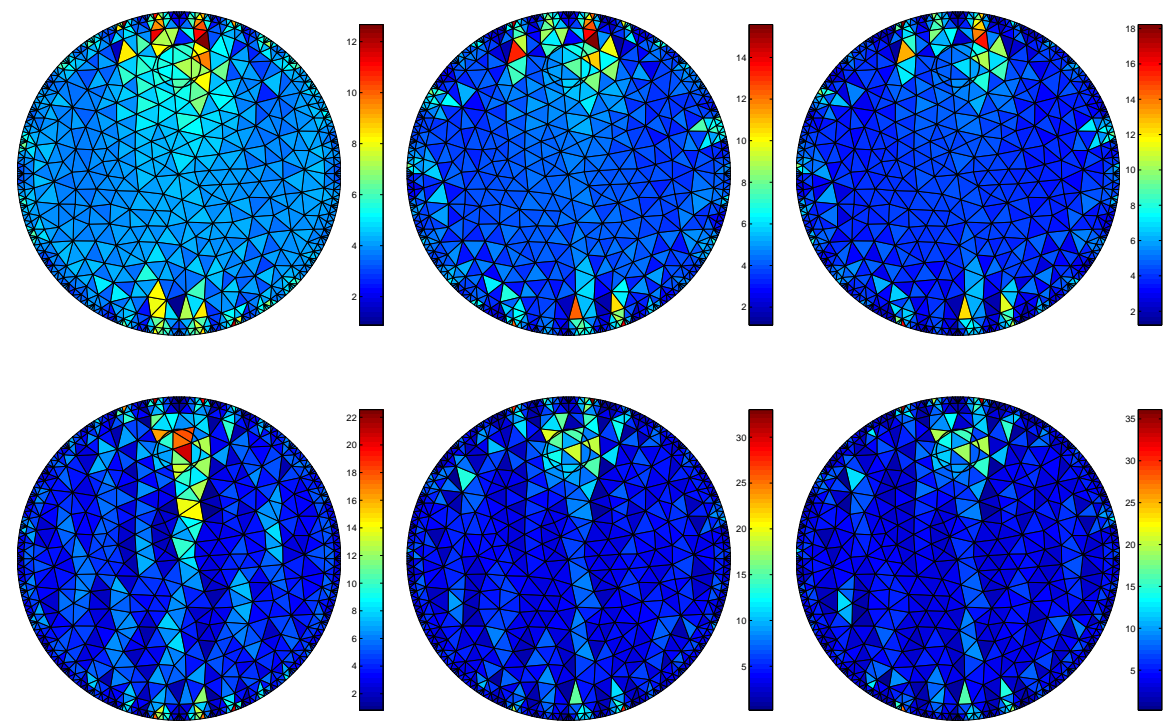

Ensaio VI

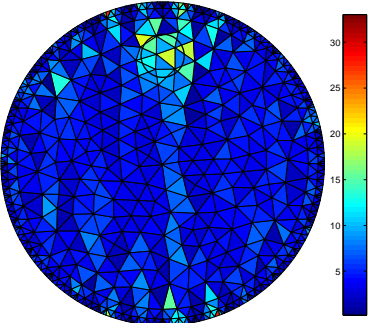

Ensaio VII

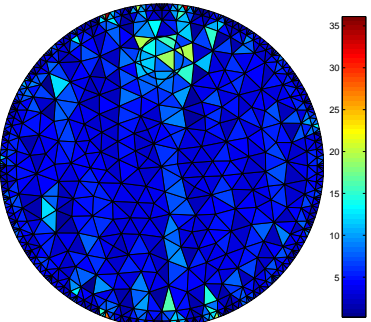

Ensaio VIII

Figura 8.7: Resultados dos Ensaios V a VIII. Primeira linha: estimação sem utilizar o método do erro de aproximação. Segunda linha: estimação utilizando o método do erro de aproximação. Valores em $\Omega \mathrm{m}$. 
Comparando-se os resultados de cada um dos ensaios, com e sem a utilização do método do erro de aproximação, nota-se que os resultados dos ensaios I e II são muito semelhantes quanto ao contraste do objeto e quantidade de artefatos5. Nestes dois ensaios, o uso do método do erro de aproximação não causa uma melhoria da imagem pois o ruído $\varepsilon$ é muito menor do que o ruído das medidas $e^{x}$.

Em compensação, os resultados dos ensaios III a VIII mostram uma expressiva melhora das imagens obtidas com o SR-UKF. Em todos os casos, há uma melhora na localização e contraste da imagem e redução do número e intensidade dos artefatos.

Nos ensaios IV e VI com o uso do método, existe um alongamento na direção radial dos objetos, porém ainda assim o objeto é identificável. Já os resultados sem o uso do método, os objetos não podem ser claramente identificados.

A deterioração das imagens quando o método não é utilizado fica ainda mais evidente nos ensaios VII e VIII, nos quais os objetos são completamente ofuscados pelos artefatos. Já com o uso do método os objetos são identificados, ainda com a presença de alguns pequenos artefatos próximos ao bordo da imagem.

Em todos os ensaios, observou-se convergência conforme os critérios estabelecidos na Seção 8.4. A Figura 8.8 mostra o histórico de estimação da distribuição de resistividade no domínio para o ensaio IV. Nestas figuras são apresentados os índices $J, J_{d}, J_{m}, C_{m}$ e $C_{v}$ apresentados na Seção 8.4. Os gráficos de todos os oito ensaios realizados são apresentados no Apêndice F.1.

\footnotetext{
${ }^{5}$ Por artefato, entende-se como a presença de uma região da imagem cujo valor de resistividade não condiz com a realidade.
} 

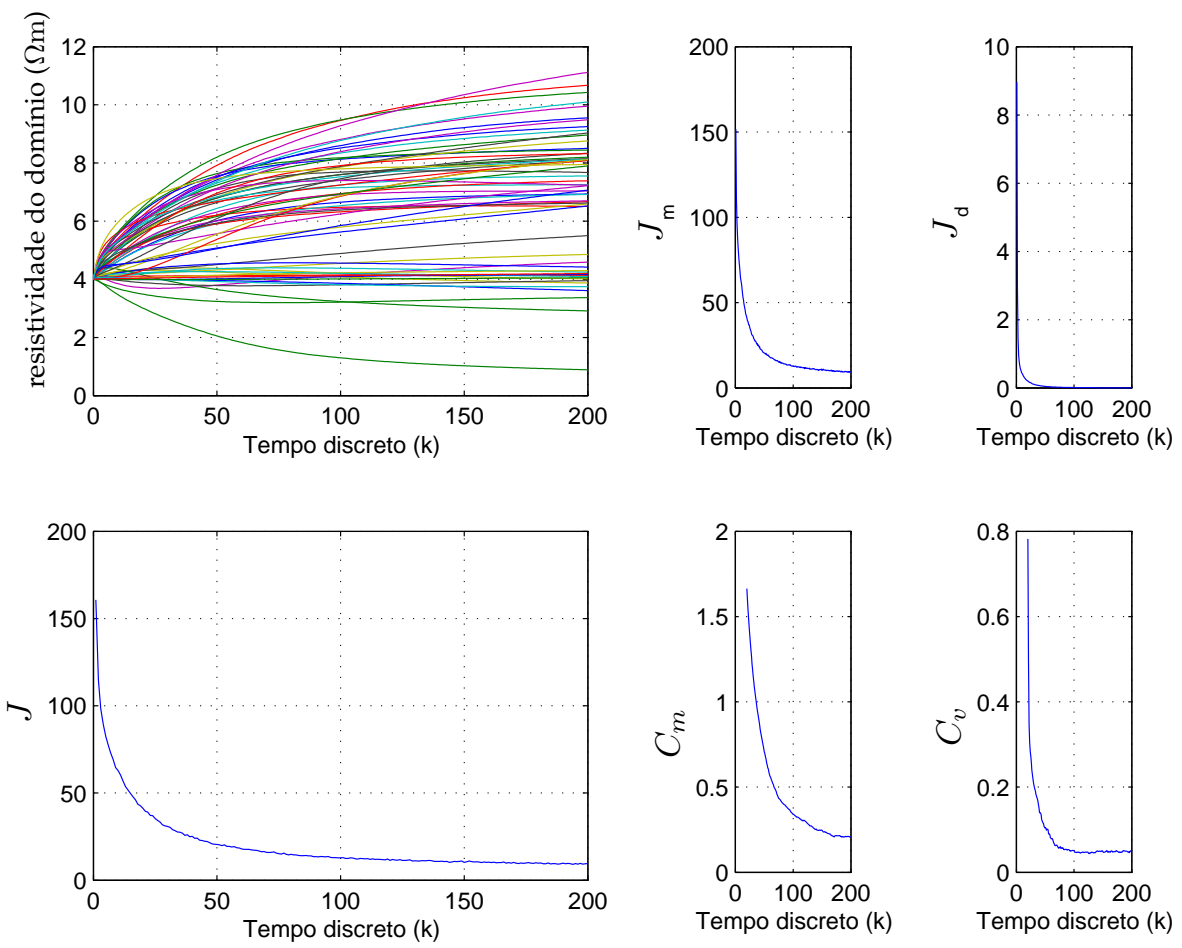

(a) Ensaio IV, sem utilizar o método do erro de aproximação.
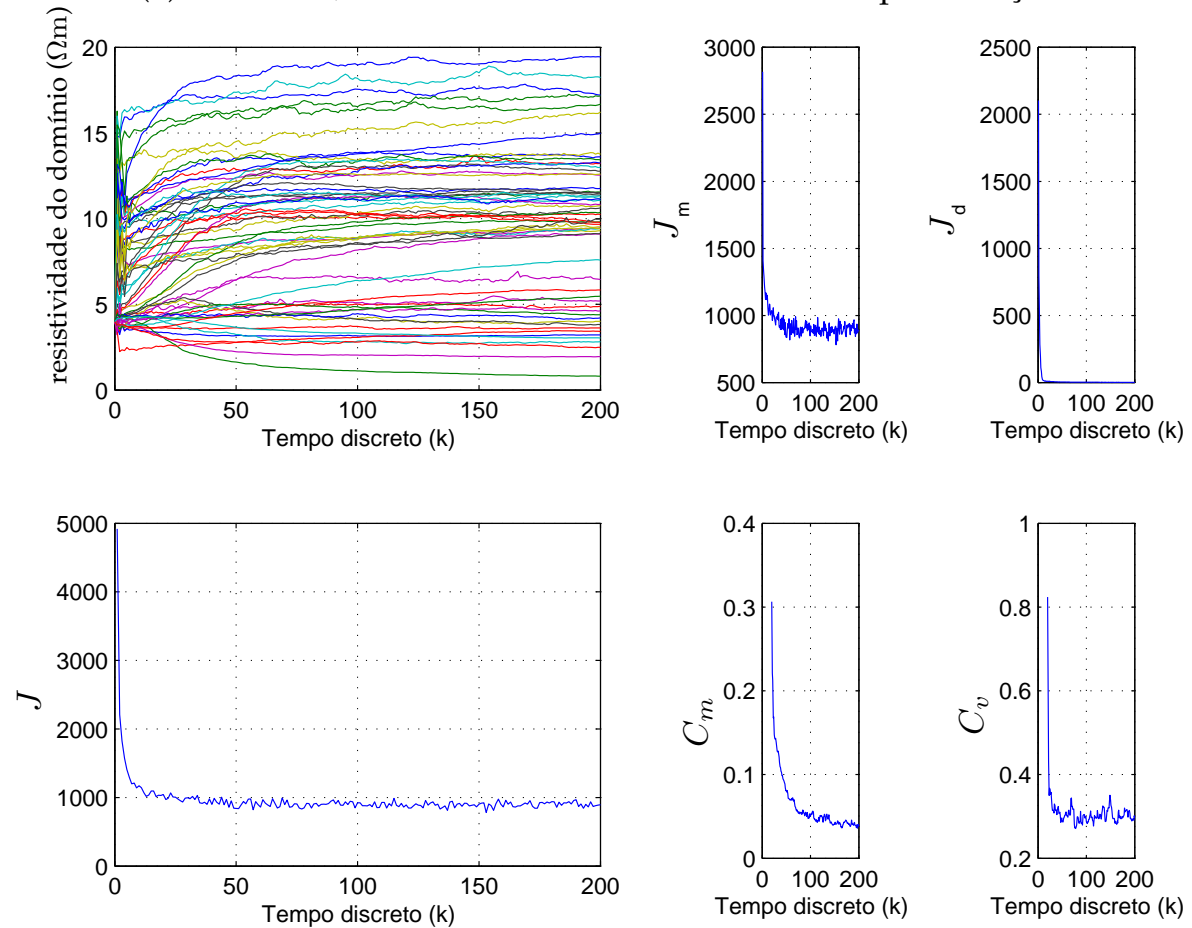

(b) Ensaio IV, utilizando o método do erro de aproximação.

Figura 8.8: Resultados do Ensaio IV. Histórico da estimação e índices de desempenho.

Pelos resultados nota-se que o uso do método do erro de aproximação acelera a convergência do filtro de Kalman. 
Quanto à presença dos artefatos e sua possível eliminação com o uso de informação a priori de suavidade, o método continua resultando em melhores imagens. A título de comparação, os mesmos ensaios foram realizados agora com o uso de informação $a$ priori de suavidade espacial (6.26), onde $\alpha=1000, \boldsymbol{L}_{r}$ é um filtro passa-altas gaussiano, com desvio padrão $\sigma=1.05 \mathrm{~cm}(7 \%$ do raio da malha $\mathrm{E})$ e $\boldsymbol{\varphi}^{-1}\left(\boldsymbol{x}_{k+1}^{*}\right)=\mathbf{0}$. Os resultados obtidos são mostrados nas Figuras 8.9 e 8.10.

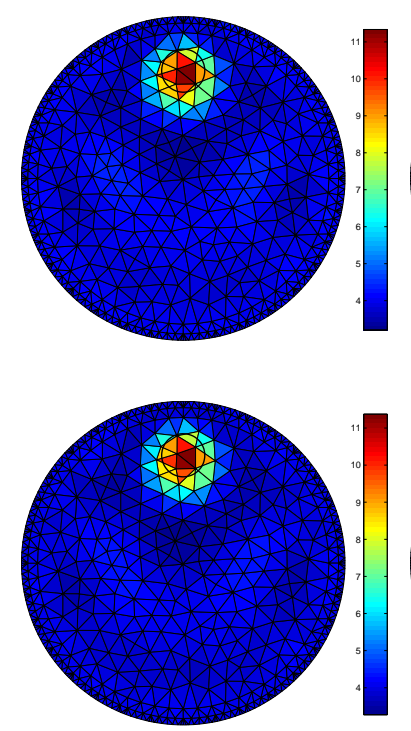

Ensaio I
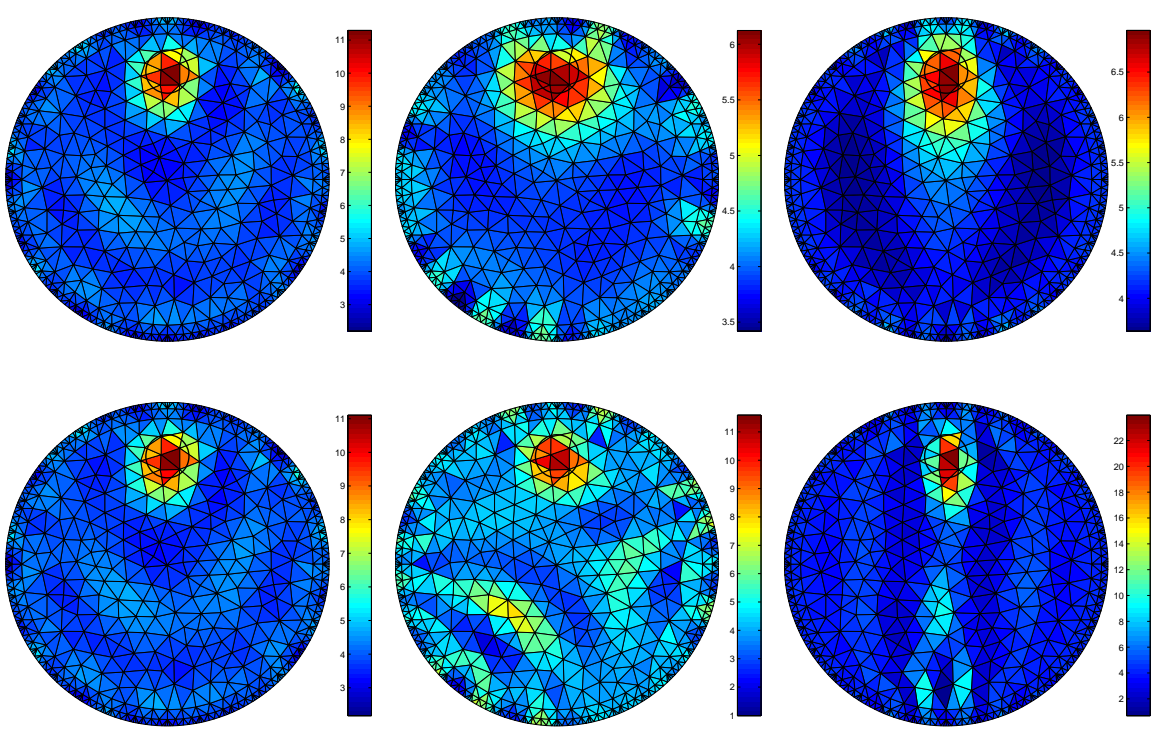

Ensaio II

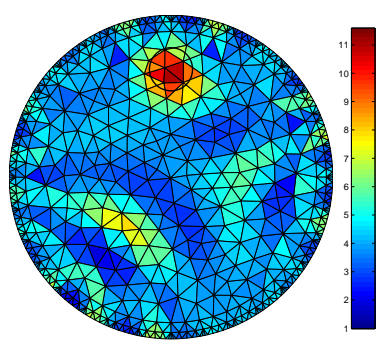

Ensaio III

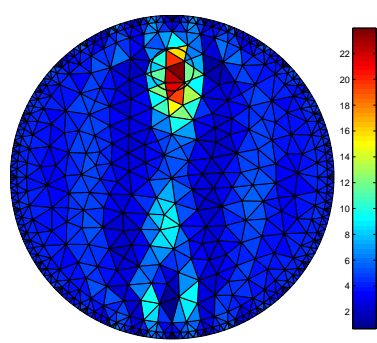

Ensaio IV

Figura 8.9: Resultados dos Ensaios I a IV. Primeira linha: estimação sem utilizar o método do erro de aproximação. Segunda linha: estimação utilizando o método do erro de aproximação. Valores em $\Omega \mathrm{m}$.

As mesmas observações continuam válidas com o uso de informação de suavidade. Um ponto importante a ser salientado é diferença de contraste das imagens quando se utiliza o método. Além disto, apesar do uso da informação de suavidade, os picos das imagens apresentam valores maiores do que os obtidos sem a informação $a$ priori. Os gráficos de convergência de todos os oito ensaios realizados são apresentados no Apêndice F.1. O mesmo número de iterações máximo foi utilizado, isto é, 200. Apesar da convergência estatística ser atingida e a função custo quase estabilizar, o valor das estimações não estabilizaram em alguns ensaios. 


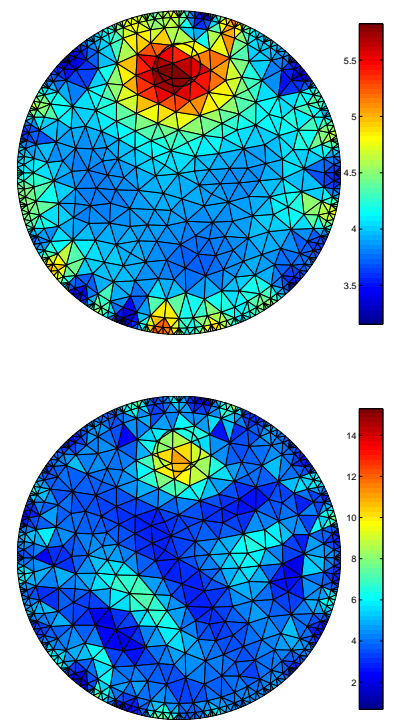

Ensaio V
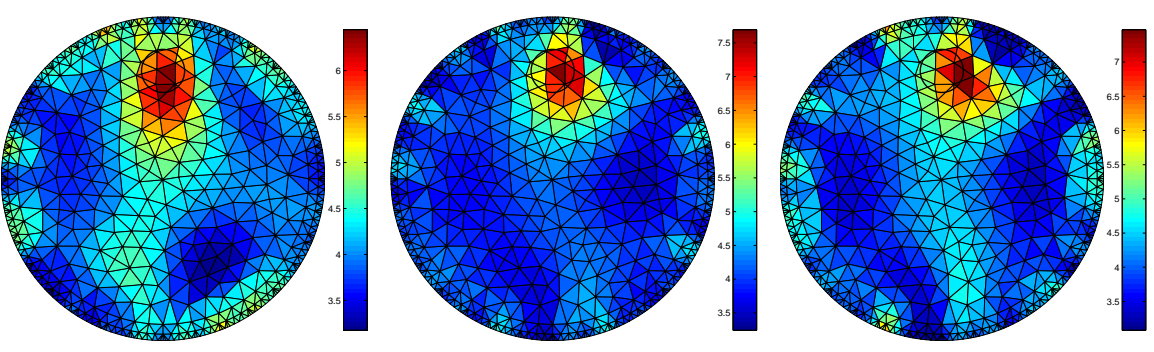

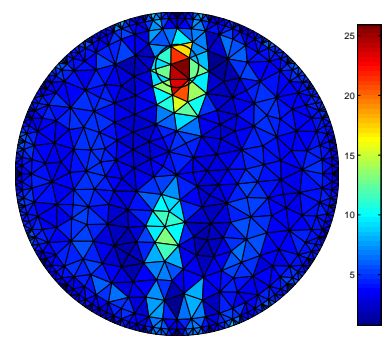

Ensaio VI

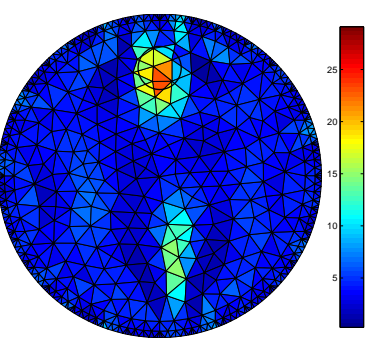

Ensaio VII

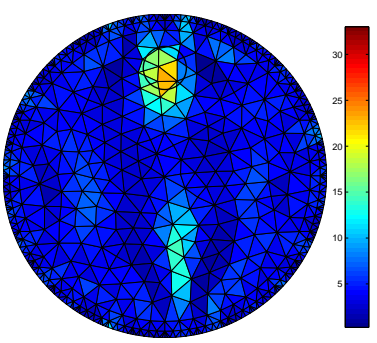

Ensaio VIII

Figura 8.10: Resultados dos Ensaios V a VIII. Primeira linha: estimação sem utilizar o método do erro de aproximação. Segunda linha: estimação utilizando o método do erro de aproximação. Valores em $\Omega \mathrm{m}$. 


\section{Capítulo 9}

\section{Modelo dinâmico}

É necessário adotar um modelo de evolução temporal para os pulmões e outro para os demais tecidos e órgãos no tórax. O modelo dinâmico adotado para os tecidos e órgãos, exceto pulmões, é o passeio aleatório, cujo termo nominal do modelo é

$$
\rho_{k}=\rho_{k-1}
$$

onde $\rho_{k}$ é a resistividade elétrica do elemento da malha em $t_{k}$, se este elemento estiver contido nesta região. Este modelo é apropriado para descrever o comportamento dos tecidos ósseos e adiposos, porém é questionável o seu uso para a modelagem dos tecidos musculares e sangue, especialmente o coração cujo comportamento temporal tem certa peridiocidade.

Apesar desta limitação, este modelo é adotado na região do coração uma vez que as variações de resistividade da região do coração durante o ciclo cardíaco são menores que as variações de resistividade da região pulmonar durante o ciclo ventilatório.

O modelo temporal adotado para os pulmões é descrito a seguir.

\subsection{Modelo dinâmico dos pulmões}

A fisiologia dos pulmões pode ser subdividida em quatro grupos. O primeiro grupo diz respeito à troca de gases por difusão passiva que ocorre nos alvéolos entre o gás no interior destes e os capilares pulmonares que os circundam. O segundo grupo é responsável pelo controle neural do ciclo respiratório. O controle, realizado no sistema 
nervoso central, depende de informações provenientes dos receptores químicos, que monitoram o nível de oxigênio e dióxido de carbono no sangue arterial e dos receptores mecânicos, que monitoram o estado de insuflamento dos pulmões. O terceiro grupo é responsável pela limpeza, lubrificação e manutenção das vias aéreas e defesa contra doenças. O quarto grupo é responsável pela mecânica da respiração. A Figura 9.1 mostra as principais estruturas do sistema respiratório.

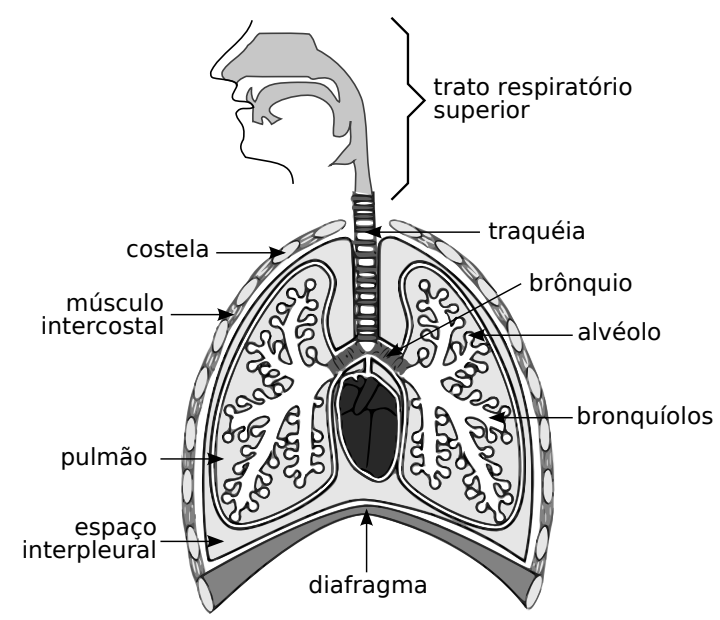

Figura 9.1: Principais componentes mecânicos do sistema respiratório.

$\mathrm{O}$ trato respiratório superior compreende nariz, boca e região da faringe. $\mathrm{O}$ trato inferior inicia após as cordas vocais, na traqueia e procede até os bronquíolos terminais, onde se localizam os alvéolos. Os dutos respiratórios são recobertos por um epitélio que secreta um muco de proteção e limpeza.

Os pulmões em humanos são divididos por fissuras em cinco lobos principais, três no pulmão direito e dois no pulmão esquerdo, como pode ser visto na Figura 9.2. A rede de bronquíolos de um lobo só se comunica com outros lobos através dos ramos principais dos brônquios.

O fluxo de ar entra e sai dos pulmões graças à ação coordenada dos músculos respiratórios. Os principais músculos responsáveis pela ventilação são o diafragma, que separa o tórax do abdômen e os músculos intercostais, localizados entre as costelas, responsáveis pela estabilização da caixa torácica.

Ao ser ativado, o diafragma desce e produz uma pressão efetiva negativa no espaço interpleural que separa a pleura visceral que recobre os pulmões e a pleura parietal que circunda a caixa torácica. Esta queda de pressão causa a entrada de ar nos pulmões pelo trato respiratório superior. A expiração em condições de descanso 


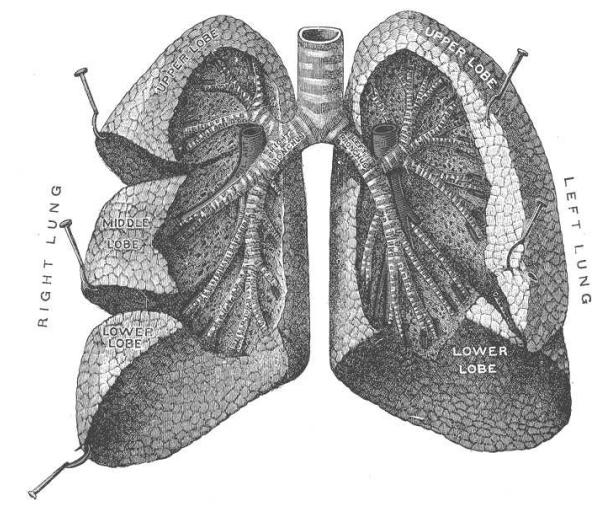

(a) Lobos pulmonares em seres humanos.

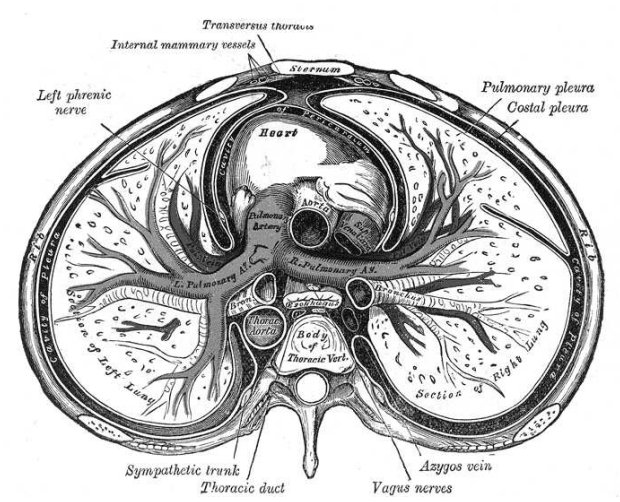

(b) Detalhe de um corte transversal de uma caixa torácica humana.

Figura 9.2: Detalhes da estrutura pulmonar e disposição interna dos órgãos na caixa torácica [115].

ocorre de forma passiva pois ao desativar os músculos respiratórios, a reconstituição elástica dos pulmões e caixa torácica força a saída de ar pelo aumento da pressão no espaço interpleural. Já no caso de expiração forçada, que ocorre por exemplo durante atividades físicas, a expiração é acelerada pela contração dos músculos abdominais.

A Figura 9.3 mostra os principais volumes pulmonares de um indivíduo ao longo do tempo durante a respiração. O volume de ar que entra dos pulmões durante respiração normal é chamado volume corrente (VC) e pode variar dependendo do nível de atividade física do indivíduo. Seu limite superior é significantemente menor que a capacidade pulmonar total (CPT). Este limite é alcançado quando a queda de pressão no espaço interpleural é cancelado pela reconstituição elástica do tecido pulmonar e pela restrição à expansão dos pulmões pela caixa torácica. A CPT é alcançada após inspiração forçada máxima e o maior volume de ar que entra ou sai dos pulmões entre uma inspiração forçada máxima e uma expiração forçada máxima é chamado capacidade vital $(\mathrm{CV})$. O volume de ar que permanece dentro dos pulmões após expiração forçada máxima é chamado volume residual (VR) e o volume que permanece dentro dos pulmões após expiração normal é chamada capacidade residual funcional (CRF). Valores usuais destes volumes em humanos adultos saudáveis são [116, 99]: $\mathrm{CPT}=6000 \mathrm{~cm}^{3}, \mathrm{CV}=4800 \mathrm{~cm}^{3}, \mathrm{VC}=400 \mathrm{~cm}^{3}$ (descanso) ou $\mathrm{VC}=2000 \mathrm{~cm}^{3}$ (atividade física intensa), $\mathrm{VR}=1200 \mathrm{~cm}^{3}$ e $\mathrm{CRF}=2400 \mathrm{~cm}^{3}$. 


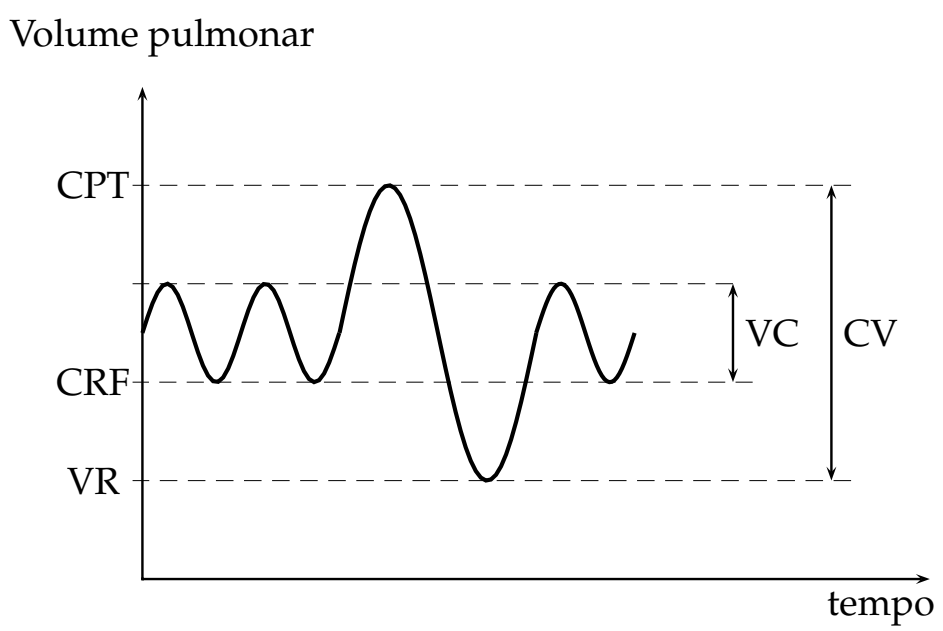

Figura 9.3: Subdivisões do volume pulmonar. VR: Volume residual CRF: Capacidade residual funcional CPT: Capacidade pulmonar total VC: Volume corrente $\mathrm{CV}$ : Capacidade vital.

Durante a respiração, os pulmões sofrem influência da pressão do espaço pleural, da resistência ao escoamento do ar nas vias aéreas, dos efeitos gravitacionais sobre sua estrutura, da elasticidade do tecido pulmonar ao estendimento e dos efeitos de tensão superficial do lubrificante dos alvéolos. Os efeitos de inércia do tecido pulmonar em geral são desprezados perante os efeitos resistivos e elásticos.

Próximo do volume máximo há grande resistência para aumentos adicionais de volume. Isto é causado pela rigidez das paredes da caixa torácica que passam a impedir aumentos de volumes adicionais, e também pelo limite de distendimento do tecido pulmonar. Próximo do volume mínimo, a pressão da cavidade interpleural passa a limitar reduções adicionais de volume. Esta relação é clarificada ao se traçar um gráfico Volume-Pressão. A curva típica deste comportamento pode ser vista na Figura 9.4

Os pulmões ainda são susceptíveis a patologias ou anormalidades, que modificam as suas propriedades mecânicas e, portanto, a curva Volume-Pressão. As patologias podem ser do tipo obstrutivas, como a asma e o enfisema pulmonar. Na asma, os músculos lisos que recobrem as vias aéreas ficam contraídos, reduzindo o calibre dos bronquíolos. No enfisema pulmonar, parte do tecido pulmonar é destruído, reduzindo a área de troca de gases e reduzindo a elasticidade dos pulmões. As patologias podem ser ainda do tipo restritiva, que reduz a CPT, como a fibrose. Nesta patologia, ocorre um depósito de proteínas, como o colágeno, aumentando a rigidez do tecido e 
reduzindo a capacidade de inflar. Em pneumonias, acúmulo de líquido nos alvéolos causa inutilização de áreas do pulmão e reduz a quantidade de ar que entra no mesmo.

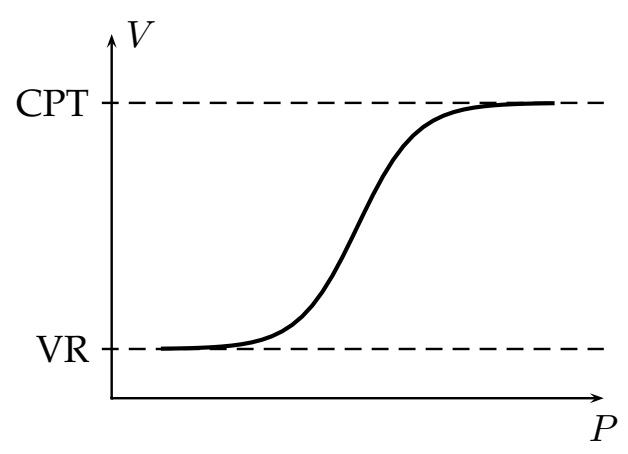

Figura 9.4: Curva Volume-Pressão do pulmão.

O modelo de evolução utilizado neste trabalho para estimação do estado é baseado nas características elásticas e resistivas da mecânica respiratória. Alguns modelos para os pulmões podem ser encontrados na literatura, como em Bates [117], Liu et al. [118], Bates e Irvin [119], Kaminsky et al. [120], Allen et al. [121], Venegas et al. [122].

Este trabalho utiliza um modelo não linear de $N_{c}$ compartimentos para os pulmões. O sistema é excitado pela pressão no trato respiratório superior e apresenta um comportamento elástico e viscoso para simular o comportamento dos pulmões.

O sistema da Figura 9.5 representa o modelo adotado para os pulmões. Neste modelo, a pressão efetiva $P(t)$ excita cada um dos compartimentos que tendem a aumentar os seus volumes $V_{i}(t)$ com aumentos de pressão. Contrária a esta tendência existe uma restituição elástica de rigidez $K_{i}\left(V_{i}\right)$, função do volume de ar no compartimento, e uma resistência viscosa $c_{i} \dot{V}_{i}$ em cada compartimento. Neste modelo não são consideradas as influências de um compartimento em outro como por exemplo a pressão que um compartimento aplica em outro vizinho quando o primeiro aumenta de volume.

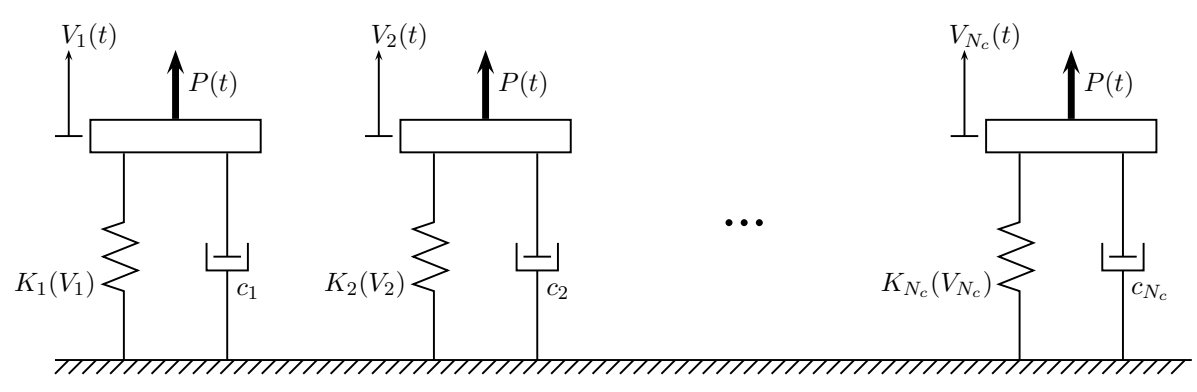

Figura 9.5: Representação dos compartimentos do modelo dinâmico dos pulmões. 
O sistema de equações do sistema de $N_{c}$ graus de liberdade é dado pela equação de Lagrange.

$$
\frac{d}{d t}\left(\frac{\partial L}{\partial \dot{V}_{i}}\right)-\frac{\partial L}{\partial V_{i}}+\frac{\partial R}{\partial \dot{V}_{i}}=Q_{i} \quad, \quad i=1, \ldots, N_{c}
$$

onde $V_{i}$ são as coordenadas generalizadas, $L$ é o lagrangiano, $R$ é a função de dissipação de Rayleigh, $Q_{i}$ são as forças generalizadas não conservativas dadas por

$$
L=-\frac{1}{2} \sum_{i=1}^{N_{c}} K_{i} \cdot V_{i}^{2} \quad, \quad R=\frac{1}{2} \sum_{i=1}^{N_{c}} c_{i} \cdot \dot{V}_{i}^{2} \quad, \quad Q_{i}=P(t)
$$

Calculando as derivações, chega-se a

$$
\frac{1}{2} \frac{\partial K_{i}}{\partial V_{i}} V_{i}^{2}+K_{i} \cdot V_{i}+c \dot{V}_{i}=P(t) \quad, \quad i=1, \ldots, N_{c}
$$

ou

$$
\dot{V}_{i}(t)=\frac{P(t)}{c_{i}}-\frac{1}{2 c_{i}} \frac{\partial K_{i}}{\partial V_{i}} V_{i}^{2}-\frac{K_{i} \cdot V_{i}}{c_{i}}
$$

Agora é necessário identificar $K_{i}\left(V_{i}\right)$ e determinar sua derivada parcial em relação à $V_{i}$. Isto pode ser determinado através do gráfico Volume-Pressão dos pulmões.

Em Venegas et al. [122] foi realizado um estudo sobre o ajuste de uma função sigmóide em curvas Volume-Pressão quase-estática em seres humanos. A curva pode ser vista na Figura 9.6 .

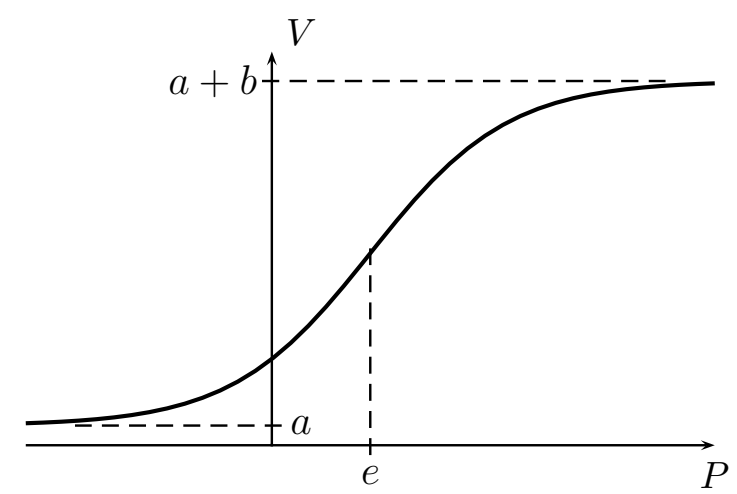

Figura 9.6: Sigmóide ajustada à curva Volume-Pressão do pulmão. 
A curva é dada por

$$
V=a+b\left[1+\exp \left(-\frac{P-e}{d}\right)\right]^{-1}
$$

onde $a$ e $a+b$ são as assíntotas da curva, e é o valor da pressão quando o volume é $(a+b) / 2$ e $d$ está relacionado com a complacência $C$ do pulmão, definida como a derivada da curva Volume-Pressão

$$
C(P)=\frac{\partial V}{\partial P}=\frac{b}{d} \frac{\exp \left(-\frac{P-e}{d}\right)}{\left[1+\exp \left(-\frac{P-e}{d}\right)\right]^{2}} .
$$

Nota-se então que $\left.K=C^{-1}, V \in\right] a, a+b[$

$$
\left.K(V)=\frac{1}{C}=\frac{b d}{(V-a)[b-(V-a)]}, \quad V \in\right] a, a+b[
$$

e sua derivada parcial com relação a $V$ é, portanto,

$$
\left.\frac{\partial K}{\partial V}=\frac{-b d[b-2(V-a)]}{\{(V-a)[b-(V-a)]\}^{2}}, \quad V \in\right] a, a+b[.
$$

O parâmetro $e$ não aparece na equação da rigidez $K$, sendo portanto irrelevante ao modelo adotado. Nesta tese assume-se que (9.9), obtida de um modelo para o volume total dos pulmões continua válida para subdivisões do volume pulmonar. Com esta hipótese, pode-se utilizar (9.9) em (9.5) utilizando o volume e demais parâmetros de cada compartimento.

Sejam dados um certo instante $t_{k}$, um intervalo de tempo $\Delta t, V_{i}\left(t_{k}\right)$, a função $P(t)$ entre $t_{k}$ e $t_{k}+\Delta t$ e os parâmetros do modelo de cada compartimento. Pode-se determinar $V_{i}\left(t_{k}+\Delta t\right)$ calculando a integração no tempo de (9.5)

$$
V_{i}\left(t_{k}+\Delta t\right)=V_{i}\left(t_{k}\right)+\int_{t_{k}}^{t_{k}+\Delta t} \dot{V}_{i}(t) d t \quad, \quad i=1, \ldots, N_{c}
$$

Diversos métodos podem ser utilizados para resolver esta integral. Aqui foi utilizado o método numérico de Runge-Kutta de quarta ordem. Desta forma, dados um certo instante no tempo discreto $k T_{s}$ e a função $P(t)$ entre $k T_{s}$ e $(k+1) T_{s}$, pode-se definir 
uma função no tempo discreto

$$
V_{i, k+1} \triangleq f\left(V_{i, k}, P_{k}, \ldots, P_{k+1}, a_{i}, b_{i}, c_{i}, d_{i}\right) \quad, \quad i=1, \ldots, N_{c}
$$

que pode ser utilizada no SR-UKF como modelo dinâmico dos pulmões. Nesta função, $f(\ldots)$ representa a integração numérica apresentada em (9.10) para $\Delta t=T_{s}$. Por fim, resta agora determinar a relação entre o volume de ar dentro dos pulmões e a resistividade elétrica.

Em Nopp et al. [123], os autores modelam a relação entre a variação de resistividade média relativa à CRF e a variação de volume de ar nos pulmões também em relação à CRF como linear na faixa de frequência utilizada. Em outras palavras,

$$
\boldsymbol{\rho}\left(V_{i}\right)-\boldsymbol{\rho}_{C R F} \approx \alpha\left(V_{i}-V_{C R F}\right)
$$

onde $\boldsymbol{\rho}_{C R F}$ é o valor da resistividade quando os pulmões contém apenas a CRF e $\alpha$ é uma constante.

Esta aproximação é feita neste trabalho, ou seja, a relação entre volume de ar e resistividade é assumida linear. Entretanto, o valor $\alpha$ deve ser ajustado caso a caso.

Finalmente, a função $\Phi_{k}\left(\mathbf{x}_{k}, \mathbf{p}_{k}, \boldsymbol{u}_{k}\right)$ em (5.1) representa o seguinte processo:

1. Determinar a resistividade média da região pulmonar em cada compartimento

$$
\tilde{\rho}_{k, i}=\frac{\sum_{j=1}^{n} A_{j} \rho_{j, k}}{\sum_{j=1}^{n} A_{j}}
$$

onde $A_{j}$ é a área (ou volume) do $j$-ésimo elemento pertencente ao $i$-ésimo compartimento e $n$ é o número de elementos dentro deste compartimento;

2. Interpolar os valores de resistividade média de cada compartimento $\tilde{\rho}_{k, i}$ para volumes $V_{i}$, utilizando (9.12);

3. Integrar no tempo a equação (9.11) para cada compartimento.

4. Interpolar o valor do novo volume para resistividade $\tilde{\rho}_{k+1, i}$, utilizando (9.12);

5. Alterar a resistividade dos elementos do vetor de estado, conforme o 
compartimento em que cada um pertence

$$
\rho_{j, k+1}=\rho_{j, k}+\frac{A_{j}}{\sum_{j=1}^{n} A_{j}}\left(\tilde{\rho}_{k+1, i}-\tilde{\rho}_{k, i}\right),
$$

que equivale a distribuir a variação de resistividade proporcionalmente à área (ou volume) de cada elemento do compartimento.

Neste processo, identifica-se $\boldsymbol{u}_{k}$ como a pressão utilizada em (9.11).

\subsection{Número de graus de liberdade no modelo dinâmico}

O número de graus de liberdade do modelo de evolução deve ser escolhido ponderando entre a simplicidade do modelo, que facilita a etapa de estimação, e a sofisticação necessária do modelo, que melhora a etapa de predição dos filtros de Kalman. A questão é determinar o número ideal de compartimentos em uma certa situação, lembrando que quando mais compartimentos, mais parâmetros devem ser estimados.

Com um modelo bastante simplificado, composto por apenas um compartimento, é possível descrever bem um par de pulmões em condições fisiológicas iguais ou muito parecidas. Entretanto não seria possível modelar anormalidades localizadas como diferenças nas capacidades pulmonares, presença de pneumotórax ou diferenças de fase entre os pulmões.

Já em um modelo com compartimentos direito/esquerdo, diferenças entre os pulmões são modeladas mas ainda assim não é possível modelar variações por efeitos gravitacionais, como a tendência de colabamento da parte posterior dos pulmões em pacientes deitados.

Em Moura et al. [38], os autores identificam uma matriz de transição para a dinâmica pulmonar, modelada como um sistema de segunda ordem não forçado. Neste modelo, com 469 graus de liberdade, mostrou-se que apenas um pequeno número de modos seria suficiente para ser utilizado nos filtro de Kalman. A Figura 9.7 mostra os modos principais da matriz de transição identificada em Moura et al. [38]. 

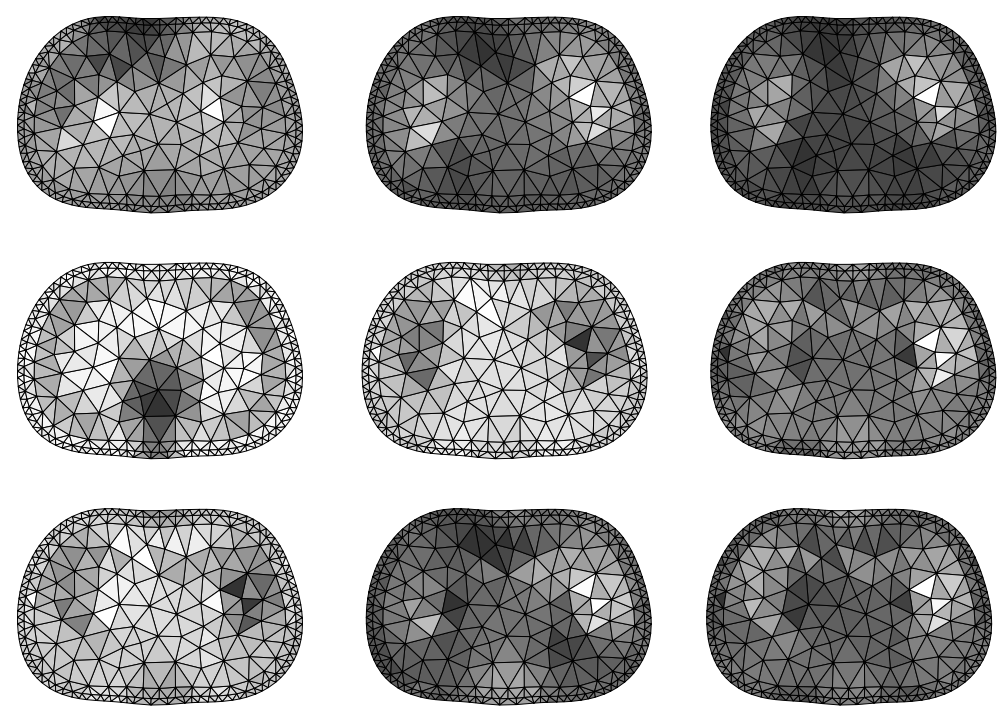

Figura 9.7: Nove modos principais identificados em Moura et al. [38].

No decorrer deste trabalho serão considerados modelos com um ou quatro compartimentos, conforme indicado em cada ensaio. Salienta-se entretanto que o método proposto nesta tese permite que o número de compartimentos seja aumentado conforme a necessidade. 


\section{Capítulo 10}

\section{Segmentação das imagens}

Segmentação é definida como a partição de um conjunto de dados em grupos cujos elementos apresentam mesmas características. A segmentação é uma forma de organizar e detectar uma estrutura em dados crus e por esse motivo é uma etapa importante no tratamento e estudo de imagens. Ela é caracterizada pela identificação e delineação de estruturas de interesse em uma imagem, seja esta 2D, 3D ou 4D (variante no tempo) que serão analisadas posteriormente.

Inúmeras técnicas de segmentação de imagens já foram desenvolvidas, dentre elas destacam-se as técnicas de segmentação por limiarização, em que a segmentação é realizada considerando um nível limite de luminosidade da imagem, as técnicas de crescimento de regiões, em que a segmentação é feita pelo agrupamento de pixels vizinhos com mesmas propriedades a partir de um pixel selecionado, as técnicas de conectividade (difusa ou não), em que a segmentação é realizada observando as similaridades entre pixels próximos e conexos, as técnicas de reconhecimento de padrões, em que a segmentação é baseada na teoria probabilística de tomada de decisões e as técnicas de contornos ativos, em que a segmentação é feita pelo ajuste de curvas/superfícies ao redor da área de interesse. Vasta literatura pode ser encontrada versando estas e outras técnicas, destaca-se [124, 125, 126, 127, 128, 129, 130, 131, 132]. Neste trabalho utiliza-se a técnica de segmentação por contornos ativos.

Dois principais algoritmos de segmentação por contornos ativos são snakes e level set. No método snake, considera-se o contorno, que define a região, uma entidade dinâmica sob ação de forças externas e internas e que deve procurar o/um ponto de equilíbrio estático. As forças internas geralmente são restrições em relação ao 
comprimento da curva ou que limitam a curvatura local da curva. Já as forças externas são geradas a partir da imagem que se quer segmentar, como por exemplo definir uma energia potencial baseada no inverso do gradiente de intensidade luminosa da imagem e então determinar o campo de forças conservativas que agem sobre a curva.

No método level set, não considera-se uma curva que separa as regiões, mas sim uma função cuja curva de nível separa as regiões. O método level set apresenta vantagens em relação ao snake: no snake é necessário parametrizar o contorno de alguma forma, já no level set não há esta necessidade pois o contorno de interesse é obtido pela curva de nível de uma função. Nos snakes, mudanças topológicas não são simples de serem implementadas em razão da parametrização da curva, como por exemplo deformar um contorno fechado por invaginação até que este seja quebrado em dois contornos fechados não conexos ou juntar duas regiões inicialmente não conexas. Já no level set, esta mudança ocorre naturalmente sem nenhuma necessidade de codificar de casos particulares.

Apesar das vantagens, o método level set tradicional sofre com o problema da reinicialização, que requer grande carga computacional. Para contornar este problema, em Li et al. [133] apresenta-se um método baseado em minimização de uma função custo que visa eliminar a reinicialização do método. Este é o método implementado neste trabalho.

A descrição completa do método level set e o desenvolvimento das equações necessárias apresentados no Apêndice E,

A imagem a ser segmentada deve ser escolhida e depende da aplicação e objetivo da segmentação. Caso se queira segmentar, por exemplo, a região cardíaca e regiões onde ocorre a perfusão sanguínea, pode-se compor uma imagem contendo apenas os coeficientes de Fourier na frequência cardíaca (e seus harmônicos). Nesta tese, o interesse é segmentar a região pulmonar.

O uso apenas dos valores de resistividade elétrica como uma imagem a ser segmentada não é adequado neste caso pois, apesar de separar os pulmões de regiões musculares, apresenta problemas para distinguir tecido ósseo do tecido pulmonar já que ambos valores podem ser similares dependendo da quantidade de ar dentro dos pulmões. Por este motivo, uma segmentação baseada apenas nestes valores tende a agrupar em uma região tanto pulmões quanto ossos e tecidos mais resistivos. 
É interessante então adicionar informação a respeito do comportamento temporal ao longo do ciclo ventilatório. A opção adotada consiste em compor uma imagem a partir da imagem de resistividade, normalizada entre 0 e 1 , e dos valores do coeficiente de correlação entre os elementos e um elemento semente, que é assumido pertencer ao pulmão.

Sejam $s$ o índice do elemento semente e $\Gamma_{n}$ a matriz de covariância do histórico dos valores de resistividade estimados até o instante da segmentaçãol. Os coeficientes de correlação $r_{i, s}$ entre os elementos da malha e o elemento de raiz são dados por

$$
r_{i, s}=\frac{\left[\boldsymbol{\Gamma}_{n}\right]_{(i, s)}}{\sigma_{i} \sigma_{s}},
$$

onde $\sigma_{i}$ é o desvio padrão do $i$-ésimo elemento e $\sigma_{s}$ é o desvio padrão do elemento raiz. Sabe-se que o coeficiente de correlação é um valor compreendido no intervalo $[-1,+1]$, sendo que se $r=0$, os elementos não apresentam correlação linear estatística, se o coeficiente se aproxima de -1 , as variáveis apresentam correlação linear negativa e se o coeficiente se aproxima de +1 , as variáveis apresentam correlação linear positiva.

Considera-se neste trabalho que elementos são pertencentes a mesma região do elemento semente se o módulo do coeficiente de correlação é próximo de um. O uso do módulo do coeficiente foi feito pois em pacientes submetidos a transplante de um pulmão, por exemplo, observa-se que o pulmão não transplantado apresenta comportamento em oposição de fase dentro do ciclo ventilatório em relação ao pulmão transplantado. O efeito indesejável desta escolha é que também incluirá na região pulmonar uma região em que ocorre pneumotórax. Apesar disto, o filtro de Kalman deve ser capaz de identificar que em tal região a resistividade é muito mais elevada.

Finalmente, a imagem usada para segmentação é composta por uma média entre a imagem de resistividade normalizada e a imagem composta pelo módulo do coeficiente de correlação.

Um ensaio numérico foi feito para avaliar o método de segmentação por level set em uma imagem de teste. Três ensaios foram realizados: Segmentar uma imagem sem adição de ruído, uma imagem com adição de ruído do tipo salt $\mathcal{E}$ pepper, comum em

\footnotetext{
${ }^{1}$ As estimativas da média e da matriz de covariância das imagens estimadas podem ser eficientemente calculadas com as fórmulas recursivas $\overline{\boldsymbol{x}}_{n}=\frac{\overline{\boldsymbol{x}}_{n-1}(n-1)+\boldsymbol{x}_{n}}{n}$ e $\boldsymbol{\Gamma}_{n}=$ $\frac{(n-2) \boldsymbol{\Gamma}_{n-1}+\left(\boldsymbol{x}_{n}-\overline{\boldsymbol{x}}_{n-1}\right)\left(\boldsymbol{x}_{n}-\overline{\boldsymbol{x}}_{n}\right)}{n-1}$.
} 
imagens digitais [134, p. 225], e uma imagem com adição de ruído salt E pepper com borramento gaussiano, que reproduz o tipo de artefato presente em imagens de TIE.

A imagem a ser segmentada apresenta 126x78 pixels e pode se vista na Figura 10.1 . Os tons de cinza foram normalizados entre 0 e 100 em todos os ensaios.

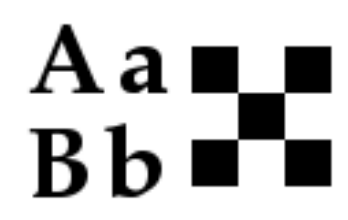

Figura 10.1: Imagem a ser segmentada.

Para os ensaios com ruído salt $\mathcal{E}$ pepper, uma imagem contendo apenas ruído foi criada com uma densidade de 0.01 , ou seja, um porcento dos pixels da imagem são modificados com o ruído. Esta imagem é então normalizada entre 0 e 100 e é somada na imagem original. Para o ensaio com ruído com borramento, convolucionou-se um núcleo gaussiano simétrico de tamanho $30 \times 30$ pixels e desvio padrão de 4 pixels sobre a imagem de ruído antes de somar na imagem original. Por último, a imagem é novamente normalizada entre 0 e 100.

Os parâmetros de (E.34) escolhidos empiricamente para os ensaios foram: $\lambda=1$ para o ensaio sem adição de ruído e com ruído salt $\mathcal{E}$ pepper e $\lambda=0.6$ para o ensaio com borramento. Os demais parâmetros utilizados nesta equação foram os mesmos para todos os ensaios: $\Delta t=5, \mu=0.04$ e $\nu=0.8$.

A diferença no valor do parâmetro $\lambda$ é explicada observando a função indicativa de contorno (E.31) em cada caso: O filtro Gaussiano utilizado para compor a função indicativa de contorno utiliza um núcleo simétrico de tamanho $5 \times 5$ e desvio padrão $\sigma=1.5$ pixel. A Figura 10.2 mostra esta função nos três casos ensaiados.

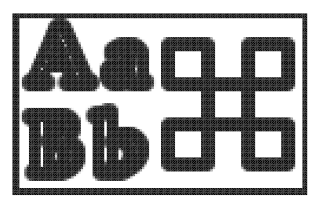

(a) Sem ruído

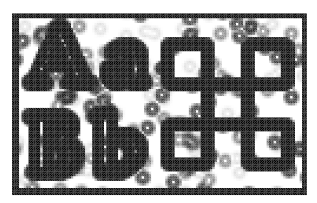

(b) salt E pepper

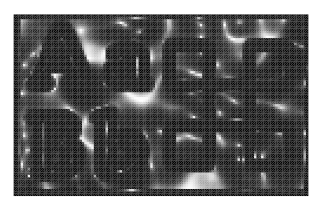

(c) salt E pepper com borramento

Figura 10.2: Funções indicativas de contorno dos ensaios. 
Nota-se por estas imagens que a função indicativa de contorno no caso de ruído com borramento é menos eficiente do que os demais ensaios para destacar o contorno de interesse. Isto acarreta em uma convergência mais lenta e um aumento da possibilidade da função custo (E.28) atingir um mínimo local não desejável. Aumentando-se ligeiramente o valor de $\lambda$ (equivalentemente pode-se aumentar $\nu$ ), regulariza-se mais a solução. Este é o motivo do aumento do valor do parâmetro $\lambda$ em relação aos demais casos.

A sugestão inicial da função distância com sinal $\phi$ segue o proposto em (E.41), com $c=4$. A curva de nível $\phi=0$ nesta sugestão inicial pode ser visualizada na Figura 10.3 , A mesma sugestão inicial foi utilizada em todos os ensaios.

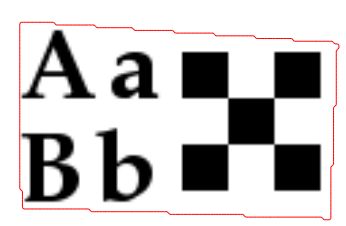

(a) Sem ruído

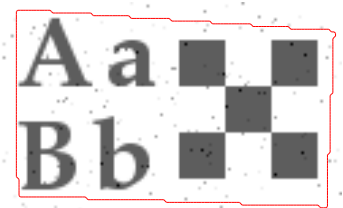

(b) salt \& pepper

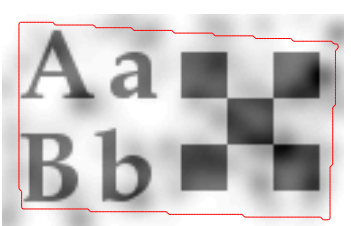

(c) salt E pepper com borramento

Figura 10.3: Sugestão inicial da função distância com sinal $\phi$ nos três ensaios.

Salienta-se que a curva de nível está envolvendo totalmente as regiões a serem segmentadas. Por conta disto, foi adotado $\nu>0$. Caso contrário, a região $\Omega^{-}$tenderia a se expandir.

Como critérios de parada, utilizou-se $\epsilon_{1}=3.0 \cdot 10^{-5}$ e $\epsilon_{2}=1.0 \cdot 10^{-5}$ em (E.35) e (E.37) respectivamente. O delta de Dirac suavizado (E.14) utiliza $\epsilon=3$ pixels.

A Figura 10.4 mostra a segmentação utilizando o algoritmo nos três casos. A primeira linha mostra a convergência para o caso sem adição de ruído, a segunda linha mostra a convergência para o caso de ruído salt $\mathcal{E}$ pepper e a terceira linha mostra a convergência para o caso de ruído salt E pepper com borramento. Em todas os casos, a coluna da direita mostra o resultado final após os critérios de parada serem satisfeitos. Os gráficos com o critério de parada $C_{1}$ e $C_{2}$ estão apresentados na Figura 10.5 .

O primeiro caso apresenta convergência mais rápida que nos demais casos. O último caso apresenta convergência mais lenta, como era esperado devido à característica da sua função indicativa de contorno. 


\section{$\underset{B b}{A a g}$ Aagd Aagd Aagd}

(a) Iteração 0

(b) Iteração 100

(c) Iteração 200

(d) Iteração 286

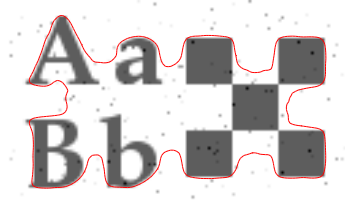

(e) Iteração 100

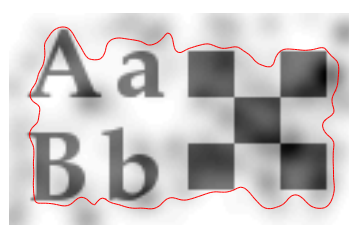

(i) Iteração 100

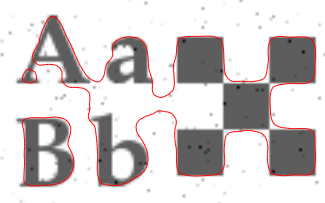

(f) Iteração 200

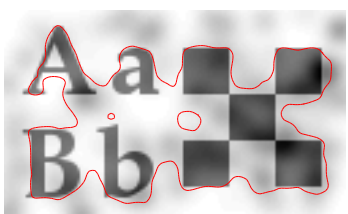

(j) Iteração 300

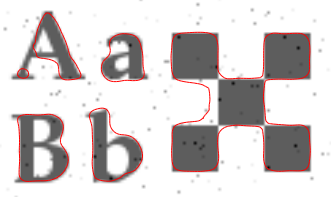

(g) Iteração 300

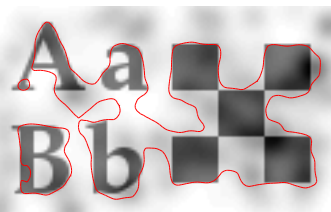

(k) Iteração 400

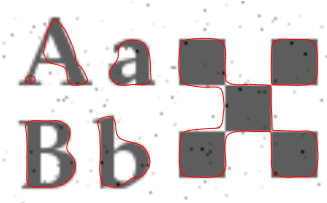

(h) Iteração 375

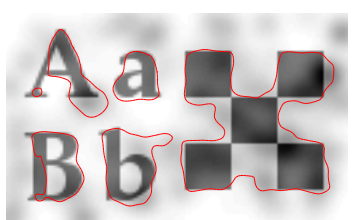

(1) Iteração 477

Figura 10.4: Resultados da segmentação de imagem. Linha 1: sem adição de ruído; Linha 2: com adição de ruído salt E pepper; Linha 3: com adição de ruído salt E pepper e borramento gaussiano.

Nota-se também a importante característica de que a curva de nível $\phi=0$ inicialmente define uma região simplesmente conexa e vai sendo contraída até que se divide em diversas regiões simplesmente conexas por estrangulamento. Esta característica é de difícil implementação numérica nos métodos de representação explícita, como no método por snakes. Tal comportamento explica também o motivo da inabilidade do algoritmo em detectar os contornos internos das letras. Para sanar tal problema seria necessário propor como sugestão inicial uma função $\phi$ que tenha uma curva de nível $\phi=0$ dentro de cada letra.

Uma boa aproximação do delineamento do bordo dos objetos foi alcançada em todos os ensaios, porém o ensaio com borramento apresentou algumas imperfeições visíveis em alguns trechos devido a presença de um ruído intenso no local.

A segmentação falhou localmente em todos os casos nas regiões com altos níveis de recortes, como na região das serifas das letras e cantos vivos do quadriculado onde a curvatura é elevada. Isto era de se esperar uma vez que foi escolhido o parâmetro $\lambda$ diferente de zero. Todavia, as limitações apresentadas não são problemáticas para a aplicação no método proposto pois a resolução espacial atual do equipamento não permite imagens com detalhes elevados e uma curvatura elevada não é esperada. 


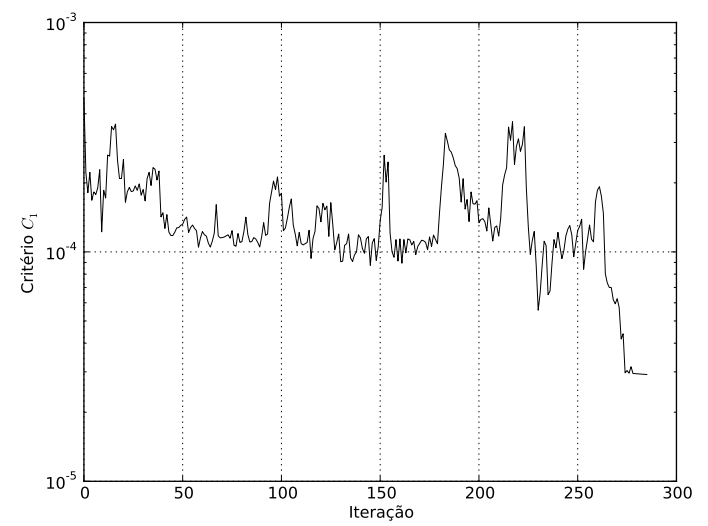

(a) Ensaio sem ruído.

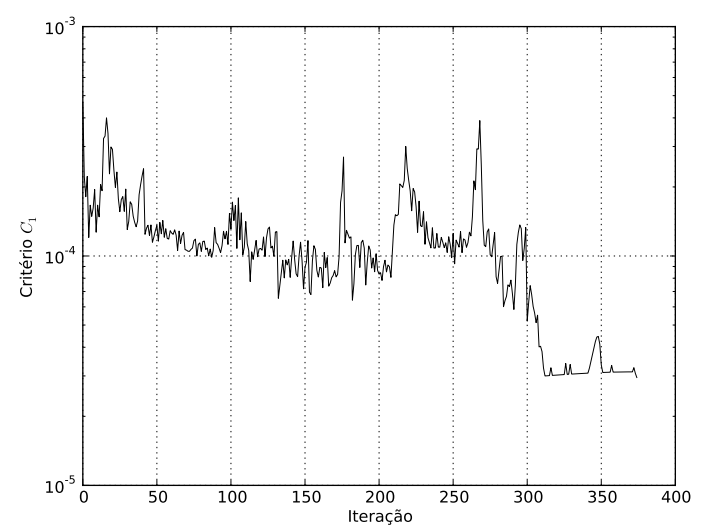

(c) Ensaio salt E pepper.

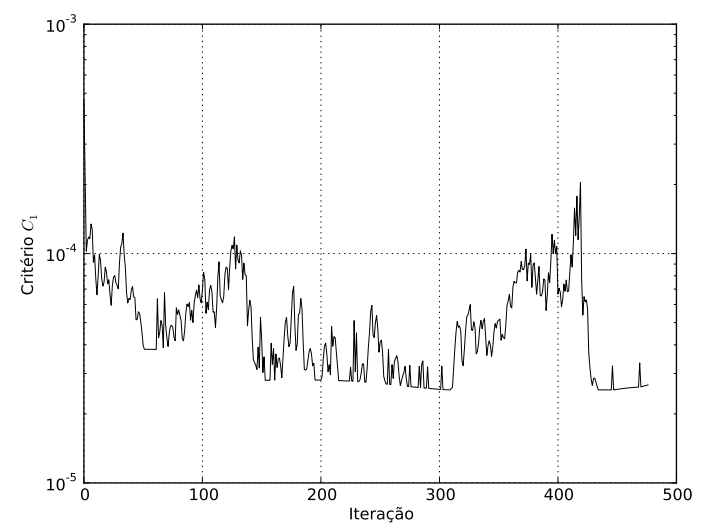

(e) Ensaio salt E pepper com borramento.

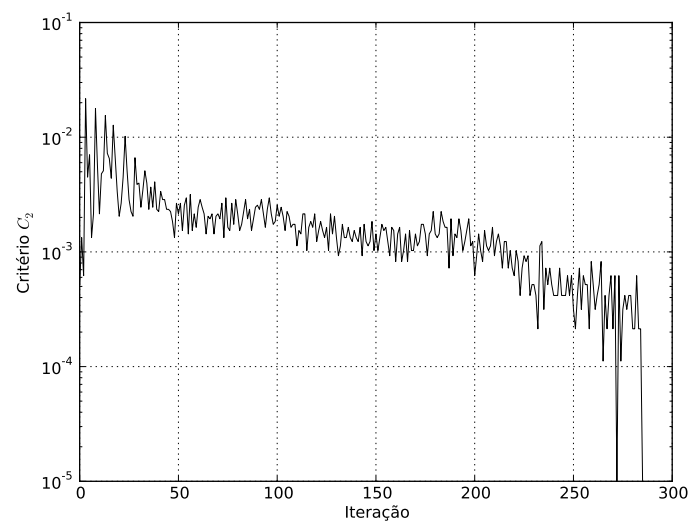

(b) Ensaio sem ruído.

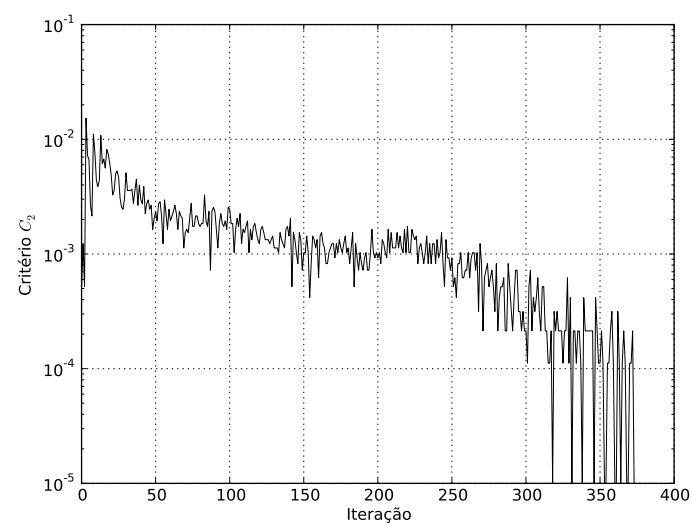

(d) Ensaio salt E pepper.

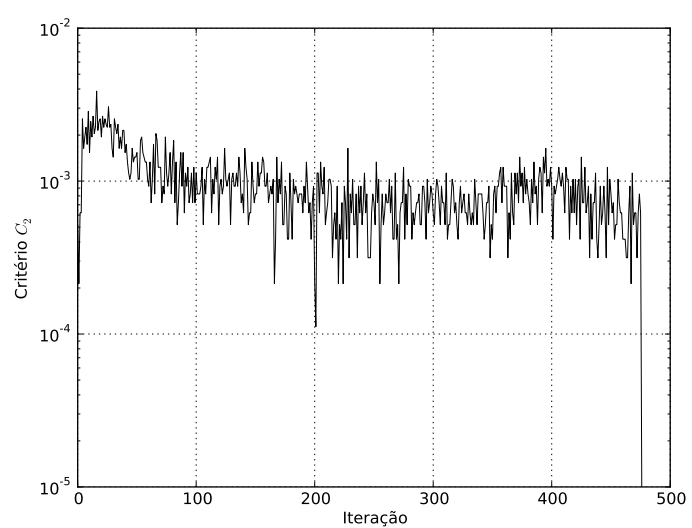

(f) Ensaio salt E pepper com borramento.

Figura 10.5: Gráficos dos critérios de parada dos ensaios realizados. 


\section{Capítulo 11}

\section{Ensaios numéricos para avaliação do método com modelo de evolução}

Este capítulo apresenta a avaliação do método proposto nesta tese com um fantoma de um tórax humano sob ventilação artificial. Os ensaios realizados avaliam o algoritmo para a estimação da distribuição de resistividade do fantoma numérico de um tórax humano em paralelo à estimação dos parâmetros dos eletrodos e dos parâmetros do modelo de evolução proposto. Os ensaios avaliam ainda a segmentação da imagem utilizando o método de level set e o uso do método do erro de aproximação para o modelo de observação.

\subsection{Fantoma numérico de um tórax humano}

O fantoma numérico do tórax consiste em emular a distribuição de resistividades dentro do tórax de um paciente conectado a um ventilador artificial.

O fantoma numérico é resolvido utilizando uma malha composta por 2556 elementos triangulares que retrata um corte transversal do tórax, que pode ser vista na Figura 11.1(a), O problema inverso é resolvido com uma malha composta por 1050 elementos triangulares, e que pode ser vista na Figura 11.1(b), Ambas as malhas possuem 32 eletrodos, dispostos ao longo de seu bordo e seguem o modelo proposto em Hua et al. [52], consistindo de quatro elementos por eletrodo. A malha é vista nestas figuras no sentido caudal-cranial e a região superior da malha na figura representa a 
região anterior do tórax 1 . Nesta simulação não são considerados erros na posição dos eletrodos e erros de formato do bordo do domínio.

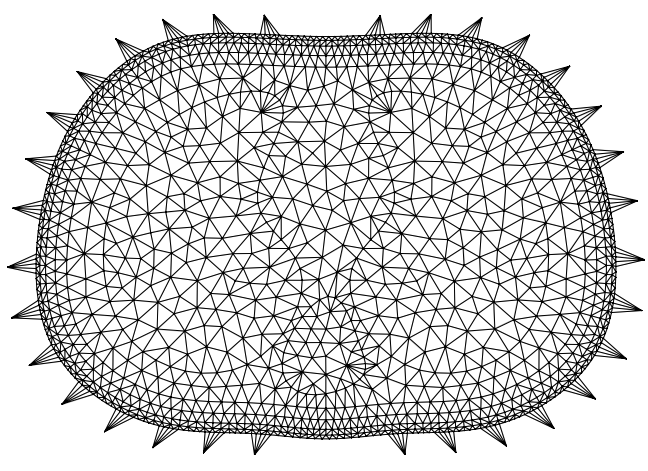

(a) Malha usada no fantoma numérico. 2556 elementos.

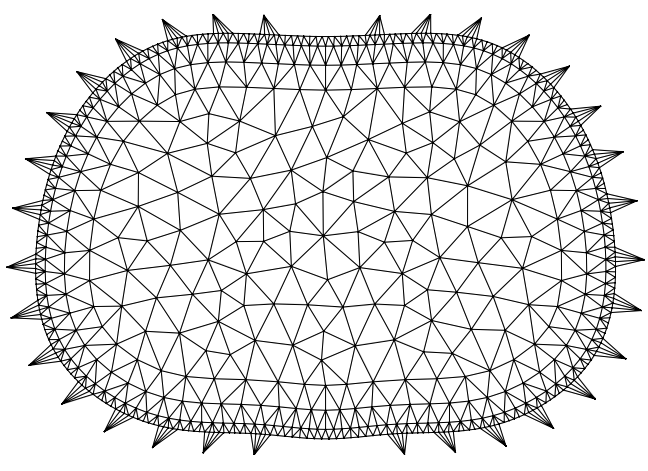

(b) Malha usada no problema inverso. 1050 elementos.

Figura 11.1: Malhas utilizadas nas simulações.

O fantoma numérico foi resolvido emulando um ciclo ventilatório com período $T=5 \mathrm{~s}$. A simulação considera que $\boldsymbol{y} \in \mathbb{R}^{\ell}, \ell=1024$ é coletado a uma taxa de $100 \mathrm{~Hz}\left(T_{s}=0.01 \mathrm{~s}\right)$. Na geração dos dados de potencial elétrico, o valor escolhido para o parâmetro de todos dos eletrodos é $\rho^{\prime}=0.02 \Omega \mathrm{m}^{2}$, pois retrata os valores típicos medidos experimentalmente em equipamentos de TIE.

O valor da amplitude da corrente atravessando o par de eletrodos de injeção é $I=4 \mathrm{~mA}$ zero-pico para todos os padrões de corrente.

A distribuição de resistividade da malha usada no fantoma numérico é particionada em três regiões. Esta divisão pode ser vista na Figura 11.2(a), As regiões geradas representam a região pulmonar, coluna vertebral e demais tecidos. Em cada uma destas regiões, um valor de resistividade diferente é utilizado:

pulmões (cinza claro): Apresenta resistividade variante no tempo, regido pela equação dinâmica (9.11).

Para gerar os valores de resistividade variante no tempo, é necessário um perfil de pressão $P(t)$. É de se esperar que um pulso de pressão proveniente do ventilador mecânico leve algum tempo para chegar aos pulmões devido à impedância mecânica imposta pelas vias aéreas e músculos da caixa torácica. Diversos modelos lineares e

\footnotetext{
${ }^{1}$ Por conta desta orientação, o lado direito do paciente aparece do lado esquerdo da imagem, e vice-versa.
} 
não lineares são apresentados em Bates [117]. Para as simulações, um comportamento linear puramente resistivo é assumido.

Modelando as vias aéreas como um sistema puramente resistivo e de primeira ordem, chega-se à seguinte resposta a um pulso quadrado do ventilador mecânico (um ciclo ventilatório), amostrado no tempo discreto $t_{k}=k T_{s}$.

$$
P\left(t_{k}\right)=\left\{\begin{array}{ll}
\left(P_{r p}-P_{0}\right)\left[1-\exp \left(-\frac{t_{k}}{\tau_{1}}\right)\right]+P_{0} \quad, \quad t_{k} \leq T_{f} \\
\left(P\left(T_{f}\right)-P_{0}\right) \exp \left(-\frac{t_{k}-T_{f}}{\tau_{2}}\right)+P_{0} \quad, \quad t_{k}>T_{f}
\end{array},\right.
$$

onde o período entre $\left[0, T_{f}\right]$ compreende o intervalo de tempo em que o ventilador está impondo uma pressão $P_{r p}$ e $\left.] T_{f}, T\right]$ é o período em que a pressão imposta vale $P_{0}$. Ainda, $\tau_{1}$ é a constante de tempo entre 0 e $T_{f}$ e $\tau_{2}$ é a constante de tempo entre $T_{f}$ e $T$. O perfil de pressão resultante pode ser visto na Figura 11.2(b).

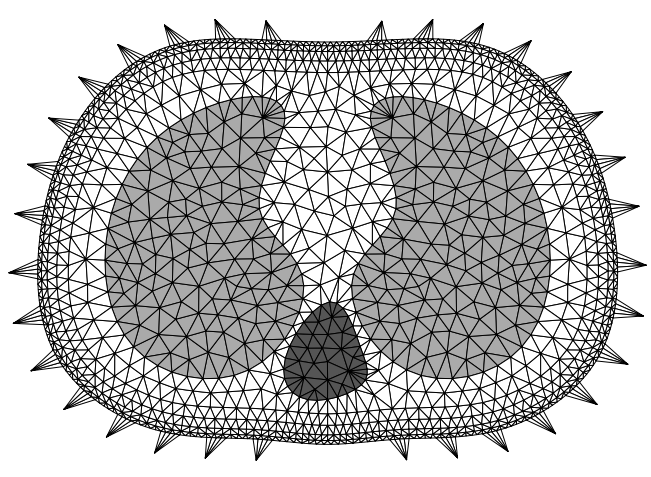

(a) Regiões do domínio na malha do problema direto.

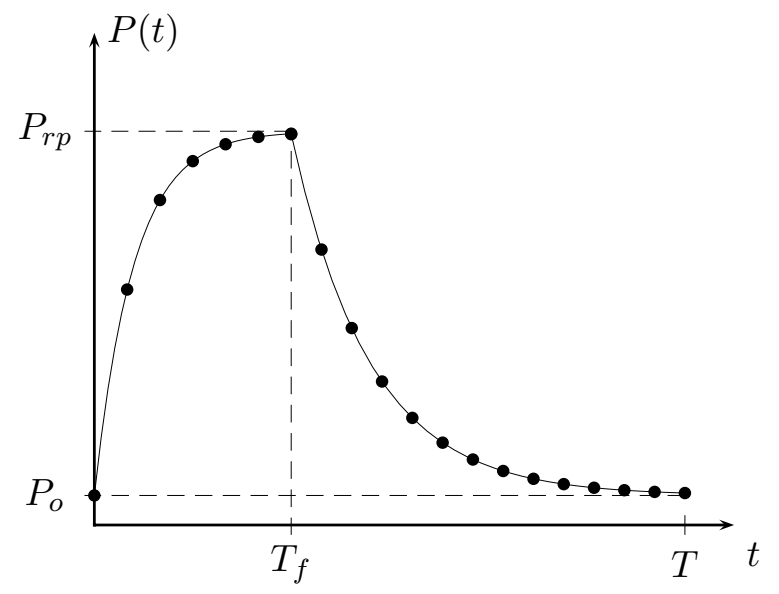

(b) Gráfico da pressão em um ciclo ventilatório.

Figura 11.2: Malha utilizadas nas simulação e gráfico da pressão $P\left(t_{k}\right)$.

Para a modelagem da pressão resultante nas vias aéreas, foram adotados $T_{f}=1.0 \mathrm{~s}$, $\tau_{1}=0.1 \mathrm{~s}, \tau_{2}=0.2 \mathrm{~s}, P_{r p}=35 \mathrm{~cm} \mathrm{H}_{2} \mathrm{O}$ e $P_{o}=20 \mathrm{~cm} H_{2} O$. Usando a curva de pressão resultante (11.1) na integral (9.11), obtém-se os valores do volume pulmonar ao longo do tempo para o fantoma.

Dois fantomas foram criados, o primeiro usa um modelo com um compartimento para os pulmões e o segundo adota um modelo com dois compartimentos (pulmão direito e esquerdo). O primeiro fantoma, mais simplificado, é utilizado para avaliar cada uma das etapas de estimação e segmentação. O segundo fantoma é utilizado para 
avaliar a capacidade do algoritmo em identificar diferenças entre compartimentos.

Para o fantoma com um compartimento, foram escolhidos os valores $a=12 \mathrm{~cm}^{3}$, $b=1200 \mathrm{~cm}^{3}, c=0.005 \mathrm{~cm} \mathrm{H}_{2} O . \mathrm{s} / \mathrm{cm}^{3}, d=5 \mathrm{~cm} \mathrm{H}_{2} \mathrm{O}$ e $e=15 \mathrm{~cm}_{2} \mathrm{O}$. Para o fantoma com dois compartimentos, foram escolhidos os mesmo valores para o pulmão esquerdo e $a=150 \mathrm{~cm}^{3}, b=800 \mathrm{~cm}^{3}, c=0.03 \mathrm{cmH}_{2} \mathrm{O} . \mathrm{s} / \mathrm{cm}^{3}, d=5 \mathrm{~cm} H_{2} \mathrm{O}$ e $e=15$ $\mathrm{cm} \mathrm{H}_{2} \mathrm{O}$ para o pulmão direito. Com esta escolha, o pulmão direito deve inflar menos que o pulmão esquerdo.

A conversão entre volume e resistividade foi feita usando uma interpolação linear

$$
\rho_{k}=\rho_{A}+\frac{\rho_{B}-\rho_{A}}{V_{B}-V_{A}}\left(V_{k}-V_{A}\right)
$$

$\operatorname{com} \rho_{A}=3.63 \Omega \mathrm{m}, \rho_{B}=9.17 \Omega \mathrm{m}$. Os valores dos volumes $V_{A}$ e $V_{B}$ são o máximo e mínimo obtidos em (11.1) em cada fantoma. Os valores de resistividade coincidem com os valores da Tabela 1.1 para pulmão desinflado e inflado respectivamente. Desta forma, os valores de resistividade obtidos na simulação emulam o enchimento e esvaziamento do pulmão.

Finalmente, os valores de resistividade médios calculados com o modelo foram utilizados nos respectivos compartimentos.

coluna vertebral (cinza escuro): A resistividade escolhida para a região da coluna vertebral é de de $40 \Omega \mathrm{m}$, constante no tempo. Tal escolha é um valor médio entre os valores dos diversos tecidos ósseos constantes na Tabela 1.1

demais regiões (branco): A resistividade escolhida para resistividade dos demais tecidos é de $4 \Omega \mathrm{m}$, constante no tempo discreto. Tal escolha é também um valor médio dos demais órgãos e tecidos na caixa torácica. Um ponto a ser observado é que o tecido adiposo apresenta valores de resistividade substancialmente maiores do que os demais. Apesar desta observação, nas simulações o tecido adiposo não foi considerado. 


\subsection{Descrição dos ensaios}

No total, seis ensaios foram realizados. Nestes ensaios, erros de parametrização de $\partial \Omega$ e posição dos eletrodos não estão presentes. Os dois primeiros ensaios avaliam apenas o uso do modelo de evolução dado por (9.11) (Ensaio II) em comparação ao passeio aleatório (Ensaio I). Nos ensaios III e IV, as estimações dos parâmetros dos eletrodos e parâmetros do modelo de evolução são adicionados e nos ensaios V e VI, as três estimações são realizadas em paralelo. Os cinco primeiro ensaios utilizam dados do fantoma com um compartimento e o ensaio VI utiliza dados do fantoma com dois compartimentos. A Tabela 11.1 apresenta uma comparação dos ensaios realizados.

Tabela 11.1: Ensaios realizados para avaliar o algoritmo proposto.

\begin{tabular}{lcccccc}
\hline Ensaio & I & II & III & IV & V & VI \\
\hline SR-UKF resistividade do domínio & $\checkmark$ & $\checkmark$ & $\checkmark$ & $\checkmark$ & $\checkmark$ & $\checkmark$ \\
SR-UKF parâmetros dos eletrodos & - & - & $\checkmark$ & - & $\checkmark$ & $\checkmark$ \\
SR-UKF parâmetros do modelo dinâmico & - & - & - & $\checkmark$ & $\checkmark$ & $\checkmark$ \\
modelo de evolução do estado (9.11) & - & $\checkmark$ & $\checkmark$ & $\checkmark$ & $\checkmark$ & $\checkmark$ \\
número de compartimentos & 1 & 1 & 1 & 1 & 1 & 2 \\
\hline
\end{tabular}

Nos ensaios I, II e IV, as estatísticas do método do erro de aproximação consideram apenas a diferença de discretização das malhas utilizadas no fantoma e no SR-UKF. Nos ensaios III, V e VI, erros dos parâmetros dos eletrodos são também considerados. A parametrização do contorno para cada realização das malhas $M_{i}$ e $M^{a}$ e a posição dos eletrodos são as mesmas da Figura 11.1. A Tabela 11.2 apresenta as estatísticas utilizadas para amostragem do ruído $\varepsilon_{i}$ do método do erro de aproximação. A amostragem segue as mesmas diretrizes apresentadas na Seção 8.2 também com $N=5000$ amostras de (8.1).

Em todos os ensaios, os parâmetros para a segmentação de imagem² foram os mesmos: $\Delta t=5, \mu=0.04, \lambda=15, \nu=1.0, \epsilon=3, \sigma=1.5$, critérios de parada $\epsilon_{1}=0.02$ e $\epsilon_{2}=0.01$ e número máximo de iterações 500. A distribuição de resistividade é interpolada em um grid de pixels de dimensões 50x50. A segmentação é realizada uma vez por ciclo ventilatório, a cada pico de inspiração.

\footnotetext{
${ }^{2}$ Para mais detalhes a respeito da implementação deste algoritmo, ver Apêndice E.2.4
} 
Tabela 11.2: Parâmetros usados no processo de amostragem do ruído $\varepsilon$.

\begin{tabular}{|c|c|c|c|c|c|}
\hline Ensaio & I II & III & IV & $\mathrm{V}$ & VI \\
\hline$\sigma_{\vartheta}(\%)$ & $-\quad-$ & - & - & - & - \\
\hline$p^{-}, p^{+}(\mathrm{mm})$ & $-\quad-$ & - & - & - & - \\
\hline param. eletrodos $\rho^{\prime}\left(\Omega \mathrm{m}^{2}\right)$ & $-\quad-$ & $\sim \mathcal{U}(0.015,0.025)$ & - & $\sim 2$ & $5,0.025)$ \\
\hline refinamento & \multicolumn{5}{|c|}{$\delta^{-}=0.9, \quad \delta^{+}=1.1$} \\
\hline ruído $\mathbf{e}^{x}$ & \multicolumn{5}{|c|}{$\sim \mathcal{N}\left(\mathbf{0},\left(1.0 \cdot 10^{-3}\right)^{2} \mathbb{I}\right)$} \\
\hline malha do fantoma num. & \multicolumn{5}{|c|}{ ver Figura 11.1(a) } \\
\hline$\rho^{\prime a}\left(\Omega \mathrm{m}^{2}\right)$ & \multicolumn{5}{|c|}{0.02} \\
\hline \multirow[t]{2}{*}{ resistividade $\boldsymbol{\rho}(\Omega \mathrm{m})$} & \multicolumn{5}{|c|}{$\sim \mathcal{N}^{L^{-}}\left(\mathbf{x}_{u} \mathbf{1}, 3.0^{2} \mathbb{I}, \mathbf{0}\right)$} \\
\hline & \multicolumn{5}{|c|}{$\mathrm{x}_{u} \sim \mathcal{U}(3.0,5.0)$} \\
\hline
\end{tabular}

A seguir apresenta-se os detalhes e parâmetros utilizados em cada ensaio.

Ensaio I: Os parâmetros utilizados para a estimação da distribuição de resistividade são apresentados na Tabela 11.3. As estimativas são realizadas a cada 5 conjuntos de medidas, iniciando em $k=5$.

Ensaio II: Neste ensaio foram utilizados os mesmos conjuntos de parâmetros do ensaio I, exceto os indicados na Tabela 11.4, referentes à mudança do modelo de evolução da região pulmonar. As estimativas são realizadas a cada 5 conjuntos de medidas, iniciando em $k=5$.

Ensaio III: Tanto a estimação da distribuição de resistividade quanto os parâmetros dos eletrodos são realizadas neste ensaio. O SR-UKF para a estimação da distribuição de resistividade utiliza o mesmo conjunto de parâmetros do ensaio II. Já o SR-UKF para estimação dos parâmetros dos eletrodos utiliza os parâmetros apresentados na Tabela 11.5. As estimativas da distribuição de resistividade são realizadas a cada 5 conjuntos de medidas, iniciando em $k=5$. As estimativas dos parâmetros dos eletrodos são realizadas a cada 2 conjuntos de medidas, iniciando em $k=20$ até $k=500$, quando foi assumida convergência. 
Tabela 11.3: Parâmetros usados no SR-UKF no ensaio I.

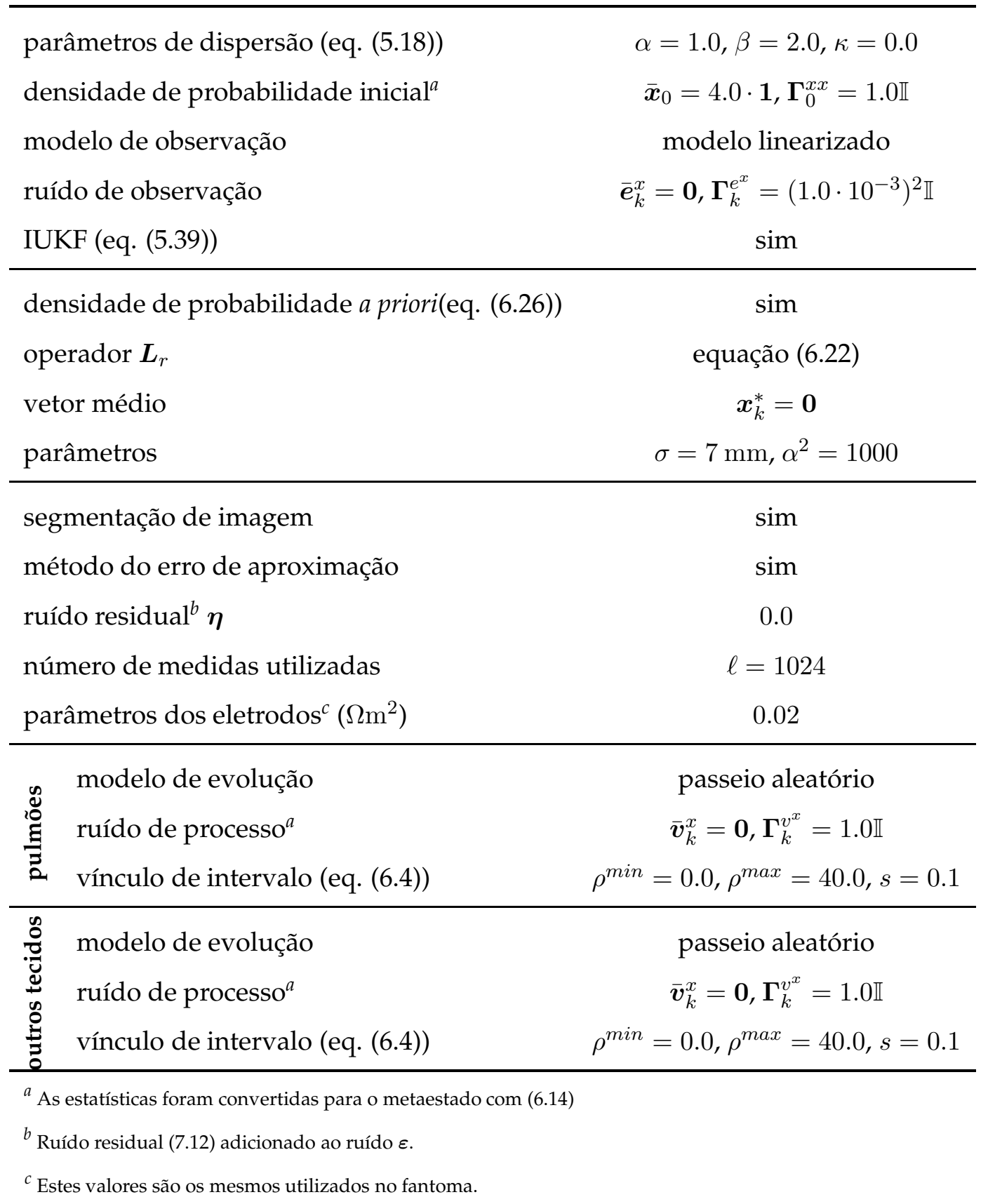

O número de medidas utilizadas para a estimação dos eletrodos é $\ell=32 * 3=96$ pois contém apenas as três medidas por padrão de injeção que envolvem o par de eletrodos de injeção. As demais medidas são ignoradas pois são pouco informativas para a estimação uma vez que a densidade de corrente nestes é muito baixa. 
Tabela 11.4: Parâmetros usados no SR-UKF no ensaio II.

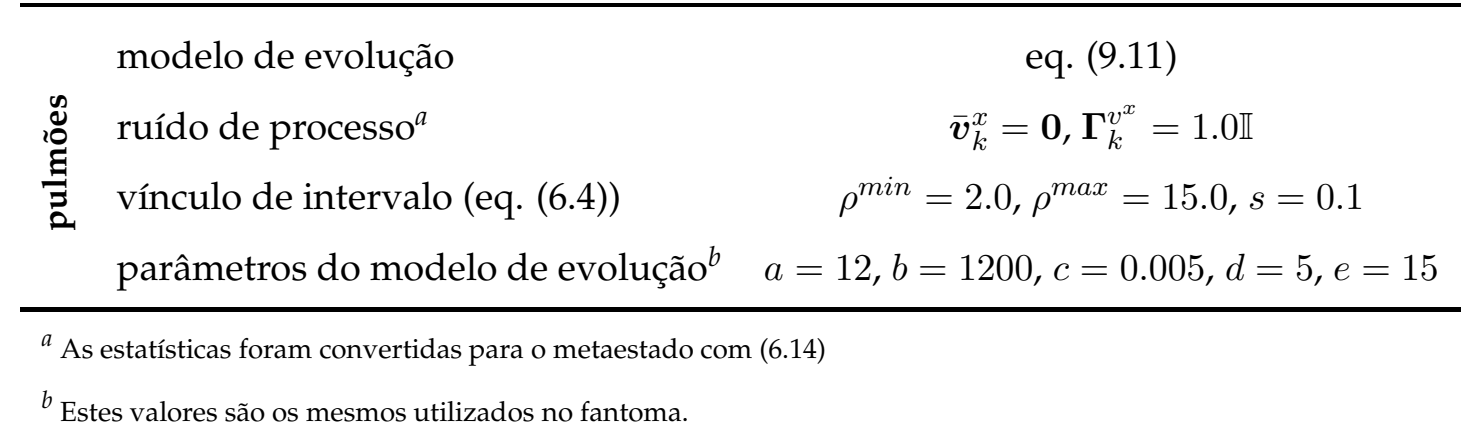

Tabela 11.5: Parâmetros usados no SR-UKF no ensaio III para estimação dos parâmetros dos eletrodos.

\begin{tabular}{|c|c|}
\hline parâmetros de dispersão (eq. (5.18) $)$ & $\alpha=0.01, \beta=2.0, \kappa=0.0$ \\
\hline 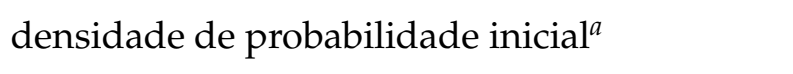 & $\overline{\boldsymbol{x}}_{0}=0.01 \cdot \mathbf{1}, \boldsymbol{\Gamma}_{0}^{x x}=1.0 \cdot 10^{-5} \mathbb{I}$ \\
\hline modelo de observação & modelo não linear \\
\hline ruído de observação & $\overline{\boldsymbol{e}}_{k}^{x}=\mathbf{0}, \boldsymbol{\Gamma}_{k}^{e^{x}}=\left(1.0 \cdot 10^{-3}\right)^{2} \mathbb{I}$ \\
\hline IUKF (eq. (5.39) ) & $\operatorname{sim}$ \\
\hline densidade de probabilidade $a$ priori(eq. (6.26) & $\operatorname{sim}$ \\
\hline operador $\boldsymbol{L}_{r}$ & identidade \\
\hline vetor médio & $\boldsymbol{x}_{k}^{*}=0.02 \cdot \mathbf{1}$ \\
\hline parâmetro & $\alpha^{2}=1000$ \\
\hline método do erro de aproximação & $\operatorname{sim}$ \\
\hline ruído residual ${ }^{b} \boldsymbol{\eta}$ & 0.0 \\
\hline número de medidas utilizadas & $\ell=96$ \\
\hline modelo de evolução & passeio aleatório \\
\hline ruído de processo ${ }^{a}$ & $\overline{\boldsymbol{v}}_{k}^{x}=\mathbf{0}, \boldsymbol{\Gamma}_{k}^{v^{x}}=1.0 \cdot 10^{-5} \mathbb{I}$ \\
\hline vínculo de intervalo (eq. (6.4)) & $\rho^{\min }=0.005, \rho^{\max }=0.035, s=0.1$ \\
\hline
\end{tabular}

Ensaio IV: Neste ensaio, a estimação dos parâmetros do modelo de evolução é realizada em paralelo à estimação da distribuição de resistividade. O SR-UKF para a estimação da distribuição de resistividade utiliza o mesmo conjunto de parâmetros do ensaio II. Já o SR-UKF para estimação dos parâmetros do modelo de evolução 
utiliza os parâmetros apresentados na Tabela 11.6. As estimativas da distribuição de resistividade são realizadas a cada 5 conjuntos de medidas, iniciando em $k=5$. As estimativas dos parâmetros do modelo de evolução são realizadas a cada 10 conjuntos de medidas, iniciando em $k=50$. Neste ensaio, apenas os parâmetros $a$ e $b$ de (9.11) são considerados na estimação $\boldsymbol{p}=\left[\begin{array}{ll}a & b\end{array}\right]^{\mathrm{T}}$, ou seja, os parâmetros $c$ e $d$ são assumidos conhecidos.

Tabela 11.6: Parâmetros usados no SR-UKF no ensaio IV para estimação dos parâmetros do modelo de evolução.

\begin{tabular}{|c|c|}
\hline parâmetros de dispersão (ver. Apêndice C.2) & $\alpha=0.1, \beta=2.0, \kappa=1.0, \eta=1.0$ \\
\hline densidade de probabilidade inicial $^{a}$ & $\overline{\boldsymbol{p}}_{0}=\left[\begin{array}{ll}20 & 1000\end{array}\right]^{\mathrm{T}}, \boldsymbol{\Gamma}_{0}^{p p}=\operatorname{diag}(10,100)$ \\
\hline modelo de observação & modelo não linear \\
\hline ruído de observação & $\overline{\boldsymbol{e}}_{k}^{p}=\mathbf{0}, \boldsymbol{\Gamma}_{k}^{e^{p}}=\left(1.0 \cdot 10^{-3}\right)^{2} \mathbb{I}$ \\
\hline IUKF (eq. (5.39)) & $\operatorname{sim}$ \\
\hline densidade de probabilidade $a$ priori $($ eq. $(\sqrt{6.26}))$ & $\operatorname{sim}$ \\
\hline operador $\boldsymbol{L}_{r}$ & identidade \\
\hline vetor médio & $\boldsymbol{p}_{k}^{*}=\left[\begin{array}{ll}12 & 1200\end{array}\right]^{\mathrm{T}}$ \\
\hline parâmetro & $\alpha^{2}=1$ \\
\hline método do erro de aproximação & $\operatorname{sim}$ \\
\hline ruído residual ${ }^{b} \boldsymbol{\eta}$ & 0.0 \\
\hline número de medidas utilizadas & $\ell=928$ \\
\hline modelo de evolução & passeio aleatório \\
\hline ruído de processo $^{a}$ & $\overline{\boldsymbol{v}}_{k}^{p}=\mathbf{0}, \boldsymbol{\Gamma}_{k}^{v^{p}}=1.0 \cdot 10^{-3} \mathbb{I}$ \\
\hline \multicolumn{2}{|l|}{ vínculo de intervalo (eq. (6.4)) } \\
\hline parâmetro $a$ & $a^{\min }=5, a^{\max }=30, s_{a}=1$ \\
\hline parâmetro $b$ & $b^{\min }=900, b^{\max }=1500, s_{b}=20$ \\
\hline
\end{tabular}

O número de medidas utilizadas para a estimação dos parâmetros do modelo é $\ell=1024-32 * 3=928$ pois não considera as medidas envolvendo os pares de injeção. A remoção destas medidas visa reduzir a influência dos parâmetros dos eletrodos na estimação dos parâmetros do modelo de evolução. 
Ensaio V: A estimação dos parâmetros do modelo de evolução é realizada em paralelo à estimação da distribuição de resistividade e dos parâmetros dos eletrodos. O SR-UKF para a estimação da distribuição de resistividade utiliza o mesmo conjunto de parâmetros do ensaio II. O SR-UKF para estimação dos parâmetros dos eletrodos são os mesmos do ensaio III e o SR-UKF para estimação dos parâmetros do modelo de evolução utiliza o mesmo conjunto de parâmetros do ensaio IV.

Ensaio VI: O último ensaio realiza as mesmas estimações do ensaio V, exceto que neste ensaio o modelo não é mais monocompartimental. Os parâmetros utilizados são os mesmo que foram utilizados no ensaio $\mathrm{V}$, exceto os parâmetros que são apresentados na Tabela 11.7, referentes aos diferentes compartimentos do pulmão. Neste ensaio, o fantoma de dois compartimentos é utilizado e o filtro de Kalman utiliza um modelo de quatro compartimentos. A divisão em quatro compartimentos no SR-UKF foi feita separando os pulmões em direito/esquerdo e cada um deles em anterior/posterior.

Tabela 11.7: Parâmetros usados no SR-UKF no ensaio VI.

\begin{tabular}{|c|c|c|}
\hline 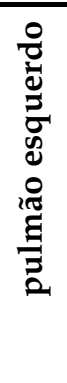 & $\begin{array}{l}\text { vetor médio (dens. a priori) } \\
\text { vínculo de intervalo (eq. (6.4)) }\end{array}$ & $\begin{array}{l}\overline{\boldsymbol{p}}_{0}=\left[\begin{array}{llll}20 & 1000 & 20 & 1000\end{array}\right]^{\mathrm{T}} \\
\boldsymbol{\Gamma}_{0}^{p p}=\operatorname{diag}(10,100,10,100) \\
\boldsymbol{p}_{k}^{*}=\left[\begin{array}{llll}12 & 1200 & 12 & 1200\end{array}\right]^{\mathrm{T}} \\
a^{\text {min }}=5, a^{\text {max }}=30, s_{a}=1 \\
b^{\text {min }}=900, b^{\text {max }}=1500, s_{b}=20\end{array}$ \\
\hline 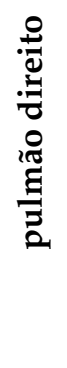 & $\begin{array}{l}\text { vetor médio (dens. a priori) } \\
\text { vínculo de intervalo (eq. (6.4)) }\end{array}$ & $\begin{array}{l}\overline{\boldsymbol{p}}_{0}=\left[\begin{array}{llll}135 & 900 & 135 & 900\end{array}\right]^{\mathrm{T}} \\
\boldsymbol{\Gamma}_{0}^{p p}=\operatorname{diag}(10,100,10,100) \\
\boldsymbol{p}_{k}^{*}=\left[\begin{array}{llll}150 & 800 & 150 & 800\end{array}\right]^{\mathrm{T}} \\
a^{\text {min }}=130, a^{\text {max }}=170, s_{a}=1 \\
b^{\text {min }}=500, b^{\text {max }}=1100, s_{b}=20\end{array}$ \\
\hline
\end{tabular}

${ }^{a}$ As estatísticas foram convertidas para o metaestado com 6.14 


\subsection{Resultados dos ensaios}

A Figura 11.3 mostra uma comparação entre as estimativas da distribuição de resistividade na malha de elementos finitos em cada um dos seis ensaios nos mesmos instantes de tempo. A primeira coluna apresenta as estimativas no início da inspiração, a segunda no início da expiração e a terceira durante a expiração. A primeira linha de imagens refere-se ao ensaio I, a segunda refere-se ao ensaio II e assim por diante. Nestas imagens, as regiões pulmonares e da coluna vertebral presentes no fantoma numérico estão destacadas com linha branca.

Observa-se que as estimativas da distribuição de resistividade no ensaio I são mais concentradas na região central da malha e nos ensaios I a V a distribuição é mais uniforme. Esta uniformidade é explicada pelo modelo adotado, monocompartimental. Tal modelo, entretanto, não impede que as estimativas apresentem sub-regiões com resistividade diferentes pois conta com a presença do ruído $\mathbf{v}_{k}^{x}$ em (5.1). Outro ponto importante a ser notado é a variação suave de resistividade dentro da região pulmonar e na região dos demais tecidos, mas uma variação abrupta no bordo das regiões. Isto é causado pela imposição da informação a prioride suavidade gaussiana (6.22) em cada região, mas não entre regiões. 

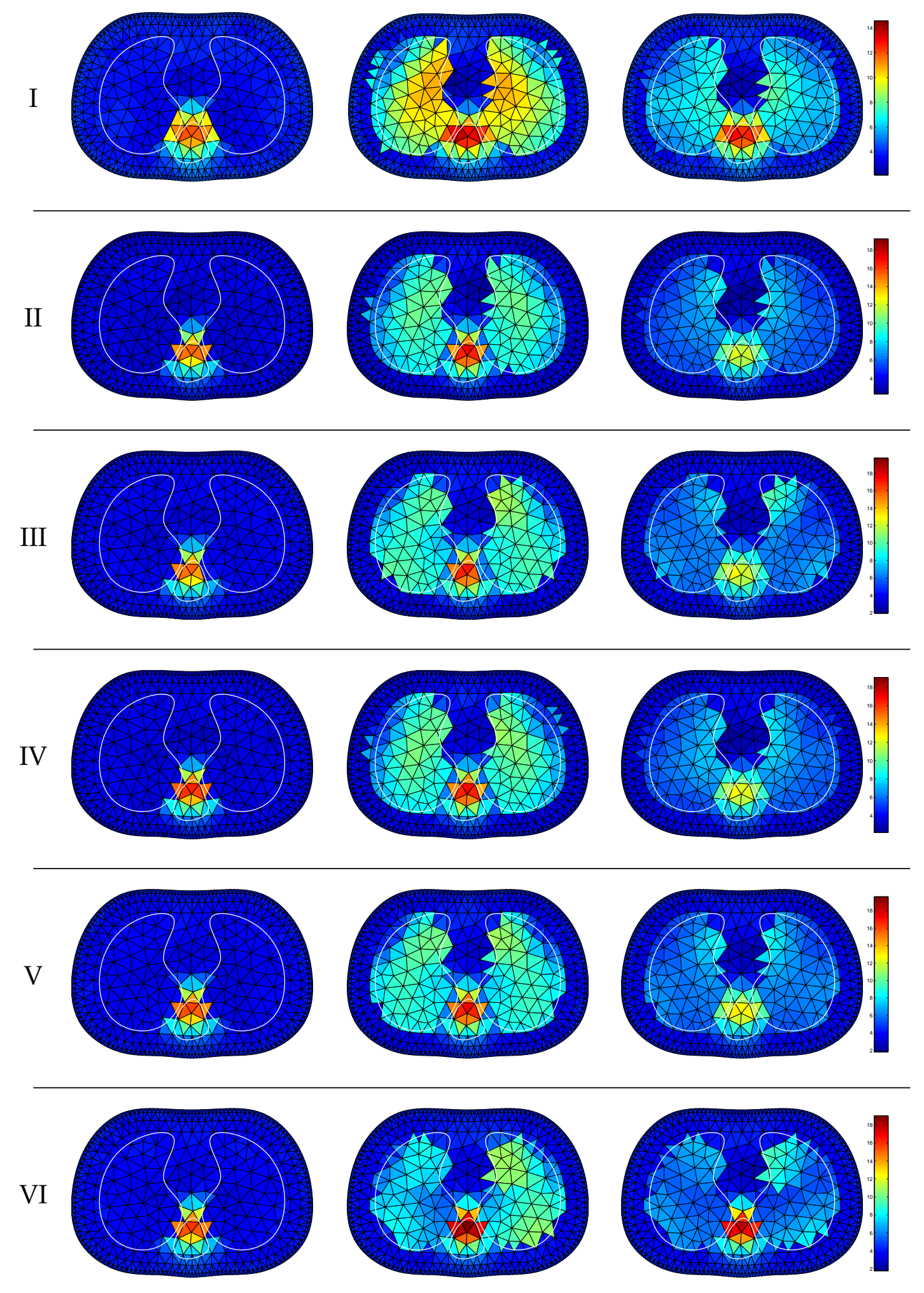

Figura 11.3: Comparação dos resultados dos Ensaios em um mesmo ciclo ventilatório. Valores em $\Omega \mathrm{m}$. 
A distribuição de resistividade no ensaio VI apresenta diferenças de valores entre os pulmões direito e esquerdo, sendo que os maiores valores estão no pulmão esquerdo (lado direito da imagem). A uniformidade agora dentro de um mesmo pulmão não é mais tão evidente quanto nos demais ensaios por conta do uso de quatro compartimentos cujos parâmetros estão sendo ajustados.

A distribuição de resistividade média na região pulmonar está próxima dos valores verdadeiros em todos os ensaios. A Figura 11.4 mostra o valor da resistividade média da região pulmonar no ensaios I a $\mathrm{V}$ ao longo do tempo discreto.

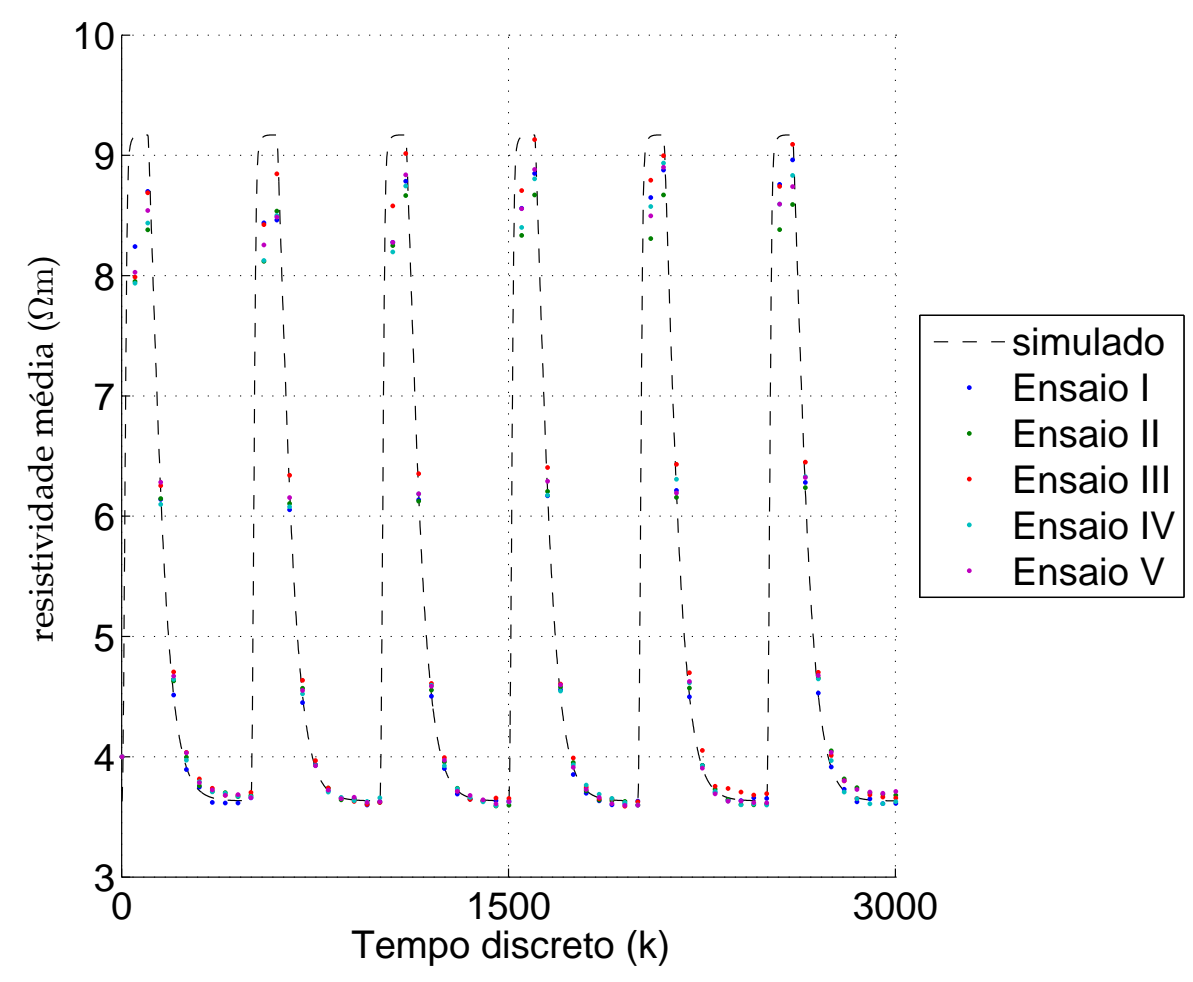

Figura 11.4: Resistividade média da região pulmonar nos ensaios I a V. A linha tracejada representa a resistividade utilizada no fantoma numérico.

A Figura 11.5 apresenta os valores das resistividades médias em cada um dos quatro compartimentos utilizados no SR-UKF do ensaio VI. Os compartimentos C1 e C4 pertencem ao pulmão esquerdo (compartimentos anterior e posterior respectivamente) e C2 e C3 pertencem ao pulmão direito (compartimentos anterior e posterior respectivamente). Pode-se notar que todos os compartimentos apresentam bom seguimento dos valores utilizados no fantoma numérico. 


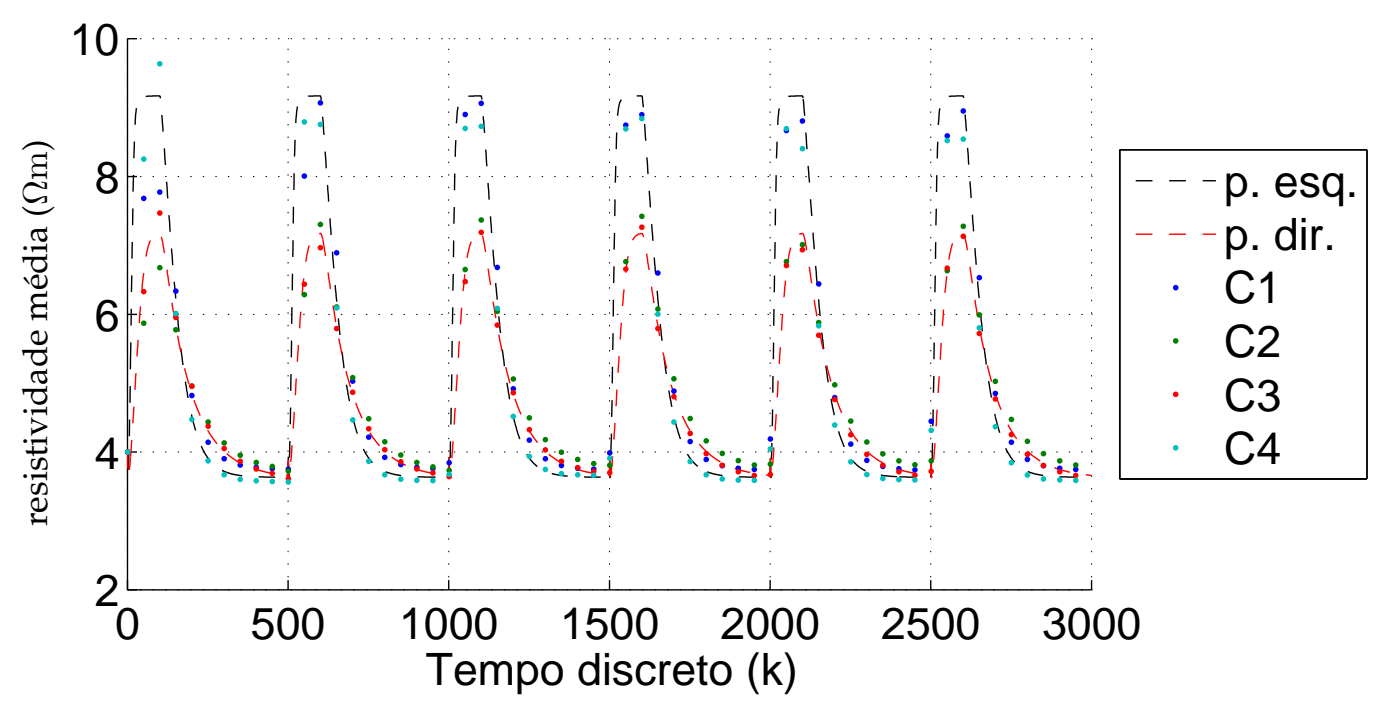

Figura 11.5: Resistividade média da região pulmonar no ensaio VI. As linhas tracejadas representam as resistividades utilizadas no fantoma numérico em cada um dos pulmões.

Todas as estimativas apresentam boa aproximação nos trechos de expiração, porém subestimam o valor da resistividade nos picos de inspiração. Este comportamento ocorre em parte por conta dos filtros não serem rápidos suficientes para acompanhar a mudança abrupta de resistividade no período de inspiração e em parte pela linearização do modelo de observação.

Estes resultados mostram que os ensaios com estimação dual (ensaio III a VI) apresentam resultados semelhantes ao ensaio II, em que apenas o estado é estimado e os demais parâmetros são corretamente informados ao filtro. Isto indica que a estimação dual foi bem sucedida, apesar das aproximações e hipóteses necessárias, apresentadas na Seção 5.2 .

Quanto à segmentação das regiões pulmonares, a Figura 11.6 mostra uma comparação das segmentações nos ensaios realizados. Nestas imagens, as regiões pulmonares e da coluna vertebral presentes no fantoma numérico estão destacadas com linha branca. 

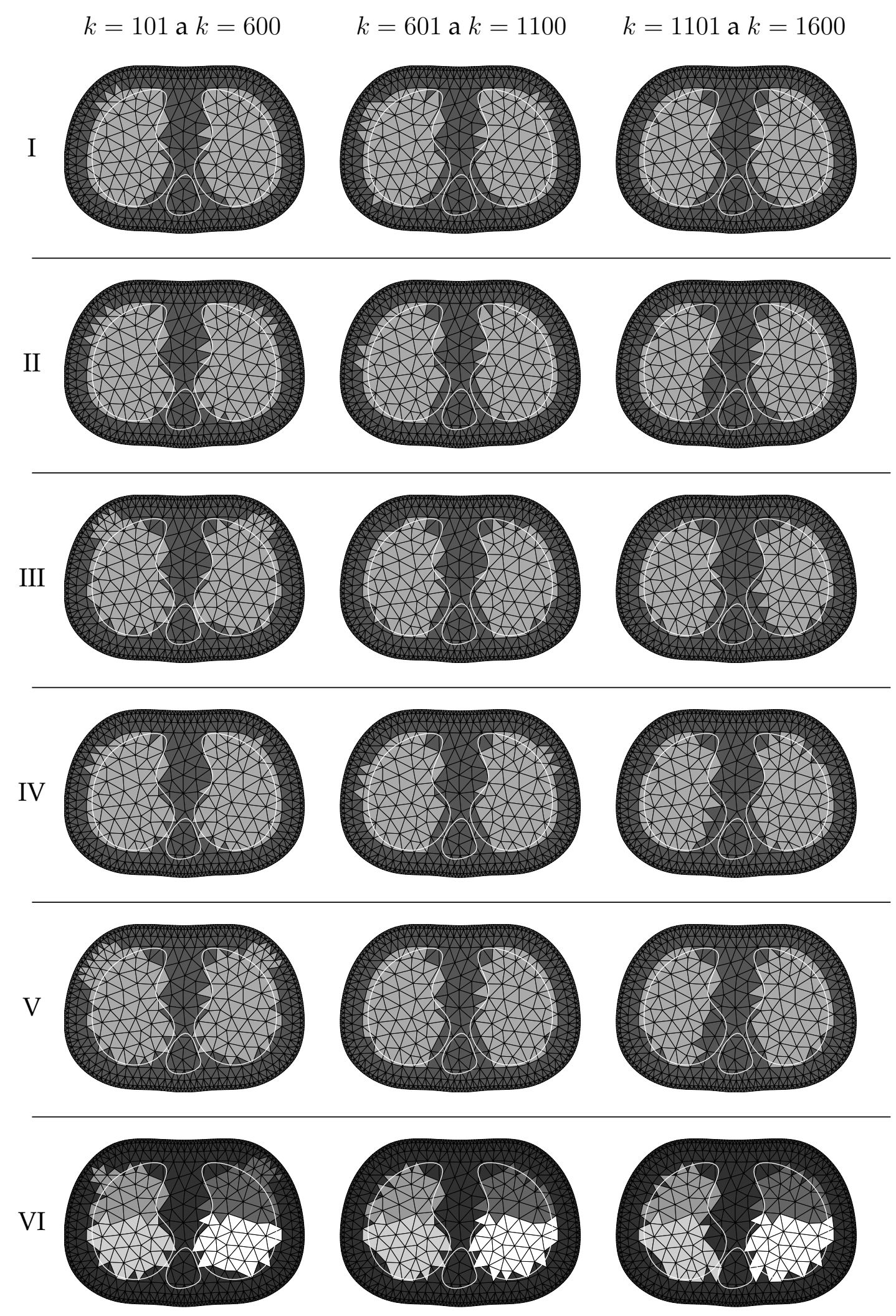

Figura 11.6: Comparação da segmentação da região pulmonar nos ensaios. Nestas imagens, diferentes tons de cinza indicam diferentes regiões. 
Todas as estimações apresentaram boa concordância entre a região pulmonar segmentada e a região pulmonar presente no fantoma numérico. Em todos os casos, houve resolução espacial suficiente para separar as duas regiões. Como apresentado no Capítulo 10, a imagem a ser segmentada é uma média entre os valores de resistividade nos elementos e a correlação entre as resistividades dos elementos para evitar que a região óssea fosse incorretamente agrupada junto os pulmões. Os resultados apresentados mostram que esta escolha permitiu que a coluna vertebral fosse corretamente deixada de fora destas regiões. Ainda, a qualidade da segmentação das imagens aumenta com o passar do tempo discreto, uma vez que estimativas mais corretas do estado e parâmetros ajudam a definir mais corretamente o contorno dos tecidos.

As figuras $11.7 \mathrm{a} 11.18$ mostram os históricos das estimações em cada um dos seis ensaios. Nestas figuras são apresentados os índices $J, J_{d}, J_{m}, C_{m}$ e $C_{v}$ descritos na Seção 8.4. Em alguns gráficos de $C_{m}$ e $C_{v}$, são traçadas linhas tracejadas para ressaltar o limite estipulado para o critério de convergência. Na estimação dos parâmetros do modelo de evolução nos ensaio IV, V e VI, os valores dos parâmetros foram normalizados com relação ao valor verdadeiro utilizado no fantoma.

Pelos gráficos apresentados, todas as estimações atingiram a convergência estatística, exceto as estimações dos parâmetros do modelo de evolução. É possível notar que a estimações destes parâmetros são influenciadas pelo ciclo ventilatório e que a estimação do parâmetro $b$ se aproxima do valor verdadeiro 1, mas $a$ não tem o mesmo comportamento. Isto pode ser explicado pela diferença de ordens de magnitudes destes parâmetros: os valores verdadeiros de $b$ são $1200 \mathrm{~cm}^{3}$ (pulmão esquerdo) e $800 \mathrm{~cm}^{3}$ (pulmão direito) enquanto que os valores verdadeiros de $a$ são $12 \mathrm{~cm}^{3}$ (pulmão esquerdo) e $150 \mathrm{~cm}^{3}$ (pulmão direito). Já os intervalos de busca são de $600 \mathrm{~cm}^{3}$ para $b$ e $25 \mathrm{~cm}^{3}$ (ou $40 \mathrm{~cm}^{3}$ no pulmão direito) para $a$. Como os dois parâmetros têm mesma natureza (volumes), variações normalizadas em $b$ são mais importantes que variações normalizadas em $a$. 


\section{Ensaio I:}
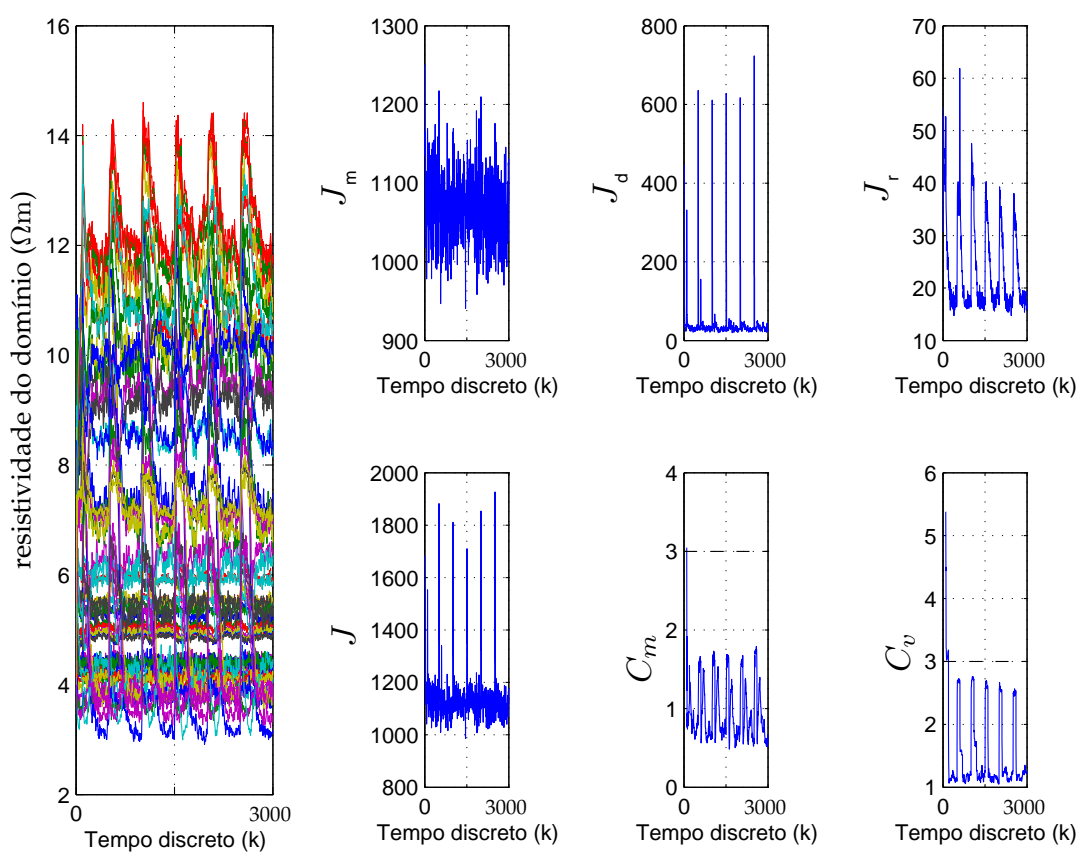

Figura 11.7: Ensaio I, SR-UKF para estimação da distribuição de resistividade.

\section{Ensaio II:}
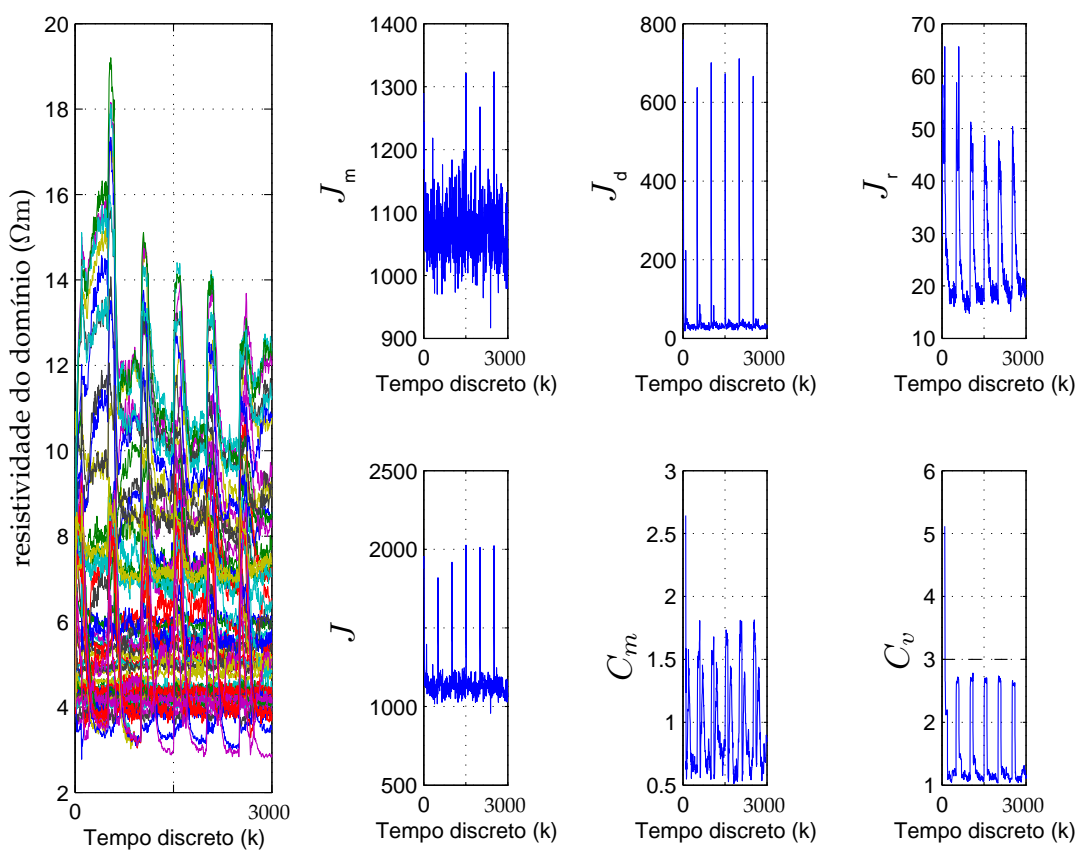

Figura 11.8: Ensaio II, SR-UKF para estimação da distribuição de resistividade. 


\section{Ensaio III:}
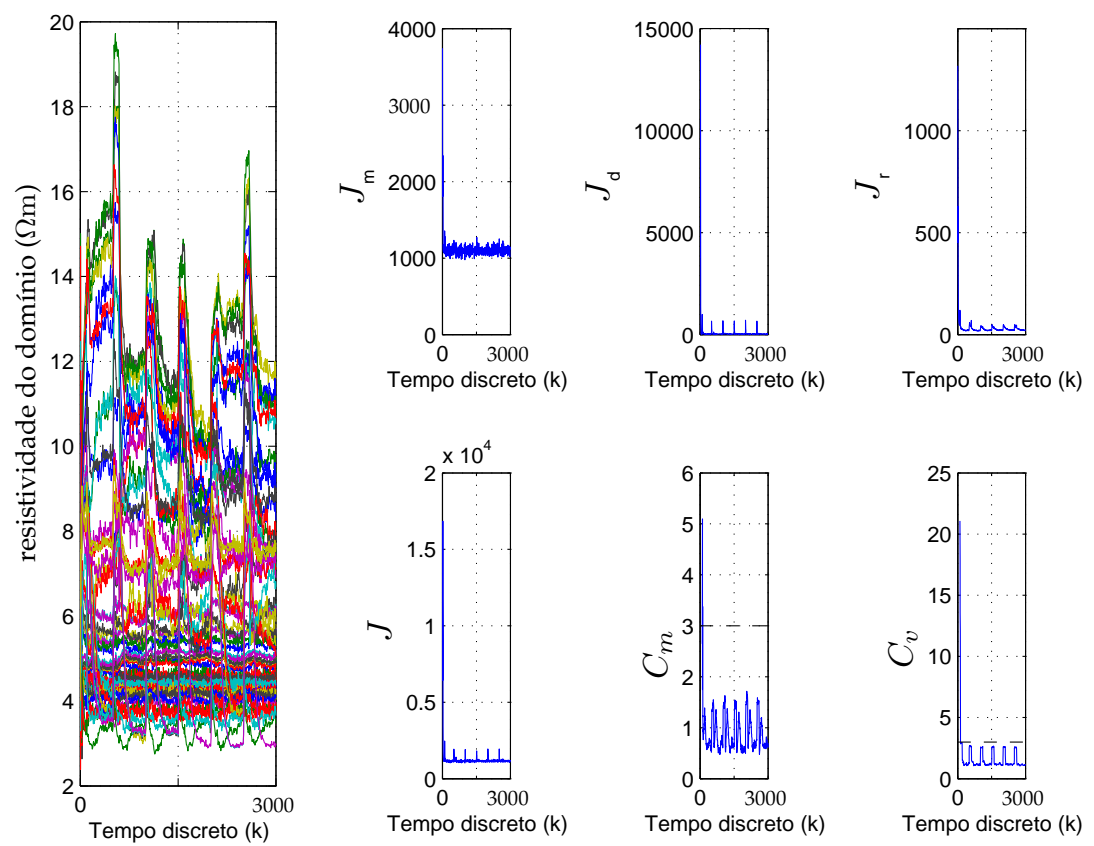

Figura 11.9: Ensaio III, SR-UKF para estimação da distribuição de resistividade.
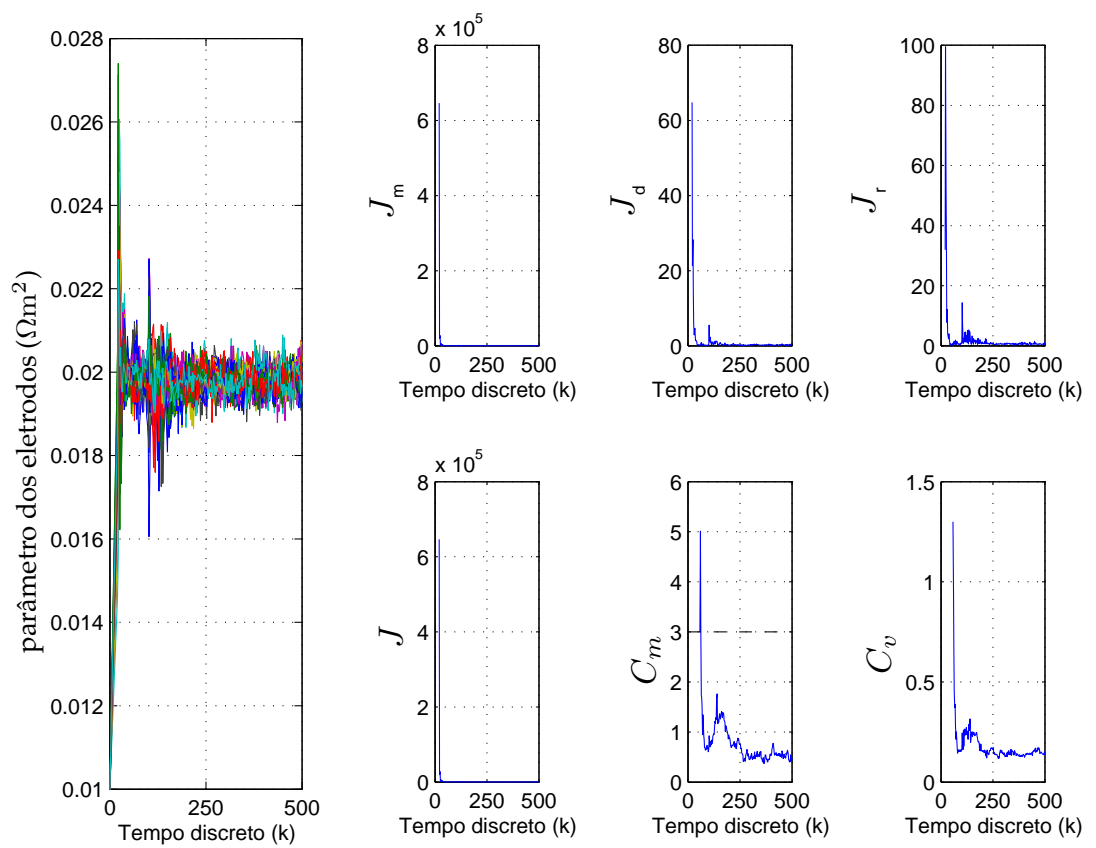

Figura 11.10: Ensaio III, SR-UKF para estimação dos parâmetros dos eletrodos. 


\section{Ensaio IV:}
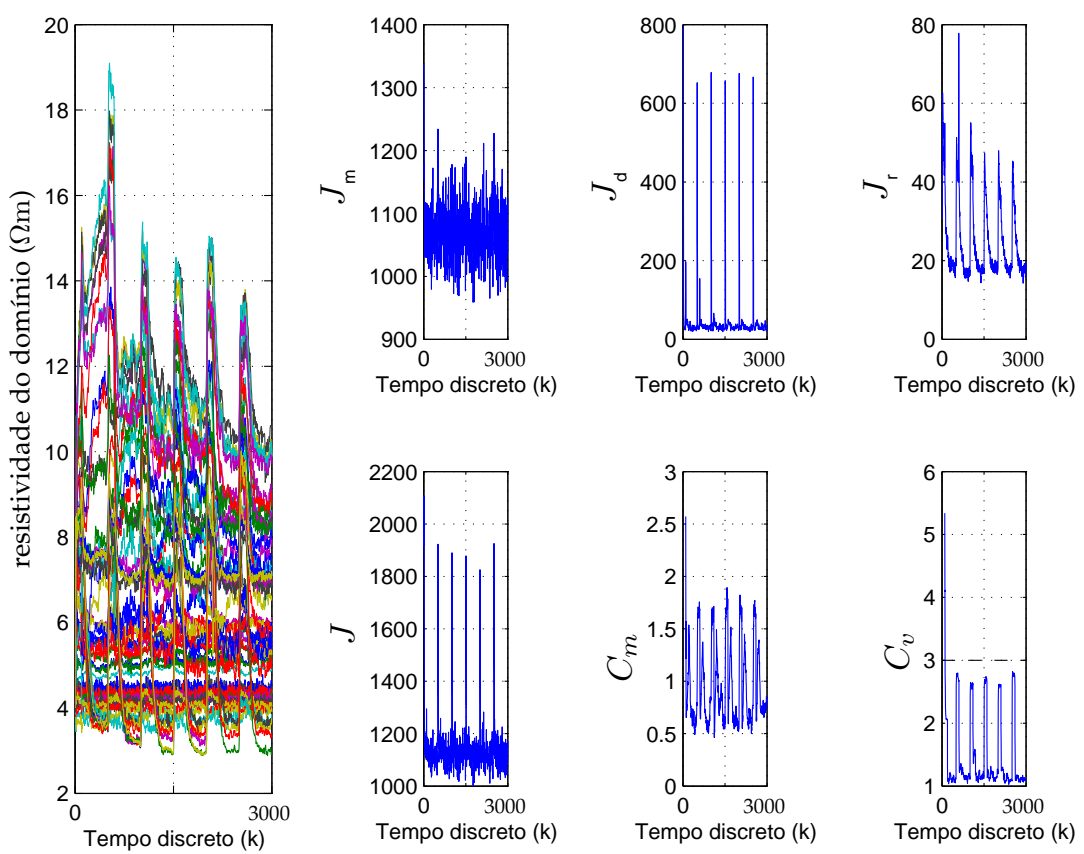

Figura 11.11: Ensaio IV, SR-UKF para estimação da distribuição de resistividade.
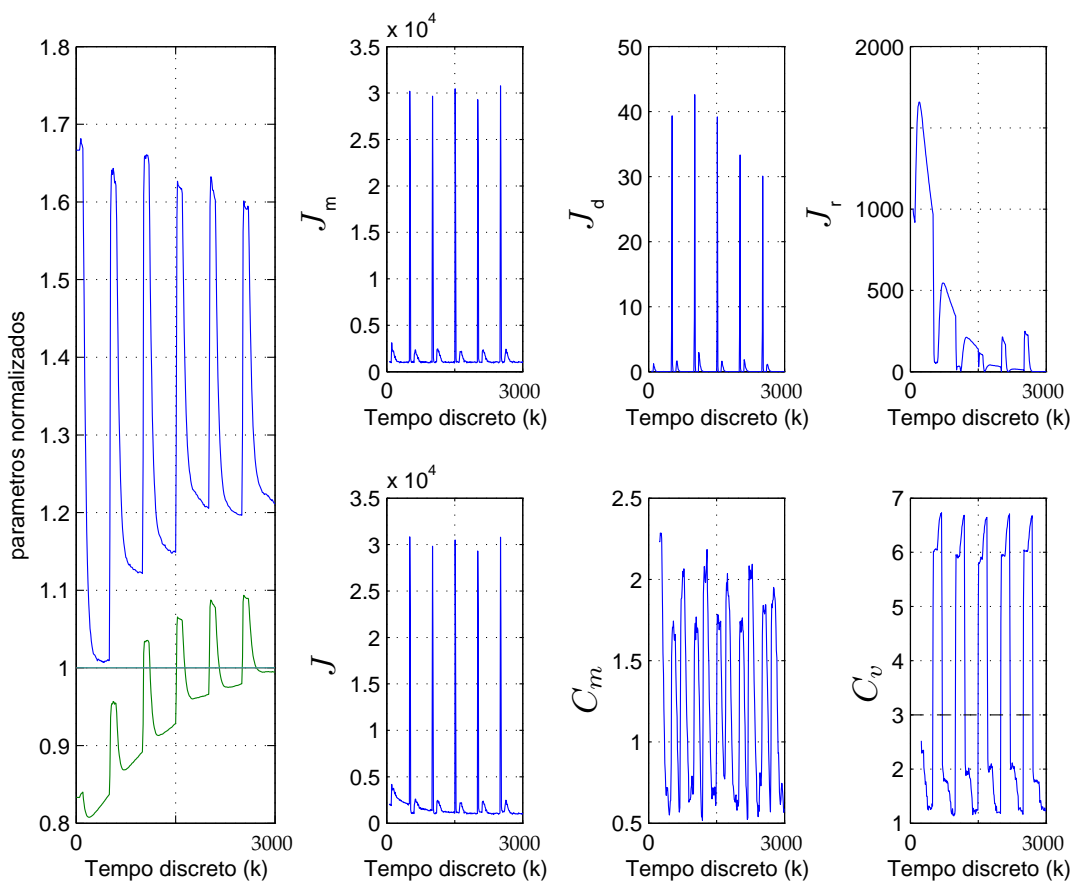

Figura 11.12: Ensaio IV, SR-UKF para estimação dos parâmetros do modelo de evolução. No gráfico da esquerda, a linha azul representa o parâmetro $a$ e a linha verde representa o parâmetro $b$. 


\section{Ensaio V:}
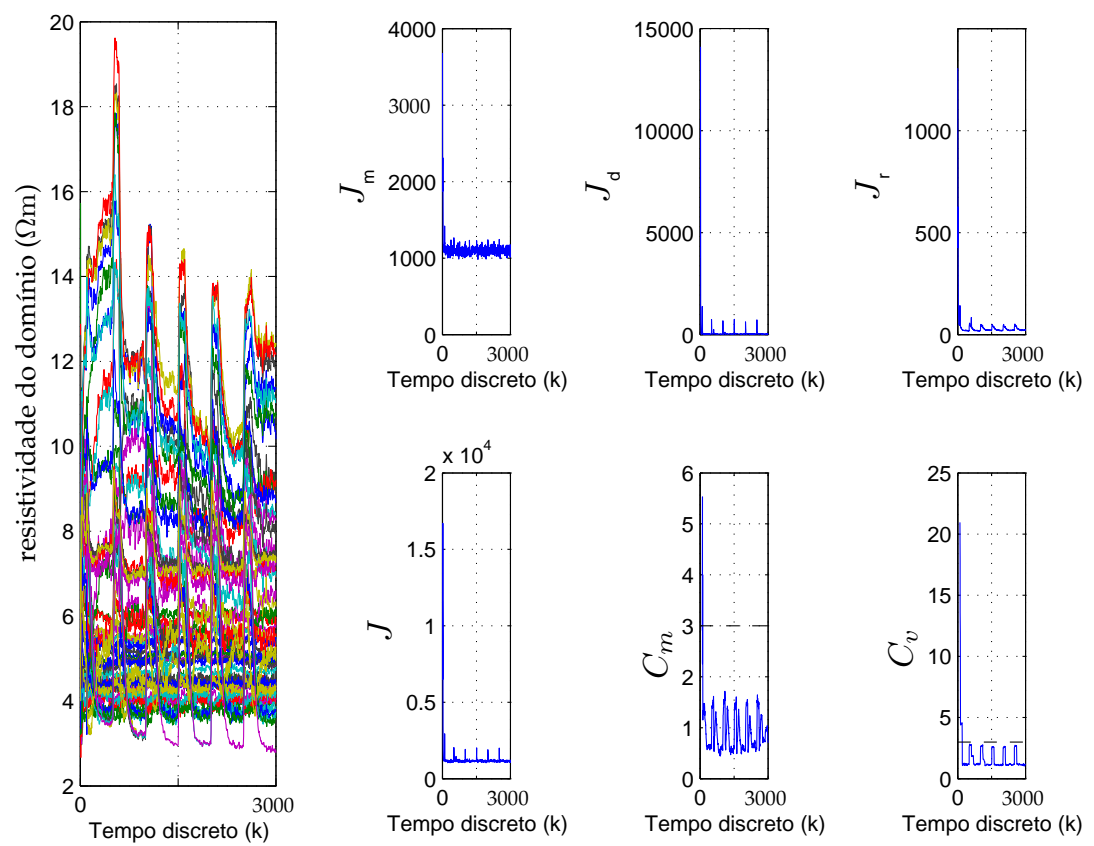

Figura 11.13: Ensaio V, SR-UKF para estimação da distribuição de resistividade.
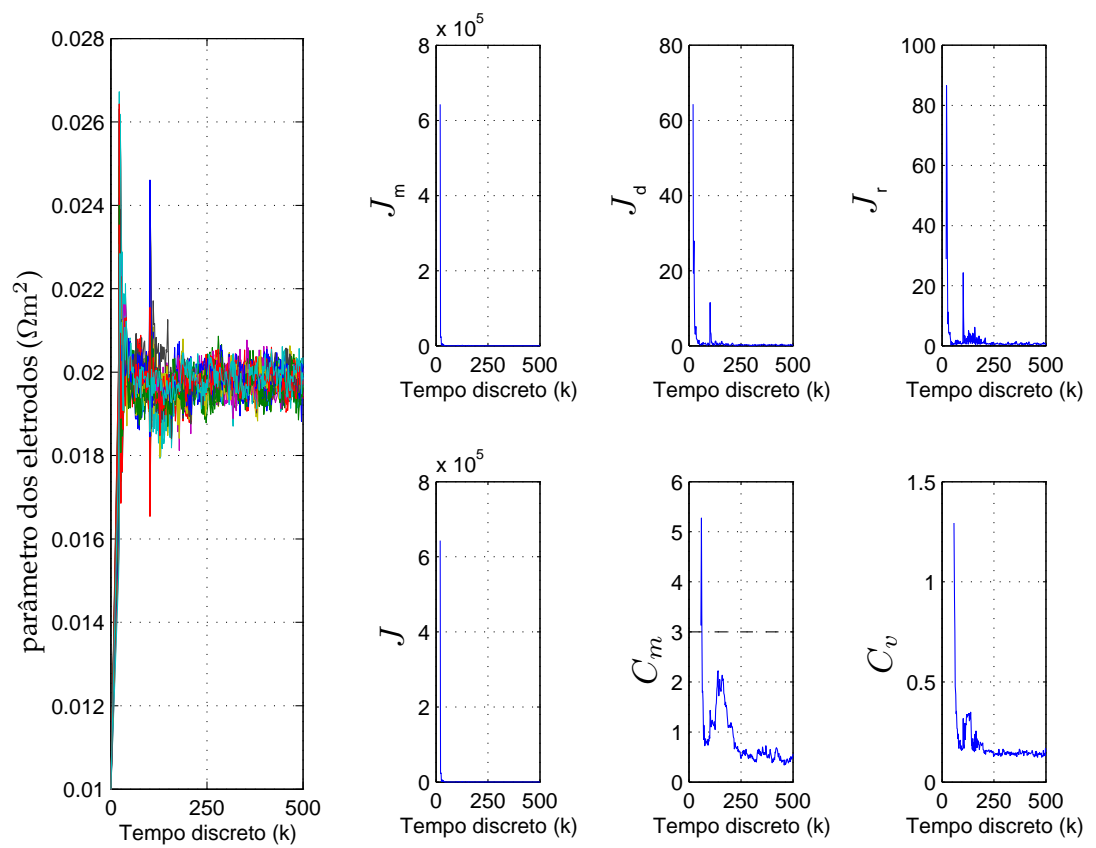

Figura 11.14: Ensaio V, SR-UKF para estimação dos parâmetros dos eletrodos. 

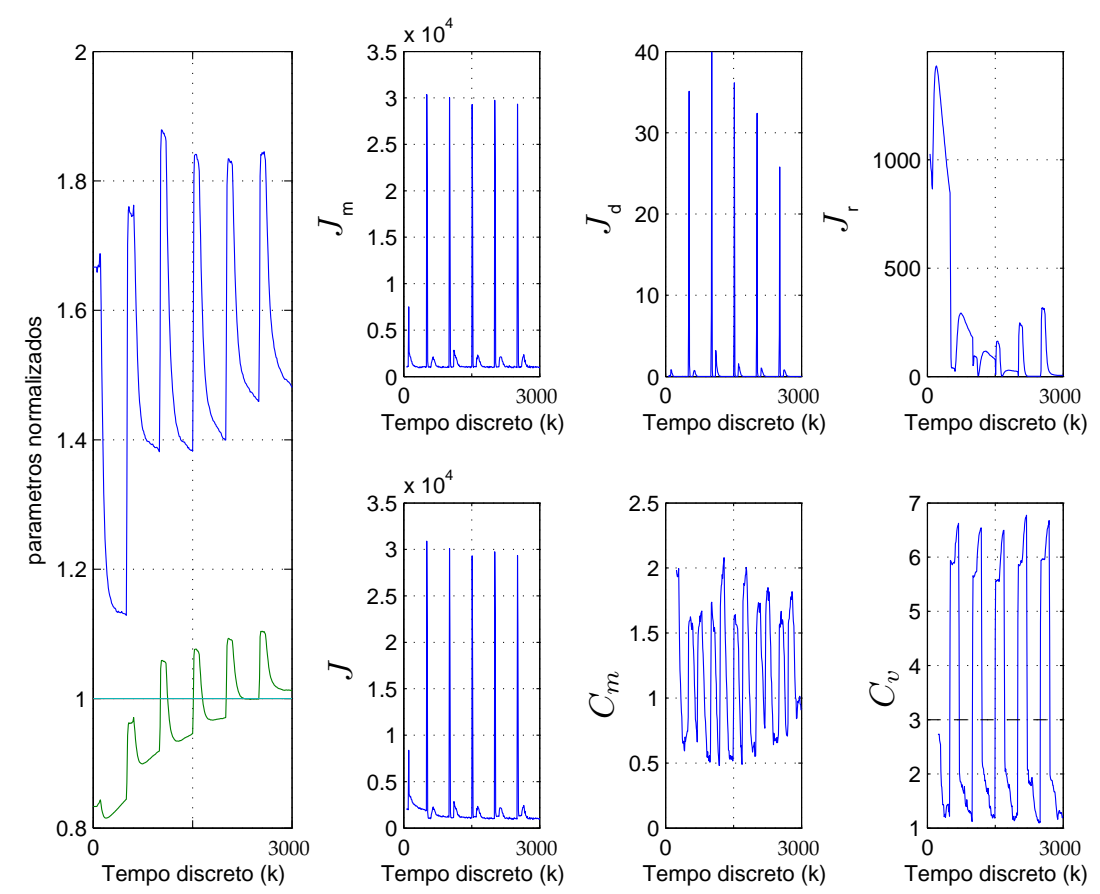

Figura 11.15: Ensaio V, SR-UKF para estimação dos parâmetros do modelo de evolução. No gráfico da esquerda, a linha azul representa o parâmetro $a$ e a linha verde representa o parâmetro $b$.

\section{Ensaio VI:}
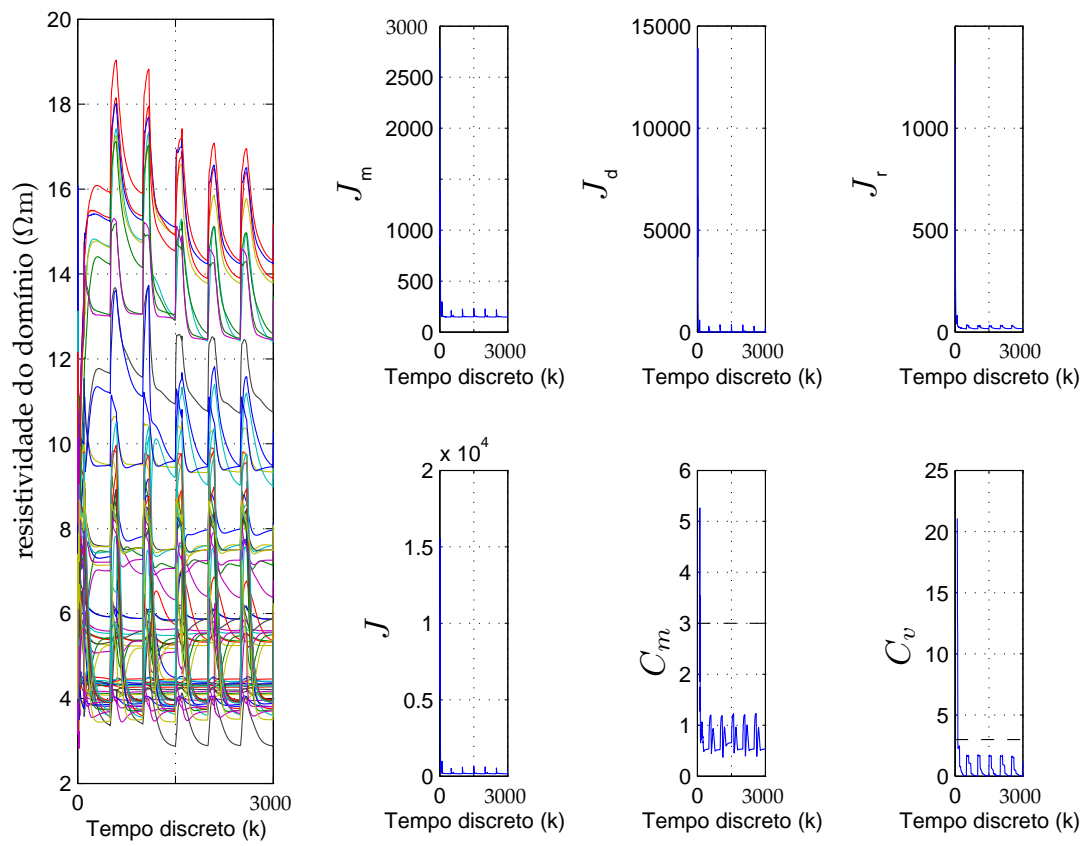

Figura 11.16: Ensaio VI, SR-UKF para estimação da distribuição de resistividade. 

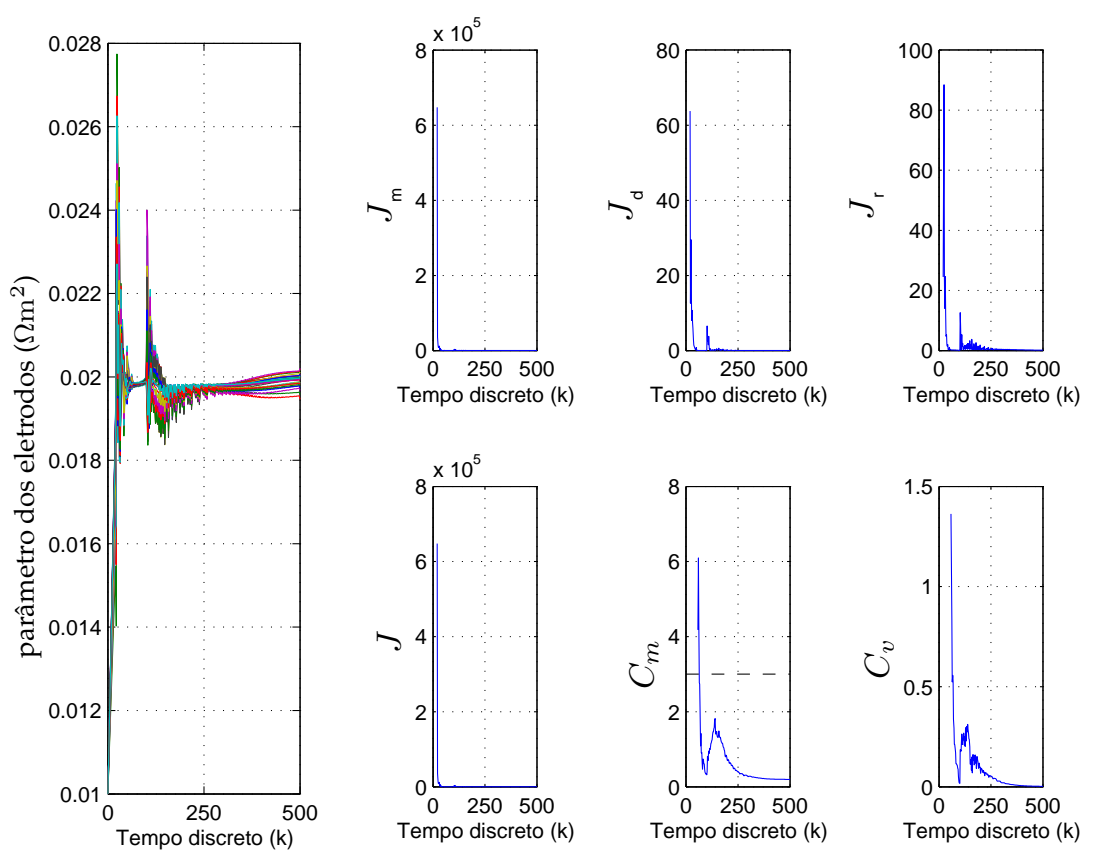

Figura 11.17: Ensaio VI, SR-UKF para estimação dos parâmetros dos eletrodos.
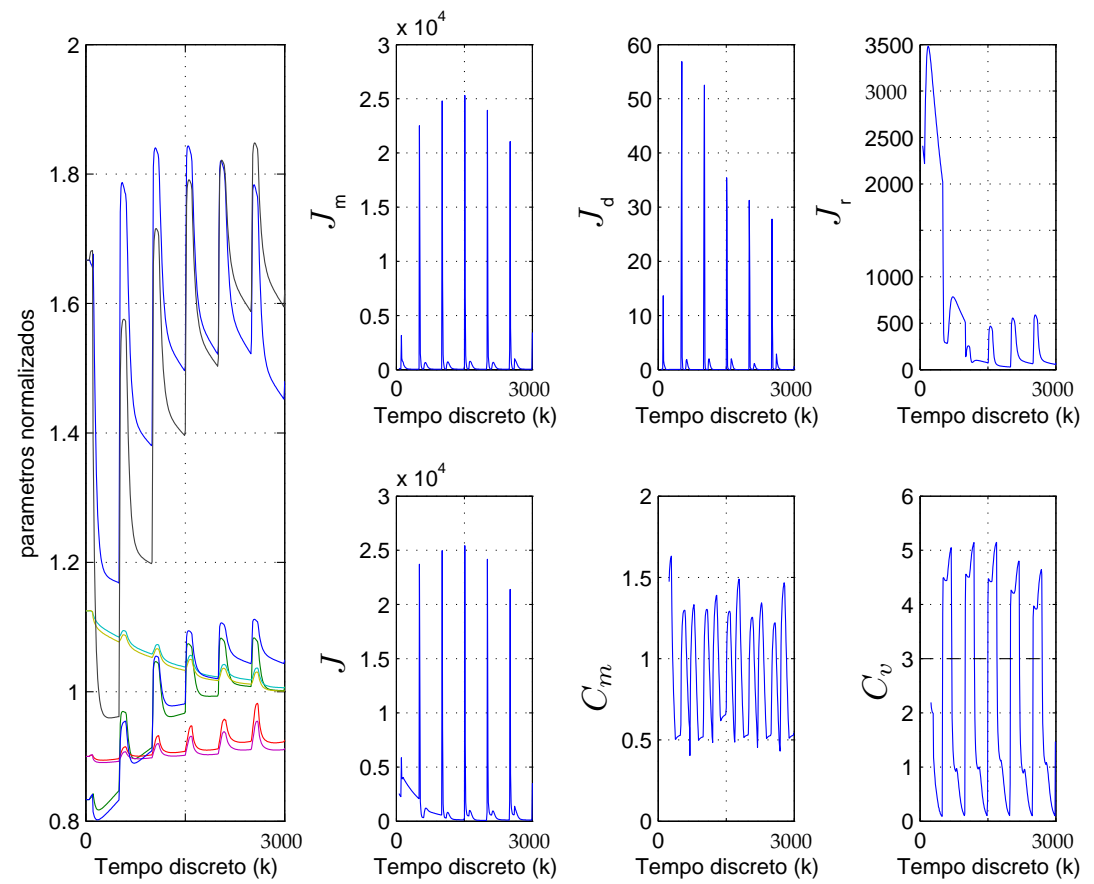

Figura 11.18: Ensaio VI, SR-UKF para estimação dos parâmetros do modelo de evolução. 


\section{Capítulo 12}

\section{Avaliação do método com dados experimentais}

Este capítulo apresenta os resultados do método utilizando dados coletados experimentalmente em um voluntário. O ensaio experimental apresenta resultados da estimação da distribuição de resistividade em um tórax humano em paralelo à estimação dos parâmetros do modelo de evolução proposto. Neste ensaio, o método do erro de aproximação e a segmentação por level set são utilizados. Não foram realizadas estimações dos parâmetros dos eletrodos já que os erros nestes parâmetros foram incluídos no método do erro de aproximação.

\subsection{Protocolo experimental, coleta e filtragem dos dados}

Um voluntário do sexo masculino de $70 \mathrm{~kg}$, não fumante e sem histórico de problemas pulmonares ou cardíacos foi selecionado para o experimento.

Trinta e dois eletrodos kendall medi-trace 200 foram posicionados em um plano perpendicular ao eixo cranial-caudal. O perímetro do tórax do voluntário neste plano é de $90 \mathrm{~cm}$. Os eletrodos foram posicionados de forma a ficarem o mais equidistantes possível ao longo do perímetro. Cada um dos trinta e dois eletrodos foram cortados de forma a ficarem com $10 \mathrm{~mm}$ de largura por $20 \mathrm{~mm}$ de altura. O posicionamento dos eletrodos pode ser visto na Figura 12.1 

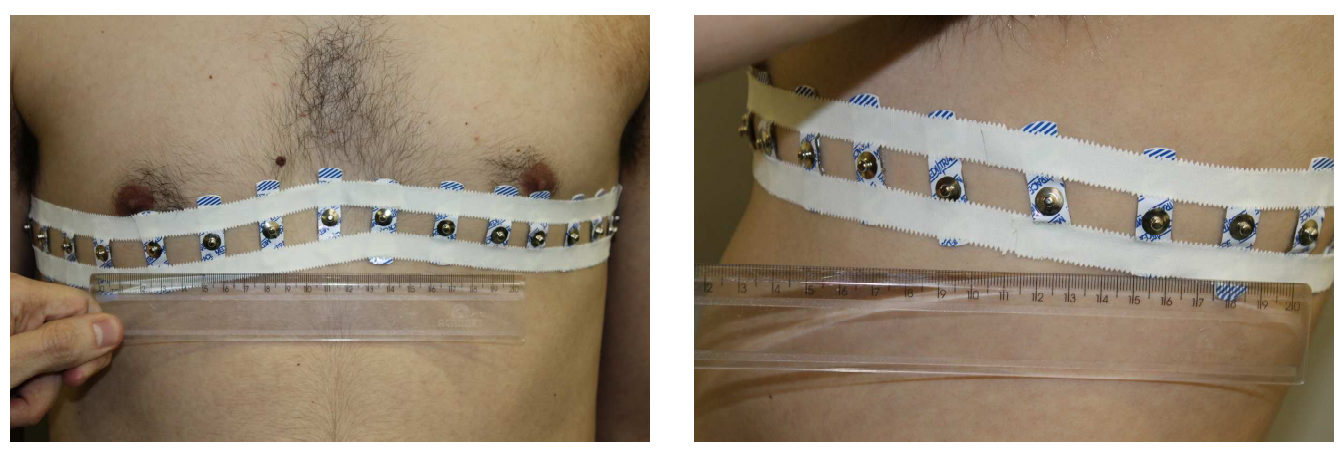

Figura 12.1: Posicionamento dos eletrodos no tórax.

O tomógrafo de impedância elétrica utilizado faz medições single-ended, utilizando padrão de corrente pula-3. Cada conjunto de 1024 medidas foi coletado a uma taxa de $35 \mathrm{~Hz}$. Um total de 3000 conjuntos de medidas foram coletados, perfazendo um período de $85 \mathrm{~s}$. Foi imposta uma corrente elétrica senoidal, com amplitude $A=0.866 \mathrm{~mA}$ zero-pico e frequência $125 \mathrm{kHz}$. Este valor foi ajustado de forma a não saturar os conversores analógico->digital do equipamento, que têm limite de $\pm 1.0 \mathrm{~V}$.

O voluntário permaneceu em pé durante a coleta de dados, sem realizar nenhuma atividade física além da respiração espontânea, inspirando e expirando profundamente a uma taxa média de 11 ciclos por minuto $(0.18 \mathrm{~Hz})$. Os dados coletados foram demodulados e filtrados com um filtro FIR equiripple passa-baixas, com região de transição entre $1.0 \mathrm{~Hz}$ e $1.2 \mathrm{~Hz}$.

O voluntário não estava sob ventilação artificial durante o experimento e não foram coletados dados da pressão nas vias aéreas. Uma forma aproximada de determinar o perfil de pressão, necessário no modelo de evolução, foi calcular a média do potencial elétrico de todas as 1024 medidas ao longo do tempo. Esta aproximação foi feita por conta da indisponibilidade de sensores de pressão durante a coleta de dados. Como a formulação do filtro de Kalman lida justamente com erros de modelagem, esta aproximação pode, em princípio, ser utilizada. Em seguida os valores foram escalados para valores de pressão usuais. A Figura 12.2 apresenta o gráfico desta média, convertidos para valores de pressão. 


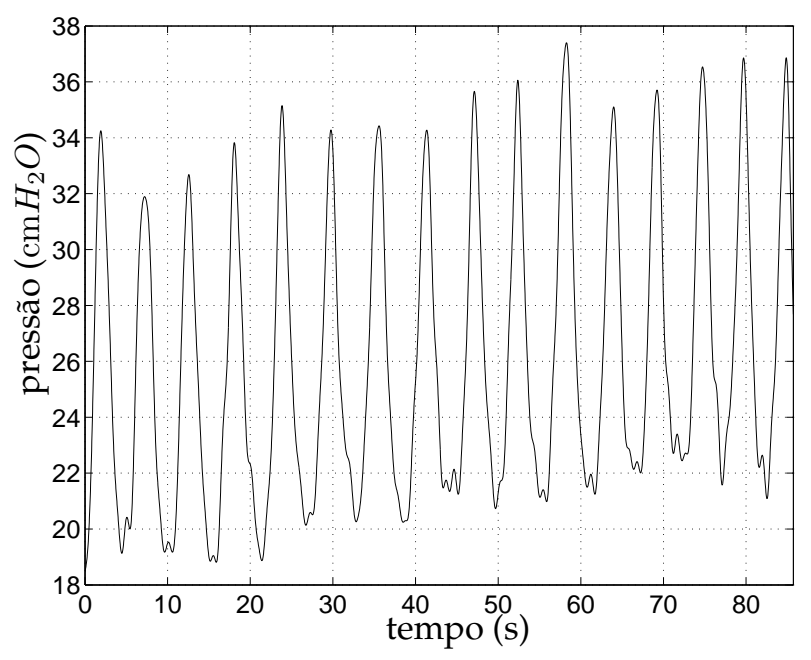

Figura 12.2: Sinal de pressão aproximado.

Foi gerada uma malha 2D para a solução do problema inverso. A malha, apresentada na Figura 12.3, é composta por 1056 elementos triangulares e aproxima o corte transversal do tórax. A malha possui 32 eletrodos, dispostos ao longo de seu bordo de forma a ficarem equidistantes. A malha é vista nestas figuras no sentido caudal-cranial e a região superior da malha na figura representa a região anterior do tóraxil.

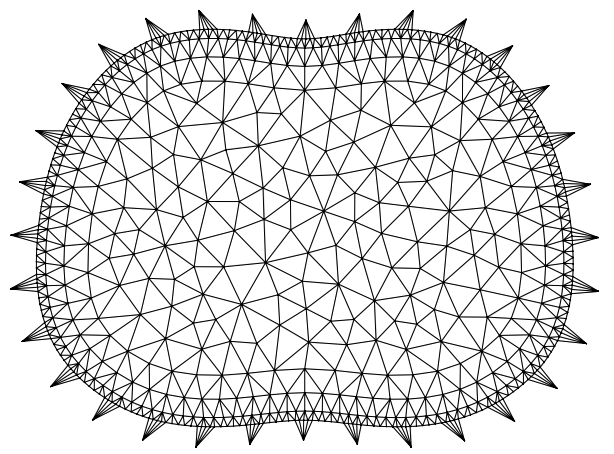

Figura 12.3: Malha 2D usada no problema inverso. 1056 elementos triangulares.

\footnotetext{
${ }^{1}$ Por conta desta orientação, o lado direito do paciente aparece do lado esquerdo da imagem, e vice-versa.
} 


\subsection{Método do erro de aproximação}

Para o cálculo das estatísticas do método do erro de aproximação, foram considerados o erro de discretização, formato do domínio, posição e parâmetros dos eletrodos. A amostragem segue as mesmas diretrizes apresentadas na Seção 8.2 , porém com algumas particularidades que serão apresentadas.

A parametrização do contorno de referência para cada realização das malhas $M_{i}$ é a mesma da Figura 12.3, porém as malhas $M_{i}$ são tridimensionais e com elementos tetraédricos lineares, como pode ser visto na Figura 12.4.

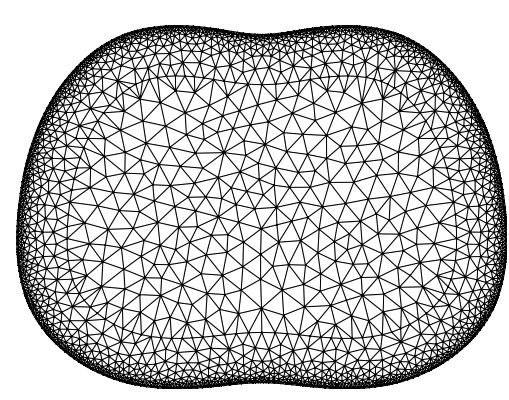

(a) Vista de topo.

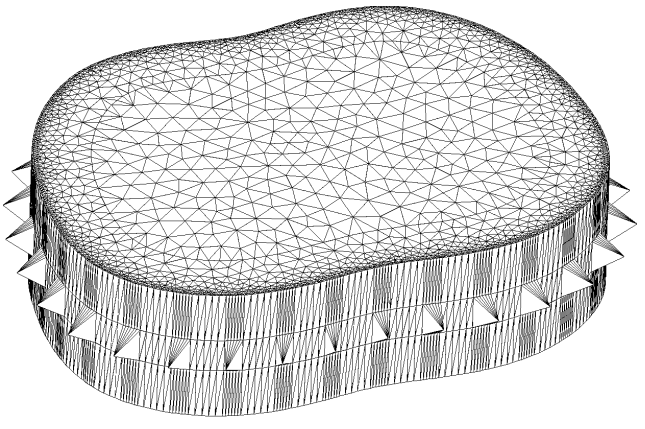

(b) Vista de perspectiva.

Figura 12.4: Malha 3D usada na amostragem das malhas $M_{i}$. 39870 elementos tetraédricos.

Amostrando malhas tridimensionais $M_{i}$ pode-se incluir no método do erro de aproximação o erro proveniente do truncamento do domínio. A malha apresentada na Figura 12.4 é utilizada como referência para a amostragem do formato da malha $M_{i}$, posição dos eletrodos e nível de discretização.

Esta malha possui $80 \mathrm{~mm}$ de altura total e as dimensões dos elementos que representam os eletrodos condizem com os eletrodos utilizados no experimento. Salienta-se que o nível de discretização da malha ainda poderia ser melhorado, principalmente na direção perpendicular ao plano dos eletrodos. 
Tabela 12.1: Parâmetros usados no processo de amostragem do ruído $\varepsilon$.

\begin{tabular}{lc}
\hline Parâmetro & valor \\
\hline$\sigma_{\vartheta}(\%)$ & 5 \\
$p^{-}, p^{+}(\mathrm{mm})$ & \pm 5 \\
param. eletrodos $\boldsymbol{\rho}^{\prime}\left(\Omega \mathrm{m}^{2}\right)$ & $\sim \mathcal{U}(0.01,0.03)$ \\
\hline refinamento & $\delta^{-}=0.0, \quad \delta^{+}=0.0$ \\
ruído $\mathbf{e}^{x}$ & $\sim \mathcal{N}\left(\mathbf{0},\left(5.0 \cdot 10^{-3}\right)^{2} \mathbb{I}\right)$ \\
$\boldsymbol{\rho}^{\prime a}\left(\Omega \mathrm{m}^{2}\right)$ & 0.02 \\
\hline resistividade $\boldsymbol{\rho}$ & $\sim \mathcal{N}^{L^{-}}\left(\mathbf{x}_{u} \mathbf{1}, 3.0^{2} \mathbb{I}, \mathbf{0}\right)$ \\
& $\mathbf{x}_{u} \sim \mathcal{U}(2.0,6.0)$ \\
\hline
\end{tabular}

A Tabela 12.1 apresenta as estatísticas utilizadas para amostragem do ruído $\varepsilon_{i}$ do método do erro de aproximação. No ensaio foram realizadas $N=4000$ amostras do ruído $\varepsilon$ em (8.1).

\subsection{Parâmetros utilizados na solução do problema inverso}

No ensaio, os parâmetros para a segmentação de imagem foram: $\Delta t=5, \mu=0.04$, $\lambda=15, \nu=1.0, \epsilon=3, \sigma=1.5$, critérios de parada $\epsilon_{1}=0.1$ e $\epsilon_{2}=0.04$ e número máximo de iterações 300. A distribuição de resistividade é interpolada em um grid de pixels de dimensões 50x50. A segmentação é realizada a cada cento e noventa dados coletados, equivalente a aproximadamente $5.4 \mathrm{~s}$.

As estimativas de distribuição de resistividade são realizadas a cada 10 conjuntos de medidas, iniciando em $k=10$. O conjunto de parâmetros utilizado nesta estimação é apresentado na Tabela 12.2. Utilizou-se como vetor de resitividade esperado $\boldsymbol{x}_{k}^{*}$ da informação a priori( (6.26) o vetor de distribuição resistividade elétrica média proveniente de um atlas anatômico da população. Para este trabalho, o atlas apresentado em Camargo et al. [41], Sousa et al. [4] para suínos foi adaptado para seres humanos. Este procedimento resulta em um aproximação do que seria o atlas 
de humanos. A Figura 12.5 apresenta a distribuição de resistividade média do atlas apresentado nos trabalhos citados e também a distribuição adaptada para seres humanos.

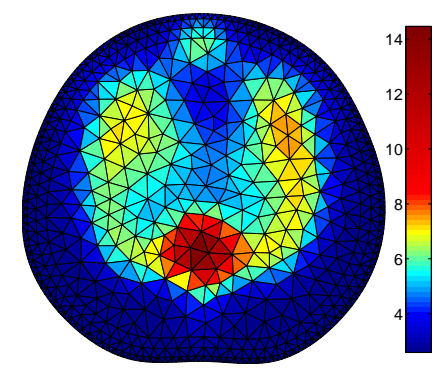

(a) Suíno

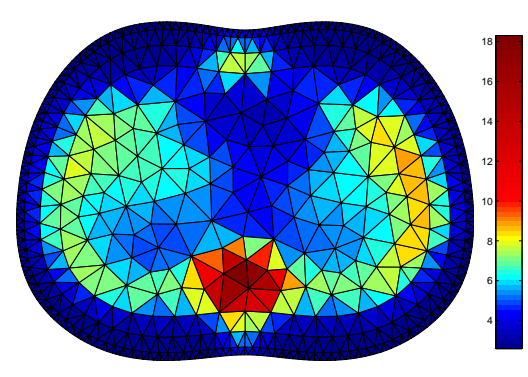

(b) Humano

Figura 12.5: Distribuição de resistividade média proveniente de um atlas anatômico em $\Omega \mathrm{m}$. A escala de cores destas imagens foi escolhida de modo a melhorar o contraste na região pulmonar.

As estimativas dos parâmetros do modelo de evolução são realizadas a cada 50 conjuntos de medidas, iniciando em $k=50$. O conjunto de parâmetros utilizado nesta estimação é apresentado na Tabela 12.3. Neste ensaio, apenas os parâmetros $a_{i}$ e $b_{i}$ de (9.11) são considerados na estimação $\boldsymbol{p}=\left[\begin{array}{llll}a_{1} & b_{1} & a_{2} & b_{2}\end{array}\right]^{\mathrm{T}}$.

A conversão entre volume e resistividade foi feita usando uma interpolação linear $\operatorname{com} \rho_{A}=3.63 \Omega \mathrm{m}, \rho_{B}=9.17 \Omega \mathrm{m}, V_{A}=100 \mathrm{~cm}^{3}$ e $V_{B}=1200 \mathrm{~cm}^{3}$. 
Tabela 12.2: Parâmetros usados no SR-UKF para estimação da distribuição de resistividade.

\begin{tabular}{|c|c|}
\hline parâmetros de dispersão (eq. (5.18)) & $\alpha=1.0, \beta=2.0, \kappa=0.0$ \\
\hline densidade de probabilidade inicial $^{a}$ & $\overline{\boldsymbol{x}}_{0}=4.0 \cdot \mathbf{1}, \boldsymbol{\Gamma}_{0}^{x x}=1.0 \mathbb{I}$ \\
\hline modelo de observação & modelo não linear \\
\hline ruído de observação & $\overline{\boldsymbol{e}}_{k}^{x}=\mathbf{0}, \boldsymbol{\Gamma}_{k}^{e^{x}}=\left(5.0 \cdot 10^{-3}\right)^{2} \mathbb{I}$ \\
\hline IUKF (eq. (5.39)) & $\operatorname{sim}$ \\
\hline densidade de probabilidade a priori(eq. (6.26) $)$ & $\operatorname{sim}$ \\
\hline operador $\boldsymbol{L}_{r}$ & equação (6.24) \\
\hline vetor médio & $\boldsymbol{x}_{k}^{*}=\overline{\boldsymbol{x}}_{\text {atlas }}$ \\
\hline parâmetros & $\sigma=8 \mathrm{~mm}, \alpha^{2}=1.0 \cdot 10^{6}$ \\
\hline segmentação de imagem & $\operatorname{sim}$ \\
\hline método do erro de aproximação & $\operatorname{sim}$ \\
\hline ruído residual $^{b} \boldsymbol{\eta}$ & 0.0 \\
\hline número de medidas utilizadas & $\ell=1024$ \\
\hline parâmetros dos eletrodos ${ }^{c}\left(\Omega \mathrm{m}^{2}\right)$ & 0.02 \\
\hline modelo de evolução & eq. (9.11) \\
\hline$\stackrel{\mathscr{Q}}{\varrho}$ número de compartimentos & 2 (direito e esquerdo) \\
\hline ruído de processo ${ }^{a}$ & $\overline{\boldsymbol{v}}_{k}^{x}=\mathbf{0}, \boldsymbol{\Gamma}_{k}^{v^{x}}=1.0 \cdot 10^{-1} \mathbb{I}$ \\
\hline vínculo de intervalo (eq. (6.4)) & $\rho^{\min }=0.0, \rho^{\max }=15.0, s_{a}=0.1$ \\
\hline parâmetros do modelo de evolução ${ }^{c}$ & $a_{i}=10, b_{i}=400, c_{i}=0.001, d_{i}=1, e_{i}=15$ \\
\hline \multirow{3}{*}{ 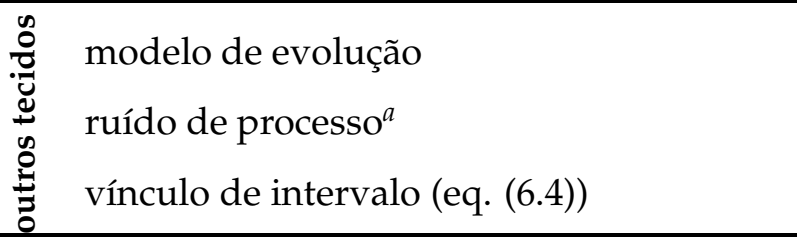 } & passeio aleatório \\
\hline & $\overline{\boldsymbol{v}}_{k}^{x}=\mathbf{0}, \boldsymbol{\Gamma}_{k}^{v^{x}}=1.0 \cdot 10^{-2} \mathbb{I}$ \\
\hline & $\rho^{\min }=0.0, \rho^{\max }=20.0, s_{a}=0.1$ \\
\hline \multicolumn{2}{|l|}{${ }^{a}$ As estatísticas foram convertidas para o metaestado com 6.14} \\
\hline \multicolumn{2}{|l|}{${ }^{b}$ Ruído residual 7.12) adicionado ao ruído $\varepsilon$. } \\
\hline $\begin{array}{l}{ }^{\circ} \text { Este conjunto de parâmetros é utilizado como primeira sugestâ } \\
\text { paralelo com o passar do tempo. }\end{array}$ & ao algoritmo. Estes valores são ajustados pelo filtro em \\
\hline
\end{tabular}


Tabela 12.3: Parâmetros usados no SR-UKF para estimação dos parâmetros do modelo de evolução.

\begin{tabular}{|c|c|}
\hline parâmetros de dispersão (ver. Apêndice C.2) & $\alpha=0.1, \beta=2.0, \kappa=1.0, \eta=1.0$ \\
\hline \multirow[t]{2}{*}{ densidade de probabilidade inicial $^{a}$} & $\overline{\boldsymbol{p}}_{0}=\left[\begin{array}{llll}10 & 400 & 10 & 400\end{array}\right]^{\mathrm{T}}$ \\
\hline & $\boldsymbol{\Gamma}_{0}^{p p}=\operatorname{diag}(10,100,10,100)$ \\
\hline modelo de observação & modelo não linear \\
\hline ruído de observação & $\overline{\boldsymbol{e}}_{k}^{p}=\mathbf{0}, \boldsymbol{\Gamma}_{k}^{e^{p}}=\left(5.0 \cdot 10^{-3}\right)^{2} \mathbb{I}$ \\
\hline IUKF (eq. (5.39)) & $\operatorname{sim}$ \\
\hline densidade de probabilidade a priori(eq. (6.26) & $\operatorname{sim}$ \\
\hline operador $\boldsymbol{L}_{r}$ & identidade \\
\hline vetor médio & $\boldsymbol{p}_{k}^{*}=\left[\begin{array}{llll}12 & 200 & 12 & 200\end{array}\right]^{1}$ \\
\hline parâmetro & $\alpha^{2}=1$ \\
\hline método do erro de aproximação & $\operatorname{sim}$ \\
\hline ruído residual ${ }^{b} \boldsymbol{\eta}$ & 0.0 \\
\hline número de medidas utilizadas & $\ell=928$ \\
\hline modelo de evolução & passeio aleatório \\
\hline ruído de processo ${ }^{a}$ & $\overline{\boldsymbol{v}}_{k}^{p}=\mathbf{0}, \boldsymbol{\Gamma}_{k}^{v^{p}}=1.0 \cdot 10^{-1} \mathbb{I}$ \\
\hline \multicolumn{2}{|l|}{ vínculo de intervalo (eq. (6.4)) } \\
\hline parâmetro do modelo de evolução $a_{i}$ & $a^{\min }=5, a^{\max }=30, s_{a}=1$ \\
\hline parâmetro do modelo de evolução $b_{i}$ & $b^{\min }=100, b^{\max }=600, s_{b}=20$ \\
\hline
\end{tabular}




\subsection{Resultados do experimento}

As figuras 12.6 e 12.7 mostram os históricos das estimações dos parâmetros do modelo de evolução e distribuição de resistividade do tórax do voluntário, assim como os respectivos índices dos critérios de convergência.

Em ambos os casos, nota-se que houve convergência estatística conforme estabelecido na Seção 8.4 para $C_{v}$, porém não para $C_{m}$. Os índices $J_{m} J_{d}$ e $J_{r}$ apresentam oscilações que coincidem com a frequência respiratória do voluntário. Nota-se também que a importância da parcela $J_{m}$ é maior que as demais parcelas na composição do custo total $J$.
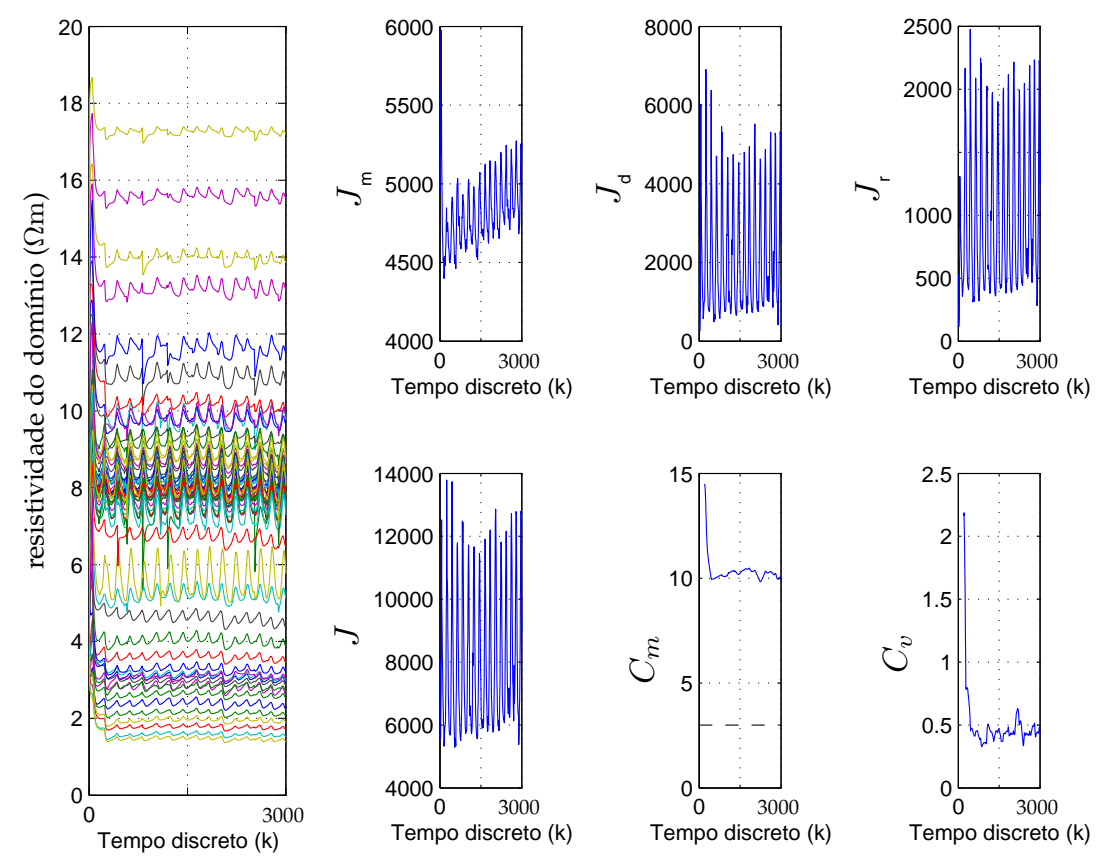

Figura 12.6: Estimação da distribuição de resistividade.

No caso da estimação dos parâmetros do modelo de evolução, as estimativas dos parâmetros $a_{i}$ ficaram próximas dos valores esperados pela informação a priori, já os parâmetros $b_{i}$ estabilizaram em torno de $150 \mathrm{~cm}^{3}$. Este estimativa está abaixo do volume corrente esperado em um ser humano sem problemas respiratórios.

A Figura 12.8 apresenta a média do valor da resistividade estimada pelo SR-UKF em cada compartimento do modelo dinâmico. Por este gráfico é possível notar que a resistividade média oscila entre 6 e $7 \Omega \mathrm{m}$ em ambos os pulmões durante o experimento. Durante os dois primeiros ciclos, as estimativas variaram mais até serem 
melhor ajustados pelo SR-UKF e segmentação. Após este período inicial, as variações temporais tornaram-se mais periódicas.
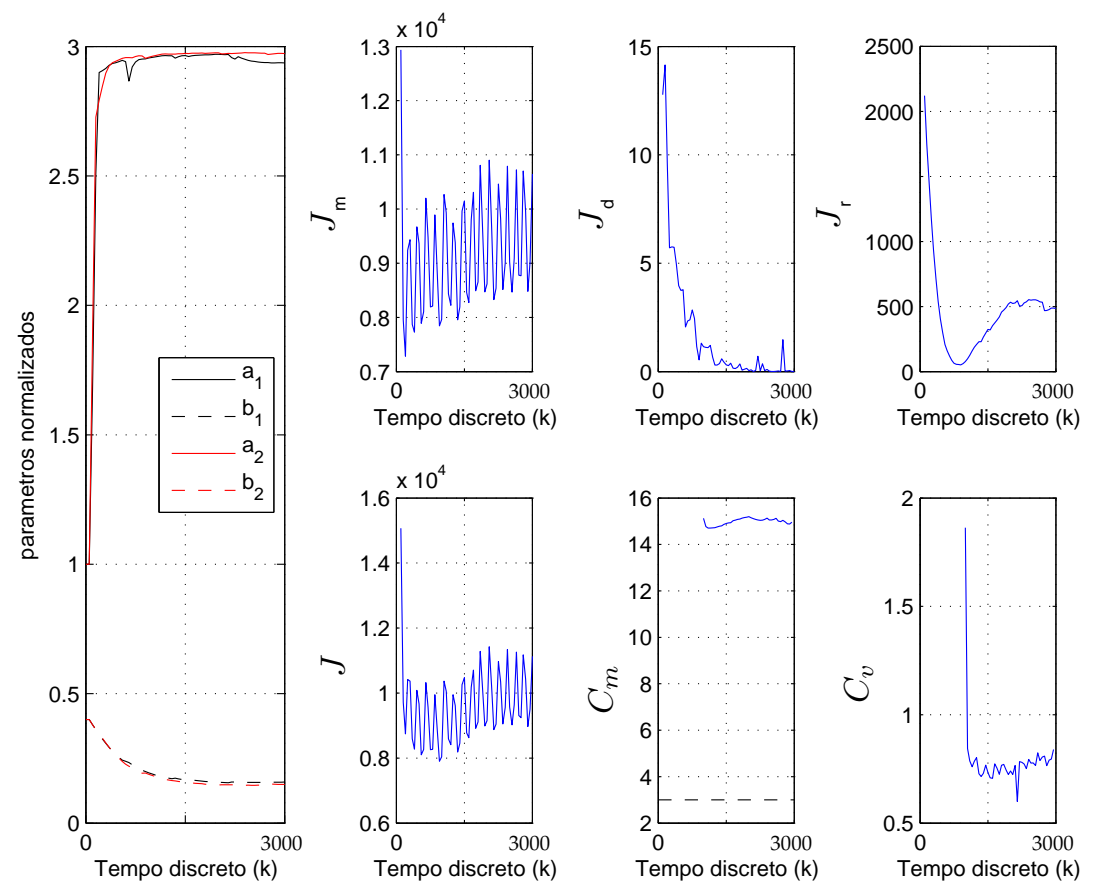

Figura 12.7: Estimação dos parâmetros do modelo de evolução. Os valores dos parâmetros $a_{i}$ e $b_{i}$ foram normalizados em relação a 10 e 1000 respectivamente.

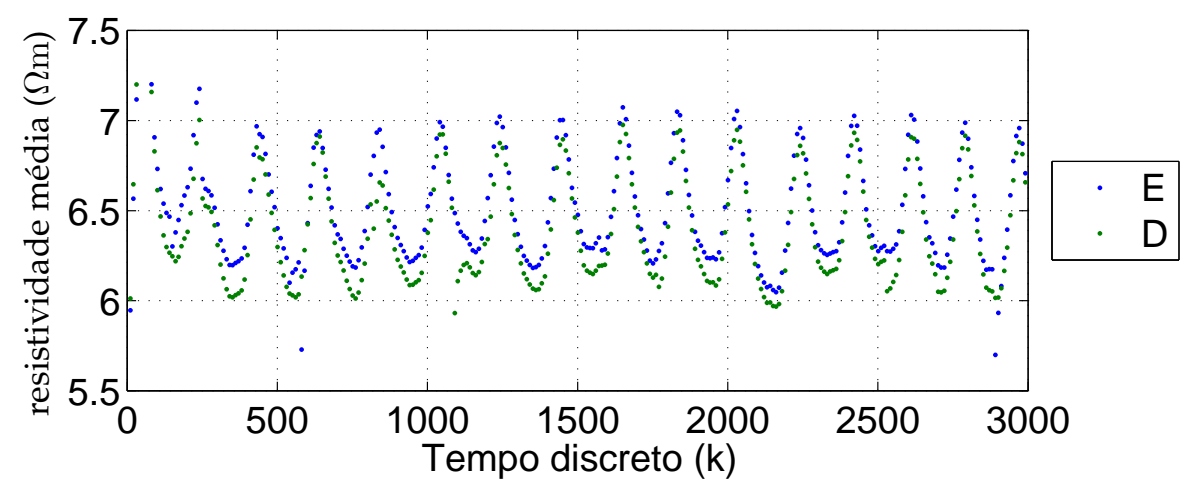

Figura 12.8: Histórico da distribuição de resistividade média estimada nos compartimentos dos pulmões.

A Figura 12.9 apresenta os resultados da segmentação da região pulmonar. É possível ver a sugestão inicial fornecida para o SR-UKF, entre $k=1$ a $k=60$. Esta segmentação inicial não é baseada em level set. As demais segmentações são todas obtidas com o método level set. Em todos os casos, os pulmões foram segmentados 
em duas regiões e não foram muito influenciados pela coluna vertebral ou esterno por conta da escolha da imagem a ser segmentada, apresentada no Capítulo 10 .

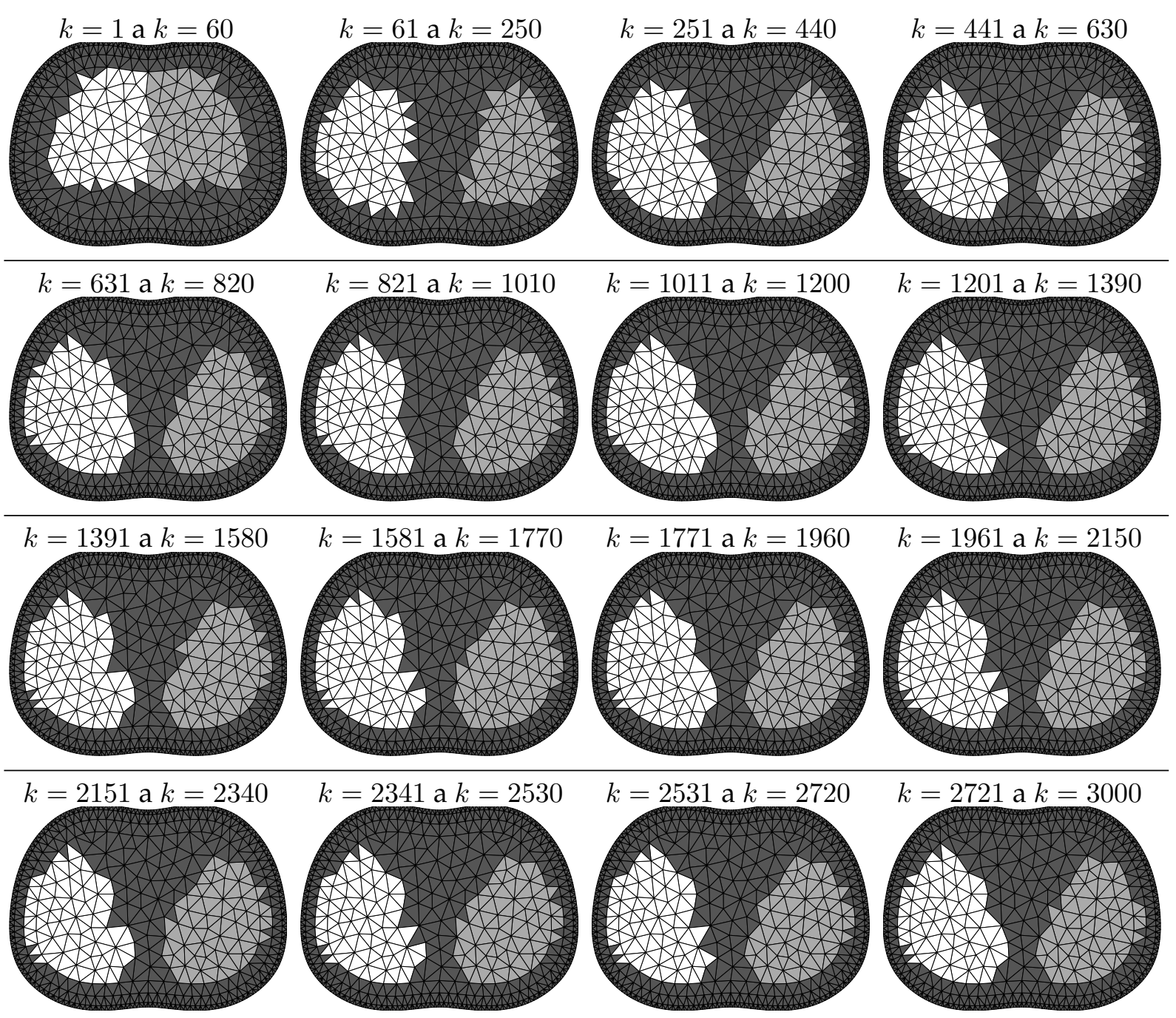

Figura 12.9: Segmentação da região pulmonar estimada pelo algoritmo. Nestas imagens, diferentes tons de cinza indicam diferentes regiões.

A Figura 12.10 mostra um ciclo respiratório entre $k=1541$ e $k=1761$. A distribuição de resistividade varia dentro das regiões pulmonares como esperado. É possível ver nestas imagens a caixa torácica, incluindo o esterno e coluna vertebral. Os valores de resistividade do tecido ósseo oscilaram levemente em torno de $17 \Omega \mathrm{m}$, valor próximo do atlas anatômico. É bastante interessante notar a ausência de grandes artefatos nas regiões próximas dos eletrodos, que costuma ser mais proeminente. 


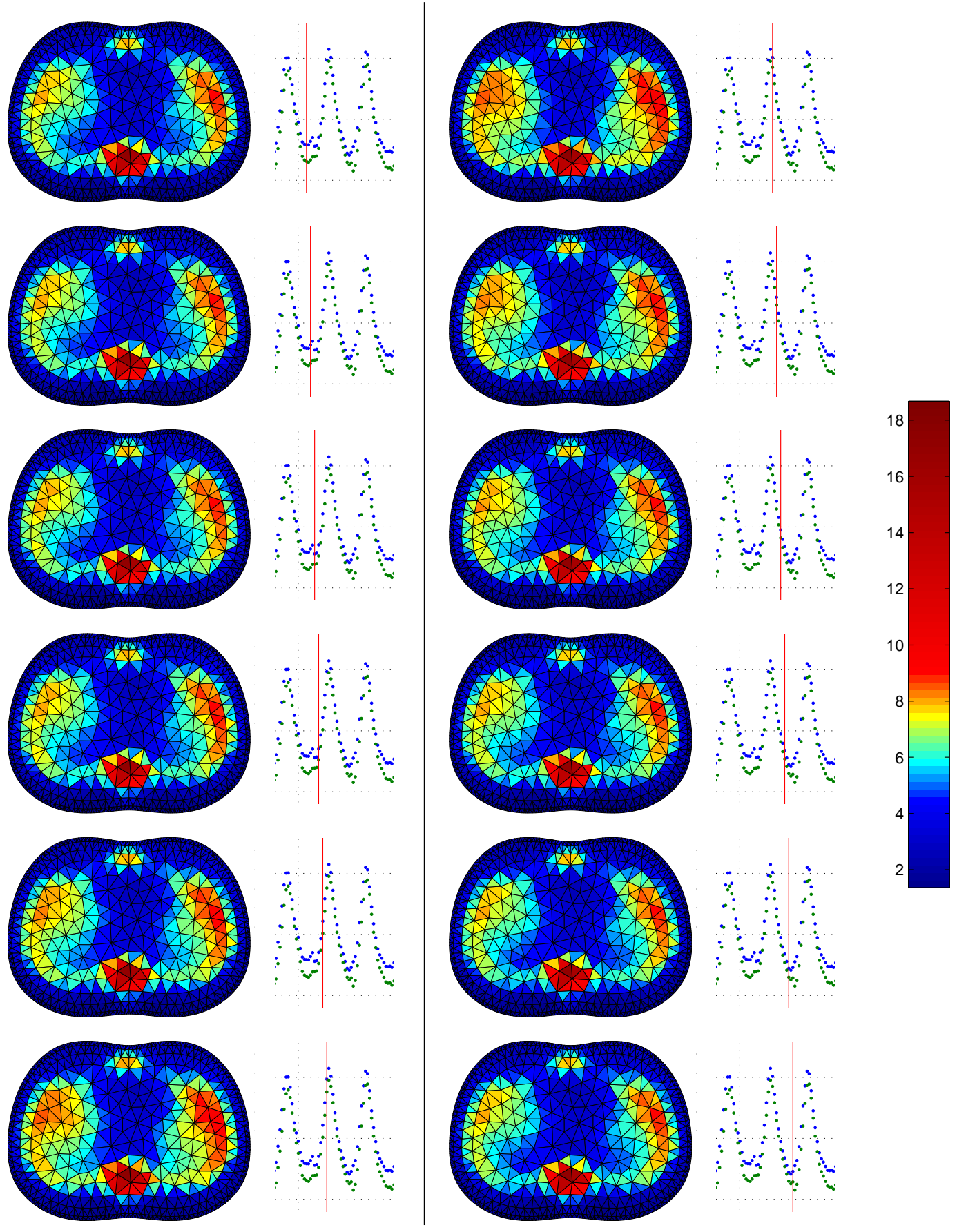

Figura 12.10: Distribuição de resistividade entre $k=1541$ e $k=1761$ e detalhe dos gráficos da resistividade média de cada compartimento dos pulmões. A escala de cores destas imagens foi ajustada de modo a melhorar o contraste na faixa de variação da resistividade na região pulmonar. Valores em $\Omega \mathrm{m}$. 


\section{Parte III}

\section{Discussões e conclusões}





\section{Capítulo 13}

\section{Discussões e conclusões}

Esta tese propôs o uso do unscented Kalman filter (UKF) para a estimação de imagens na tomografia de impedância elétrica (TIE). O uso do UKF tem como vantagem não necessitar o cálculo de jacobianos e permite estimar as estatísticas do estado condicionado às medidas com menos erros que o filtro estendido de Kalman (FEK). O cerne do UKF está na escolha dos pontos sigma para transformar uma variável aleatória através da função não linear original ao invés de uma aproximação como no FEK. Tal escolha permite que toda a não linearidade da função seja levada em consideração.

Por conta da falta de uma solução analítica para o problema da TIE em um domínio de formato arbitrário, uma discretização do domínio é necessária para o uso do método dos elementos finitos. Ao realizar tal discretização, erros são introduzidos nas equações do modelo de observação. Nesta tese, o método do erro de aproximação é utilizado para lidar com tais incertezas. Além do problema da discretização, a forma do domínio pode não ser conhecida com precisão necessária nem a posição dos eletrodos. Outras fontes de erros são os valores das impedâncias de contato, as distâncias entre os eletrodos, erros de eletrônica, interferências e erros de truncamento do domínio. Todas estas de fontes de erros reduzem a qualidade do modelo de observação.

O método do erro de aproximação não elimina os erros de modelagem do problema mas permite estimar as estatísticas do erro total causado pelas diferentes fontes de erros identificadas. Todos os cálculos necessários no método do erro de aproximação são feitos antes da realização das medidas e o resultado é armazenado para uso futuro. Durante a etapa de estimação, pouco esforço computacional adicional é necessário 
para incluir o método do erro de aproximação. As estatísticas do erro total resultante permanecem válidas contanto que as estatísticas das fontes de erros permaneçam as mesmas e o modelo de observação não mude. O método foi utilizado com sucesso nesta tese para mitigar erros de posição e parâmetros dos eletrodos, formato do domínio, nível de discretização e truncamento do domínio.

O método do erro de aproximação é especialmente interessante nos casos de redução da dimensionalidade do problema. No ensaio experimental, por exemplo, a reconstrução foi feita em uma malha $2 \mathrm{D}$, com formato apenas aproximado do voluntário. Na Figura 12.1 é possível ver que os eletrodos não estão em um mesmo plano e estão visivelmente não equidistantes entre si. Como os erros de posicionamento dos eletrodos e de formato do tórax são grandes fontes de artefatos na imagem final, o método do erro de aproximação é uma ferramenta útil para a estimação do estado. Os resultados das simulações mostraram que o uso do método do erro de aproximação reduziu os artefatos e melhorou a resolução espacial nas imagens estimadas, entretanto alguns artefatos persistiram na imagem, especialmente no caso de erros de formato do domínio.

Nesta tese foi proposto o uso de um modelo de evolução para a variação de volume de ar nos pulmões durante a respiração. Tal modelo difere do usual passeio aleatório comumente utilizado nos filtro de Kalman para obtenção de imagens médicas na da TIE. O modelo proposto utiliza a informação da pressão nas vias aéreas como forçante do sistema mecânico, composto pelos pulmões, caixa torácica e musculatura. O modelo ainda considera a presença de uma rigidez não linear, relacionada com a complacência pulmonar observada experimentalmente.

O modelo proposto permite a escolha do número de compartimentos pulmonares. Esta tese não propôs modelos para os demais tecidos e órgãos que participam ou influenciam a dinâmica da respiração, porém o método proposto nesta tese permite que tais modelos sejam adicionados posteriormente.

A delimitação das regiões pulmonares é feita de forma automática através de um algoritmo de segmentação por level set. A segmentação é feita em uma imagem em tons de cinza da média entre a distribuição de resistividade normalizada e a correlação entre os pixels da imagem. A escolha desta imagem composta é apenas uma das possibilidades de segmentação e pode ser facilmente modificada. Como exemplo, 
no caso de se constatar a ocorrência de pneumotórax no paciente, seria importante incluir também informação da fase do comportamento temporal da resistividade pois é comum haver uma inversão de fase entre o volume de ar no pulmão e o volume ocupado pelo pneumotórax. Esta inversão de fase também costuma acontecer em casos de transplante de pulmão.

A escolha do número de graus de liberdade do modelo de evolução dos pulmões é um passo importante pois um grande número de compartimentos resulta em um modelo mais fidedigno, porém aumenta do número de parâmetros a serem estimados. Uma possibilidade para selecionar o número de compartimento seria adicionar este número ao conjunto de parâmetros a serem estimados. Tal algoritmo poderia identificar as regiões pulmonares com diferentes comportamentos temporais e criar um novo compartimento para cada região. Outra possibilidade não explorada nesta tese seria utilizar também o método do erro de aproximação no modelo de evolução, teoricamente evitando que o modelo precisasse ser ajustado ao paciente.

Foi ainda proposto nesta tese um outro UKF rodando em paralelo à estimação da distribuição de resistividade na caixa torácica para o ajuste dos parâmetros do modelo de evolução ao paciente sob monitoração e um terceiro filtro para a estimação dos parâmetros dos eletrodos caso seja de interesse. As estimações seguem um esquema dual como uma aproximação para a estimação conjunta.

Com o intuito de melhorar a solução do problema mal-posto, além do modelo dinâmico, outras formas de informações a prioriforam utilizadas. Este trabalho propôs a inclusão de informação a respeito do intervalo de valores que o estado pode assumir. Tal informação é adicionada na forma de uma transformação de variáveis tal que o novo estado, denominado nesta tese metaestado, possa assumir valores em $\mathbb{R}$, enquanto que a grandeza física permanece dentro de um intervalo especificado. A inclusão deste vínculo de intervalo não necessita aumentar o vetor de medidas para incluir as novas medidas de não conformidade e, portanto, não acarreta em um aumento do tamanho das matrizes e vetores utilizados no filtro de Kalman. Entretanto, tal vínculo aumenta a não linearidade do problema, especialmente próximo dos limites do intervalo.

A razão para a degradação das estimações usando a separação de interfaces na informação a priori (6.21) com dados experimentais é o nível de contraste da distribuição de resistividade estimada. A segmentação por level set torna-se mais 
sensível à artefatos quando a imagem a ser segmentada possui menor contraste. Os artefatos forçam o level set a delinear trechos que não deveriam pertencer à região interna $\Omega^{-}$(região pulmonar). Após a segmentação, a densidade de probabilidade a prioriassume que o trecho que foi classificado erroneamente como região pulmonar não deve manter a relação de suavidade com outros elementos que não estão na região pulmonar. Por este motivo, foi escolhida uma densidade de probabilidade que não faz distinção de interface nos ensaios experimentais.

Quanto aos parâmetros do modelo de evolução, as estimações não estabilizaram nas simulações como esperado. Apenas os parâmetros $a$ e $b$ foram incluídos nas estimações pois a inclusão dos parâmetros $c$ e $d$ resultaram em algumas instabilidades na estimação dos parâmetros em alguns casos. Apesar da redução do número de parâmetros a serem ajustados nos modelos, os resultados das estimações da distribuições de resistividade resultaram em boas imagens. A aproximação dos valores de pressão através das medidas elétricas no ensaio experimental pode ter influenciado nesta instabilidade, mas não deve ser a única causa de tal comportamento. Mais estudos serão necessários para identificar a causa do problema.

É possível identificar os pulmões, caixa torácica, coluna vertebral e a região do coração nas imagens obtidas com os dados experimentais. Este nível de detalhe ainda não tinha sido obtido com dados experimentais em humanos. Um dos fatores importantes para a obtenção de tais imagens foi o uso da informação $a$ prioriproveniente do um atlas anatômico. Mesmo utilizando um atlas proveniente de suínos, esta informação pôde ser utilizada em seres humanos após algumas pequenas mudanças de escala para compatibilizar as dimensões e proporções do tórax. A informação proveniente do atlas foi apenas parcialmente utilizada pois apenas o vetor médio foi utilizado. A sua matriz de covariância também poderia ser utilizada como informação a priori. Neste caso, é possível mostrar que a matriz $\boldsymbol{L}_{r}$ da informação $a$ priorié composta por uma raiz quadrada da inversa da matriz de covariância.

Quanto à estabilidade do algoritmo, foram observados alguns problemas ao longo da tese. As principais instabilidades observadas foram: (i) a definição dos pontos sigma utilizados nos filtros, apresentados em (5.24) - (5.26), podem apresentar valores de resistividade negativas dependendo dos valores das variâncias. Isto foi resolvido ao utilizar os vínculos de intervalo como informação a priori. (ii) o filtro pode se fechar 
para novas medidas e divergir devido à rápida convergência da matriz de covariância do erro de estimação. Como já apresentado na Seção 4.5, a divergência é causada pela rápida contração da matriz de covariância do estado estimado em comparação com a distância entre o estado verdadeiro e o estado estimado, e também pelo possível aumento do seu número de condição. Com esta rápida contração e aumento do número de condição, o estado verdadeiro passa a localizar-se fora do intervalo de confiança da estimativa após algumas iterações. Para procurar abrandar o problema, foi utilizado o IUKF, do inglês improved UKF, apresentado inicialmente em Perea e Elosegui [93]. (iii) é necessário manter aberto o filtro para a estimação dos parâmetros do modelo de evolução para novas medidas. Em várias simulações constatou-se que o filtro se fecha muito rápido, resultando em divergências.

Ainda com a implementação destas modificações, o ajuste dos filtros continua sendo crucial para o desempenho e sucesso do filtro na estimação. Avaliar os modelos de evolução e observação nos pontos sigma, calcular as fatorações QR e as atualizações de Cholesky são etapas de grande custo computacional, especialmente quando o vetor de medidas deve ser aumentado para incluir a informação a priori.

Para sumarizar, as contribuições desta tese foram (i) o desenvolvimento de um novo modelo de evolução paramétrico da mecânica da respiração em humanos e a adição deste modelo no filtro de Kalman. Tal modelo é importante para aumentar a velocidade de seguimento do filtro. (ii) A utilização do algoritmo de segmentação por level set em paralelo aos filtros de Kalman para identificar as regiões pulmonares. (iii) A inclusão do vínculo de intervalo como forma de limitar os valores esperado das estimativas sem aumentar o tamanho do vetor de medidas. Tal vínculo reduziu a frequência de divergência do filtro, além de permitir impor limites clinicamente significativos para os valores a serem estimados. (iv) A utilização de um atlas anatômico experimental como informação a priorina estimação das imagens com dados coletados de um voluntário. Tal atlas foi provavelmente o principal responsável pelo nível de detalhe dos resultados experimentais. (v) Utilizar o método do erro de aproximação com dados experimentais de humanos. Todas estas contribuições resultaram na melhoria das imagens de TIE apresentadas na tese. 


\section{Parte IV}

Apêndice 



\section{Apêndice A}

\section{Formulação da matriz local dos elementos finitos}

\section{A.1 Elementos que compõem o interior do domínio}

Nesta seção é mostrada a formulação das matrizes das malhas de elementos finitos 2D e 3D.

\section{A.1.1 Domínio 2D - Elementos Triangulares}

Considere um elemento triangular, com vértices numerados conforme a Figura A.1,

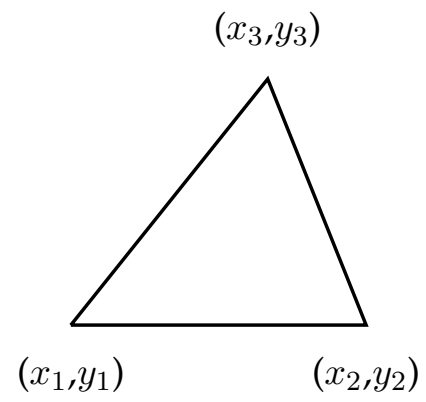

Figura A.1: Elemento triangular: numeração dos nós.

Ao longo de todo o domínio, a função que descreve o potencial elétrico é dada pela composição do potencial elétrico em cada elemento,

$$
\Psi(x, y) \simeq \sum_{i=1}^{n} w_{i} v_{i}(x, y)
$$


onde $n$ é o número de elementos finitos da malha, $w_{i}$ vale 1 quando $(x, y)$ estiver contido no $i$-ésimo elemento e vale 0 caso contrário e $v_{i}(x, y)$ é o potencial elétrico dentro do $i$-ésimo elemento da malha, sendo aproximado por uma função interpoladora linear do tipo

$$
v_{i}(x, y)=a_{i}+b_{i} x+c_{i} y=\left[\begin{array}{lll}
1 & x & y
\end{array}\right]\left[\begin{array}{l}
a_{i} \\
b_{i} \\
c_{i}
\end{array}\right],
$$

cujos os coeficientes $a_{i}, b_{i}$ e $c_{i}$ são funções dos potenciais elétricos nos três vértices do elemento [135].

Para escrever estes coeficientes em função dos potenciais elétricos dos 3 vértices que compõem o elemento, utiliza-se (A.2) para montar o sistema linear

$$
\left[\begin{array}{ccc}
1 & x_{1} & y_{1} \\
1 & x_{2} & y_{2} \\
1 & x_{3} & y_{3}
\end{array}\right]\left[\begin{array}{l}
a_{i} \\
b_{i} \\
c_{i}
\end{array}\right]=\left[\begin{array}{c}
v_{i 1} \\
v_{i 2} \\
v_{i 3}
\end{array}\right]
$$

onde $x_{k}$ e $y_{k}$, com $k \in\{1,2,3\}$, são as coordenadas de cada um dos vértices e $v_{i k} \triangleq v_{i}\left(x_{k}, y_{k}\right)$. As constantes $a_{i}, b_{i}$ e $c_{i}$ são calculadas resolvendo o sistema acima

$$
\left[\begin{array}{l}
a_{i} \\
b_{i} \\
c_{i}
\end{array}\right]=\left[\begin{array}{lll}
1 & x_{1} & y_{1} \\
1 & x_{2} & y_{2} \\
1 & x_{3} & y_{3}
\end{array}\right]^{-1}\left[\begin{array}{l}
v_{i 1} \\
v_{i 2} \\
v_{i 3}
\end{array}\right]
$$

Seja $\mathbb{A}_{i}$ a área do elemento triangular, a inversa desta matriz é dada por

$$
\begin{gathered}
{\left[\begin{array}{lll}
1 & x_{1} & y_{1} \\
1 & x_{2} & y_{2} \\
1 & x_{3} & y_{3}
\end{array}\right]^{-1}=\frac{1}{2 \mathbb{A}_{i}}\left[\begin{array}{ccc}
\alpha_{1} & \alpha_{2} & \alpha_{3} \\
\beta_{1} & \beta_{2} & \beta_{3} \\
\gamma_{1} & \gamma_{2} & \gamma_{3}
\end{array}\right]} \\
\alpha_{1}=\left|\begin{array}{ll}
x_{2} & y_{2} \\
x_{3} & y_{3}
\end{array}\right|=x_{2} y_{3}-x_{3} y_{2}, \quad \alpha_{2}=-\left|\begin{array}{ll}
x_{1} & y_{1} \\
x_{3} & y_{3}
\end{array}\right|=x_{3} y_{1}-x_{1} y_{3}, \quad \alpha_{3}=\left|\begin{array}{ll}
x_{1} & y_{1} \\
x_{2} & y_{2}
\end{array}\right|=x_{1} y_{2}-x_{2} y_{1}
\end{gathered}
$$




$$
\begin{gathered}
\beta_{1}=-\left|\begin{array}{ll}
1 & y_{2} \\
1 & y_{3}
\end{array}\right|=y_{2}-y_{3}, \quad \beta_{2}=\left|\begin{array}{ll}
1 & y_{1} \\
1 & y_{3}
\end{array}\right|=y_{3}-y_{1}, \quad \beta_{3}=-\left|\begin{array}{ll}
1 & y_{1} \\
1 & y_{2}
\end{array}\right|=y_{1}-y_{2} \\
\gamma_{1}=\left|\begin{array}{ll}
1 & x_{2} \\
1 & x_{3}
\end{array}\right|=x_{3}-x_{2}, \quad \gamma_{2}=-\left|\begin{array}{ll}
1 & x_{1} \\
1 & x_{3}
\end{array}\right|=x_{1}-x_{3}, \quad \gamma_{3}=\left|\begin{array}{ll}
1 & x_{1} \\
1 & x_{2}
\end{array}\right|=x_{2}-x_{1} .
\end{gathered}
$$

Subsistindo (A.4) em (A.2), obtém-se

$$
v_{i}(x, y)=\left[\begin{array}{lll}
1 & x & y
\end{array}\right] \frac{1}{2 \mathbb{A}_{i}}\left[\begin{array}{ccc}
\alpha_{1} & \alpha_{2} & \alpha_{3} \\
\beta_{1} & \beta_{2} & \beta_{3} \\
\gamma_{1} & \gamma_{2} & \gamma_{3}
\end{array}\right]\left[\begin{array}{c}
v_{i 1} \\
v_{i 2} \\
v_{i 3}
\end{array}\right]=\left[\begin{array}{lll}
f_{1} & f_{2} & f_{3}
\end{array}\right]\left[\begin{array}{c}
v_{i 1} \\
v_{i 2} \\
v_{i 3}
\end{array}\right]=\boldsymbol{f}_{i}^{\mathrm{T}} \tilde{\boldsymbol{v}}_{i}=\tilde{\boldsymbol{v}}_{i}^{\mathrm{T}} \boldsymbol{f}_{i}
$$

onde as funções $f_{k}$ são denominadas funções de forma,

$$
f_{k}=\frac{1}{2 \mathbb{A}_{i}}\left(\alpha_{k}+x \beta_{k}+y \gamma_{k}\right), \quad k=1,2,3
$$

$\tilde{\boldsymbol{v}}_{i}$ é vetor de potenciais elétricos nodais do $i$-ésimo elemento e $\boldsymbol{f}_{i}$ é o vetor de funções de forma.

Para associar o potencial elétrico do elemento com sua resistividade, usa-se o princípio variacional correspondente à (2.17) [52],[136, p. 231],[137] para materiais isotrópicos

$$
\pi_{i}=\frac{1}{2} \int_{\mathbb{V}_{i}} \frac{1}{\rho_{i}}\|\boldsymbol{E}\|^{2} d V-\int_{\partial \mathbb{V}_{i}} v_{i}\left(-\boldsymbol{J}_{i} \cdot \hat{\boldsymbol{n}}_{i}\right) d A
$$

onde $\mathbb{V}_{i}$ é o volume do $i$-ésimo elemento, $\hat{\boldsymbol{n}}_{i}$ é o versor normal às arestas do elemento e $\boldsymbol{J}_{i}$ é o vetor densidade de corrente elétrica livre£ imposta nas arestas do elemento. A primeira parcela representa a energia armazenada em virtude do campo potencial dentro do $i$-ésimo elemento, a segunda parcela representa a energia que atravessa os bordos do elemento através da corrente elétrica imposta (condição de contorno).

\footnotetext{
${ }^{1} \mathrm{O}$ sinal negativo dentro da segunda integral é usado para manter a convenção de que correntes positivas entram no elemento e correntes negativas saem do elemento.
} 
Usando (A.9) para aproximar $\Psi$ em (2.9) e substituindo em (A.11), chega-se a

$$
\pi_{i}=\frac{1}{2} \int_{\mathbb{V}_{i}} \frac{1}{\rho_{i}}\left\|-\nabla v_{i}\right\|^{2} d V-\int_{\partial \mathbb{V}_{i}} \tilde{\boldsymbol{v}}_{i}^{\mathrm{T}} \boldsymbol{f}_{i}\left(-\boldsymbol{J}_{i} \cdot \hat{\boldsymbol{n}}_{i}\right) d A,
$$

cujo gradiente da função potencial elétrico do elemento é dado pela derivação de (A.9)

$$
\nabla v_{i}=\left[\begin{array}{c}
\frac{\partial v_{i}}{\partial x} \\
\frac{\partial v_{i}}{\partial y}
\end{array}\right]=\left[\begin{array}{lll}
\frac{\partial f_{1}}{\partial x} & \frac{\partial f_{2}}{\partial x} & \frac{\partial f_{3}}{\partial x} \\
\frac{\partial f_{1}}{\partial y} & \frac{\partial f_{2}}{\partial y} & \frac{\partial f_{3}}{\partial y}
\end{array}\right]\left[\begin{array}{c}
v_{i 1} \\
v_{i 2} \\
v_{i 3}
\end{array}\right]=\boldsymbol{F}_{i} \tilde{\boldsymbol{v}}_{i} .
$$

Substituindo (A.13) em (A.12) e notando que $\left\|-\nabla v_{i}\right\|^{2}=\nabla v_{i}^{\mathrm{T}} \nabla v_{i}$, obtém-se

$$
\pi_{i}=\frac{1}{2} \tilde{\boldsymbol{v}}_{i}^{\mathrm{T}}\left[\int_{\mathbb{V}_{i}} \frac{1}{\rho_{i}} \boldsymbol{F}_{i}^{\mathrm{T}} \boldsymbol{F}_{i} d V\right] \tilde{\boldsymbol{v}}_{i}-\tilde{\boldsymbol{v}}_{i}^{\mathrm{T}}\left[\int_{\partial \mathbb{V}_{i}} \boldsymbol{f}_{i}\left(-\boldsymbol{J}_{i} \cdot \hat{\boldsymbol{n}}_{i}\right) d A\right] .
$$

O ponto estacionário deste funcional é encontrado impondo $\frac{\partial \pi_{i}}{\partial \tilde{v}_{i}}=\mathbf{0}$, o que resulta em

$$
\begin{aligned}
{\left[\int_{\mathbb{V}_{i}} \frac{1}{\rho_{i}} \boldsymbol{F}_{i}^{\mathrm{T}} \boldsymbol{F}_{i} d V\right] \tilde{\boldsymbol{v}}_{i} } & =\left[\int_{\partial \mathbb{V}_{i}} \boldsymbol{f}_{i}\left(-\boldsymbol{J}_{i} \cdot \hat{\boldsymbol{n}}_{i}\right) d A\right] \\
\tilde{\boldsymbol{Y}}_{i} \tilde{\boldsymbol{v}}_{i} & =\tilde{\boldsymbol{I}}_{i},
\end{aligned}
$$

onde $\tilde{\boldsymbol{Y}}_{i} \in \mathbb{R}^{3 \times 3}$ é chamada matriz de condutividade local do $i$-ésimo elemento e $\tilde{\boldsymbol{I}}_{i} \in \mathbb{R}^{3 \times 1}$ é chamado de vetor de correntes externas local.

Como a imposição de corrente elétrica é feita através de eletrodos posicionados em $\partial \boldsymbol{\Omega}$, somente os elementos finitos que representam a interface dos eletrodos apresentam $\boldsymbol{J}_{i} \neq \mathbf{0}$. Para os elementos que representam o interior do domínio, $\boldsymbol{J}_{i}=\mathbf{0}$ e, por consequência, $\tilde{\boldsymbol{I}}_{i}=\mathbf{0}$.

Realizando a integração de $\tilde{\boldsymbol{Y}}_{i}$, adotando uma espessura $h$ uniforme para o elemento e considerando resistividade homogênea e isotrópica, chega-se a

$$
\tilde{\boldsymbol{Y}}_{i}\left(\rho_{i}\right)=\frac{h}{4 \mathbb{A}_{i} \rho_{i}}\left[\begin{array}{ccc}
\beta_{1}^{2}+\gamma_{1}^{2} & \beta_{1} \beta_{2}+\gamma_{1} \gamma_{2} & \beta_{1} \beta_{3}+\gamma_{1} \gamma_{3} \\
& \beta_{2}^{2}+\gamma_{2}^{2} & \beta_{2} \beta_{3}+\gamma_{2} \gamma_{3} \\
\operatorname{sim} . & & \beta_{3}^{2}+\gamma_{3}^{2}
\end{array}\right] .
$$




\section{A.1.2 Domínio 3D - Elementos Tetraédricos}

Considere um elemento tetraédrico, com vértices numerados conforme Figura A.2

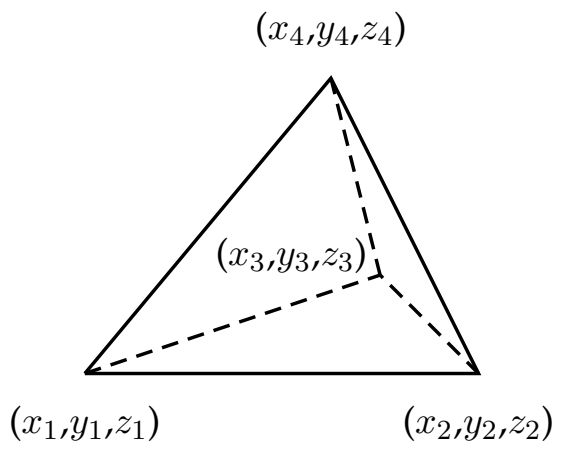

Figura A.2: Elemento tetraédrico: numeração dos nós.

Ao longo de todo o domínio, a função que descreve o potencial elétrico é dada pela composição do potencial elétrico em cada elemento,

$$
\Psi(x, y, z) \simeq \sum_{i=1}^{n} w_{i} v_{i}(x, y, z)
$$

onde $w_{i}$ vale 1 quando $(x, y, z)$ estiver contido no $i$-ésimo elemento e vale 0 caso contrário e $v_{i}(x, y, z)$ é o potencial elétrico dentro do $i$-ésimo elemento da malha, sendo aproximado por uma função interpoladora linear do tipo

$$
v_{i}(x, y)=a_{i}+b_{i} x+c_{i} y+d_{i} z
$$

cujos os coeficientes $a_{i}, b_{i}, c_{i}$ e $d_{i}$ são funções dos potenciais elétricos nos quatro vértices do elemento. Seguindo os mesmos passos do caso 2D, chega-se em

$$
v_{i}(x, y, z)=\left[\begin{array}{llll}
f_{1} & f_{2} & f_{3} & f_{4}
\end{array}\right]\left[\begin{array}{c}
v_{i 1} \\
v_{i 2} \\
v_{i 3} \\
v_{i 4}
\end{array}\right]=\boldsymbol{f}_{i}^{\mathrm{T}} \tilde{\boldsymbol{v}}_{i}=\tilde{\boldsymbol{v}}_{i}^{\mathrm{T}} \boldsymbol{f}_{i}
$$

cujas funções de forma $f_{k}$ são

$$
f_{k}=\frac{1}{6 \mathbb{V}_{i}}\left(\alpha_{k}+x \beta_{k}+y \gamma_{k}+z \delta_{k}\right), \quad k=1,2,3,4
$$


onde $\mathbb{V}_{i}$ é o volume deste tetraedro e

$$
\begin{gathered}
\alpha_{1}=\left|\begin{array}{lll}
x_{2} & y_{2} & z_{2} \\
x_{3} & y_{3} & z_{3} \\
x_{4} & y_{4} & z_{4}
\end{array}\right|, \quad \alpha_{2}=-\left|\begin{array}{lll}
x_{1} & y_{1} & z_{1} \\
x_{3} & y_{3} & z_{3} \\
x_{4} & y_{4} & z_{4}
\end{array}\right|, \quad \alpha_{3}=\left|\begin{array}{lll}
x_{1} & y_{1} & z_{1} \\
x_{2} & y_{2} & z_{2} \\
x_{4} & y_{4} & z_{4}
\end{array}\right|, \quad \alpha_{4}=-\left|\begin{array}{lll}
x_{1} & y_{1} & z_{1} \\
x_{2} & y_{2} & z_{2} \\
x_{3} & y_{3} & z_{3}
\end{array}\right| \\
\beta_{1}=-\left|\begin{array}{lll}
1 & y_{2} & z_{2} \\
1 & y_{3} & z_{3} \\
1 & y_{4} & z_{4}
\end{array}\right|, \quad \beta_{2}=\left|\begin{array}{lll}
1 & y_{1} & z_{1} \\
1 & y_{3} & z_{3} \\
1 & y_{4} & z_{4}
\end{array}\right|, \quad \beta_{3}=-\left|\begin{array}{lll}
1 & y_{1} & z_{1} \\
1 & y_{2} & z_{2} \\
1 & y_{4} & z_{4}
\end{array}\right|, \quad \beta_{4}=\left|\begin{array}{lll}
1 & y_{1} & z_{1} \\
1 & y_{2} & z_{2} \\
1 & y_{3} & z_{3}
\end{array}\right| \\
\gamma_{1}=\left|\begin{array}{lll}
1 & x_{2} & z_{2} \\
1 & x_{3} & z_{3} \\
1 & x_{4} & z_{4}
\end{array}\right|, \quad \gamma_{2}=-\left|\begin{array}{lll}
1 & x_{1} & z_{1} \\
1 & x_{3} & z_{3} \\
1 & x_{4} & z_{4}
\end{array}\right|, \quad \gamma_{3}=\left|\begin{array}{lll}
1 & x_{1} & z_{1} \\
1 & x_{2} & z_{2} \\
1 & x_{4} & z_{4}
\end{array}\right|, \quad \gamma_{4}=-\left|\begin{array}{lll}
1 & x_{1} & z_{1} \\
1 & x_{2} & z_{2} \\
1 & x_{3} & z_{3}
\end{array}\right| \\
\delta_{1}=-\left|\begin{array}{lll}
1 & x_{3} & y_{3} \\
1 & x_{4} & y_{4}
\end{array}\right|, \quad \delta_{2}=\left|\begin{array}{lll}
1 & x_{1} & y_{1} \\
1 & x_{3} & y_{3} \\
1 & x_{4} & y_{4}
\end{array}\right|, \quad \delta_{3}=-\left|\begin{array}{lll}
1 & x_{1} & y_{1} \\
1 & x_{2} & y_{2} \\
1 & x_{4} & y_{4}
\end{array}\right|, \quad \delta_{4}=\left|\begin{array}{lll}
1 & x_{1} & y_{1} \\
1 & x_{2} & y_{2} \\
1 & x_{3} & y_{3}
\end{array}\right| .
\end{gathered}
$$

O princípio variacional é dado por (A.11), porém o gradiente do potencial neste caso é

$$
\nabla v_{i}=\left[\begin{array}{c}
\frac{\partial v_{i}}{\partial x} \\
\frac{\partial v_{i}}{\partial y} \\
\frac{\partial v_{i}}{\partial z}
\end{array}\right]=\left[\begin{array}{cccc}
\frac{\partial f_{1}}{\partial x} & \frac{\partial f_{2}}{\partial x} & \frac{\partial f_{3}}{\partial x} & \frac{\partial f_{4}}{\partial x} \\
\frac{\partial f_{1}}{\partial y} & \frac{\partial f_{2}}{\partial y} & \frac{\partial f_{3}}{\partial y} & \frac{\partial f_{4}}{\partial y} \\
\frac{\partial f_{1}}{\partial z} & \frac{\partial f_{2}}{\partial z} & \frac{\partial f_{3}}{\partial z} & \frac{\partial f_{4}}{\partial z}
\end{array}\right]\left[\begin{array}{c}
v_{i 1} \\
v_{i 2} \\
v_{i 3} \\
v_{i 4}
\end{array}\right]=\boldsymbol{F}_{i} \tilde{\boldsymbol{v}}_{i}
$$

Realizando a integração de (A.15), assumindo que a condutividade elétrica do elemento seja isotrópica, chega-se na matriz de condutividade local

$$
\tilde{\boldsymbol{Y}}_{i}\left(\rho_{i}\right)=\frac{1}{36 \mathbb{V}_{i} \rho_{i}}\left[\begin{array}{cccc}
\beta_{1}^{2}+\gamma_{1}^{2}+\delta_{1}^{2} & \beta_{1} \beta_{2}+\gamma_{1} \gamma_{2}+\delta_{1} \delta_{2} & \beta_{1} \beta_{3}+\gamma_{1} \gamma_{3}+\delta_{1} \delta_{3} & \beta_{1} \beta_{4}+\gamma_{1} \gamma_{4}+\delta_{1} \delta_{4} \\
& \beta_{2}^{2}+\gamma_{2}^{2}+\delta_{2}^{2} & \beta_{2} \beta_{3}+\gamma_{2} \gamma_{3}+\delta_{2} \delta_{3} & \beta_{2} \beta_{4}+\gamma_{2} \gamma_{4}+\delta_{2} \delta_{4} \\
& & \beta_{3}^{2}+\gamma_{3}^{2}+\delta_{3}^{2} & \beta_{3} \beta_{4}+\gamma_{3} \gamma_{4}+\delta_{3} \delta_{4} \\
\operatorname{sim} . & & & \beta_{4}^{2}+\gamma_{4}^{2}+\delta_{4}^{2}
\end{array}\right] .
$$




\section{A.1.3 Domínio 3D - Elementos prismáticos de base triangular}

No caso de malhas 3D extrudadas de uma malha triangular 2D, pode-se encontrar, para cada elemento triangular da projeção, três elementos tetraédricos que juntos preenchem o volume de um prisma de base triangular. Com isto em mente, pode-se trabalhar com elementos prismáticos ao invés de tetraédricos, com o intuito de reduzir o número de incógnitas do problema inverso. Entretanto esta redução impõe que os elementos que compõem este volume prismático apresentem a mesma resistividade.

A matriz de condutividade local do elemento prismático pode ser determinada a partir da matriz local de cada um dos tetraedros que o compõe. Fazendo isto, o elemento prismático resultante apresenta funções de interpolação do potencial elétrico linear por partes.
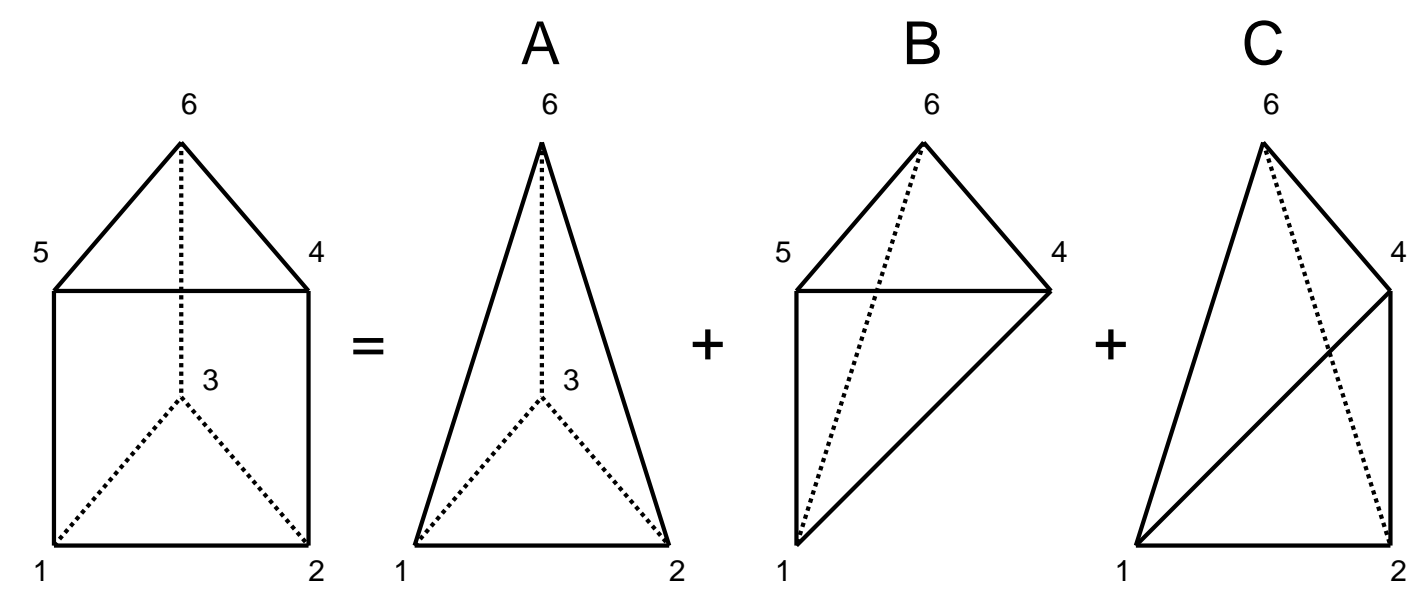

Figura A.3: Divisão do prisma em três tetraedros: numeração dos nós.

Para calcular a matriz de condutividade do prisma de bases triangulares e paralelas, primeiro ele é dividido em 3 tetraedros, conforme a Figura A.3. Eles serão identificados por sobrescritos ${ }^{A},{ }^{B} \mathrm{e}^{C}$ respectivamente. A Tabela A.1 mostra como é dividido o prisma em 3 elementos.

Tabela A.1: Numeração dos nós.

\begin{tabular}{ccccc}
\hline elemento & nó 1 & nó 2 & nó 3 & nó 4 \\
\hline A & 1 & 2 & 3 & 6 \\
B & 4 & 5 & 6 & 1 \\
C & 4 & 1 & 6 & 2 \\
\hline
\end{tabular}


Identificando os elementos da matriz de condutividade (A.27) por $y_{i j}$, com $i, j \in$ $\{1,2,3,4\}$, as matrizes locais dos tetraedros, considerando a numeração do elemento prismático, são

$$
\begin{aligned}
& \tilde{\boldsymbol{Y}}_{i}^{A}=\frac{1}{36 \mathbb{V}_{i}^{A} \rho_{i}^{A}}\left[\begin{array}{cccccc}
y_{11}^{A} & y_{12}^{A} & y_{13}^{A} & 0 & 0 & y_{14}^{A} \\
& y_{22}^{A} & y_{23}^{A} & 0 & 0 & y_{24}^{A} \\
& & y_{33}^{A} & 0 & 0 & y_{34}^{A} \\
& & & 0 & 0 & 0 \\
& & & & 0 & 0 \\
& & & & & y_{44}^{A}
\end{array}\right]=\frac{1}{36 \mathbb{V}_{i}^{A} \rho_{i}^{A}} \boldsymbol{M}^{A}
\end{aligned}
$$

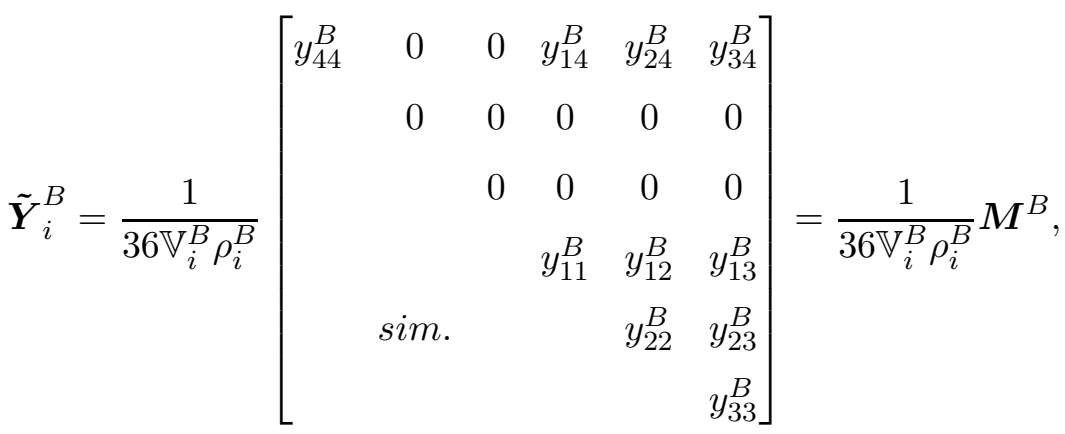

$$
\begin{aligned}
& \tilde{\boldsymbol{Y}}_{i}^{C}=\frac{1}{36 \mathbb{V}_{i}^{C} \rho_{i}^{C}}\left[\begin{array}{cccccc}
y_{22}^{C} & y_{24}^{C} & 0 & y_{21}^{C} & 0 & y_{23}^{C} \\
& y_{44}^{C} & 0 & y_{41}^{C} & 0 & y_{43}^{C} \\
& & 0 & 0 & 0 & 0 \\
& & & y_{11}^{C} & 0 & y_{13}^{C} \\
& & & & 0 & 0 \\
& & & & & y_{33}^{C}
\end{array}\right]=\frac{1}{36 \mathbb{V}_{i}^{C} \rho_{i}^{C}} \boldsymbol{M}^{C} .
\end{aligned}
$$

Sabe-se que $\mathbb{V}_{i}^{A}=\mathbb{V}_{i}^{B}=\mathbb{V}_{i}^{C}=\mathbb{V}_{i}$ pois o prisma tem bases paralelas?. Assumindo que $\rho_{i}^{A}=\rho_{i}^{B}=\rho_{i}^{C}=\rho_{i}$, a matriz de condutividade local do elemento prismático é dada por

$$
\tilde{\boldsymbol{Y}}_{i}\left(\rho_{i}\right)=\frac{1}{36 \mathbb{V}_{i} \rho_{i}}\left(\boldsymbol{M}^{A}+\boldsymbol{M}^{B}+\boldsymbol{M}^{C}\right)
$$

onde $\mathbb{V}_{i}$ é o volume dos tetraedros.

\footnotetext{
${ }^{2}$ Note que os tetraedros A e B têm bases iguais à base do prisma então, pela fórmula do volume de pirâmides, seus volumes são de um terço do volume do prisma cada um. Com isto, o volume do terceiro prisma deve ser também de um terço do volume do prisma.
} 


\section{A.1.4 Matriz Global}

O princípio variacional aplicado em todo o domínio discretizado corresponde à somatória 3 de todas as matrizes locais e vetores de corrente dos elementos

$$
\begin{array}{r}
\boldsymbol{Y}(\boldsymbol{\rho})=\sum_{i=1}^{n} \tilde{\boldsymbol{Y}}_{i}\left(\rho_{i}\right) \\
\boldsymbol{I}=\sum_{i=1}^{n} \tilde{\boldsymbol{I}}_{i}=\mathbf{0},
\end{array}
$$

onde $\boldsymbol{Y} \in \mathbb{R}^{m \times m}$ é a matriz de condutividade global, $\boldsymbol{I} \in \mathbb{R}^{m}$ é o vetor de correntes externas impostas, $\boldsymbol{\rho} \in \mathbb{R}^{n}$ é o vetor de resistividade dos elementos, $m$ é o número de nós e $n$ é o número de elementos da malha.

\section{A.2 Elementos que compõem a interface dos eletrodos}

A discretização dos eletrodos segue Hua et al. [52]. Neste trabalho, o modelo completo de eletrodos é discretizado em elementos finitos de duas dimensões. Neste trabalho, uma extensão 3D é feita para este modelo.

Cada interface de eletrodo é dividida em elementos quadriláteros planos (2D) ou hexaédricos (3D). A Figura A.4mostra uma interface 2D, composta por dois elementos. Neste caso, os nós 1, 2 e 3 estão em contato com o domínio e os nós 4, 5 e 6 estão em contato com a superfície metálica do eletrodo.

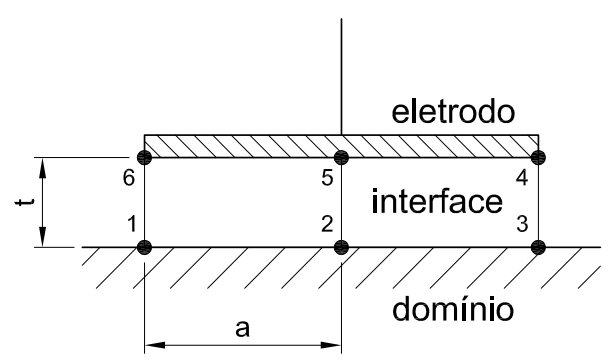

Figura A.4: Discretização da interface. (adaptado de Hua et al. [52]).

Duas hipóteses são usadas pelos autores:

1. Considera-se que os potenciais elétricos nos nós que estão em contato com o

\footnotetext{
${ }^{3}$ Antes da somatória é necessário mudar a numeração dos nós do sistema local para a numeração global da malha.
} 
eletrodo (no caso 4, 5 e 6) são iguais devido à pequena resistividade elétrica do material do eletrodo;

2. A espessura $t$ da interface tem dimensão muito menor que a largura $a$ do eletrodo $(t \ll a)$.

A seguir mostra-se o equacionamento dos elementos quadriláteros e hexaédricos do modelo de interface de eletrodo.

\section{A.2.1 Modelo da interface dos eletrodos 2D}

Seja o elemento retangular que compõe o $i$-ésimo eletrodo, $i=1,2, \ldots, e$, cujos nós são numerados conforme a Figura A.5. Com esta numeração, os nós 1 e 2 estão em contato com $\partial \boldsymbol{\Omega}$ e os nós 3 e 4 estão em contato com a parte metálica do eletrodo.

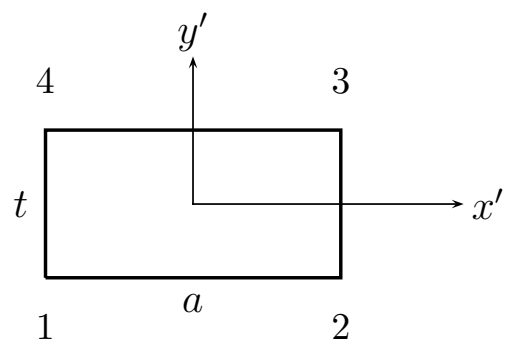

Figura A.5: Elemento retangular: numeração dos nós e dimensões.

Criando um sistema de coordenadas local $\left(O x^{\prime} y^{\prime}\right)$, com origem no centro do elemento e com os eixos paralelos às arestas, pode-se utilizar as funções de forma

$$
\begin{gathered}
f_{1}=\frac{1}{a t}\left(\frac{1}{2} a-x^{\prime}\right)\left(\frac{1}{2} t-y^{\prime}\right), \quad f_{2}=\frac{1}{a t}\left(\frac{1}{2} a+x^{\prime}\right)\left(\frac{1}{2} t-y^{\prime}\right) \\
f_{3}=\frac{1}{a t}\left(\frac{1}{2} a+x^{\prime}\right)\left(\frac{1}{2} t+y^{\prime}\right), \quad f_{4}=\frac{1}{a t}\left(\frac{1}{2} a-x^{\prime}\right)\left(\frac{1}{2} t+y^{\prime}\right) \\
f_{r}=\left[\begin{array}{llll}
f_{1} & f_{2} & f_{3} & f_{4}
\end{array}\right]^{\mathrm{T}}
\end{gathered}
$$

para interpolar o potencial elétrico dentro do elemento. $f_{r}$ é o vetor função de forma do elemento retangular. A matriz de condutividade local $\tilde{\boldsymbol{Y}}_{r}$ é determinada através do princípio variacional (A.11). Integrando-se o termo da esquerda de (A.15), mostra-se que a matriz de condutividade local do elemento retangular, com 
resistividade isotrópica é dada por

$$
\tilde{\boldsymbol{Y}}_{r}=\int_{\mathbb{V}_{r e t}} \frac{1}{\rho} \boldsymbol{F}_{r}^{\mathrm{T}} \boldsymbol{F}_{r} d V=b \int_{-\frac{a}{2}}^{\frac{a}{2}} \int_{-\frac{t}{2}}^{\frac{t}{2}} \frac{1}{\rho} \boldsymbol{F}_{r}^{\mathrm{T}} \boldsymbol{F}_{r} d y^{\prime} d x^{\prime}
$$

onde $b$ é a largura do eletrodo, perpendicular a $a$ e $t$, e

$$
\boldsymbol{F}_{r}=\left[\begin{array}{cccc}
\frac{\partial f_{1}}{\partial x^{\prime}} & \frac{\partial f_{2}}{\partial x^{\prime}} & \frac{\partial f_{3}}{\partial x^{\prime}} & \frac{\partial f_{4}}{\partial x^{\prime}} \\
\frac{\partial f_{1}}{\partial y^{\prime}} & \frac{\partial f_{2}}{\partial y^{\prime}} & \frac{\partial f_{3}}{\partial y^{\prime}} & \frac{\partial f_{4}}{\partial y^{\prime}} .
\end{array}\right]
$$

Integrando-se A.37 no volume do elemento, chega-se a

$$
\tilde{\boldsymbol{Y}}_{r}=\frac{b}{6 a t \rho}\left[\begin{array}{cccc}
a_{1} & a_{2} & a_{3} & a_{4} \\
& a_{1} & a_{4} & a_{3} \\
& a_{1} & a_{2} \\
\text { sim. } & & a_{1}
\end{array}\right]
$$

Agora, considerando que $t \ll a$,

$$
\begin{array}{ll}
a_{1}=2 a^{2} & , \quad a_{2}=a^{2} \\
a_{3}=-a^{2} & , \quad a_{4}=-2 a^{2} .
\end{array}
$$

No caso de elementos da interface, a matriz de correntes externas local não será mais o vetor nulo. A determinação do vetor de correntes local é feita através da integração do termo da direita de (A.15).

Como apresentado, a corrente elétrica é imposta ao longo da aresta 3-4 do elemento, que está em contato com a superfície metálica do eletrodo. Desta forma, a integral reduz-se a

$$
\tilde{\boldsymbol{I}}_{r}=b \int_{-\frac{a}{2}}^{\frac{a}{2}} \boldsymbol{f}_{r}\left(x, y^{\prime}=t / 2\right)(-\boldsymbol{J} . \hat{\boldsymbol{n}}) d x^{\prime}
$$

Considera-se também que o vetor densidade de corrente elétrica que atravessa a interface é perpendicular à aresta 3-4, então o produto escalar $(-\boldsymbol{J} . \hat{\boldsymbol{n}})$ é $J$ se a corrente 
atravessa a interface entrando no domínio, ou $-J$ se a corrente atravessa a interface saindo do domínio. Por último, admite-se que a densidade de corrente seja uniforme ao longo de toda a aresta. Isto resulta em

$$
\tilde{\boldsymbol{I}}_{r}= \pm \frac{J a b}{2}\left[\begin{array}{llll}
0 & 0 & 1 & 1
\end{array}\right]^{\mathrm{T}}= \pm \frac{I}{2}\left[\begin{array}{llll}
0 & 0 & 1 & 1
\end{array}\right]^{\mathrm{T}}
$$

onde $I=J a b$ é a corrente total imposta no eletrodo.

Como os nós 3 e 4 estão em contato com a superfície metálica, que apresenta resistividade elétrica muito menor que a interface, pode-se supor que $v_{3}=v_{4}$ e pode-se criar um nó virtual 3' que representa ambos, conforme Figura A.6.

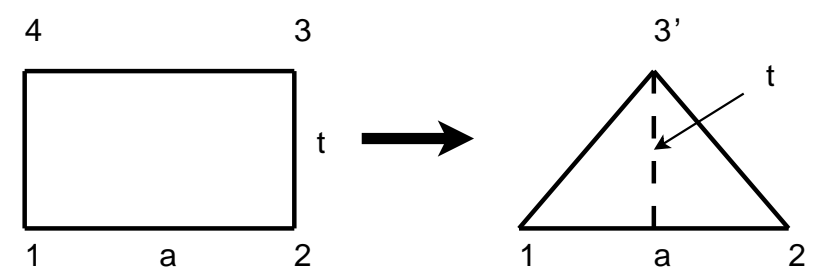

Figura A.6: Elemento retangular: união dos nós.

Ao impor que $v_{3}=v_{4}$, chega-se a

$$
\begin{aligned}
\tilde{\boldsymbol{Y}}_{r} \tilde{\boldsymbol{v}}_{r} & =\tilde{\boldsymbol{I}}_{r} \\
\tilde{\boldsymbol{v}}_{r} & =\left[\begin{array}{lll}
v_{1} & v_{2} & v_{3^{\prime}}
\end{array}\right]^{\mathrm{T}} \\
\tilde{\boldsymbol{Y}}_{r} & =\frac{b a}{6 t \rho}\left[\begin{array}{ccc}
2 & 1 & -3 \\
& 2 & -3 \\
\text { sim. } & & 6
\end{array}\right] \\
\tilde{\boldsymbol{I}}_{r} & = \pm I\left[\begin{array}{lll}
0 & 0 & 1
\end{array}\right]^{\mathrm{T}} .
\end{aligned}
$$

Pode-se isolar o termo t $\rho$ e considerá-lo uma única grandeza física que caracteriza a resistência por unidade de área de contato do eletrodo (unidade SI $\Omega \mathrm{m}^{2}$ ), que será nomeado $\rho^{\prime}$ parâmetro de eletrodo.

Por fim, a matriz local da interface do $i$-ésimo eletrodo, $\tilde{\boldsymbol{Y}}_{i}^{\text {elet }}\left(\rho_{i}^{\prime}\right), i=1,2, \ldots, e$ é composta pela soma das matrizes de todos os elementos retangulares, considerando um mesmo parâmetro de eletrodo $\rho_{i}^{\prime}$ para todos os elementos. A Figura A.7 mostra 
uma interface composta por $n-1$ elementos retangulares. O nó virtual é o mesmo para todos os elementos quadriláteros e é o último na numeração local do eletrodo.

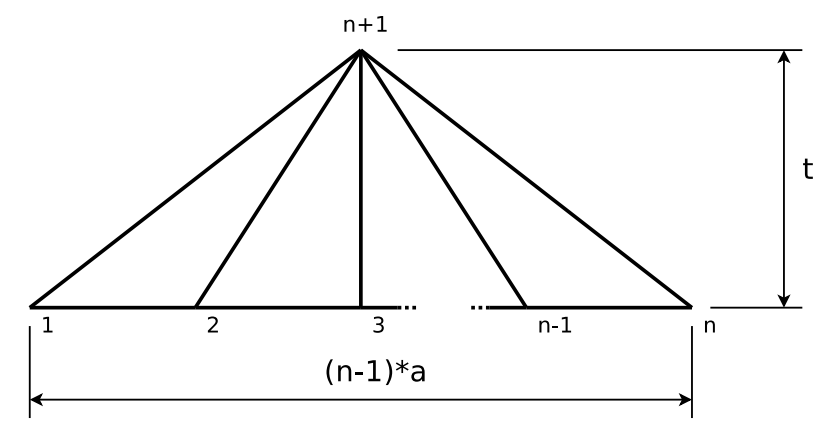

Figura A.7: Modelo de eletrodo com $n-1$ sub elementos quadriláteros.

A corrente é injetada através do nó virtual $n+1$, então $\tilde{\boldsymbol{I}}_{i}^{\text {elet }}=\left[\begin{array}{lllll}0 & 0 & \ldots & 0 & I_{i}\end{array}\right]^{\mathrm{T}}$, onde $I_{i}$ é a corrente elétrica total injetada no $i$-ésimo eletrodo.

\section{A.2.2 Modelo da interface dos eletrodos 3D}

Seja o elemento hexaédrico de faces paralelas, que compõe o eletrodo, numerado conforme a Figura A.8. Com esta numeração, os nós 1 a 4 estão em contato com $\partial \Omega \mathrm{e}$ os nós 5 a 8 estão em contato com o eletrodo metálico.

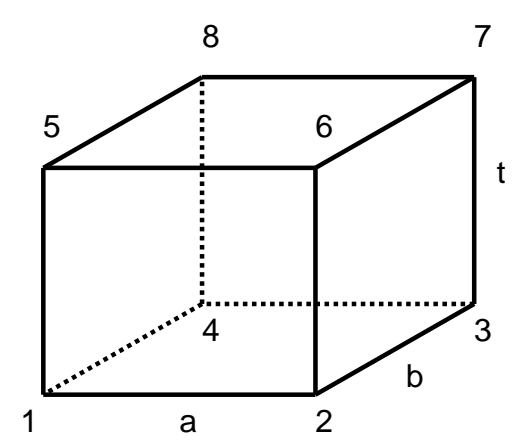

Figura A.8: Elemento hexaédrico: numeração dos nós e dimensões.

Criando um sistema de coordenadas local com origem no centro do elemento hexaédrico e os eixos paralelos às arestas do mesmo $\left(O x^{\prime} y^{\prime} z^{\prime}\right)$, pode-se utilizar as 
seguintes funções de forma

$$
\begin{aligned}
& f_{1}=\frac{1}{a b t}\left(\frac{1}{2} a-x^{\prime}\right)\left(\frac{1}{2} b-y^{\prime}\right)\left(\frac{1}{2} t-z^{\prime}\right), \quad f_{2}=\frac{1}{a b t}\left(\frac{1}{2} a+x^{\prime}\right)\left(\frac{1}{2} b-y^{\prime}\right)\left(\frac{1}{2} t-z^{\prime}\right) \\
& f_{3}=\frac{1}{a b t}\left(\frac{1}{2} a+x^{\prime}\right)\left(\frac{1}{2} b+y^{\prime}\right)\left(\frac{1}{2} t-z^{\prime}\right), \quad f_{4}=\frac{1}{a b t}\left(\frac{1}{2} a-x^{\prime}\right)\left(\frac{1}{2} b+y^{\prime}\right)\left(\frac{1}{2} t-z^{\prime}\right) \\
& f_{5}=\frac{1}{a b t}\left(\frac{1}{2} a-x^{\prime}\right)\left(\frac{1}{2} b-y^{\prime}\right)\left(\frac{1}{2} t+z^{\prime}\right), \quad f_{6}=\frac{1}{a b t}\left(\frac{1}{2} a+x^{\prime}\right)\left(\frac{1}{2} b-y^{\prime}\right)\left(\frac{1}{2} t+z^{\prime}\right) \\
& f_{7}=\frac{1}{a b t}\left(\frac{1}{2} a+x^{\prime}\right)\left(\frac{1}{2} b+y^{\prime}\right)\left(\frac{1}{2} t+z^{\prime}\right), \quad f_{8}=\frac{1}{a b t}\left(\frac{1}{2} a-x^{\prime}\right)\left(\frac{1}{2} b+y^{\prime}\right)\left(\frac{1}{2} t+z^{\prime}\right) \\
& \boldsymbol{f}_{h}=\left[\begin{array}{llll}
f_{1} & f_{2} & \cdots & f_{8}
\end{array}\right]^{\mathrm{T}}
\end{aligned}
$$

para interpolar o potencial elétrico dentro do elemento. A matriz de condutividade local $\tilde{\boldsymbol{Y}}_{h}$ é calculada com o princípio variacional (A.11). Procedendo de maneira análoga ao caso 2D, mostra-se que a matriz de condutividade local é dada por

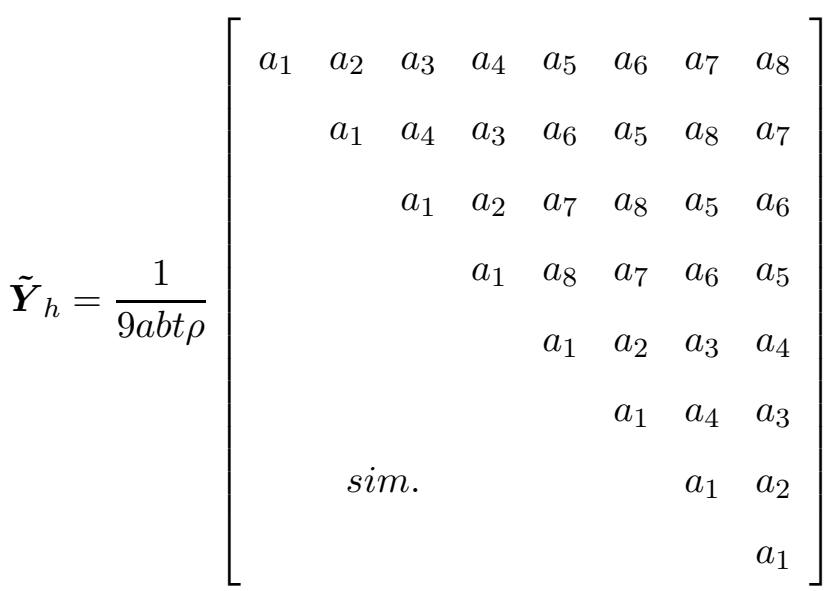

$$
\begin{aligned}
& a_{1}=a^{2} b^{2}+b^{2} t^{2}+a^{2} t^{2} \\
& a_{1}=a^{2} b^{2} \\
& a_{2}=\frac{1}{2}\left(a^{2} b^{2}-2 b^{2} t^{2}+a^{2} t^{2}\right) \\
& a_{2}=\frac{1}{2}\left(a^{2} b^{2}\right) \\
& a_{3}=\frac{1}{4}\left(a^{2} b^{2}-2 b^{2} t^{2}-2 a^{2} t^{2}\right) \\
& a_{3}=\frac{1}{4}\left(a^{2} b^{2}\right) \\
& a_{4}=\frac{1}{2}\left(a^{2} b^{2}+b^{2} t^{2}-2 a^{2} t^{2}\right) \\
& \Rightarrow t \ll a, b \Rightarrow a_{4}=\frac{1}{2}\left(a^{2} b^{2}\right) \\
& a_{5}=-\frac{1}{2}\left(2 a^{2} b^{2}-b^{2} t^{2}-a^{2} t^{2}\right) \\
& a_{5}=-\left(a^{2} b^{2}\right) \\
& a_{6}=-\frac{1}{4}\left(2 a^{2} b^{2}+2 b^{2} t^{2}-a^{2} t^{2}\right) \\
& a_{6}=-\frac{1}{2}\left(a^{2} b^{2}\right) \\
& a_{7}=-\frac{1}{4}\left(a^{2} b^{2}+b^{2} t^{2}+a^{2} t^{2}\right) \\
& a_{7}=-\frac{1}{4}\left(a^{2} b^{2}\right) \\
& a_{8}=-\frac{1}{4}\left(2 a^{2} b^{2}-b^{2} t^{2}+2 a^{2} t^{2}\right) \\
& a_{8}=-\frac{1}{2}\left(a^{2} b^{2}\right)
\end{aligned}
$$

A determinação do vetor de correntes local é feito através da integração do termo da direita de (A.15). Procedendo como no caso 2D, chega-se em

$$
\tilde{\boldsymbol{I}}_{h}= \pm \frac{J a b}{4}\left[\begin{array}{llllllll}
0 & 0 & 0 & 0 & 1 & 1 & 1 & 1
\end{array}\right]^{\mathrm{T}}
$$


Similar ao caso 2D, os nós que estão em contato com o eletrodo possuem o mesmo potencial elétrico (nós 5 a 8) e podem ser representados por um único nó virtual 5', conforme Figura A.9. Com isto, as quatro últimas colunas e linhas da matriz de condutividade local podem ser somadas.

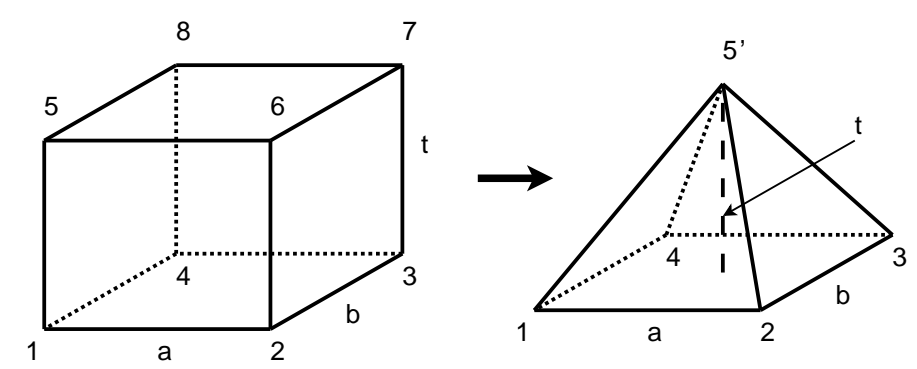

Figura A.9: Elemento hexaédrico: união dos nós.

Com esta modificação, o sistema local (

$$
\begin{aligned}
& \tilde{\boldsymbol{Y}}_{h} \tilde{\boldsymbol{v}}_{h}=\tilde{\boldsymbol{I}}_{h} \\
& \tilde{\boldsymbol{v}}_{h}=\left[\begin{array}{lllll}
v_{1} & v_{2} & v_{3} & v_{4} & v_{5^{\prime}}
\end{array}\right]^{\mathrm{T}} \\
& \tilde{\boldsymbol{Y}}_{h}=\frac{a b}{9 t \rho}\left[\begin{array}{rrrrr}
1 & \frac{1}{2} & \frac{1}{4} & \frac{1}{2} & -\frac{9}{4} \\
& 1 & \frac{1}{2} & \frac{1}{4} & -\frac{9}{4} \\
& 1 & \frac{1}{2} & -\frac{9}{4} \\
& & & 1 & -\frac{9}{4} \\
\operatorname{sim} . & & & 9
\end{array}\right] \\
& \tilde{\boldsymbol{I}}_{h}= \pm J a b\left[\begin{array}{lllll}
0 & 0 & 0 & 0 & 1
\end{array}\right]^{\mathrm{T}}= \pm I\left[\begin{array}{lllll}
0 & 0 & 0 & 0 & 1
\end{array}\right]^{\mathrm{T}} .
\end{aligned}
$$

Por fim, a matriz local da interface do eletrodo $\tilde{\boldsymbol{Y}}_{i}^{\text {elet }}\left(\rho_{i}^{\prime}\right)$ é composta pela soma das matrizes de todos elementos hexaédricos que a compõe, considerando um mesmo parâmetro de eletrodo $\rho_{i}^{\prime}$ para todos. A Figura A.10 mostra uma interface composta por $n-1$ elementos hexaédricos. O nó virtual é o mesmo para todos os elementos e é o último na numeração local do eletrodo. 


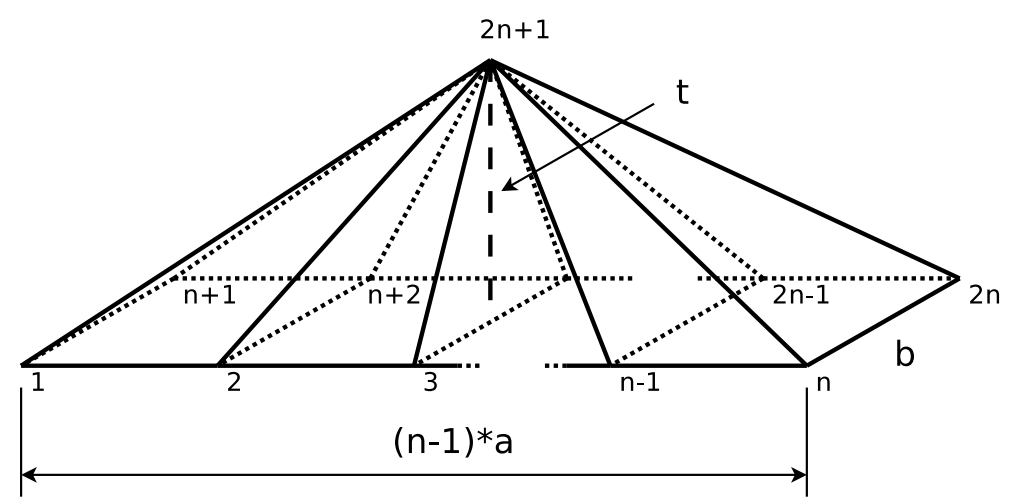

Figura A.10: Modelo de eletrodo com $n-1$ sub elementos hexaédricos.

A corrente é injetada através do nó virtual $2 n+1$, então $\tilde{\boldsymbol{I}}_{i}^{\text {elet }}=\left[\begin{array}{lllll}0 & 0 & \ldots & 0 & I_{i}\end{array}\right]^{\mathrm{T}}$, onde $I_{i}$ é a corrente elétrica total injetada no $i$-ésimo eletrodo

\section{A.2.3 Matriz Global}

Após montadas as matrizes locais $\tilde{\boldsymbol{Y}}_{i}^{\text {elet }}\left(\rho_{i}^{\prime}\right)$ de cada uma das $i=1,2, \ldots$, interfaces e os vetores de correntes $\tilde{\boldsymbol{I}}_{i}^{\text {elet }}$, pode-se somá-los à (A.32) e (A.33)

$$
\begin{aligned}
\boldsymbol{Y}(\boldsymbol{\rho}) \boldsymbol{v} & =\boldsymbol{I} \\
\boldsymbol{Y}(\boldsymbol{\rho}) & =\sum_{i=1}^{n} \tilde{\boldsymbol{Y}}_{i}\left(\rho_{i}\right)+\sum_{i=1}^{e} \tilde{\boldsymbol{Y}}_{i}^{\text {elet }}\left(t \rho_{i}\right) \\
\boldsymbol{I} & =\sum_{i=1}^{n} \tilde{\boldsymbol{I}}_{i}+\sum_{i=1}^{e} \tilde{\boldsymbol{I}}_{i}^{\text {elet }},
\end{aligned}
$$

onde $\boldsymbol{Y} \in \mathbb{R}^{m \times m}, \boldsymbol{I} \in \mathbb{R}^{m}$ e $\boldsymbol{\rho} \in \mathbb{R}^{n+e}$ é o vetor composto pelas resistividade dos elementos e pelos parâmetros de eletrodo. 


\section{Apêndice B}

\section{Autoadjuntividade do operador $A_{\rho}$}

Define-se o operador linear $A_{\rho}: S_{J} \rightarrow S_{\Psi}$ por

$$
\left.A_{\rho} J \triangleq \Psi\right|_{\partial \Omega}(\rho, J(s))
$$

onde $S_{J}$ e $S_{\Psi}$ são os espaços de funções da corrente $J(s)$ e potencial elétrico $\left.\Psi\right|_{\partial \Omega}$ respectivamente.

É de interesse mostrar que este operador é autoadjunto. Para tal é necessário identificar os espaços duais $S_{J}^{*}$ e $S_{\Psi}^{*}$ e mostrar que o operador dual $A_{\rho}^{*}: S_{\Psi}^{*} \rightarrow S_{J}^{*}$ é igual a $A_{\rho}$.

Pode-se considerar que $J$ e $\left.\Psi\right|_{\partial \Omega}$ pertencem a $L^{2}$, espaço de funções com norma

$$
\|v\|_{2}=\left(\int_{\partial \boldsymbol{\Omega}}|v|^{2} d x\right)^{1 / 2}<\infty
$$

Fisicamente falando, este é o espaço de correntes e potenciais elétricos com energia finita. Com esta hipótese, pode-se identificar prontamente os espaços duais por

$$
S_{J}^{*} \simeq S_{\Psi} \quad \text { e } \quad S_{\Psi}^{*} \simeq S_{J}
$$

É possível dar uma interpretação física para os espaços duais apresentados. Um funcional linear e limitado qualquer $F(v): V \rightarrow \mathbb{R}$ é uma função linear que leva de $V$ a $\mathbb{R}$. 
Costuma-se representar este funcional através da notação de chaves, também denotado por par dual

$$
F_{v^{*}}(v)=\left\langle v, v^{*}\right\rangle
$$

onde $v^{*}$ é um vetor do espaço dual $V^{*}$ a ser determinado. De fato, mostra-se que é possível identificar univocamente cada funcional com um elemento $v^{*}$ deste espaço. Além disto, quando $V$ for um espaço de Hilbert, pelo teorema de Riesz, o funcional linear e limitado pode ser representado pelo produto interno

$$
F_{v^{*}}(v)=\left(v, v^{*}\right)
$$

Para o espaço $L^{2} \subset H^{2}$, esta equação assume a forma

$$
F_{v^{*}}(v)=\int_{\partial \boldsymbol{\Omega}} v \cdot \bar{v}^{*} d A
$$

Voltando à física do problema, a potência instantânea $P: S_{J} \times S_{\Psi} \rightarrow \mathbb{R}$ que entra em $\Omega$ cé calculada através da integral

$$
P=\int_{\partial \boldsymbol{\Omega}} J .\left.\bar{\Psi}\right|_{\partial \boldsymbol{\Omega}} d x
$$

que é um produto interno em $L^{2}$. Fixando por hora o potencial elétrico $\left.\Psi\right|_{\partial \Omega}$, a potência $P$ pode ser vista como um funcional que leva do espaço de correntes para os números reais. Comparando (B.6) com (B.7) identifica-se prontamente que

$$
J^{*}=\left.\Psi\right|_{\partial \Omega}
$$

e, por consequência,

$$
S_{J}^{*} \simeq S_{\Psi}
$$


Fixando agora a corrente $J$, (B.7) pode ser vista como um funcional que leva do espaço dos potenciais elétricos para os número reais. Identifica-se então que

$$
\left.\Psi\right|_{\partial \Omega} ^{*}=J
$$

e

$$
S_{\Psi}^{*} \simeq S_{J}
$$

Para mostrar agora que $A_{\rho}$ é autoadjunto, parte-se da identidade bilinear

$$
\left\langle\left.\Psi\right|_{\partial \boldsymbol{\Omega}},\left.\Psi\right|_{\partial \boldsymbol{\Omega}} ^{*}\right\rangle=\left\langle J, J^{*}\right\rangle \quad \Leftrightarrow \quad\left\langle A_{\rho} J,\left.\Psi\right|_{\partial \boldsymbol{\Omega}} ^{*}\right\rangle=\left\langle J,\left.A_{\rho}^{*} \Psi\right|_{\partial \boldsymbol{\Omega}} ^{*}\right\rangle .
$$

Substituindo (B.10)

$$
\left\langle A_{\rho} J, J\right\rangle=\left\langle J, A_{\rho}^{*} J\right\rangle
$$

Substituindo os pares duais pelos equivalentes produtos internos e assumindo grandezas reais, tem-se

$$
\begin{aligned}
& \int_{\partial \boldsymbol{\Omega}} J . A_{\rho}^{*} J d x=\int_{\partial \boldsymbol{\Omega}} A_{\rho} J . J d x \\
& \int_{\partial \boldsymbol{\Omega}}\left(J \cdot A_{\rho}^{*} J-A_{\rho} J . J\right) d x=0 \\
& \int_{\partial \boldsymbol{\Omega}} J .\left(A_{\rho}^{*} J-A_{\rho} J\right) d x=0 .
\end{aligned}
$$

Considerando um $J$ arbitrário, para que a integral se anule sempre é necessário que

$$
A_{\rho}^{*} J-A_{\rho} J=0 \quad \Leftrightarrow \quad A_{\rho}^{*}=A_{\rho}
$$

mostrando que o operador $A_{\rho}$ é autoadjunto. 


\section{Apêndice $C$}

\section{Detalhes de implementação do SR-UKF}

\section{C.1 Equações do cálculo da matriz de covariância do algoritmo SR-UKF}

O algoritmo do SR-UKF utiliza uma fatoração QR e uma (des)atualização de Cholesky para o cálculo dos fatores de Cholesky das matrizes de covariância do estado e das medidas. Esta seção detalha o processo.

Inicialmente, seja a variável aleatória $\mathbf{y} \in \mathbb{R}^{n}$, relaciona com outras duas variáveis aleatórias $\mathbf{x} \in \mathbb{R}^{n} \mathrm{e} \mathbf{v} \in \mathbb{R}^{n}$, assumidas independentes, através de $\mathbf{y}=\mathbf{x}+\mathbf{v}$. Deseja-se determinar $\boldsymbol{S}^{y}$, fator de Cholesky (inferior) da matriz de covariância de $\mathbf{y}$, a partir de $N$ amostras de $\mathbf{x}$, cuja média é conhecida $\overline{\boldsymbol{x}}$ e de $\mathbf{v}$ que já possui covariância conhecida $\Gamma^{v}$. A estimativa da covariância de $\mathbf{y}$ é dada por

$$
\boldsymbol{\Gamma}^{y}=\frac{1}{N-1} \sum_{i=0}^{N-1}\left(\boldsymbol{x}_{i}-\overline{\boldsymbol{x}}\right)\left(\boldsymbol{x}_{i}-\overline{\boldsymbol{x}}\right)^{\mathrm{T}}+\boldsymbol{\Gamma}^{v}
$$

Definindo $\boldsymbol{\Delta} \boldsymbol{x}_{i}=\frac{\boldsymbol{x}_{i}-\overline{\boldsymbol{x}}}{\sqrt{N-1}}$, pode-se mostrar que

$$
\begin{aligned}
\boldsymbol{\Gamma}^{y} & =\boldsymbol{\Delta} \boldsymbol{x}_{0} \boldsymbol{\Delta} \boldsymbol{x}_{0}^{\mathrm{T}}+\boldsymbol{\Delta} \boldsymbol{X} \boldsymbol{\Delta} \boldsymbol{X}^{\mathrm{T}}+\boldsymbol{\Gamma}^{v} \\
\boldsymbol{\Delta} \boldsymbol{X} & =\left[\begin{array}{llll}
\boldsymbol{\Delta} \boldsymbol{x}_{1} & \boldsymbol{\Delta} \boldsymbol{x}_{2} & \ldots & \boldsymbol{\Delta} \boldsymbol{x}_{N-1}
\end{array}\right]
\end{aligned}
$$

onde $\boldsymbol{\Delta} \boldsymbol{X} \in \mathbb{R}^{n \times N-1}$ é composta pelos vetores $\boldsymbol{\Delta} \boldsymbol{x}_{i}$ com exceçãd1 1 de $\boldsymbol{\Delta} \boldsymbol{x}_{0}$. Calculando

\footnotetext{
${ }^{1} \mathrm{O}$ termo com $\boldsymbol{\Delta} \boldsymbol{x}_{0}$ foi deixado propositalmente fora de $\boldsymbol{\Delta} \boldsymbol{X}$ por hora. Ele será tratado separadamente mais a frente.
} 
uma raiz quadrada de $\Gamma^{v}=\sqrt{\Gamma^{v}} \cdot \sqrt{\Gamma^{v}}$ e substituindo na equação anterior, pode-se agrupar os dois últimos termos da direita em uma matriz $\boldsymbol{B} \in \mathbb{R}^{n \times(n+N-1)}$

$$
\begin{aligned}
\boldsymbol{\Gamma}^{y} & =\boldsymbol{\Delta} \boldsymbol{x}_{0} \boldsymbol{\Delta} \boldsymbol{x}_{0}^{\mathrm{T}}+\boldsymbol{B} \boldsymbol{B}^{\mathrm{T}} \\
\boldsymbol{B} & =\left[\begin{array}{ll}
\boldsymbol{\Delta} \boldsymbol{X} & \sqrt{\boldsymbol{\Gamma}^{v}}
\end{array}\right]
\end{aligned}
$$

A decomposição QR de $\boldsymbol{B}^{\mathrm{T}}$ é dada por

$$
\boldsymbol{B}^{\mathrm{T}}=\boldsymbol{Q} \boldsymbol{R}=\left[\begin{array}{ll}
\boldsymbol{Q}_{1} & \boldsymbol{Q}_{2}
\end{array}\right]\left[\begin{array}{c}
\boldsymbol{R}_{1} \\
\mathbf{0}
\end{array}\right]=\boldsymbol{Q}_{1} \boldsymbol{R}_{1}=\left[\begin{array}{c}
\boldsymbol{\Delta} \boldsymbol{X}^{\mathrm{T}} \\
\sqrt{\boldsymbol{\Gamma}^{\mathrm{T}}}
\end{array}\right]
$$

onde $\boldsymbol{R}_{1} \in \mathbb{R}^{n \times n}$ é triangular superior, $\boldsymbol{Q}_{1} \in \mathbb{R}^{(n+N-1) \times n}$ e $\boldsymbol{Q}_{2} \in \mathbb{R}^{(n+N-1) \times(N-1)}$ são matrizes ortogonais [138, sec. 4.1.1]. As matrizes $\boldsymbol{R}_{1}$ e $\boldsymbol{Q}_{1}$ são únicas e $\boldsymbol{R}_{1}$ coincide com o transposto da fatoração de Cholesky (inferior) de $\boldsymbol{B} \boldsymbol{B}^{\mathrm{T}}$, denominada nesta tese $\boldsymbol{S}$.

Substituindo esta decomposição em ( (C.4), tem-se

$$
\boldsymbol{\Gamma}^{y}=\boldsymbol{\Delta} \boldsymbol{x}_{0} \boldsymbol{\Delta} \boldsymbol{x}_{0}^{\mathrm{T}}+\boldsymbol{R}^{\mathrm{T}} \boldsymbol{Q}^{\mathrm{T}} \boldsymbol{Q} \boldsymbol{R}=\boldsymbol{\Delta} \boldsymbol{x}_{0} \boldsymbol{\Delta} \boldsymbol{x}_{0}^{\mathrm{T}}+\boldsymbol{R}_{1}^{\mathrm{T}} \boldsymbol{R}_{1}=\boldsymbol{\Delta} \boldsymbol{x}_{0} \boldsymbol{\Delta} \boldsymbol{x}_{0}^{\mathrm{T}}+\boldsymbol{S} \boldsymbol{S}^{\mathrm{T}}
$$

Neste ponto, pode-se fazer uma relação direta deste resultado com (5.22) ao identificar

$$
\begin{aligned}
\boldsymbol{\Delta} \boldsymbol{X} & =\sqrt{W_{i}^{c}}\left(\mathcal{X}_{k+1 \mid k}^{1: 2 L}-\overline{\boldsymbol{x}}_{k+1 \mid k}\right) \\
\sqrt{\boldsymbol{\Gamma}^{v}} & =\boldsymbol{A}_{k}^{x} \sqrt{\boldsymbol{\Gamma}_{k}^{v^{x}}}
\end{aligned}
$$

ou seja, a equação (5.22) computa $\boldsymbol{R}_{1}^{\mathrm{T}}$ de (C.5).

A última etapa necessária é a inclusão do primeiro termo $\boldsymbol{\Delta} \boldsymbol{x}_{0} \boldsymbol{\Delta} \boldsymbol{x}_{0}^{\mathrm{T}}$ em $\boldsymbol{S}$. Esta etapa pode ser apresentada da seguinte maneira:

Dada a fatoração QR de $\boldsymbol{B}^{\mathrm{T}}=\boldsymbol{Q} \boldsymbol{R}$, determinar a nova fatoração da matriz $\left(\boldsymbol{B}^{\prime}\right)^{\mathrm{T}}$ onde

$$
\boldsymbol{B}^{\prime}=\left[\begin{array}{lll}
\Delta \boldsymbol{x}_{0} & \boldsymbol{\Delta} \boldsymbol{X} & \sqrt{\boldsymbol{\Gamma}^{v}}
\end{array}\right]=\left[\begin{array}{ll}
\Delta \boldsymbol{x}_{0} & \boldsymbol{B}
\end{array}\right]
$$


A inclusão deste último termo em $S$ é visto, portanto, como a atualização da fatoração QR 2 de uma matriz cujo número de colunas foi aumentado de um.

Esta inclusão consiste de uma atualização de posto 1 de $\boldsymbol{R}_{1}^{\mathrm{T}}=\boldsymbol{S}$ e pode ser obtida através da atualização de Cholesky 3 , representada por

$$
\begin{aligned}
& \boldsymbol{S}^{y}=\text { cholupdate }\left(\boldsymbol{S}, \boldsymbol{\Delta} \boldsymbol{x}_{0}, 1\right) \\
& \boldsymbol{\Gamma}^{y}=\boldsymbol{S}^{y}\left(\boldsymbol{S}^{y}\right)^{\mathrm{T}}
\end{aligned}
$$

Finalmente, pode-se fazer a relação direta deste resultado com (5.23) ao identificar

$$
\boldsymbol{\Delta} \boldsymbol{x}_{0}=\sqrt{W_{0}^{c}}\left(\mathcal{X}_{k+1 \mid k}^{0}-\overline{\boldsymbol{x}}_{k+1 \mid k}\right)
$$

A justificativa para a separação da primeira realização $\boldsymbol{x}_{0}$ das demais realizações no cálculo da matriz $\Gamma^{y}$ é devido à problemas numéricos da UT.

\section{C.2 Equações do SR-UKF para estimação dos parâmetros do modelo de evolução}

\section{Inicialização}

Seja $\boldsymbol{p} \in \mathbb{R}^{r}$ o vetor de parâmetros a ser estimado e

$$
\begin{aligned}
\overline{\boldsymbol{p}}_{0} & =E\left\{\mathbf{p}_{0}\right\} \\
\boldsymbol{S}_{0}^{p} & =\operatorname{chol}\left(\boldsymbol{\Gamma}_{0}^{p p}\right),
\end{aligned}
$$

onde $\operatorname{chol}(\boldsymbol{M})$ representa uma função que retorna o fator (triangular inferior) de Cholesky de uma matriz $M$.

Após a inicialização, para $k=0,1, \ldots$ as fases de propagação, predição das medidas e atualização do filtro são repetidas sucessivamente:

\footnotetext{
${ }^{2}$ Neste problema, apenas a atualização do fator $\boldsymbol{R}$ é necessária.

${ }^{3}$ Este algoritmo é descrito em detalhes no Apêndice D.
} 


\section{Propagação do filtro}

Em vista da falta de dinâmica na componente nominal da equação de evolução dos parâmetros (5.2), a fase de propagação pode ser simplificada.

As estatísticas de $\boldsymbol{p}_{k+1 \mid k}$ são calculadas com

$$
\begin{aligned}
& \overline{\boldsymbol{p}}_{k+1 \mid k}=\overline{\boldsymbol{p}}_{k}+\boldsymbol{A}_{k}^{p} \overline{\boldsymbol{v}}_{k}^{p} \\
& \boldsymbol{S}_{k+1 \mid k}^{p}=\frac{1}{\sqrt{\eta}} \boldsymbol{S}_{k}^{p},
\end{aligned}
$$

onde $\eta \in] 0,1]$ é um fator de esquecimento análogo ao utilizado em filtros RLS. Esta abordagem impõe um esquecimento exponencial em medidas passadas, mantendo o filtro aberto para novas medidas. É equivalente a escolher uma matriz $\Gamma_{k}^{v^{p}}=\left(\eta^{-1}-\right.$ 1) $\Gamma_{k}^{p p}$, como apresentado em Wan e Merwe [103]. Outra opção é utilizar

$$
\begin{aligned}
\boldsymbol{S}_{k+1 \mid k}^{p} & =\boldsymbol{S}_{k}^{p}+\boldsymbol{D}_{k} \\
\boldsymbol{D}_{k} & =-\operatorname{diag}\left(\boldsymbol{S}_{k}^{p}\right)+\left(\operatorname{diag}\left(\boldsymbol{S}_{k}^{p}\right)^{2}+\operatorname{diag}\left(\boldsymbol{A}_{k}^{p} \boldsymbol{\Gamma}_{k}^{v^{p}}\left(\boldsymbol{A}_{k}^{p}\right)^{\mathrm{T}}\right)\right)^{1 / 2}
\end{aligned}
$$

Nesta segunda opção, $\operatorname{diag}(\boldsymbol{A})$ é uma função que retorna uma matriz contendo apenas

a digonal da matriz $\boldsymbol{A}$ e $\boldsymbol{\Gamma}_{k}^{v^{p}}$ é a matriz de covariância do ruído do processo $\mathbf{v}_{k}^{p}$. A matriz $\boldsymbol{D}_{k}$ aproxima o efeito de reduzir a matriz de covariância do ruído de processo $\Gamma_{k}^{v^{p}}$ conforme o filtro converge.

\section{Predição das medidas}

1-) O conjunto de $2 r+1$ pontos sigma $\left\{\mathcal{P}_{k+1 \mid k}^{i}\right\}_{i=0}^{2 r}$ e seus pesos $W_{i}$ são amostrados da seguinte forma

$$
\begin{aligned}
\mathcal{P}_{k+1 \mid k}^{i} & =\overline{\boldsymbol{p}}_{k+1 \mid k} \\
\mathcal{P}_{k+1 \mid k}^{i} & =\overline{\boldsymbol{p}}_{k+1 \mid k}+\gamma\left[\boldsymbol{S}_{k+1 \mid k}^{p}\right]_{(:, i)}, \quad i=1, \ldots, r \\
\mathcal{P}_{k+1 \mid k}^{i+L} & =\overline{\boldsymbol{p}}_{k+1 \mid k}-\gamma\left[\boldsymbol{S}_{k+1 \mid k}^{p}\right]_{(:, i)}, \quad i=1, \ldots, r \\
W_{0}^{m} & =\frac{\lambda}{(r+\lambda)} \\
W_{0}^{c} & =\frac{\lambda}{(r+\lambda)}+1-\alpha^{2}+\beta \\
W_{i}^{m} & =W_{i}^{c}=\frac{1}{2(r+\lambda)}, \quad i=1, \ldots, 2 r
\end{aligned}
$$


onde $\gamma=\sqrt{r+\lambda}$ e $\lambda=\alpha^{2}(2 r+\kappa)-r$.

2-) O conjunto de pontos sigma é observado pelo modelo (5.8)

$$
\mathcal{Y}_{k+1 \mid k}^{i}=\boldsymbol{h}_{k+1}\left(\boldsymbol{\Phi}_{k}\left(\hat{\boldsymbol{x}}_{k}, \mathcal{P}_{k+1 \mid k}^{i}, \boldsymbol{u}_{k}\right)+\boldsymbol{B}_{k+1}^{p} \overline{\boldsymbol{e}}_{k+1}^{p}, \quad i=1, \ldots, 2 r\right.
$$

onde $\hat{\boldsymbol{x}}_{k}$ é a ultima estimativa obtida com o filtro em paralelo para estimação do estado.

3-) As estatísticas de $\boldsymbol{y}_{k+1 \mid k}$ são calculadas com

$$
\begin{aligned}
& \overline{\boldsymbol{y}}_{k+1 \mid k}=\sum_{i=0}^{2 r} W_{i}^{m} \mathcal{Y}_{k+1 \mid k}^{i} \\
& \boldsymbol{S}_{k+1 \mid k}^{y}=\operatorname{qr}\left(\left[\sqrt{W_{i}^{c}}\left(\mathcal{Y}_{k+1 \mid k}^{1: 2 r}-\overline{\boldsymbol{y}}_{k+1 \mid k}\right) \quad \boldsymbol{B}_{k+1}^{p} \sqrt{\boldsymbol{\Gamma}_{k+1}^{e^{p}}}\right]\right) \\
& \boldsymbol{S}_{k+1 \mid k}^{y}=\text { cholupdate }\left(\boldsymbol{S}_{k+1 \mid k}^{y y}, \mathcal{Y}_{k+1 \mid k}^{0}-\overline{\boldsymbol{y}}_{k+1 \mid k}, W_{0}^{c}\right) \text {, }
\end{aligned}
$$

onde $\sqrt{\Gamma_{k+1}^{e^{p}}}$ é uma matriz raiz quadrada da matriz de covariância do ruído de observação $\mathbf{e}_{k+1}^{p}$.

\section{Atualização do filtro}

1-) A covariância cruzada entre $\mathbf{p}_{k}$ e $\mathbf{y}_{k}$ é determinada por

$$
\Gamma_{k+1 \mid k}^{p y}=\sum_{i=0}^{2 r} W_{i}^{c}\left(\mathcal{P}_{k+1 \mid k}^{i}-\overline{\boldsymbol{p}}_{k+1 \mid k}\right)\left(\mathcal{Y}_{k+1 \mid k}^{i}-\overline{\boldsymbol{y}}_{k+1 \mid k}\right)^{\mathrm{T}}
$$

2-) As atualizações de $\overline{\boldsymbol{p}}$ e $\boldsymbol{S}^{p}$ são calculadas com

$$
\begin{aligned}
\boldsymbol{U} & =\boldsymbol{\Gamma}_{k+1 \mid k}^{p y} /\left(\boldsymbol{S}_{k+1 \mid k}^{y}\right)^{\mathrm{T}} \\
\boldsymbol{K}_{k+1} & =\boldsymbol{U} / \boldsymbol{S}_{k+1 \mid k}^{y} \\
\overline{\boldsymbol{p}}_{k+1} & =\overline{\boldsymbol{p}}_{k+1 \mid k}+\boldsymbol{K}_{k+1}\left(\boldsymbol{y}_{k+1}^{\text {medido }}-\overline{\boldsymbol{y}}_{k+1 \mid k}\right) \\
\boldsymbol{S}_{k+1}^{p} & =\text { cholupdate }\left\{\boldsymbol{S}_{k+1 \mid k}^{p}, \boldsymbol{U},-1\right\},
\end{aligned}
$$

onde a operação $\boldsymbol{B} / \boldsymbol{A}$ representa a solução eficiente do sistema linear $\boldsymbol{X} \boldsymbol{A}=\boldsymbol{B}$, onde $A$ é triangular. 


\section{C.3 Modelo linearizado de observação utilizando a mudança de variável}

A seguir é apresentada a linearização da equação de observação (2.40), em relação à $\zeta$.

\section{C.3.1 Regra da cadeia para derivativos matriciais}

Sejam os vetores $\boldsymbol{x} \in \mathbb{R}^{n}, \boldsymbol{y} \in \mathbb{R}^{m}$ e $\boldsymbol{z} \in \mathbb{R}^{p}$ relacionados por

$$
\begin{aligned}
\boldsymbol{y} & =\boldsymbol{y}(\boldsymbol{x}) \\
z & =\boldsymbol{z}(\boldsymbol{y}) .
\end{aligned}
$$

Por definição, o derivativo de um vetor $\boldsymbol{z}$ com relação a um vetor $\boldsymbol{x}$ é

$$
\frac{\partial \boldsymbol{z}}{\partial \boldsymbol{x}} \triangleq\left[\begin{array}{ccc}
\frac{\partial z_{1}}{\partial x_{1}} & \cdots & \frac{\partial z_{1}}{\partial x_{n}} \\
\vdots & \ddots & \vdots \\
\frac{\partial z_{p}}{\partial x_{1}} & \cdots & \frac{\partial z_{p}}{\partial x_{n}}
\end{array}\right]_{p \times n}
$$

Os termos desta matriz podem ser expandidos pela regra da cadeia para variáveis escalares

$$
\frac{\partial z_{i}}{\partial x_{j}}=\sum_{k=1}^{m} \frac{\partial z_{i}}{\partial y_{k}} \frac{\partial y_{k}}{\partial x_{j}}, \quad i=1, \ldots, p, \quad j=1, \ldots, n
$$

Substituindo novamente em (C.37), chega-se ao resultado

$$
\frac{\partial \boldsymbol{z}}{\partial \boldsymbol{x}}=\left[\begin{array}{ccc}
\sum_{k} \frac{\partial z_{1}}{\partial y_{k}} \frac{\partial y_{k}}{\partial x_{1}} & \ldots & \sum_{k} \frac{\partial z_{1}}{\partial y_{k}} \frac{\partial y_{k}}{\partial x_{n}} \\
\vdots & \ddots & \vdots \\
\sum_{k} \frac{\partial z_{p}}{\partial y_{k}} \frac{\partial y_{k}}{\partial x_{1}} & \ldots & \sum_{k} \frac{\partial z_{p}}{\partial y_{k}} \frac{\partial y_{k}}{\partial x_{n}}
\end{array}\right]=\left[\begin{array}{ccc}
\frac{\partial z_{1}}{\partial y_{1}} & \ldots & \frac{\partial z_{1}}{\partial y_{m}} \\
\vdots & \ddots & \vdots \\
\frac{\partial z_{p}}{\partial y_{1}} & \ldots & \frac{\partial z_{p}}{\partial y_{m}}
\end{array}\right]\left[\begin{array}{ccc}
\frac{\partial y_{1}}{\partial x_{1}} & \ldots & \frac{\partial y_{1}}{\partial x_{n}} \\
\vdots & \ddots & \vdots \\
\frac{\partial y_{m}}{\partial x_{1}} & \ldots & \frac{\partial y_{m}}{\partial x_{n}}
\end{array}\right]=\frac{\partial \boldsymbol{z}}{\partial \boldsymbol{y}} \frac{\partial \boldsymbol{y}}{\partial \boldsymbol{x}}
$$




\section{C.3.2 Modelo linearizado de observação}

Expandindo (2.40) em série de Taylor em torno de um vetor $\zeta_{0}$ qualquer e desprezando os termos de ordem superior, tem-se

$$
\boldsymbol{v}_{j}(\boldsymbol{\zeta})=\boldsymbol{v}_{j}\left(\boldsymbol{\zeta}_{0}\right)+\left.\frac{\partial \boldsymbol{h}_{j}(\boldsymbol{\rho})}{\partial \boldsymbol{\zeta}}\right|_{\boldsymbol{\zeta}_{0}}\left(\boldsymbol{\zeta}-\boldsymbol{\zeta}_{0}\right)
$$

onde $\boldsymbol{v}_{j}\left(\boldsymbol{\zeta}_{0}\right)$ é notação simplificada para $\boldsymbol{v}_{j}\left(\boldsymbol{\varphi}\left(\boldsymbol{\zeta}_{0}\right)\right)$. A derivada parcial da equação acima pode ser reescrita com o uso da regra da cadeia (C.39)

$$
\tilde{\boldsymbol{H}}_{j}^{\zeta}=\frac{\partial \boldsymbol{h}_{j}(\boldsymbol{\rho})}{\partial \boldsymbol{\zeta}}=\frac{\partial \boldsymbol{h}_{j}(\boldsymbol{\rho})}{\partial \boldsymbol{\rho}} \frac{\partial \boldsymbol{\rho}}{\partial \boldsymbol{\zeta}}
$$

A primeira derivada parcial é dada por (2.45). A segunda pode ser reescrita

$$
\frac{\partial \boldsymbol{\rho}}{\partial \boldsymbol{\zeta}}=\boldsymbol{J}^{\zeta}=\left[\begin{array}{ccc}
\frac{\partial \rho_{1}}{\partial \zeta_{1}} & \cdots & \frac{\partial \rho_{1}}{\partial \zeta_{n}} \\
\vdots & \ddots & \vdots \\
\frac{\partial \rho_{n}}{\partial \zeta_{1}} & \cdots & \frac{\partial \rho_{n}}{\partial \zeta_{n}}
\end{array}\right]_{n \times n}
$$

cujas derivadas parciais de seus elementos são

$$
\frac{\partial \rho_{i}}{\partial \zeta_{j}}= \begin{cases}\frac{b_{i}-a_{i}}{c_{i}} \frac{\exp \left(-\frac{\zeta_{i}}{c_{i}}\right)}{\left[1+\exp \left(-\frac{\zeta_{i}}{c_{i}}\right)\right]^{2}} & , i=j \\ 0 & , i \neq j\end{cases}
$$

Portanto a matriz Jacobiana modificada para a linearização em torno de $\boldsymbol{\zeta}_{0}$ é dada pela matriz jacobiana original multiplicada pela matriz jacobiana da função $\rho=\varphi(\zeta)$

$$
\left.\tilde{\boldsymbol{H}}_{j}^{\zeta}\right|_{\zeta_{0}}=\left.\frac{\partial \boldsymbol{h}_{j}(\boldsymbol{\rho})}{\partial \boldsymbol{\zeta}}\right|_{\zeta_{0}}=\left.\left.\tilde{\boldsymbol{H}}_{j}\right|_{\boldsymbol{\rho}_{0}} \boldsymbol{J}^{\zeta}\right|_{\zeta_{0}}
$$




\section{Apêndice D}

\section{Atualização e desatualização de Cholesky}

Seja $\boldsymbol{A} \in \mathbb{R}^{n \times n}$ uma matriz positiva semi-definida, cujo fator de Cholesky $\boldsymbol{S} \in \mathbb{R}^{n \times n}$ é conhecido.

Suponha agora o problema de se calcular o novo fator de Cholesky da matriz modificada $\boldsymbol{A}^{\prime}=\boldsymbol{A}+\alpha \boldsymbol{u} \boldsymbol{u}^{\mathrm{T}}$, onde $\boldsymbol{u} \in \mathbb{R}^{n}$ é um vetor e $\alpha>0$ é um escalar. O modo mais imediato seria calcular a matriz $\boldsymbol{A}^{\prime}$ e, por fim, o novo fator de Cholesky. Entretanto, esta operação requer um número de operações da ordem de $n^{3}$.

A atualização de Cholesky lida com este problema sem a necessidade de calcular novamente o fator de Cholesky, mas através de modificações na matriz $\boldsymbol{S}$ diretamente, utilizando apenas $n^{2}$ operações. Este processo também não necessita o armazenamento de $\boldsymbol{A}$ ou $\boldsymbol{A}^{\prime}$.

A atualização de Cholesky determina o novo fator de Cholesky da matriz $\boldsymbol{A}^{\prime}=\boldsymbol{A}+\alpha \boldsymbol{u} \boldsymbol{u}^{\mathrm{T}}$, enquanto que a desatualização de Cholesky determina o fator de Cholesky da matriz $\boldsymbol{A}^{\prime}=\boldsymbol{A}-\alpha \boldsymbol{u} \boldsymbol{u}^{\mathrm{T}}$, contanto que esta continuar positiva definida.

A propriedade utilizada para deduzir a atualização de Cholesky diz respeito à sua relação com a fatoração QR: mostra-se que o fator de Cholesky (inferior) de uma matriz $\boldsymbol{M}^{\mathrm{T}} \boldsymbol{M}$ é o transposto do fator $\boldsymbol{R}$ da fatoração QR de $\boldsymbol{M}$ [139, p. 230]. Utilizando esta relação, a atualização de Cholesky é feita através da atualização da fatoração QR. Algoritmos para atualizações QR de diversas formas podem ser encontradas, como adição/remoção de linhas ou colunas, permutação de linhas ou colunas, entre outras. Para mais detalhes, ver Stewart [138, sec. 4.3]. Para esta tese, apenas modificações para 
a adição e remoção de linhas são necessárias.

Os algoritmos utilizam duas operações ortogonais em matrizes, a rotação de Givens e a reflexão de Householder. Estas operações são apresentadas a seguir e na sequência são também apresentados os algoritmos para a (des)atualização de Cholesky.

Para mais propriedades sobres os algoritmos e detalhes de implementação, ver Stewart [138, cap. 4.1 e 4.3] e Golub e Loan [139, cap. 5 e seç. 12.5].

\section{D.1 Rotação de Givens}

Inicialmente para um caso em $\mathbb{R}^{2}$, a matriz de rotação no plano ou matriz de rotação de Givens é dada por

$$
\boldsymbol{G}=\left[\begin{array}{cc}
c & s \\
-s & c
\end{array}\right]
$$

onde $s$ e $c$ são notações simplificadas para $\sin \theta$ e $\cos \theta$, e $\theta \in[0,2 \pi$ [ é chamado ângulo de rotação.

Aplicando em um vetor $\boldsymbol{v}=\left[\begin{array}{ll}a & b\end{array}\right]^{\mathrm{T}} \neq \mathbf{0}$, obtém-se

$$
\boldsymbol{v}^{\prime}=\boldsymbol{G} \boldsymbol{v}=\left[\begin{array}{l}
a c+b s \\
b c-a s
\end{array}\right]
$$

que representa uma rotação do vetor $\boldsymbol{v}$ no sentido anti-horário de um ângulo $\theta$. É de interesse encontrar o valor de $\theta$ que rotaciona o vetor para a orientação horizontal, pois neste caso introduz-se zero em sua sua segunda componente. O ângulo $\theta$ para tal é encontrado igualando a componente $y$ do vetor rotacionado $\boldsymbol{y}^{\prime}$ a zero e resolvendo a equação para $s$ e $c$, com a condição extra $s^{2}+c^{2}=1$. Uma das soluções é

$$
c=\frac{a}{r}, \quad s=\frac{b}{r}
$$

que resulta em

$$
\boldsymbol{v}^{\prime}=\left[\begin{array}{l}
r \\
0
\end{array}\right], \quad r=\sqrt{a^{2}+b^{2}}=\|\boldsymbol{v}\|_{2}
$$


Esta rotação é de interesse pois pode ser utilizada para introduzir zeros em elementos específicos de uma matriz. Doravante, $(c, s)=$ GeraTheta $(a, b)$ representa a função que, dados os valores $a$ e $b$, calcula $c$ e $s$ que zeram a componente $b$.

Para um caso em $\mathbb{R}^{n}$, pode-se utilizar a rotação de Givens para zerar um elemento específico de uma matriz. O operador rotação no plano $(i, j)$ de uma matriz é dado por

$$
\boldsymbol{G}_{i, j} \triangleq\left(\begin{array}{ccccc}
\mathbb{I} & & & \\
& c & & s & \\
& & \mathbb{I} & & \\
-s & & c & \\
& & & & \mathbb{I} \\
i & & j &
\end{array}\right)_{j}^{i}
$$

cujas matrizes identidade possuem dimensões compatíveis e os demais componentes de $\boldsymbol{G}_{i, j}$ são preenchidos com zeros. Nota-se que esta matriz é composta pela matriz identidade, com a inclusão da matriz de rotação $G$ nos elementos correspondentes às linhas e colunas $i$ e $j$, portanto define um operador ortogonal.

Considere, como exemplo, uma matriz $\boldsymbol{A} \in \mathbb{R}^{4 \times 3}$

$$
\boldsymbol{A}=\left[\begin{array}{ccc}
X & a & X \\
X & X & X \\
X & b & X \\
X & X & X
\end{array}\right]
$$

onde $X$ representa um valor numérico arbitrário. Deseja-se deseja zerar o componente $b=[\boldsymbol{A}]_{(3,2)}$, utilizando também o elemento $a=[\boldsymbol{A}]_{(1,2)}$ para calcular o ângulo da rotaçãd1.

\footnotetext{
${ }^{1}$ Qualquer outro elemento da coluna 2 poderia ser utilizado.
} 
Criando o operador $\boldsymbol{G}_{1,3}$ com os valores $(c, s)=\operatorname{GeraTheta}(a, b)$ e aplicando o operado em $\boldsymbol{A}$, obtém-se

$$
\boldsymbol{A}^{\prime}=\boldsymbol{G}_{1,3} \boldsymbol{A}=\left[\begin{array}{cccc}
c & & s & \\
& 1 & & \\
-s & & c & \\
& & & 1
\end{array}\right]\left[\begin{array}{ccc}
X & a & X \\
X & X & X \\
X & b & X \\
X & X & X
\end{array}\right]=\left[\begin{array}{ccc}
Y & r & Y \\
X & X & X \\
Y & 0 & Y \\
X & X & X
\end{array}\right]
$$

Somente as linhas de $\boldsymbol{A}$ com índice $i$ e $j$ são modificadas neste processo e o elemento $[\boldsymbol{A}]_{3,2}$ é zerado como esperado. Em uma implementação eficiente da rotação de Givens, a multiplicação $\boldsymbol{G}_{i, j} \boldsymbol{A}$ não é realizada, pois basta notar que as linhas modificadas são dadas por

$$
\begin{gathered}
{\left[\boldsymbol{A}^{\prime}\right]_{(i,:)}=c *[\boldsymbol{A}]_{(i,:)}+s *[\boldsymbol{A}]_{(j,:)}} \\
{\left[\boldsymbol{A}^{\prime}\right]_{(j,:)}=-s *[\boldsymbol{A}]_{(i,:)}+c *[\boldsymbol{A}]_{(j,:)},}
\end{gathered}
$$

onde $[\cdot]_{(i,:)}$ representa a $i$-ésima linha de uma matriz.

Doravante, $\boldsymbol{A}^{\prime}=\operatorname{Givens}(\boldsymbol{A}, i, j, k)$ designa a função que calcula a rotação de Givens em uma matriz $\boldsymbol{A}$, cujas linhas a serem modificadas são $i$ e $j$, zerando o componente $(j, k)$.

A rotação de Givens pode ser utilizada sucessivamente para triangularizar eficientemente uma matriz de Hessenberg superior2. A seguir mostra-se um diagrama do algoritmo para transformar uma matriz de Hessenberg superior em uma matriz triangular superior $\boldsymbol{T}$. Em cada passo, os termos $a$ e $b$ da rotação estão destacados.

$$
\left[\begin{array}{ccc}
a_{1} & X & X \\
b_{1} & X & X \\
0 & X & X \\
0 & 0 & X
\end{array}\right] \stackrel{\boldsymbol{G}_{1,2}}{\rightarrow}\left[\begin{array}{ccc}
X & X & X \\
0 & a_{2} & X \\
0 & b_{2} & X \\
0 & 0 & X
\end{array}\right] \stackrel{\boldsymbol{G}_{2,3}}{\rightarrow}\left[\begin{array}{ccc}
X & X & X \\
0 & X & X \\
0 & 0 & a_{3} \\
0 & 0 & b_{3}
\end{array}\right] \stackrel{\boldsymbol{G}_{3,4}}{\rightarrow}\left[\begin{array}{ccc}
X & X & X \\
0 & X & X \\
0 & 0 & X \\
0 & 0 & 0
\end{array}\right]=\boldsymbol{T} .
$$

Givens $(\boldsymbol{A}, 1,2,1) \quad \operatorname{Givens}\left(\boldsymbol{A}^{\prime}, 2,3,2\right) \quad \operatorname{Givens}\left(\boldsymbol{A}^{\prime \prime}, 3,4,3\right)$

\footnotetext{
${ }^{2}$ Uma matriz de Hessenberg superior tem zeros em todos os elementos abaixo da primeira sub-diagonal principal.
} 
Nas seções posteriores, $\boldsymbol{T}=\operatorname{TriHessGivens}(\boldsymbol{M})$ representa a função que triangulariza uma matriz de Hessenberg $\boldsymbol{M}$ superior, utilizando rotações de Givens seguindo o algoritmo descrito.

No caso de matrizes cheias, que não estão prontamente na forma de Hessenberg, a rotação de Givens também pode ser usada. Para a primeira e segunda colunas de $\boldsymbol{A}$, tem-se

$$
\left[\begin{array}{ccc}
a_{1} & X & X \\
X & X & X \\
X & X & X \\
b_{1} & X & X
\end{array}\right] \stackrel{\boldsymbol{G}_{1,4}}{\rightarrow}\left[\begin{array}{ccc}
a_{2} & X & X \\
X & X & X \\
b_{2} & X & X \\
0 & X & X
\end{array}\right] \stackrel{\boldsymbol{G}_{133}}{\rightarrow}\left[\begin{array}{ccc}
a_{3} & X & X \\
b_{3} & X & X \\
0 & X & X \\
0 & X & X
\end{array}\right] \stackrel{G_{1,2}}{\rightarrow}\left[\begin{array}{ccc}
X & X & X \\
0 & X & X \\
0 & X & X \\
0 & X & X
\end{array}\right]
$$

Givens $(\boldsymbol{A}, 1,4,1) \quad \operatorname{Givens}\left(\boldsymbol{A}^{\prime}, 1,3,1\right) \quad \operatorname{Givens}\left(\boldsymbol{A}^{\prime \prime}, 1,2,1\right)$

$$
\left[\begin{array}{ccc}
X & X & X \\
0 & a_{4} & X \\
0 & X & X \\
0 & b_{4} & X
\end{array}\right] \stackrel{\boldsymbol{G}_{2,4}}{\rightarrow}\left[\begin{array}{ccc}
X & X & X \\
0 & a_{5} & X \\
0 & b_{5} & X \\
0 & 0 & X
\end{array}\right] \stackrel{\boldsymbol{G}_{2,3}}{\rightarrow}\left[\begin{array}{ccc}
X & X & X \\
0 & X & X \\
0 & 0 & X \\
0 & 0 & X
\end{array}\right],
$$

Givens $\left(\boldsymbol{A}^{\prime \prime \prime}, 2,4,2\right) \quad$ Givens $\left(\boldsymbol{A}^{\prime \prime \prime \prime}, 2,3,2\right)$

e assim sucessivamente até que a matriz esteja triangularizada.

Esta operação em matrizes cheias, entretanto, requer muitas operações algébricas. Uma forma alternativa e mais econômica nestes casos utiliza a reflexão de Householder, que será apresentada sa seção seguinte.

Como última observação, nota-se que

$$
\begin{aligned}
A^{\prime} & =G_{1} A \\
A^{\prime \prime} & =G_{2} A^{\prime}=G_{2} G_{1} A \\
& \vdots \\
& \\
T & =G_{p} G_{p-1} \ldots G_{2} G_{1} A .
\end{aligned}
$$

Seja $p$ o numero de operadores $\boldsymbol{G}_{i}$ aplicados em $\boldsymbol{A}$ necessários para torná-la triangular superior. Então a matriz

$$
\boldsymbol{P}=\boldsymbol{G}_{p} \boldsymbol{G}_{p-1} \ldots \boldsymbol{G}_{2} \boldsymbol{G}_{1}
$$


é ortogonal e

$$
\boldsymbol{T}=\boldsymbol{P} \boldsymbol{A} \Leftrightarrow \boldsymbol{P}^{\mathrm{T}} \boldsymbol{T}=\boldsymbol{A}
$$

com $\boldsymbol{P}^{\mathrm{T}}$ ortogonal e $\boldsymbol{T}$ triangular superior. Em outras palavras, esta é a fatoração QR de $\boldsymbol{A}$.

\section{D.2 Reflexão de Householder}

Seja uma matriz $\boldsymbol{H} \in \mathbb{R}^{n \times n}$, dada por

$$
\boldsymbol{H} \triangleq \mathbb{I}-\frac{2}{\|\boldsymbol{v}\|_{2}^{2}} \boldsymbol{v} \boldsymbol{v}^{\mathrm{T}}
$$

Esta matriz tem a propriedade de refletir um vetor $\boldsymbol{x}$ em relação ao plano ortogonal ao vetor $\boldsymbol{v}$. Mostra-se também que $\boldsymbol{H}$ é um operador simétrico e ortogonal. Um pequeno exemplo em $\mathbb{R}^{2}$ clarifica a apresentação.

Seja $\boldsymbol{v}=\left[\begin{array}{ll}-1 & 1\end{array}\right]^{\mathrm{T}}$ e $\boldsymbol{x}=\left[\begin{array}{ll}3 & 1\end{array}\right]^{\mathrm{T}}$. Neste caso,

$$
\boldsymbol{x}^{\prime}=\boldsymbol{H} \boldsymbol{x}=\left(\mathbb{I}-\frac{2}{\|\boldsymbol{v}\|_{2}^{2}} \boldsymbol{v} \boldsymbol{v}^{\mathrm{T}}\right) \boldsymbol{x}=\left[\begin{array}{ll}
0 & 1 \\
1 & 0
\end{array}\right]\left[\begin{array}{l}
3 \\
1
\end{array}\right]=\left[\begin{array}{l}
1 \\
3
\end{array}\right]
$$

A figura Figura D.1 mostra a reflexão no plano $\mathbb{R}^{2}$.

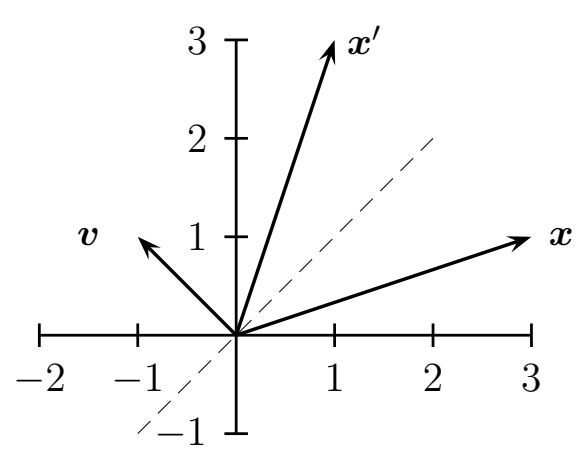

Figura D.1: Representação gráfica da reflexão de Householder do vetor $\boldsymbol{x}$ em relação ao plano ortogonal ao vetor $\boldsymbol{v}$. A linha tracejada indica o plano de reflexão.

Pode-se então encontrar um vetor $\boldsymbol{v}$ que seja capaz de refletir o vetor $\boldsymbol{x}$ sobre o eixo $\hat{\boldsymbol{e}}_{1}=\left[\begin{array}{llll}1 & 0 & \ldots & 0\end{array}\right]$, zerando os demais elementos deste vetor. 
A partir da definição (D.17), chega-se a

$$
\boldsymbol{x}^{\prime}=\boldsymbol{H} \boldsymbol{x}=\left(\mathbb{I}-\frac{2}{\|\boldsymbol{v}\|_{2}^{2}} \boldsymbol{v} \boldsymbol{v}^{\mathrm{T}}\right) \boldsymbol{x}=\boldsymbol{x}-\left(\frac{2 \boldsymbol{v}^{\mathrm{T}} \boldsymbol{x}}{\|\boldsymbol{v}\|_{2}^{2}}\right) \boldsymbol{v}
$$

onde o termo entre parênteses na direita é um valor escalar.

Como $\boldsymbol{x}^{\prime} \in \operatorname{span}\left\{\hat{\boldsymbol{e}}_{1}\right\}$, então obrigatoriamente $\boldsymbol{v} \in \operatorname{span}\left\{\boldsymbol{x}, \hat{\boldsymbol{e}}_{1}\right\}$. Desta forma, escolhendo-se $\boldsymbol{v}=\boldsymbol{x}+\alpha \hat{\boldsymbol{e}}_{1}$, substituindo na equação anterior e coletando os termos, chega-se a

$$
\boldsymbol{x}^{\prime}=\left(1-\frac{2\left(\|\boldsymbol{x}\|_{2}^{2}+\alpha x_{1}\right)}{\|\boldsymbol{x}\|_{2}^{2}+2 \alpha x_{1}+\alpha^{2}}\right) \boldsymbol{x}-\left(\frac{2\left(\boldsymbol{x}+\alpha \hat{\boldsymbol{e}}_{\mathbf{1}}\right)^{\mathrm{T}} \boldsymbol{x} \alpha}{\boldsymbol{x}^{\mathrm{T}} \boldsymbol{x}+2 \alpha \boldsymbol{x} \hat{\boldsymbol{e}}_{\mathbf{1}}+\alpha^{2}}\right) \hat{\boldsymbol{e}}_{\mathbf{1}}
$$

onde $x_{1}$ é o primeiro componente do vetor $\boldsymbol{x}$. Para que $\boldsymbol{x}^{\prime} \in \operatorname{span}\left\{\hat{\boldsymbol{e}}_{1}\right\}$, é necessário que o primeiro termo entre parênteses seja zero, logo

$$
1-\frac{2\left(\|\boldsymbol{x}\|_{2}^{2}+\alpha x_{1}\right)}{\|\boldsymbol{x}\|_{2}^{2}+2 \alpha x_{1}+\alpha^{2}}=0 \Leftrightarrow \alpha= \pm\|\boldsymbol{x}\|_{2}
$$

e portanto

$$
\boldsymbol{v}=\boldsymbol{x} \pm\|\boldsymbol{x}\|_{2} \hat{e}_{1}
$$

Finalmente, substituindo o valor de $\boldsymbol{v}$ em (D.20), chega-se a

$$
\boldsymbol{x}^{\prime}=\boldsymbol{H} \boldsymbol{x}=\mp\|\boldsymbol{x}\|_{2} \hat{\boldsymbol{e}}_{\mathbf{1}} .
$$

A escolha do sinal para $\alpha$ não é importante sob ponto de vista teórico, mas pode fazer diferença numericamente. Para mais detalhes e para métodos alternativos, ver Stewart [138, p. 289] e Golub e Loan [139, p. 210].

Doravante, $\boldsymbol{v}=\operatorname{GenHouse}(\boldsymbol{x})$ designa a função que calcula o vetor $\boldsymbol{v}$ a partir do vetor $\boldsymbol{x}$ de tal forma que $\boldsymbol{x}^{\prime}$ seja múltiplo de $\hat{\boldsymbol{e}}_{1}$. Em outras palavras, zera todas as componentes de $\boldsymbol{x}^{\prime}$ exceto a primeira.

Seja uma matriz $\boldsymbol{X} \in \mathbb{R}^{n \times m}$ particionada em vetores-coluna de tal sorte que $\boldsymbol{X}=\left[\begin{array}{ll}\boldsymbol{x}_{1} & \tilde{\boldsymbol{X}}_{1}\end{array}\right]$. Identificando a primeira coluna com um vetor a ser refletido, pode-se montar uma matriz $\boldsymbol{H}_{1} \operatorname{com} \boldsymbol{v}_{1}=\operatorname{GenHouse}\left(\boldsymbol{x}_{1}\right)$. 
Aplicando esta reflexão em $\boldsymbol{X}$ resulta em

$$
\boldsymbol{H}_{1} \boldsymbol{X}=\left[\begin{array}{ll}
\boldsymbol{H}_{1} \boldsymbol{x}_{1} & \boldsymbol{H}_{1} \tilde{\boldsymbol{X}}_{1}
\end{array}\right]=\left[\begin{array}{ll}
\boldsymbol{x}_{1}^{\prime} & \boldsymbol{H}_{1} \tilde{\boldsymbol{X}}_{1}
\end{array}\right]=\left[\begin{array}{cc}
\mp\left\|\boldsymbol{x}_{1}\right\|_{2} & \boldsymbol{r}_{1} \\
\mathbf{0} & \boldsymbol{X}_{2}
\end{array}\right]
$$

onde $\boldsymbol{r}_{1} \in \mathbb{R}^{1 \times(m-1)}$ e $\boldsymbol{X}_{2} \in \mathbb{R}^{(n-1) \times m-1}$ compõem a partição $\boldsymbol{H}_{1} \tilde{\boldsymbol{X}}_{1}$.

Seja agora a partição de $\boldsymbol{X}_{2}$ em vetores-coluna $\boldsymbol{X}_{2}=\left[\begin{array}{ll}\boldsymbol{x}_{2} & \tilde{\boldsymbol{X}}_{\mathbf{2}}\end{array}\right]$. Identificando a primeira coluna com um vetor a ser refletido, pode-se montar uma segunda reflexão no subespaço $\mathbb{R}^{(n-1)}$, dado por $\hat{\boldsymbol{H}}_{2} \in \mathbb{R}^{(n-1) \times(n-1)} \operatorname{com} \boldsymbol{v}_{2}=\operatorname{GenHouse}\left(\boldsymbol{x}_{2}\right)$ e expandir para $\mathbb{R}^{n}$

$$
\boldsymbol{H}_{2}=\left[\begin{array}{cc}
1 & 0 \\
\mathbf{0} & \hat{\boldsymbol{H}}_{2}
\end{array}\right]
$$

Aplicando este operador em $\boldsymbol{H}_{1} \boldsymbol{X}$, tem-se

$$
\boldsymbol{H}_{2} \boldsymbol{H}_{1} \boldsymbol{X}=\boldsymbol{H}_{2}\left[\begin{array}{ccc}
\mp\|\boldsymbol{x}\|_{2} & \boldsymbol{r}_{1} \\
\mathbf{0} & \boldsymbol{X}_{2}
\end{array}\right]=\left[\begin{array}{cc}
\mp\left\|\boldsymbol{x}_{1}\right\|_{2} & \boldsymbol{r}_{1} \\
\mathbf{0} & \hat{\boldsymbol{H}}_{2} \boldsymbol{X}_{2}
\end{array}\right]=\left[\begin{array}{c}
\mp\left\|\boldsymbol{x}_{1}\right\|_{2} \\
\mathbf{0}
\end{array}\left[\begin{array}{cc}
\mp\left\|\boldsymbol{x}_{2}\right\|_{2} & \boldsymbol{r}_{2} \\
\mathbf{0} & \boldsymbol{X}_{3}
\end{array}\right]\right]
$$

Doravante, $\boldsymbol{X}^{\prime}=\operatorname{House}(\boldsymbol{X}, i, j)$ designa a função que calcula a reflexão de Householder de uma matriz $\boldsymbol{X}$, identificando como vetor $\boldsymbol{x}$ o trecho da $j$-ésima coluna, iniciando na $i$-ésima linha.

A seguir mostra-se um diagrama do algoritmo para transformar eficientemente uma matriz em uma matriz triangular superior. Em cada passo, o vetor $\boldsymbol{x}$ utilizado é destacado.

$$
\left[\begin{array}{lll}
X & X & X \\
X & X & X \\
X & X & X \\
X & X & X
\end{array}\right] \stackrel{\boldsymbol{H}_{1}}{\rightarrow}\left[\begin{array}{ccc}
X & X & X \\
0 & X & X \\
0 & X & X \\
0 & X & X
\end{array}\right] \stackrel{\boldsymbol{H}_{2}}{\rightarrow}\left[\begin{array}{ccc}
X & X & X \\
0 & X & X \\
0 & 0 & X \\
0 & 0 & X
\end{array}\right] \stackrel{\boldsymbol{H}_{3}}{\rightarrow}\left[\begin{array}{ccc}
X & X & X \\
0 & X & X \\
0 & 0 & X \\
0 & 0 & 0
\end{array}\right]=\boldsymbol{T} .
$$

House $(\boldsymbol{A}, 1,1) \quad \operatorname{House}(\boldsymbol{A}, 2,2) \quad \operatorname{House}(\boldsymbol{A}, 3,3)$ 
A mesma observação feita para a rotação de Givens é valida aqui: Seja $p$ o numero de operadores $\boldsymbol{H}_{i}$ aplicados em $\boldsymbol{A}$ necessários para torná-la triangular superior. Então a matriz

$$
\boldsymbol{P}=\boldsymbol{H}_{p} \boldsymbol{H}_{p-1} \ldots \boldsymbol{H}_{2} \boldsymbol{H}_{1}
$$

é ortogonal e também define a fatoração QR de $\boldsymbol{A}$.

Nas seções posteriores, $\boldsymbol{T}=\operatorname{TriHouse}(\boldsymbol{M})$ representa a função que triangulariza uma matriz $M$, utilizando reflexões de Householder seguindo o algoritmo descrito.

\section{D.3 Atualização de Cholesky}

Sejam $\boldsymbol{A}=\boldsymbol{X} \boldsymbol{X}^{\mathrm{T}}$, onde $\boldsymbol{A} \in \mathbb{R}^{n \times n}$ e $\boldsymbol{X} \in \mathbb{R}^{n \times m}, m \geq n$, e o fator de Cholesky (inferior) de $\boldsymbol{A}=\boldsymbol{S} \boldsymbol{S}^{\mathrm{T}}$.

A fatoração QR equivalente ao problema é dada por

$$
\boldsymbol{Q R}=\boldsymbol{X}^{\mathrm{T}}
$$

onde $\boldsymbol{R}=\boldsymbol{S}^{\mathrm{T}}$

A determinação do novo fator de Cholesky de $\boldsymbol{A}^{\prime}=\boldsymbol{A}+\alpha \boldsymbol{u} \boldsymbol{u}^{\mathrm{T}}$ é equivalente a achar a nova matriz $\boldsymbol{R}^{\prime}$ de

$$
\begin{aligned}
& \boldsymbol{Q}^{\prime} \boldsymbol{R}^{\prime}=\boldsymbol{X}^{\prime} \\
& \boldsymbol{X}^{\prime}=\left[\begin{array}{c}
\sqrt{\alpha} \boldsymbol{u}^{\mathrm{T}} \\
\boldsymbol{X}^{\mathrm{T}}
\end{array}\right] .
\end{aligned}
$$

Incluindo a nova linha na fatoração QR, tem-se

$$
\begin{aligned}
{\left[\begin{array}{ll}
1 & \mathbf{0} \\
\mathbf{0} & \boldsymbol{Q}
\end{array}\right]\left[\begin{array}{c}
\sqrt{\alpha} \boldsymbol{u}^{\mathrm{T}} \\
\boldsymbol{R}
\end{array}\right] } & =\left[\begin{array}{c}
\sqrt{\alpha} \boldsymbol{u}^{\mathrm{T}} \\
\boldsymbol{X}^{\mathrm{T}}
\end{array}\right] \\
\tilde{\boldsymbol{Q}} \tilde{\boldsymbol{R}} & =\boldsymbol{X}^{\prime},
\end{aligned}
$$

onde $\tilde{Q}$ continua ortogonal, mas $\tilde{R}$ não é mais triangular superior. 
Multiplicando pela esquerda por $\tilde{\boldsymbol{Q}}^{\mathrm{T}}$ em ambos os lados,

$$
\tilde{\boldsymbol{R}}=\tilde{\boldsymbol{Q}}^{\mathrm{T}} \boldsymbol{X}^{\prime}
$$

Resta agora encontra uma série de transformações ortogonais que transformem o termo da esquerda em triangular superior. Determinadas estas transformações, a matriz $\boldsymbol{P}$ que as contém pode ser usada para encontrar a nova fatoração $Q R$

$$
\begin{aligned}
\boldsymbol{P} \tilde{\boldsymbol{R}} & =\boldsymbol{P} \tilde{\boldsymbol{Q}}^{\mathrm{T}} \boldsymbol{X}^{\prime} \\
\boldsymbol{R}^{\prime} & =\left(\boldsymbol{Q}^{\prime}\right)^{\mathrm{T}} \boldsymbol{X}^{\prime}
\end{aligned}
$$

onde $\boldsymbol{R}^{\prime}$ é triangular superior e

$$
\boldsymbol{S}^{\prime}=\left(\boldsymbol{R}^{\prime}\right)^{\mathrm{T}}
$$

A determinação de $\boldsymbol{R}^{\prime}$ pode ser feita utilizando rotações de Givens. De fato, $\tilde{\boldsymbol{R}}$ é Hessenberg superior e pode ser triangularizada com $\boldsymbol{R}^{\prime}=\operatorname{TriHessGivens}(\tilde{\boldsymbol{R}})$.

Caso a atualização for do tipo $\boldsymbol{A}^{\prime}=\boldsymbol{A}+\alpha \boldsymbol{U} \boldsymbol{U}^{\mathrm{T}}$, onde $\boldsymbol{U} \in \mathbb{R}^{n \times p}$, ou seja, uma atualização de posto $p>1$, pode-se proceder da mesma maneira para cada coluna de $\boldsymbol{U}$. Porém neste caso é preferível, montar a matriz $\tilde{\boldsymbol{R}}$ como

$$
\tilde{\boldsymbol{R}}=\left[\begin{array}{c}
\sqrt{\alpha} \boldsymbol{U}^{\mathrm{T}} \\
\boldsymbol{R}
\end{array}\right]
$$

e proceder a triangularização $\operatorname{com} \boldsymbol{R}^{\prime}=\operatorname{TriHouse}(\tilde{\boldsymbol{R}})$ para reduzir o número de operações.

\section{D.4 Desatualização de Cholesky}

Para $\boldsymbol{A}=\boldsymbol{X} \boldsymbol{X}^{\mathrm{T}}$, cujo fator de Cholesky é $\boldsymbol{S}=\boldsymbol{R}^{\mathrm{T}}$, o problema de encontrar o novo fator de Cholesky de $\boldsymbol{A}^{\prime}=\boldsymbol{A}-\alpha \boldsymbol{u} \boldsymbol{u}^{\mathrm{T}}$ é de difícil solução pois $\boldsymbol{X}$ não é conhecida em geral.

Como a remoção de uma linha de $\boldsymbol{X}^{\mathrm{T}}$ é equivalente ao produto externo $\boldsymbol{X} \boldsymbol{X}^{\mathrm{T}}-\boldsymbol{x} \boldsymbol{x}^{\mathrm{T}}$, a determinação do novo fator de Cholesky de $\boldsymbol{A}^{\prime}=\boldsymbol{A}-\alpha \boldsymbol{u} \boldsymbol{u}^{\mathrm{T}}$ é 
equivalente a achar a nova fatoração $\mathrm{QR}$ de $\boldsymbol{X}^{\mathrm{T}}$ com esta linha removida.

A desatualização pode ser feita com $\boldsymbol{u}$ arbitrário, contanto que $\boldsymbol{A}^{\prime}$ continue positiva definida.

O ponto chave do método é notar que supondo $\boldsymbol{A}^{\prime}=\boldsymbol{A}-\alpha \boldsymbol{u} \boldsymbol{u}^{\mathrm{T}}$ positiva definida, esta matriz apresenta fatoração de Cholesky $\boldsymbol{A}^{\prime}=\left(\boldsymbol{R}^{\prime}\right)^{\mathrm{T}} \boldsymbol{R}^{\prime}$ e poderíamos fazer uma atualização de Cholesky para encontrar $\boldsymbol{R}$ de $\boldsymbol{A}$, como descrita na seção anterior. Resta agora inverter o processo.

Seja o operador ortogonal $\boldsymbol{P}$ obtido pela atualização de Cholesky para adicionar $\sqrt{\alpha} \boldsymbol{u}$ a $\boldsymbol{R}$, então 3

$$
\begin{aligned}
\boldsymbol{P}\left[\begin{array}{c}
\boldsymbol{R}^{\prime} \\
\sqrt{\alpha} \boldsymbol{u}
\end{array}\right] & =\left[\begin{array}{l}
\boldsymbol{R} \\
0
\end{array}\right] \\
\boldsymbol{P} \tilde{\boldsymbol{R}} & =\boldsymbol{R} .
\end{aligned}
$$

Considere agora o primeira passo desse algoritmo, que gera a rotação para a primeira coluna de $\tilde{\boldsymbol{R}}$. Seja a seguinte partição das matrizes deste primeiro passo:

$$
\left[\begin{array}{ccc}
c & 0 & s \\
0 & \mathbb{I} & 0 \\
-s & 0 & c
\end{array}\right]\left[\begin{array}{cc}
r_{11}^{\prime} & \boldsymbol{r}_{12}^{\prime \mathrm{T}} \\
0 & \boldsymbol{R}_{22}^{\prime} \\
u_{1} & \boldsymbol{u}_{2}^{\mathrm{T}}
\end{array}\right]=\left[\begin{array}{cc}
r_{11} & \boldsymbol{r}_{12}^{\mathrm{T}} \\
0 & \boldsymbol{R}_{22} \\
0 & \tilde{\boldsymbol{u}}_{2}^{\mathrm{T}}
\end{array}\right]
$$

Este é o passo que zera o elemento $u_{1}$ através de uma rotação com $r_{11}^{\prime}$. Salienta-se que nesta equação $r_{11}^{\prime}, c, s, \boldsymbol{r}_{12}^{\prime}$ e $\tilde{\boldsymbol{u}}_{2}$ devem ser determinados a partir dos valores conhecidos $r_{11}, \boldsymbol{r}_{12}, u_{1}$ e $\boldsymbol{u}_{2}$.

Inicialmente, a transformação ortogonal não altera a norma dos vetores, então

$$
r_{11}^{\prime 2}+u_{1}^{2}=r_{11}^{2} \Rightarrow r_{11}^{\prime}=\sqrt{r_{11}^{2}-u_{1}^{2}}
$$

Conhecendo $r_{11}^{\prime}$, c e $s$ podem ser determinados

$$
c=\frac{r_{11}^{\prime}}{r_{11}}, \quad s=\frac{u_{1}}{r_{11}}
$$

\footnotetext{
${ }^{3}$ Nesta equação, ao contrário do apresentado na atualização de Cholesky, a linha adicionada é a última. Esta mudança é feita para facilitar a explicação e não afeta o resultado.
} 
De (D.41) obtém-se também

$$
\boldsymbol{r}_{12}^{\mathrm{T}}=c \boldsymbol{r}_{12}^{\prime \mathrm{T}}+s \boldsymbol{u}_{2}^{\mathrm{T}} \quad \Leftrightarrow \quad \boldsymbol{r}_{12}^{\prime \mathrm{T}}=\frac{\boldsymbol{r}_{12}^{\mathrm{T}}-s \boldsymbol{u}_{2}^{\mathrm{T}}}{c}
$$

$\mathrm{e}$

$$
\tilde{\boldsymbol{u}}_{2}^{\mathrm{T}}=c \boldsymbol{u}_{2}^{\mathrm{T}}-s \boldsymbol{r}_{12}^{\prime \mathrm{T}}
$$

Com isto, determina-se a primeira linha de $\boldsymbol{R}^{\prime}$.

$$
\boldsymbol{R}^{\prime}=\left[\begin{array}{cc}
r_{11}^{\prime} & \boldsymbol{r}_{12}^{\prime \mathrm{T}} \\
& \\
&
\end{array}\right]
$$

Uma vez que $\tilde{\boldsymbol{u}}_{2}^{\mathrm{T}}$ foi determinado, pode-se repetir o processo com a matriz

$$
\tilde{\boldsymbol{R}}=\left[\begin{array}{c}
\boldsymbol{R}_{22} \\
\tilde{\boldsymbol{u}}_{2}^{\mathrm{T}}
\end{array}\right]
$$

cuja partição agora é

$$
\left[\begin{array}{ccc}
c & 0 & s \\
0 & \mathbb{I} & 0 \\
-s & 0 & c
\end{array}\right]\left[\begin{array}{cc}
r_{22}^{\prime} & \boldsymbol{r}_{23}^{\prime \mathrm{T}} \\
0 & \boldsymbol{R}_{33}^{\prime} \\
u_{2} & \boldsymbol{u}_{3}^{\mathrm{T}}
\end{array}\right]=\left[\begin{array}{cc}
r_{22} & \boldsymbol{r}_{23}^{\mathrm{T}} \\
0 & \boldsymbol{R}_{33} \\
0 & \tilde{\boldsymbol{u}}_{3}^{\mathrm{T}}
\end{array}\right],
$$

para obter a segunda linha de $\boldsymbol{R}^{\prime}$

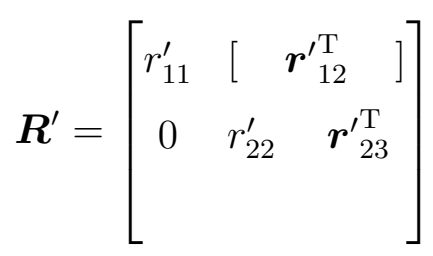

e assim sucessivamente até completar a matriz $\boldsymbol{R}^{\prime}$.

O algoritmo falha se o termo dentro da raiz quadrada em (D.42) tornar-se negativo. Isto significa que a matriz deixou de ser positiva definida. Este algoritmo também falha se (D.42) for igual a zero pois neste caso $c=0$ e as divisões não pode ser calculadas em (D.44). 


\section{Apêndice E}

\section{Método Level Set para segmentação de imagens}

Nesta parte do apêndice apresenta-se a formulação do método de segmentação por curvas de nível (Level set) utilizada neste trabalho. Inicia-se discutindo as formas de representação implícita e explícita do bordo de uma região fechada e alguns resultados do cálculo que servirão de auxílio. Após é apresentado o método Level set e a formulação que não necessita de reinicialização. Por fim critérios de parada para o algoritmo iterativo e demais detalhes de implementação. Para mais informações a respeito do método, ver Osher e Fedkiw [140], Osher e Paragios [141], Sethian [142], Li et al. [133].

\section{E.1 Funções explícitas e implícitas}

Seja o problema unidimensional de particionar a reta real em duas regiões, limitadas pelos os pontos $x=-1$ e $x=1$. Uma região compreendida entres estes valores, $\mathrm{e}$ a outra região composta pela união das duas semi-retas restantes. Neste problema, identifica-se e nomeia-se as duas regiões e sua interface: $\Omega^{-}=(-1,1)$, nomeando-a interior, $\Omega^{+}=(-\infty, 1) \cup(1, \infty)$ nomeando-a exterior e a interface $\partial \Omega=\{-1,1\}$. No caso unidimensional, as regiões apresentam dimensão 1 e a interface dimensão 0. Mais genericamente, as regiões estarão em $\mathbb{R}^{n}$ e a interface em $\mathbb{R}^{n-1}$.

Pode-se representar esta interface de duas maneiras: a representação explícita e a representação implícita. No primeiro caso, identifica-se explicitamente todos os pontos 
que compõem a interface. Para este primeiro exemplo $\partial \Omega=\{-1,1\}$. Na representação implícita, identificamos a interface como uma curva de nível de uma função $\phi(x) \in \mathbb{R}^{n}$, definida em todo o domínio. Uma possível função para este exemplo seja $\phi=x^{2}-1$, cuja curva de nível $\phi(x)=0$ representa a interface desejada! 1 .

A Figura E.1 mostra a função $\phi$ definindo a interface das regiões $\Omega^{+}$e $\Omega^{-}$em $\phi(x)=$ 0 .

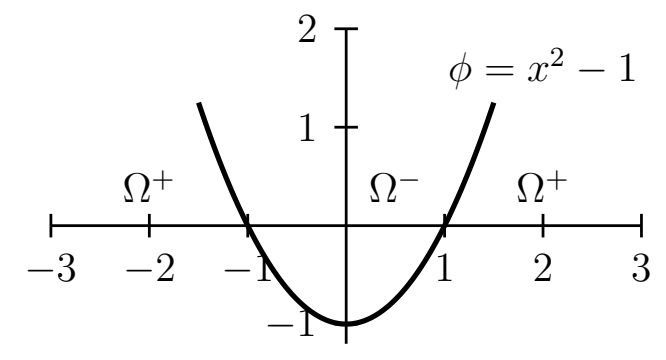

Figura E.1: Função implícita $\phi=x^{2}-1$ definindo as regiões $\Omega^{+}$e $\Omega^{-}$.

Em duas dimensões, seja o problema de separar uma região circular, de raio unitário centrado na origem. Usando a representação explícita, a interface é descrita por $\partial \Omega=\{x \mid\|x\|=1\}$. Na representação implícita pode-se adotar a função $\phi(x, y)=x^{2}+y^{2}-1$, definida em todo $\mathbb{R}^{2}$, cuja curva de nível $\phi(x, y)=0$ define a interface desejada.

$\mathrm{Na}$ representação explícita, é necessária a parametrização da interface para sua manipulação e estudo. Neste mesmo exemplo a parametrização é dada por $\partial \Omega=(\cos (s), \sin (s)), s \in[0,2 \pi[$, porém a determinação de uma parametrização para uma interface de formato arbitrário não é simples de ser obtida, sendo geralmente aproximada por splines.

No caso mais geral em $\mathbb{R}^{n}$, a representação explícita necessita de uma parametrização de uma hiper-curva em $\mathbb{R}^{n-1}$ enquanto que na representação implícita, é necessária a definição de uma função em $\mathbb{R}^{n}$ (ou em parte dele).

Na prática sempre trabalha-se com discretizações destas entidades, desta forma algum tipo de interpolação deverá ser usada para encontrar ou aproximar todos os pontos da interface. No caso da representação explícita, uma forma aproximada

\footnotetext{
${ }^{1} \mathrm{O}$ valor da curva de nível que representa a interface é arbitrariamente definido. Usualmente é
} atribuído o nível zero. 
de descrever a interface é através do conhecimento de um número finito de pontos pertencentes à interface e usando uma interpolação para aproximar a hiper-curva nos demais pontos. No caso da representação implícita pode-se também fazer uma discretização, porém serão necessários pontos amostrados em todo o $\mathbb{R}^{n}$ para aproximar a função. Para reduzir o número de pontos, pode-se discretizar uma região menor $D \subset \mathbb{R}^{n}$ usando um número finito de pontos, ou ainda aproximar a função $\phi$ apenas em uma vizinhança apropriada da hiper-curva de nível de interesse.

\section{E.1.1 Propriedades}

A representação implícita tem uma série de características geométricas que a torna mais vantajosa quando comparada com a representação explícita:

1. Mesmo nos casos em que a parametrização da interface é possível na representação explícita, classificar os demais pontos $\boldsymbol{x} \notin \boldsymbol{x}_{I}$ como pertencente a $\Omega^{+}$ou a $\Omega^{-}$necessita um grande esforço computacional. Já na representação implícita, basta observar o sinal de $\phi(\boldsymbol{x})$ : se $\phi(\boldsymbol{x})<0$, então $\boldsymbol{x} \in \Omega^{-}$e se $\phi(\boldsymbol{x})>0$, então $\boldsymbol{x} \in \Omega^{+}$;

2. Operações booleanas com regiões na representação implícita são elementares. Sejam $\phi_{1}$ e $\phi_{2}$ duas funções implícitas que destacam duas regiões. Então $f=$ $\min \left(\phi_{1}, \phi_{2}\right)$ representa a união das duas regiões, $f=\max \left(\phi_{1}, \phi_{2}\right)$ representa a intersecção das duas regiões, $f=-\phi_{1}$ representa o complemento de $\phi_{1}$ e $f=\max \left(\phi_{1},-\phi_{2}\right)$ representa subtrair a região 2 da região 1 . Na representação explícita estas operações não são possíveis de serem obtidas apenas com as parametrizações $\partial \Omega_{1}(s)$ e $\partial \Omega_{2}(s)$, um grande trabalho computacional é necessário antes da operação booleana;

3. O vetor gradiente

$$
\nabla \phi=\left(\frac{\partial \phi}{\partial x}, \frac{\partial \phi}{\partial y}, \frac{\partial \phi}{\partial z}, \ldots\right) \in \mathbb{R}^{n}
$$

é perpendicular às curvas de nível e aponta2 2 para o sentido de $\Omega^{+}$.

\footnotetext{
${ }^{2}$ Exceto nos pontos de estacionariedade de $\phi$ onde o gradiente é o vetor zero. Mostra-se que estes casos não são problemáticos e pode-se arbitrar uma direção para $\nabla \phi$.
} 
4. Definindo o versor $\hat{\boldsymbol{n}}=\frac{\nabla \phi}{\|\nabla \phi\|_{2}}$, a curvatura local $\kappa(\boldsymbol{x})$ de uma curva de nível pode ser definida pelo divergente de $\hat{\boldsymbol{n}}$

$$
\kappa(\boldsymbol{x})=\nabla \cdot \hat{\boldsymbol{n}}=\frac{\partial n_{x}}{\partial x}+\frac{\partial n_{y}}{\partial y}+\frac{\partial n_{z}}{\partial z}+\ldots
$$

Com esta definição, a curva de nível será localmente convexa se $\kappa(\boldsymbol{x})>0$, côncava se $\kappa(\boldsymbol{x})<0$ e plana se $\kappa(\boldsymbol{x})=0$. A Figura E.2 mostra estas regiões.

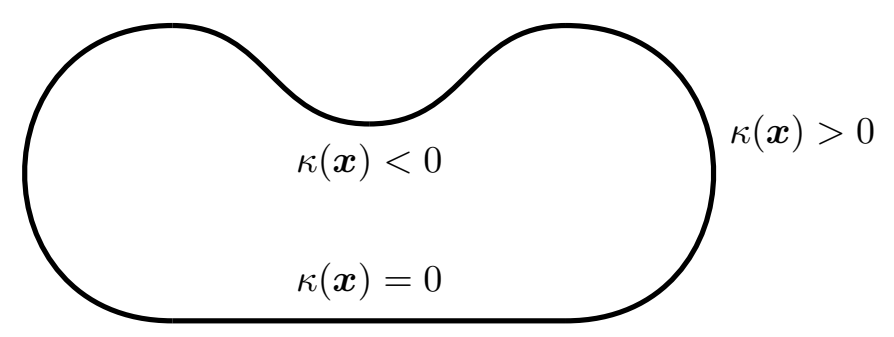

Figura E.2: Curva de nível de $\phi$ destacando regiões com curvaturas diferentes.

\section{E.1.2 Ferramentas}

Algumas ferramentas do cálculo são úteis no tratamento da representação implícita.

A função característica $\chi^{-}$da região interior $\Omega^{-}$é definida por

$$
\chi^{-}(\boldsymbol{x}) \triangleq \begin{cases}1 & , \quad \phi(\boldsymbol{x}) \leq 0 \\ 0 & , \quad \phi(\boldsymbol{x})>0\end{cases}
$$

Similarmente, a função característica $\chi^{+}$da região exterior $\Omega^{+}$é definida por

$$
\chi^{+}(\boldsymbol{x}) \triangleq \begin{cases}0 & , \quad \phi(\boldsymbol{x}) \leq 0 \\ 1 & , \quad \phi(\boldsymbol{x})>0\end{cases}
$$

Definindo-se a distribuição Heaviside unidimensional por

$$
H(\phi) \triangleq \begin{cases}0 & , \quad \phi(\boldsymbol{x}) \leq 0 \\ 1 & , \quad \phi(\boldsymbol{x})>0\end{cases}
$$


pode-se reescrever as funções características como

$$
\begin{aligned}
& \chi^{+}=H(\phi(\boldsymbol{x})) \\
& \chi^{-}=1-H(\phi(\boldsymbol{x}))=H(-\phi(\boldsymbol{x})),
\end{aligned}
$$

que são formas mais convenientes de se trabalhar.

A integral de uma função $f(\boldsymbol{x})$ sobre a região $\Omega^{-}$é definida por 3

$$
V_{f}^{-} \triangleq \int_{\Omega} f(\boldsymbol{x}) \chi^{-}(\boldsymbol{x}) d \boldsymbol{x}=\int_{\Omega} f(\boldsymbol{x}) H(-\phi(\boldsymbol{x})) d \boldsymbol{x} .
$$

Nota-se que a integral é calculada sobre todo domínio $\Omega$ e que $\chi^{-}(\boldsymbol{x})$ elimina da integral toda a região $\Omega^{+}$. Similarmente, a integral de $f(\boldsymbol{x})$ sobre a região $\Omega^{+}$é definida por

$$
V_{f}^{+} \triangleq \int_{\Omega} f(\boldsymbol{x}) \chi^{+}(\boldsymbol{x}) d \boldsymbol{x}=\int_{\Omega} f(\boldsymbol{x}) H(\phi(\boldsymbol{x})) d \boldsymbol{x}
$$

Uma última integral necessária é aquela calculada sobre a interface $\partial \Omega$. Para isto, utiliza-se a Delta de Dirac n-dimensional.

Por definição, a derivada direcional da distribuição de Heaviside na direção de um vetor $\boldsymbol{n}$ qualquer é a distribuição Delta de Dirac $n$-dimensional $\hat{\delta}(\boldsymbol{x})$ :

$$
\hat{\delta}(\boldsymbol{x}) \triangleq \nabla H(\phi(\boldsymbol{x})) \cdot \hat{\boldsymbol{n}}
$$

Usando a definição anterior para $H(\phi)$, a distribuição $\hat{\delta}(\boldsymbol{x})$ será não nula somente na interface $\partial \Omega$. Pode-se expandir esta última expressão, através da regra da cadeia aplicada a uma distribuição [143] e usando a definição do versor normal já definido anteriormente

$$
\hat{\delta}(\boldsymbol{x})=H^{\prime}(\phi(\boldsymbol{x})) \nabla \phi(\boldsymbol{x}) \cdot \frac{\nabla \phi(\boldsymbol{x})}{\|\nabla \phi(\boldsymbol{x})\|_{2}}=H^{\prime}(\phi(\boldsymbol{x}))\|\nabla \phi(\boldsymbol{x})\|_{2},
$$

\footnotetext{
${ }^{3}$ Chamada integral de volume no caso de regiões 3D, integral de área no caso de regiões 2D ou integral de comprimento no caso de regiões 1D.
} 
ou ainda, usando a relação entre o de delta de Dirac e a Heaviside unidimensionais,

$$
\hat{\delta}(\boldsymbol{x})=\delta(\phi(\boldsymbol{x}))\|\nabla \phi(\boldsymbol{x})\|_{2}
$$

A integral de uma função $f(\boldsymbol{x})$ sobre a interface $\partial \Omega$ é definida então port 4

$$
S_{f} \triangleq \int_{\Omega} f(\boldsymbol{x}) \hat{\delta}(\boldsymbol{x}) d \boldsymbol{x}=\int_{\Omega} f(\boldsymbol{x}) \delta(\phi(\boldsymbol{x}))\|\nabla \phi(\boldsymbol{x})\|_{2} d \boldsymbol{x}
$$

Note que a integral é calculada sobre todo domínio $\Omega$.

As equações (E.8), (E.9) e (E.13) eliminam a necessidade de separar as regiões de interesse do domínio para calcular algumas integrais, sendo então adequadas para uso com a representação implícita.

Por último, um comentário de ordem prática. Ao invés de utilizar o delta de Dirac, opta-se por uma aproximação suavizada:

$$
\delta(\phi)= \begin{cases}0 & ,|\phi|<\epsilon \\ \frac{1}{2 \epsilon}(1+\cos (\pi \phi / \epsilon)) & ,|\phi| \leq \epsilon\end{cases}
$$

onde $\epsilon$ é um parâmetro escolhido.

\section{E.1.3 Função distância com sinal}

Até o momento, foi escolhida como função implícita a família de funções que apresentam a curva de nível $\phi=0$ delimitando a interface desejada e que $\phi<0$ em $\Omega^{-}$e $\phi>0$ em $\Omega^{+}$. Nada além disto foi imposto. Nesta seção introduz-se uma sub família de funções que apresentam algumas características que permitem um melhor comportamento numérico dos algoritmos.

Considere a função distância, definida por

$$
d(\boldsymbol{x})=\min _{\boldsymbol{x}_{I} \in \partial \Omega}\left(\left\|\boldsymbol{x}-\boldsymbol{x}_{I}\right\|_{2}\right)
$$

implicando que $d(\boldsymbol{x})=0$ se $\boldsymbol{x} \in \partial \Omega$.

\footnotetext{
${ }^{4}$ Chamada integral de superfície no caso de regiões 3D, integral de comprimento no caso de regiões $2 \mathrm{D}$ ou integral de ponto no caso de regiões 1D.
} 
Para um dado $\boldsymbol{x}$, seja $\boldsymbol{x}_{c}$ o ponto na interface mais próximo de $\boldsymbol{x}$ (ver Figura E.3).

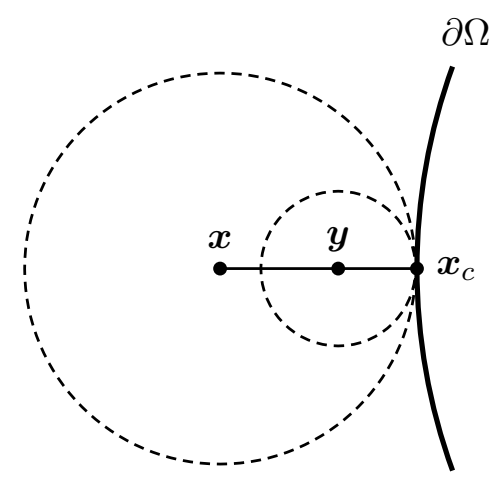

Figura E.3: Vizinhança de um ponto $\boldsymbol{x}$.

Pode-se reescrever a equação anterior como $d(\boldsymbol{x})=\left\|\boldsymbol{x}-\boldsymbol{x}_{c}\right\|_{2}$. Além disto, ligando-se o ponto $\boldsymbol{x}$ ao ponto $\boldsymbol{x}_{c}$ por um segmento de reta, nota-se que para todo ponto $\boldsymbol{y}$ sobre este segmento, $\boldsymbol{x}_{c}$ é também o ponto da interface mais próximo de $\boldsymbol{y}$. Este segmento é o caminho de menor distância entre $\boldsymbol{x}$ e a interface, ou seja, este é o caminho steepest descent de $d(\boldsymbol{x})$. Calculando o vetor gradiente $\nabla d$ verifica-se que este aponta de $\boldsymbol{x}_{c}$ para $\boldsymbol{x}$ e a sua norma é unitária: $\|\nabla d\|_{2}=1$.

Este raciocínio é apenas geralmente válido pois pode-se construir exemplos onde existam mais de um ponto com distância mínima. Neste caso, o vetor $\nabla d$ não é único. Em Osher e Fedkiw [140, p. 19], mostra-se que o problema de não unicidade de solução para $\nabla d$ nestes casos não é grave e basta adotar uma das soluções para o gradiente que o método convergirá.

Usando a definição de função distância, pode-se definir a função distância com sinal que será usada como função $\phi$ no método:

$$
\phi(\boldsymbol{x})=\left\{\begin{array}{lll}
d(\boldsymbol{x}) & , & \boldsymbol{x} \in \Omega^{+} \\
0 & , \quad \boldsymbol{x} \in \partial \Omega . \\
-d(\boldsymbol{x}) & , \quad \boldsymbol{x} \in \Omega^{-}
\end{array}\right.
$$

Esta função apresenta duas características importantes a mais das apresentadas na seção anterior:

1. O módulo do gradiente é unitário: $\|\nabla \phi\|_{2}=1$;

2. Dado um ponto $\boldsymbol{x}$, pode-se escrever $\boldsymbol{x}_{c}=\boldsymbol{x}-\phi(\boldsymbol{x}) \hat{\boldsymbol{n}}$. 
Voltando ao exemplo $1 \mathrm{D}$ da seção anterior, a função implícita que representa $\partial \Omega$ é dada por $\phi(x)=|x|-1$, como pode ser visto na Figura E.4

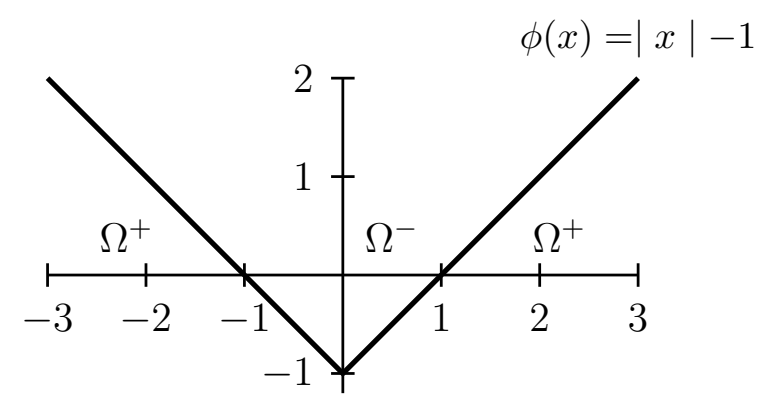

Figura E.4: Função implícita $\phi(x)=|x|-1$ definindo as regiões $\Omega^{+}$e $\Omega^{-}$.

Nota-se que $\|\nabla \phi\|_{2}=1$, exceto para $x=0$, onde a derivada não está definida. Esta singularidade não acarreta problemas na implementação numérica pois devido à amostragem em intervalos discretos dos pontos no domínio, pode-se mostrar que $\|\nabla \phi\|_{2} \in[0,1]$, o que torna o método numérico estável, tomando-se as devidas precauções.

No caso 2D da seção anterior, a função implícita é $\phi(x)=\sqrt{x^{2}+y^{2}}-1$. A mesma observação vale aqui para o ponto $(0,0)$ onde a derivada direcional desta função não existe.

\section{E.2 Método level set e segmentação de imagem}

O método level set aplicado à segmentação de imagem modifica iterativamente a função $\phi$ de modo que sua curva de nível $\phi=0$ posicione-se ao longo do bordo da entidade na imagem que se quer segmentar. Por bordo, considera-se uma diferença contrastante entre pixels próximos. O exemplo mais simples é o caso de uma imagem em tons de cinza, onde se quer segmentar um objeto destacado do resto da imagem. Neste caso, o bordo é identificado por uma variação do nível de cinza da imagem.

Uma imagem $I \in \mathbb{R}^{n}$ pode ser vista como uma função $I: D \rightarrow \mathbb{R}$, onde o domínio $D$ é o conjunto de índices dos pixels da imagem, usualmente amostrados a intervalos regulares no hiper-prisma $D=\left[0, r_{1}\right] \times\left[0, r_{2}\right] \times \ldots \times\left[0, r_{n}\right]$, onde $r_{i}$ é a resolução da imagem na $i$-ésima dimensão. Desta forma, cada pixel pode ser identificado como 
um ponto $x \in D$. O contra domínio pode ser toda a reta real, porém usualmente I apresenta um supremo e um mínimo finito e pode ser então normalizado para o intervalo $[0,1]$. Aqui considera-se o problema de segmentar uma imagem em tons de cinza.

Seja agora $\boldsymbol{v}(\boldsymbol{x}): D \rightarrow \mathbb{R}^{n}$ um campo vetorial, função da imagem e definido de forma que $\boldsymbol{v}(\boldsymbol{x})=\mathbf{0} \mathrm{em}$ todos os pontos no bordo $\partial \Omega$ que se quer segmentar.

O interesse é resolver o problema dinâmico

$$
\frac{d \boldsymbol{x}}{d t}=\boldsymbol{v}(\boldsymbol{x}), \quad \forall \boldsymbol{x} \in \partial \Omega,
$$

com condições iniciais $\partial \Omega(t=0)=\partial \Omega_{0}$, até a convergência. Em outras palavras, deseja-se utilizar $v$ como um campo de velocidades agindo nos pontos do contorno, em um problema de valores iniciais. Nota-se porém que esta formulação necessita de uma representação explícita de $\partial \Omega$. Diz-se que esta equação está na descrição Lagrangeana do movimento.

Para se trabalhar com a representação implícita, é necessário transformá-la na descrição Euleriana do movimento. A seguir algumas propriedades de ambas as descrições:

Descrição Lagrangeana do movimento: Descreve as propriedades de cada ponto, acompanhando-as em suas trajetórias ao longo do tempo.

- O observador (sensor) desloca-se junto com o ponto;

- Os pontos são observados individualmente como uma função do tempo;

- As propriedades de cada ponto são funções exclusiva do tempo, por exemplo: $\boldsymbol{P}_{i}(t), \boldsymbol{v}_{i}(t)$ para posição e velocidade do $i$-ésimo ponto no instante $t$.

Descrição Euleriana do movimento: Consiste em fixar os sensores em um local no espaço e considerar todos os pontos que passam por esse local em um intervalo de tempo.

- O observador (sensor) não desloca-se junto com a partícula;

- As propriedades de interesse são medidas em um local fixo no espaço, enquanto os pontos passam; 
- As propriedades de cada ponto são função do espaço (posição de observação) e do tempo, por exemplo: $\boldsymbol{P}(\boldsymbol{x}, t), \boldsymbol{v}(\boldsymbol{x}, t)$ para posição e velocidade na posição $\boldsymbol{x}$ e instante $t$.

A relação entre as duas representações pode ser facilmente desenvolvida. Seja $F$ uma propriedade qualquer, função da posição e do tempo. Considera-se aqui um domínio em $\mathbb{R}^{2}$ por simplicidade. O seu diferencial em torno de um ponto, em um determinado instante , e do tempo é (representação Euleriana)

$$
d F=\frac{\partial F}{\partial t} d t+\frac{\partial F}{\partial x} d x+\frac{\partial F}{\partial y} d y
$$

Restringindo os aumentos $d x$ e $d y$ de forma que eles sigam a trajetória de um ponto no intervalo $d t: d x=u(x, y) . d t, d y=v(x, y) . d t$, onde $u$ e $v$ são as velocidades em $x$ e $y$ do ponto, chega-se na representação Lagrangeana

$$
d F=\frac{\partial F}{\partial t} d t+\frac{\partial F}{\partial x} u(x, y) \cdot d t+\frac{\partial F}{\partial y} v(x, y) \cdot d t .
$$

Finalmente, dividindo os dois lados por $d t$

$$
\frac{d F}{d t}=\frac{\partial F}{\partial t}+\frac{\partial F}{\partial x} u(x, y)+\frac{\partial F}{\partial y} v(x, y)=\frac{\partial F}{\partial t}+\nabla F \cdot \boldsymbol{v}(x, y),
$$

que é a relação entre as duas representações. Considerando $F=\phi$ e sabendo que $\phi(\boldsymbol{x})=0$ para toto $\boldsymbol{x} \in \partial \Omega$, em todos os instantes, chega-se a

$$
\frac{d \phi}{d t}=0 \Rightarrow \frac{\partial \phi}{\partial t}+\nabla \phi \cdot \boldsymbol{v}=0
$$

Agora, ao invés de resolver (E.17), resolve-se

$$
\frac{\partial \phi}{\partial t}+\nabla \phi \cdot \boldsymbol{v}=0, \quad \boldsymbol{x} \in \Omega
$$

que é mais estável numericamente, apesar do aumento da carga computacional. Esta equação é conhecida como level set equation. 
Usando a relação $\hat{\boldsymbol{n}}=\frac{\nabla \phi}{\|\nabla \phi\|_{2}}$, chega-se a

$$
\frac{\partial \phi}{\partial t}+\nabla \phi \cdot \boldsymbol{v}=\frac{\partial \phi}{\partial t}+\left(\|\nabla \phi\|_{2} \hat{\boldsymbol{n}}\right) \cdot\left(v_{n} \hat{\boldsymbol{n}}+v_{t} \hat{\boldsymbol{t}}\right)=\frac{\partial \phi}{\partial t}+v_{n}\|\nabla \phi\|_{2}=0
$$

onde $v_{n}$ e $v_{t}$ são as componentes normal e tangencial da velocidade $\boldsymbol{v}$ em relação à $\partial \Omega$ e $\hat{\boldsymbol{t}}$ é o versor tangencial à mesma. Nota-se, portanto, que a componente tangencial da velocidade $\boldsymbol{v}$ em relação ao bordo $\partial \Omega$ é naturalmente eliminada pelo produto escalar.

\section{E.2.1 Reinicialização}

Ao inicializar a função $\phi$ com a função distância com sinal e usar a equação de evolução (E.22) iterativamente, a cada nova iteração $\phi$ será apenas aproximadamente uma função distância com sinal. Conforme as iterações avançam, esta aproximação torna-se cada vez pior, o que pode tornar-se catastrófico. Por este motivo, a formulação clássica do método necessita reinicializar, de tempos em tempos, a função $\phi$ para corrigir a sua forma e manter a estabilidade do algoritmo. É interessante que a reinicialização mantenha o valor atual de $\phi$ nos pontos em $\partial \Omega$ e corrigir os demais pontos.

Quer-se que a função tenha a propriedade $\|\nabla \phi\|_{2}=1$. Então pode-se considerar resolver a equação

$$
\frac{\partial \phi}{\partial t}+\|\nabla \phi\|_{2}=1
$$

iterativamente até a convergência para uma nova função distância com sinal, com a esperança de que não haja mudança nos pontos onde $\phi=0$ e que nos demais pontos, $\phi$ seja corrigida. Porém não há garantias de que não haja mudanças da curva $\phi=0$, o que de fato ocorre.

Para contornar isto, pode-se resolver dois problemas de contorno

$$
\begin{aligned}
& \frac{\partial \phi}{\partial t}+\|\nabla \phi\|_{2}=1, \quad x \in \Omega^{+} \\
& \frac{\partial \phi}{\partial t}-\|\nabla \phi\|_{2}=-1, \quad x \in \Omega^{-}
\end{aligned}
$$

e os valores $\phi(\boldsymbol{x}), \boldsymbol{x} \in \partial \Omega$ (ou próximos dele) são usados como condição de contorno. 
Usando a função sinal $\operatorname{sign}(\phi)$, pode-se juntar as duas

$$
\frac{\partial \phi}{\partial t}=\operatorname{sign}(\phi)\left(1-\|\nabla \phi\|_{2}\right)
$$

Infelizmente ainda restam alguns problemas: (i) A reinicialização é muito custosa computacionalmente, (ii) ainda podem ocorrer influências da reinicialização na interface $\partial \Omega$ em alguns casos, (iii) se $\phi$ estiver muito distante de uma função distância com sinal, pode ser que a reinicialização falhe e (iv) não há um método robusto de decidir quando há necessidade de reinicialização.

Por conta de todos estes desafios, um equacionamento que evita a reinicialização de $\phi$ foi desenvolvido em Li et al. [133]

\section{E.2.2 Formulação sem reinicialização}

O novo equacionamento baseia-se em uma formulação variacional do problema. $\mathrm{O}$ Objetivo é criar uma função custo e minimizá-la em função de $\phi$. Três parcelas são consideradas

$$
\mathcal{E}(\phi)=\mu \mathcal{P}(\phi)+\lambda \mathcal{L}_{g}(\phi)+\nu \mathcal{A}_{g}(\phi)
$$

onde $\mu, \lambda, \nu$ são parâmetros a serem ajustados. Nesta equação, a primeira parcela $\mathcal{P}(\phi)$ é um custo de afastamento de $\phi$ em relação à função distância com sinal,

$$
\mathcal{P}(\phi)=\int_{\Omega} \frac{1}{2}\left(\|\nabla \phi\|_{2}-1\right)^{2} d \boldsymbol{x}
$$

A segunda parcela $\mathcal{L}_{g}$ penaliza soluções com grande comprimento da hiper-curva $\phi=0$. O objetivo é penalizar soluções com $\partial \Omega$ muito recortado.

$$
\mathcal{L}_{g}=\int_{\Omega} g(\boldsymbol{x}) \delta(\phi(\boldsymbol{x}))\|\nabla \phi(\boldsymbol{x})\|_{2} d \boldsymbol{x}
$$

onde a função $g$ é a função indicativa de contorno.

$$
g(\boldsymbol{x})=\left(1+\left\|\nabla\left(\boldsymbol{G}_{\sigma} * I\right)\right\|_{2}^{2}\right)^{-1}
$$

Definindo $g(x)$ desta maneira, a função apresenta mínimos locais onde o gradiente 
da imagem $I$ é elevado, ela destaca os contornos a serem usados na segmentação. $\mathrm{O}$ termo $\nabla\left(\boldsymbol{G}_{\sigma} * I\right)=\nabla \boldsymbol{G}_{\sigma} * I$ é a convolução da imagem $I$ com um filtro passa-baixas gaussiano usado para suavizar a imagem e reduzir a influência de ruído.

A terceira parcela $\mathcal{A}_{g}$ penaliza soluções nas quais $\Omega^{-}$apresenta pequenas áreas (se $\nu<0$ ) ou grandes áreas (se $\nu>0$ )

$$
\mathcal{A}_{g}=\int_{\Omega} g(\boldsymbol{x}) H(-\phi(\boldsymbol{x})) d \boldsymbol{x} .
$$

Pode-se então usar o método steepest descent para encontrar o(um) mínimo de $\mathcal{E}(\phi)$ iterativamente

$$
\begin{aligned}
\phi_{k} & =\phi_{k-1}-\Delta t \frac{\partial \mathcal{E}}{\partial \phi} \\
\frac{\partial \mathcal{E}}{\partial \phi} & =-\mu[\Delta \phi-\nabla \cdot \boldsymbol{n}]-\lambda \delta(\phi) \nabla \cdot(g \boldsymbol{n})-\nu g \delta(\phi),
\end{aligned}
$$

onde $\triangle \phi$ é o laplaciano de $\phi$.

\section{E.2.3 Critério de parada}

O método iterativo para a segmentação deve ser interrompido por algum critério de parada. Dois critérios foram utilizados neste trabalho. A parada ocorre se ambos os critérios forem satisfeitos.

O primeiro critério é baseado no tamanho da atualização da função implícita. Foi usada a norma $L_{\infty}$ na matriz que contém a atualização da função $\phi$ entre duas iterações sucessivas. Esta matriz é formada considerando que a (E.34) resulta em uma matriz $\Delta \phi_{k}$, cujos elementos contém a atualização de $\phi$ na $k$-ésima iteração. O primeiro critério é satisfeito se a seguinte inequação for verdadeira

$$
C_{1}=\frac{\left\|\Delta \phi_{k}\right\|_{\infty}}{r_{1} r_{2} \ldots r_{n}} \leq \epsilon_{1}
$$

\footnotetext{
${ }^{5}$ Para a avaliação do critério de parada, considera-se a norma elemento a elemento de uma matriz, ou seja $\|\boldsymbol{A}\|_{p}=\left(\sum_{i, j}\left|[\boldsymbol{A}]_{(i, j)}\right|^{p}\right)^{1 / p}$. Assim,

$$
\begin{aligned}
\|\boldsymbol{A}\|_{\infty} & =\max _{i, j}\left\{\left|[\boldsymbol{A}]_{(i, j)}\right|\right\} \\
\|\boldsymbol{A}\|_{1} & =\sum_{i, j}\left|[\boldsymbol{A}]_{(i, j)}\right|
\end{aligned}
$$
}


onde $\Delta \phi_{k}=\phi_{k}-\phi_{k-1}=-\Delta t \frac{\partial \mathcal{E}}{\partial \phi}$ é uma matriz cujos elementos apresentam as mudanças de $\phi$ entre a iteração atual e a anterior, $\epsilon_{1}$ é um parâmetro para ser ajustado e $r_{i}$ é a resolução da imagem na $i$-ésima dimensão.

O segundo critério é baseado na mudança da região segmentada. Considerando duas funções implícitas em iterações sucessivas $\phi_{k}$ e $\phi_{k-1}$, calcula-se a operação XOR entre os respectivos elementos das duas funções características $\chi_{k}^{-}$e $\chi_{k-1}^{-}$, compondo uma imagem $I_{X O R}$

$$
I_{X O R}=X O R\left(\chi_{n}^{-}, \chi_{n-1}^{-}\right)
$$

Usa-se então a norma $L_{1}$ nesta imagem. O segundo critério é satisfeito se a seguinte inequação for verdadeira

$$
C_{2}=\frac{\left\|I_{X O R}\right\|_{1}}{r_{1} r_{2} \ldots r_{n}} \leq \epsilon_{2}
$$

O exemplo a seguir mostra o cálculo destes critérios para uma imagem $3 \times 3$ $\left(r_{1}=3, r_{2}=3\right)$.

Sejam duas matrizes que representam $\phi_{n}$ e $\phi_{n-1}$ :

$$
\text { função } \phi_{n}=\begin{array}{|c|c|c|}
\hline-1.5 & 0.5 & 1.0 \\
\hline-2.0 & 0.4 & 0.8 \\
\hline-0.5 & 0.3 & 1.2 \\
\hline
\end{array}
$$$$
\text { função } \phi_{n-1}=\begin{array}{|c|c|c|}
\hline-1.4 & -0.1 & 0.9 \\
\hline-1.8 & 0.3 & 0.7 \\
\hline 0.2 & 0.2 & 1.1 \\
\hline
\end{array}
$$

Critério 1

$$
\begin{gathered}
\Delta \phi=\phi_{n}-\phi_{n-1}=\left(\begin{array}{ccc}
-0.1 & 0.6 & 0.1 \\
-0.2 & 0.1 & 0.1 \\
-0.7 & 0.1 & 0.1
\end{array}\right) \\
C_{1}=\frac{\left\|\Delta \phi_{n}\right\|_{\infty}}{r_{1} r_{2}}=\frac{\max \left(\left|[\Delta \phi]_{(i, j)}\right|\right)}{9}=\frac{0.7}{9} .
\end{gathered}
$$


Critério 2

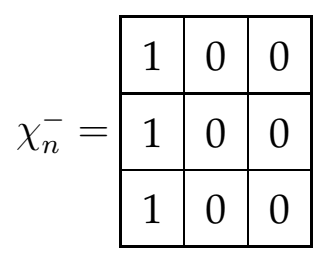

$$
\chi_{n-1}^{-}=\begin{array}{|l|l|l|}
\hline 1 & 1 & 0 \\
\hline 1 & 0 & 0 \\
\hline 0 & 0 & 0 \\
\hline
\end{array}
$$

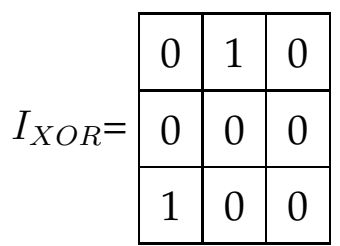

$$
C_{2}=\frac{\left\|I_{X O R}\right\|_{1}}{r_{1} r_{2}}=\frac{1}{9} \sum_{i, j}\left|\left[I_{X O R}\right]_{(i, j)}\right|=\frac{2}{9}
$$

\section{E.2.4 Detalhes da implementação}

Nesta seção são apresentados alguns detalhes de implementação do método.

A-) Devido ao termo de difusão adicional em relação à formulação original, não é necessário utilizar o método upwind para resolver a equação de evolução, porém deve-se selecionar um passo de tempo $\Delta t$, que obedeça à condição $\Delta t . \mu<0.25$.

B-) A inicialização da função $\phi$ pode ser mais relaxada. Usualmente basta utilizar

$$
\phi_{0}(\boldsymbol{x})= \begin{cases}-c & , \quad \boldsymbol{x} \in \Omega_{0}^{-} \\ 0 & , \quad \boldsymbol{x} \in \partial \Omega_{0} \\ c & , \quad \boldsymbol{x} \in \partial \Omega_{0}^{+}\end{cases}
$$

onde $c$ é um parâmetro a ser ajustado. Em Li et al. [133] sugere-se a relação $c>2 \epsilon$, onde $\epsilon$ é o parâmetro usado no delta de Dirac suavizado (E.14).

C-) As intensidades dos pixels foram normalizadas entre 0 e 100 e um filtro gaussiano foi utilizado para suavizar a imagem antes da segmentação.

D-) O filtro gaussiano 2D isotrópico, isto é, circularmente simétrico, de dimensões 
$[2 N+1] \times[2 N+1], N>1$ é dado por

$$
\begin{aligned}
{\left[\boldsymbol{G}_{\sigma}\right]_{(i, j)} } & =\frac{\frac{1}{2 \pi \sigma} \exp \left(-\frac{i^{2}+j^{2}}{2 \sigma^{2}}\right)}{\sum_{i, j} \frac{1}{2 \pi \sigma} \exp \left(-\frac{i^{2}+j^{2}}{2 \sigma^{2}}\right)}=\frac{\exp \left(-\frac{i^{2}+j^{2}}{2 \sigma^{2}}\right)}{S}, i, j=-N, \ldots, N \\
S & =\sum_{i, j} \exp \left(-\frac{i^{2}+j^{2}}{2 \sigma^{2}}\right)
\end{aligned}
$$

onde $S$ é a soma dos elementos da matriz $\boldsymbol{G}_{\sigma}$, usada para manter ganho unitário na filtragem e $\sigma$ é o parâmetro de suavização do filtro. Esta função é convolucionada na imagem com o pixel de referência centrado na matriz $\boldsymbol{G}_{\sigma}$. Esta filtragem necessita de um número de operações da ordem de $(2 N+1)^{2}$, porém pode-se reduzir para $2(2 N+1)$ operações com o uso da propriedade da separabilidade do núcleo gaussiano:

$$
\left[\boldsymbol{G}_{\sigma}\right]_{(i, j)}=\frac{\exp \left(-\frac{i^{2}+j^{2}}{2 \sigma^{2}}\right)}{\sum_{i, j} \exp \left(-\frac{i^{2}+j^{2}}{2 \sigma^{2}}\right)}=\frac{\exp \left(-\frac{i^{2}}{2 \sigma^{2}}\right)}{\sum_{i} \exp \left(-\frac{i^{2}}{2 \sigma^{2}}\right)} \frac{\exp \left(-\frac{j^{2}}{2 \sigma^{2}}\right)}{\sum_{j} \exp \left(-\frac{j^{2}}{2 \sigma^{2}}\right)}=\left[\boldsymbol{G}_{\sigma}^{y}\right]_{i}\left[\boldsymbol{G}_{\sigma}^{x}\right]_{j} .
$$

Portanto o filtro original $\boldsymbol{G}_{\sigma}$ pode ser dividido em dois outros filtros $\boldsymbol{G}_{\sigma}^{y}$ e $\boldsymbol{G}_{\sigma}^{x}$, unidimensionais com núcleos de dimensões $[2 N+1] \times 1$ e $1 \times[2 N+1]$ respectivamente. A operação de convolução, usando os filtros unidimensionais é

$$
\boldsymbol{G}_{\sigma} * I=\left(\boldsymbol{G}_{\sigma}^{y} \boldsymbol{G}_{\sigma}^{x}\right) * I=\boldsymbol{G}_{\sigma}^{y} *\left(\boldsymbol{G}_{\sigma}^{x} * I\right),
$$

ou seja, a convolução original é dividida em dois filtros em cascata, um na direção horizontal e outro na direção vertical. A ordem dos filtro não é importante pela associatividade e comutatividade do operador convolução.

E-) É utilizada uma interpolação do mapa de resistividade elétrica nos centróides dos $n$ elementos da malha de elementos finitos para um grid de pixels em coordenadas cartesianas na segmentação das imagens. Para cada pixel da imagem interpolada $[I]_{(i, j)}, i=1, \ldots, m, j=1, \ldots, n$, o valor da intensidade do pixel (resitividade) é dado por

$$
[I]_{(i, j)}=\frac{\sum_{k=1}^{n} \exp \left(-\frac{d_{i j}\left(c_{k}\right)}{2 \sigma^{2}}\right) \rho_{k}}{\sum_{k=1}^{n} \exp \left(-\frac{d_{i j}\left(c_{k}\right)}{2 \sigma^{2}}\right)}
$$


onde $d_{i j}\left(c_{k}\right)$ é a distância, em unidades métricas da malha entre o centróide do $k$-ésimo elemento da malha e o pixel $[I]_{(i, j)}$. A dimensão de cada pixel em unidades métricas é calculada através da divisão das dimensões da malha, pela resolução em pixels da imagem. O parâmetro $\sigma$ regula a suavização da imagem na interpolação.

F-) O cálculo numérico das derivadas parciais em (E.34) foram realizadas através de diferenças finitas. Seja uma função $f$, definida em pontos $i=1,2, \ldots, m$, distantes entre si $\Delta x$. As aproximações por diferenças finitas, com erro de ordem $\Delta x^{2}$ são

$$
\begin{aligned}
& \left.\frac{\partial f}{\partial x}\right|_{i}=\frac{f(i+1)-f(i-1)}{2 \Delta x} \\
& \left.\frac{\partial f}{\partial x}\right|_{1}=\frac{-3 f(1)+4 f(2)-f(3)}{2 \Delta x} \\
& \left.\frac{\partial f}{\partial x}\right|_{m}=\frac{f(m-2)-4 f(m-1)+3 f(m)}{2 \Delta x} \\
& \left.\frac{\partial^{2} f}{\partial x^{2}}\right|_{i}=\frac{f(i-1)-2 f(i)+f(i+1)}{\Delta x^{2}} \\
& \left.\frac{\partial^{2} f}{\partial x^{2}}\right|_{1}=\frac{2 f(1)-5 f(2)+4 f(3)-f(4)}{\Delta x^{2}} \\
& \left.\frac{\partial^{2} f}{\partial x^{2}}\right|_{m}=\frac{-f(m-3)+4 f(m-2)-5 f(m-1)+2 f(m)}{\Delta x^{2}} .
\end{aligned}
$$

Para as demais coordenadas, as fórmulas são análogas.

G-) Após a determinação da função $\phi$ no grid que representa os pixels da imagem, é necessário avaliar se um determinado elemento da malha de elementos finitos pertence à região $\Omega^{-}$ou $\Omega^{+}$. Isto foi feito utilizando uma interpolação bilinear para avaliar o valor de $\phi$ no centróide $P$ de um elemento.

Dado o centróide $P=\left(x_{P}, y_{p}\right)$, denomina-se $A, B, C$ e $D$ os quatro centros dos pixels que determinam o menor retângulo ao redor de $P$, como na Figura E.5, Sejam ainda $\phi_{A}, \phi_{B}, \phi_{C}$ e $\phi_{D}$ os valores de $\phi$ avaliados nestes pontos. 


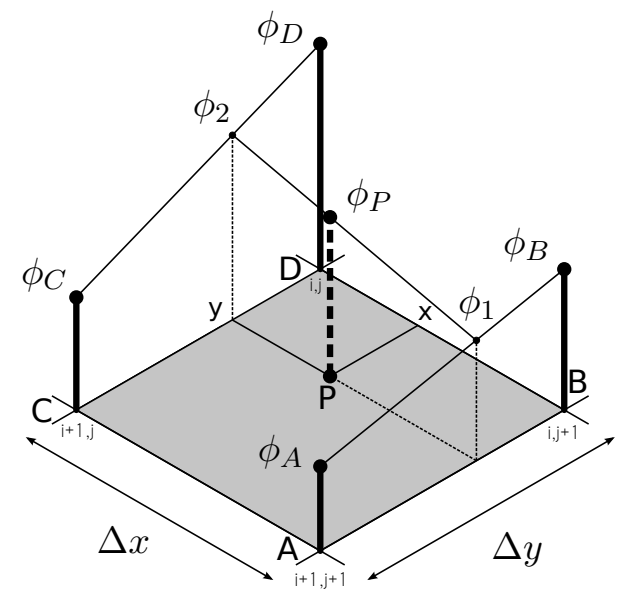

Figura E.5: Interpolação bilinear.

A interpolação é realizada em dois passos. No primeiro, interpola-se, sobre a reta que conecta $\phi_{A}$ e $\phi_{B}$, o valor de $\phi_{1}$ no ponto de coordenada $y=y_{P}$. O mesmo é feito sobre a reta que conecta $\phi_{C}$ e $\phi_{D}$ para determinar $\phi_{2}$. No segundo, interpola-se, sobre a reta que conecta $\phi_{1}$ e $\phi_{2}$, o valor de $\phi_{P}$ no ponto $P$

$$
\begin{aligned}
& \phi_{1}=\left(\frac{\phi_{A}-\phi_{B}}{\Delta y}\right)\left(y_{P}-y_{D}\right)+\phi_{B} \\
& \phi_{2}=\left(\frac{\phi_{C}-\phi_{D}}{\Delta y}\right)\left(y_{P}-y_{D}\right)+\phi_{D} \\
& \phi_{P}=\left(\frac{\phi_{1}-\phi_{2}}{\Delta x}\right)\left(x_{P}-x_{D}\right)+\phi_{2} .
\end{aligned}
$$




\section{Apêndice F}

\section{Resultados dos ensaios}

\section{F.1 Resultados dos ensaios para avaliação do método do erro de aproximação}

\section{F.1.1 Sem o uso de densidade a priori}

As figuras a seguir mostram os históricos das estimações nos ensaios. Nestas figuras são apresentados os índices $J, J_{d}, J_{m}, C_{m}$ e $C_{v}$ apresentados na Seção 8.4. Em alguns gráficos de $C_{m}$ e $C_{v}$, são traçadas linhas tracejadas para ressaltar o limite estipulado para o critério de convergência. 

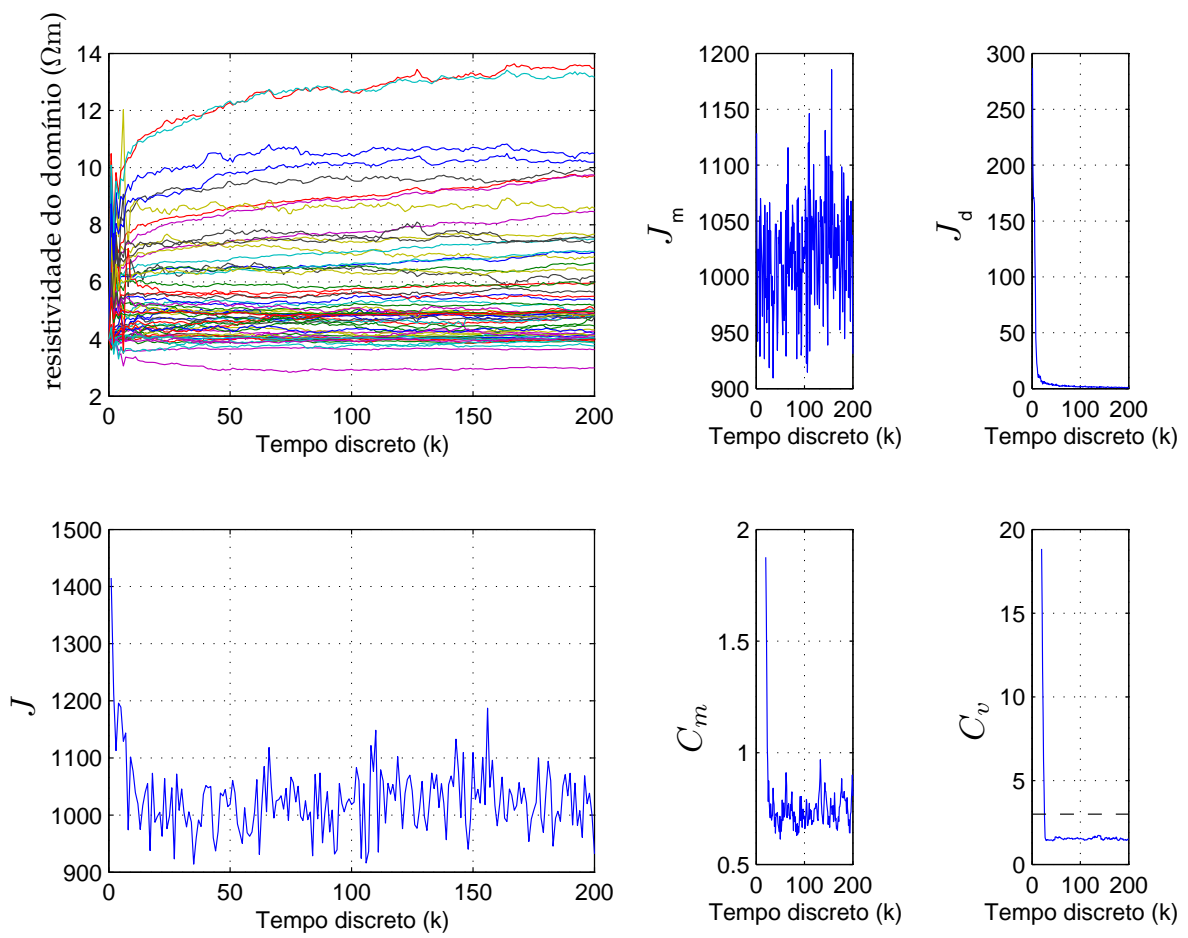

(a) Ensaio 1, sem utilizar o método do erro de aproximação.
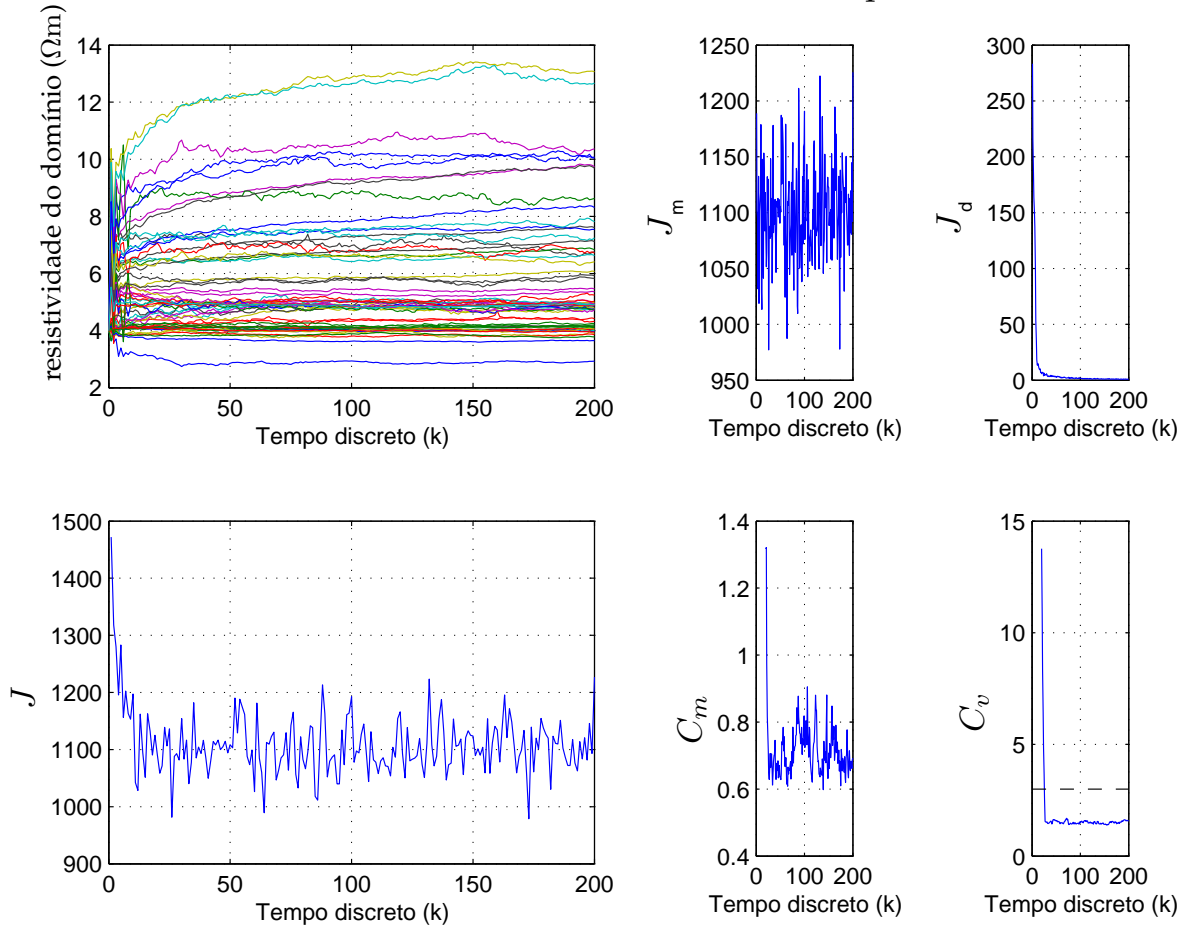

(b) Ensaio 1, utilizando o método do erro de aproximação.

Figura F.1: Resultados do Ensaio 1. Histórico da estimação e índices de desempenho. 

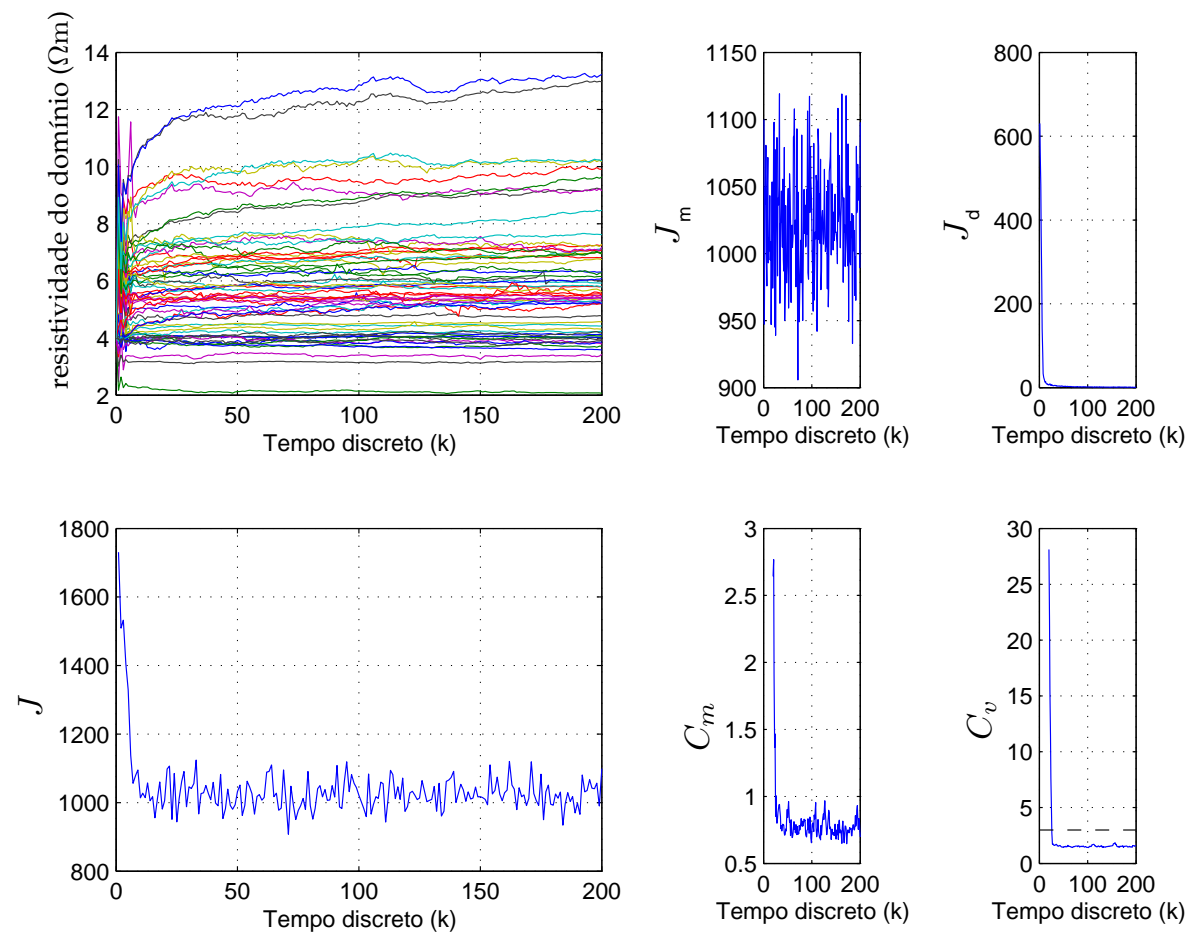

(a) Ensaio 2, sem utilizar o método do erro de aproximação.
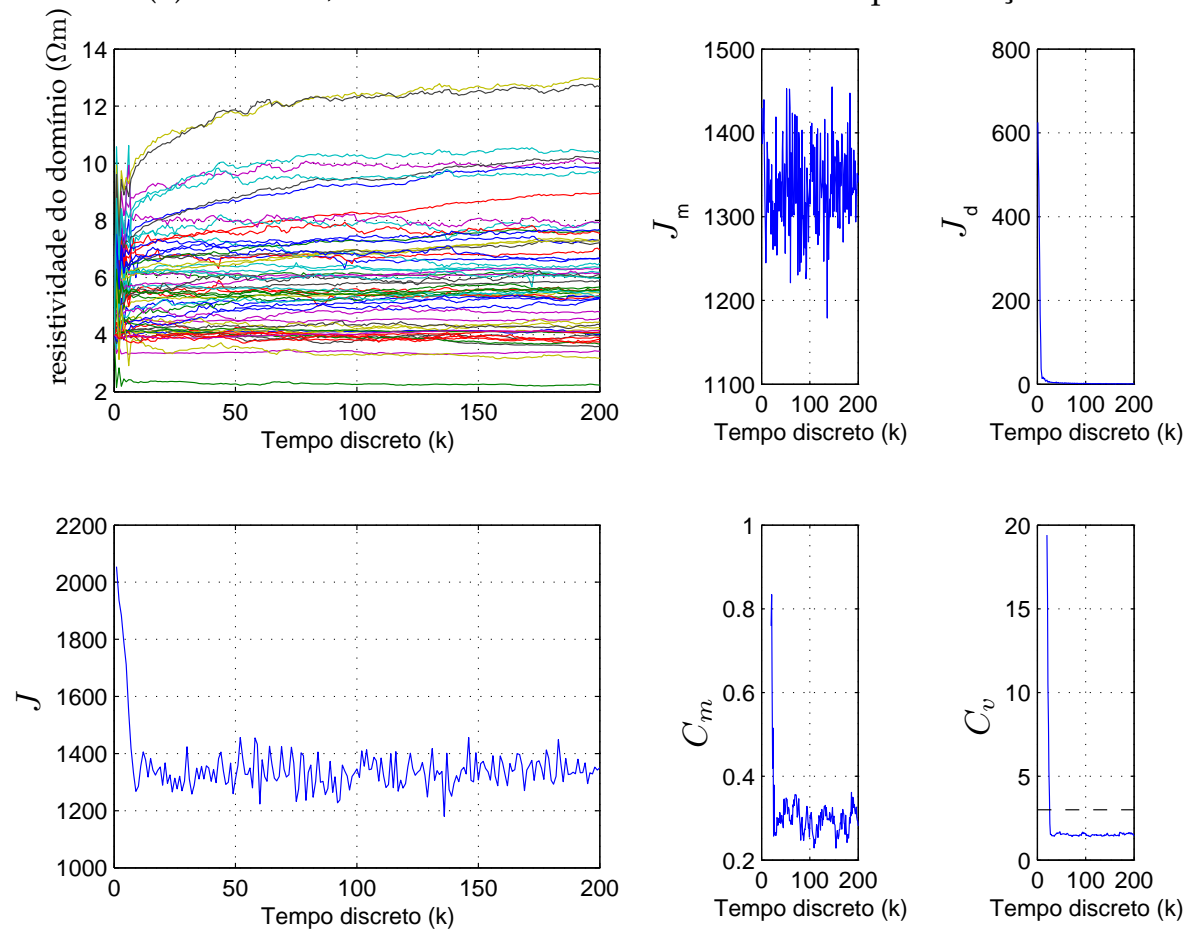

(b) Ensaio 2, utilizando o método do erro de aproximação.

Figura F.2: Resultados do Ensaio 2. Histórico da estimação e índices de desempenho. 

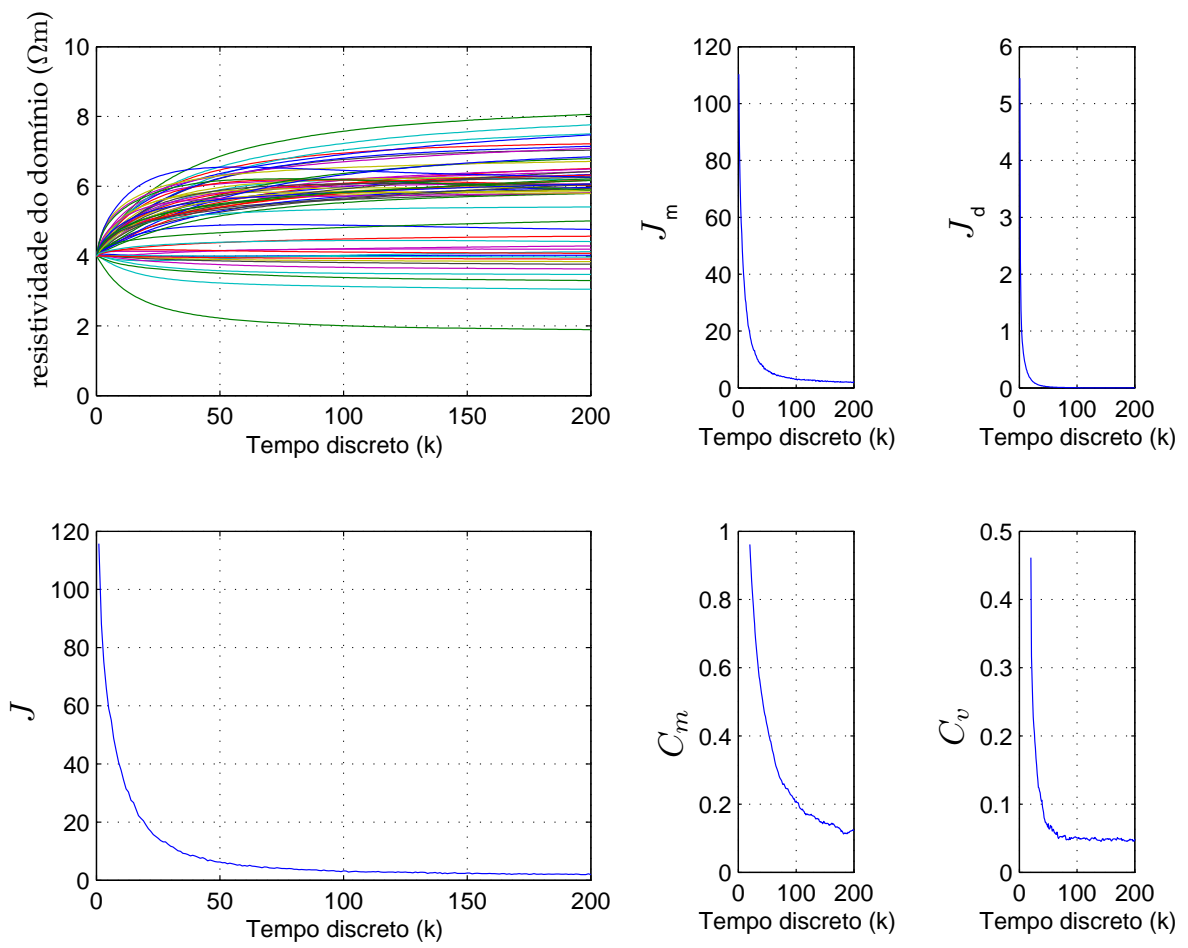

(a) Ensaio 3, sem utilizar o método do erro de aproximação.
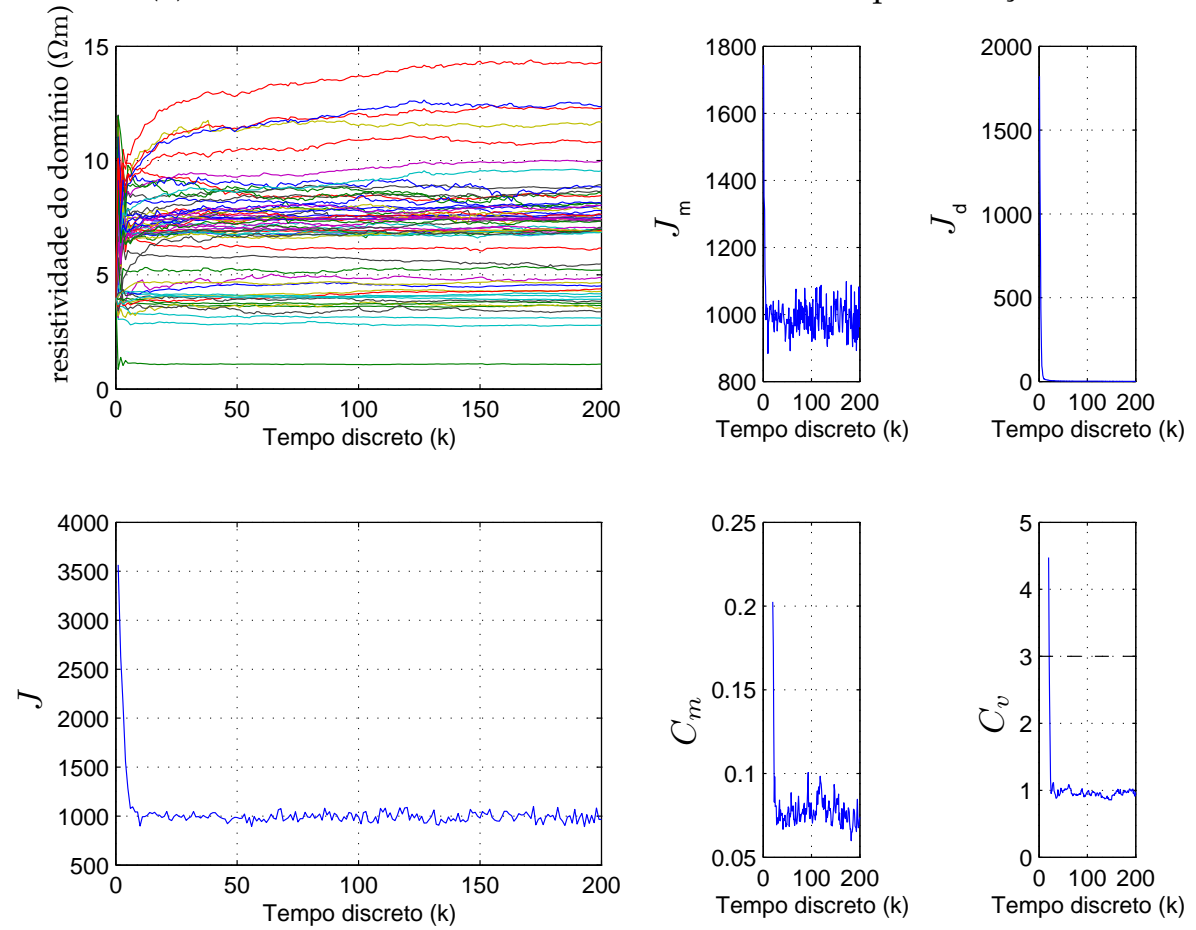

(b) Ensaio 3, utilizando o método do erro de aproximação.

Figura F.3: Resultados do Ensaio 3. Histórico da estimação e índices de desempenho. 

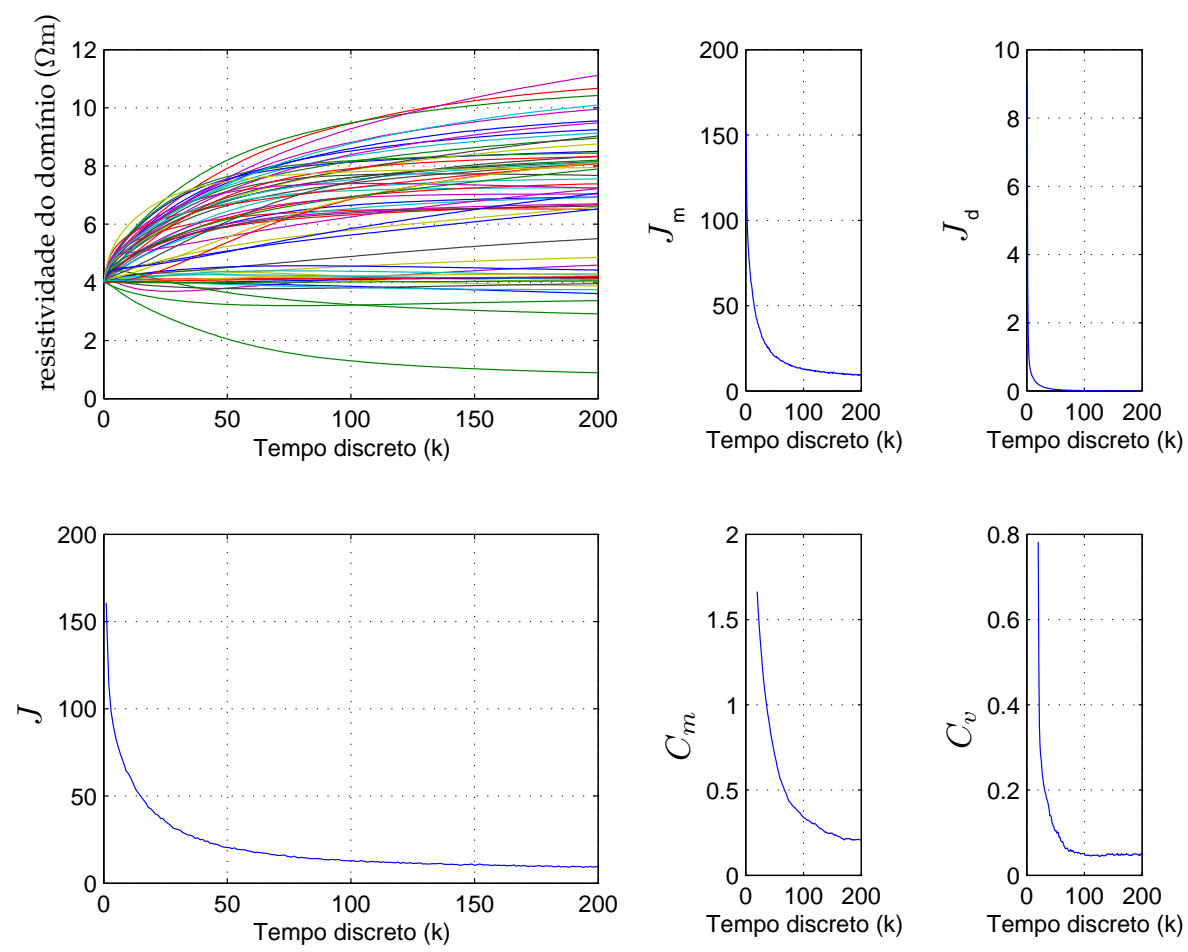

(a) Ensaio 4, sem utilizar o método do erro de aproximação.
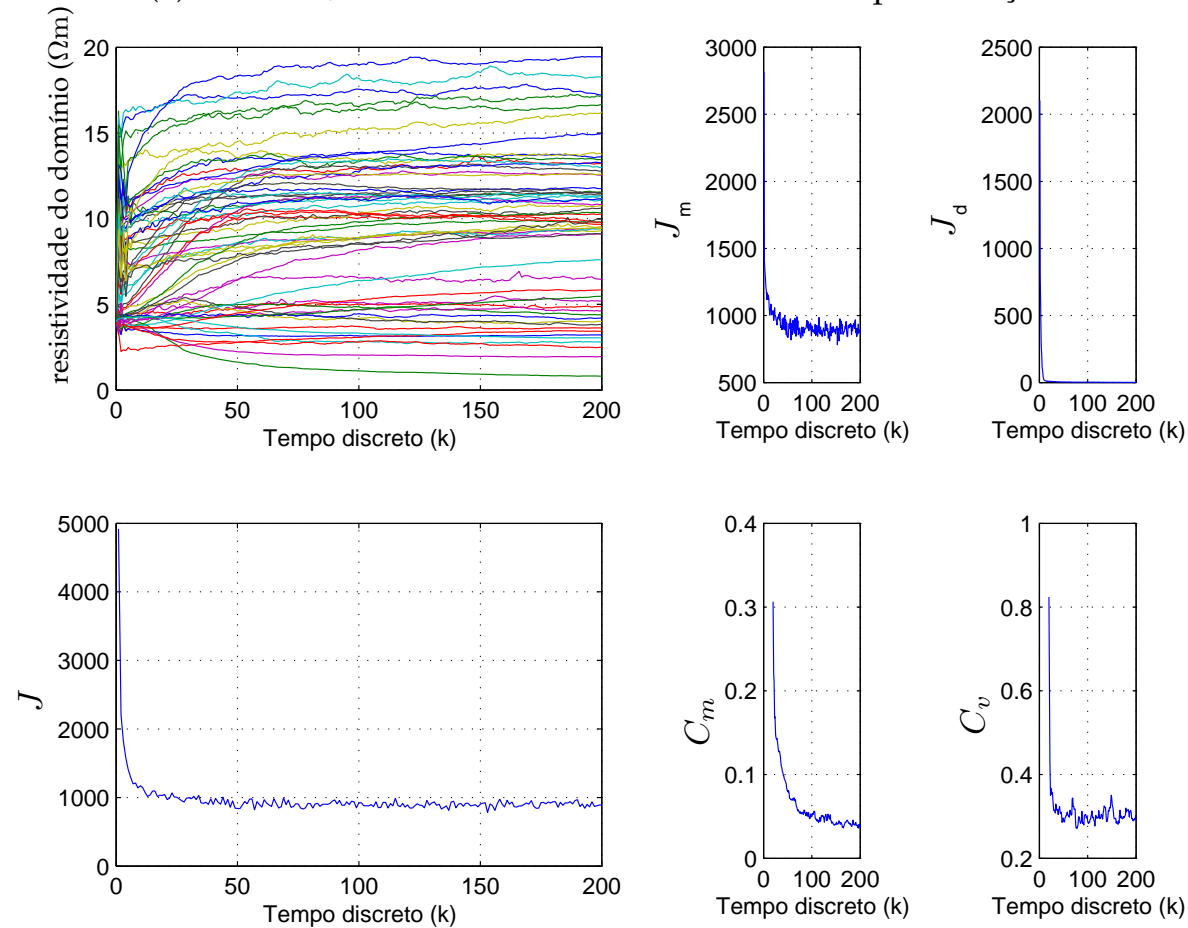

(b) Ensaio 4, utilizando o método do erro de aproximação.

Figura F.4: Resultados do Ensaio 4. Histórico da estimação e índices de desempenho. 

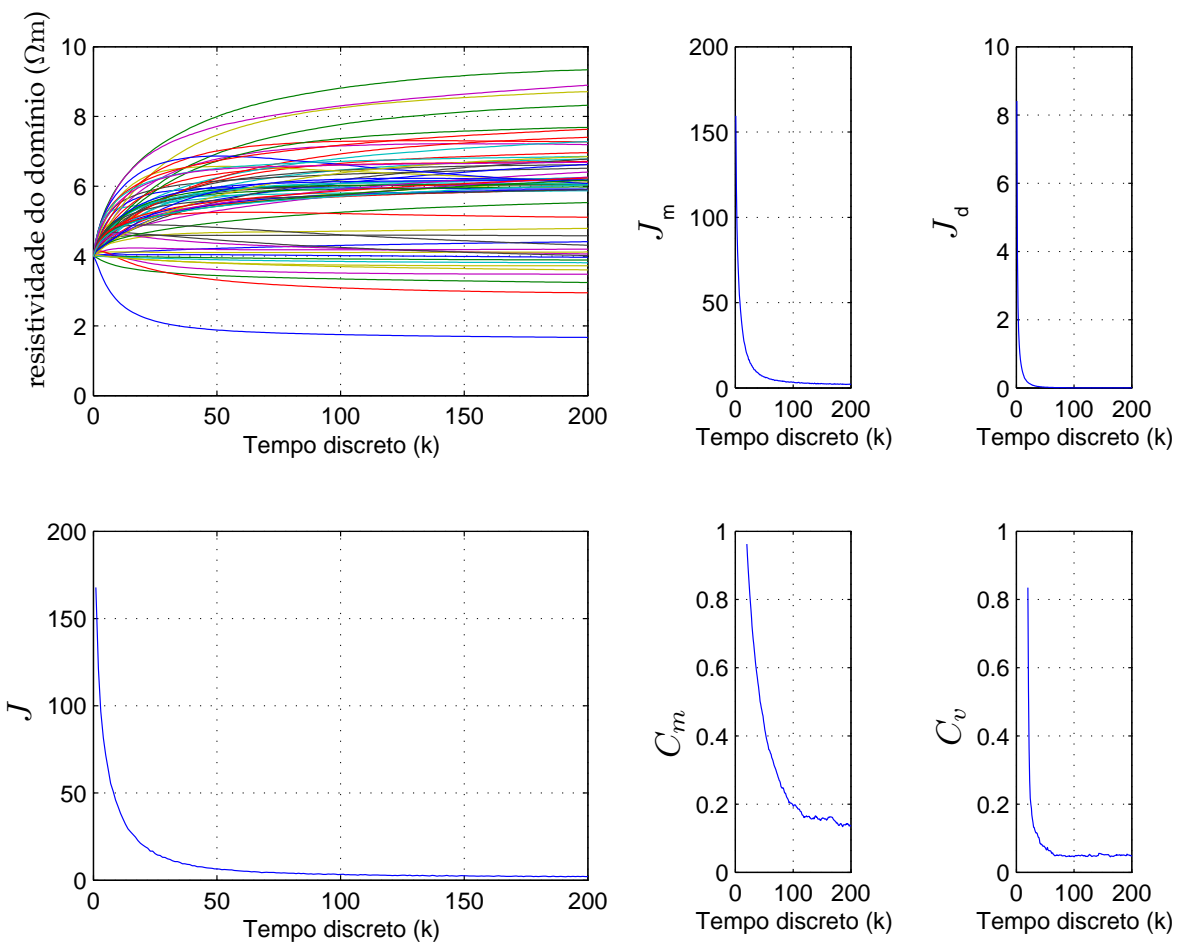

(a) Ensaio 5, sem utilizar o método do erro de aproximação.
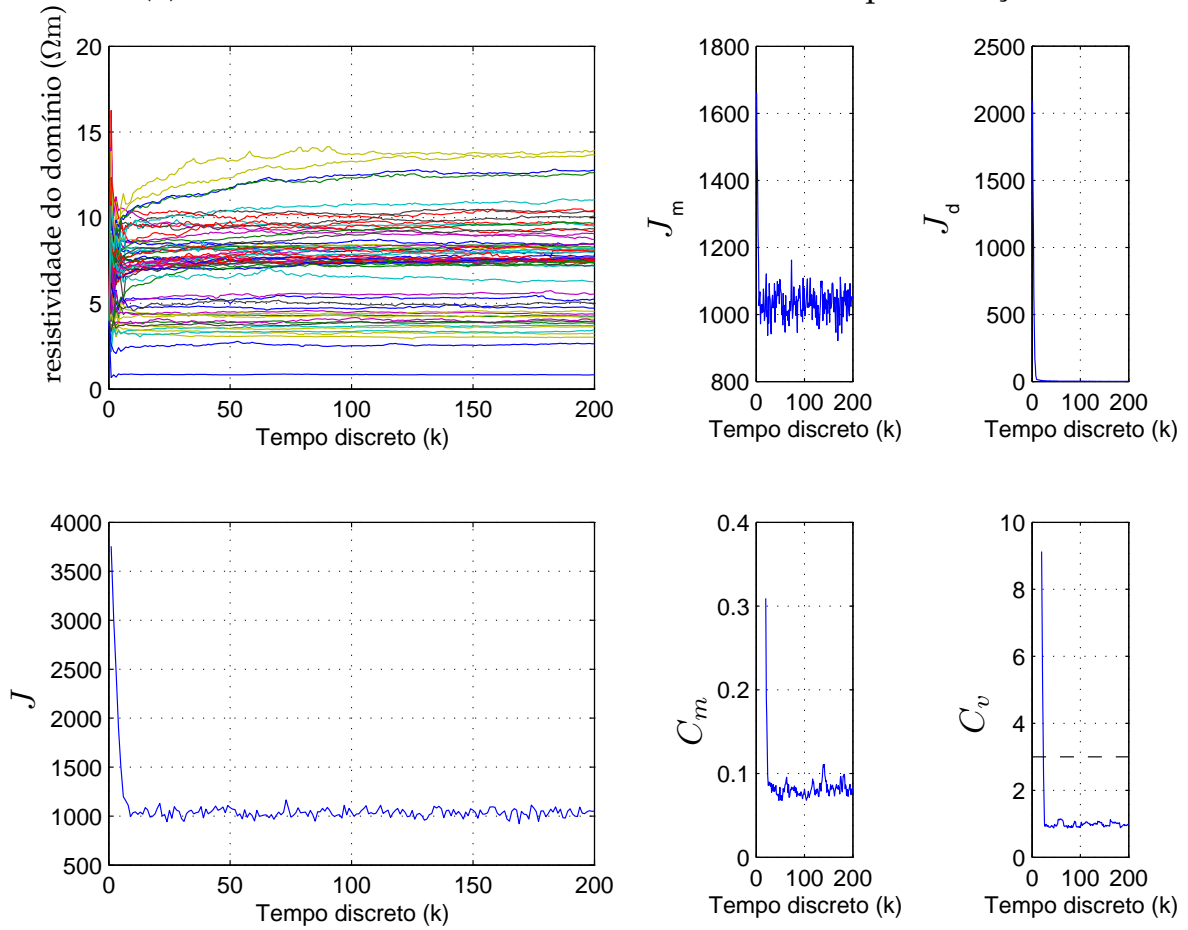

(b) Ensaio 5, utilizando o método do erro de aproximação.

Figura F.5: Resultados do Ensaio 5. Histórico da estimação e índices de desempenho. 

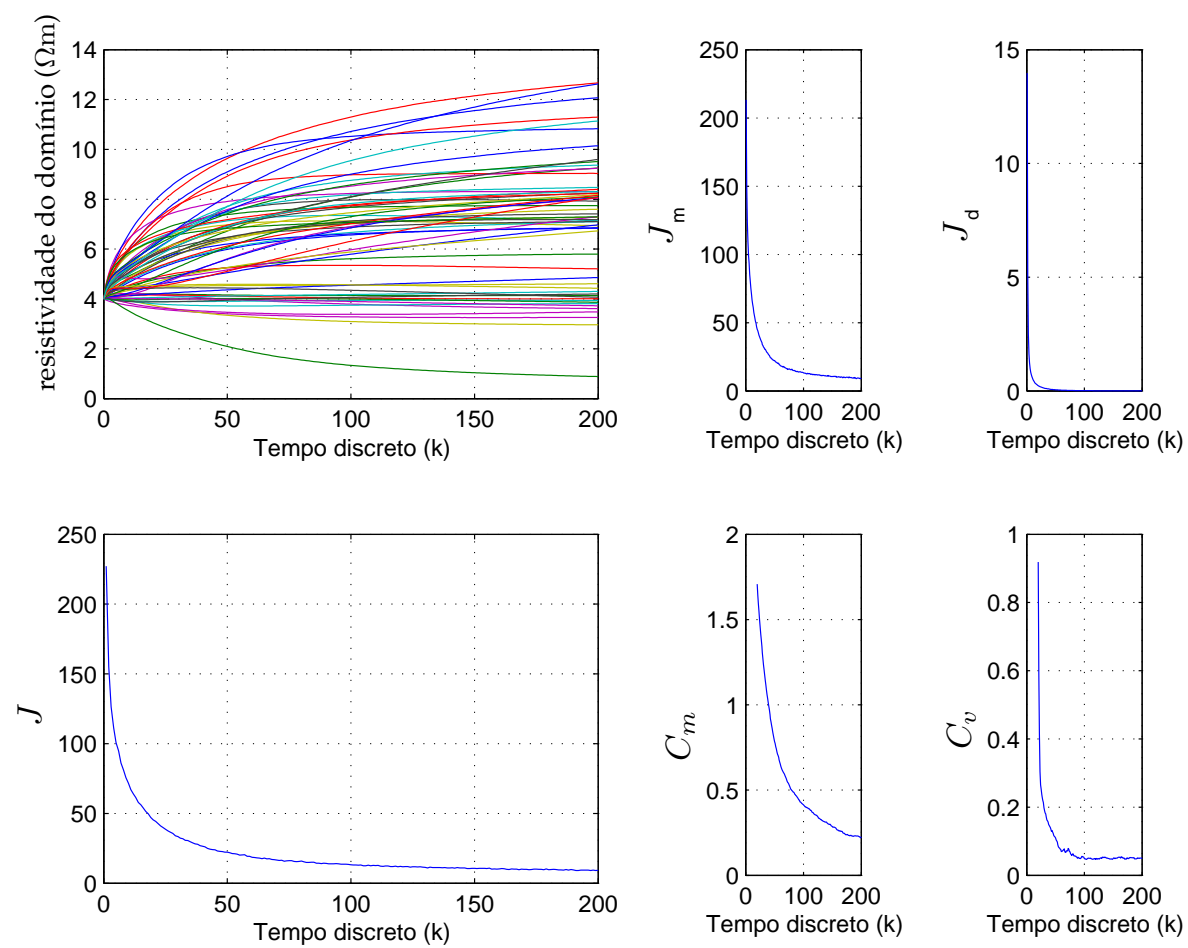

(a) Ensaio 6, sem utilizar o método do erro de aproximação.
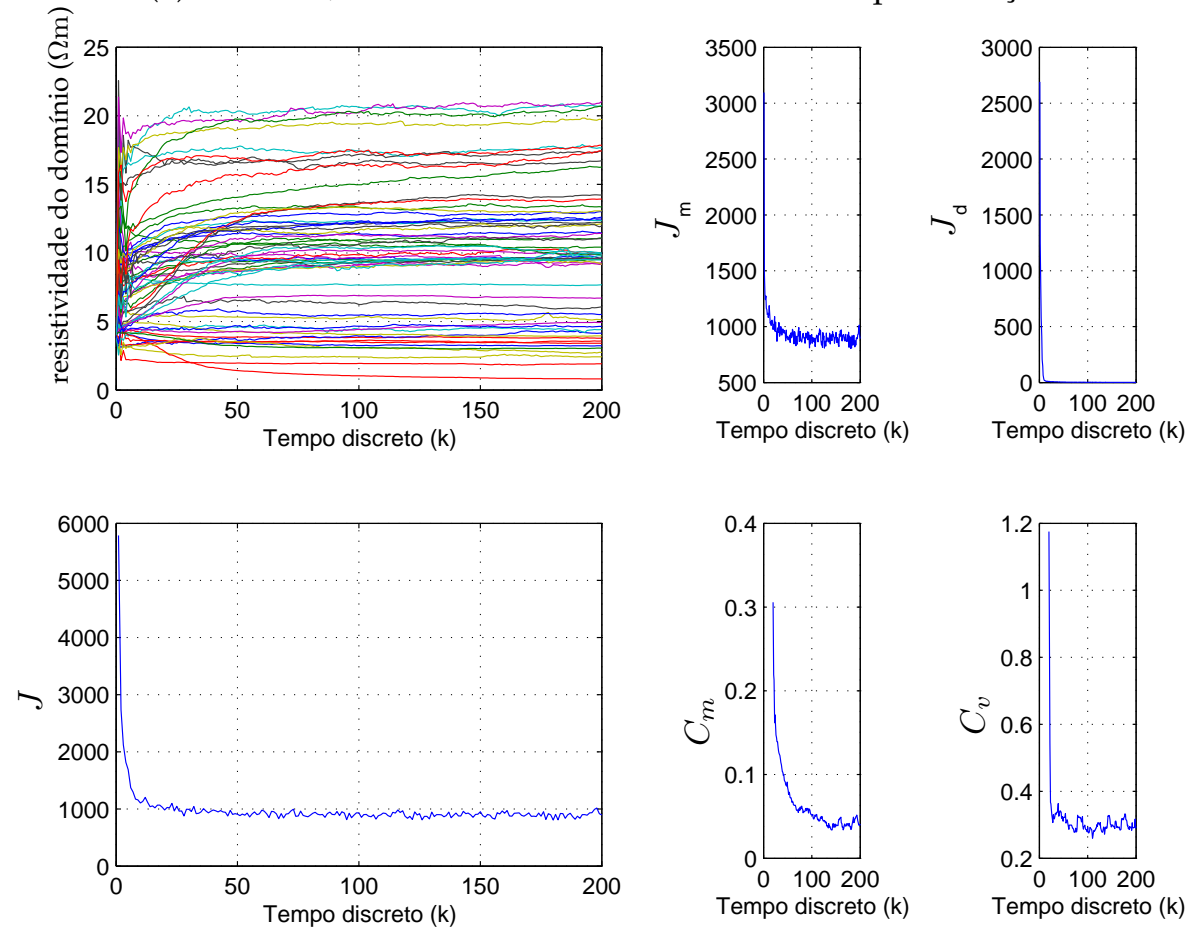

(b) Ensaio 6, utilizando o método do erro de aproximação.

Figura F.6: Resultados do Ensaio 6. Histórico da estimação e índices de desempenho. 

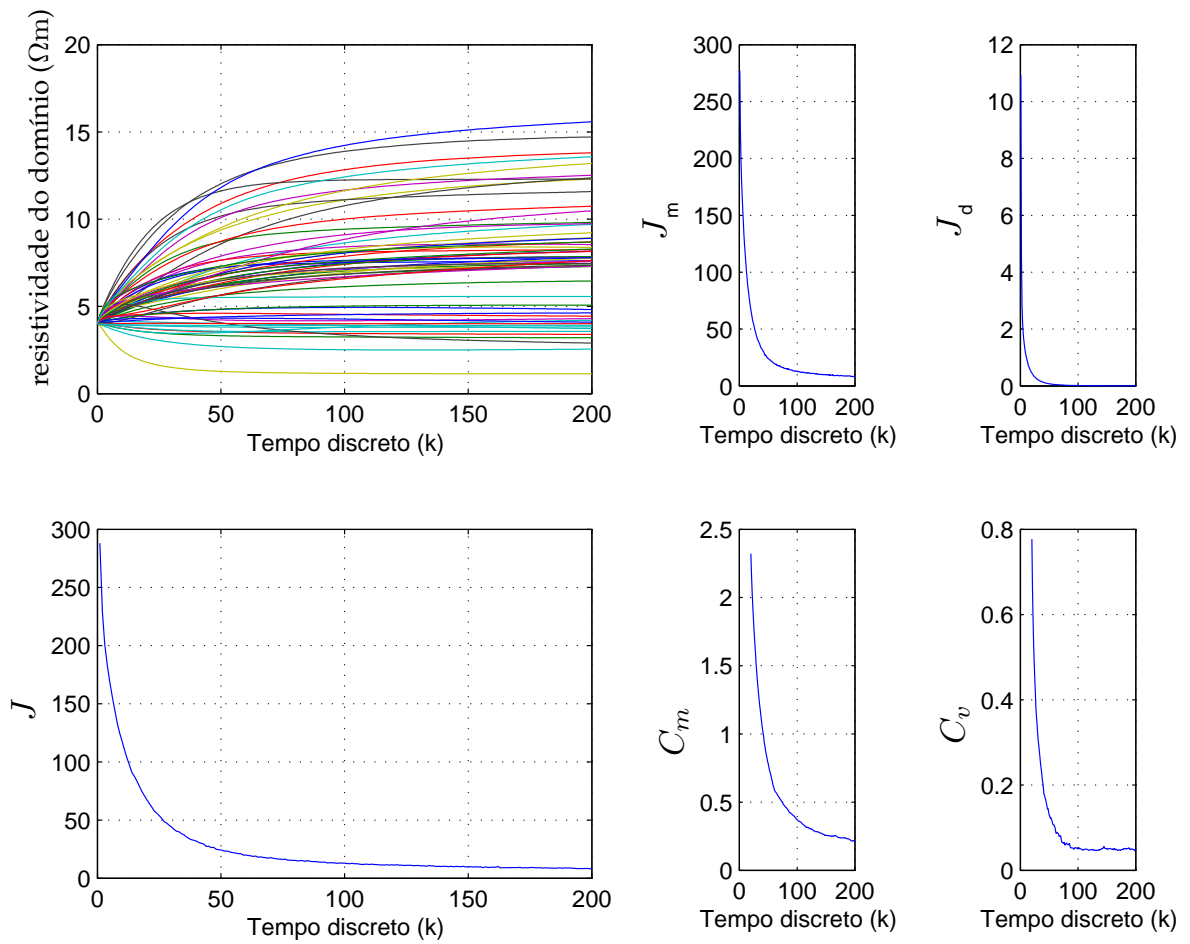

(a) Ensaio 7, sem utilizar o método do erro de aproximação.
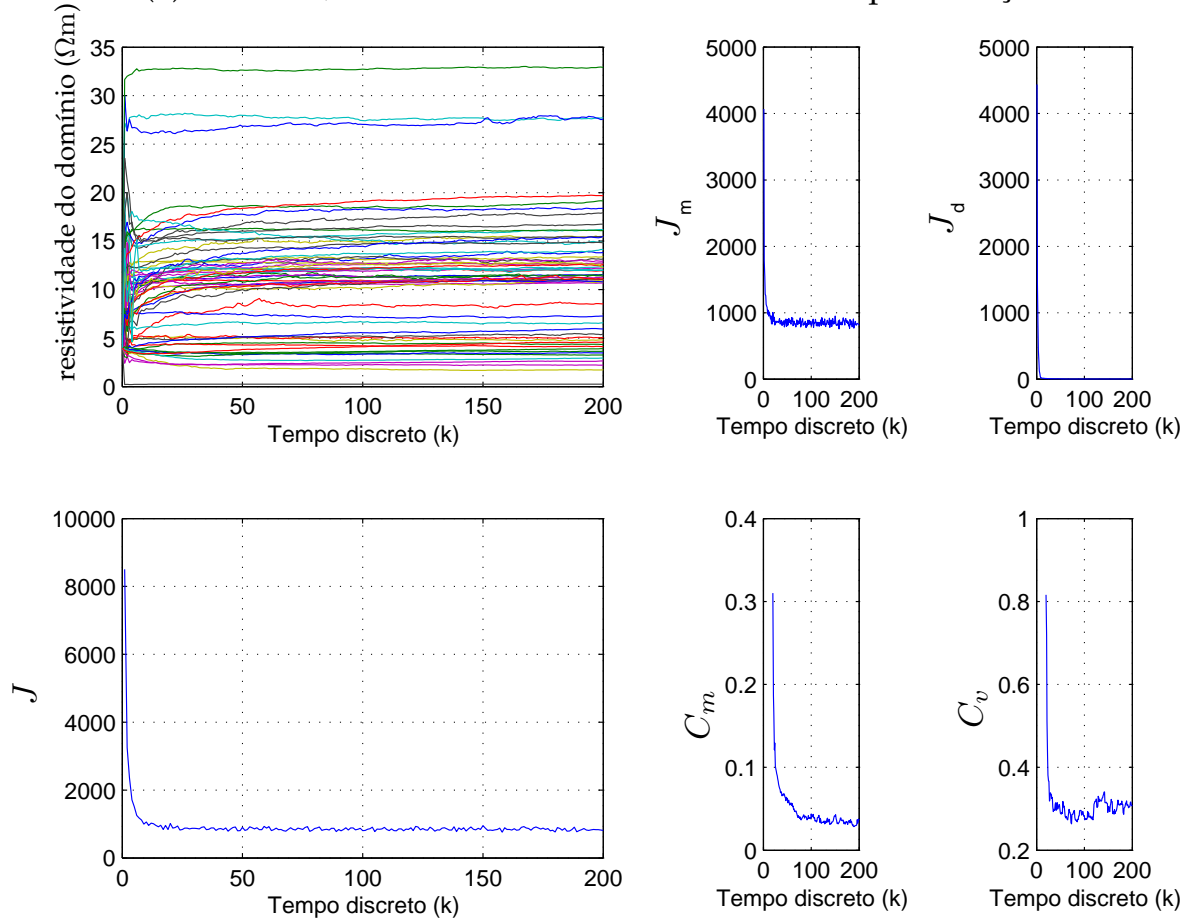

(b) Ensaio 7, utilizando o método do erro de aproximação.

Figura F.7: Resultados do Ensaio 7. Histórico da estimação e índices de desempenho. 

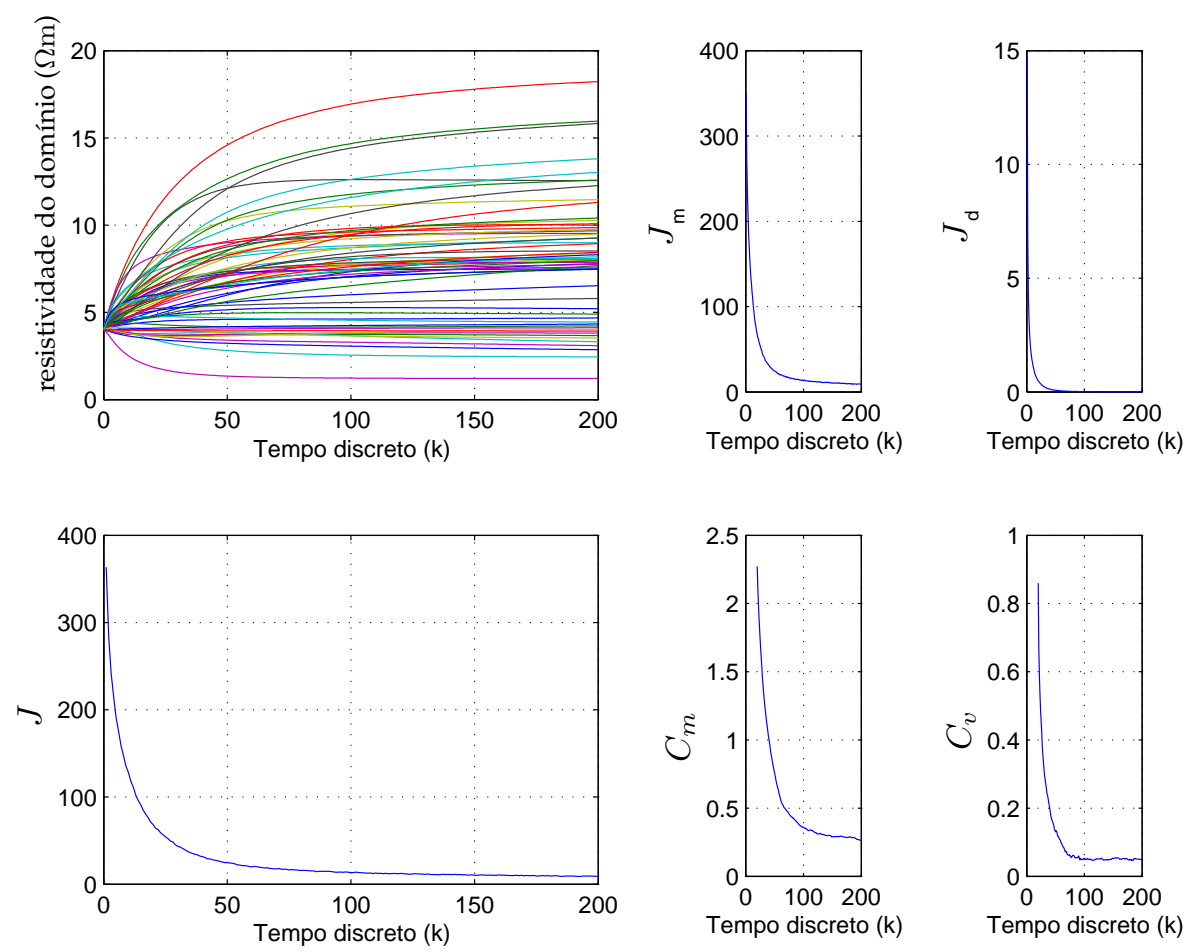

(a) Ensaio 8, sem utilizar o método do erro de aproximação.
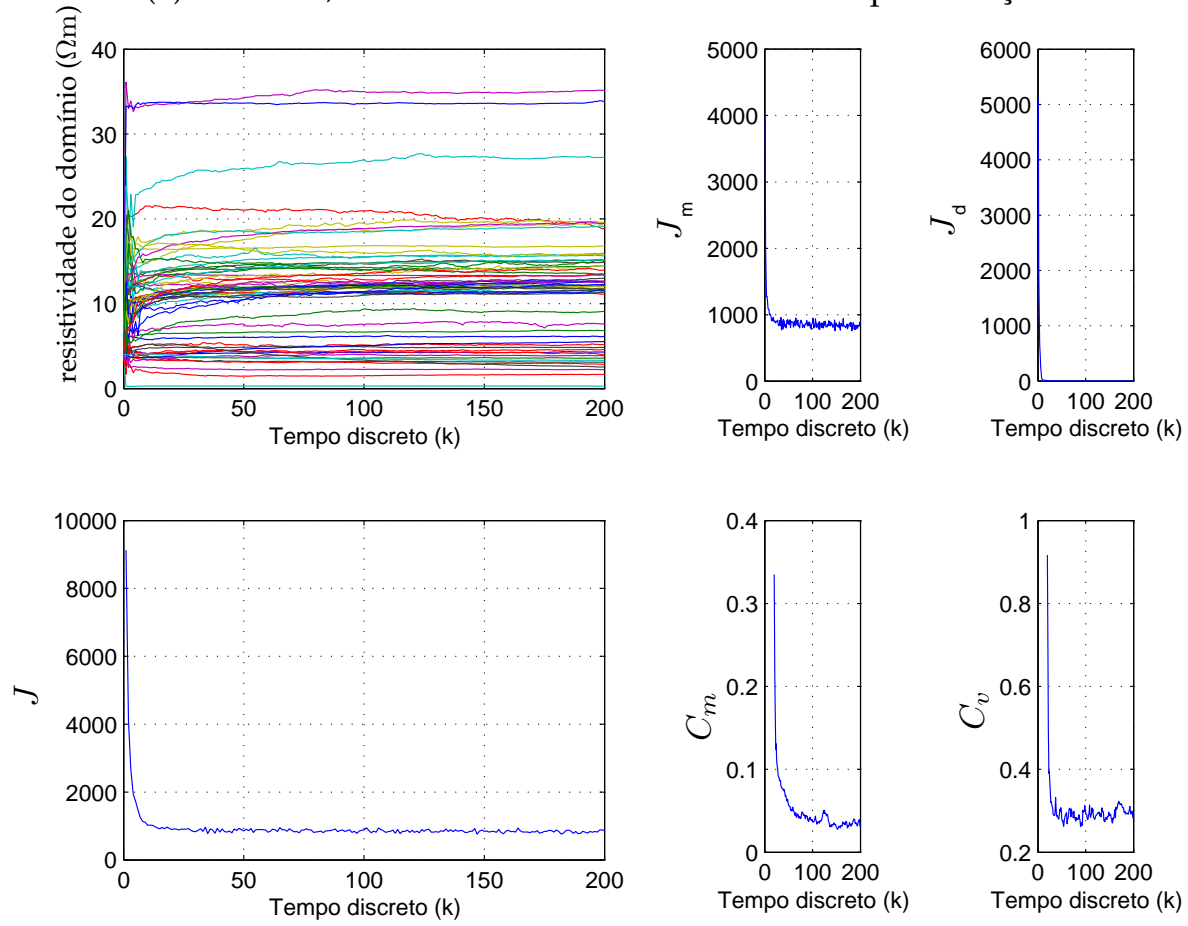

(b) Ensaio 8, utilizando o método do erro de aproximação.

Figura F.8: Resultados do Ensaio 8. Histórico da estimação e índices de desempenho. 


\section{F.1.2 Com o uso de densidade a priori}
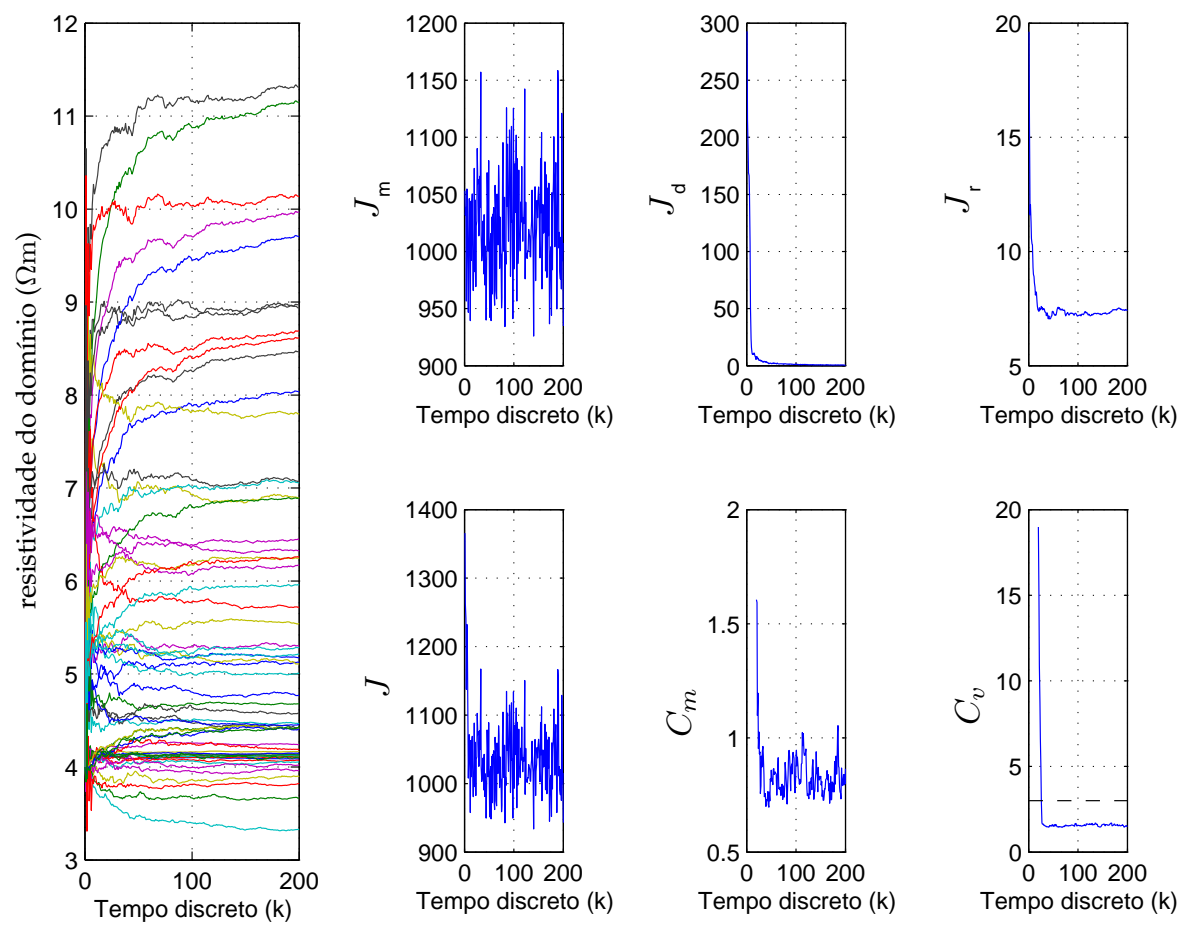

(a) Ensaio 1, sem utilizar o método do erro de aproximação.
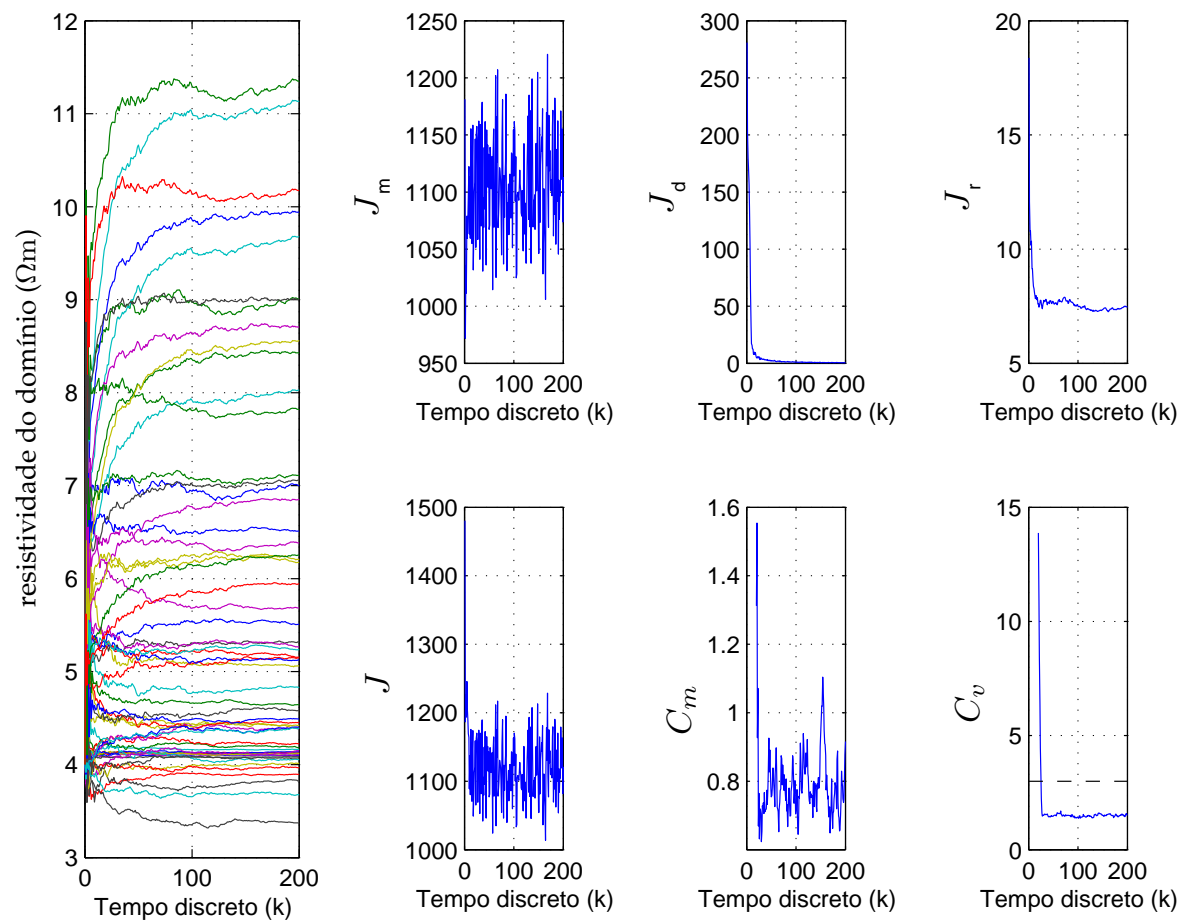

(b) Ensaio 1, utilizando o método do erro de aproximação.

Figura F.9: Resultados do Ensaio 1. Histórico da estimação e índices de desempenho. 

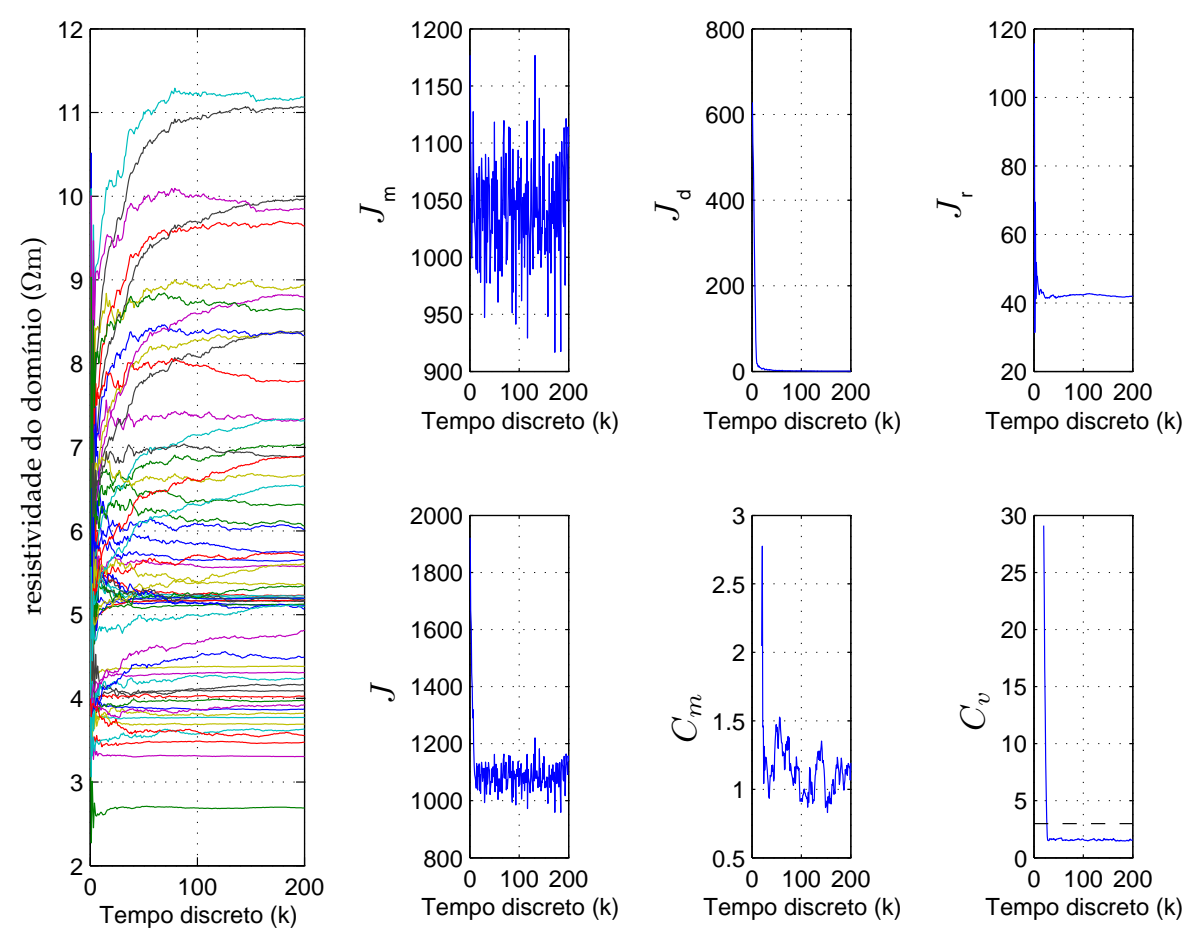

(a) Ensaio 2, sem utilizar o método do erro de aproximação.
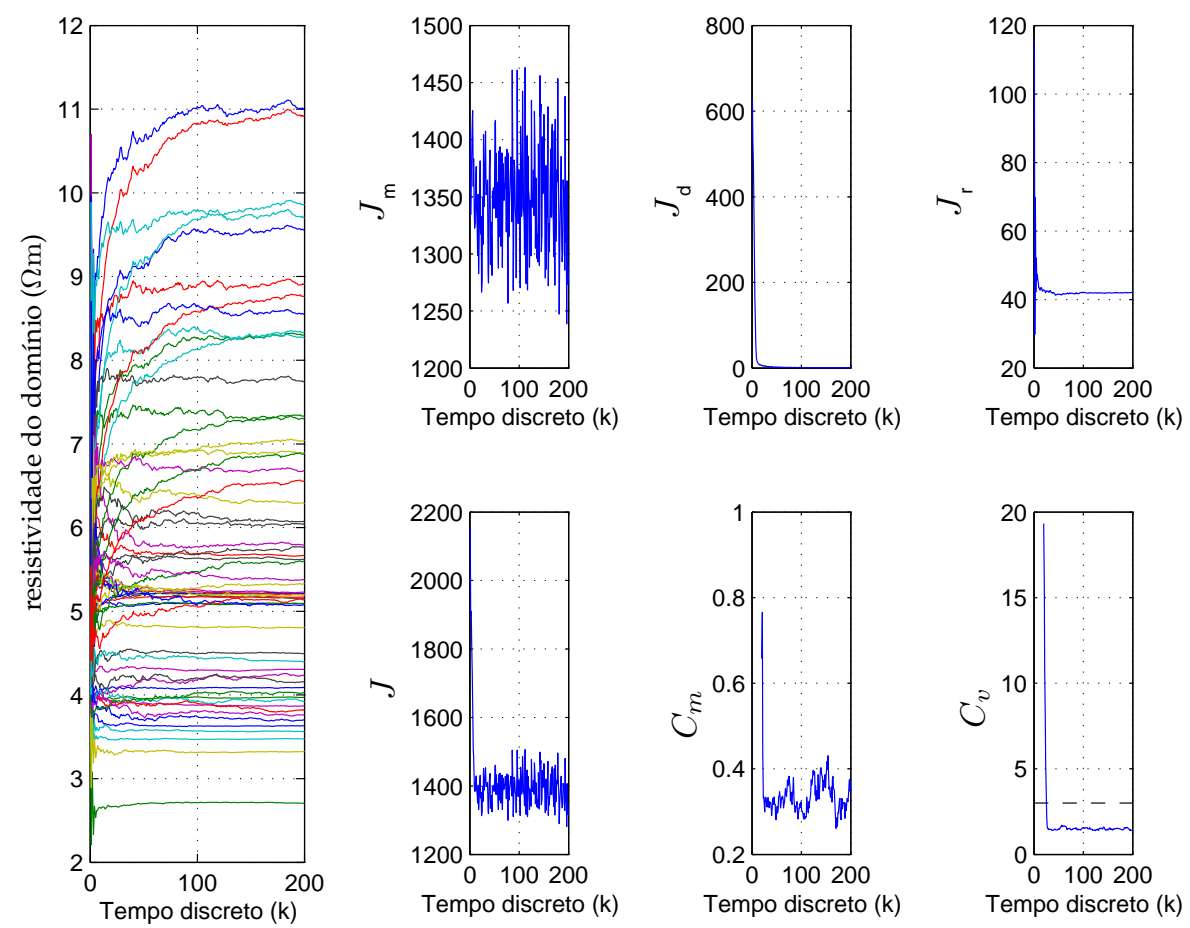

(b) Ensaio 2, utilizando o método do erro de aproximação.

Figura F.10: Resultados do Ensaio 2. Histórico da estimação e índices de desempenho. 

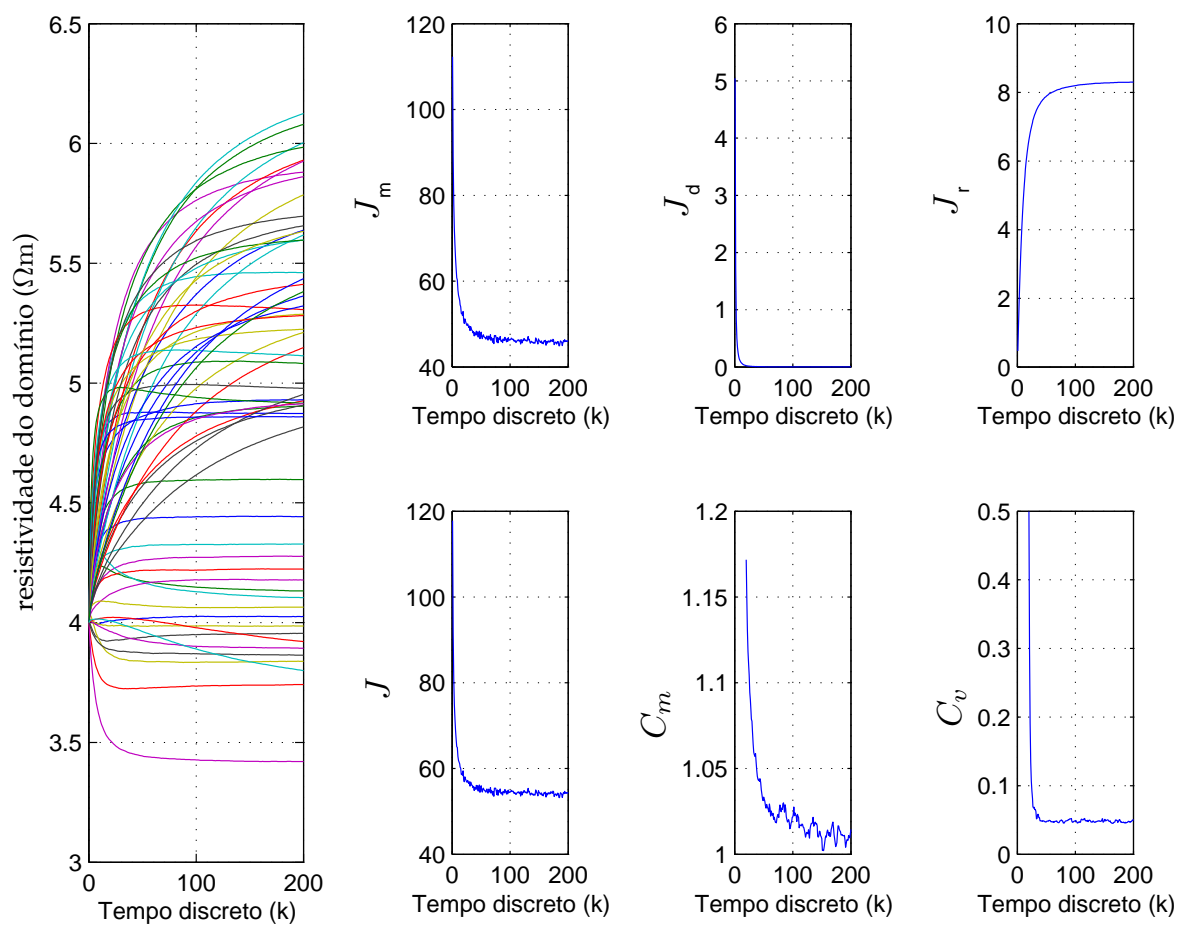

(a) Ensaio 3, sem utilizar o método do erro de aproximação.
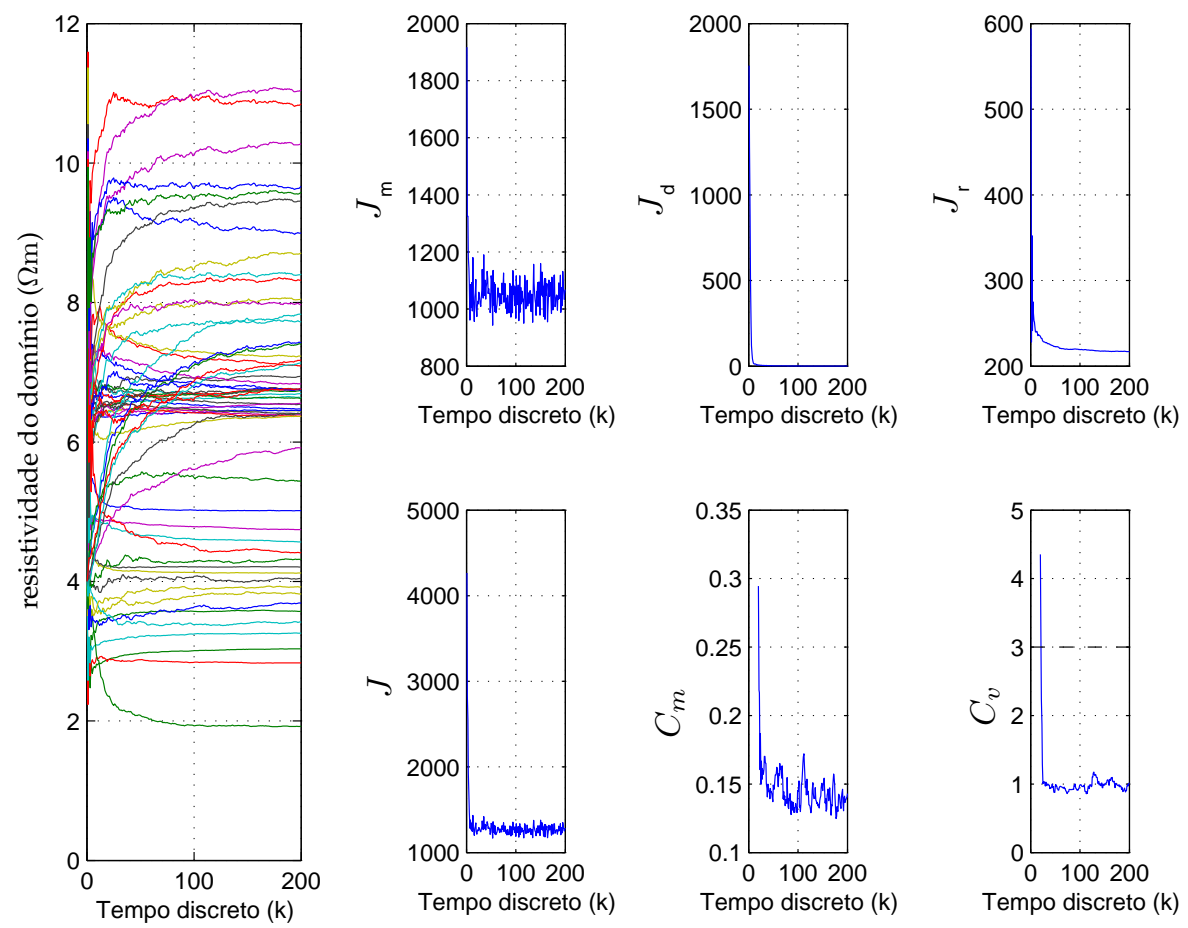

(b) Ensaio 3, utilizando o método do erro de aproximação.

Figura F.11: Resultados do Ensaio 3. Histórico da estimação e índices de desempenho. 

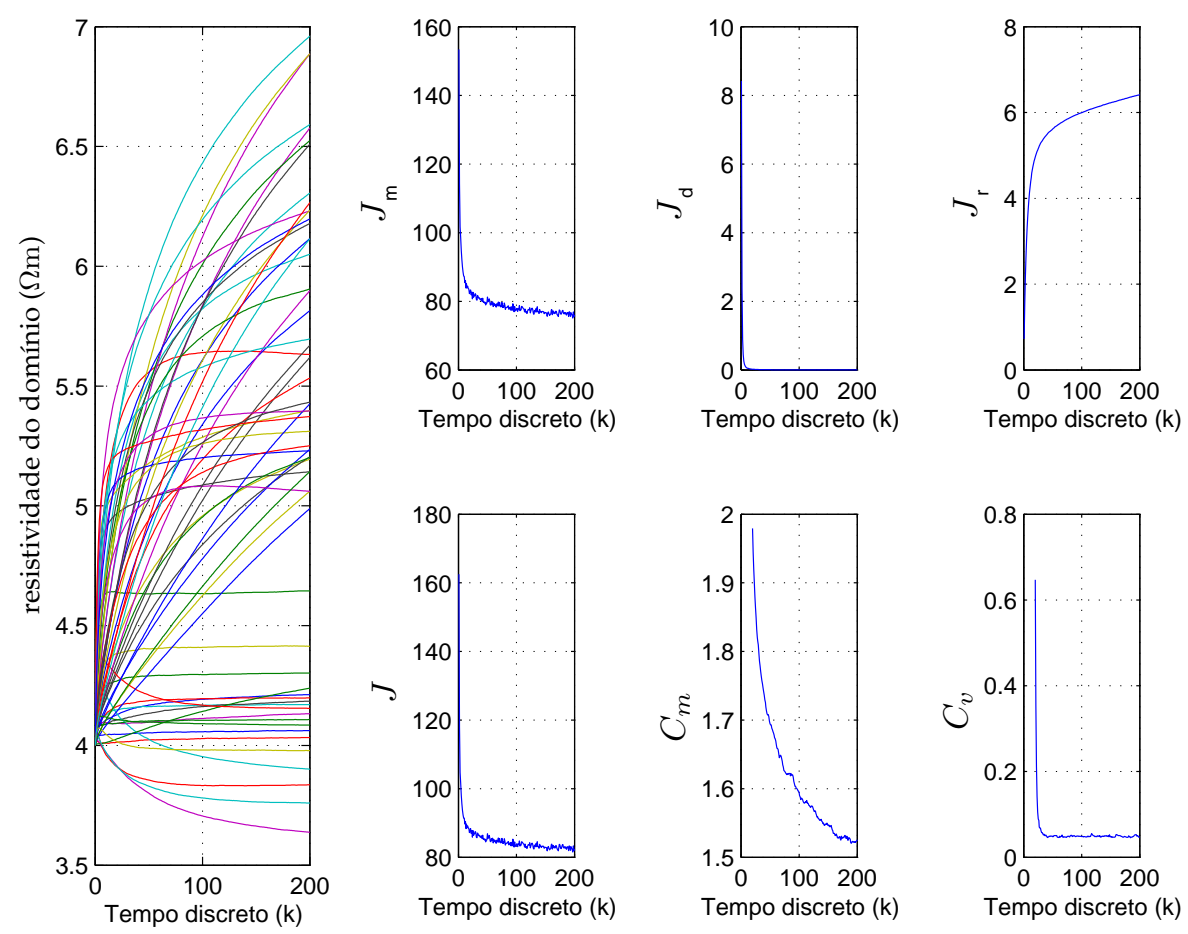

(a) Ensaio 4, sem utilizar o método do erro de aproximação.
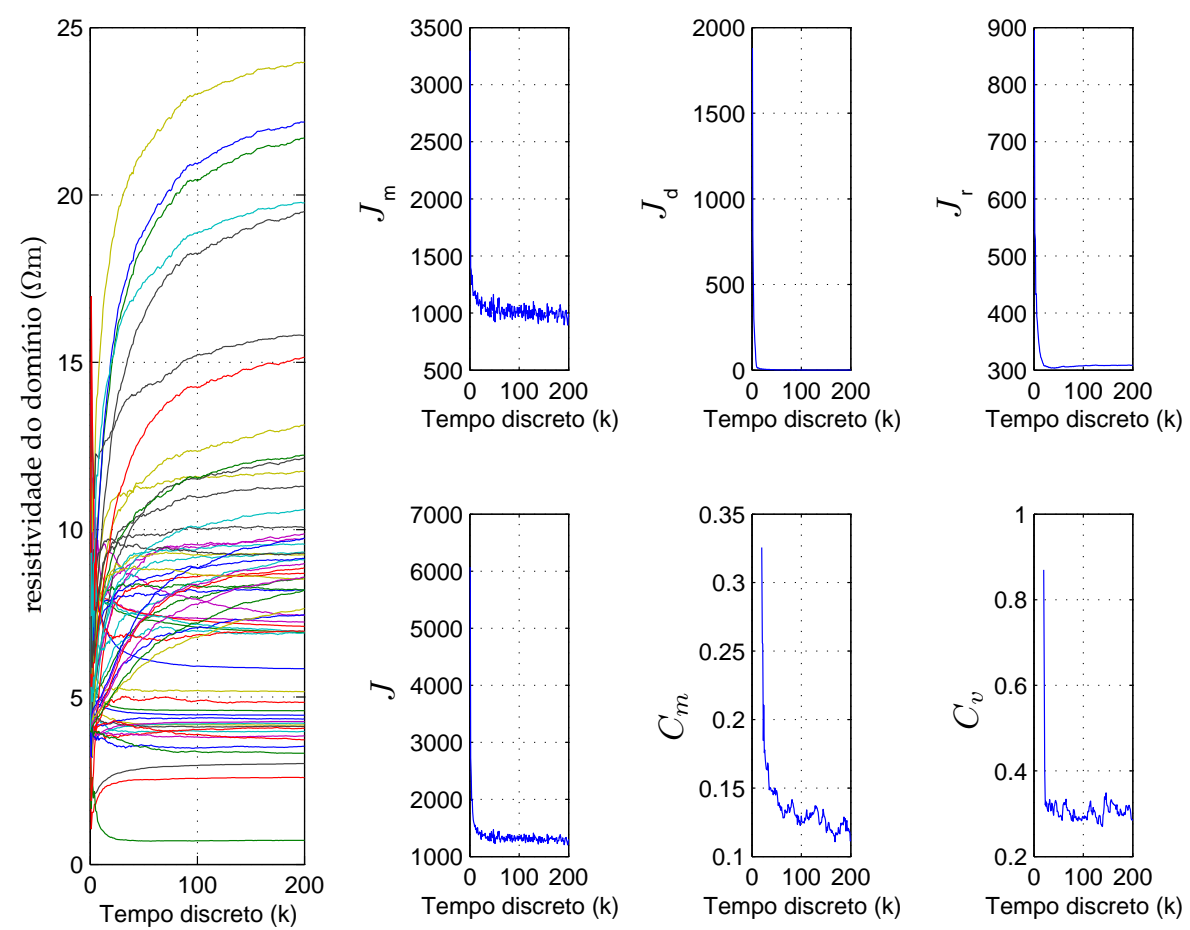

(b) Ensaio 4, utilizando o método do erro de aproximação.

Figura F.12: Resultados do Ensaio 4. Histórico da estimação e índices de desempenho. 

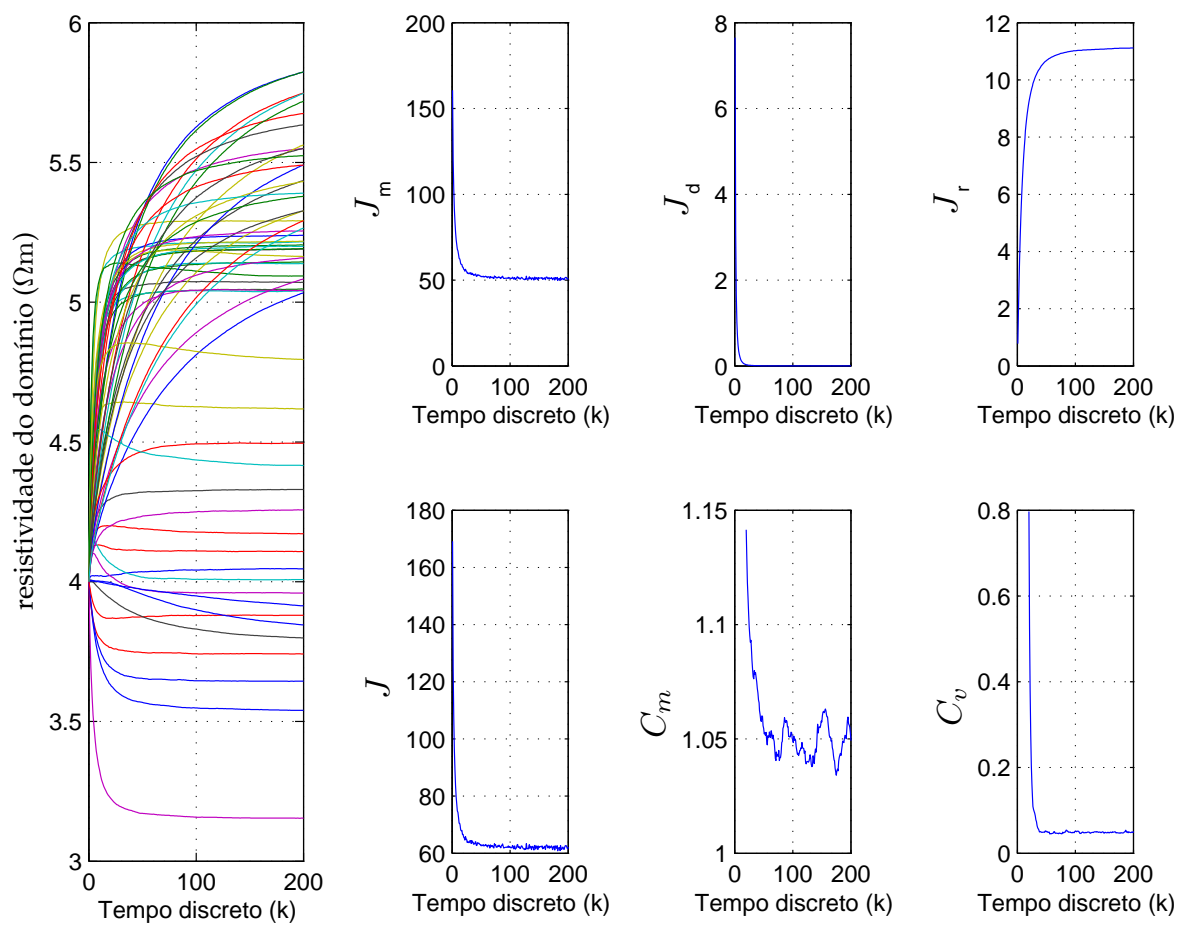

(a) Ensaio 5, sem utilizar o método do erro de aproximação.
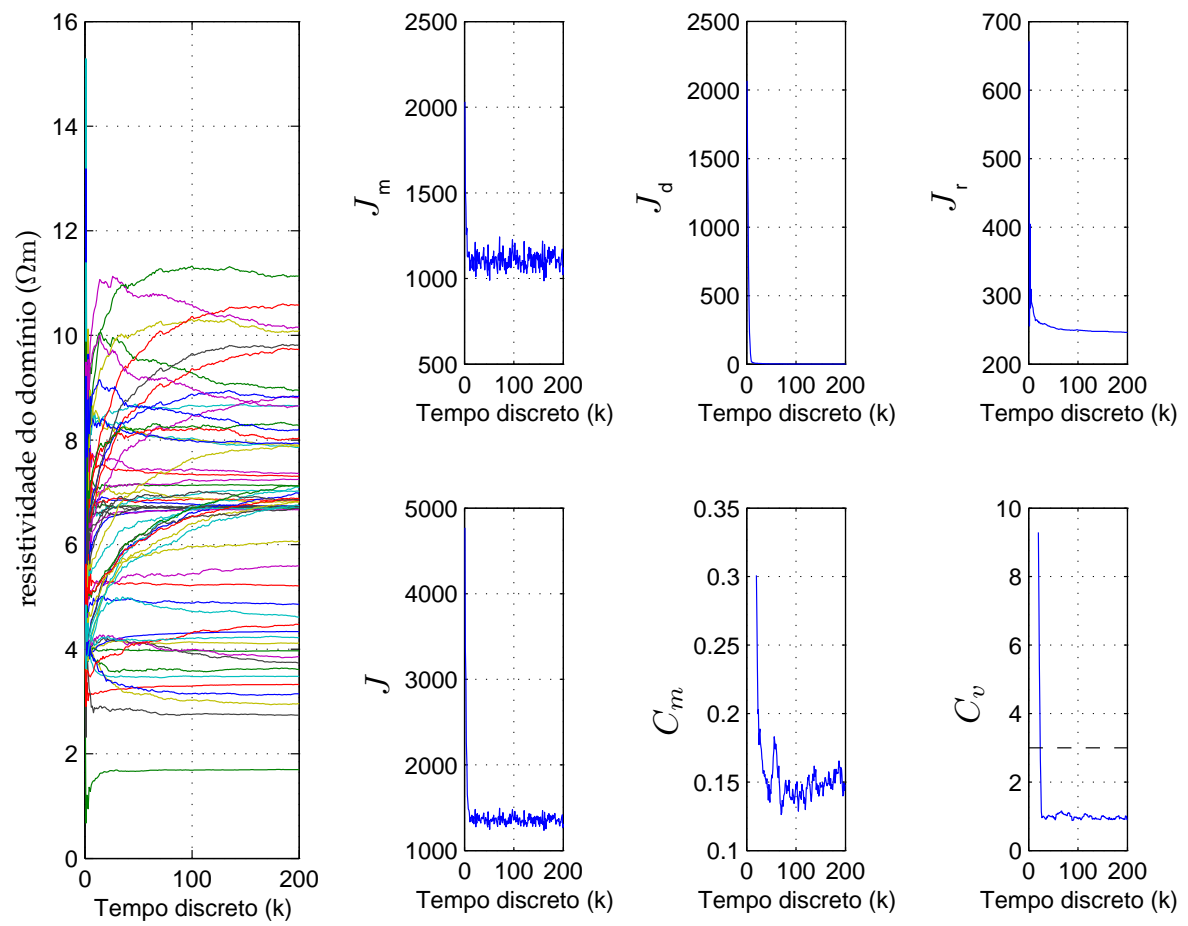

(b) Ensaio 5, utilizando o método do erro de aproximação.

Figura F.13: Resultados do Ensaio 5. Histórico da estimação e índices de desempenho. 

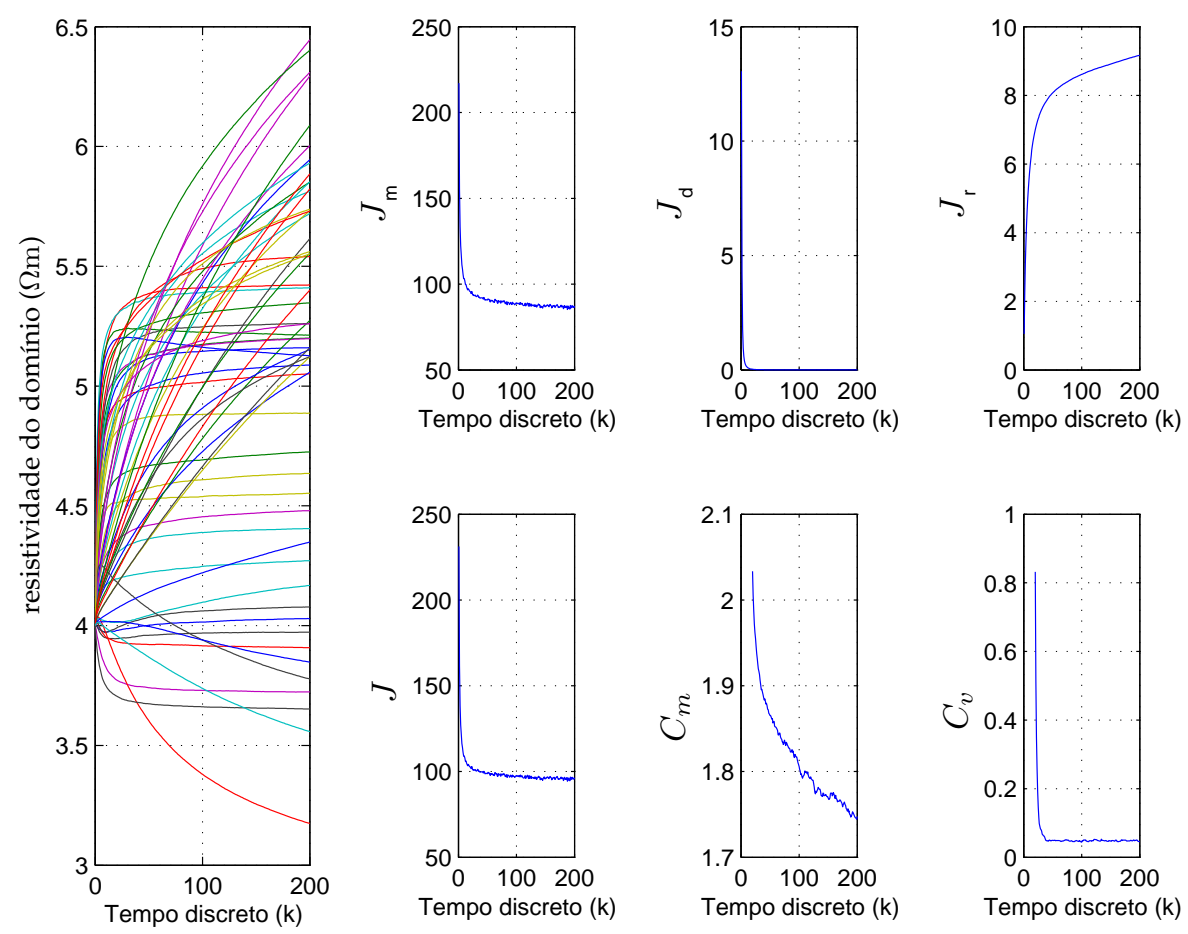

(a) Ensaio 6, sem utilizar o método do erro de aproximação.
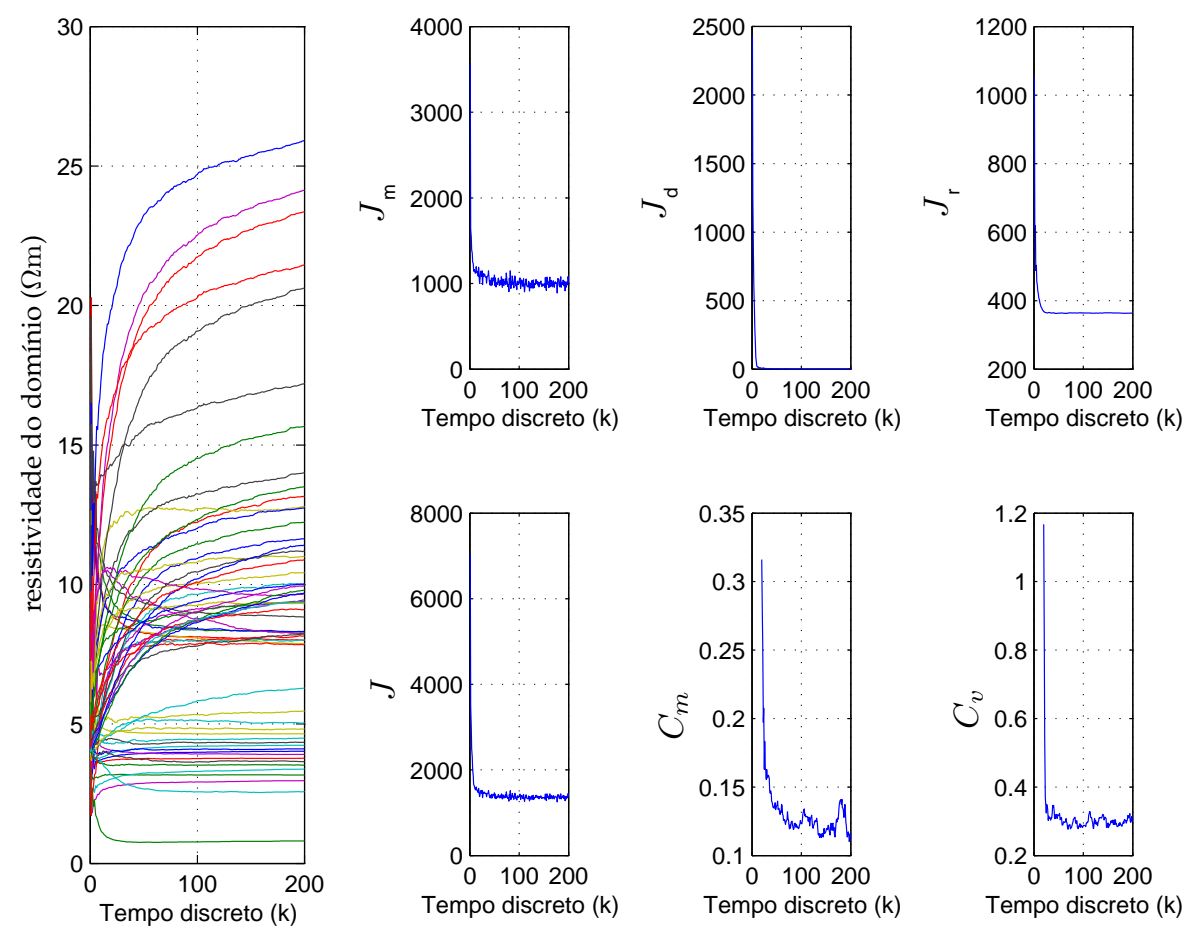

(b) Ensaio 6, utilizando o método do erro de aproximação.

Figura F.14: Resultados do Ensaio 6. Histórico da estimação e índices de desempenho. 

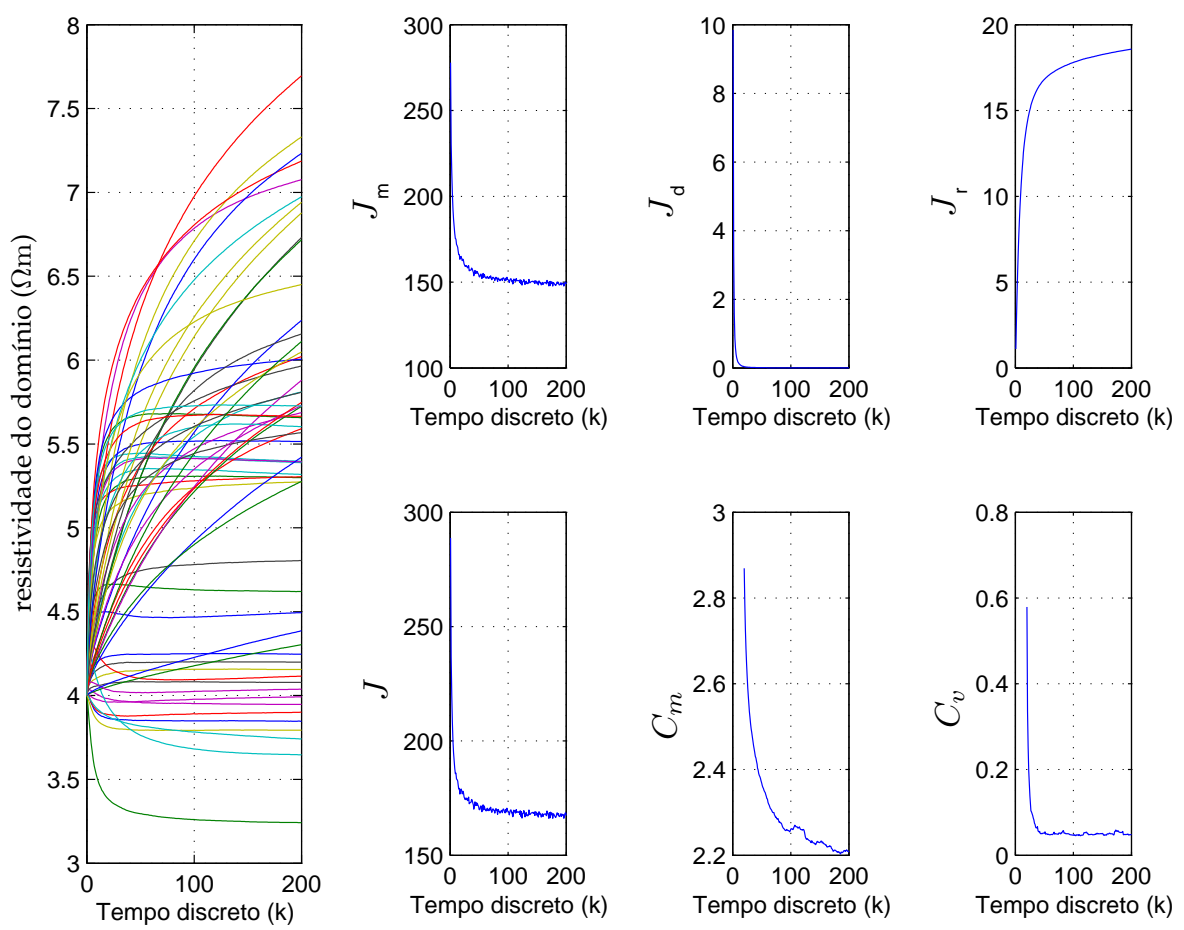

(a) Ensaio 7, sem utilizar o método do erro de aproximação.
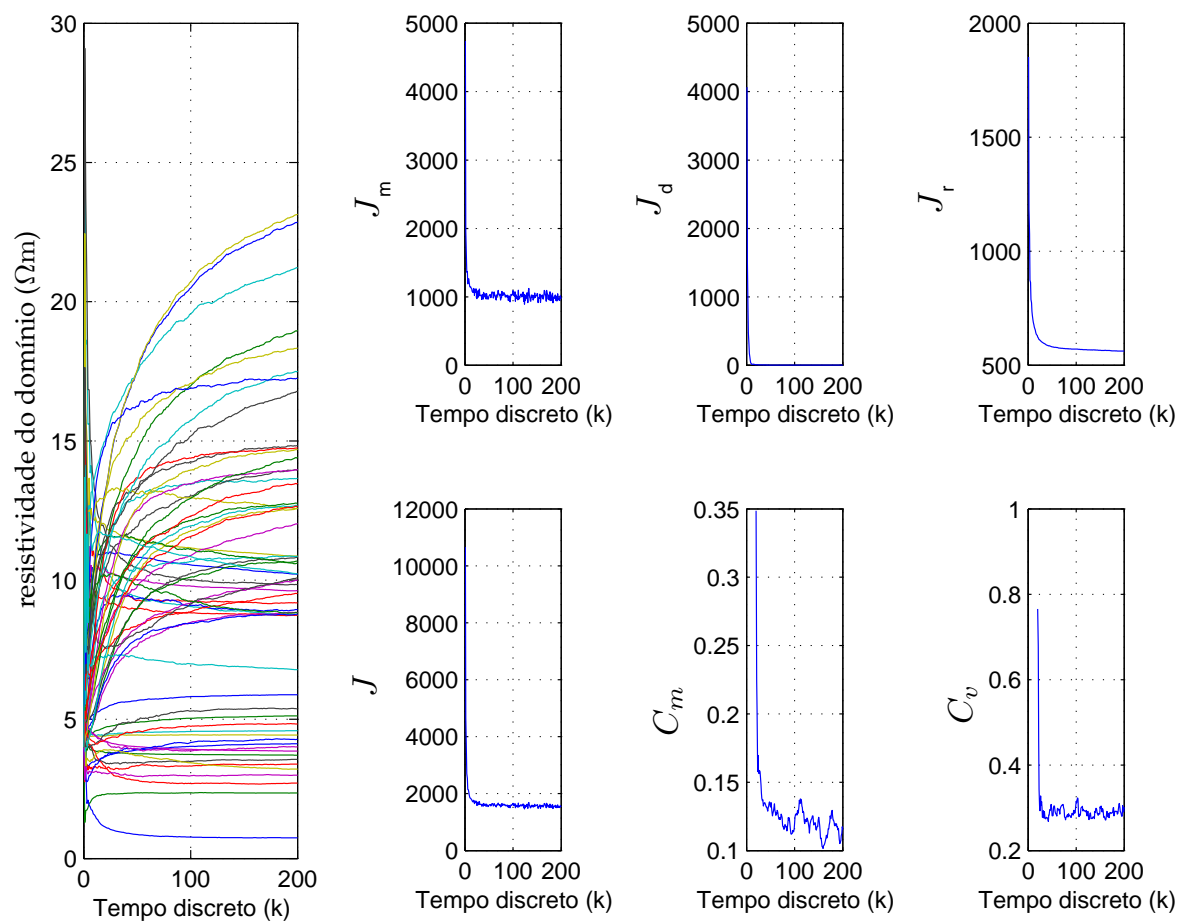

(b) Ensaio 7, utilizando o método do erro de aproximação.

Figura F.15: Resultados do Ensaio 7. Histórico da estimação e índices de desempenho. 

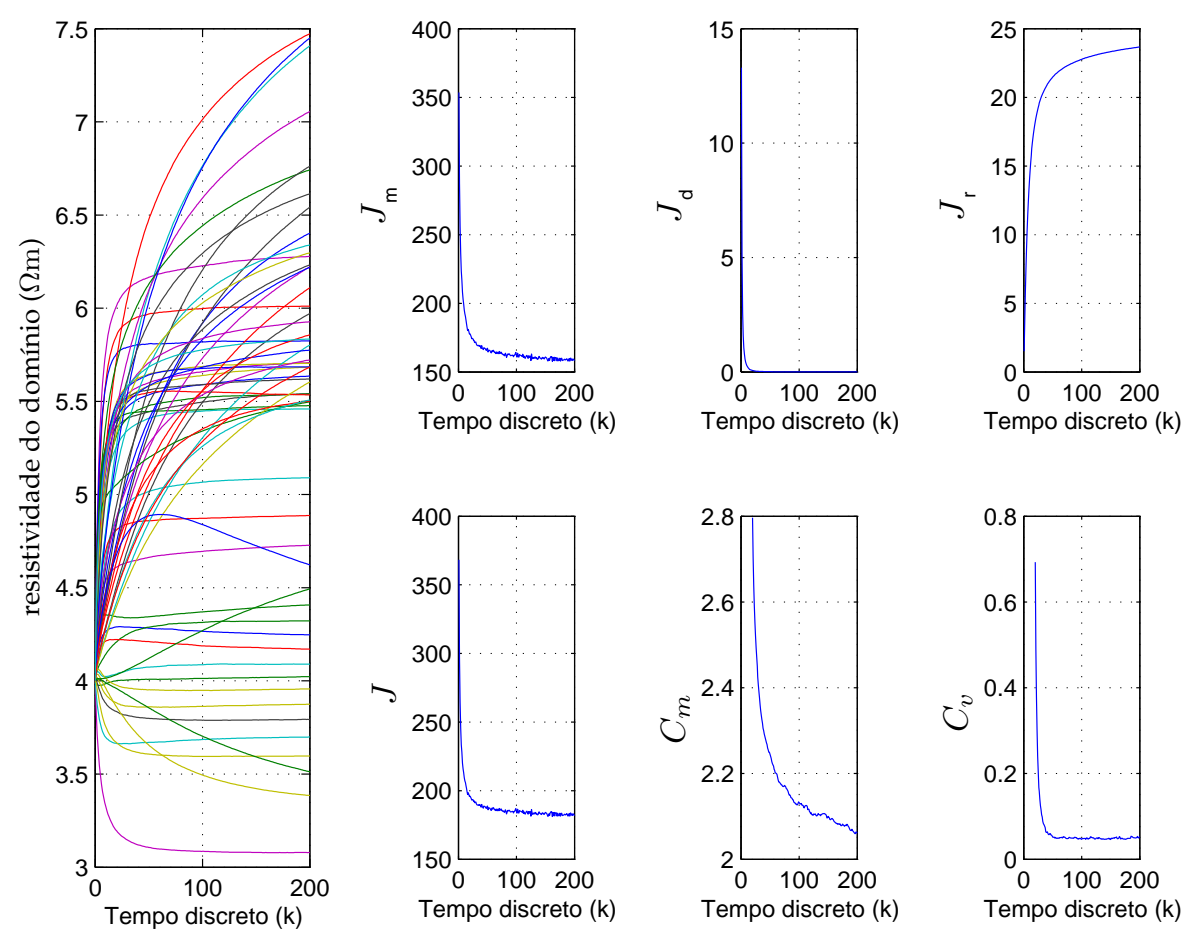

(a) Ensaio 8, sem utilizar o método do erro de aproximação.
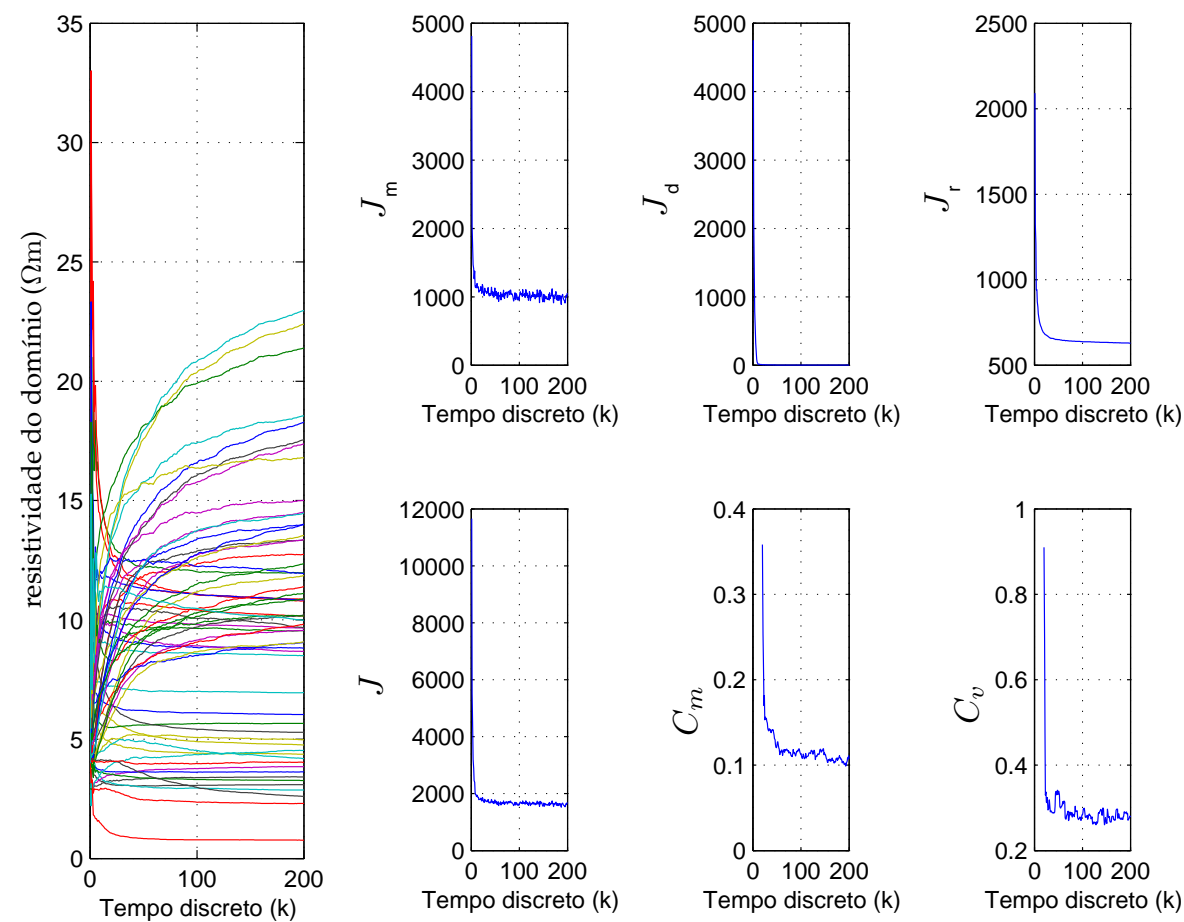

(b) Ensaio 8, utilizando o método do erro de aproximação.

Figura F.16: Resultados do Ensaio 8. Histórico da estimação e índices de desempenho. 


\section{Referências Bibliográficas}

[1] M. Cheney, D. Isaacson, e J. C. Newell. Electrical impedance tomography. SIAM Review, 41(1):85-101, 1999.

[2] M. B. P. Amato, C. S. Barbas, D. M. Medeiros, R. B Magaldi, G. P. Schettino, G. Lorenzi Filho, R. A. Kairalla, D. Deheinzelin, C. Munoz, R. Oliveira, T. Y. Takagaki, e C. R. R. Carvalho. Effect of a protective-ventilation strategy on mortality in the acute respiratory distress syndrome. New England Journal of Medicine, 338(6):347-354, 1998.

[3] F. C. Trigo, R. G. Lima, e M. B. P. Amato. Electrical impedance tomography using the extended kalman filter. IEEE Transactions on Biomedical Engineering, 51(1):72-81, january 2004.

[4] T. H. S. Sousa, F. S. Moura, I. O. Hoffmann, E. D. L. B. Camargo, R. G. Lima, A. R. C. Martins, D. T. Fantoni, e C. Biasi. In vivo admitivity measurements for regularizations in electrical impedance tomography. In Proceedings of 21st International Congress of Mechanical Engineering, 2011.

[5] P. Nopp, E. Rapp, H. Pfützner, H. Nakesch, e C. Rushsam. Dieletric properties of lung tissue as a function of air content. Physics in Medicine and Biology, 38(6):699-716, 1993.

[6] S. Gabriel, R. W. Lau, e C. Gabriel. The dielectric properties of biological tissues: III. parametric models for the dielectric spectrum of tissues. Physics In Medicine And Biology, 41(11):2271-2293, november 1996. doi: 10.1088/0031-9155/41/11/003.

[7] M. B. P. Amato, C. S. Barbas, D. M. Medeiros, G. P. Schettino, G. Lorenzi Filho, R. A. Kairalla, D. Deheinzelin, C. Moraes, E. O. Fernandes, T. Y. Takagaki, e C. R. R. Carvalho. Beneficial effects of the "open lung approach"with low distending pressures in acute respiratory distress syndrome. a prospective randomized study on mechanical ventilation. American Journal of Respiratory and Critical Care Medicine, 152(1):1835-1846, 1995.

[8] E. V. C. Leite, S. Gomes, M. A. Beraldo, M. S. Volpe, I. A. Schettino, M. R. Tucci, S. H. Böhm, H. Tanaka, R. G. Lima, C. R. R. Carvalho, e M. B. P. Amato. Real-time detection of pneumothorax using electrical impedance tomography. Critical Care Medicine, 36(4): 1230-1238, april 2008.

[9] E. V. L. Costa, J. B. Borges, A. Melo, F. Suarez-Sipmann, C. Toufen, S. H. Böhm, e M. B. P. Amato. Bedside estimation of recruitable alveolar collapse and hyperdistension by electrical impedance tomography. Intensive Care Medicine, 35(6):1132-1137, june 2009.

[10] I. K. Ike e R. G. Lima. ABCM Symposium Series in Bioengineering, volume 1, chapter Effects of static condensation on electrical impedance tomography. Brazilian Society of Mechanical Sciences and Engineering, Rio de Janeiro, 2007. 
[11] R. A. Hellmuth, R. G. Lima, e M. B. P. Amato. Pulmonary alveolus' model including surface tension effects. In Proceedings of the 19th International Congress on mechanical Engineering, Brasília, 2007. ABCM.

[12] D. A. A. Miranda. Tomografia por impedância elétrica utilizando o método de newton-raphson. Dissertação de Mestrado, Escola Politécnica da Universidade de São Paulo, São Paulo, 2002.

[13] M. F. M. Vallejo, F. S. Moura, C. N. L. Herrera, J. C. C. Aya, e R. G. Lima. An algorithm for real time estimation of electrode position in electrical impedance tomography. In Proceedings of the Technology and Medical Sciences International 4, Dundee, 2006.

[14] M. F. M. Vallejo, C. N. L. Herrera, F. S. Moura, J. C. C. Aya, e R. G. Lima. The use of linear programming as search method of images in electrical impedance tomography. In Proceedings of the 19th International Congress of mechanical Engineering, Brasilia, 2007. ABCM.

[15] M. F. M. Vallejo. Algoritmo de tomografia por impedância elétrica utilizando programação linear omo método de busca de imagem. Dissertação de Mestrado, Escola Politécnica da Universidade de São Paulo, São Paulo, 2007.

[16] O. L. Silva. Muscle contraction detection using electrical impedance tomography. Tese de doutorado, Escola Politécnica da Universidade de São Paulo, São Paulo, 2012.

[17] C. R. Lima, L. A. M. Mello, e E. C. N. Silva. Node-based distribution of material properties for topology optimization algorithm applied to electrical impedance tomography imaging. In Proceedings of the XXVI Iberian Latin-American Congress on Computational Methods in Engineering, Guaraparí, Brazil, october 2005.

[18] C. R. Lima, L. A. M. Mello, e E. C. N. Silva. Topology optimization applied to electrical impedance tomography. In Proceedings of 6th World Congress on Structural and Multidisciplinary Optimization, Rio de Janeiro, may 2005.

[19] C. R. Lima, L. A. M. Mello, e E. C. N. Silva. ABCM Symposium Series in Mechatronics, volume 2, chapter Topology optimization applied to $2 \mathrm{~d}$ and $3 \mathrm{~d}$ electrical impedance tomography. Brazilian Society of Mechanical Sciences and Engineering, Rio de Janeiro, 2006.

[20] L. A. Motta-Mello, C. R. Lima, J. C. Aya, C. N. Pai, F. S. Moura, E. C. N. Silva, e R. G. Lima. Comparing two electrical impedance tomography algorithms: Gauss-newton and topology optimization. In A. Manduca, editor, Proceedings of SPIE Medical Imaging 2006: Physiology, Function, and Structure from Medical Images, volume 6143, pages 1013-1021, 2006. doi: $10.1117 / 12.654059$.

[21] C. R. Lima, L. M. Mello, R. G. Lima, e E. N. Silva. Electrical impedance tomography through constrained sequential linear programming: a topology optimization approach. Measurement Science and Technology, 18:2847-2858, 2007. doi: 10.1088/0957-0233/18/9/ 014.

[22] L. M. Mello, C. R. Lima, M. B. P. Amato, R. G. Lima, e E. N. Silva. Three-dimensional electrical impedance tomography: a topology optimization approach. IEEE Transactions on Biomedical Engineering, 55(2):531-540, 2008. doi: 10.1109/TBME.2007.912637. 
[23] L. A. M. Mello, R. G. Lima, M. B. P. Amato, e E. C. N. Silva. On the design of electrodes through the topology optimization method applied to electrical impedance tomography. In Proceedings of EngOpt 2008 - International Conference on Engineering Optimization, Rio de Janeiro, Brazil, june 2008.

[24] L. A. Mello, E. C. N. Silva, E. Sturler, e G. H. Paulino. Topology optimization method utilizing iterative solvers with subspace recycling applied to high-resolution electrical impedance tomography. In J. F. Abel e J. R. Cooke, editors, Proceedings of the 6th International Conference on Computation of Shell and Spatial Structures, Ithaca, USA, May 2008.

[25] L. A. M. Mello e E. C. N. Silva. An electrical impedance tomography algorithm based on reliability-based topology optimization. In Proceedings of 8th World Congress on Structural and Multidisciplinary Optimization, Lisbon, Portugal, june 2009.

[26] L. A. M. Mello. Estudo do aumento do desempenho de um sistema de tomografia de impedância elétrica através do método de otimização topológica. Tese de doutorado, Escola Politécnica da Universidade de São Paulo, São Paulo, 2010.

[27] P. C. Nan, J. C. C. Aya, e R. G. Lima. ABCM Symposium Series in Bioengineering, volume 1, chapter An implementation of the back-projection algorithm according to Santosa and Vogelius. Brazilian Society of Mechanical Sciences and Engineering, Rio de Janeiro, 2007.

[28] C. N. L. Herrera, M. F. M. Vallejo, F. S. Moura, J. C. C. Aya, e R. G. Lima. Electrical impedance tomography algorithm based on simulated annealing search method. In Proceeedings of the 19th International Congress of mechanical Engineering, Brasília, 2007. ABCM.

[29] C. N. L. Herrera. Algoritmo de tomografia por impedância elétrica baseado em simulated annealing. Dissertação de Mestrado, Escola Politécnica da Universidade de São Paulo, São Paulo, 2007.

[30] P. C. Nan, F. S. Moura, R. K. Schweder, L. A. S. Mirandola, J. C. C. Aya, e R. G. Lima. A black-box back projection algorithm for electrical impedance tomography. In Proc. International Congress of Mechanical Engineering, 2005.

[31] J. C. C. Aya, F. S. Moura, P. C. Nan, R. K. Schweder, e R. G. Lima. ABCM Symposium Series in Bioengineering, volume 1, chapter Regularizations for a Black Box Back-projection EIT algorithm. Brazilian Society of Mechanical Sciences and Engineering, Rio de Janeiro, 2007.

[32] F. C. Trigo. Filtro estendido de kalman aplicado à tomografia por impedância elétrica. Dissertação de Mestrado, Escola Politécnica da Universidade de São Paulo, São Paulo, 2001.

[33] N. V. Molina. Redução de erro numérico no filtro de kalman estendido aplicado a tomografia por impedância elétrica. Dissertação de Mestrado, Escola Politécnica da Universidade de São Paulo, São Paulo, 2002.

[34] F. C. Trigo. Estimação não linear de parâmetros através dos filtros de Kalman na tomografia por impedância elétrica. Tese de doutorado, Escola Politécnica da Universidade de São Paulo, São Paulo, 2005. 
[35] F. C. Trigo e R. G. Lima. ABCM Symposium Series in Bioengineering, volume 1, chapter Iterated Extended Kalman Filter with adaptive state noise estimation for electrical impedance tomography. Brazilian Society of Mechanical Sciences and Engineering, Rio de Janeiro, 2007.

[36] F. S. Moura, J. C. C. Aya, A. T. Fleury, e R. G. Lima. On line transition matrix identification of the state evolution model for the extended kalman filter in electrical impedance tomography. Journal of Physics: Conference Series, 124, august 2008. doi: 10.1088/1742-6596/124/1/012052.

[37] F. S. Moura, J. C. C. Aya, A. T. Fleury, e R. G. Lima. Dynamic electrical impedance tomography of a human chest with transition matrix identification. In Proceedings of 10th International Conference on Biomedical Applications of Electrical Impedance Tomography, Manchester, UK, june 2009.

[38] F. S. Moura, J. C. C. Aya, A. T. Fleury, M. B. P. Amato, e R. G. Lima. Dynamic imaging in electrical impedance tomography of the human chest with online transition matrix identification. IEEE Transactions on Biomedical Engineering, 57(2):422-431, February 2010. doi: 10.1109/TBME.2009.2032529.

[39] C. N. L. Herrera. Um método D-bar para estimar admitividade em 2-D através de tomografia por impedância elétrica. Tese de doutorado, Escola Politécnica da Universidade de São Paulo, São Paulo, 2012.

[40] M. F. M. Vallejo. Reconstrução não-linear completa de imagens de tomografia por impedância elétrica utilizando o método D-bar 2D. Tese de doutorado, Escola Politécnica da Universidade de São Paulo, São Paulo, 2012.

[41] E. D. L. B. Camargo, F. S. Moura, O. L. Luppi, F. P. R. Martins, R. G. Lima, M. B. P. Amato, e A. C. B. C. F. Pinto. Converting ct scan images into impeditivity measurements to form an anatomical atlas for electrical impedance tomography. In Proceedings of 21st International Congress of Mechanical Engineering, 2011.

[42] F. L. H. Gielen, W. W. Jonge, e K. L. Boon. Electrical conductivity of skeletal muscle tissue: Experimental results from different musclesin vivo. Medical and Biological Engineering and Computing, 22(6):569-577, november 1984. doi: 10.1007/BF02443872.

[43] J. P. J. Abascal, S. R. Arridge, W. R. B. Lionheart, R. H. Bayford, e D. S. Holder. Validation of a finite-element solution for electrical impedance tomography in an anisotropic medium. Physiological Measurement, 28(7):S129-S140, june 2007. doi: 10.1088/0967-3334/ 28/7/S10.

[44] J. F. Abascal, S.R. Arridge, D. Atkinson, R. Horesh, L. Fabrizi, M. De Lucia, L. Horesh, R. H. Bayford, e Holder D. S. Use of anisotropic modelling in electrical impedance tomography: description of method and preliminary assessment of utility in imaging brain function in the adult human head. Neuroimage, 43(2):258-268, 2008.

[45] M. E. Glidewell e T. N Kwong. Anatomically constrained electrical impedance tomography for three-dimensional anisotropic bodies. IEEE Transactions on Medical Imaging, 16(5):572-580, october 1997.

[46] P. L. Nunez e R. Srinivasan. Electric fields of the brain: the neurophysics of EEG. Oxford University Press, 2 edição, 2006. 
[47] S. Gabriel, R. W. Lau, e C. Gabriel. The dielectric properties of biological tissues: II. measurements in the frequency range $10 \mathrm{~Hz}$ to $20 \mathrm{GHz}$. Physics In Medicine And Biology, 41(11):2251-2269, november 1996. doi: 10.1088/0031-9155/41/11/002.

[48] S. Gabriel, R. W. Lau, e C. Gabriel. The dielectric properties of biological tissues: I. literature survey. Physics In Medicine And Biology, 41(11):2231-2249, november 1996. doi: 10.1088/0031-9155/41/11/001.

[49] C. Gabriel, A. Peyman, e E. H. Grant. Electrical conductivity of tissue at frequencies below 1 mhz. Physics in Medicine and Biology, 54:4863-4878, 2009. doi: 10.1088/ 0031-9155/54/16/002.

[50] W. Lionheart, N. Polydorides, e A. Borsic. Electrical Impedance Tomography: Methods, History And Applications. Institute of Physics Publishing, London, 1 edição, 2005.

[51] K. S. Cheng, D. Isaacson, J. C. Newell, e D. G. Gisser. Electrode models for electric current computed tomography. IEEE Transactions on Biomedical Engineering, 36(9):918-924, 1989.

[52] P. Hua, E. J. Woo, J. G. Webster, e W. J. Tompkins. Finite element modeling of electrode-skin contact impedance in electrical impedance tomography. IEEE Transactions on Biomedical Engineering, 40(4):335-343, april 1993.

[53] D. Isaacson. Distinguishability of conductivities by electric current computed tomography. IEEE Transactions on Medical Imaging, 5(2):91-95, june 1986.

[54] G. A. Kyriacou, C. S. Koukourlis, e J. N. Sahalos. A reconstruction algorithm of electrical impedance tomography with optimal configuration of the driven electrodes. IEEE Transactions on Medical Imaging, 12(3):430-438, september 1993.

[55] A. D. Seagar, D. C. Barber, e B. H. Brown. Theoretical limits to sensitivity and resolution in impedance imaging. Clinical Physics and Physiological Measurement, 8(4A):13-31, january 1987. doi: http://dx.doi.org/10.1088/0143-0815/8/4A/301.

[56] M. Cheney e D. Isaacson. Distinguishability in impedance imaging. IEEE Transactions in Biomedical Enginnering, 39(8):852-860, august 1992.

[57] B M. Eyüboğlu e T. C. Pilkington. Comments on distinguishability in electrical impedance imaging. IEEE Transactions on Biomedical Engineering, 40(12):1328-1330, december 1993.

[58] B M. Eyüboğlu, A. Köksal, e M. Demirbilek. Distinguishability analysis of an induced current eit system using discrete coils. Physics In Medicine And Biology, 45(7):1997-2009, 2000. doi: http://dx.doi.org/10.1088/0031-9155/45/7/321.

[59] J. Malmivuo e R. Plonsey. Bioelectromagnetism - Principles and Applications of Bioelectric and Biomagnetic Fields. Oxford University Press, Oxford, 1 edição, 1995. URL http://www.bem.fi/book/index.htm.

[60] K. B. Petersen e M. S. Pedersen. The matrix cookbook, october 2008. URL http://www2.imm.dtu.dk/pubdb/p.php?3274. Version 20081110.

[61] J. Kaipio e E. Somersalo. Statistical and Computational Inverse Problems. Springer, New York, 1 edição, 2004.

[62] A. N. Tikhonov e V. Y. Arsenin. Solutions of ill posed problems. Bulletin of The American Mathematical Society, 1(3):521-524, 1979. 
[63] K. Astala e L. Päivärinta. Calderón's inverse conductivity problem in the plane. The Annals of Mathematics, 163(1):265-299, jan. 2006. ISSN 0003486X. URL http://www.jstor.org/stable/20159954.

[64] E. Francini. Recovering a complex coefficient in a planar domain from the dirichlet-to-neumann map. Inverse Problems, 16(1):107-119, 2000. URL http://stacks.iop.org/0266-5611/16/i=1/a=309.

[65] R. M. Brown e R. H. Torres. Uniqueness in the inverse conductivity problem for conductivities with $3 / 2$ derivatives in $l^{p}, p>2$. Journal of Fourier Analysis and Applications, 9:563-574, 2003. ISSN 1069-5869. doi: 10.1007/s00041-003-0902-3. URL http://dx.doi.org/10.1007/s00041-003-0902-3.

[66] A. Greenleaf, M. Lassas, e G. Uhlmann. Anisotropic conductivities that cannot be detected by eit. Physiological Measurement, 24(2):413-419, 2003. URL http://stacks.iop.org/0967-3334/24/i=2/a=353.

[67] G. Uhlmann. Commentary on Calderón's paper (29), on an inverse boundary value problem. In Selected papers of Alberto P. Calderón, pages 623-636. American Mathematical Society, Providence, RI, 2008.

[68] H. W. Engl, K. Kunisch, e A. Neubauer. Convergence rates for tikhonov regularisation of non-linear ill-posed problems. Inverse Problems, 5(4):523-540, 1989. URL http://stacks.iop.org/0266-5611/5/i=4/a=007.

[69] P. C. Hansen. Rank-deficient and discrete ill-posed problems: numerical aspects of linear inversion. SIAM Monographs on Mathematical Modeling and Computation. SIAM, 1 edição, 1998.

[70] C. Hansen. Regularization tools: A matlab package for analysis and solution of discrete ill-posed problems. Technical report, Informatics and Mathematical Modelling, Technical University of Denmark, Lyngby, Denmark, march 2008.

[71] A. Quarteroni, R. Sacco, e F. Saleri. Numerical mathematics. Number 37 in Texts in applied mathematics. Springer-Verlag New York, Inc., Secaucus, NJ, USA, 2000.

[72] A. G. Ramm. Inverse Problems: Mathematical and Analytical Techniques With Applications to Engineering. Springer, 1 edição, 2004.

[73] R. C. Aster, B. Borchers, e C. H. Thurber. Parameter Estimation and Inverse Problems, volume 90 of International Geophysics Series. Elsevier Academic Press Publications, 1 edição, 2005.

[74] B. Kaltenbacher, A. Neubauer, e O. Scherzer. Iterative Regularization Methods for Nonlinear Ill-Posed Problems. Walter de Gruyter, 1 edição, 2008.

[75] H. W. Engl, M. Hanke, e A. Neubauer. Regularization of Inverse Problems, volume 375 of Mathematics and Its Applications. Kluwer Academic Publishers, 1996.

[76] R. E. Kalman. A new approach to linear filtering and prediction problems. Transactions of the ASME - Journal of Basic Engineering, 82(Series D):35-45, 1960.

[77] R. E. Kalman e R. S. Bucy. New results in linear filtering and prediction theory. Transactions of the ASME. - Journal of Basic Engineering, 83(Series D):95-107, 1961. 
[78] Petter S. Maybeck. Stochastic models, estimation and control, volume 1. Academic Press Inc., New York, 1 edição, 1979.

[79] Petter S. Maybeck. Stochastic models, estimation and control, volume 2. Academic Press Inc., New York, 1 edição, 1982.

[80] Petter S. Maybeck. Stochastic models, estimation and control, volume 3. Academic Press Inc., New York, 1 edição, 1982.

[81] A. H. Jazwinski. Stochastic Processes and Filtering Theory. Academic Press Inc., New York, 1970.

[82] J. P. Kaipio, P. A. Karjalainen, e E. Somersalo. State estimation in time-varying electrical impedance tomography. Annals of the New York Academy Of Sciences, 873:430-439, april 1999. doi: 10.1111/j.1749-6632.1999.tb09492.x.

[83] K. Y. Kim, B. S. Kim, M. C. Kim, Y. J. Lee, e M. Vauhkonen. Image reconstruction in time-varying electrical impedance tomography based on the extended kalman filter. Measurement Science and Technology, 12(8):1032-1039, august 2001.

[84] K. Y. Kim, S. I. Kang, M. C. Kim, S. Kim, Y.J. Lee, e M. Vauhkonen. Dynamic image reconstruction in electrical impedance tomography with known internal structure. IEEE Transactions on Magnetics, 38(2):1301-1304, march 2002.

[85] A. Papoulis. Probability, random variables, and stochastic processes. McGraw-Hill, New York, 3 edição, 1991.

[86] M. Vauhkonen, P. A. Karjalainen, e J. P. Kaipio. A kalman filter aproach to track fast impedance changes in electrical impedance tomography. IEEE Transactions on Biomedical Engineering, 45(4):486-493, april 1998.

[87] V. Kolehmainen, A. Voutilainen, e J. P. Kaipio. Estimation of non-stationary region boundaries in EIT-state estimation approach. Inverse Problems, 17(6):1937-1956, december 2001. doi: 10.1088/0266-5611/17/6/324.

[88] D. J. Ewins. Modal Testing: Theory and Practice. Research Studies Press LTD., New York, 1 edição, 1984.

[89] S. R. Ibrahim e E. C. Mikulcik. A time domain modal vibration test technique. Shock and Vibration Bulletin, 43(4):21-37, 1973.

[90] S. J. Julier e J. K. Uhlmann. A general method for approximating nonlinear transformations of probability distributions. Technical report, Department of Engineering Science,University of Oxford, Los Angeles, november 1996.

[91] L. Perea, J. How, L. Breger, e P. Elosegui. Nonlinearity in sensor fusion: Divergence issues in ekf, modified truncated sof, and ukf. In Proceedings of the AIAA Guidance Navigation and Control Conference and Exhibit, pages 1-16, august 2007.

[92] L. Perea. Design and evaluation of navigation and control algorithms for spacecraft formation flying missions. Tese de doutorado, Universitat de Barcelona, june 2010.

[93] L. Perea e P. Elosegui. New state update equation for the unscented kalman filter. Journal of Guidance, Control, and Dynamics, 31(5):1500-1504, september 2008. doi: 10.2514/1. 36824 . 
[94] W. F. Leven e A. D. Lanterman. Unscented kalman filters for multiple target tracking with symmetric measurement equations. IEEE Transactions on Automatic Control, 54(2): 370-375, february 2009. doi: 10.1109/TAC.2008.2008327.

[95] S. J. Julier. The scaled unscented transformation. 1999.

[96] S. J. Julier. The scaled unscented transformation. In Proceedings of the 2002 American Control Conference, pages 4555-4559, 2002.

[97] S. J. Julier e J. K. Uhlmann. Reduced sigma point filters for the propagation of means and covariances through nonlinear transformations. In Proceedings of the 2002 American Control Conference, pages 887-892, 2002.

[98] S. J. Julier. The spherical simplex unscented transformation. In Proceedings of the 2003 American Control Conference, pages 2430-2434, 2003. doi: 10.1109/ACC.2003.1243439.

[99] L. Padilla e C. W. Rowley. An adaptive-covariance-rank algorithm for the unscented kalman filter. In Proccedings of 49th IEEE Conference on Decision and Control, 2010.

[100] S. A. Banani e M. A. Masnadi-Shirazi. A new version of unscented kalman filter. World Academy Of Science, Engineering And Technology, 26:192-197, 2007.

[101] B. O. S. Teixeira, L. A. B. Tôrres, L. A. Aguirre, e D. S. Bernstein. On unscented kalman filtering with state interval constraints. Journal of Process Control, 20(1):45-57, january 2010.

[102] S. Haykin, G. V. Puskorius, L. A. Feldkamp, G. S. Patel, S. Becker, R. Racine, E. A. Wan, A. T. Nelson, S. T. Roweis, Z. Ghahramani, e R. Merwe. Kalman Filtering and Neural Networks. Wiley-Interscience Publications, 1 edição, 2001.

[103] E. A. Wan e R Merwe. The square-root unscented kalman filter for state and parameter-estimation. In Proceedings of International Conference on Acoustics, Speech, and Signal Processing, pages 3461-3464, 2001.

[104] J. Huttunen. Approximation and modelling errors in nonstationary inverse problems. Tese de doutorado, Department of Physics of University of Kuopio, Kuopio, january 2008.

[105] A. Lipponen, A. Seppanen, J. Hamalainen, e J. P. Kaipio. Nonstationary inversion of convection-diffusion problems - recovery from unknown nonstationary velocity fields. Inverse Problems and Imaging, 4(3):463-483, 2010.

[106] A. Lehikoinen, J. M. J. Huttunen, S. Finsterle, M. B. Kowalsky, e J. P. Kaipio. Dynamic inversion for hydrological process monitoring with electrical resistance tomography under model uncertainties. Water Resources Research, 46, 2010.

[107] L. Nelson e E. Stear. The simultaneous on-line estimation of parameters and states in linear systems. IEEE Transactions on Automatic Control, 21(1):94-98, february 1976.

[108] E. A. Wan e A. T. Nelson. Dual kalman filtering methods for nonlinear prediction, smoothing, and estimation. In Proceedings In Advances in Neural Information Processing Systems 9. Cambridge, 1997.

[109] A. Hegyi, D. Girimonte, R. Babuăka, e B. Schutter. A comparison of filter configurations for freeway traffic state estimation. In Proceedings of IEEE Intelligent Transportation Systems Conference (ITSC 2006), pages 1029-1034, september 2006. 
[110] E. A. Wan, R. Merwe, e A. T. Nelson. Dual estimation and the unscented transformation. In in Neural Information Processing Systems, pages 666-672. MIT Press, 2000.

[111] A. Nissinen, V. Kolehmainen, e J. P. Kaipio. Reconstruction of domain boundary and conductivity in electrical impedance tomography using the approximation error approach. International Journal for Uncertainty Quantification, 1(3):203-222, 2011. ISSN 2152-5080.

[112] A. Nissinen, V. Kolehmainen, e J. Kaipio. Compensation of modelling errors due to unknown domain boundary in electrical impedance tomography. IEEE Transaction on Medical Imaging, 30(2):231-242, 2011.

[113] A. Nissinen, L. M. Heikkinen, V. Kolehmainen, e J. P. Kaipio. Compensation of errors due to discretization, domain truncation and unknown contact impedances in electrical impedance tomography. Measurement Science and Technology, 20(10):105504, 2009.

[114] A. Nissinen. Modelling Errors in Electrical Impedance Tomography. Tese de doutorado, University of Eastern Finland, Kuopio, Finland, 2011.

[115] H. Gray. Anatomy of the human body. Lea \& Febiger, Philadelphia, 1 edição, 1918. URL http://www.bartleby.com/107/.

[116] B. Palsson, J. A. Hubbell, R. Plonsey, e J. D. Bronzino, editors. Tissue Engineering. Principles and Applications in Engineering Series. CRC Press, 2003.

[117] J. H. T. Bates. Lung Mechanics: An Inverse Modeling Approach. Cambridge University Press, 1 edição, 2009.

[118] C. H. Liu, S. C. Niranjan, Jr. Clark J. W., K. Y. San, J. B. Zwischenberger, e A. Bidani. Airway mechanics, gas exchange, and blood flow in a nonlinear model of the normal human lung. Journal of Applied Physiology, 84(4):1447-1469, 1998.

[119] J. H. T. Bates e C. G. Irvin. Time dependence of recruitment and derecruitment in the lung: a theoretical model. Journal of Applied Physiology, 93(2):705-713, 2002. doi: 10.1152/ japplphysiol.01274.2001.

[120] D. A. Kaminsky, C. G. Irvin, L. Lundblad, H. T. Moriya, S. Lang, J. Allen, T. Viola, M. Lynn, e J. H. T. Bates. Oscillation mechanics of the human lung periphery in asthma. Journal of Applied Physiology, 97:1849-1858, june 2004. doi: 10.1152/japplphysiol.00300. 2004.

[121] G. B. Allen, L. A. Pavone, J. D. DiRocco, J. H. T. Bates, e G. F. Nieman. Pulmonary impedance and alveolar instability during injurious ventilation in rats. Journal of Applied Physiology, 99(2):723-730, 2005. doi: 10.1152/japplphysiol.01339.2004.

[122] J. G. Venegas, R. S. Harris, e B. A. Simon. A comprehensive equation for the pulmonary pressure-volume curve. Journal of Applied Physiology, 84(1):389-395, january 1998.

[123] P. Nopp, N. D. Harris, T. X. Zhao, e B. H. Brown. Model for the dielectric properties of human lung tissue against frequency and air content. Medical and Biological Engineering and Computing, 35(6):695-702, november 1997.

[124] W. K. Pratt. Digital Image Processing. Wiley-Interscience, 4 edição, 2007.

[125] I. N. Bankman, editor. Handbook of Medical Imaging: Processing and Analysis Management. Academic Press Series in Biomedical Engineering. Academic Press, 1 edição, 2000. 
[126] J. S. Suri, D. Wilson, e S. Laxminarayan, editors. Handbook of Biomedical Image Analysis: Volume 1: Segmentation Models Part A, volume 1 of Topics in Biomedical Engineering. Springer, 2005.

[127] J. S. Suri, D. Wilson, e S. Laxminarayan, editors. Handbook of Biomedical Image Analysis: Volume 2: Segmentation Models Part B, volume 2 of Topics in Biomedical Engineering. Springer, 2005.

[128] J. S. Suri, D. Wilson, e S. Laxminarayan, editors. Handbook of Biomedical Image Analysis: Volume 3: Registration Models, volume 3 of Topics in Biomedical Engineering. Springer, 2005.

[129] T. S. Yoo, editor. Insight Into Images: Principles and Practice for Segmentation, Registration and Image Analysis. A K Peters, 1 edição, 2004.

[130] K. Fukunaga. Introduction to Statistical Pattern Recognition. Computer Science and Scientific Computing Series. Academic Press, 2 edição, 1990.

[131] R. O. Duda, P. E. Hart, e D. G. Stork. Pattern Classification. Wiley-Interscience, 2 edição, 2000.

[132] S. Theodoridis e K. Koutroumbas. Pattern Recognition. Academic Press, 4 edição, 2009.

[133] C. Li, C. Xu, C. Gui, e M. D. Fox. Level set evolution without re-initialization: A new variational formulation. In Proceedings of the 2005 IEEE Computer Society Conference on Computer Vision and Pattern Recognition, volume 1, pages 430-436, june 2005. doi: 10. 1109/CVPR.2005.213.

[134] R. C. Gonzalez e R. E. Woods. Digital Image Processing. Prentice-Hall, Englewood Clifs, 2 edição, 2002.

[135] T. J. Yorkey, J. G. Webster, e W. J. Tompkins. Comparing reconstruction algorithms for electrical impedance tomography. IEEE Transactions on Biomedical Engineering, 34(11): 843-852, november 1987.

[136] O. C. Zienkiewicz, R. L. Taylor, e J. Z. Zhu. The Finite Element Method: Its Basis E Fundamentals, volume 1. Butterworth-Heinemann, 6 edição, 2005.

[137] D. L. Logan. A First Course in the Finite Element Method. PWS-KENT Publishing Company, Boston, 1986.

[138] G. W. Stewart. Matrix Algorithms: Basic Decompositions, volume 1. SIAM, Philadelphia, 1998.

[139] G. H. Golub e C. F. V. Loan. Matrix Computations. The Johns Hopkins University Press, 3 edição, 1996.

[140] S. Osher e R. Fedkiw. Level Set Methods and Dynamic Implicit Surfaces. Springer, New York, 1 edição, 2003.

[141] S. Osher e N. Paragios. Geometric Level Set Methods in Imaging,Vision, and Graphics. Springer-Verlag New York, Inc., Secaucus, NJ, USA, 1 edição, 2003. ISBN 0387954880.

[142] J. A. Sethian. Level Set Methods and Fast Marching Methods - Evolving interfaces in computational geometry, fluid mechanics, computer vision, and material science. Cambridge monographs on applied and computational mathematics. Cambridge University Press, 2 edição, 1999. 
[143] P. Cordaro e A. Kawano. O Delta de Dirac. Editora Livraria da Física, São Paulo, 1 edição, 2002. 